\title{
Chemo- and Regioselective Direct Functional Group Installation through Catalytic Hydroxy Group-Selective Conjugate Addition of Amino Alcohols to $\alpha, \beta$ - Unsaturated Sulfonyl Compounds
}

\author{
Zhao Li, Ryo Yazaki* and Takashi Ohshima* \\ Graduate School of Pharmaceutical Sciences, Kyushu University, Fukuoka, Japan \\ yazaki@phar.kyushu-u.ac.jp, ohshima@phar.kyushu-u.ac.jp
}

\author{
1. General \\ 2. Instrumentation \\ 3. Materials \\ 4. Substrate Syntheses and Characterization \\ 5. General Procedure and Characterization of the Products \\ 6. Proposed Catalytic Cycle \\ 7. Reference \\ 8. NMR Spectra of New Compounds
}

\section{General}

All reactions were carried out using heat gun dried glassware under a positive pressure of dry argon unless otherwise noted. The test tubes were fitted with a 3-way glass stopcock and catalytic reactions 
were run under argon atmosphere. Air- and moisture-sensitive liquids were transferred via a syringe and a stainless-steel needle. Reactions were magnetically stirred and monitored by thin layer chromatography using Merck Silica Gel 60 F254 plates. All work-up and purification procedures were carried out with reagent-grade solvents under ambient atmosphere. Flash chromatography was performed using silica gel 60N (spherical neutral, particle size $40-50 \mu \mathrm{m}$ ) purchased from Kanto Chemical Co. Ltd.

\section{Instrumentation}

NMR was recorded on $400 \mathrm{MHz}$ Varian Unity instruments and $500 \mathrm{MHz}$ Bruker Advanced III. Chemical shifts for proton are reported in parts per million downfield from tetramethylsilane and are referenced to residual protium in the $\mathrm{NMR}$ solvent $\left(\mathrm{CDCl}_{3}: \delta 7.26 \mathrm{ppm}\right)$. For ${ }^{13} \mathrm{C} \mathrm{NMR}$, chemical shifts were reported in the scale relative to $\mathrm{NMR}$ solvent $\left(\mathrm{CDCl}_{3}: 77.0 \mathrm{ppm} ; \mathrm{CD}_{3} \mathrm{OD}: 49.0 \mathrm{ppm}\right)$ as an internal reference. NMR data are reported as follows: chemical shifts, multiplicity (s: singlet, d: doublet, dd: doublet of doublets, t: triplet, q: quartet, sep: septet, m: multiplet, br: broad signal), coupling constant $(\mathrm{Hz})$, and integration. Infrared (IR) spectra were recorded on with Shimadzu FTIR-8400. High-resolution mass spectroscopy (HRMS) was obtained with Waters ACQUITY UPLC ${ }^{\circledR}-$ LCT-Premier $^{\text {TM }}$ XE system and Bruker MicrOTOF II. Optical rotation was measured with JASCO P2200 polarimeter.

\section{Materials}

DMF ( $N, N$-dimethylformamide), DMA (dimethylacetamide), DMPU ( $N, N$ '-dimethylpropyleneurea), DMSO (dimethyl sulfoxide) and NMP ( $\mathrm{N}$-methylpyrrolidone) were distilled from $\mathrm{CaH}_{2}$ and stored in schlenk flask. Cu(MeSal), LiHMDS, KHMDS and NaHMDS were purchased from Aldrich and used as received. ${ }^{n} \mathrm{BuLi}$ in $n$-hexane (1.6 M), ethylenebis(diphenylphosphine) (dppe) and $\mathrm{Boc}_{2} \mathrm{O}$ were purchased from Kanto Chemical Co. Ltd. and used as received. CuOAc and commercially available amino alcohols were purchased from $\mathrm{TCl}$ and used as received. Hydroxychloroquine was purchased from $\mathrm{TCl}$ as salt, which were extracted with chloroform from $2.0 \mathrm{M} \mathrm{NaOH}$ solution and purified with column chromatography $\left(1 / 2 / 20={ }^{i} \mathrm{PrNH}_{2} / \mathrm{MeOH} / \mathrm{CH}_{2} \mathrm{Cl}_{2}\right)$. Ambroxol was also purchased from $\mathrm{TCl}$ as salt, which were extracted with chloroform from $2.0 \mathrm{M} \mathrm{NaOH}$ solution and purified with column chromatography $(1 / 20=$ $\mathrm{MeOH} / \mathrm{CH}_{2} \mathrm{Cl}_{2}$ ). AgOAc, Sodium azide, THF (Super Dehydrated, Stabilizer Free) and palladium on carbon (10\%) were purchased from Wako Pure Chemical Industries, Ltd. and used as received. All other 
commercially available reagents were used without further purification. (vinylsulfonyl)benzene ${ }^{1}$ (1a), 1bromo-4-(vinylsulfonyl)benzene ${ }^{2}$ (1) $), 2$-(vinylsulfonyl)pyridine ${ }^{3}$ (1c), 2-(benzylamino)ethanol ${ }^{4}$ (2k), (4amino-3-iodophenyl)methanol $^{5}(\mathbf{2 o})$, 1-(4-aminophenyl)ethan-1-ol ${ }^{6}(\mathbf{2} \mathbf{p})$, racemic propranolol ${ }^{7}(\mathbf{2 q})$ and 2-aminopentane-1,5-diol ${ }^{8}(\mathbf{2 w})$ were prepared according to the literature. 2-aminopentane-1,5-diol (2w) was purified with column chromatography (1/2/8 $\left.={ }^{i} \mathrm{PrNH}_{2} / \mathrm{MeOH} / \mathrm{CH}_{2} \mathrm{Cl}_{2}\right)$. Amino group derived products were prepared from corresponding amines with $\alpha, \beta$-unsaturated sulfonyl compounds in EtOH at room temperature or reflux conditions ${ }^{9}$.

\section{Substrate Syntheses and Characterization}

\section{General procedure for synthesis of unsaturated sulfonamide ${ }^{10}$}

2-Chloroethanesulfonyl chloride (1.0 equiv.) was added drop wise to a stirred solution of secondary amine (1.0 equiv) with $\mathrm{Et}_{3} \mathrm{~N}$ (3.5 equiv) in $\mathrm{CH}_{2} \mathrm{Cl}_{2}$ at $0{ }^{\circ} \mathrm{C}$. After addition, stirring was continued at $0{ }^{\circ} \mathrm{C}$ overnight. The mixture then was diluted with $\mathrm{CH}_{2} \mathrm{Cl}_{2}$, then washed with brine, dried over $\mathrm{MgSO}_{4}$, and concentrated. Crude mixture was purified by silica gel column chromatography to give the desired product. If the product was solid, the product was further purified by recrystallization. If the amine hydrochloride was used, the 4.5 equivalents of $\mathrm{Et}_{3} \mathrm{~N}$ were used. Compounds $\mathbf{1 d}, \mathbf{1 e}, \mathbf{1} \mathbf{f}, \mathbf{1} \mathbf{g}, \mathbf{1} \mathbf{h}, \mathbf{1 i}, \mathbf{1} \mathbf{j}$ and 1l were also synthesized according to the same procedure and the corresponding amine used for synthesis of $\mathbf{1} \mathbf{d}^{11}$ and $\mathbf{1} \mathbf{l}^{12}$ were prepared according to the literature.

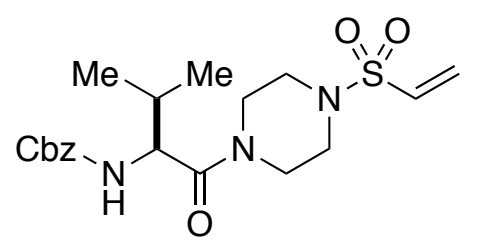

To a solution of Z-L-Val-OH (3.60 g, $15 \mathrm{mmol})$ in DMF (15 ml) was added EDC (3.45 g, $18 \mathrm{mmol})$ and HOBt $(2.00 \mathrm{~g}, 15 \mathrm{mmo})$ followed by tert-butyl piperazine-1-carboxylate $(2.79 \mathrm{ml}, 15 \mathrm{mmol})$ and NMM (3.30 ml, $30 \mathrm{mmol}$ ) at $0^{\circ} \mathrm{C}$. After $1 \mathrm{~h}$ stirring at $0^{\circ} \mathrm{C}$, ice bath was removed. After stirring for $24 \mathrm{~h}$ at room temperature, it was quenched by $1 \mathrm{~N} \mathrm{HCl}$. The resultant mixture was extracted with EtOAc and combined organic layers were washed with sat. $\mathrm{NaHCO}_{3}$ solution and brine successively. The resulting organic layers were dried over $\mathrm{Na}_{2} \mathrm{SO}_{4}$. After removal of solvent under reduced pressure, corresponding amide was obtained as white foam $(5.76 \mathrm{~g}, 96 \%$ yield), which was used for next step without further 
purification. To a flask with amide $(5.76 \mathrm{~g}, 14.4 \mathrm{mmol})$ was added $20 \%$ TFA in $\mathrm{CH}_{2} \mathrm{Cl}_{2}(125 \mathrm{~mL})$ and the reaction mixture was stirred under argon atmosphere overnight. Evaporation of the organic solvent under reduced pressure gave the trifluoroacetate salt as yellow oil in quantitative yield, which was used for the next step without further purification. A solution of above trifluoroacetate salt in $\mathrm{CH}_{2} \mathrm{Cl}_{2}(100.0$ $\mathrm{mL}$ ) was washed with saturated aqueous $\mathrm{Na}_{2} \mathrm{CO}_{3}$ solution $(100.0 \mathrm{~mL})$, and the aqueous phase was extracted with $\mathrm{CH}_{2} \mathrm{Cl}_{2}(2 \times 50.0 \mathrm{~mL})$. The organic phase was combined, dried over $\mathrm{Na}_{2} \mathrm{SO}_{4}$ and concentrated to give the amine as yellow oil. A solution of above amine and $\mathrm{Et}_{3} \mathrm{~N}$ (15 mL, $107.8 \mathrm{mmol}$ ) in $\mathrm{CH}_{2} \mathrm{Cl}_{2}(100.0 \mathrm{~mL})$ was cooled to $0{ }^{\circ} \mathrm{C}$ and treated with 2-chloroethanesulfonyl chloride ( $\left.3 \mathrm{~mL}, 28.3 \mathrm{mmol}\right)$ and stirred at the same temperature overnight. The solution was washed with saturated aqueous $\mathrm{NH}_{4} \mathrm{Cl}$ solution $(100.0 \mathrm{~mL})$ and the aqueous phase was extracted with $\mathrm{CH}_{2} \mathrm{Cl}_{2}(2 \times 50.0 \mathrm{~mL})$. The organic phase was combined, dried over $\mathrm{Na}_{2} \mathrm{SO}_{4}$ and concentrated. Flash chromatography of the residue on silica gel (ethyl acetate : $n$-hexanes $=1: 6$ to $2: 1$ ) afforded benzyl (S)-(3-methyl-1-oxo-1-(4(vinylsulfonyl)piperazin-1-yl)butan-2-yl)carbamate (1j) (2.50 g, 44\% yield) as a pale yellow foam.

benzyl (S)-(3-methyl-1-oxo-1-(4-(vinylsulfonyl)piperazin-1-yl)butan-2-yl)carbamate (1j): (pale yellow foam); ${ }^{1} \mathrm{H}-\mathrm{NMR}\left(500 \mathrm{MHz}, \mathrm{CDCl}_{3}\right)(\mathrm{ppm}) \delta 7.40-7.30(\mathrm{~m}, 5 \mathrm{H}, \mathrm{ArH}), 6.39$ (dd, $1 \mathrm{H}, J=10.0,16.5 \mathrm{~Hz}$, $\mathrm{SO}_{2} \mathrm{CHCH}_{2}$ ), $6.27\left(\mathrm{~d}, 1 \mathrm{H}, J=16.5 \mathrm{~Hz}, \mathrm{SO}_{2} \mathrm{CHCH}_{2}\right.$ ), 6.08 (d, $1 \mathrm{H}, J=10.0 \mathrm{~Hz}, \mathrm{SO}_{2} \mathrm{CHCH}_{2}$ ), $5.51(\mathrm{~d}, 1 \mathrm{H}, J=9.0$ $\mathrm{Hz}, \mathrm{NH}), 5.09\left(\mathrm{~s}, 2 \mathrm{H}, \mathrm{PhCH}_{2} \mathrm{O}\right), 4.47$ (dd, $\left.1 \mathrm{H}, \mathrm{J}=6.0,9.0 \mathrm{~Hz}, \mathrm{NHCHCO}\right), 4.03-3.93\left(\mathrm{~m}, 1 \mathrm{H}, \mathrm{N}\left(\mathrm{CH}_{2} \mathrm{CH}_{2}\right)_{2} \mathrm{~N}\right)$, 3.80-3.72 (m, $\left.1 \mathrm{H}, \mathrm{N}\left(\mathrm{CH}_{2} \mathrm{CH}_{2}\right)_{2} \mathrm{~N}\right)$, 3.62-3.44 (m, 2H, N( $\left.\left.\mathrm{CH}_{2} \mathrm{CH}_{2}\right)_{2} \mathrm{~N}\right), 3.35-3.22\left(\mathrm{~m}, 2 \mathrm{H}, \mathrm{N}\left(\mathrm{CH}_{2} \mathrm{CH}_{2}\right)_{2} \mathrm{~N}\right)$, 3.15$2.98\left(\mathrm{~m}, 2 \mathrm{H}, \mathrm{N}\left(\mathrm{CH}_{2} \mathrm{CH}_{2}\right)_{2} \mathrm{~N}\right), 2.00-1.86\left(\mathrm{~m}, 1 \mathrm{H}, \mathrm{CH}\left(\mathrm{CH}_{3}\right)_{2}\right), 0.97\left(\mathrm{~d}, 3 \mathrm{H}, J=6.5 \mathrm{~Hz}, \mathrm{CH}\left(\mathrm{CH}_{3}\right)_{2}\right), 0.90(\mathrm{~d}, 3 \mathrm{H}, J=$ $\left.6.5 \mathrm{~Hz}, \mathrm{CH}\left(\mathrm{CH}_{3}\right)_{2}\right) ;{ }^{13} \mathrm{C} \mathrm{NMR}\left(125 \mathrm{MHz}, \mathrm{CDCl}_{3}\right) \delta 170.7,156.6,136.4,132.2,129.8,128.7,128.3,128.1$, 67.1, 55.5, 45.9, 45.6, 45.5, 41.7, 31.6, 19.7, 17.3; IR (KBr) 3034, 2933, 1716, 1348, $1159 \mathrm{~cm}^{-1}$; HRMS $(\mathrm{ESI}, \mathrm{H}) \mathrm{m} / z$ calc'd for $\mathrm{C}_{19} \mathrm{H}_{28} \mathrm{~N}_{3} \mathrm{O}_{5} \mathrm{~S}(\mathrm{M}+\mathrm{H})^{+} 410.1744$, found $410.1748 .[\alpha]_{\mathrm{D}}{ }^{25}+29.8\left(c 1.00, \mathrm{CHCl}_{3}\right)$.

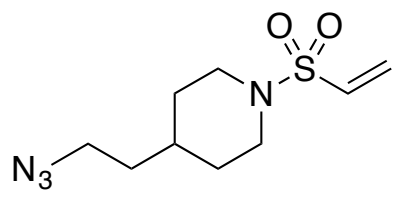

The synthesis of compound $\mathbf{1} \mathbf{k}$ was prepared according to the literature. ${ }^{10,13} \mathbf{A}$ solution of 4 piperidineethanol $(1.30 \mathrm{~g}, 10.0 \mathrm{mmol})$ was dissolved in $\mathrm{CH}_{2} \mathrm{Cl}_{2}(25.0 \mathrm{~mL})$ and treated with di-tert-butyl dicarbonate $(2.22 \mathrm{~g}, 10.0 \mathrm{mmol})$ at $0{ }^{\circ} \mathrm{C}$. The solution was warmed to room temperature and stirred for $1.5 \mathrm{~h}$. The solution was then washed with water, $10 \% \mathrm{KHSO}_{4}$, and brine, dried over $\mathrm{Na}_{2} \mathrm{SO}_{4}$, filtered, and concentrated to give 2-( $N$-Boc-piperidin-4-yl)ethanol $(2.15 \mathrm{~g}, 98 \%)$ as a colorless oil, which was used for 
the next step without further purification. A solution of the above alcohol $(1.19 \mathrm{~g}, 5.46 \mathrm{mmol})$ in $23.0 \mathrm{~mL}$ of toluene was treated with imidazole $(530.6 \mathrm{mg}, 7.74 \mathrm{mmol})$, triphenylphosphine $(1.74 \mathrm{~g}, 5.69 \mathrm{mmol})$, and iodine $(1.39 \mathrm{~g}, 5.46 \mathrm{mmol})$. The resulting mixture was heated at $80{ }^{\circ} \mathrm{C}$ for $1 \mathrm{~h}$, then filtered, and concentrated. Flash chromatography of the residue on silica gel (ethyl acetate : $n$-hexanes $=1: 4$ ) afforded 2-(N-Boc-piperidin-4-yl)ethyl lodide $(1.31 \mathrm{~g}, 75 \%)$ as a colorless oil. A solution of above iodide $(1.45 \mathrm{~g}, 4.28 \mathrm{mmol})$ in DMSO $(14.0 \mathrm{~mL})$ was treated with $\mathrm{NaN}_{3}(553.0 \mathrm{mg}, 9.56 \mathrm{mmol})$ and stirred at room temperature for $18 \mathrm{~h}$. The reaction mixture was diluted with ethyl acetate $(25.0 \mathrm{~mL})$, washed with $\mathrm{H}_{2} \mathrm{O}(4 \times 10.0 \mathrm{~mL})$ and brine $(10.0 \mathrm{~mL})$, then dried over $\mathrm{Na}_{2} \mathrm{SO}_{4}$, filtered, and concentrated to give the azide $(1.06 \mathrm{~g}, 97 \%)$ as colorless oil, which was used for the next step without further purification. A solution of above azide $(490.4 \mathrm{mg}, 1.92 \mathrm{mmol})$ in $\mathrm{CH}_{2} \mathrm{Cl}_{2}(25.0 \mathrm{~mL})$ was cooled to $0{ }^{\circ} \mathrm{C}$, and treated with TFA $(2.5 \mathrm{~mL})$. The solution was warmed to room temperature and stirred overnight. Evaporation of the organic solvent under reduced pressure provided the trifluoroacetate salt as yellow oil in quantitative yield, which was used for the next step without further purification. A solution of above trifluoroacetate salt $(545.5 \mathrm{mg}, 2.03 \mathrm{mmol})$ in $\mathrm{CH}_{2} \mathrm{Cl}_{2}(10.0 \mathrm{~mL})$ was washed with saturated aqueous $\mathrm{Na}_{2} \mathrm{CO}_{3}$ solution (10.0 mL) and the aqueous phase was extracted with $\mathrm{CH}_{2} \mathrm{Cl}_{2}(2 \times 10.0 \mathrm{~mL})$. The organic phase was combined, dried over $\mathrm{Na}_{2} \mathrm{SO}_{4}$ and concentrated to give the amine as yellow oil. A solution of above amine and $\mathrm{Et}_{3} \mathrm{~N}(650.0 \mu \mathrm{L}, 4.67 \mathrm{mmol})$ in $\mathrm{CH}_{2} \mathrm{Cl}_{2}(6.0 \mathrm{~mL})$ was cooled to $-20{ }^{\circ} \mathrm{C}$ and treated with 2chloroethanesulfonyl chloride $(215 \mu \mathrm{L}, 2.03 \mathrm{mmol})$ and stirred the same temperature for 30 minutes. The solution was warmed to room temperature slowly and stirred for $4 \mathrm{~h}$. The solution was washed with saturated aqueous $\mathrm{NH}_{4} \mathrm{Cl}$ solution $(10.0 \mathrm{~mL})$ and the aqueous phase was extracted with $\mathrm{CH}_{2} \mathrm{Cl}_{2}(2 \times 10.0$ $\mathrm{mL}$ ). The organic phase was combined, dried over $\mathrm{Na}_{2} \mathrm{SO}_{4}$ and concentrated. Flash chromatography of the residue on silica gel (ethyl acetate $: n$-hexanes $=1: 6$ to $1: 3$ ) afforded 4-(2-azidoethyl)-1(vinylsulfonyl)piperidine (1k) $(246.9 \mathrm{mg}, 50 \%$ yield) as a yellow oil.

4-(2-azidoethyl)-1-(vinylsulfonyl)piperidine (1k): (pale yellow oil); ${ }^{1} \mathrm{H}-\mathrm{NMR}\left(500 \mathrm{MHz}, \mathrm{CDCl}_{3}\right)(\mathrm{ppm}) \delta$ $6.42\left(\mathrm{dd}, 1 \mathrm{H}, J=10.0,16.5 \mathrm{~Hz}, \mathrm{CH}_{2} \mathrm{CHSO}_{2}\right.$ ), 6.23 (dd, $1 \mathrm{H}, J=16.5 \mathrm{~Hz}, \mathrm{CH}_{2} \mathrm{CHSO}_{2}$ ), 6.01 (dd, $1 \mathrm{H}, J=10.0 \mathrm{~Hz}$, $\mathrm{CH}_{2} \mathrm{CHSO}_{2}$ ), 3.82-3.68 (m, $\left.2 \mathrm{H}, \mathrm{N}\left(\mathrm{CH}_{2} \mathrm{CH}_{2}\right)_{2} \mathrm{CH}\right), 3.44$ (t, $\left.2 \mathrm{H}, J=6.5 \mathrm{~Hz}, \mathrm{CH}_{2} \mathrm{~N}_{3}\right), 2.59$ (td, $2 \mathrm{H}, J=2.5,12.0 \mathrm{~Hz}$, $\left.\mathrm{N}\left(\mathrm{CH}_{2} \mathrm{CH}_{2}\right)_{2} \mathrm{CH}\right), 1.86-1.73\left(\mathrm{~m}, 2 \mathrm{H}, \mathrm{N}\left(\mathrm{CH}_{2} \mathrm{CH}_{2}\right)_{2} \mathrm{CH}\right), 1.63-1.42\left(\mathrm{~m}, 3 \mathrm{H}, \mathrm{N}\left(\mathrm{CH}_{2} \mathrm{CH}_{2}\right)_{2} \mathrm{CH}\right), 1.38-1.28(\mathrm{~m}, 2 \mathrm{H}$, $\left.\mathrm{CH}_{2} \mathrm{CH}_{2} \mathrm{~N}_{3}\right) ;{ }^{13} \mathrm{C}$ NMR $\left(125 \mathrm{MHz}, \mathrm{CDCl}_{3}\right) \delta 132.8,128.4,48.8,46.0,35.0,32.8,31.5$; IR (thin film, $\mathrm{NaCl}$ ) 3107, 2096, 1635, 1336, $935 \mathrm{~cm}^{-1}$; HRMS (ESI, H) $\mathrm{m} / z$ calc'd for $\mathrm{C}_{9} \mathrm{H}_{17} \mathrm{~N}_{4} \mathrm{O}_{2} \mathrm{~S}(\mathrm{M}+\mathrm{H})^{+} 245.1067$, found 245.2076. 
<smiles>C=CS(=O)(=O)N1CCN(S(=O)(=O)c2cccc3c(NC)cccc23)CC1</smiles>

N,N-dimethyl-5-((4-(vinylsulfonyl)piperazin-1-yl)sulfonyl)naphthalen-1-amine (11): (pale green solid, EtOAc:Hexane = 2:1, 50\% yield, $592.0 \mathrm{mg}) ;{ }^{1} \mathrm{H}-\mathrm{NMR}\left(500 \mathrm{MHz}, \mathrm{CDCl}_{3}\right)(\mathrm{ppm}) \delta 8.59(\mathrm{~d}, 1 \mathrm{H}, J=8.5 \mathrm{~Hz}$, $\operatorname{ArH}), 8.33(\mathrm{~d}, 1 \mathrm{H}, J=9.0 \mathrm{~Hz}, \mathrm{ArH}), 8.19(\mathrm{dd}, 1 \mathrm{H}, J=1.5,7.5 \mathrm{~Hz}, \mathrm{ArH}) 7.56-7.50(\mathrm{~m}, 2 \mathrm{H}, \operatorname{ArH}), 7.20(\mathrm{~d}, 1 \mathrm{H}, J$ $=7.5 \mathrm{~Hz}, \mathrm{ArH}), 6.36\left(\mathrm{dd}, 1 \mathrm{H}, J=10.0,16.5 \mathrm{~Hz}, \mathrm{SO}_{2} \mathrm{CHCH}_{2}\right.$ ), $6.22\left(\mathrm{~d}, 1 \mathrm{H}, J=16.5 \mathrm{~Hz}, \mathrm{SO}_{2} \mathrm{CHCH}_{2}\right.$ ), 6.05 (d, $\left.1 \mathrm{H}, J=10.0 \mathrm{~Hz}, \mathrm{SO}_{2} \mathrm{CHCH}_{2}\right), 3.31\left(\mathrm{t}, 4 \mathrm{H}, \mathrm{J}=5.0 \mathrm{~Hz}, \mathrm{~N}\left(\mathrm{CH}_{2} \mathrm{CH}_{2}\right)_{2} \mathrm{~N}\right), 3.21\left(\mathrm{t}, 4 \mathrm{H}, \mathrm{J}=5.0 \mathrm{~Hz}, \mathrm{~N}\left(\mathrm{CH}_{2} \mathrm{CH}_{2}\right)_{2} \mathrm{~N}\right)$, $2.90\left(\mathrm{~s}, 6 \mathrm{H}, \mathrm{N}\left(\mathrm{CH}_{3}\right)_{2}\right) ;{ }^{13} \mathrm{C} \mathrm{NMR}\left(125 \mathrm{MHz}, \mathrm{CDCl}_{3}\right) \delta 152.1,132.4,132.3,131.3,130.9,130.4,130.3,129.6$, $128.5,123.3,119.4,115.5,45.6,45.4,45.4$; IR (KBr) 3053, 1867, 1454, 1336, 941 $\mathrm{cm}^{-1}$; HRMS (ESI, H) m/z calc'd for $\mathrm{C}_{18} \mathrm{H}_{24} \mathrm{~N}_{3} \mathrm{O}_{4} \mathrm{~S}_{2}^{+}(\mathrm{M}+\mathrm{H})^{+} 410.1203$, found 410.1206 .

\section{General Procedure and Characterization of the Products}

\section{5-1. General procedure for catalytic chemoselective conjugate addition reaction:}

A flame-dried $20 \mathrm{~mL}$ test tube equipped with a magnetic stirring bar and 3-way glass stopcock was charged with AgOAc $(4.0 \mathrm{mg}, 24.0 \mu \mathrm{mol})$ and dppe $(9.6 \mathrm{mg}, 24.1 \mu \mathrm{mol})$ and $2(0.96 \mathrm{mmol})$. To the test tube was added distilled DMF $(0.8 \mathrm{~mL})$ via syringe. After stirred at room temperature for about 10 minutes, the resulting mixture was stirred at $-20{ }^{\circ} \mathrm{C}$. Then KHMDS solution $(32.0 \mu \mathrm{l}, 1.0 \mathrm{M}$ in THF, 32 $\mu \mathrm{mol})$, which was prepared freshly in Glove Box using KHMDS solid and deoxidized THF, was added followed by the addition of solution of $1(0.8 \mathrm{mmol}$ in $0.8 \mathrm{~mL} \mathrm{DMF})$. The resulting solution was stirred at the same temperature for 30 min under Ar (note: efficient stirring was important.) and diluted with $\mathrm{CH}_{2} \mathrm{Cl}_{2} / \mathrm{MeOH}(4 / 1)$ solution. The diluted solution was filtered through silica short column and washed with $\mathrm{CH}_{2} \mathrm{Cl}_{2} / \mathrm{MeOH}$ (4/1) (ca. $80 \mathrm{ml}$ ). After evaporation of the organic solvent under reduced pressure, $\mathrm{O} / \mathrm{N}$ product ratio and yield were determined by ${ }^{1} \mathrm{H}-\mathrm{NMR}$ analysis. Then the crude mixture was purified by silica gel column chromatography to give the desired product.

\section{Catalytic chemoselective conjugate addition reaction using AgHMDS}

\section{Reaction conditions}

1a $(0.8 \mathrm{mmol})$ in DMF $(0.8 \mathrm{~mL}), 2 \mathrm{a}(0.96 \mathrm{mmol}), \mathrm{DMF}(0.8 \mathrm{~mL}),-20{ }^{\circ} \mathrm{C}, 0.5 \mathrm{~h}, \mathrm{AgHMDS} / \mathrm{dppe}(120.0$ $\mu \mathrm{mol})$. 
AgHMDS used for catalytic reaction was prepared according to the literature ${ }^{11}$ and stored in Glove Box. Elemental analysis of obtained AgHMDS $\left(\mathrm{C}_{6} \mathrm{H}_{18} \mathrm{AgNSi}_{2}\right)$ : calcd. C 26.86, H 6.76, N, 5.22; found C 26.76, H 6.75, N 5.19.

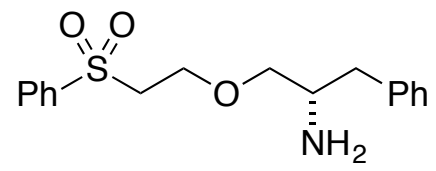

\section{Reaction conditions}

1a $(0.8 \mathrm{mmol})$ in $0.8 \mathrm{~mL} \mathrm{DMF}, 2 \mathrm{a}(0.96 \mathrm{mmol}), \mathrm{DMF}(0.8 \mathrm{~mL}),-20^{\circ} \mathrm{C}, 0.5 \mathrm{~h}, \mathrm{AgOAc} / \mathrm{dppe}(24.0 \mu \mathrm{mol})$, KHMDS $(32 \mu \mathrm{mol})$.

(S)-1-phenyl-3-(2-(phenylsulfonyl)ethoxy)propan-2-amine (3aa): (pale yellow oil, $\mathrm{CH}_{2} \mathrm{Cl}_{2} / \mathrm{MeOH}=10 / 1$, $80 \%$ yield, $204.0 \mathrm{mg}) ;{ }^{1} \mathrm{H}-\mathrm{NMR}\left(500 \mathrm{MHz}, \mathrm{CDCl}_{3}\right)(\mathrm{ppm}) \delta$ 7.98-7.88 (m, 2H, $\left.\mathrm{ArH}\right), 7.68-7.50(\mathrm{~m}, 3 \mathrm{H}, \mathrm{ArH})$, 7.33-7.18 (m, $3 \mathrm{H}, \mathrm{ArH})$, 7.16-7.08 (m, $2 \mathrm{H}, \mathrm{ArH}), 3.86-3.76\left(\mathrm{~m}, 2 \mathrm{H}, \mathrm{SO}_{2} \mathrm{CH}_{2} \mathrm{CH}_{2} \mathrm{O}\right), 3.42(\mathrm{t}, 2 \mathrm{H}, J=6.0 \mathrm{~Hz}$, $\mathrm{OCH}_{2} \mathrm{CHNH}_{2}$ ), 3.30 (dd, $1 \mathrm{H}, J=4.0,9.0 \mathrm{~Hz}, \mathrm{SO}_{2} \mathrm{CH}_{2} \mathrm{CH}_{2} \mathrm{O}$ ), 3.15 (dd, $1 \mathrm{H}, J=7.0,9.0 \mathrm{~Hz}, \mathrm{SO}_{2} \mathrm{CH}_{2} \mathrm{CH}_{2} \mathrm{O}$ ), 3.05$2.97\left(\mathrm{~m}, 1 \mathrm{H}, \mathrm{OCH}_{2} \mathrm{CHNH}_{2}\right.$ ), 2.60 (dd, $1 \mathrm{H}, J=5.5,13.5 \mathrm{~Hz}, \mathrm{CH}_{2} \mathrm{Ph}$ ), 2.42 (dd, $1 \mathrm{H}, J=8.0,13.5 \mathrm{~Hz}, \mathrm{CH}_{2} \mathrm{Ph}$ ), $1.23\left(\mathrm{br}, 2 \mathrm{H}, \mathrm{NH}_{2}\right) ;{ }^{13} \mathrm{C} \mathrm{NMR}\left(125 \mathrm{MHz}, \mathrm{CDCl}_{3}\right) \delta 140.1,138.7,133.8,129.3$ (overlapped), 128.6, 128.1, 126.5, 75.9, 64.6, 56.4, 52.2, 40.7; R (thin film, NaCl) 3373, 2918, 1359, 1178, $833 \mathrm{~cm}^{-1}$; HRMS (ESI, H) $\mathrm{m} / \mathrm{z}$ calc'd for $\mathrm{C}_{17} \mathrm{H}_{22} \mathrm{~N}_{2} \mathrm{O}_{3} \mathrm{~S}(\mathrm{M}+\mathrm{H})^{+} 320.1315$, found 320.1311. $[\alpha]_{D}{ }^{25}-4.1$ (c 1.00, $\left.\mathrm{CHCl}_{3}\right)$.

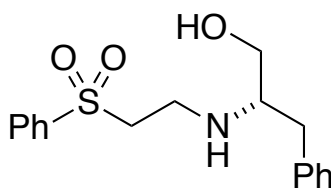

(S)-3-phenyl-2-((2-(phenylsulfonyl)ethyl)amino)propan-1-ol (4aa): (white solid); ${ }^{1} \mathrm{H}-\mathrm{NMR}$ (500 MHz, $\left.\mathrm{CDCl}_{3}\right)(\mathrm{ppm}) \delta$ 7.95-7.75 (m, 2H, ArH), 7.69-7.63 (m, 1H, ArH), 7.60-7.52 (m, 2H, ArH), 7.34-7.20 (m, 3H, ArH), 7.19-7.13 (m, 2H, ArH), $3.57\left(\mathrm{dd}, 1 \mathrm{H}, J=4.0,11.0 \mathrm{~Hz}, \mathrm{HOCH}_{2}\right), 3.33-3.13\left(\mathrm{~m}, 3 \mathrm{H}, \mathrm{HOCH}_{2}\right.$, $\left.\mathrm{SO}_{2} \mathrm{CH}_{2} \mathrm{CH}_{2} \mathrm{NH}\right)$, 3.08-3.00 (m, $\left.1 \mathrm{H}, \mathrm{SO}_{2} \mathrm{CH}_{2} \mathrm{CH}_{2} \mathrm{NH}\right)$, 2.98-2.90 (m, $\left.1 \mathrm{H}, \mathrm{SO}_{2} \mathrm{CH}_{2} \mathrm{CH}_{2} \mathrm{NH}\right), 2.88-2.80(\mathrm{~m}, 1 \mathrm{H}$, $\left.\mathrm{HOCH}_{2} \mathrm{CH}\right), 2.71\left(\mathrm{~d}, 2 \mathrm{H}, \mathrm{J}=7.0 \mathrm{~Hz}, \mathrm{CH}_{2} \mathrm{Ph}\right), 2.53(\mathrm{br}, 1 \mathrm{H}, \mathrm{NH}), 1.55(\mathrm{br}, 1 \mathrm{H}, \mathrm{OH}) ;{ }^{13} \mathrm{C} \mathrm{NMR}\left(125 \mathrm{MHz}, \mathrm{CDCl}_{3}\right)$ $\delta 139.4,138.3,134.0,129.5,129.3,128.8,128.1,126.7,62.8,60.6,56.7,40.9,38.3$; IR (KBr) 3525, 1344, 1124, 1080, $1062 \mathrm{~cm}^{-1}$; HRMS (ESI, H) m/z calc'd for $\mathrm{C}_{17} \mathrm{H}_{22} \mathrm{~N}_{2} \mathrm{O}_{3} \mathrm{~S}(\mathrm{M}+\mathrm{H})^{+} 320.1315$, found 320.13119 . 
$[\alpha]_{\mathrm{D}}^{25}+8.6\left(c 1.00, \mathrm{CHCl}_{3}\right)$.<smiles>N[C@@H](COCCS(=O)(=O)c1ccc(Br)cc1)Cc1ccccc1</smiles>

\section{Reaction conditions}

1b $(0.8 \mathrm{mmol})$ in DMF (0.8 mL), 2a (0.96 mmol), DMF $(0.8 \mathrm{~mL}),-20^{\circ} \mathrm{C}, 0.5 \mathrm{~h}, \mathrm{AgOAc} / \mathrm{dppe}(24.0 \mu \mathrm{mol})$, $\operatorname{KHMDS}(24 \mu \mathrm{mol})$.

(S)-1-(2-((4-bromophenyl)sulfonyl)ethoxy)-3-phenylpropan-2-amine (3ba): (pale yellow oil, $\mathrm{CH}_{2} \mathrm{Cl}_{2} / \mathrm{MeOH}=20 / 1,69 \%$ yield, $\left.221.2 \mathrm{mg}\right) ;{ }^{1} \mathrm{H}-\mathrm{NMR}\left(500 \mathrm{MHz}, \mathrm{CDCl}_{3}\right)(\mathrm{ppm}) \delta$ 7.82-7.75 (m, 2H, $\left.\operatorname{ArH}\right)$, 7.73-7.65 (m, 2H, ArH), 7.34-7.20 (m, 3H, ArH), 7.18-7.10 (m, $2 \mathrm{H}, \mathrm{ArH}), 3.81(\mathrm{t}, 2 \mathrm{H}, J=6.0 \mathrm{~Hz}$, $\mathrm{SO}_{2} \mathrm{CH}_{2} \mathrm{CH}_{2} \mathrm{O}$ ), 3.40 (t, $2 \mathrm{H}, J=6.0 \mathrm{~Hz}, \mathrm{SO}_{2} \mathrm{CH}_{2} \mathrm{CH}_{2} \mathrm{O}$ ), 3.30 (dd, $1 \mathrm{H}, J=4.5,8.7 \mathrm{~Hz}, \mathrm{OCH}_{2} \mathrm{CHCH}_{2} \mathrm{Ph}$ ), 3.17 (dd, $\left.1 \mathrm{H}, J=7.0,9.0 \mathrm{~Hz}, \mathrm{OCH}_{2} \mathrm{CHCH}_{2} \mathrm{Ph}\right), 3.05-2.96\left(\mathrm{~m}, 1 \mathrm{H}, \mathrm{CHNH}_{2}\right), 2.61\left(\mathrm{dd}, 1 \mathrm{H}, J=6.0,13.3 \mathrm{~Hz}, \mathrm{CH}_{2} \mathrm{Ph}\right), 2.42$ (dd, $\left.1 \mathrm{H}, J=8.5,13.3 \mathrm{~Hz}, \mathrm{CH}_{2} \mathrm{Ph}\right), 1.15\left(\mathrm{br}, 2 \mathrm{H}, \mathrm{NH}_{2}\right) ;{ }^{13} \mathrm{C} \mathrm{NMR}\left(125 \mathrm{MHz}, \mathrm{CDCl}_{3}\right) \delta 139.2,138.6,132.6$, $129.8,129.3,129.2,128.7,126.6,76.0,64.5,56.5,52.2,40.8$; IR (thin film, NaCl) 3350, 3086, 1357, 1145, $1114 \mathrm{~cm}^{-1}$; HRMS (ESI, H) $\mathrm{m} / z$ calc'd for $\mathrm{C}_{17} \mathrm{H}_{21} \mathrm{BrNO}_{3} \mathrm{~S}^{+}(\mathrm{M}+\mathrm{H})^{+} 398.0420$, found 398.0420. $[\alpha]_{\mathrm{D}}{ }^{25}+10.1$ (c $\left.1.00, \mathrm{CHCl}_{3}\right)$.<smiles>O=S(=O)(CCN[C@H](CO)Cc1ccccc1)c1ccc(Br)cc1</smiles>

(S)-2-((2-((4-bromophenyl)sulfonyl)ethyl)amino)-3-phenylpropan-1-ol (4ba): (white solid); ${ }^{1} \mathrm{H}-\mathrm{NMR}$ (500 MHz, $\left.\mathrm{CDCl}_{3}\right)(\mathrm{ppm})$ 8 7.73-7.63 (m, 4H, ArH), 7.35-7.20 (m, 3H, ArH), 7.19-7.12 (m, 2H. ArH), 3.59 (dd, $1 \mathrm{H}, J=4.0,11.0 \mathrm{~Hz}, \mathrm{CH}_{2} \mathrm{OH}$ ), 3.15 (dd, $\left.1 \mathrm{H}, \mathrm{J}=6.0,11.0 \mathrm{~Hz}, \mathrm{CH}_{2} \mathrm{OH}\right), 3.26-3.11\left(\mathrm{~m}, 2 \mathrm{H}, \mathrm{SO}_{2} \mathrm{CH}_{2} \mathrm{CH}_{2} \mathrm{NH}\right.$ ), 3.05-2.90 (m, $\left.2 \mathrm{H}, \mathrm{SO}_{2} \mathrm{CH}_{2} \mathrm{CH}_{2} \mathrm{NH}\right), 2.89-2.80\left(\mathrm{~m}, 1 \mathrm{H}, \mathrm{NHCHCH}_{2} \mathrm{Ph}\right), 2.78-2.66\left(\mathrm{~m}, 2 \mathrm{H}, \mathrm{NHCHCH}_{2} \mathrm{Ph}\right) ; 2.54$ (br, 1H, NH), $1.60(\mathrm{br}, 1 \mathrm{H}, \mathrm{OH}){ }^{13} \mathrm{C}$ NMR (125 MHz, CD $\left.3 \mathrm{OD}\right) \delta 140.2,139.6,133.8,131.0,130.3,130.0$, 129.6, 127.4, 64.0, 62.1, 56.5, 42.1, 38.8; IR (KBr) 3360, 1346, 1128, 1085, $1045 \mathrm{~cm}^{-1}$; HRMS (ESI, H) $\mathrm{m} / z$ calc'd for $\mathrm{C}_{17} \mathrm{H}_{21} \mathrm{BrNO}_{3} \mathrm{~S}^{+}(\mathrm{M}+\mathrm{H})^{+}$398.0420, found 398.0417. [ $\left.\alpha\right]_{\mathrm{D}}^{25}+3.9$ (c 1.00, $\left.\mathrm{CHCl}_{3}\right)$. 


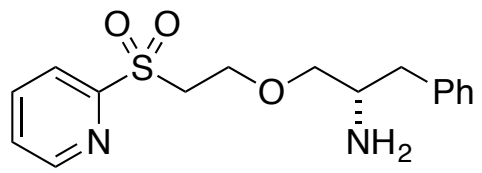

\section{$\underline{\text { Reaction conditions }}$}

1c $(0.8 \mathrm{mmol})$ in DMF $(0.8 \mathrm{~mL}), 2 \mathrm{a}(0.96 \mathrm{mmol}), \mathrm{DMF}(0.8 \mathrm{~mL}),-20{ }^{\circ} \mathrm{C}, 0.5 \mathrm{~h}, \mathrm{AgOAc} / \mathrm{dppe}(24.0 \mu \mathrm{mol})$, KHMDS $(32 \mu \mathrm{mol})$.

(S)-1-phenyl-3-(2-(pyridin-2-ylsulfonyl)ethoxy)propan-2-amine (3ca): (pale yellow oil, $\mathrm{CH}_{2} \mathrm{Cl}_{2} / \mathrm{MeOH}=$ 50/1, 55\% yield, $140.0 \mathrm{mg}) ;{ }^{1} \mathrm{H}-\mathrm{NMR}\left(500 \mathrm{MHz}^{\mathrm{C} C \mathrm{CDC}_{3}}\right)$ (ppm) 8.78-8.72 (m, 1H, ArH), 8.09 (dd, $1 \mathrm{H}, J=7.5$ $\mathrm{Hz}, \mathrm{ArH})$, 7.98-7.90 (m, 1H, ArH), 7.55-7.50 (m, 1H, ArH), 7.33-7.20 (m, 3H, ArH), 7.15-7.09 (m, 2H, ArH), $3.88\left(\mathrm{t}, 2 \mathrm{H}, \mathrm{J}=6.5 \mathrm{~Hz}, \mathrm{SO}_{2} \mathrm{CH}_{2} \mathrm{CH}_{2} \mathrm{O}\right), 3.73\left(\mathrm{t}, 2 \mathrm{H}, \mathrm{J}=5.5 \mathrm{~Hz}, \mathrm{SO}_{2} \mathrm{CH}_{2} \mathrm{CH}_{2} \mathrm{OCH}_{2}\right), 3.30(\mathrm{dd}, 1 \mathrm{H}, J=4.0,9.0 \mathrm{~Hz}$, $\mathrm{SO}_{2} \mathrm{CH}_{2} \mathrm{CH}_{2} \mathrm{O}$ ), $3.15\left(\mathrm{dd}, 1 \mathrm{H}, J=7.0,8.8 \mathrm{~Hz}, \mathrm{SO}_{2} \mathrm{CH}_{2} \mathrm{CH}_{2} \mathrm{O}\right), 2.99-2.90\left(\mathrm{~m}, 1 \mathrm{H}, \mathrm{CHNH}_{2}\right), 2.55(\mathrm{dd}, 1 \mathrm{H}, J=5.5$, $13.5 \mathrm{~Hz}, \mathrm{CH}_{2} \mathrm{Ph}$ ), 2.39 (dd, $\left.1 \mathrm{H}, J=8.0,13.5 \mathrm{~Hz}, \mathrm{CH}_{2} \mathrm{Ph}\right), 1.22\left(\mathrm{br}, 2 \mathrm{H}, \mathrm{NH}_{2}\right) ;{ }^{13} \mathrm{CNMR}\left(125 \mathrm{MHz}, \mathrm{CDCl}_{3}\right) \delta$ $158.2,150.2,138.7,138.2,129.3,128.7,127.4,126.5,121.9$, 75.9, $64.5,52.2,52.1$, 40.6; IR (thin film, $\mathrm{NaCl}$ ) 3373, 1600, 1454, 1317, $1161 \mathrm{~cm}^{-1}$; HRMS (ESI, H) $\mathrm{m} / z$ calc'd for $\mathrm{C}_{16} \mathrm{H}_{21} \mathrm{~N}_{2} \mathrm{O}_{3} \mathrm{~S}^{+}(\mathrm{M}+\mathrm{H})^{+} 321.1267$, found 321.1264. $[\alpha]_{\mathrm{D}}{ }^{25}-3.7\left(\mathrm{c} 1.00, \mathrm{CHCl}_{3}\right)$.<smiles>O=S(=O)(CCN[C@H](CO)Cc1ccccc1)c1ccccn1</smiles>

(S)-3-phenyl-2-((2-(pyridin-2-ylsulfonyl)ethyl)amino)propan-1-ol (4ca): (white solid); ${ }^{1} \mathrm{H}-\mathrm{NMR}$ (500 MHz, $\left.\mathrm{CDCl}_{3}\right)(\mathrm{ppm}) \delta 8.78-8.70(\mathrm{~m}, 1 \mathrm{H}, \mathrm{ArH}), 8.08-7.92(\mathrm{~m}, 2 \mathrm{H}, \mathrm{ArH}), 7.60-7.52(\mathrm{~m}, 1 \mathrm{H}, \mathrm{ArH}), 7.32-7.18(\mathrm{~m}, 3 \mathrm{H}$, ArH), 7.16-7.10 (m, 2H, ArH), 3.63-3.45 (m, 3H, $\left.\mathrm{CH}_{2} \mathrm{OH}, \mathrm{SO}_{2} \mathrm{CH}_{2} \mathrm{CH}_{2} \mathrm{NH}\right), 3.26(\mathrm{dd}, 1 \mathrm{H}, J=6.0,10.8 \mathrm{~Hz}$, $\mathrm{SO}_{2} \mathrm{CH}_{2} \mathrm{CH}_{2} \mathrm{NH}$ ), 3.17-2.98 (m, $\left.2 \mathrm{H}, \mathrm{SO}_{2} \mathrm{CH}_{2} \mathrm{CH}_{2} \mathrm{NH}\right), 2.89-2.80\left(\mathrm{~m}, 1 \mathrm{H}, \mathrm{NHCHCH}_{2} \mathrm{Ph}\right), 2.69$ (d, $2 \mathrm{H}, \mathrm{J}=7.0 \mathrm{~Hz}$, $\left.\mathrm{CH}_{2} \mathrm{Ph}\right), 2.56(\mathrm{br}, 1 \mathrm{H}, \mathrm{NH}), 1.37(\mathrm{br}, 1 \mathrm{H}, \mathrm{OH}) ;{ }^{13} \mathrm{C} \mathrm{NMR}\left(125 \mathrm{MHz}, \mathrm{CDCl}_{3}\right) \delta 157.8,150.3,138.4,138.3$, 129.3, 128.8, 127.5, 126.7, 122.0, 62.7, 60.4, 52.5, 40.6, 38.2; IR (KBr) 3657, 3294, 1382, 1226, 1155 $\mathrm{cm}^{-1}$; HRMS (ESI, H) $\mathrm{m} / z$ calc'd for $\mathrm{C}_{16} \mathrm{H}_{21} \mathrm{~N}_{2} \mathrm{O}_{3} \mathrm{~S}^{+}(\mathrm{M}+\mathrm{H})^{+}$321.1267, found 321.1272. [ $\left.\alpha\right]_{\mathrm{D}}{ }^{25}-0.9$ (c 1.00, $\left.\mathrm{CHCl}_{3}\right)$. 


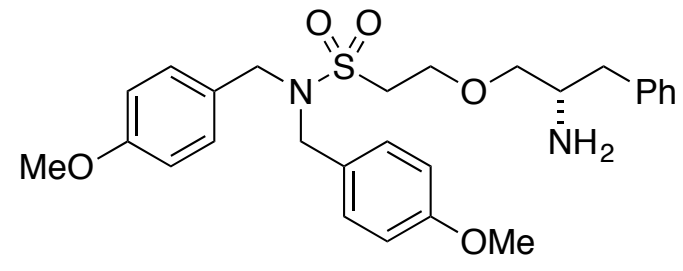

\section{$\underline{\text { Reaction conditions }}$}

1d $(0.8 \mathrm{mmol})$ in DMF $(0.8 \mathrm{~mL}), 2 \mathrm{a}(0.96 \mathrm{mmol}), \mathrm{DMF}(0.8 \mathrm{~mL}),-20{ }^{\circ} \mathrm{C}, 48 \mathrm{~h}, \mathrm{AgOAc} / \mathrm{dppe}(24.0 \mu \mathrm{mol})$, KHMDS $(32 \mu \mathrm{mol})$.

(S)-2-(2-amino-3-phenylpropoxy)- $N, N$-bis(4-methoxybenzyl)ethane-1-sulfonamide (3da): (pale yellow oil, $\mathrm{CH}_{2} \mathrm{Cl}_{2} / \mathrm{MeOH}=20 / 1,88 \%$ yield, $\left.352.2 \mathrm{mg}\right) ;{ }^{1} \mathrm{H}-\mathrm{NMR}\left(500 \mathrm{MHz}, \mathrm{CDCl}_{3}\right)(\mathrm{ppm}) \delta$ 7.33-7.28 (m, $2 \mathrm{H}$, ArH), 7.25-7.15 (m, 7H, ArH), 6.89-6.84 (m, $4 \mathrm{H}, \mathrm{ArH}), 4.26\left(\mathrm{~d}, 4 \mathrm{H}, J=1.5 \mathrm{~Hz}, \mathrm{~N}\left(\mathrm{CH}_{2} \mathrm{Ar}\right)_{2}\right), 3.88-3.78(\mathrm{~m}$, $8 \mathrm{H}, \mathrm{OCH}_{3}, \mathrm{OCH}_{3}, \mathrm{SO}_{2} \mathrm{CH}_{2} \mathrm{CH}_{2} \mathrm{O}$ ), $3.41\left(\mathrm{dd}, 1 \mathrm{H}, J=4.0,8.8 \mathrm{~Hz}, \mathrm{OCH}_{2} \mathrm{CHNH}_{2}\right), 3.28-3.16\left(\mathrm{~m}, 4 \mathrm{H}, \mathrm{OCH}_{2} \mathrm{CHNH}_{2}\right.$, $\mathrm{SO}_{2} \mathrm{CH}_{2} \mathrm{CH}_{2} \mathrm{O}$ ), $2.75\left(\mathrm{dd}, 1 \mathrm{H}, J=5.5,13.5 \mathrm{~Hz}, \mathrm{CH}_{2} \mathrm{Ph}\right.$ ), $2.53\left(\mathrm{dd}, 1 \mathrm{H}, J=8.0,13.5 \mathrm{~Hz}, \mathrm{CH}_{2} \mathrm{Ph}\right.$ ), $1.35(\mathrm{br}, 2 \mathrm{H}$, $\left.\mathrm{NH}_{2}\right) ;{ }^{13} \mathrm{CNMR}\left(125 \mathrm{MHz}, \mathrm{CDCl}_{3}\right) \delta 159.5,138.8,130.2,129.4,128.7,127.8,126.5,114.2,76.1,56.2,55.4$, 53.7, 52.3, 49.4, 40.8; IR (thin film, NaCl) 3375, 1330, 1209, 1176, $1143 \mathrm{~cm}^{-1}$; HRMS (ESI, H) $\mathrm{m} / z$ calc'd for

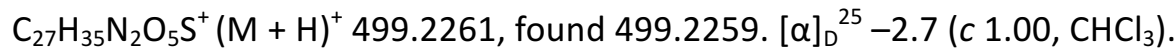

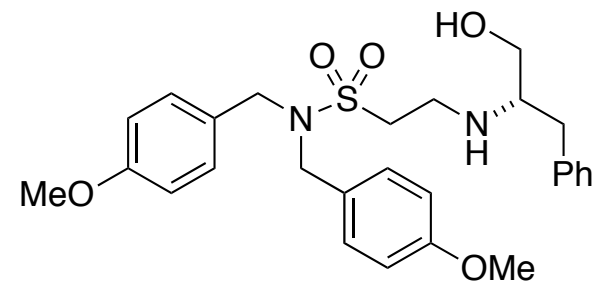

(S)-2-((1-hydroxy-3-phenylpropan-2-yl)amino)-N,N-bis(4-methoxybenzyl)ethane-1-sulfonamide (4da): (white solid); ${ }^{1} \mathrm{H}-\mathrm{NMR}\left(500 \mathrm{MHz}, \mathrm{CDCl}_{3}\right)(\mathrm{ppm}) \delta$ 7.32-7.26 (m, 2H, ArH), 7.22-7.15 (m, 7H, ArH), 6.90$6.85(\mathrm{~m}, 4 \mathrm{H}, \mathrm{ArH}), 4.21\left(\mathrm{~d}, 4 \mathrm{H}, J=2.5 \mathrm{~Hz},\left(\mathrm{ArCH}_{2}\right)_{2} \mathrm{~N}\right), 3.81\left(\mathrm{~s}, 6 \mathrm{H}, \mathrm{OCH}_{3}\right), 3.56(\mathrm{dd}, 1 \mathrm{H}, J=3.5,11.0 \mathrm{~Hz}$, $\left.\mathrm{CH}_{2} \mathrm{OH}\right), 3.29\left(\mathrm{dd}, 1 \mathrm{H}, J=6.0,10.5 \mathrm{~Hz}, \mathrm{CH}_{2} \mathrm{OH}\right), 3.13-3.04\left(\mathrm{~m}, 1 \mathrm{H}, \mathrm{SO}_{2} \mathrm{CH}_{2} \mathrm{CH}_{2} \mathrm{~N}\right), 2.98-2.80(\mathrm{~m}, 4 \mathrm{H}$, $\left.\mathrm{SO}_{2} \mathrm{CH}_{2} \mathrm{CH}_{2} \mathrm{NHCH}\right), 2.78-2.52\left(\mathrm{~m}, 3 \mathrm{H}, \mathrm{NH}, \mathrm{CH}_{2} \mathrm{Ph}\right), 1.58(\mathrm{br}, 1 \mathrm{H}, \mathrm{OH}) ;{ }^{13} \mathrm{C} \mathrm{NMR}\left(125 \mathrm{MHz}, \mathrm{CDCl}_{3}\right) \delta 159.5$, $138.4,130.2$, 129.3, 128.8, 127.7, 126.7, 114.3, 62.7, 60.5, 55.4, 54.1, 49.3, 41.5, 38.3; IR (KBr) 3408, 3219, 1249, 1172, $1064 \mathrm{~cm}^{-1}$; HRMS (ESI, H) $\mathrm{m} / \mathrm{z}$ calc'd for $\mathrm{C}_{27} \mathrm{H}_{35} \mathrm{~N}_{2} \mathrm{O}_{5} \mathrm{~S}^{+}(\mathrm{M}+\mathrm{H})^{+} 499.2261$, found 499.2266. $[\alpha]_{\mathrm{D}}^{25}+5.3\left(c 1.00, \mathrm{CHCl}_{3}\right)$. 


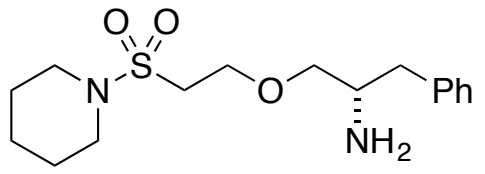

\section{Reaction conditions}

1e $(0.8 \mathrm{mmol})$ in DMF (0.8 mL), $2 \mathrm{a}(0.96 \mathrm{mmol}), \mathrm{DMF}(0.8 \mathrm{~mL}),-20^{\circ} \mathrm{C}, 1 \mathrm{~h}, \mathrm{AgOAc} / \mathrm{dppe}(24.0 \mu \mathrm{mol})$, KHMDS $(32 \mu \mathrm{mol})$.

(S)-1-phenyl-3-(2-(piperidin-1-ylsulfonyl)ethoxy)propan-2-amine (3ea): (pale orange oil, $\mathrm{CH}_{2} \mathrm{Cl}_{2} / \mathrm{MeOH}$

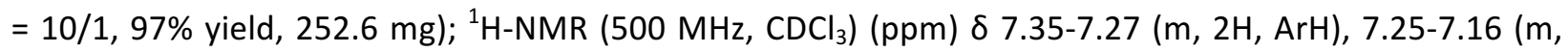

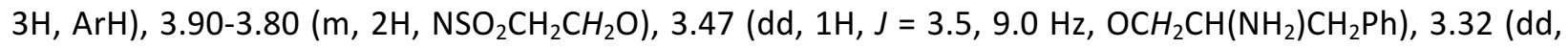
$\left.1 \mathrm{H}, J=7.0,9.0 \mathrm{~Hz},, \mathrm{OCH} \mathrm{CH}_{2}\left(\mathrm{NH}_{2}\right) \mathrm{CH}_{2} \mathrm{Ph}\right), 3.28-3.31\left(\mathrm{~m}, 5 \mathrm{H}, \mathrm{OCH}_{2} \mathrm{CH}\left(\mathrm{NH}_{2}\right) \mathrm{CH}_{2} \mathrm{Ph}_{2} \mathrm{CH}_{2}\left(\mathrm{CH}_{2} \mathrm{CH}_{2}\right)_{2} \mathrm{~N}\right), 3.19$ (t, $\left.2 \mathrm{H}, J=6.0 \mathrm{~Hz}, \mathrm{NSO}_{2} \mathrm{CH}_{2} \mathrm{CH}_{2} \mathrm{O}\right), 2.78\left(\mathrm{dd}, 1 \mathrm{H}, J=5.5,13.5 \mathrm{~Hz}, \mathrm{OCH}_{2} \mathrm{CH}\left(\mathrm{NH}_{2}\right) \mathrm{CH}_{2} \mathrm{Ph}\right), 2.57(\mathrm{dd}, 1 \mathrm{H}, J=8.5$, $\left.13.5 \mathrm{~Hz}, \mathrm{OCH}_{2} \mathrm{CH}\left(\mathrm{NH}_{2}\right) \mathrm{CH}_{2} \mathrm{Ph}\right), 1.70-1.40\left(\mathrm{~m}, 8 \mathrm{H}, \mathrm{OCH}_{2} \mathrm{CH}\left(\mathrm{NH}_{2}\right) \mathrm{CH}_{2} \mathrm{Ph}, \mathrm{CH}_{2}\left(\mathrm{CH}_{2} \mathrm{CH}_{2}\right)_{2} \mathrm{~N}\right) ;{ }^{13} \mathrm{C} N M R(125$ $\left.\mathrm{MHz}_{2} \mathrm{CDCl}_{3}\right) \delta 138.7,129.4,128.7,126.6,76.1,64.9,52.4,49.4,46.6,40.8,25.8,24.0$; IR (thin film, NaCl) $3375,1676,1357,1163,1053 \mathrm{~cm}^{-1}$; HRMS (ESI, H) $\mathrm{m} / \mathrm{z}$ calc'd for $\mathrm{C}_{16} \mathrm{H}_{27} \mathrm{~N}_{2} \mathrm{O}_{3} \mathrm{~S}(\mathrm{M}+\mathrm{H})^{+} 327.1737$, found 327.1737. $[\alpha]_{D}^{25}-1.2\left(c 1.00, \mathrm{CHCl}_{3}\right)$.

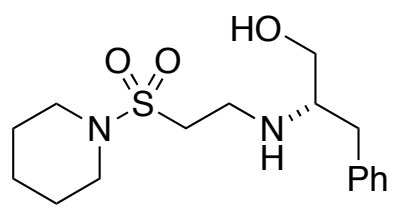

(S)-3-phenyl-2-((2-(piperidin-1-ylsulfonyl)ethyl)amino)propan-1-ol (4ea): (white solid); ${ }^{1} \mathrm{H}-\mathrm{NMR}$ (500 $\left.\mathrm{MHz}, \mathrm{CDCl}_{3}\right)(\mathrm{ppm}) \delta$ 7.33-7.22 (m, 2H, ArH), 7.25-7.17 (m, 3H, ArH), $3.60(\mathrm{dd}, 1 \mathrm{H}, J=4.0,10.5 \mathrm{~Hz}$, $\left.\mathrm{HOCH}_{2} \mathrm{CH}(\mathrm{NH}) \mathrm{CH}_{2} \mathrm{Ph}\right), 3.32\left(\mathrm{dd}, 1 \mathrm{H}, J=6.0,10.5 \mathrm{~Hz}, \mathrm{HOCH}_{2} \mathrm{CH}(\mathrm{NH}) \mathrm{CH}_{2} \mathrm{Ph}\right), 3.21-3.09(\mathrm{~m}, 5 \mathrm{H}$, $\left.\mathrm{CH}_{2}\left(\mathrm{CH}_{2} \mathrm{CH}_{2}\right)_{2} \mathrm{NSO}_{2} \mathrm{CH}_{2} \mathrm{CH}_{2} \mathrm{NH}\right), \quad 3.05-2.85\left(\mathrm{~m}, 4 \mathrm{H}\right.$, $\left.\mathrm{SO}_{2} \mathrm{CH}_{2} \mathrm{CH}_{2} \mathrm{NHCH}\right), \quad 2.80-2.30 \quad(\mathrm{~m}, \quad 3 \mathrm{H}$, $\left.\mathrm{HOCH}_{2} \mathrm{CH}(\mathrm{NH}) \mathrm{CH}_{2} \mathrm{Ph}\right), 1.70-1.50\left(\mathrm{~m}, 7 \mathrm{H}, \mathrm{HOCH}_{2} \mathrm{CH}(\mathrm{NH}) \mathrm{CH}_{2} \mathrm{Ph}, \mathrm{CH}_{2}\left(\mathrm{CH}_{2} \mathrm{CH}_{2}\right)_{2} \mathrm{NSO}_{2}\right) ;{ }^{13} \mathrm{C}$ NMR (125 MHz, $\left.\mathrm{CDCl}_{3}\right) \delta 138.4,129.3,128.8,126.7,62.8,60.6,49.5,47.0,41.3,38.4,25.7,23.9 ; \mathrm{IR}(\mathrm{KBr}) 3689,3354$, $1161,1057,1028 \mathrm{~cm}^{-1}$; HRMS (ESI, H) $\mathrm{m} / z$ calc'd for $\mathrm{C}_{16} \mathrm{H}_{27} \mathrm{~N}_{2} \mathrm{O}_{3} \mathrm{~S}(\mathrm{M}+\mathrm{H})^{+} 327.1737$, found 327.1742 . $[\alpha]_{\mathrm{D}}^{25}+3.3\left(c 1.00, \mathrm{CHCl}_{3}\right)$.

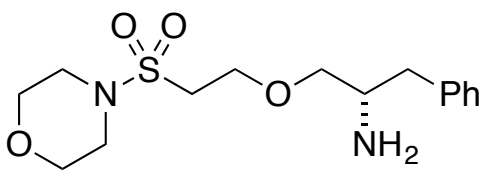




\section{Reaction conditions}

1f $(0.8 \mathrm{mmol})$ in DMF $(0.8 \mathrm{~mL}), 2 \mathrm{a}(0.96 \mathrm{mmol})$, DMF $(0.8 \mathrm{~mL}),-20{ }^{\circ} \mathrm{C}, 1 \mathrm{~h}, \mathrm{AgOAc} / \mathrm{dppe}(24.0 \mu \mathrm{mol})$, KHMDS $(32 \mu \mathrm{mol})$.

(S)-1-(2-(morpholinosulfonyl)ethoxy)-3-phenylpropan-2-amine (3fa): (pale yellow oil, $\mathrm{CH}_{2} \mathrm{Cl}_{2} / \mathrm{MeOH}=$ 10/1, 88\% yield, $232.2 \mathrm{mg}) ;{ }^{1} \mathrm{H}-\mathrm{NMR}\left(500 \mathrm{MHz}_{\mathrm{CDCl}}\right.$ ) (ppm) $\delta$ 7.33-7.27 (m, 2H, ArH), 7.25-7.17 (m, 3H, ArH), 3.90-3.81 (m, $\left.2 \mathrm{H}, \mathrm{SO}_{2} \mathrm{CH}_{2} \mathrm{CH}_{2} \mathrm{O}\right), 3.75\left(\mathrm{t}, 4 \mathrm{H}, J=5.0 \mathrm{~Hz}, \mathrm{O}\left(\mathrm{CH}_{2} \mathrm{CH}_{2}\right)_{2} \mathrm{~N}\right), 3.49(\mathrm{dd}, 1 \mathrm{H}, J=4.0,9.0 \mathrm{~Hz}$, $\mathrm{OCH}_{2} \mathrm{CHNH}_{2}$ ), 3.38-3.19 (m, 8H, OCH $\left.\mathrm{CHNH}_{2}, \mathrm{O}\left(\mathrm{CH}_{2} \mathrm{CH}_{2}\right)_{2} \mathrm{~N}, \mathrm{SO}_{2} \mathrm{CH}_{2} \mathrm{CH}_{2} \mathrm{O}\right), 2.78(\mathrm{dd}, 1 \mathrm{H}, J=5.5,13.5 \mathrm{~Hz}$, $\mathrm{CH}_{2} \mathrm{Ph}$ ), 2.57 (dd, $\left.1 \mathrm{H}, J=8.0,13.5 \mathrm{~Hz}, \mathrm{CH}_{2} \mathrm{Ph}\right), 1.54\left(\mathrm{br}, 2 \mathrm{H}, \mathrm{NH}_{2}\right) ;{ }^{13} \mathrm{C} \mathrm{NMR}\left(125 \mathrm{MHz}, \mathrm{CDCl}_{3}\right) \delta 138.6$, $129.3,128.7,126.6,76.2,66.7,64.7,52.4,49.4,45.7,40.8$; IR (thin film, NaCl) 3373, 1454, 1147, 1112, $1074 \mathrm{~cm}^{-1}$; HRMS (ESI, H) $\mathrm{m} / z$ calc'd for $\mathrm{C}_{15} \mathrm{H}_{25} \mathrm{~N}_{2} \mathrm{O}_{4} \mathrm{~S}(\mathrm{M}+\mathrm{H})^{+} 329.1530$, found 329.1530. [ $\left.\alpha\right]_{D}{ }^{25}-0.8(c$ $\left.1.00, \mathrm{CHCl}_{3}\right)$.

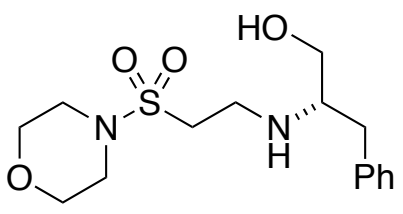

(S)-2-((2-(morpholinosulfonyl)ethyl)amino)-3-phenylpropan-1-ol (4fa): (white solid); ${ }^{1} \mathrm{H}-\mathrm{NMR}(500 \mathrm{MHz}$, $\left.\mathrm{CDCl}_{3}\right)(\mathrm{ppm})$ 87.34-7.27 (m, 2H, ArH), 7.25-7.17 (m, 3H, $\left.\mathrm{ArH}\right), 3.73\left(\mathrm{t}, 4 \mathrm{H}, J=5.0 \mathrm{~Hz}, \mathrm{O}\left(\mathrm{CH}_{2} \mathrm{CH}_{2}\right)_{2} \mathrm{~N}\right), 3.62$ (dd, $\left.1 \mathrm{H}, J=3.5,11.0 \mathrm{~Hz}, \mathrm{HOCH}_{2}\right), 3.34(\mathrm{dd}, 1 \mathrm{H}, \mathrm{J}=6.0,11.0 \mathrm{~Hz}, \mathrm{HOCH})_{2}, 3.23-3.10\left(\mathrm{~m}, 5 \mathrm{H}, \mathrm{O}\left(\mathrm{CH}_{2} \mathrm{CH}_{2}\right)_{2} \mathrm{~N}\right.$, $\mathrm{SO}_{2} \mathrm{CH}_{2} \mathrm{CH}_{2} \mathrm{~N}$ ), 3.08-2.86 (m, 4H, $\mathrm{SO}_{2} \mathrm{CH}_{2} \mathrm{CH}_{2} \mathrm{~N}, \mathrm{HNCHCH}_{2} \mathrm{Ph}$ ), $2.50\left(\mathrm{~d}, 2 \mathrm{H}, J=6.5 \mathrm{~Hz}, \mathrm{CH}_{2} \mathrm{Ph}\right), 2.57(\mathrm{br}, 1 \mathrm{H}$, $\mathrm{NH}), 1.55(\mathrm{br}, 1 \mathrm{H}, \mathrm{OH}) ;{ }^{13} \mathrm{C}$ NMR $\left(125 \mathrm{MHz}, \mathrm{CDCl}_{3}\right) \delta 138.4,129.3,128.8,126.7,66.6,63.0,60.6,49.2$, 45.8, 41.2, 38.4; IR (KBr) 3722, 3292, 1238, 1147, $1066 \mathrm{~cm}^{-1}$; HRMS (ESI, H) $\mathrm{m} / z$ calc'd for $\mathrm{C}_{15} \mathrm{H}_{25} \mathrm{~N}_{2} \mathrm{O}_{4} \mathrm{~S}$ (M $+\mathrm{H})^{+}$329.1530, found 329.1531. $[\alpha]_{\mathrm{D}}{ }^{25}-2.4\left(c 1.00, \mathrm{CHCl}_{3}\right)$.

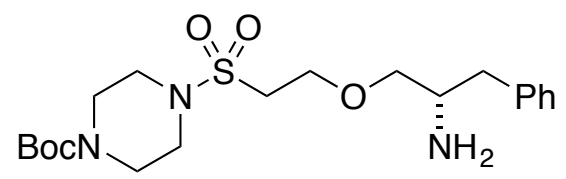

\section{$\underline{\text { Reaction conditions }}$}

$1 \mathrm{~g}(0.8 \mathrm{mmol})$ in DMF $(0.8 \mathrm{~mL}), 2 \mathrm{a}(0.96 \mathrm{mmol}), \mathrm{DMF}(0.8 \mathrm{~mL}),-20^{\circ} \mathrm{C}, 1 \mathrm{~h}, \mathrm{AgOAc} / \mathrm{dppe}(24.0 \mu \mathrm{mol})$, KHMDS $(32 \mu \mathrm{mol})$.

tert-butyl (S)-4-((2-(2-amino-3-phenylpropoxy)ethyl)sulfonyl)piperazine-1-carboxylate (3ga): (pale 
yellow oil, $\mathrm{CH}_{2} \mathrm{Cl}_{2} / \mathrm{MeOH}=10 / 1,86 \%$ yield, $\left.293.3 \mathrm{mg}\right) ;{ }^{1} \mathrm{H}-\mathrm{NMR}\left(500 \mathrm{MHz}, \mathrm{CDCl}_{3}\right)(\mathrm{ppm}) \delta$ 7.35-7.27 (m, $2 \mathrm{H}, \mathrm{ArH}), 7.25-7.15(\mathrm{~m}, 3 \mathrm{H}, \mathrm{ArH}), 3.90-3.78\left(\mathrm{~m}, 2 \mathrm{H}, \mathrm{SO}_{2} \mathrm{CH}_{2} \mathrm{CH}_{2} \mathrm{O}\right), 3.58-3.44\left(\mathrm{~m}, 5 \mathrm{H}, \operatorname{BocN}\left(\mathrm{CH}_{2} \mathrm{CH}_{2}\right)_{2} \mathrm{~N}\right.$, $\left.\mathrm{OCH}_{2} \mathrm{CH}\left(\mathrm{NH}_{2}\right) \mathrm{CH}_{2} \mathrm{Ph}\right), 3.55-3.15\left(\mathrm{~m}, 8 \mathrm{H}, \mathrm{BocN}\left(\mathrm{CH}_{2} \mathrm{CH}_{2}\right)_{2} \mathrm{~N}, \mathrm{SO}_{2} \mathrm{CH}_{2} \mathrm{CH}_{2} \mathrm{O}, \mathrm{CHNH} \mathrm{NH}_{2}, \mathrm{OCH} \mathrm{CH}_{2}\left(\mathrm{NH}_{2}\right) \mathrm{CH}_{2} \mathrm{Ph}\right), 2.76$ (dd, $1 \mathrm{H}, J=5.5,13.5 \mathrm{~Hz}, \mathrm{OCH}_{2} \mathrm{CH}\left(\mathrm{NH}_{2}\right) \mathrm{CH}_{2} \mathrm{Ph}$ ), 2.56 (dd, $1 \mathrm{H}, J=8.0,13.5 \mathrm{~Hz}, \mathrm{OCH}_{2} \mathrm{CH}\left(\mathrm{NH}_{2}\right) \mathrm{CH}_{2} \mathrm{Ph}$ ), 1.62$1.30\left(\mathrm{~m}, 11 \mathrm{H}, \mathrm{NH}_{2}, \mathrm{C}\left(\mathrm{CH}_{3}\right)_{3}\right) ;{ }^{13} \mathrm{C} \mathrm{NMR}\left(125 \mathrm{MHz}_{\mathrm{CDCl}}\right) \delta 154.4,138.5,129.3,128.6,126.5,80.5,76.0$, 64.6, 52.2, 49.8, 45.5, 43.5, 40.8, 28.4; IR (KBr) 3381, 1689, 1365, 1153, $1055 \mathrm{~cm}^{-1}$; HRMS (ESI, H) $\mathrm{m} / z$ calc'd for $\mathrm{C}_{20} \mathrm{H}_{34} \mathrm{~N}_{3} \mathrm{O}_{5} \mathrm{~S}^{+}(\mathrm{M}+\mathrm{H})^{+}$428.2214, found 428.2213. $[\alpha]_{D}{ }^{25}-3.5$ (c 1.00, $\left.\mathrm{CHCl}_{3}\right)$.

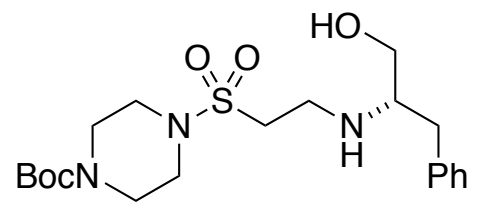

tert-butyl (S)-4-((2-((1-hydroxy-3-phenylpropan-2-yl)amino)ethyl)sulfonyl)piperazine-1-carboxylate (4ga): ( pale yellow solid); ${ }^{1} \mathrm{H}-\mathrm{NMR}\left(500 \mathrm{MHz}, \mathrm{CDCl}_{3}\right.$ ) (ppm) $\delta$ 7.34-7.27 (m, 2H, ArH), 7.25-7.15 (m, 3H, $\operatorname{ArH}), 3.61\left(\mathrm{dd}, 1 \mathrm{H}, J=3.5,11.0 \mathrm{~Hz}, \mathrm{HOCH}_{2}\right), 3.49\left(\mathrm{t}, 4 \mathrm{H}, J=5.0 \mathrm{~Hz}, \operatorname{BocN}\left(\mathrm{CH}_{2} \mathrm{CH}_{2}\right)_{2} \mathrm{~N}\right), 3.37(\mathrm{dd}, 1 \mathrm{H}, J=6.0$, $\left.11.5 \mathrm{~Hz}, \mathrm{HOCH}_{2}\right)$ 3.20-3.08 (m, 5H, SO $\mathrm{CH}_{2} \mathrm{CH}_{2} \mathrm{~N}$, $\left.\operatorname{BocN}\left(\mathrm{CH}_{2} \mathrm{CH}_{2}\right)_{2} \mathrm{~N}\right)$, 3.05-2.85 (m, 4H, $\left.\mathrm{SO}_{2} \mathrm{CH}_{2} \mathrm{CH}_{2} \mathrm{NCH}\right)$, $2.74\left(\mathrm{dd}, 2 \mathrm{H}, \mathrm{J}=7.0 \mathrm{~Hz}, \mathrm{CH}_{2} \mathrm{Ph}\right), 2.55(\mathrm{br}, 1 \mathrm{H}, \mathrm{NH}), 1.56(\mathrm{br}, 1 \mathrm{H}, \mathrm{OH}), 1.47\left(\mathrm{~s}, 9 \mathrm{H}, \mathrm{C}\left(\mathrm{CH}_{3}\right)_{3}\right) ;{ }^{13} \mathrm{C} \mathrm{NMR}(125$ $\left.\mathrm{MHz}, \mathrm{CDCl}_{3}\right) \delta 154.4,138.4,129.3,128.8,126.7,80.6,62.9,60.6,49.7,45.6,43.6,41.2,38.3,28.5 ; \mathrm{IR}$ (KBr) 3282, 1689, 1602, 1049, $1030 \mathrm{~cm}^{-1}$; HRMS (ESI, H) $\mathrm{m} / z$ calc'd for $\mathrm{C}_{20} \mathrm{H}_{34} \mathrm{~N}_{3} \mathrm{O}_{5} \mathrm{~S}^{+}(\mathrm{M}+\mathrm{H})^{+} 428.2214$, found 428.2214. $[\alpha]_{D}^{25}-0.4$ (c 1.00, $\left.\mathrm{CHCl}_{3}\right)$.

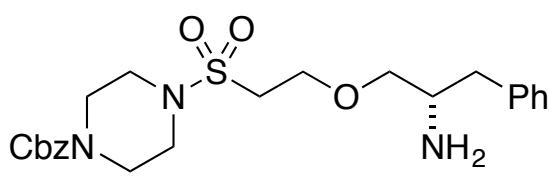

\section{Reaction conditions}

1h $(0.8 \mathrm{mmol})$ in DMF (0.8 mL), $2 \mathrm{a}(0.96 \mathrm{mmol}), \mathrm{DMF}(0.8 \mathrm{~mL}),-20^{\circ} \mathrm{C}, 1 \mathrm{~h}, \mathrm{AgOAc} / \mathrm{dppe}(24.0 \mu \mathrm{mol})$, KHMDS $(32 \mu \mathrm{mol})$.

benzyl (S)-4-((2-(2-amino-3-phenylpropoxy)ethyl)sulfonyl)piperazine-1-carboxylate (3ha): (white solid, $\mathrm{CH}_{2} \mathrm{Cl}_{2} / \mathrm{MeOH}=10 / 1,80 \%$ yield, $\left.296.8 \mathrm{mg}\right) ;{ }^{1} \mathrm{H}-\mathrm{NMR}\left(500 \mathrm{MHz}, \mathrm{CDCl}_{3}\right)(\mathrm{ppm}) \delta$ 7.40-7.14 (m, 10H, $\left.\mathrm{ArH}\right)$, 3.88-3.78 (m, $\left.2 \mathrm{H}, \mathrm{SO}_{2} \mathrm{CH}_{2} \mathrm{CH}_{2} \mathrm{O}\right), 5.12\left(\mathrm{~s}, 2 \mathrm{H}, \mathrm{PhCH} \mathrm{H}_{2} \mathrm{OCON}\right) 3.59\left(\mathrm{t}, 4 \mathrm{H}, J=4.5 \mathrm{~Hz}, \mathrm{CbzN}\left(\mathrm{CH}_{2} \mathrm{CH}_{2}\right)_{2} \mathrm{~N}\right), 3.45$ (dd, $1 \mathrm{H}, \mathrm{J}=4.0,18.0 \mathrm{~Hz}, \mathrm{OCH}_{2} \mathrm{CHN}$ ), 3.35-3.16 (m, 8H, OCH$\left.{ }_{2} \mathrm{CHN}, \mathrm{CbzN}\left(\mathrm{CH}_{2} \mathrm{CH}_{2}\right)_{2} \mathrm{~N}, \mathrm{SO}_{2} \mathrm{CH}_{2} \mathrm{CH}_{2} \mathrm{O}\right), 2.75$ 
(dd, $1 \mathrm{H}, J=5.5,13.5 \mathrm{~Hz}, \mathrm{PhCH}_{2} \mathrm{CHN}$ ), 2.54 (dd, $1 \mathrm{H}, J=8.5,13.5 \mathrm{~Hz}, \mathrm{PhCH}_{2} \mathrm{CHN}$ ), $1.51\left(\mathrm{br}, 2 \mathrm{H}, \mathrm{NH}_{2}\right) ;{ }^{13} \mathrm{C}$ NMR $\left(125 \mathrm{MHz}, \mathrm{CDCl}_{3}\right) \delta 151.1,138.6,136.4,129.3,128.7,128.4,128.2,126.6,76.2,67.7,64.7,52.3$, 50.0, 45.4, 43.9, 40.9; IR (KBr) 3354, 1689, 1367, 1105, $1058 \mathrm{~cm}^{-1}$; HRMS (ESI, H) $\mathrm{m} / z$ calc'd for $\mathrm{C}_{23} \mathrm{H}_{32} \mathrm{~N}_{3} \mathrm{O}_{5} \mathrm{~S}(\mathrm{M}+\mathrm{H})^{+} 462.2058$, found 462.2059. $[\alpha]_{D}^{25}-4.0\left(c 1.00, \mathrm{CHCl}_{3}\right)$.

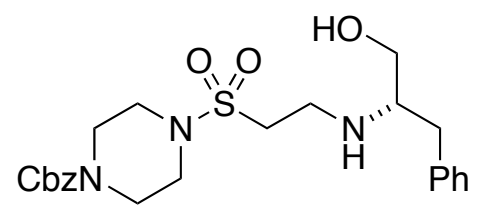

benzyl (S)-4-((2-((1-hydroxy-3-phenylpropan-2-yl)amino)ethyl)sulfonyl)piperazine-1-carboxylate (4ha): (white soild); ${ }^{1} \mathrm{H}-\mathrm{NMR}\left(500 \mathrm{MHz}, \mathrm{CDCl}_{3}\right)(\mathrm{ppm}) \delta$ 7.40-7.16 (m, 10H, $\left.\mathrm{ArH}\right), 5.15$ (s, 2H, $\left.\mathrm{PhCH}_{2} \mathrm{OCON}\right), 3.64-$ $3.50\left(\mathrm{~m}, 5 \mathrm{H}, \mathrm{OCH}_{2} \mathrm{CHN}, \mathrm{CbzN}\left(\mathrm{CH}_{2} \mathrm{CH}_{2}\right)_{2} \mathrm{~N}\right), 3.38-3.30\left(\mathrm{~m}, 1 \mathrm{H}, \mathrm{OCH}_{2} \mathrm{CHN}\right), 3.24-3.07(\mathrm{~m}, 5 \mathrm{H}$, $\left.\mathrm{CbzN}\left(\mathrm{CH}_{2} \mathrm{CH}_{2}\right)_{2} \mathrm{~N}, \mathrm{SO}_{2} \mathrm{CH}_{2} \mathrm{CH}_{2} \mathrm{NHCH}\right), 3.04-2.85\left(\mathrm{~m}, 4 \mathrm{H}, \mathrm{SO}_{2} \mathrm{CH}_{2} \mathrm{CH}_{2} \mathrm{NHCH}\right), 2.74(\mathrm{~d}, 2 \mathrm{H}, J=7.0 \mathrm{~Hz}$, $\left.\mathrm{PhCH}_{2} \mathrm{CHN}\right), 2.53(\mathrm{br}, 1 \mathrm{H}, \mathrm{NH}), 1.58(\mathrm{br}, 1 \mathrm{H}, \mathrm{OH}) ;{ }^{13} \mathrm{C} \mathrm{NMR}\left(125 \mathrm{MHz}, \mathrm{CDCl}_{3}\right) \delta$ 155.1. 138.4, 136.4, 129.3, $128.8,128.7,128.5,128.3,126.7,67.8,63.0,60.6,49.9,45.5,43.9,41.2,38.4 ; \mathrm{IR}$ (KBr) 3749, 1687, 1585,

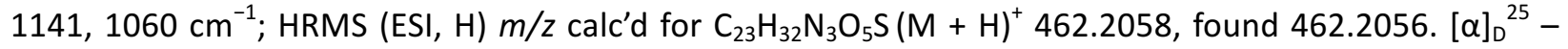
1.2 (c 1.00, $\left.\mathrm{CHCl}_{3}\right)$.

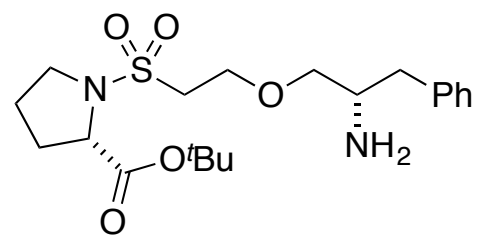

\section{Reaction conditions}

1i $(0.8 \mathrm{mmol})$ in DMF $(0.8 \mathrm{~mL}), 2 \mathrm{a}(0.96 \mathrm{mmol})$, DMF $(0.8 \mathrm{~mL}),-20{ }^{\circ} \mathrm{C}, 1 \mathrm{~h}$, AgOAc/dppe $(24.0 \mu \mathrm{mol})$, KHMDS $(32 \mu \mathrm{mol})$.

tert-butyl ((2-((S)-2-amino-3-phenylpropoxy)ethyl)sulfonyl)-S-prolinate (3ia): (pale yellow oil, $\mathrm{CH}_{2} \mathrm{Cl}_{2} / \mathrm{MeOH}=10 / 1,84 \%$ yield, $\left.277.8 \mathrm{mg}\right) ;{ }^{1} \mathrm{H}-\mathrm{NMR}\left(500 \mathrm{MHz}, \mathrm{CDCl}_{3}\right)(\mathrm{ppm}) \delta$ 7.34-7.27 (m, 2H, $\left.\mathrm{ArH}\right)$, 7.25-7.16 (m, 3H, ArH), 4.39 (dd, $1 \mathrm{H}, J=3.0,9.0 \mathrm{~Hz}, \mathrm{NCHCOO}{ }^{\mathrm{Bu}}$ ), $3.99\left(\mathrm{t}, 2 \mathrm{H}, J=6.5 \mathrm{~Hz}, \mathrm{SO}_{2} \mathrm{CH}_{2} \mathrm{CH}_{2} \mathrm{O}\right.$ ), 3.60-3.20 (m, 7H, $\mathrm{CH}_{2} \mathrm{~N}\left(\mathrm{SO}_{2}\right) \mathrm{CH}, \mathrm{SO}_{2} \mathrm{CH}_{2} \mathrm{CH}_{2} \mathrm{O}, \mathrm{OCH}_{2} \mathrm{CHNH}_{2}$ ), 2.77 (dd, $1 \mathrm{H}, J=5.5,13.5 \mathrm{~Hz}, \mathrm{CH}_{2} \mathrm{Ph}$ ), 2.56 (dd, $\left.1 \mathrm{H}, J=8.0,13.5 \mathrm{~Hz}, \mathrm{CH}_{2} \mathrm{Ph}\right), 2.29-2.17\left(\mathrm{~m}, 1 \mathrm{H}, \mathrm{SO}_{2} \mathrm{NCH}\left(\mathrm{COO}^{t} \mathrm{Bu}\right) \mathrm{CH}_{2} \mathrm{CH}_{2}\right), 2.05-1.92(\mathrm{~m}, 3 \mathrm{H}$, $\left.\mathrm{SO}_{2} \mathrm{NCH}\left(\mathrm{COO}^{t} \mathrm{Bu}\right) \mathrm{CH}_{2} \mathrm{CH}_{2}\right), 1.60-1.32\left(\mathrm{~m}, 11 \mathrm{H}, \mathrm{O}\left(\mathrm{CH}_{3}\right)_{3}, \mathrm{NH}_{2}\right) ;{ }^{13} \mathrm{CNMR}\left(125 \mathrm{MHz}, \mathrm{CDCl}_{3}\right) \delta 171.7,138.8$, 
129.3, 128.6, 126.4, 81.9, 75.8, 64.9, 61.2, 52.7, 52.3, 48.2, 40.8, 31.2, 28.0, 24.9; IR (thin film, NaCl) $3377,1367,1197,1141,1080 \mathrm{~cm}^{-1}$; HRMS $(E S I, H) \mathrm{m} / z$ calc'd for $\mathrm{C}_{20} \mathrm{H}_{34} \mathrm{~N}_{3} \mathrm{O}_{5} \mathrm{~S}^{+}(\mathrm{M}+\mathrm{H})^{+} 413.2105$, found 413.2101. $[\alpha]_{D}^{25}-30.0\left(c 1.00, \mathrm{CHCl}_{3}\right)$.

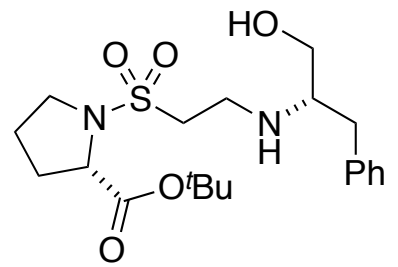

tert-butyl ((2-(((S)-1-hydroxy-3-phenylpropan-2-yl)amino)ethyl)sulfonyl)-S-prolinate (4ia): (pale yellow oil); ${ }^{1} \mathrm{H}-\mathrm{NMR}\left(500 \mathrm{MHz}, \mathrm{CDCl}_{3}\right.$ ) (ppm) 8 7.32-7.27 (m, 2H, ArH), 7.24-7.18 (m, 3H, ArH), 3.39 (dd, $1 \mathrm{H}, J=$ 3.0, 8.5 Hz, NCHCOO ${ }^{t} \mathrm{Bu}$ ), 3.60 (dd, $\left.1 \mathrm{H}, J=3.5,11.0 \mathrm{~Hz}, \mathrm{HOCH}_{2}\right), 3.56-3.48\left(\mathrm{~m}, 1 \mathrm{H}, \mathrm{SO}_{2} \mathrm{CH}_{2} \mathrm{CH}_{2} \mathrm{NH}\right), 3.38-$ $3.14\left(\mathrm{~m}, 5 \mathrm{H}, \mathrm{SO}_{2} \mathrm{CH}_{2} \mathrm{CH}_{2} \mathrm{NH}, \mathrm{HOCH}_{2}, \mathrm{SO}_{2} \mathrm{NCH}_{2}\right), 3.08-3.00\left(\mathrm{~m}, 1 \mathrm{H}, \mathrm{SO}_{2} \mathrm{NCH}_{2}\right), 2.93-2.86(\mathrm{~m}, 1 \mathrm{H}$, $\left.\mathrm{NHCHCH}_{2} \mathrm{Ph}\right), 2.84-2.76\left(\mathrm{~m}, 3 \mathrm{H}, \mathrm{NH}, \mathrm{CH}_{2} \mathrm{Ph}\right), 2.29-2.18\left(\mathrm{~m}, 1 \mathrm{H}, \mathrm{SO}_{2} \mathrm{NCH}\left(\mathrm{COO}^{t} \mathrm{Bu}^{2} \mathrm{CH}_{2} \mathrm{CH}_{2}\right), 2.03-1.90(\mathrm{~m}\right.$, $\left.3 \mathrm{H}, \mathrm{SO}_{2} \mathrm{NCH}\left(\mathrm{COO}^{t} \mathrm{Bu}\right) \mathrm{CH}_{2} \mathrm{CH}_{2}\right), 1.63(\mathrm{br}, 1 \mathrm{H}, \mathrm{OH}), 1.47\left(\mathrm{~s}, 9 \mathrm{H}, \mathrm{O}\left(\mathrm{CH}_{3}\right)_{3}\right) ;{ }^{13} \mathrm{C} \mathrm{NMR}\left(125 \mathrm{MHz}, \mathrm{CDCl}_{3}\right) \delta 172.0$, 138.6, 129.4, 128.7, 126.6, 82.2, 62.6, 61.2, 60.6, 53.5, 48.4, 41.5, 38.3, 31.2, 28.1, 25.1; IR (KBr) 3649, $3365,1683,1145,1058 \mathrm{~cm}^{-1}$; HRMS (ESI, H) $\mathrm{m} / z$ calc'd for $\mathrm{C}_{20} \mathrm{H}_{34} \mathrm{~N}_{3} \mathrm{O}_{5} \mathrm{~S}^{+}(\mathrm{M}+\mathrm{H})^{+}$413.2105, found 413.2106. $[\alpha]_{D}^{25}-36.8\left(c 1.00, \mathrm{CHCl}_{3}\right)$.

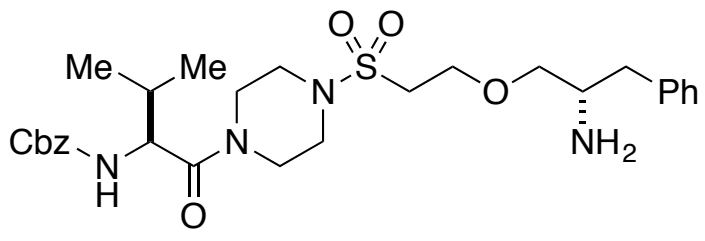

\section{$\underline{\text { Reaction conditions }}$}

1j $(0.8 \mathrm{mmol})$ in DMF (0.8 mL), $2 \mathrm{a}(0.96 \mathrm{mmol}), \mathrm{DMF}(0.8 \mathrm{~mL}),-20^{\circ} \mathrm{C}, 24 \mathrm{~h}$, AgOAc/dppe $(24.0 \mu \mathrm{mol})$, KHMDS $(32 \mu \mathrm{mol})$

benzyl ((S)-1-(4-((2-((S)-2-amino-3-phenylpropoxy)ethyl)sulfonyl)piperazin-1-yl)-3-methyl-1-oxobutan2-yl)carbamate (3ja): (pale yellow oil, $\mathrm{CH}_{2} \mathrm{Cl}_{2} / \mathrm{MeOH}=20 / 1,60 \%$ yield, $270.4 \mathrm{mg}$ ); ${ }^{1} \mathrm{H}-\mathrm{NMR}$ (500 MHz, $\left.\mathrm{CDCl}_{3}\right)(\mathrm{ppm})$ 8 7.40-7.10 (m, 10H, $\left.\mathrm{ArH}\right), 6.05$ (d, $\left.1 \mathrm{H}, \mathrm{J}=9.5 \mathrm{~Hz}, \mathrm{NHC}(\mathrm{O}) \mathrm{O}\right), 5.09$ (dd, $2 \mathrm{H}, \mathrm{J}=12.5,30.3 \mathrm{~Hz}$, $\mathrm{PhCH}_{2} \mathrm{O}$ ), $4.42(\mathrm{dd}, 1 \mathrm{H}, J=6.0,9.0 \mathrm{~Hz}, \mathrm{CHNHC}(\mathrm{O}) \mathrm{O}), 4.05\left(\mathrm{~d}, 1 \mathrm{H}, J=13.0 \mathrm{~Hz}, \mathrm{SO}_{2}\left(\mathrm{CH}_{2} \mathrm{CH}_{2}\right)_{2} \mathrm{SO}_{2}\right), 3.85-3.75$ (m, $\left.3 \mathrm{H}, \mathrm{SO}_{2}\left(\mathrm{CH}_{2} \mathrm{CH}_{2}\right)_{2} \mathrm{SO}_{2}, \mathrm{SO}_{2} \mathrm{CH}_{2} \mathrm{CH}_{2} \mathrm{O}\right)$, 3.55-3.05 (m, $11 \mathrm{H}, \mathrm{SO}_{2}\left(\mathrm{CH}_{2} \mathrm{CH}_{2}\right)_{2} \mathrm{SO}_{2}, \mathrm{SO}_{2} \mathrm{CH}_{2} \mathrm{CH}_{2} \mathrm{OCH}_{2} \mathrm{CHNH}_{2}$ ), 
$2.70\left(\mathrm{dd}, 1 \mathrm{H}, J=5.5,13.3 \mathrm{~Hz}, \mathrm{NH}_{2} \mathrm{CHCH}_{2} \mathrm{Ph}\right.$ ), 2.52 (dd, $1 \mathrm{H}, J=8.5,13.3 \mathrm{~Hz}, \mathrm{NH}_{2} \mathrm{CHCH}_{2} \mathrm{Ph}$ ), 2.00-1.90 (m, $\left.1 \mathrm{H}, \mathrm{CH}\left(\mathrm{CH}_{3}\right)_{2}\right), 1.44\left(\mathrm{br}, 2 \mathrm{H}, \mathrm{NH}_{2}\right), 0.95\left(\mathrm{~d}, 3 \mathrm{H}, J=6.5 \mathrm{~Hz}, \mathrm{CH}\left(\mathrm{CH}_{3}\right)_{2}\right), 0.90\left(\mathrm{~d}, 3 \mathrm{H}, J=6.5 \mathrm{~Hz}, \mathrm{CH}\left(\mathrm{CH}_{3}\right)_{2}\right) ;{ }^{13} \mathrm{C}$ $\operatorname{NMR}\left(125 \mathrm{MHz}, \mathrm{CDCl}_{3}\right) \delta 170.5,156.6,138.5,136.5,129.3,128.7,128.7,128.4,128.4,126.7,76.1,67.1$, $64.7,55.7,52.3,50.3,46.0,45.9,45.4,42.2,40.6,31.5,19.8,17.5$; IR (thin film, NaCl) 3302, 1643, 1344,

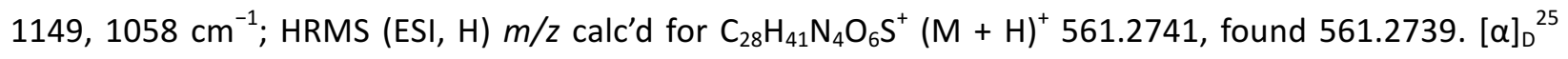
$+7.3\left(c 1.00, \mathrm{CHCl}_{3}\right)$.

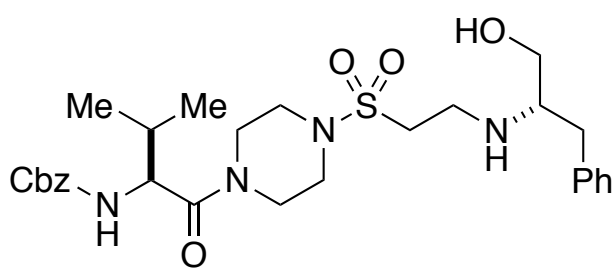

benzyl ((S)-1-(4-((2-(((S)-1-hydroxy-3-phenylpropan-2-yl)amino)ethyl)sulfonyl)piperazin-1-yl)-3methyl-1-oxobutan-2-yl)carbamate (4ja): (pale yellow foam); ${ }^{1} \mathrm{H}-\mathrm{NMR}\left(500 \mathrm{MHz}, \mathrm{CDCl}_{3}\right.$ ) (ppm) $\delta 7.40$ $7.15(\mathrm{~m}, 10 \mathrm{H}, \mathrm{ArH}), 5.63$ (dd, $1 \mathrm{H}, J=9.0 \mathrm{~Hz}, \mathrm{NHCO}), 5.09$ (dd, $2 \mathrm{H}, J=12.5,14.3 \mathrm{~Hz}, \mathrm{PhCH}_{2} \mathrm{O}$ ), 4.77 (dd, $1 \mathrm{H}, J=4.0,9.0 \mathrm{~Hz}, \mathrm{CONHCHCO}), 3.95\left(\mathrm{~d}, 1 \mathrm{H}, J=13.0 \mathrm{~Hz}, \mathrm{~N}\left(\mathrm{CH}_{2} \mathrm{CH}_{2}\right)_{2} \mathrm{~N}\right), 3.73(\mathrm{~d}, 1 \mathrm{H}, J=13.5 \mathrm{~Hz}, \mathrm{~N}$ $\left.\left(\mathrm{CH}_{2} \mathrm{CH}_{2}\right)_{2} \mathrm{~N}\right), 3.60\left(\mathrm{dd}, 1 \mathrm{H}, J=4.0,11.0 \mathrm{~Hz}, \mathrm{CH}_{2} \mathrm{OH}\right), 3.55-2.82\left(\mathrm{~m}, 13 \mathrm{H}, \mathrm{CH}_{2} \mathrm{OH}\right.$, $\left.\left.\mathrm{N}\left(\mathrm{CH}_{2} \mathrm{CH}_{2}\right)_{2} \mathrm{~N}\right), \mathrm{SO}_{2} \mathrm{CH}_{2} \mathrm{CH}_{2} \mathrm{NHCH}\right), 2.73\left(\mathrm{~d}, 2 \mathrm{H}, J=7.0 \mathrm{~Hz}, \mathrm{CH}_{2} \mathrm{Ph}\right), 2.10-1.50\left(\mathrm{~m}, 2 \mathrm{H}, \mathrm{OH}, \mathrm{CH}\left(\mathrm{CH}_{3}\right)_{2}\right), 0.94$ (dd, $\left.6 \mathrm{H}, J=7.0,32.0 \mathrm{~Hz}, \mathrm{CH}\left(\mathrm{CH}_{3}\right)_{2}\right) ;{ }^{13} \mathrm{C} \mathrm{NMR}\left(125 \mathrm{MHz}, \mathrm{CDCl}_{3}\right) \delta 170.7,156.6,138.3,136.3,129.3,128.7$, 128.6, 128.2, 128.0, 126.6, 67.1, 63.0, 60.5, 55.5, 50.0, 45.9, 45.8, 45.5, 42.0, 41.1, 38.2, 31.4, 19.7, 17.4; IR (KBr) 3309, 1645, 1232, 1151, $1028 \mathrm{~cm}^{-1}$; HRMS (ESI, H) $\mathrm{m} / z$ calc'd for $\mathrm{C}_{28} \mathrm{H}_{41} \mathrm{~N}_{4} \mathrm{O}_{6} \mathrm{~S}^{+}(\mathrm{M}+\mathrm{H})^{+} 561.2741$, found 561.2740. $[\alpha]_{D}^{25}+22.6\left(c 1.00, \mathrm{CHCl}_{3}\right)$.

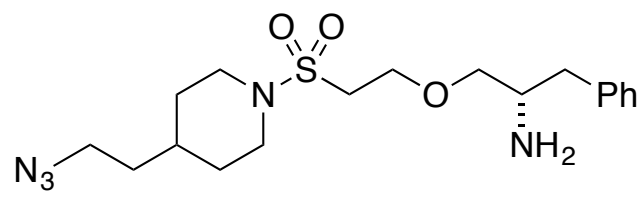

\section{$\underline{\text { Reaction conditions }}$}

1k $(0.8 \mathrm{mmol})$ in DMF $(0.8 \mathrm{~mL}), 2 \mathrm{a}(0.96 \mathrm{mmol}), \mathrm{DMF}(0.8 \mathrm{~mL}),-20^{\circ} \mathrm{C}, 1 \mathrm{~h}, \mathrm{AgOAc} / \mathrm{dppe}(24.0 \mu \mathrm{mol})$, KHMDS $(32 \mu \mathrm{mol})$.

(S)-1-(2-((4-(2-azidoethyl)piperidin-1-yl)sulfonyl)ethoxy)-3-phenylpropan-2-amine (3ka): (pale yellow oil, $\mathrm{CH}_{2} \mathrm{Cl}_{2} / \mathrm{MeOH}=20 / 1,56 \%$ yield, $176.7 \mathrm{mg}$ ); ${ }^{1} \mathrm{H}-\mathrm{NMR}\left(500 \mathrm{MHz}, \mathrm{CDCl}_{3}\right)(\mathrm{ppm}) \delta 7.30(\mathrm{t}, 2 \mathrm{H}, J=7.5 \mathrm{~Hz}$, 
ArH), 7.26-7.18 (m, 3H, ArH), 3.90-3.72 (m, 4H, $\left.\mathrm{SO}_{2} \mathrm{CH}_{2} \mathrm{CH}_{2} \mathrm{O}, \mathrm{N}_{3} \mathrm{CH}_{2} \mathrm{CH}_{2} \mathrm{CH}\left(\mathrm{CH}_{2} \mathrm{CH}_{2}\right)_{2} \mathrm{~N}\right), 3.46$ (dd, $1 \mathrm{H}, J=$ 4.0, 9.0 Hz, OCH $\mathrm{CHNH}_{2}$ ), 3.39-3.15 (m, 6H, OCH $\left.\mathrm{CHNH}_{2}, \mathrm{~N}_{3} \mathrm{CH}_{2} \mathrm{CH}_{2} \mathrm{CH}\left(\mathrm{CH}_{2} \mathrm{CH}_{2}\right)_{2} \mathrm{~N}\right)$, 2.82- $2.68(\mathrm{~m}, 3 \mathrm{H}$, $\mathrm{CH}_{2} \mathrm{Ph}, \mathrm{SO}_{2} \mathrm{CH}_{2} \mathrm{CH}_{2} \mathrm{O}$ ), 2.56 (dd, $1 \mathrm{H}, J=8.5,13.5 \mathrm{~Hz}, \mathrm{CH}_{2} \mathrm{Ph}$ ), 1.84-1.20 (m, 9H, , $\mathrm{N}_{3} \mathrm{CH}_{2} \mathrm{CH}_{2} \mathrm{CH}\left(\mathrm{CH}_{2} \mathrm{CH}_{2}\right)_{2} \mathrm{~N}$, $\left.\mathrm{NH}_{2}\right) ;{ }^{13} \mathrm{C}$ NMR $\left(125 \mathrm{MHz}, \mathrm{CDCl}_{3}\right) \delta 138.7,129.3,128.7,126.6,76.1,64.8,52.3,49.6,48.7,45.9,40.8$, 35.0, 32.9, 31.8; IR (thin film, NaCl) 3373, 2096, 1332, 1111, $1053 \mathrm{~cm}^{-1}$; HRMS (ESI, H) $\mathrm{m} / z$ calc'd for $\mathrm{C}_{18} \mathrm{H}_{30} \mathrm{~N}_{5} \mathrm{O}_{3} \mathrm{~S}(\mathrm{M}+\mathrm{H})^{+}$396.2064, found 396.2069. $[\alpha]_{D}^{25}-2.0\left(c 1.00, \mathrm{CHCl}_{3}\right)$.

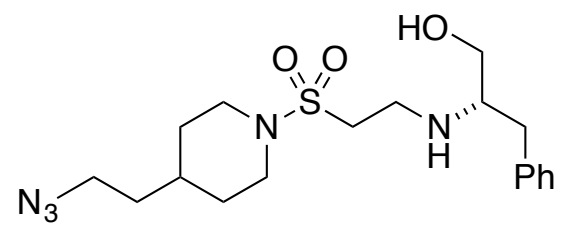

(S)-2-((2-((4-(2-azidoethyl)piperidin-1-yl)sulfonyl)ethyl)amino)-3-phenylpropan-1-ol $\quad$ (4ka): (white solid); ${ }^{1} \mathrm{H}-\mathrm{NMR}\left(500 \mathrm{MHz}, \mathrm{CDCl}_{3}\right)(\mathrm{ppm}) \delta$ 7.36-7.28 (m, 2H, ArH), 7.25-7.78 (m, 3H, ArH), 3.80-3.68 (m, $\left.2 \mathrm{H}, \mathrm{N}_{3} \mathrm{CH}_{2} \mathrm{CH}_{2} \mathrm{CH}\left(\mathrm{CH}_{2} \mathrm{CH}_{2}\right)_{2} \mathrm{~N}\right), 3.60(\mathrm{dd}, 1 \mathrm{H}, \mathrm{J}=3.5,10.5 \mathrm{~Hz}, \mathrm{HOCH})_{2}, 3.37-3.30\left(\mathrm{~m}, 3 \mathrm{H}, \mathrm{HOCH}_{2}, \mathrm{~N}_{3} \mathrm{CH}_{2}\right)$, 3.16-2.64 (m, $\left.10 \mathrm{H}, \quad \mathrm{SO}_{2} \mathrm{CH}_{2} \mathrm{CH}_{2} \mathrm{NHCHCH}_{2} \mathrm{Ph}, \quad \mathrm{N}_{3} \mathrm{CH}_{2} \mathrm{CH}_{2} \mathrm{CH}\left(\mathrm{CH}_{2} \mathrm{CH}_{2}\right)_{2} \mathrm{~N}\right), \quad 2.00-1.23(\mathrm{~m}, 8 \mathrm{H}$, $\left.\mathrm{N}_{3} \mathrm{CH}_{2} \mathrm{CH}_{2} \mathrm{CH}\left(\mathrm{CH}_{2} \mathrm{CH}_{2}\right)_{2} \mathrm{~N}, \mathrm{OH}\right) ;{ }^{13} \mathrm{C}$ NMR $\left(125 \mathrm{MHz}, \mathrm{CDCl}_{3}\right) \delta 138.4,129.3,128.8,126.6,62.8,60.5,49.6$, 48.7, 46.0, (45.9), 41.2, 38.3, 35.0, 32.8, 31.7, (31.7); IR (KBr) 3649, 3329, 2104, 1143, $1049 \mathrm{~cm}^{-1}$; HRMS $(E S I, H) m / z$ calc'd for $\mathrm{C}_{18} \mathrm{H}_{30} \mathrm{~N}_{5} \mathrm{O}_{3} \mathrm{~S}(\mathrm{M}+\mathrm{H})^{+} 396.2064$, found 396.2057. $[\alpha]_{D}^{25}+1.0$ (c 1.00, $\mathrm{CHCl}_{3}$ ).

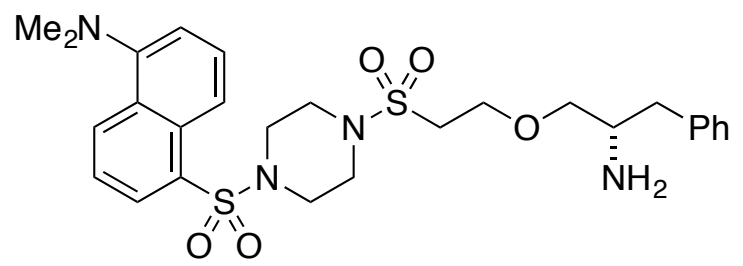

\section{$\underline{\text { Reaction conditions }}$}

1l (0.8 mmol) in mixture of DMF and THF (1.6 mL, DMF:THF=1:1), 2a (0.96 mmol), DMF $(0.8 \mathrm{~mL}),-20^{\circ} \mathrm{C}$, $24 \mathrm{~h}, \mathrm{AgOAc} / \mathrm{dppe}(24.0 \mu \mathrm{mol}), \mathrm{KHMDS}(32 \mu \mathrm{mol})$.

\section{(S)-5-((4-((2-(2-amino-3-phenylpropoxy)ethyl)sulfonyl)piperazin-1-yl)sulfonyl)- $N, N$ -}

dimethylnaphthalen-1-amine (3la): (pale green foam, $\mathrm{CH}_{2} \mathrm{Cl}_{2} / \mathrm{MeOH}=20 / 1,77 \%$ yield, $381.7 \mathrm{mg}$ with DMF); ${ }^{1} \mathrm{H}-\mathrm{NMR}\left(500 \mathrm{MHz}, \mathrm{CDCl}_{3}\right.$ ) (ppm) (with DMF) $\delta 8.58$ (d, $\left.1 \mathrm{H}, J=8.5 \mathrm{~Hz}, \mathrm{ArH}\right), 8.34(\mathrm{~d}, 1 \mathrm{H}, J=8.5 \mathrm{~Hz}$, ArH), 8.19 (dd, $1 \mathrm{H}, J=1.0,7.5 \mathrm{~Hz}, \mathrm{ArH}), 8.02(\mathrm{~s}, 1 \mathrm{H}, \mathrm{DMF}), 7.58-7.50(\mathrm{~m}, 2 \mathrm{H}, \mathrm{ArH}), 7.35-7.15(\mathrm{~m}, 6 \mathrm{H}, \mathrm{ArH})$, 
3.82-3.74, $\left(\mathrm{m}, 2 \mathrm{H}, \mathrm{SO}_{2} \mathrm{CH}_{2} \mathrm{CH}_{2} \mathrm{O}\right), 3.43\left(\mathrm{dd}, 1 \mathrm{H}, J=3.5,8.5 \mathrm{~Hz}, \mathrm{OCH}_{2} \mathrm{CHNH}_{2}\right), 3.36-3.08(\mathrm{~m}, 12 \mathrm{H}$, $\mathrm{SO}_{2} \mathrm{CH}_{2} \mathrm{CH}_{2} \mathrm{OCH}_{2} \mathrm{CHNH}_{2}, \mathrm{~N}\left(\mathrm{CH}_{2} \mathrm{CH}_{2}\right)_{2} \mathrm{~N}$ ), 2.96 (s, 3H, DMF), 2.89 (s, 6H, N( $\left.\left.\mathrm{CH}_{3}\right)_{2}\right), 2.88$ (s, 3H, DMF), 2.74 (dd, $\left.1 \mathrm{H}, J=5.5,13.5 \mathrm{~Hz}, \mathrm{CH}_{2} \mathrm{Ph}\right), 2.54\left(\mathrm{dd}, 1 \mathrm{H}, J=8.0,13.5 \mathrm{~Hz}, \mathrm{CH}_{2} \mathrm{Ph}\right), 1.45\left(\mathrm{br}, 2 \mathrm{H}, \mathrm{NH}_{2}\right) ;{ }^{13} \mathrm{C} \mathrm{NMR}(125$ $\mathrm{MHz}, \mathrm{CDCl}_{3}$ ) (with DMF) $\delta 162.7$ (DMF), 152.1, 138.5, 132.4, 131.3, 130.9, 130.4, 130.3, 129.4, 128.7, 128.4, 126.6, 123.3, 119.4, 115.5, 76.3, 64.7, 52.3, 50.4, 45.8, 45.6, 45.3, 40.9, 36.6 (DMF), 31.6 (DMF); IR (KBr) 3379, 1334, 1161, 1085, $1055 \mathrm{~cm}^{-1}$; HRMS (ESI, H) $\mathrm{m} / \mathrm{z}$ calc'd for $\mathrm{C}_{27} \mathrm{H}_{37} \mathrm{~N}_{4} \mathrm{O}_{5} \mathrm{~S}_{2}{ }^{+}(\mathrm{M}+\mathrm{H})^{+}$ 561.2200, found 561.2205. $[\alpha]_{D}^{25}-3.1$ (c 1.00, MeOH).<smiles>CNc1cccc2c(S(=O)(=O)N3CCN(S(=O)(=O)CCN[C@H](CO)Cc4ccccc4)CC3)cccc12</smiles>

(S)-2-((2-((4-((5-(dimethylamino)naphthalen-1-yl)sulfonyl)piperazin-1-yl)sulfonyl)ethyl)amino)-3-

phenylpropan-1-ol (4la): (light green solid); ${ }^{1} \mathrm{H}-\mathrm{NMR}\left(500 \mathrm{MHz}, \mathrm{CDCl}_{3}\right)$ (ppm) $\delta 8.59$ (d, $1 \mathrm{H}, J=8.5 \mathrm{~Hz}$, $\operatorname{ArH}), 8.33(\mathrm{~d}, 1 \mathrm{H}, J=9.0 \mathrm{~Hz}, \mathrm{ArH}), 8.19(\mathrm{dd}, 1 \mathrm{H}, J=1.0,7.3 \mathrm{~Hz}, \mathrm{ArH}), 7.60-7.50(\mathrm{~m}, 2 \mathrm{H}, \mathrm{ArH}), 7.32-7.13$ $(\mathrm{m}, 6 \mathrm{H}, \mathrm{ArH}), 3.58\left(\mathrm{dd}, 1 \mathrm{H}, J=4.0,10.8 \mathrm{~Hz}, \mathrm{CH} \mathrm{H}_{2} \mathrm{OH}\right), 3.35-3.15\left(\mathrm{~m}, 9 \mathrm{H}, \mathrm{CH}_{2} \mathrm{OH}, \mathrm{SO}_{2} \mathrm{CH}_{2} \mathrm{CH}_{2} \mathrm{~N}\right.$, $\left.\mathrm{SO}_{2} \mathrm{~N}\left(\mathrm{CH}_{2} \mathrm{CH}_{2}\right)_{2} \mathrm{SO}_{2}\right), 3.10-2.80\left(\mathrm{~m}, 11 \mathrm{H}, \mathrm{SO}_{2} \mathrm{~N}\left(\mathrm{CH}_{2} \mathrm{CH}_{2}\right)_{2} \mathrm{SO}_{2}, \mathrm{SO}_{2} \mathrm{CH}_{2} \mathrm{CH}_{2} \mathrm{~N}, \mathrm{NHCHCH} 2 \mathrm{OH}, \mathrm{N}\left(\mathrm{CH}_{3}\right)_{2}\right), 2.71$ (dd, $\left.2 \mathrm{H}, J=6.5 \mathrm{~Hz}, \mathrm{CH}_{2} \mathrm{Ph}\right), 2.48(\mathrm{br}, 1 \mathrm{H}, \mathrm{NH}), 1.54(\mathrm{br}, 1 \mathrm{H}, \mathrm{OH}) ;{ }^{13} \mathrm{C} \mathrm{NMR}\left(125 \mathrm{MHz}, \mathrm{CDCl}_{3}\right) \delta 152.1,138.3$, 123.4, 131.3, 130.9, 130.4, 130.3, 129.3, 128.8, 128.5, 126.7, 123.3, 119.3, 115.6, 63.0, 60.6, 50.2, 45.7, 45.6, 45.4, 41.2, 38.3; IR (KBr) 3616, 3365, 1230, 1161, $1049 \mathrm{~cm}^{-1}$; HRMS (ESI, H) $\mathrm{m} / \mathrm{z}$ calc'd for $\mathrm{C}_{27} \mathrm{H}_{37} \mathrm{~N}_{4} \mathrm{O}_{5} \mathrm{~S}_{2}^{+}(\mathrm{M}+\mathrm{H})^{+} 561.2200$, found 561.2202. [ $\left.\alpha\right]_{D}^{25}+1.5$ (c 1.00, $\left.\mathrm{CHCl}_{3}\right)$.

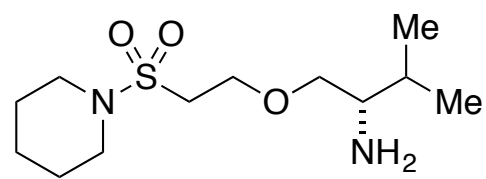

\section{Reaction conditions}

1e $(0.8 \mathrm{mmol})$ in DMF $(0.8 \mathrm{~mL}), 2 \mathrm{~b}(0.96 \mathrm{mmol}), \mathrm{DMF}(0.8 \mathrm{~mL}),-20^{\circ} \mathrm{C}, 8 \mathrm{~h}, \mathrm{AgOAc} / \mathrm{dppe}(24.0 \mu \mathrm{mol})$, KHMDS $(32 \mu \mathrm{mol})$.

(S)-3-methyl-1-(2-(piperidin-1-ylsulfonyl)ethoxy)butan-2-amine (3eb): (pale yellow oil, $\mathrm{CH}_{2} \mathrm{Cl}_{2} / \mathrm{MeOH}=$ 9/1, 90\% yield, $199.9 \mathrm{mg}$ ); ${ }^{1} \mathrm{H}-\mathrm{NMR}\left(500 \mathrm{MHz}, \mathrm{CDCl}_{3}\right.$ ) (ppm) $\delta$ 3.92-3.78 (m, 2H, $\mathrm{SO}_{2} \mathrm{CH}_{2} \mathrm{CH}_{2} \mathrm{O}$ ), 3.53 (dd, $\left.1 \mathrm{H}, J=9.3,6.0 \mathrm{~Hz}, \mathrm{OCH}_{2} \mathrm{CHN}\right), 3.35-3.15\left(\mathrm{~m}, 7 \mathrm{H}, \mathrm{OCH}_{2} \mathrm{CHN}, \mathrm{CH}_{2}\left(\mathrm{CH}_{2} \mathrm{CH}_{2}\right)_{2} \mathrm{~N}, \mathrm{SO}_{2} \mathrm{CH}_{2}\right), 2.81-2.73(\mathrm{~m}, 1 \mathrm{H}$, 
$\left.\mathrm{NH}_{2} \mathrm{CH}\right), 1.82\left(\mathrm{br}, 2 \mathrm{H}, \mathrm{NH}_{2}\right), 1.70-1.50\left(\mathrm{~m}, 7 \mathrm{H}, \mathrm{CH}_{2}\left(\mathrm{CH}_{2} \mathrm{CH}_{2}\right)_{2} \mathrm{~N}, \mathrm{CH}\left(\mathrm{CH}_{3}\right)_{2}\right), 0.93\left(\mathrm{~d}, 6 \mathrm{H}, J=6.5 \mathrm{~Hz}, \mathrm{CH}\left(\mathrm{CH}_{3}\right)_{2}\right)$;

${ }^{13} \mathrm{CNMR}\left(125 \mathrm{MHz}, \mathrm{CDCl}_{3}\right) \delta 74.7,64.8,56.2,49.4,46.5,30.8,25.7,23.9,19.4,18.2 ; \mathrm{IR}$ (thin film, $\mathrm{NaCl}$ ) $3379,1467,1357,1163,1109 \mathrm{~cm}^{-1}$; HRMS (ESI, H) m/z calc'd for $\mathrm{C}_{12} \mathrm{H}_{27} \mathrm{~N}_{2} \mathrm{O}_{3} \mathrm{~S}(\mathrm{M}+\mathrm{H})^{+} 279.1737$, found 279.1738. $[\alpha]_{D}^{25}+10.5\left(c 1.00, \mathrm{CHCl}_{3}\right)$.<smiles>CC(C)[C@H](CO)NCCS(=O)(=O)N1CCCCC1</smiles>

(S)-3-methyl-2-((2-(piperidin-1-ylsulfonyl)ethyl)amino)butan-1-ol (4eb): (white solid); ${ }^{1} \mathrm{H}-\mathrm{NMR}$ (500 $\left.\mathrm{MHz}_{2} \mathrm{CDCl}_{3}\right)(\mathrm{ppm}) \delta 3.6\left(\mathrm{dd}, 1 \mathrm{H}, \mathrm{J}=6.0,10.5 \mathrm{~Hz}, \mathrm{CH}_{2} \mathrm{OH}\right.$ ), 3.33 (dd, $1 \mathrm{H}, \mathrm{J}=6.0,10.8 \mathrm{~Hz}, \mathrm{CH}_{2} \mathrm{OH}$ ), 3.28$3.18\left(\mathrm{~m}, 5 \mathrm{H}, \mathrm{SO}_{2} \mathrm{CH}_{2} \mathrm{CH}_{2} \mathrm{~N}, \mathrm{CH}_{2}\left(\mathrm{CH}_{2} \mathrm{CH}_{2}\right)_{2} \mathrm{~N}\right)$, 3.10-2.96 (m, 3H, $\left.\mathrm{SO}_{2} \mathrm{CH}_{2} \mathrm{CH}_{2} \mathrm{~N}\right), 2.84(\mathrm{br}, 1 \mathrm{H}, \mathrm{NH}), 2.40-2.35$ $(\mathrm{m}, 1 \mathrm{H}, \mathrm{HNCH}), 1.85-1.76\left(\mathrm{~m}, 1 \mathrm{H}, \mathrm{CH}\left(\mathrm{CH}_{3}\right)_{2}\right), 1.70-1.62\left(\mathrm{~m}, 4 \mathrm{H}, \mathrm{CH}_{2}\left(\mathrm{CH}_{2} \mathrm{CH}_{2}\right)_{2} \mathrm{~N}\right), 1.60-1.52(\mathrm{~m}, 3 \mathrm{H}$, $\left.\mathrm{CH}_{2}\left(\mathrm{CH}_{2} \mathrm{CH}_{2}\right)_{2} \mathrm{~N}, \mathrm{OH}\right), 0.95$ (dd, $\left.6 \mathrm{H}, J=7.0,36.0 \mathrm{~Hz}, \mathrm{CH}\left(\mathrm{CH}_{3}\right)_{2}\right) ;{ }^{13} \mathrm{CNMR}\left(125 \mathrm{MHz}, \mathrm{CDCl}_{3}\right) \delta 64.8,60.9$, 49.6, 46.8, 41.6, 29.3, 25.8, 23.9, 19.6, 18.7; IR (KBr) 3643, 3356, 1157, 1057, $1028 \mathrm{~cm}^{-1}$; HRMS (ESI, H) $\mathrm{m} / \mathrm{z}$ calc'd for $\mathrm{C}_{12} \mathrm{H}_{27} \mathrm{~N}_{2} \mathrm{O}_{3} \mathrm{~S}^{+}(\mathrm{M}+\mathrm{H})^{+} 279.1737$, found 279.1735. [ $\left.\alpha\right]_{D}{ }^{25}+21.0$ (c 1.00, $\mathrm{CHCl}_{3}$ ).

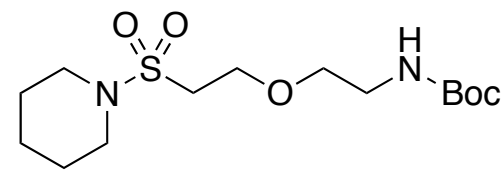

\section{Reaction conditions}

1e $(0.8 \mathrm{mmol})$ in DMF $(0.8 \mathrm{~mL}), 2 \mathrm{c}(0.96 \mathrm{mmol}), \mathrm{DMF}(0.8 \mathrm{~mL}),-20^{\circ} \mathrm{C}, 4.5 \mathrm{~h}, \mathrm{AgOAc} / \mathrm{dppe}(24.0 \mu \mathrm{mol})$, KHMDS $(32 \mu \mathrm{mol}) ;$ Boc protection: $\mathrm{Boc}_{2} \mathrm{O}(1.1 \mathrm{mmol}), \mathrm{Et}_{3} \mathrm{~N}(1.4 \mathrm{mmol}), \mathrm{CH}_{2} \mathrm{Cl}_{2}(10 \mathrm{~mL}), 4{ }^{\circ} \mathrm{C}$ to $\mathrm{RT}$, overnight.

tert-butyl (2-(2-(piperidin-1-ylsulfonyl)ethoxy)ethyl)carbamate (3ec): (white semisolid, EtOAC/ Hexane $=1 / 1,86 \%$ yield for two steps, $232.5 \mathrm{mg}) ;{ }^{1} \mathrm{H}-\mathrm{NMR}\left(500 \mathrm{MHz}, \mathrm{CDCl}_{3}\right)(\mathrm{ppm}) \delta 4.92(\mathrm{br}, 1 \mathrm{H}, \mathrm{NH}), 3.84(\mathrm{t}$, $2 \mathrm{H}, J=6.0 \mathrm{~Hz}, \mathrm{SO}_{2} \mathrm{CH}_{2} \mathrm{CH}_{2} \mathrm{O}$, ), $3.53\left(\mathrm{t}, 2 \mathrm{H}, J=5.0 \mathrm{~Hz}, \mathrm{OCH}_{2} \mathrm{CH}_{2} \mathrm{NH}\right), 3.33\left(\mathrm{~d}, 2 \mathrm{H}, J=5.0 \mathrm{~Hz}, \mathrm{OCH}_{2} \mathrm{CH}_{2} \mathrm{NH}\right.$ ), $3.24\left(\mathrm{t}, 4 \mathrm{H}, J=5.5 \mathrm{~Hz}, \mathrm{CH}_{2}\left(\mathrm{CH}_{2} \mathrm{CH}_{2}\right)_{2} \mathrm{~N}\right), 3.17\left(\mathrm{t}, 2 \mathrm{H}, J=6.0 \mathrm{~Hz}, \mathrm{SO}_{2} \mathrm{CH}_{2} \mathrm{CH}_{2} \mathrm{O}\right), 1.70-1.55(\mathrm{~m}, 6 \mathrm{H}$, $\left.\mathrm{CH}_{2}\left(\mathrm{CH}_{2} \mathrm{CH}_{2}\right)_{2} \mathrm{~N}\right), 1.45\left(\mathrm{~s}, 9 \mathrm{H}, \mathrm{C}\left(\mathrm{CH}_{3}\right)_{3}\right) ;{ }^{13} \mathrm{C}$ NMR $\left(125 \mathrm{MHz}, \mathrm{CDCl}_{3}\right) \delta$ 156.1, 79.5, 70.4, 64.7, 49.5, 46.6, 40.4, 28.5, 25.8, 24.0; IR (KBr) 3360, 1680, 1336, 1157, $1141 \mathrm{~cm}^{-1}$; HRMS (ESI, H) $\mathrm{m} / \mathrm{z}$ calc'd for $\mathrm{C}_{14} \mathrm{H}_{29} \mathrm{~N}_{2} \mathrm{O}_{5} \mathrm{~S}(\mathrm{M}+\mathrm{H})^{+}$337.1792, found 337.1791. 


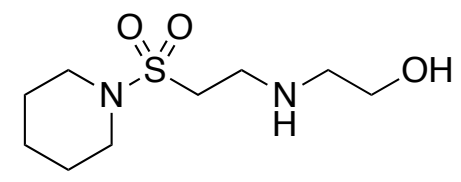

2-((2-(piperidin-1-ylsulfonyl)ethyl)amino)ethan-1-ol (4ec): (white solid); ${ }^{1} \mathrm{H}-\mathrm{NMR}$ (500 $\mathrm{MHz}, \mathrm{CDCl}_{3}$ ) (ppm) $\delta 3.65$ (t, 2H, J=5.0 Hz, CH $\left.\mathrm{Hz}, \mathrm{CH}_{2} \mathrm{NHCH}_{2}\right), 2.81\left(\mathrm{t}, 2 \mathrm{H}, J=5.0 \mathrm{~Hz}, \mathrm{SO}_{2} \mathrm{CH}_{2}\right), 2.40-1.40\left(\mathrm{~m}, 8 \mathrm{H}, \mathrm{OH}, \mathrm{NH}, \mathrm{CH}_{2}\left(\mathrm{CH}_{2} \mathrm{CH}_{2}\right)_{2} \mathrm{~N}\right) ;{ }^{13} \mathrm{C} \mathrm{NMR}(125$ $\left.\mathrm{MHz}, \mathrm{CDCl}_{3}\right) \delta 61.0,50.9,49.1,46.8,43.3,25.8,23.9$; IR (KBr) 3381, 1616, 1159, 1064, $1022 \mathrm{~cm}^{-1}$; HRMS (ESI, H) $m / z$ calc'd for $\mathrm{C}_{9} \mathrm{H}_{21} \mathrm{~N}_{2} \mathrm{O}_{3} \mathrm{~S}^{+}(\mathrm{M}+\mathrm{H})^{+}$237.1267, found 237.1265.

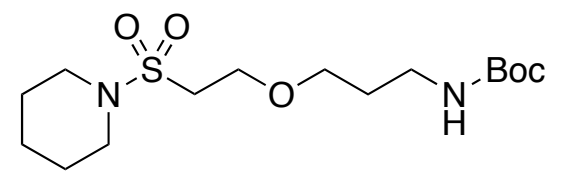

Reaction conditions

1e $(0.8 \mathrm{mmol})$ in DMF $(0.8 \mathrm{~mL}), 2 \mathrm{~d}(0.96 \mathrm{mmol}), \mathrm{DMF}(0.8 \mathrm{~mL}),-20^{\circ} \mathrm{C}, 4 \mathrm{~h}, \mathrm{AgOAc} / \mathrm{dppe}(24.0 \mu \mathrm{mol})$, KHMDS $(32 \mu \mathrm{mol})$; Boc protection: $\mathrm{Boc}_{2} \mathrm{O}(1.1 \mathrm{mmol}), \mathrm{Et}_{3} \mathrm{~N}(1.4 \mathrm{mmol}), \mathrm{CH}_{2} \mathrm{Cl}_{2}(10 \mathrm{~mL}), 4{ }^{\circ} \mathrm{C}$ to $\mathrm{RT}$, overnight.

tert-butyl (3-(2-(piperidin-1-ylsulfonyl)ethoxy)propyl)carbamate (3ed): (white semisolid, EtOAC/ Hexane $=1 / 1,77 \%$ yield, $215.5 \mathrm{mg}) ;{ }^{1} \mathrm{H}-\mathrm{NMR}\left(500 \mathrm{MHz}, \mathrm{CDCl}_{3}\right)(\mathrm{ppm}) \delta 4.83(1 \mathrm{H}, \mathrm{br}, \mathrm{NH}), 3.81(\mathrm{t}, 2 \mathrm{H}, J=$ $\left.6.5 \mathrm{~Hz}, \mathrm{SO}_{2} \mathrm{CH}_{2} \mathrm{CH}_{2} \mathrm{O}\right), 3.52\left(\mathrm{t}, 2 \mathrm{H}, J=6.0 \mathrm{~Hz}, \mathrm{OCH}_{2} \mathrm{CH}_{2} \mathrm{CH}_{2} \mathrm{NH}\right), 3.30-3.10\left(\mathrm{~m}, 8 \mathrm{H}, \mathrm{SO}_{2} \mathrm{CH}_{2} \mathrm{CH}_{2} \mathrm{O}\right.$, $\left.\mathrm{OCH}_{2} \mathrm{CH}_{2} \mathrm{CH}_{2} \mathrm{NH}, \mathrm{CH}_{2}\left(\mathrm{CH}_{2} \mathrm{CH}_{2}\right)_{2} \mathrm{~N}\right), 1.80-1.73\left(\mathrm{~m}, 2 \mathrm{H}, \mathrm{OCH}_{2} \mathrm{CH}_{2} \mathrm{CH}_{2} \mathrm{~N}\right), 1.70-1.53\left(\mathrm{~m}, 6 \mathrm{H}, \mathrm{CH}_{2}\left(\mathrm{CH}_{2} \mathrm{CH}_{2}\right)_{2} \mathrm{~N}\right)$, $1.44\left(\mathrm{~s}, 9 \mathrm{H}, \mathrm{C}\left(\mathrm{CH}_{3}\right)_{3}\right) ;{ }^{13} \mathrm{C} \mathrm{NMR}\left(125 \mathrm{MHz}, \mathrm{CDCl}_{3}\right) \delta 156.1,79.2,69.4,64.7,49.5,46.6,38.3,30.0,28.6$, 25.8, 24.0; IR (KBr) 3379, 1687, 1325, 1157, $1143 \mathrm{~cm}^{-1}$; HRMS (ESI, H) $\mathrm{m} / \mathrm{z}$ calc'd for $\mathrm{C}_{15} \mathrm{H}_{31} \mathrm{~N}_{2} \mathrm{O}_{5} \mathrm{~S}(\mathrm{M}+\mathrm{H})^{+}$ 351.1948 , found 351.1945 .<smiles>O=S(=O)(CCNCCCO)N1CCCCC1</smiles>

3-((2-(piperidin-1-ylsulfonyl)ethyl)amino)propan-1-ol (4ed): (colourless oil); ${ }^{1} \mathrm{H}-\mathrm{NMR}\left(500 \mathrm{MHz}, \mathrm{CDCl}_{3}\right.$ ) 
(ppm) $\delta 3.79\left(\mathrm{t}, 2 \mathrm{H}, J=5.0 \mathrm{~Hz}, \mathrm{HOCH}_{2}\right), 3.23\left(\mathrm{t}, 4 \mathrm{H}, J=5.0 \mathrm{~Hz}, \mathrm{CH}_{2}\left(\mathrm{CH}_{2} \mathrm{CH}_{2}\right)_{2} \mathrm{~N}\right), 3.08(\mathrm{dt}, 4 \mathrm{H}, J=29.5,6.0$ $\left.\mathrm{Hz}, \mathrm{CH}_{2} \mathrm{NHCH}_{2}\right), 2.88\left(\mathrm{t}, 2 \mathrm{H}, J=5.5 \mathrm{~Hz}, \mathrm{SO}_{2} \mathrm{CH}_{2}\right), 1.78-1.44\left(\mathrm{~m}, 10 \mathrm{H}, \mathrm{OH}, \mathrm{NH}, \mathrm{CH}_{2}\left(\mathrm{CH}_{2} \mathrm{CH}_{2}\right)_{2} \mathrm{~N}\right.$, $\mathrm{NCH}_{2} \mathrm{CH}_{2} \mathrm{CH}_{2} \mathrm{O}$ ); ${ }^{13} \mathrm{C}$ NMR $\left(125 \mathrm{MHz}, \mathrm{CDCl}_{3}\right) \delta$ 64.0, 49.3, 48.6, 46.7, 43.6, 30.9, 25.7, 23.9; IR (thin film, $\mathrm{NaCl}$ ) 3524, 3309, 1161, 1053, $1028 \mathrm{~cm}^{-1}$; HRMS (ESI, H) $\mathrm{m} / z$ calc'd for $\mathrm{C}_{10} \mathrm{H}_{23} \mathrm{~N}_{2} \mathrm{O}_{3} \mathrm{~S}^{+}(\mathrm{M}+\mathrm{H})^{+} 251.1424$, found 251.1423 .

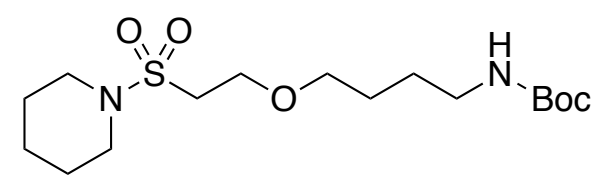

\section{$\underline{\text { Reaction conditions }}$}

1e $(0.8 \mathrm{mmol})$ in DMF (0.8 mL), $2 \mathrm{e}(0.96 \mathrm{mmol}), \mathrm{DMF}(0.8 \mathrm{~mL}),-20^{\circ} \mathrm{C}, 8 \mathrm{~h}, \mathrm{AgOAc} / \mathrm{dppe}(24.0 \mu \mathrm{mol})$, KHMDS $(32 \mu \mathrm{mol}) ;$ Boc protection: $\mathrm{Boc}_{2} \mathrm{O}(1.1 \mathrm{mmol}), \mathrm{Et}_{3} \mathrm{~N}(1.4 \mathrm{mmol}), \mathrm{CH}_{2} \mathrm{Cl}_{2}(10 \mathrm{~mL}), 4{ }^{\circ} \mathrm{C}$ to $\mathrm{RT}$, overnight.

tert-butyl (4-(2-(piperidin-1-ylsulfonyl)ethoxy)butyl)carbamate (3ee): (white solid, EtOAc/ Hexane = 1/1, $76 \%$ yield, $222.7 \mathrm{mg}) ;{ }^{1} \mathrm{H}-\mathrm{NMR}\left(500 \mathrm{MHz}, \mathrm{CDCl}_{3}\right)(\mathrm{ppm}) \delta 4.60(1 \mathrm{H}, \mathrm{br}, \mathrm{NH}), 3.79(\mathrm{t}, 2 \mathrm{H}, J=6.5 \mathrm{~Hz}$, $\left.\mathrm{SO}_{2} \mathrm{CH}_{2} \mathrm{CH}_{2} \mathrm{O}\right), 3.47\left(\mathrm{t}, 2 \mathrm{H}, J=6.0 \mathrm{~Hz}, \mathrm{OCH}_{2} \mathrm{CH}_{2} \mathrm{CH}_{2} \mathrm{CH}_{2} \mathrm{NH}\right), 3.30-3.08\left(\mathrm{~m}, 8 \mathrm{H}, \mathrm{SO}_{2} \mathrm{CH}_{2}, \mathrm{CH}_{2} \mathrm{NH}\right.$, $\left.\mathrm{CH}_{2}\left(\mathrm{CH}_{2} \mathrm{CH}_{2}\right)_{2} \mathrm{~N}\right), 1.70-1.50\left(\mathrm{~m}, 10 \mathrm{H}, \mathrm{CH}_{2}\left(\mathrm{CH}_{2} \mathrm{CH}_{2}\right)_{2} \mathrm{~N}, \mathrm{OCH}_{2} \mathrm{CH}_{2} \mathrm{CH}_{2} \mathrm{CH}_{2} \mathrm{NH}\right), 1.44\left(\mathrm{~s}, 9 \mathrm{H}, \mathrm{C}\left(\mathrm{CH}_{3}\right)_{3}\right) ;{ }^{13} \mathrm{C} \mathrm{NMR}$ $\left(125 \mathrm{MHz}, \mathrm{CDCl}_{3}\right) \delta$ 156.1, 79.2, 71.1, 64.5, 49.7, 46.6, 40.4, 28.6, 28.6, 27.0, 25.8, 34.0; IR (KBr) 3358, 1681, 1323, 1159, $1139 \mathrm{~cm}^{-1}$; HRMS (ESI, H) $\mathrm{m} / \mathrm{z}$ calc'd for $\mathrm{C}_{16} \mathrm{H}_{33} \mathrm{~N}_{2} \mathrm{O}_{5} \mathrm{~S}(\mathrm{M}+\mathrm{H})^{+} 365.2105$, found 365.2106.

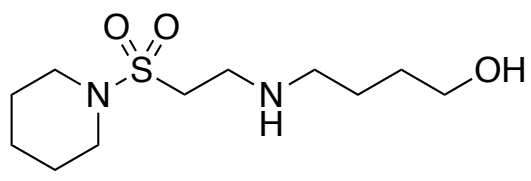

4-((2-(piperidin-1-ylsulfonyl)ethyl)amino)butan-1-ol (4ee): (pale yellow oil); ${ }^{1} \mathrm{H}-\mathrm{NMR}\left(500 \mathrm{MHz}, \mathrm{CDCl}_{3}\right.$ ) (ppm) $\delta 3.59$ (t, $\left.2 \mathrm{H}, J=5.0 \mathrm{~Hz}, \mathrm{HOCH}_{2}\right), 3.23\left(\mathrm{t}, 4 \mathrm{H}, J=5.5 \mathrm{~Hz}, \mathrm{CH}_{2}\left(\mathrm{CH}_{2} \mathrm{CH}_{2}\right){ }_{2} \mathrm{~N}\right), 3.15-3.00(\mathrm{~m}, 4 \mathrm{H}$, $\mathrm{CH}_{2} \mathrm{NHCH}_{2}$ ), $2.68\left(\mathrm{t}, 2 \mathrm{H}, \mathrm{J}=5.5 \mathrm{~Hz}, \mathrm{SO}_{2} \mathrm{CH}_{2}\right), 1.72-1.50\left(\mathrm{~m}, 1 \mathrm{OH}, \mathrm{CH}_{2}\left(\mathrm{CH}_{2} \mathrm{CH}_{2}\right)_{2} \mathrm{~N}, \mathrm{OCH}_{2} \mathrm{CH}_{2} \mathrm{CH}_{2} \mathrm{CH}_{2} \mathrm{~N}\right)$, 5.5$0.5(\mathrm{br}, 2 \mathrm{H}, \mathrm{OH}, \mathrm{NH}){ }^{13} \mathrm{C}$ NMR $\left(125 \mathrm{MHz}, \mathrm{CDCl}_{3}\right) \delta 62.8,49.6,48.4,46.7,43.3,32.3,28.4,25.7,23.9 ; \mathrm{IR}$ (KBr) 3649, 1608, 1163, 1053, $1028 \mathrm{~cm}^{-1}$; HRMS (ESI, H) $\mathrm{m} / z$ calc'd for $\mathrm{C}_{11} \mathrm{H}_{25} \mathrm{~N}_{2} \mathrm{O}_{3} \mathrm{~S}^{+}(\mathrm{M}+\mathrm{H})^{+} 265.1580$, found 265.1579 . 


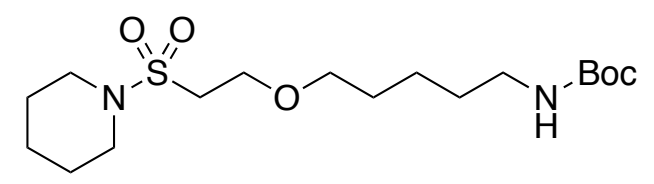

\section{$\underline{\text { Reaction conditions }}$}

1e $(0.8 \mathrm{mmol})$ in DMF $(0.8 \mathrm{~mL}), 2 \mathrm{f}(0.96 \mathrm{mmol}), \mathrm{DMF}(0.8 \mathrm{~mL}),-20^{\circ} \mathrm{C}, 8 \mathrm{~h}, \mathrm{AgOAc} / \mathrm{dppe}(24.0 \mu \mathrm{mol})$, KHMDS $(32 \mu \mathrm{mol}) ;$ Boc protection: $\mathrm{Boc}_{2} \mathrm{O}(1.1 \mathrm{mmol}), \mathrm{Et}_{3} \mathrm{~N}(1.4 \mathrm{mmol}), \mathrm{CH}_{2} \mathrm{Cl}_{2}(10 \mathrm{~mL}), 4{ }^{\circ} \mathrm{C}$ to $\mathrm{RT}$, overnight.

tert-butyl (5-(2-(piperidin-1-ylsulfonyl)ethoxy)pentyl)carbamate (3ef): (white solid, EtOAc/ Hexane = 1/1, 80\% yield, $243.4 \mathrm{mg}) ;{ }^{1} \mathrm{H}-\mathrm{NMR}\left(500 \mathrm{MHz}^{\mathrm{C} C D C l}\right)(\mathrm{ppm}) \delta 4.53(1 \mathrm{H}, \mathrm{br}, \mathrm{NH}), 3.78(\mathrm{t}, 2 \mathrm{H}, J=6.5 \mathrm{~Hz}$, $\left.\mathrm{SO}_{2} \mathrm{CH}_{2} \mathrm{CH}_{2} \mathrm{O}\right), 3.45\left(\mathrm{t}, 2 \mathrm{H}, J=6.5 \mathrm{~Hz}, \mathrm{OCH}_{2} \mathrm{CH}_{2} \mathrm{CH}_{2} \mathrm{CH}_{2} \mathrm{CH}_{2} \mathrm{NH}\right), 3.30-3.08\left(\mathrm{~m}, 8 \mathrm{H}, \mathrm{SO}_{2} \mathrm{CH}_{2}, \mathrm{CH}_{2} \mathrm{NH}\right.$, $\left.\mathrm{CH}_{2}\left(\mathrm{CH}_{2} \mathrm{CH}_{2}\right)_{2} \mathrm{~N}\right)$, 1.70-1.30 (m, $\left.21 \mathrm{H}, \mathrm{CH}_{2}\left(\mathrm{CH}_{2} \mathrm{CH}_{2}\right)_{2} \mathrm{~N}, \mathrm{OCH}_{2} \mathrm{CH}_{2} \mathrm{CH}_{2} \mathrm{CH}_{2} \mathrm{NH}, \mathrm{C}\left(\mathrm{CH}_{3}\right)_{3}\right) ;{ }^{13} \mathrm{C}$ NMR (125 MHz, $\left.\mathrm{CDCl}_{3}\right) \delta 156.1,79.2,71.3,64.5,49.7,46.5,40.6,30.0,29.3,28.6,25.8,24.0,23.5 ; \mathrm{IR}(\mathrm{KBr}) 3375,1687$, 1325, 1157, $1141 \mathrm{~cm}^{-1}$; HRMS (ESI, H) $\mathrm{m} / z$ calc'd for $\mathrm{C}_{17} \mathrm{H}_{35} \mathrm{~N}_{2} \mathrm{O}_{5} \mathrm{~S}(\mathrm{M}+\mathrm{H})^{+} 379.2261$, found 379.2266 .

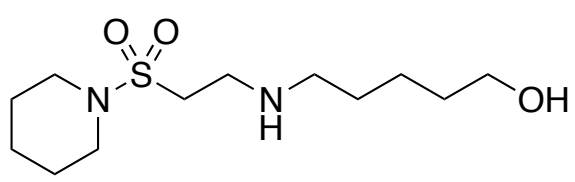

5-((2-(piperidin-1-ylsulfonyl)ethyl)amino)pentan-1-ol (4ef): (colorless oil); ${ }^{1} \mathrm{H}-\mathrm{NMR}\left(500 \mathrm{MHz}, \mathrm{CDCl}_{3}\right.$ ) (ppm) $\delta 3.65\left(\mathrm{t}, 2 \mathrm{H}, J=56.5 \mathrm{~Hz}, \mathrm{HOCH}_{2}\right), 3.24\left(\mathrm{t}, 4 \mathrm{H}, J=5.5 \mathrm{~Hz}, \mathrm{CH}_{2}\left(\mathrm{CH}_{2} \mathrm{CH}_{2}\right)_{2} \mathrm{~N}\right), 3.12-3.02(\mathrm{~m}, 4 \mathrm{H}$, $\mathrm{CH}_{2} \mathrm{NHCH}_{2}$ ), $2.64\left(\mathrm{t}, 2 \mathrm{H}, J=7.0 \mathrm{~Hz}, \mathrm{SO}_{2} \mathrm{CH}_{2}\right), 1.72-1.35\left(\mathrm{~m}, 14 \mathrm{H}, \mathrm{CH}_{2}\left(\mathrm{CH}_{2} \mathrm{CH}_{2}\right){ }_{2} \mathrm{~N}, \mathrm{OCH}_{2} \mathrm{CH}_{2} \mathrm{CH}_{2} \mathrm{CH}_{2} \mathrm{CH}_{2} \mathrm{~N}\right)$, $\mathrm{OH}, \mathrm{NH}){ }^{13} \mathrm{CNMR}\left(125 \mathrm{MHz}, \mathrm{CDCl}_{3}\right) \delta 62.9,49.7,49.0,46.7,43.7,32.6,29.7,25.8,23.9,23.5$; IR (thin film, $\mathrm{NaCl}$ ) 3520, 1244, 1111, 1053, $1028 \mathrm{~cm}^{-1}$; HRMS (ESI, H) $\mathrm{m} / z$ calc'd for $\mathrm{C}_{12} \mathrm{H}_{27} \mathrm{~N}_{2} \mathrm{O}_{3} \mathrm{~S}^{+}(\mathrm{M}+\mathrm{H})^{+} 279.1737$, found 279.1739 .

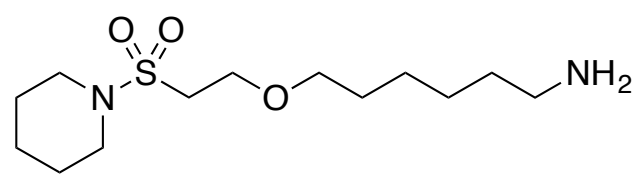

$\underline{\text { Reaction conditions }}$ 
1e $(0.8 \mathrm{mmol})$ in DMF $(0.8 \mathrm{~mL}), 2 \mathrm{~g}(0.96 \mathrm{mmol}), \mathrm{DMF}(0.8 \mathrm{~mL}),-20^{\circ} \mathrm{C}, 1 \mathrm{~h}, \mathrm{AgOAc} / \mathrm{dppe}(24.0 \mu \mathrm{mol})$, KHMDS $(32 \mu \mathrm{mol})$.

6-(2-(piperidin-1-ylsulfonyl)ethoxy)hexan-1-amine (3eg): (pale yellow oil, $\mathrm{CH}_{2} \mathrm{Cl}_{2} / \mathrm{MeOH} / \mathrm{PrNH}_{2}=$ 200/10/5, 79\% yield, $185.9 \mathrm{mg}) ;{ }^{1} \mathrm{H}-\mathrm{NMR}\left(500 \mathrm{MHz}, \mathrm{CDCl}_{3}\right)(\mathrm{ppm}) \delta 3.78\left(\mathrm{t}, 2 \mathrm{H}, J=6.0 \mathrm{~Hz}, \mathrm{SO}_{2} \mathrm{CH}_{2} \mathrm{CH}_{2} \mathrm{O}\right)$, $3.45\left(\mathrm{t}, 2 \mathrm{H}, J=7.0 \mathrm{~Hz}, \mathrm{OCH}_{2} \mathrm{CH}_{2} \mathrm{CH}_{2} \mathrm{CH}_{2} \mathrm{CH}_{2} \mathrm{CH}_{2} \mathrm{NH}_{2}\right.$ ), 3.28-3.15 (m, $6 \mathrm{H}, \mathrm{OCH}_{2} \mathrm{CH}_{2} \mathrm{CH}_{2} \mathrm{CH}_{2} \mathrm{CH}_{2} \mathrm{CH}_{2} \mathrm{NH}_{2}$, $\left.\mathrm{CH}_{2}\left(\mathrm{CH}_{2} \mathrm{CH}_{2}\right)_{2} \mathrm{NSO}_{2}\right), 2.69\left(\mathrm{t}, 2 \mathrm{H}, J=7.0 \mathrm{~Hz}, \mathrm{SO}_{2} \mathrm{CH}_{2} \mathrm{CH}_{2} \mathrm{O}\right), 1.69-1.20\left(\mathrm{~m}, 16 \mathrm{H}, \mathrm{OCH}_{2} \mathrm{CH}_{2} \mathrm{CH}_{2} \mathrm{CH}_{2} \mathrm{CH}_{2} \mathrm{CH}_{2} \mathrm{NH}_{2}\right.$, $\left.\mathrm{CH}_{2}\left(\mathrm{CH}_{2} \mathrm{CH}_{2}\right)_{2} \mathrm{NSO}_{2}\right) ;{ }^{13} \mathrm{C} \mathrm{NMR}\left(125 \mathrm{MHz}, \mathrm{CDCl}_{3}\right) \delta 71.6,64.5,49.8,46.5,42.3,33.9,29.7,26.9,26.2,25.8$, 24.0; IR (KBr) 3348, 1371, 1217, 1155, $1116 \mathrm{~cm}^{-1}$; HRMS (ESI, H) $\mathrm{m} / \mathrm{z}$ calc'd for $\mathrm{C}_{13} \mathrm{H}_{29} \mathrm{~N}_{2} \mathrm{O}_{3} \mathrm{~S}(\mathrm{M}+\mathrm{H})^{+}$ 293.1893, found 293.1892.

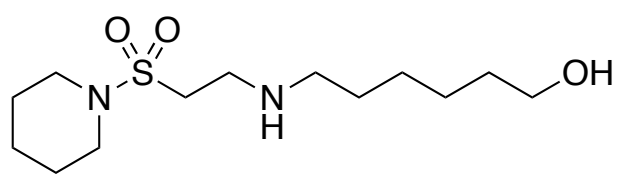

6-((2-(piperidin-1-ylsulfonyl)ethyl)amino)hexan-1-ol (4eg): (white solid); ${ }^{1} \mathrm{H}-\mathrm{NMR}$ (500 $\mathrm{MHz}, \mathrm{CDCl}_{3}$ ) (ppm) $\delta 3.64\left(\mathrm{t}, 2 \mathrm{H}, J=7.0 \mathrm{~Hz}, \mathrm{HOCH}_{2} \mathrm{CH}_{2} \mathrm{CH}_{2} \mathrm{CH}_{2} \mathrm{CH}_{2} \mathrm{CH}_{2} \mathrm{NH}\right), 3.24(\mathrm{t}, 4 \mathrm{H}, J=5.0 \mathrm{~Hz}$, $\mathrm{HOCH}_{2} \mathrm{CH}_{2} \mathrm{CH}_{2} \mathrm{CH}_{2} \mathrm{CH}_{2} \mathrm{CH}_{2} \mathrm{NH}, \mathrm{SO}_{2} \mathrm{CH}_{2} \mathrm{CH}_{2} \mathrm{~N}$ ), 3.12-3.02 (m, $\left.4 \mathrm{H}, \mathrm{CH}_{2}\left(\mathrm{CH}_{2} \mathrm{CH}_{2}\right)_{2} \mathrm{NSO}_{2}\right), 2.62(\mathrm{t}, 2 \mathrm{H}, \mathrm{J}=7.0 \mathrm{~Hz}$, $\left.\mathrm{SO}_{2} \mathrm{CH}_{2} \mathrm{CH}_{2} \mathrm{~N}\right), \quad 1.70-1.64\left(\mathrm{~m}, 4 \mathrm{H}, \quad \mathrm{CH}_{2}\left(\mathrm{CH}_{2} \mathrm{CH}_{2}\right)_{2} \mathrm{NSO}_{2}\right), \quad 1.61-1.44\left(\mathrm{~m}, 7 \mathrm{H}, \mathrm{CH}_{2}\left(\mathrm{CH}_{2} \mathrm{CH}_{2}\right)_{2} \mathrm{NSO}_{2}\right.$, $\mathrm{HOCH}_{2} \mathrm{CH}_{2} \mathrm{CH}_{2} \mathrm{CH}_{2} \mathrm{CH}_{2} \mathrm{CH}_{2} \mathrm{NH}$ ), 1.43-1.32 (m, $5 \mathrm{H}, \mathrm{HOCH}_{2} \mathrm{CH}_{2} \mathrm{CH}_{2} \mathrm{CH}_{2} \mathrm{CH}_{2} \mathrm{CH}_{2} \mathrm{NH}$ ); ${ }^{13} \mathrm{C} \mathrm{NMR}\left(125 \mathrm{MHz}, \mathrm{CDCl}_{3}\right.$ ) $\delta$ 62.8, 49.7, 48.9, 46.7, 43.7, 32.8, 30.0, 27.1, 25.7, 25.7, 23.9; IR (KBr) 3329, 1637, 1157, 1062, 1026 $\mathrm{cm}^{-1}$; HRMS (ESI, H) $\mathrm{m} / z$ calc'd for $\mathrm{C}_{13} \mathrm{H}_{29} \mathrm{~N}_{2} \mathrm{O}_{3} \mathrm{~S}(\mathrm{M}+\mathrm{H})^{+}$293.1893, found 293.1899.

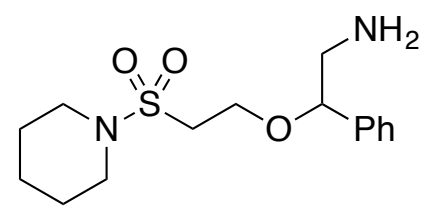

Reaction conditions

1e (0.8 mmol) DMF (0.8 mL), $2 \mathrm{~h}(0.96 \mathrm{mmol}), \operatorname{DMF}(0.8 \mathrm{~mL}),-20{ }^{\circ} \mathrm{C}, 2 \mathrm{~h}$, AgOAc/dppe $(24.0 \mu \mathrm{mol})$, KHMDS $(32 \mu \mathrm{mol})$.

2-phenyl-2-(2-(piperidin-1-ylsulfonyl)ethoxy)ethan-1-amine (3eh): (pale orange oil, $\mathrm{CH}_{2} \mathrm{Cl}_{2} / \mathrm{MeOH}=$ 10/1, 81\% yield, $202.2 \mathrm{mg}$ ); ${ }^{1} \mathrm{H}-\mathrm{NMR}\left(500 \mathrm{MHz}^{\mathrm{CDCl}} 3\right.$ ) (ppm) $\delta$ 7.42-7.28 (m, 5H, ArH), 4.34 (dd, $1 \mathrm{H}, J=$ 4.5, 7.8 Hz, PhCHO), 3.86-3.69 (m, $\left.2 \mathrm{H}, \mathrm{SO}_{2} \mathrm{CH}_{2} \mathrm{CH}_{2} \mathrm{O}\right)$, 3.29-3.11 (m, $\left.6 \mathrm{H}, \mathrm{CH}_{2}\left(\mathrm{CH}_{2} \mathrm{CH}_{2}\right)_{2} \mathrm{NSO}_{2}, \mathrm{H}_{2} \mathrm{NCH}_{2} \mathrm{CHPh}\right)$, 
3.00-2.85 (m, $\left.2 \mathrm{H}, \mathrm{SO}_{2} \mathrm{CH}_{2} \mathrm{CH}_{2} \mathrm{O}\right), 2.09\left(\mathrm{br}, 2 \mathrm{H}, \mathrm{NH}_{2}\right), 1.69-1.48\left(\mathrm{~m}, 6 \mathrm{H}, \mathrm{CH}_{2}\left(\mathrm{CH}_{2} \mathrm{CH}_{2}\right){ }_{2} \mathrm{NSO}_{2}\right) ;{ }^{13} \mathrm{C} \mathrm{NMR}(125$ $\left.\mathrm{MHz}, \mathrm{CDCl}_{3}\right) \delta 139.4,128.8,128.3,126.9,84.6,62,8,49.3,48.7,46.6,25.7,23.9 ;$ IR (thin film, $\mathrm{NaCl}$ ) 3375, 1359, 1161, 1105, $1072 \mathrm{~cm}^{-1}$; HRMS (ESI, H) $\mathrm{m} / \mathrm{z}$ calc'd for $\mathrm{C}_{15} \mathrm{H}_{25} \mathrm{~N}_{2} \mathrm{O}_{3} \mathrm{~S}(\mathrm{M}+\mathrm{H})^{+} 313.1580$, found 313.1584 .<smiles>O=S(=O)(CCNCC(O)c1ccccc1)N1CCCCC1</smiles>

1-phenyl-2-((2-(piperidin-1-ylsulfonyl)ethyl)amino)ethan-1-ol (4eh): (white solid); ${ }^{1} \mathrm{H}-\mathrm{NMR}$ (500 MHz, $\left.\mathrm{CDCl}_{3}\right)(\mathrm{ppm}) \delta$ 7.49-7.32 (m, 4H, ArH), 7.30-7.27 (m, 1H, $\left.\mathrm{ArH}\right), 4.71(\mathrm{dd}, 1 \mathrm{H}, J=3.5,9.0 \mathrm{~Hz}$, $\left.\mathrm{HNCH}_{2} \mathrm{CH}(\mathrm{Ph}) \mathrm{OH}\right), 3.37(\mathrm{br}, 1 \mathrm{H}, \mathrm{NH}), 3.25-3.10\left(\mathrm{~m}, 6 \mathrm{H}, \mathrm{HNCH} \mathrm{CH}_{2}(\mathrm{Ph}) \mathrm{OH}, \mathrm{CH}_{2}\left(\mathrm{CH}_{2} \mathrm{CH}_{2}\right)_{2} \mathrm{NSO}_{2}\right), 3.06(\mathrm{t}, 2 \mathrm{H}$, $\left.J=6.0 \mathrm{~Hz}, \mathrm{SO}_{2} \mathrm{CH}_{2} \mathrm{CH}_{2} \mathrm{~N}\right), 2.95\left(\mathrm{dd}, 1 \mathrm{H}, J=3.5,12.5 \mathrm{~Hz}, \mathrm{SO}_{2} \mathrm{CH}_{2} \mathrm{CH}_{2} \mathrm{~N}\right), 2.73(\mathrm{dd}, 1 \mathrm{H}, J=9.0,12.5 \mathrm{~Hz}$, $\left.\mathrm{SO}_{2} \mathrm{CH}_{2} \mathrm{CH}_{2} \mathrm{~N}\right), 1.95-1.48\left(\mathrm{~m}, 7 \mathrm{H}, \mathrm{CH}_{2}\left(\mathrm{CH}_{2} \mathrm{CH}_{2}\right)_{2} \mathrm{NSO}_{2}, \mathrm{OH}\right) ;{ }^{13} \mathrm{C} \mathrm{NMR}\left(125 \mathrm{MHz}, \mathrm{CDCl}_{3}\right) \delta 142.3,128.6$, 127.8, 125.9, 72.0, 57.0, 49.0, 46.7, 43.5, 25.7, 23.9; IR (KBr) 3649, 3362, 1155, 1053, $1030 \mathrm{~cm}^{-1}$; HRMS $(E S I, H) m / z$ calc'd for $\mathrm{C}_{15} \mathrm{H}_{25} \mathrm{~N}_{2} \mathrm{O}_{3} \mathrm{~S}(\mathrm{M}+\mathrm{H})^{+} 313.1580$, found 313.1578 .

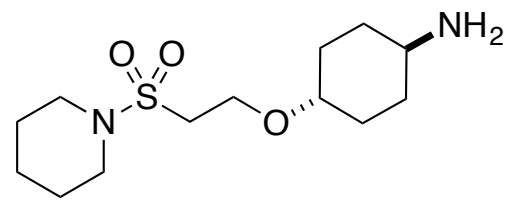

\section{$\underline{\text { Reaction conditions }}$}

1e $(0.8 \mathrm{mmol})$ in DMF (0.8 mL), $2 \mathrm{i}(0.96 \mathrm{mmol}), \mathrm{DMF}(0.8 \mathrm{~mL}),-20^{\circ} \mathrm{C}, 1 \mathrm{~h}, \mathrm{AgOAc} / \mathrm{dppe}(24.0 \mu \mathrm{mol})$, KHMDS $(32 \mu \mathrm{mol})$.

(1r,4r)-4-(2-(piperidin-1-ylsulfonyl)ethoxy)cyclohexan-1-amine (3ei): (pale yellow solid, $\mathrm{CH}_{2} \mathrm{Cl}_{2} / \mathrm{MeOH}=$ 10/1 to 5/1, 82\% yield, $191.3 \mathrm{mg}$ ); ${ }^{1} \mathrm{H}-\mathrm{NMR}\left(500 \mathrm{MHz}, \mathrm{CDCl}_{3}\right)(\mathrm{ppm}) \delta 3.83(\mathrm{t}, 2 \mathrm{H}, J=6.5, \mathrm{~Hz}$ $\left.\mathrm{CH}_{2} \mathrm{CH}_{2} \mathrm{SO}_{2} \mathrm{~N}\right), 3.31-3.20\left(\mathrm{~m}, 5 \mathrm{H}, \mathrm{CH}_{2}\left(\mathrm{CH}_{2} \mathrm{CH}_{2}\right){ }_{2} \mathrm{NSO}_{2} \mathrm{CH}_{2} \mathrm{CH}_{2} \mathrm{OCH}\left(\mathrm{CH}_{2} \mathrm{CH}_{2}\right)_{2} \mathrm{CHNH}_{2}\right), 3.16(\mathrm{t}, 2 \mathrm{H}, J=6.5, \mathrm{~Hz}$ $\left.\mathrm{CH}_{2} \mathrm{CH}_{2} \mathrm{SO}_{2} \mathrm{~N}\right), 2.75-2.66\left(\mathrm{~m}, 1 \mathrm{H}, \mathrm{OCH}\left(\mathrm{CH}_{2} \mathrm{CH}_{2}\right)_{2} \mathrm{CHNH}_{2}\right), 2.07-1.85\left(\mathrm{~m}, 4 \mathrm{H}, \mathrm{OCH}\left(\mathrm{CH}_{2} \mathrm{CH}_{2}\right) 2 \mathrm{CHNH}_{2}\right), 1.68-$ $1.40\left(\mathrm{~m}, 8 \mathrm{H}, \mathrm{CH}_{2}\left(\mathrm{CH}_{2} \mathrm{CH}_{2}\right)_{2} \mathrm{NSO}_{2} \mathrm{CH}_{2} \mathrm{CH}_{2} \mathrm{OCH}\left(\mathrm{CH}_{2} \mathrm{CH}_{2}\right)_{2} \mathrm{CHNH}_{2}\right), 1.35-1.08\left(\mathrm{~m}, 4 \mathrm{H}, \mathrm{OCH}\left(\mathrm{CH}_{2} \mathrm{CH}_{2}\right)_{2} \mathrm{CHNH}_{2}\right) ;{ }^{13} \mathrm{C}$ NMR (125 MHz, $\left.\mathrm{CDCl}_{3}\right) \delta 78.3,61.9,50.2,50.0,46.6,34.4,30.8,25.8,24.0$; IR (KBr) 3350, 1647, 1323, $1161,1053 \mathrm{~cm}^{-1}$; HRMS (ESI, H) $\mathrm{m} / z$ calc'd for $\mathrm{C}_{13} \mathrm{H}_{27} \mathrm{~N}_{2} \mathrm{O}_{3} \mathrm{~S}(\mathrm{M}+\mathrm{H})^{+}$291.1737, found 291.1738. 


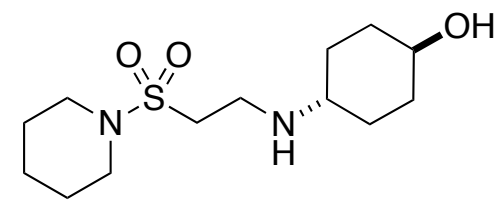

(1r,4r)-4-((2-(piperidin-1-ylsulfonyl)ethyl)amino)cyclohexan-1-ol (4ei): (white solid); ${ }^{1} \mathrm{H}-\mathrm{NMR}$ (500 MHz, $\left.\mathrm{CDCl}_{3}\right) \quad(\mathrm{ppm}) \quad \delta \quad 3.66-3.55\left(\mathrm{~m}, \quad 1 \mathrm{H}, \quad \mathrm{HOCH}\left(\mathrm{CH}_{2} \mathrm{CH}_{2}\right)_{2} \mathrm{CHNH}\right), \quad 3.22 \quad(\mathrm{t}, 4 \mathrm{H}$, $\left.\mathrm{CH}_{2}\left(\mathrm{CH}_{2} \mathrm{CH}_{2}\right)_{2} \mathrm{NSO}_{2} \mathrm{CH}_{2} \mathrm{CH}_{2} \mathrm{NHCH}\left(\mathrm{CH}_{2} \mathrm{CH}_{2}\right)_{2} \mathrm{CHOH}\right)$, 3.13-2.98 (m, $\left.4 \mathrm{H}, \mathrm{NSO}_{2} \mathrm{CH}_{2} \mathrm{CH}{ }_{2} \mathrm{NHCH}\left(\mathrm{CH}_{2} \mathrm{CH}_{2}\right)_{2} \mathrm{CHOH}\right)$, 2.52-2.38 (m, $\left.1 \mathrm{H}, \mathrm{HOCH}\left(\mathrm{CH}_{2} \mathrm{CH}_{2}\right)_{2} \mathrm{CHNH}\right), 2.04-1.04\left(\mathrm{~m}, 16 \mathrm{H}, \mathrm{CH}_{2}\left(\mathrm{CH}_{2} \mathrm{CH}_{2}\right)_{2} \mathrm{~N}, \mathrm{NHCH}\left(\mathrm{CH}_{2} \mathrm{CH}_{2}\right)_{2} \mathrm{CHOH}\right) ;{ }^{13} \mathrm{C}$ $\operatorname{NMR}\left(125 \mathrm{MHz}, \mathrm{CDCl}_{3}\right) \delta 70.4,55.8,49.3,46.7,41.1,34.0,31.2,25.7,23.9 ; \mathrm{IR}(\mathrm{KBr}) 3327,1647,1161$, $1058,1028 \mathrm{~cm}^{-1}$; HRMS (ESI, H) $\mathrm{m} / z$ calc'd for $\mathrm{C}_{13} \mathrm{H}_{27} \mathrm{~N}_{2} \mathrm{O}_{3} \mathrm{~S}(\mathrm{M}+\mathrm{H})^{+}$291.1737, found 291.1738.

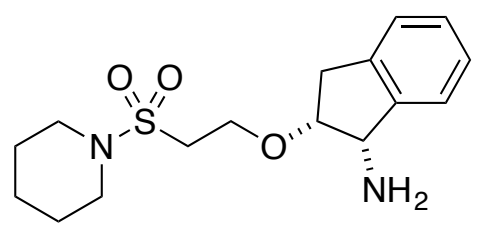

\section{$\underline{\text { Reaction conditions }}$}

1e $(0.8 \mathrm{mmol})$ in DMF (0.8 mL), $2 \mathrm{j}(0.96 \mathrm{mmol}), \operatorname{DMF}(0.8 \mathrm{~mL}),-20{ }^{\circ} \mathrm{C}, 4 \mathrm{~h}, \mathrm{AgOAc} / \mathrm{dppe}(24.0 \mu \mathrm{mol})$, KHMDS $(32 \mu \mathrm{mol})$.

(1S,2R)-2-(2-(piperidin-1-ylsulfonyl)ethoxy)-2,3-dihydro-1H-inden-1-amine (3ej): (pale yellow oil, $\mathrm{CH}_{2} \mathrm{Cl}_{2} / \mathrm{MeOH}=10 / 1,87 \%$ yield, $\left.224.9 \mathrm{mg}\right) ;{ }^{1} \mathrm{H}-\mathrm{NMR}\left(500 \mathrm{MHz}, \mathrm{CDCl}_{3}\right)(\mathrm{ppm}) \delta 7.37(\mathrm{~d}, 1 \mathrm{H}, J=6.5 \mathrm{~Hz}$, $\mathrm{ArH})$, 7.25-7.12 (m, 3H, ArH), $4.32\left(\mathrm{~d}, 1 \mathrm{H}, J=5.0 \mathrm{~Hz}, \mathrm{CHNH}_{2}\right), 4.20-4.07\left(\mathrm{~m}, 1 \mathrm{H}, \mathrm{SO}_{2} \mathrm{CH}_{2} \mathrm{CH}_{2} \mathrm{OCH}\right), 4.05-$ $3.80\left(\mathrm{~m}, 2 \mathrm{H}, \mathrm{SO}_{2} \mathrm{CH}_{2} \mathrm{CH}_{2} \mathrm{OCH}\right), 3.18\left(\mathrm{t}, 2 \mathrm{H}, \mathrm{J}=6.0 \mathrm{~Hz}, \mathrm{SO}_{2} \mathrm{CH}_{2} \mathrm{CH}_{2} \mathrm{OCH}\right), 3.12-2.90\left(\mathrm{~m}, 6 \mathrm{H}, \mathrm{OCH}\left(\mathrm{CHNH}_{2}\right) \mathrm{CH}_{2}\right.$, $\left.\mathrm{CH}_{2}\left(\mathrm{CH}_{2} \mathrm{CH}_{2}\right)_{2} \mathrm{NSO}_{2}\right), 1.69\left(\mathrm{br}, 2 \mathrm{H}, \mathrm{NH}_{2}\right), 1.60-1.35\left(\mathrm{~m}, 6 \mathrm{H}, \mathrm{CH}_{2}\left(\mathrm{CH}_{2} \mathrm{CH}_{2}\right)_{2} \mathrm{NSO}_{2}\right) ;{ }^{13} \mathrm{C} \mathrm{NMR}\left(125 \mathrm{MHz}, \mathrm{CDCl}_{3}\right)$ $\delta 144.8,139.7,127.8,127.1,125.1,124.0,83.6,63.4,59.1,49.7,46.4,35.6,25.7,23.8$; IR (KBr) 3390, 1348, 1161, 1112, $1097 \mathrm{~cm}^{-1}$; HRMS (ESI, H) m/z calc'd for $\mathrm{C}_{16} \mathrm{H}_{25} \mathrm{~N}_{2} \mathrm{O}_{3} \mathrm{~S}(\mathrm{M}+\mathrm{H})^{+} 325.1580$, found 325.1580. $[\alpha]_{D}^{25}-33.5\left(c 1.00, \mathrm{CHCl}_{3}\right)$.<smiles>O=S(=O)(CCN[C@H]1c2ccccc2C[C@H]1O)N1CCCCC1</smiles>

(1S,2R)-1-((2-(piperidin-1-ylsulfonyl)ethyl)amino)-2,3-dihydro-1H-inden-2-ol (4ej): (yellow solid); ${ }^{1} \mathrm{H}$ $\operatorname{NMR}\left(500 \mathrm{MHz}_{\mathrm{CDCl}}\right.$ ) (ppm) 8 7.35-7.28 (m, 1H, ArH), 7.25-7.16 (m, 3H, ArH), 4.35 (dd, $1 \mathrm{H}, J=6.5,12.5$ 
$\mathrm{Hz}, \mathrm{CHOH}), 4.06\left(\mathrm{~d}, 1 \mathrm{H}, J=5.5 \mathrm{~Hz}, \mathrm{SO}_{2} \mathrm{CH}_{2} \mathrm{CH}_{2} \mathrm{NHCH}\right), 3.40-3.08\left(\mathrm{~m}, 9 \mathrm{H}, \mathrm{CH}_{2}\left(\mathrm{CH}_{2} \mathrm{CH}_{2}\right)_{2} \mathrm{NSO}_{2}\right.$, $\left.\mathrm{SO}_{2} \mathrm{CH}_{2} \mathrm{CH}_{2} \mathrm{NHCH}, \mathrm{OCH}\left(\mathrm{CHNH}_{2}\right) \mathrm{CH}_{2}\right), 2.80\left(\mathrm{dd}, 1 \mathrm{H}, J=6.5,16.0 \mathrm{~Hz}, \mathrm{OCH}\left(\mathrm{CHNH}_{2}\right) \mathrm{CH}_{2}\right), 2.05(\mathrm{br}, 2 \mathrm{H}, \mathrm{OH}$, $\mathrm{NH}), 1.70-1.51\left(\mathrm{~m}, 6 \mathrm{H}, \mathrm{CH}_{2}\left(\mathrm{CH}_{2} \mathrm{CH}_{2}\right)_{2} \mathrm{NSO}_{2}\right) ;{ }^{13} \mathrm{C} \mathrm{NMR}\left(125 \mathrm{MHz}, \mathrm{CDCl}_{3}\right) \delta 142.0,139.8,128.1,127.1$, 125.2, 124.3, 79.2, 70.2, 49.4, 46.7, 41.4, 39.2, 25.7, 23.6; IR (KBr) 3284, 1627, 1159, 1053, $1028 \mathrm{~cm}^{-1}$; HRMS (ESI, H) $m / z$ calc'd for $\mathrm{C}_{16} \mathrm{H}_{25} \mathrm{~N}_{2} \mathrm{O}_{3} \mathrm{~S}(\mathrm{M}+\mathrm{H})^{+} 325.1580$, found 325.1578. $[\alpha]_{D}{ }^{25}+2.9\left(c 1.00, \mathrm{CHCl}_{3}\right)$.

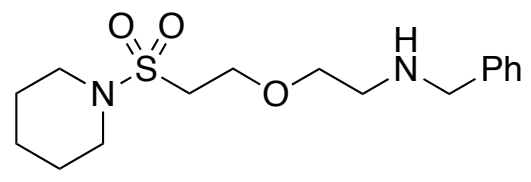

\section{$\underline{\text { Reaction conditions }}$}

1e $(0.8 \mathrm{mmol})$ in DMF, $2 \mathrm{k}(0.96 \mathrm{mmol}), \mathrm{DMF}(0.8 \mathrm{~mL}),-20^{\circ} \mathrm{C}, 24 \mathrm{~h}, \mathrm{AgOAc} / \mathrm{dppe}(24.0 \mu \mathrm{mol}), \mathrm{KHMDS}$ (32 $\mu \mathrm{mol})$.

N-benzyl-2-(2-(piperidin-1-ylsulfonyl)ethoxy)ethan-1-amine (3ek): (colorless oil, $\mathrm{CH}_{2} \mathrm{Cl}_{2} / \mathrm{MeOH}=10 / 1$, 77\% yield, $202.0 \mathrm{mg}) ;{ }^{1} \mathrm{H}-\mathrm{NMR}\left(500 \mathrm{MHz}, \mathrm{CDCl}_{3}\right)(\mathrm{ppm}) \delta$ 7.36-7.29 (m, 4H, ArH), 7.28-7.22 (m, $\left.1 \mathrm{H}, \mathrm{ArH}\right)$, 3.86-3.78 (m, $\left.4 \mathrm{H}, \mathrm{SO}_{2} \mathrm{CH}_{2} \mathrm{CH}_{2} \mathrm{O}, \mathrm{NCH}_{2} \mathrm{Ph}\right), 3.60\left(\mathrm{t}, 2 \mathrm{H}, J=5.0 \mathrm{~Hz}, \mathrm{OCH}_{2} \mathrm{CH}_{2} \mathrm{~N}\right), 3.25-3.15(\mathrm{~m}, 6 \mathrm{H}$, $\left.\mathrm{CH}_{2}\left(\mathrm{CH}_{2} \mathrm{CH}_{2}\right)_{2} \mathrm{~N}, \mathrm{OCH}_{2} \mathrm{CH}_{2} \mathrm{~N}\right), 2.82\left(\mathrm{t}, 2 \mathrm{H}, \mathrm{J}=6.0 \mathrm{~Hz}, \mathrm{SO}_{2} \mathrm{CH}_{2} \mathrm{CH}_{2} \mathrm{O}\right), 1.72-1.44\left(\mathrm{~m}, 7 \mathrm{H}, \mathrm{NH}, \mathrm{CH}_{2}\left(\mathrm{CH}_{2} \mathrm{CH}_{2}\right)_{2} \mathrm{~N}\right)$;

${ }^{13} \mathrm{CNMR}\left(125 \mathrm{MHz}, \mathrm{CDCl}_{3}\right) \delta 140.3,128.6,128.3,127.1$, 70.8, 64.7, 54.1, 49.6, 48.7, 46.6, 25.8, 23.9; IR (thin film, NaCl) 3325, 1357, 1163,1112, $1053 \mathrm{~cm}^{-1}$; HRMS (ESI, H) $\mathrm{m} / z$ calc'd for $\mathrm{C}_{16} \mathrm{H}_{27} \mathrm{~N}_{2} \mathrm{O}_{3} \mathrm{~S}^{+}(\mathrm{M}+\mathrm{H})^{+}$ 327.1737, found 327.1740.

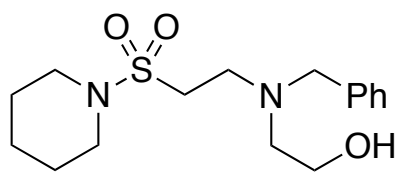

2-(benzyl(2-(piperidin-1-ylsulfonyl)ethyl)amino)ethan-1-ol (4ek): (colorless oil); ${ }^{1} \mathrm{H}-\mathrm{NMR}$ (500 MHz, $\left.\mathrm{CDCl}_{3}\right)(\mathrm{ppm}) \delta$ 7.40-7.23 (m, 5H, $\left.\mathrm{ArH}\right), 3.67\left(\mathrm{~s}, 2 \mathrm{H}, \mathrm{CH}_{2} \mathrm{Ph}\right), 3.65$ (dd, $\left.2 \mathrm{H}, J=5.5,10.5 \mathrm{~Hz}, \mathrm{CH}_{2} \mathrm{OH}\right), 3.13(\mathrm{t}$, $\left.4 \mathrm{H}, J=5.0 \mathrm{~Hz}, \mathrm{CH}_{2}\left(\mathrm{CH}_{2} \mathrm{CH}_{2}\right)_{2} \mathrm{~N}\right), 3.05-2.94\left(\mathrm{~m}, 4 \mathrm{H}, \mathrm{SO}_{2} \mathrm{CH}_{2} \mathrm{CH}_{2} \mathrm{NCH}_{2} \mathrm{CH}_{2} \mathrm{OH}\right), 2.78(\mathrm{br}, 1 \mathrm{H}, \mathrm{OH}), 2.69(\mathrm{t}, 2 \mathrm{H}, J$ $\left.=5.5 \mathrm{~Hz}, \mathrm{SO}_{2} \mathrm{CH}_{2} \mathrm{CH}_{2} \mathrm{~N}\right), 1.65-1.45\left(\mathrm{~m}, 6 \mathrm{H}, \mathrm{CH}_{2}\left(\mathrm{CH}_{2} \mathrm{CH}_{2}\right)_{2} \mathrm{~N}\right) ;{ }^{13} \mathrm{C} \mathrm{NMR}\left(125 \mathrm{MHz}, \mathrm{CDCl}_{3}\right) \delta$ 138.2, 129.1, 128.7, 127.6, 59.1, 59.0, 56.0, 48.0, 46.9, 46.6, 25.7, 23.8; IR (thin film, NaCl) 3500, 1602, 1161, 1053, $1028 \mathrm{~cm}^{-1}$; HRMS (ESI, H) m/z calc'd for $\mathrm{C}_{16} \mathrm{H}_{27} \mathrm{~N}_{2} \mathrm{O}_{3} \mathrm{~S}^{+}(\mathrm{M}+\mathrm{H})^{+} 327.1737$, found 327.1739. 


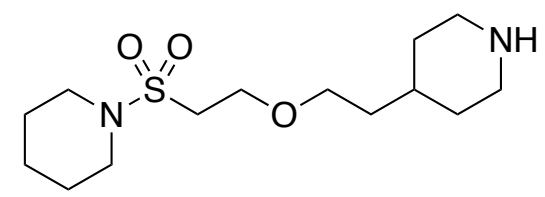

\section{Reaction conditions}

1e $(0.8 \mathrm{mmol})$ in DMF (0.8 mL), $2 \mathrm{l}(0.96 \mathrm{mmol}), \mathrm{DMF}(0.8 \mathrm{~mL}),-20^{\circ} \mathrm{C}, 22.5 \mathrm{~h}, \mathrm{AgOAc} / \mathrm{dppe}(48.0 \mu \mathrm{mol})$, KHMDS $(64 \mu \mathrm{mol})$.

1-((2-(2-(piperidin-4-yl)ethoxy)ethyl)sulfonyl)piperidine (3el): (pale orange oil, $\mathrm{CH}_{2} \mathrm{Cl}_{2} / \mathrm{MeOH} / \mathrm{iPrNH}{ }_{2}=$ 20/1/0.5, 74\% yield, $180.9 \mathrm{mg}) ;{ }^{1} \mathrm{H}-\mathrm{NMR}\left(500 \mathrm{MHz}, \mathrm{CDCl}_{3}\right)(\mathrm{ppm}) \delta 3.78\left(\mathrm{t}, 2 \mathrm{H}, J=6.5 \mathrm{~Hz}, \mathrm{SO}_{2} \mathrm{CH}_{2} \mathrm{CH}_{2} \mathrm{O}\right)$, $3.49\left(\mathrm{t}, 2 \mathrm{H}, J=6.5 \mathrm{~Hz}, \mathrm{OCH}_{2} \mathrm{CH}_{2} \mathrm{CH}\right.$ ), $3.24\left(\mathrm{t}, 4 \mathrm{H}, J=5.0 \mathrm{~Hz}, \mathrm{CH}_{2}\left(\mathrm{CH}_{2} \mathrm{CH}_{2}\right)_{2} \mathrm{NSO}_{2}\right), 3.18$ (t, $2 \mathrm{H}, J=6.5 \mathrm{~Hz}$, $\left.\mathrm{CH}\left(\mathrm{CH}_{2} \mathrm{CH}_{2}\right)_{2} \mathrm{NH}\right), 3.09-3.02\left(\mathrm{~m}, 2 \mathrm{H}, \mathrm{SO}_{2} \mathrm{CH}_{2} \mathrm{CH}_{2} \mathrm{O}\right), 2.63-2.52\left(\mathrm{~m}, 2 \mathrm{H}, \mathrm{CH}\left(\mathrm{CH}_{2} \mathrm{CH}_{2}\right)_{2} \mathrm{NH}\right), 1.85-1.42(\mathrm{~m}, 12 \mathrm{H}$, $\left.\mathrm{CH}\left(\mathrm{CH}_{2} \mathrm{CH}_{2}\right)_{2} \mathrm{NH}, \mathrm{CH}_{2}\left(\mathrm{CH}_{2} \mathrm{CH}_{2}\right)_{2} \mathrm{NSO}_{2}\right), 1.20-1.05\left(\mathrm{~m}, 2 \mathrm{H}, \mathrm{OCH}_{2} \mathrm{CH}_{2} \mathrm{CH}\right) ;{ }^{13} \mathrm{C} \mathrm{NMR}\left(125 \mathrm{MHz}, \mathrm{CDCl}_{3}\right) \delta 69.0$, 64.5, 49.7, 46.8, 46.5, 36.9, 33.7, 33.3, 35.8, 24.0; IR (KBr) 3346, 1635, 1323, 1111, $1053 \mathrm{~cm}^{-1}$; HRMS (ESI, H) $\mathrm{m} / \mathrm{z}$ calc'd for $\mathrm{C}_{14} \mathrm{H}_{29} \mathrm{~N}_{2} \mathrm{O}_{3} \mathrm{~S}^{+}(\mathrm{M}+\mathrm{H})^{+} 305.1893$, found 305.1892 .

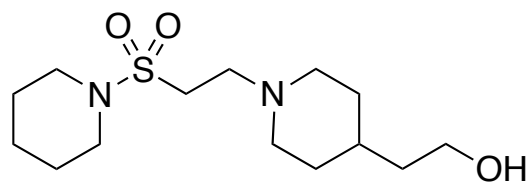

2-(1-(2-(piperidin-1-ylsulfonyl)ethyl)piperidin-4-yl)ethan-1-ol (4el): (white solid); ${ }^{1} \mathrm{H}-\mathrm{NMR}$ (500 MHz, $\left.\mathrm{CDCl}_{3}\right)(\mathrm{ppm}) \delta 3.70\left(\mathrm{t}, 2 \mathrm{H}, J=6.5 \mathrm{~Hz}, \mathrm{CH}_{2} \mathrm{OH}\right), 3.24\left(\mathrm{t}, 4 \mathrm{H}, J=5.0 \mathrm{~Hz}, \mathrm{CH}_{2}\left(\mathrm{CH}_{2} \mathrm{CH}_{2}\right)_{2} \mathrm{~N}\right), 3.14-3.05(\mathrm{~m}, 2 \mathrm{H}$, $\left.\mathrm{N}\left(\mathrm{CH}_{2} \mathrm{CH}_{2}\right)_{2} \mathrm{CH}\right), 2.91-2.77\left(\mathrm{~m}, 4 \mathrm{H}, \mathrm{SO}_{2} \mathrm{CH}_{2} \mathrm{CH}_{2} \mathrm{~N}, \mathrm{~N}\left(\mathrm{CH}_{2} \mathrm{CH}_{2}\right)_{2} \mathrm{CH}\right), 2.10-2.00\left(\mathrm{~m}, 2 \mathrm{H}, \mathrm{SO}_{2} \mathrm{CH}_{2} \mathrm{CH}_{2} \mathrm{~N}\right), 1.76-$ $1.38\left(\mathrm{~m}, 11 \mathrm{H}, \mathrm{CH}_{2}\left(\mathrm{CH}_{2} \mathrm{CH}_{2}\right)_{2} \mathrm{~N}, \mathrm{~N}\left(\mathrm{CH}_{2} \mathrm{CH}_{2}\right)_{2} \mathrm{CH}, \mathrm{OH}\right), 1.33-1.20\left(\mathrm{~m}, 3 \mathrm{H}, \mathrm{N}\left(\mathrm{CH}_{2} \mathrm{CH}_{2}\right)_{2} \mathrm{CH}, \mathrm{CH}_{2} \mathrm{CH}_{2} \mathrm{OH}\right) ;{ }^{13} \mathrm{C} \mathrm{NMR}$ (125 MHz, $\left.\mathrm{CDCl}_{3}\right) \delta 60.6,54.0,52.1,47.1,46.7,39.5,32.5,32.3,25.8,24.0 ; \mathrm{IR}(\mathrm{KBr}) 3649,1610,1157$, 1055, $1024 \mathrm{~cm}^{-1}$; HRMS (ESI, H) $\mathrm{m} / z$ calc'd for $\mathrm{C}_{14} \mathrm{H}_{29} \mathrm{~N}_{2} \mathrm{O}_{3} \mathrm{~S}^{+}(\mathrm{M}+\mathrm{H})^{+}$305.1893, found 305.1894 .

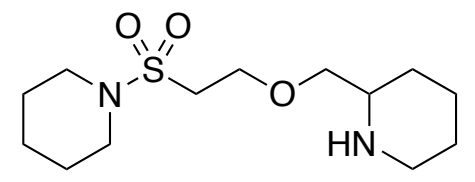

\section{Reaction conditions}


1e $(0.8 \mathrm{mmol})$ in DMF $(0.8 \mathrm{~mL}), 2 \mathrm{~m}(0.96 \mathrm{mmol})$, DMF $(0.8 \mathrm{~mL}),-20^{\circ} \mathrm{C}, 24 \mathrm{~h}, \mathrm{AgOAc} / \mathrm{dppe}(48.0 \mu \mathrm{mol})$, KHMDS (64 $\mu \mathrm{mol})$.

1-((2-(piperidin-2-ylmethoxy)ethyl)sulfonyl)piperidine (3em): (pale yellow oil, $\mathrm{CH}_{2} \mathrm{Cl}_{2} / \mathrm{MeOH}=9 / 1$ to 4/1, 71\% yield, $164.4 \mathrm{mg}$ ); ${ }^{1} \mathrm{H}-\mathrm{NMR}\left(500 \mathrm{MHz}, \mathrm{CDCl}_{3}\right.$ ) (ppm) $\delta$ 3.84-3.70 (m, $\left.2 \mathrm{H}, \mathrm{SO}_{2} \mathrm{CH}_{2} \mathrm{CH}_{2} \mathrm{O}\right), 3.44-2.50$ (m, $\left.12 \mathrm{H}, \quad \mathrm{CH}_{2}\left(\mathrm{CH}_{2} \mathrm{CH}_{2}\right)_{2} \mathrm{~N}, \quad \mathrm{SO}_{2} \mathrm{CH}_{2} \mathrm{CH}_{2} \mathrm{O}, \quad \mathrm{OCH}_{2} \mathrm{CHNHCH}\right), 1.80-1.00 \quad\left(\mathrm{~m}, 12 \mathrm{H}, \mathrm{CH}_{2}\left(\mathrm{CH}_{2} \mathrm{CH}_{2}\right)_{2} \mathrm{~N}\right.$, $\left.\mathrm{NHCH}_{2} \mathrm{CH}_{2} \mathrm{CH}_{2} \mathrm{CH}_{2} \mathrm{CH}\right)$; ${ }^{13} \mathrm{C}$ NMR $\left(125 \mathrm{MHz}, \mathrm{CDCl}_{3}\right) \delta 75.8,64.8,56.1$, 49.3, 46.6, 46.4, 28.5, 26.1, 25.7, 24.3, 23.9; IR (KBr) 3371, 1332, 1161, 1141, $1105 \mathrm{~cm}^{-1}$; HRMS (ESI, H) m/z calc'd for $\mathrm{C}_{13} \mathrm{H}_{27} \mathrm{~N}_{2} \mathrm{O}_{3} \mathrm{~S}^{+}(\mathrm{M}+$ $H)^{+}$291.1737, found 291.1734 .

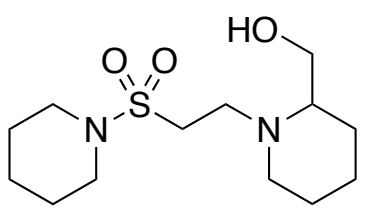

(1-(2-(piperidin-1-ylsulfonyl)ethyl)piperidin-2-yl)methanol (4em): (pale yellow oil); ${ }^{1} \mathrm{H}-\mathrm{NMR}(500 \mathrm{MHz}$, $\left.\mathrm{CDCl}_{3}\right)(\mathrm{ppm}) \delta 3.73\left(\mathrm{dd}, 1 \mathrm{H}, J=3.5,11.0 \mathrm{~Hz}, \mathrm{CH}_{2} \mathrm{OH}\right.$ ), 3.54 (dd, $1 \mathrm{H}, J=4.5,11.0 \mathrm{~Hz}, \mathrm{CH}_{2} \mathrm{OH}$ ), 3.30-2.85 (m, 9H, $\left.\mathrm{CH}_{2}\left(\mathrm{CH}_{2} \mathrm{CH}_{2}\right)_{2} \mathrm{~N}, \mathrm{SO}_{2} \mathrm{CH}_{2} \mathrm{CH}_{2} \mathrm{~N}, \mathrm{CHCH}_{2} \mathrm{OH}, \mathrm{NCH}_{2}\right), 2.51(\mathrm{br}, 1 \mathrm{H}, \mathrm{OH}), 2.44-2.28\left(\mathrm{~m}, 2 \mathrm{H}, \mathrm{SO}_{2} \mathrm{CH}_{2} \mathrm{CH}_{2} \mathrm{~N}\right.$, $\left.\mathrm{NCH}_{2}\right), 1.80-1.19\left(\mathrm{~m}, 12 \mathrm{H}, \mathrm{CH}_{2}\left(\mathrm{CH}_{2} \mathrm{CH}_{2}\right)_{2} \mathrm{~N}, \mathrm{NCH}_{2} \mathrm{CH}_{2} \mathrm{CH}_{2} \mathrm{CH}_{2} \mathrm{CH}\right) ;{ }^{13} \mathrm{C} \mathrm{NMR}\left(125 \mathrm{MHz}, \mathrm{CDCl}_{3}\right) \delta 62.8,60.7$, 51.6, 47.3, 46.7, 46.3, 27.3, 25.8, 24.4, 23.9, 23.4; IR (KBr) 3614, 3356, 1159, 1053, $1028 \mathrm{~cm}^{-1}$; HRMS (ESI, H) $m / z$ calc'd for $\mathrm{C}_{13} \mathrm{H}_{27} \mathrm{~N}_{2} \mathrm{O}_{3} \mathrm{~S}^{+}(\mathrm{M}+\mathrm{H})^{+} 291.1737$, found 291.1739 .

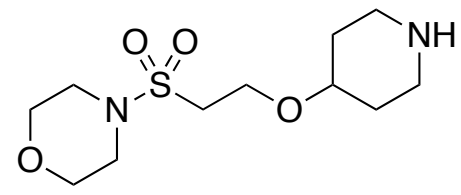

\section{Reaction conditions}

1f $(0.8 \mathrm{mmol})$ in DMF $(0.8 \mathrm{~mL}), 2 \mathrm{n}(0.96 \mathrm{mmol}), \operatorname{DMF}(0.8 \mathrm{~mL}),-20^{\circ} \mathrm{C}, 24 \mathrm{~h}$, AgOAc/dppe $(48.0 \mu \mathrm{mol})$, KHMDS $(64 \mu \mathrm{mol})$

4-((2-(piperidin-4-yloxy)ethyl)sulfonyl)morpholine (3fn): (pale yellow oil, $\mathrm{CH}_{2} \mathrm{Cl}_{2} / \mathrm{MeOH} / \mathrm{iPrNH}{ }_{2}=$ 40/1/0.5 to 20/1/0.5, 74\% yield, $164.0 \mathrm{mg}$ ); ${ }^{1} \mathrm{H}-\mathrm{NMR}\left(500 \mathrm{MHz}, \mathrm{CDCl}_{3}\right)(\mathrm{ppm}) \delta 3.84(\mathrm{t}, 2 \mathrm{H}, J=6.0 \mathrm{~Hz}$, $\left.\mathrm{SO}_{2} \mathrm{CH}_{2} \mathrm{CH}_{2} \mathrm{O}\right), 3.75\left(\mathrm{t}, 4 \mathrm{H}, J=4.5 \mathrm{~Hz}, \mathrm{O}\left(\mathrm{CH}_{2} \mathrm{CH}_{2}\right)_{2} \mathrm{~N}\right), 3.46-3.02\left(\mathrm{~m}, 9 \mathrm{H}, \mathrm{SO}_{2} \mathrm{CH}_{2} \mathrm{CH}_{2} \mathrm{O}, \mathrm{O}\left(\mathrm{CH}_{2} \mathrm{CH}_{2}\right)_{2} \mathrm{~N}\right.$, $\left.\mathrm{OCH}\left(\mathrm{CH}_{2} \mathrm{CH}_{2}\right)_{2} \mathrm{NH}\right), 2.68-2.55\left(\mathrm{~m}, 2 \mathrm{H}, \mathrm{OCH}\left(\mathrm{CH}_{2} \mathrm{CH}_{2}\right)_{2} \mathrm{NH}\right), 2.00-1.90\left(\mathrm{~m}, 2 \mathrm{H}, \mathrm{OCH}\left(\mathrm{CH}_{2} \mathrm{CH}_{2}\right)_{2} \mathrm{NH}\right), 1.60-1.35$ (m, 3H, OCH $\left.\left(\mathrm{CH}_{2} \mathrm{CH}_{2}\right)_{2} \mathrm{NH}\right) ;{ }^{13} \mathrm{C}$ NMR $\left(125 \mathrm{MHz}, \mathrm{CDCl}_{3}\right) \delta 76.8,66.8,61.3,50.3,45.7,44.6$, 33.0; IR (thin film, $\mathrm{NaCl}$ ) 3331, 1342, 1147, 1112, $1045 \mathrm{~cm}^{-1}$; HRMS (ESI, H) $\mathrm{m} / z$ calc'd for $\mathrm{C}_{11} \mathrm{H}_{23} \mathrm{~N}_{2} \mathrm{O}_{4} \mathrm{~S}^{+}(\mathrm{M}+\mathrm{H})^{+}$ 279.1373, found 279.1376 . 


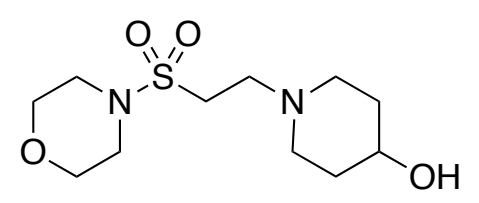

1-(2-(morpholinosulfonyl)ethyl)piperidin-4-ol (4fn): (white solid); ${ }^{1} \mathrm{H}-\mathrm{NMR}\left(500 \mathrm{MHz}, \mathrm{CDCl}_{3}\right)(\mathrm{ppm}) \delta$ 3.84-3.68 (m, 5H, O $\left.\left(\mathrm{CH}_{2} \mathrm{CH}_{2}\right)_{2} \mathrm{~N}, \mathrm{CHOH}\right), 3.28\left(\mathrm{t}, 4 \mathrm{H}, J=4.5 \mathrm{~Hz}, \mathrm{O}\left(\mathrm{CH}_{2} \mathrm{CH}_{2}\right)_{2} \mathrm{~N}\right), 3.15-3.08(\mathrm{t}, 2 \mathrm{H}, J=7.0 \mathrm{~Hz}$, $\left.\mathrm{SO}_{2} \mathrm{CH}_{2} \mathrm{CH}_{2} \mathrm{~N}\right), 2.90-2.70\left(\mathrm{~m}, 4 \mathrm{H}, \mathrm{N}\left(\mathrm{CH}_{2} \mathrm{CH}_{2}\right)_{2} \mathrm{CH}, \mathrm{SO}_{2} \mathrm{CH}_{2} \mathrm{CH}_{2} \mathrm{~N}\right), 2.29-2.19\left(\mathrm{~m}, 2 \mathrm{H}, \mathrm{N}\left(\mathrm{CH}_{2} \mathrm{CH}_{2}\right){ }_{2} \mathrm{CH}\right), 1.94-$ $1.85\left(\mathrm{~m}, 2 \mathrm{H}, \mathrm{N}\left(\mathrm{CH}_{2} \mathrm{CH}_{2}\right)_{2} \mathrm{CH}\right), 1.80-1.50\left(\mathrm{~m}, 3 \mathrm{H}, \mathrm{N}\left(\mathrm{CH}_{2} \mathrm{CH}_{2}\right)_{2} \mathrm{CH}, \mathrm{CHOH}\right) ;{ }^{13} \mathrm{C} \mathrm{NMR}\left(125 \mathrm{MHz}, \mathrm{CDCl}_{3}\right) \delta 67.5$, 66.7, 51.6, 51.0, 47.0, 45.8, 34.4; IR (KBr) 3313, 1647, 1246, 1159, $1068 \mathrm{~cm}^{-1}$; HRMS (ESI, H) $\mathrm{m} / z$ calc'd for $\mathrm{C}_{11} \mathrm{H}_{23} \mathrm{~N}_{2} \mathrm{O}_{4} \mathrm{~S}^{+}(\mathrm{M}+\mathrm{H})^{+} 279.1373$, found 279.1374 .

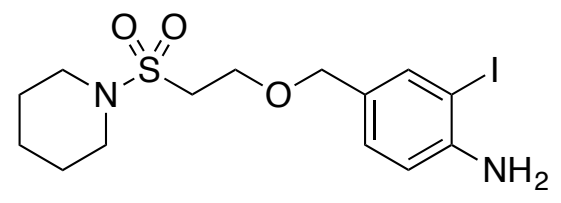

\section{Reaction conditions}

1e $(0.8 \mathrm{mmol})$ in DMF $(0.8 \mathrm{~mL}), 20(0.96 \mathrm{mmol}), \mathrm{DMF}(0.8 \mathrm{~mL}),-20{ }^{\circ} \mathrm{C}, 1 \mathrm{~h}, \mathrm{AgOAc} / \mathrm{dppe}(24.0 \mu \mathrm{mol})$, KHMDS $(32 \mu \mathrm{mol})$;

2-iodo-4-((2-(piperidin-1-ylsulfonyl)ethoxy)methyl)aniline (3eo): (pale yellow oil, $\mathrm{CH}_{2} \mathrm{Cl}_{2} / \mathrm{MeOH}=100 / 1$, $86 \%$ yield, $293.1 \mathrm{mg}) ;{ }^{1} \mathrm{H}-\mathrm{NMR}\left(500 \mathrm{MHz}, \mathrm{CDCl}_{3}\right)(\mathrm{ppm}) \delta 7.62(\mathrm{~d}, 1 \mathrm{H}, J=2.0 \mathrm{~Hz}, \mathrm{ArH}), 7.10(\mathrm{dd}, 1 \mathrm{H}, J=$ 2.0, 8.3 Hz, ArH), $6.71(\mathrm{~d}, 1 \mathrm{H}, J=8.5 \mathrm{~Hz}, \mathrm{ArH}), 4.37\left(\mathrm{~s}, 2 \mathrm{H}, \mathrm{OCH} \mathrm{Ar}_{2}\right), 4.12\left(\mathrm{br}, 2 \mathrm{H}, \mathrm{NH}_{2}\right), 3.80(\mathrm{t}, 2 \mathrm{H}, J=6.0$ $\left.\mathrm{Hz}, \mathrm{SO}_{2} \mathrm{CH}_{2} \mathrm{CH}_{2} \mathrm{O}\right)$, 3.26-3.15 (m, $\left.6 \mathrm{H}, \mathrm{SO}_{2} \mathrm{CH}_{2} \mathrm{CH}_{2} \mathrm{O}, \mathrm{CH}_{2}\left(\mathrm{CH}_{2} \mathrm{CH}_{2}\right)_{2} \mathrm{~N}\right)$, 1.66-1.49 (m, 6H, $\left.\mathrm{CH}_{2}\left(\mathrm{CH}_{2} \mathrm{CH}_{2}\right)_{2} \mathrm{~N}\right) ;{ }^{13} \mathrm{C}$ NMR $\left(125 \mathrm{MHz}, \mathrm{CDCl}_{3}\right) \delta 146.7,138.9,129.6,128.8,114.4,83.7,72.4,63.5,49.7,46.4,25.6,23.8$; IR (KBr) 3439, 1361, 1155, 1055, $1028 \mathrm{~cm}^{-1}$; HRMS (ESI, H) $\mathrm{m} / z$ calc'd for $\mathrm{C}_{14} \mathrm{H}_{22} \mathrm{IN}_{2} \mathrm{O}_{3} \mathrm{~S}(\mathrm{M}+\mathrm{H})^{+} 425.0390$, found 425.0390 .<smiles>CC(OCCS(=O)(=O)N1CCCCC1)c1ccc(N)cc1</smiles>

$\underline{\text { Reaction conditions }}$ 
1e $(0.8 \mathrm{mmol})$ in DMF $(0.8 \mathrm{~mL}), 2 \mathrm{p}(0.96 \mathrm{mmol}), \mathrm{DMF}(0.8 \mathrm{~mL}),-20^{\circ} \mathrm{C}, 21 \mathrm{~h}$, AgOAc/dppe $(24.0 \mu \mathrm{mol})$, KHMDS $(32 \mu \mathrm{mol})$.

4-(1-(2-(piperidin-1-ylsulfonyl)ethoxy)ethyl)aniline (3ep): (pale yellow oil, $\mathrm{CH}_{2} \mathrm{Cl}_{2} / \mathrm{MeOH}=50 / 1,87 \%$ yield, $218.5 \mathrm{mg}) ;{ }^{1} \mathrm{H}-\mathrm{NMR}\left(500 \mathrm{MHz}, \mathrm{CDCl}_{3}\right)(\mathrm{ppm}) \delta$ 7.12-7.06 (m, 2H, ArH), 6.70-6.02 (m, 2H, ArH), 4.33 (dd, $1 \mathrm{H}, J=6.0,10.8 \mathrm{~Hz}, \mathrm{OCHAr}$ ), 3.74-3.56 (m, $\left.4 \mathrm{H}, \mathrm{SO}_{2} \mathrm{CH}_{2} \mathrm{CH}_{2} \mathrm{O}, \mathrm{NH}_{2}\right), 3.30-3.08\left(\mathrm{~m}, 6 \mathrm{H}, \mathrm{SO}_{2} \mathrm{CH}_{2} \mathrm{CH}_{2} \mathrm{O}\right.$, $\left.\mathrm{CH}_{2}\left(\mathrm{CH}_{2} \mathrm{CH}_{2}\right)_{2} \mathrm{~N}\right), 1.66-1.50\left(\mathrm{~m}, 6 \mathrm{H}, \mathrm{CH}_{2}\left(\mathrm{CH}_{2} \mathrm{CH}_{2}\right)_{2} \mathrm{~N}\right), 1.43\left(\mathrm{~d}, 3 \mathrm{H}, J=6.5 \mathrm{~Hz}, \mathrm{CH}_{3}\right) ;{ }^{13} \mathrm{C} \mathrm{NMR}(125 \mathrm{MHz}$, $\left.\mathrm{CDCl}_{3}\right) \delta 146.3,132.6,127.7,115.2,78.6,61.9,50.0,46.5,25.8,24.0,23.7$; IR (thin film, $\mathrm{NaCl}$ ) 3462, 1356, 1161, 1053, $1028 \mathrm{~cm}^{-1}$; HRMS (ESI, H) m/z calc'd for $\mathrm{C}_{15} \mathrm{H}_{25} \mathrm{~N}_{2} \mathrm{O}_{3} \mathrm{~S}^{+}(\mathrm{M}+\mathrm{H})^{+} 313.1581$, found 313.1582.

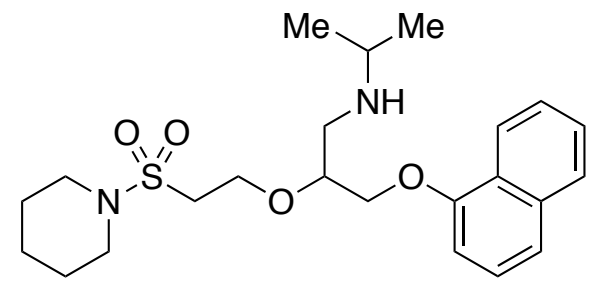

\section{Reaction conditions}

1e $(0.8 \mathrm{mmol})$ in DMF $(0.8 \mathrm{~mL}), \mathbf{2 q}(0.96 \mathrm{mmol}), \mathrm{DMF}(0.8 \mathrm{~mL}),-20^{\circ} \mathrm{C}, 1 \mathrm{~h}, \mathrm{AgOAc} / \mathrm{dppe}(24.0 \mu \mathrm{mol})$, KHMDS $(32 \mu \mathrm{mol})$.

$\mathrm{N}$-isopropyl-3-(naphthalen-1-yloxy)-2-(2-(piperidin-1-ylsulfonyl)ethoxy)propan-1-amine (3eq): (pale yellow oil, $\mathrm{CH}_{2} \mathrm{Cl}_{2} / \mathrm{MeOH}=10 / 1,84 \%$ yield, $290.6 \mathrm{mg}$ ); ${ }^{1} \mathrm{H}-\mathrm{NMR}\left(500 \mathrm{MHz}, \mathrm{CDCl}_{3}\right)(\mathrm{ppm}) \delta$ 8.25-8.19 (m, $1 \mathrm{H}, \mathrm{ArH}), 7.83-7.76(\mathrm{~m}, 1 \mathrm{H}, \mathrm{ArH}), 7.55-7.32(\mathrm{~m}, 4 \mathrm{H}, \mathrm{ArH}), 6.82(\mathrm{dd}, 1 \mathrm{H}, \mathrm{J}=7.0 \mathrm{~Hz}, \mathrm{ArH}), 4.29-4.15(\mathrm{~m}, 3 \mathrm{H}$, $\mathrm{SO}_{2} \mathrm{CH}_{2} \mathrm{CH}_{2} \mathrm{OCHCH} \mathrm{OAr}_{2}$, 4.12-3.98 (m, $\left.2 \mathrm{H}, \mathrm{SO}_{2} \mathrm{CH}_{2} \mathrm{CH}_{2} \mathrm{OCHCH}{ }_{2} \mathrm{OAr}\right), 3.25-3.10\left(\mathrm{~m}, 6 \mathrm{H}, \mathrm{OCHCH}{ }_{2} \mathrm{NH}\right.$, $\left.\mathrm{CH}_{2}\left(\mathrm{CH}_{2} \mathrm{CH}_{2}\right)_{2} \mathrm{~N}\right), 3.00-2.80\left(\mathrm{~m}, 3 \mathrm{H}, \mathrm{SO}_{2} \mathrm{CH}_{2} \mathrm{CH}_{2} \mathrm{O}, \mathrm{NHCH}\left(\mathrm{CH}_{3}\right)_{2}\right), 1.70-1.40\left(\mathrm{~m}, 7 \mathrm{H}, \mathrm{CH}_{2}\left(\mathrm{CH}_{2} \mathrm{CH}_{2}\right)_{2} \mathrm{~N}, \mathrm{NH}\right)$, $1.10\left(\mathrm{dd}, 6 \mathrm{H}, J=2.5,6.0 \mathrm{~Hz}, \mathrm{NHCH}\left(\mathrm{CH}_{3}\right)_{2}\right) ;{ }^{13} \mathrm{C} \mathrm{NMR}\left(125 \mathrm{MHz}, \mathrm{CDCl}_{3}\right) \delta 154.4,134.7,127.7,126.6,125.9$, $125.7,125.5,122.0,120.8,104.9,79.1,69.6,64.5,49.7,49.0,48.8,46.6,25.7,23.9,23.1$, 23.0; IR (thin film, $\mathrm{NaCl}$ ) 3317, 1381, 1240, 1163, $1053 \mathrm{~cm}^{-1}$; HRMS (ESI, H) $\mathrm{m} / \mathrm{z}$ calc'd for $\mathrm{C}_{23} \mathrm{H}_{35} \mathrm{~N}_{2} \mathrm{O}_{4} \mathrm{~S}^{+}(\mathrm{M}+\mathrm{H})^{+}$ 435.2312, found 435.2313 . 


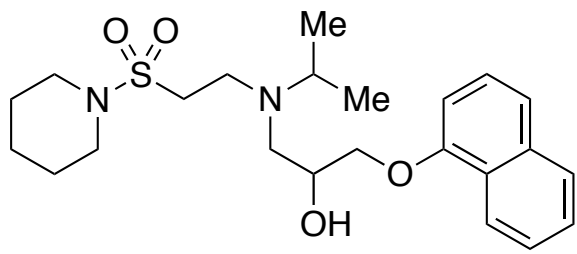

1-(isopropyl(2-(piperidin-1-ylsulfonyl)ethyl)amino)-3-(naphthalen-1-yloxy)propan-2-ol $\quad$ (4eq): (pale yellow oil); ${ }^{1} \mathrm{H}-\mathrm{NMR}\left(500 \mathrm{MHz}, \mathrm{CDCl}_{3}\right)$ (ppm) $\delta$ 8.28-8.21 (m, $\left.1 \mathrm{H}, \mathrm{ArH}\right), 7.83-7.75(\mathrm{~m}, 1 \mathrm{H}, \mathrm{ArH}), 7.52-7.40$ (m, 3H, ArH), 7,37 (t, 1H, J= 7.5 Hz, ArH), $6.84(\mathrm{~d}, 1 \mathrm{H}, J=7.5 \mathrm{~Hz}, \mathrm{ArH}), 4.28-4.20\left(\mathrm{~m}, 1 \mathrm{H}, \operatorname{ArOCH}_{2}\right), 4.19$ $4.06\left(\mathrm{~m}, 2 \mathrm{H}, \mathrm{ArOCH}_{2} \mathrm{CHOH}\right), 3.59(\mathrm{~s}, 1 \mathrm{H}, \mathrm{OH}), 3.20-2.90\left(\mathrm{~m}, 9 \mathrm{H}, \mathrm{CH}_{2} \mathrm{NCH}_{2}, \mathrm{~N}\left(\mathrm{CH}_{2} \mathrm{CH}_{2}\right)_{2} \mathrm{CH}_{2}, \mathrm{NCH}\left(\mathrm{CH}_{3}\right)_{2}\right)$, $2.84\left(\mathrm{dd}, 1 \mathrm{H}, J=4.5,13.3 \mathrm{~Hz}, \mathrm{SO}_{2} \mathrm{CH}_{2}\right), 2.67\left(\mathrm{dd}, 1 \mathrm{H}, J=9.0,13.3 \mathrm{~Hz}, \mathrm{SO}_{2} \mathrm{CH}_{2}\right), 1.65-1.45(\mathrm{~m}, 6 \mathrm{H}$, $\left.\mathrm{N}\left(\mathrm{CH}_{2} \mathrm{CH}_{2}\right)_{2} \mathrm{CH}_{2}\right), 1.10\left(\mathrm{dd}, 6 \mathrm{H}, J=6.5,29.3 \mathrm{~Hz}, \mathrm{NCH}\left(\mathrm{CH}_{3}\right)_{2}\right) ;{ }^{13} \mathrm{C} \mathrm{NMR}\left(125 \mathrm{MHz}, \mathrm{CDCl}_{3}\right) \delta 154.4,134.5$, 127.5, 126.4, 125.9, 125.6, 125.3, 121.8, 120.6, 104.9, 70.0, 66.9, 53.4, 51.8, 48.1, 46.5, 44.5, 25.6, 23.7, 19.7, 17.0; IR (thin film, NaCl) 3458, 1627, 1161, 1053, $1020 \mathrm{~cm}^{-1}$; HRMS (ESI, H) $\mathrm{m} / z$ calc'd for $\mathrm{C}_{23} \mathrm{H}_{35} \mathrm{~N}_{2} \mathrm{O}_{4} \mathrm{~S}^{+}(\mathrm{M}+\mathrm{H})^{+} 435.2312$, found 435.2313 .

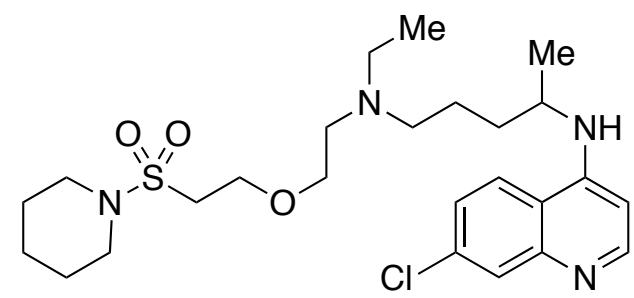

\section{$\underline{\text { Reaction conditions }}$}

1e $(0.8 \mathrm{mmol})$ in DMF (0.8 mL), $2 \mathrm{r}(0.96 \mathrm{mmol}), \operatorname{DMF}(2.4 \mathrm{~mL}),-20{ }^{\circ} \mathrm{C}, 9 \mathrm{~h}, \mathrm{AgOAc} / \mathrm{dppe}(24.0 \mu \mathrm{mol})$, KHMDS $(32 \mu \mathrm{mol})$.

\section{$N^{4}$-(7-chloroquinolin-4-yl)- $N^{1}$-ethyl- $N^{1}$-(2-(2-(piperidin-1-ylsulfonyl)ethoxy)ethyl)pentane-1,4-diamine}

(3er): (pale yellow oil, $\mathrm{CH}_{2} \mathrm{Cl}_{2} / \mathrm{MeOH}=9 / 1$ to 4/1, 92\% yield, $376.8 \mathrm{mg}$ ); ${ }^{1} \mathrm{H}-\mathrm{NMR}\left(500 \mathrm{MHz}, \mathrm{CDCl}_{3}\right)$ (ppm) $\delta 8.51(\mathrm{~d}, 1 \mathrm{H}, J=5.5 \mathrm{~Hz}, \operatorname{ArH}), 7.93(\mathrm{~d}, 1 \mathrm{H}, J=2.0 \mathrm{~Hz}, \operatorname{ArH}), 7.72(\mathrm{~d}, 1 \mathrm{H}, J=9.0 \mathrm{~Hz}, \operatorname{ArH}), 7.34(\mathrm{dd}, 1 \mathrm{H}, J=$ 2.0, 8.5 Hz, ArH); $6.20(\mathrm{~d}, 1 \mathrm{H}, J=5.5 \mathrm{~Hz}, \mathrm{ArH}), 5.21(\mathrm{~d}, 1 \mathrm{H}, J=6.5 \mathrm{~Hz}, \mathrm{NH}), 3.81(\mathrm{t}, 2 \mathrm{H}, J=6.5 \mathrm{~Hz}$, $\mathrm{SO}_{2} \mathrm{CH}_{2} \mathrm{CH}_{2} \mathrm{O}$ ), 3.76-3.66 (m, $\left.1 \mathrm{H}, \mathrm{NHCH}\right), 3.54\left(\mathrm{t}, 2 \mathrm{H}, J=6.0 \mathrm{~Hz}, \mathrm{OCH}_{2} \mathrm{CH}_{2} \mathrm{~N}\right), 3.17(\mathrm{t}, 4 \mathrm{H}, J=5.0 \mathrm{~Hz}$, $\left.\mathrm{N}\left(\mathrm{CH}_{2} \mathrm{CH}_{2}\right)_{2} \mathrm{CH}_{2}\right), 3.12\left(\mathrm{t}, 2 \mathrm{H}, J=6.5 \mathrm{~Hz}, \mathrm{SO}_{2} \mathrm{CH}_{2} \mathrm{CH}_{2} \mathrm{O}\right), 2.70-2.43\left(\mathrm{~m}, 6 \mathrm{H}, \mathrm{OCH}_{2} \mathrm{CH}_{2} \mathrm{~N}\left(\mathrm{CH}_{2} \mathrm{CH}_{3}\right) \mathrm{CH}_{2}\right), 1.80-$ $1.46\left(\mathrm{~m}, 10 \mathrm{H}, \mathrm{N}\left(\mathrm{CH}_{2} \mathrm{CH}_{2}\right)_{2} \mathrm{CH}_{2}, \mathrm{NCH}_{2} \mathrm{CH}_{2} \mathrm{CH}_{2} \mathrm{CHNH}\right), 1.31$ (d, $\left.3 \mathrm{H}, J=6.5 \mathrm{~Hz}, \mathrm{CHCH}_{3}\right), 1.00$ (t, 3H, J = 7.0 Hz, $\left.\mathrm{CH}_{2} \mathrm{CH}_{3}\right) ;{ }^{13} \mathrm{C}$ NMR $\left(125 \mathrm{MHz}, \mathrm{CDCl}_{3}\right) \delta 152.2,149.6,149.2,134.9,129.0,125.1,121.4,117.5,99.4,69.9$, $64.8,53.8,52.9,49.4,48.5,48.3,46.6,34.5,25.7,24.0,23.9,20.4,11.5$; IR (thin film, NaCl) 3398, 1359, 


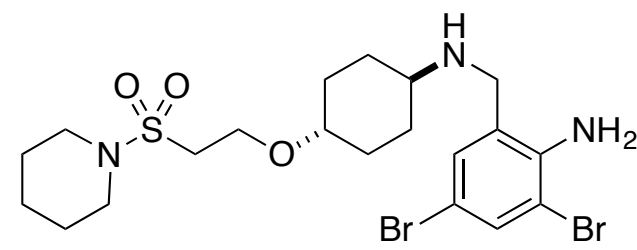

\section{$\underline{\text { Reaction conditions }}$}

1e $(0.8 \mathrm{mmol})$ in DMF $(0.8 \mathrm{~mL}), 2 \mathrm{~s}(0.96 \mathrm{mmol}), \mathrm{DMF}(1.6 \mathrm{~mL}),-20^{\circ} \mathrm{C}, 4 \mathrm{~h}, \mathrm{AgOAc} / \mathrm{dppe}(24.0 \mu \mathrm{mol})$, KHMDS $(32 \mu \mathrm{mol})$.

\section{2,4-dibromo-6-((((1r,4r)-4-(2-(piperidin-1-ylsulfonyl)ethoxy)cyclohexyl)amino)methyl)aniline}

(3es):

(pale yellow oil, $\mathrm{CH}_{2} \mathrm{Cl}_{2} / \mathrm{MeOH}=50 / 1,80 \%$ yield, $353.2 \mathrm{mg}$ ); ${ }^{1} \mathrm{H}-\mathrm{NMR}\left(500 \mathrm{MHz}, \mathrm{CDCl}_{3}\right.$ ) (ppm) $\delta 7.47$ (d, $1 \mathrm{H}, J=2.5 \mathrm{~Hz}, \operatorname{ArH}), 7.08(\mathrm{~d}, 1 \mathrm{H}, J=2.0 \mathrm{~Hz}, \operatorname{ArH}), 5.33,\left(\mathrm{br}, 2 \mathrm{H}, \operatorname{ArNH} \mathrm{H}_{2}\right), 3.83(\mathrm{t}, 2 \mathrm{H}, J=6.5 \mathrm{~Hz}$, $\mathrm{SO}_{2} \mathrm{CH}_{2} \mathrm{CH}_{2} \mathrm{O}$ ), $3.78\left(\mathrm{~s}, 2 \mathrm{H}, \mathrm{ArCH}_{2} \mathrm{NH}\right), 3.34-3.06\left(\mathrm{~m}, 7 \mathrm{H}, \mathrm{SO}_{2} \mathrm{CH}_{2} \mathrm{CH}_{2} \mathrm{O}, \mathrm{N}\left(\mathrm{CH}_{2} \mathrm{CH}_{2}\right)_{2} \mathrm{CH}_{2}, \mathrm{NCH}\left(\mathrm{CH}_{2} \mathrm{CH}_{2}\right)_{2} \mathrm{CHO}\right)$, 2.51-2.42 (m, $\left.1 \mathrm{H}, \mathrm{NCH}\left(\mathrm{CH}_{2} \mathrm{CH}_{2}\right)_{2} \mathrm{CHO}\right), 2.08-1.96\left(\mathrm{~m}, 4 \mathrm{H}, \mathrm{NCH}\left(\mathrm{CH}_{2} \mathrm{CH}_{2}\right)_{2} \mathrm{CHO}\right), 1.70-1.53(\mathrm{~m}, 6 \mathrm{H}$, $\left.\mathrm{N}\left(\mathrm{CH}_{2} \mathrm{CH}_{2}\right)_{2} \mathrm{CH}_{2}\right), 1.34-1.22\left(\mathrm{~m}, 2 \mathrm{H}, \mathrm{NCH}\left(\mathrm{CH}_{2} \mathrm{CH}_{2}\right)_{2} \mathrm{CHO}\right), 1.16-1.05\left(\mathrm{~m}, 2 \mathrm{H}, \mathrm{NCH}\left(\mathrm{CH}_{2} \mathrm{CH}_{2}\right)_{2} \mathrm{CHO}\right), 0.93$ (br, $\left.1 \mathrm{H}, \mathrm{CH}_{2} \mathrm{NH}\right) ;{ }^{13} \mathrm{C} \mathrm{NMR}\left(125 \mathrm{MHz}, \mathrm{CDCl}_{3}\right) \delta 144.0,133.4,131.4,126.9,110.5,108.4,78.3,61.9,55.6,50.8$, 50.1, 46.6, 31.1, 30.5, 25.8, 24.0; IR (KBr) 3394, 1379, 1159, 1053, $1028 \mathrm{~cm}^{-1}$; HRMS (ESI, H) m/z calc'd for $\mathrm{C}_{20} \mathrm{H}_{32} \mathrm{Br}_{2} \mathrm{~N}_{3} \mathrm{O}_{3} \mathrm{~S}^{+}(\mathrm{M}+\mathrm{H})^{+}$552.0526, found 552.0532 .<smiles>Nc1c(Br)cc(Br)cc1CN(CCS(=O)(=O)N1CCCCC1)C1CCC(O)CC1</smiles>

(1r,4r)-4-((2-amino-3,5-dibromobenzyl)(2-(piperidin-1-ylsulfonyl)ethyl)amino)cyclohexan-1-ol (4es): (pale yellow oil); ${ }^{1} \mathrm{H}-\mathrm{NMR}\left(500 \mathrm{MHz}, \mathrm{CDCl}_{3}\right.$ ) (ppm) (with DMF) $\delta 7.51$ (d, $\left.1 \mathrm{H}, J=2.5 \mathrm{~Hz}, \mathrm{ArH}\right), 7.08(\mathrm{~d}, 1 \mathrm{H}$, $J=2.0 \mathrm{~Hz}, \mathrm{ArH}), 5.27\left(\mathrm{br}, 2 \mathrm{H}, \operatorname{ArNH}_{2}\right), 3.65\left(\mathrm{~s}, 2 \mathrm{H}, \operatorname{ArCH}_{2} \mathrm{~N}\right), 3.60-3.50(\mathrm{~m}, 1 \mathrm{H}, \mathrm{CHOH}), 3.06(\mathrm{t}, 4 \mathrm{H}, J=5.0$ $\left.\mathrm{Hz}, \mathrm{CH}_{2}\left(\mathrm{CH}_{2} \mathrm{CH}_{2}\right)_{2} \mathrm{~N}\right), 2.92-2.82\left(\mathrm{~m}, 4 \mathrm{H}, \mathrm{SO}_{2} \mathrm{CH}_{2} \mathrm{CH}_{2} \mathrm{~N}\right), 2.60-2.50(\mathrm{~m}, 1 \mathrm{H}, \mathrm{NCH}), 2.10-2.00(\mathrm{~m}, 2 \mathrm{H}$, $\left.\mathrm{NCH}\left(\mathrm{CH}_{2} \mathrm{CH}_{2}\right)_{2} \mathrm{CHOH}\right), 1.90-1.80\left(\mathrm{~m}, 2 \mathrm{H}, \mathrm{NCH}\left(\mathrm{CH}_{2} \mathrm{CH}_{2}\right)_{2} \mathrm{CHOH}\right), 1.62-1.34\left(\mathrm{~m}, 9 \mathrm{H}, \mathrm{NCH}\left(\mathrm{CH}_{2} \mathrm{CH}_{2}\right)_{2} \mathrm{CHOH}\right.$, $\left.\mathrm{CH}_{2}\left(\mathrm{CH}_{2} \mathrm{CH}_{2}\right)_{2} \mathrm{~N}\right)$, 1.33-1.22(m, $\left.\left.2 \mathrm{H}, \mathrm{NCH}\left(\mathrm{CH}_{2} \mathrm{CH}_{2}\right)_{2} \mathrm{CHOH}\right) ;{ }^{13} \mathrm{C} \mathrm{NMR} \mathrm{(125} \mathrm{MHz,} \mathrm{CDCl}_{3}\right) \delta 144.0,133.8$, 
132.0, 125.1, 110.5, 108.6, 70.4, 59.8, 55.4, 48.3, 46.6, 44.4, 34.7, 26.1, 25.7, 23.9; IR (thin film, NaCl) $3435,1608,1161,1055,1028 \mathrm{~cm}^{-1}$; HRMS (ESI, H) $\mathrm{m} / z$ calc'd for $\mathrm{C}_{20} \mathrm{H}_{32} \mathrm{Br}_{2} \mathrm{~N}_{3} \mathrm{O}_{3} \mathrm{~S}^{+}(\mathrm{M}+\mathrm{H})^{+} 552.0526$, found 552.0524 .

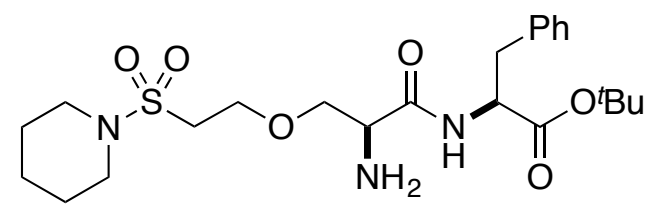

\section{$\underline{\text { Reaction conditions }}$}

1e $(0.57 \mathrm{mmol})$ in DMF $(0.57 \mathrm{~mL}), 2 \mathrm{t}(0.68 \mathrm{mmol})$, DMF $(1.8 \mathrm{~mL}),-20^{\circ} \mathrm{C}, 33 \mathrm{~h}, \mathrm{AgOAc} / \mathrm{dppe}(34.0 \mu \mathrm{mol})$, KHMDS $(46 \mu \mathrm{mol})$.

tert-butyl $\quad$ O-(2-(piperidin-1-ylsulfonyl)ethyl)-L-seryl-L-phenylalaninate $\quad$ (3et): (colorless oil, $\mathrm{CH}_{2} \mathrm{Cl}_{2} / \mathrm{MeOH}=50 / 1,76 \%$ yield, $\left.208.4 \mathrm{mg}\right) ;{ }^{1} \mathrm{H}-\mathrm{NMR}\left(500 \mathrm{MHz}, \mathrm{CDCl}_{3}\right)(\mathrm{ppm}) \delta 7.89(\mathrm{~d}, 1 \mathrm{H}, J=8.5 \mathrm{~Hz}$, $\mathrm{NHCO})$, 7.30-7.15 (m, 5H, ArH), 4.77-4.70 (m, $\left.1 \mathrm{H}, \mathrm{CHNH}_{2}\right)$, 3.89-3.79 (m, 2H, SO $\left.\mathrm{CH}_{2} \mathrm{CH}_{2} \mathrm{O}\right)$, 3.65-3.50 (m, $\left.3 \mathrm{H}, \mathrm{OCH}_{2} \mathrm{CHNH}_{2}, \mathrm{NHCHCH}_{2} \mathrm{Ph}\right), 3.25-3.01\left(\mathrm{~m}, 8 \mathrm{H}, \mathrm{CH}_{2}\left(\mathrm{CH}_{2} \mathrm{CH}_{2}\right)_{2} \mathrm{~N}, \mathrm{SO}_{2} \mathrm{CH}_{2} \mathrm{CH}_{2} \mathrm{O}, \mathrm{CH}_{2} \mathrm{Ph}\right), 1.70-1.50(\mathrm{~m}, 8 \mathrm{H}$, $\left.\mathrm{NH}_{2}, \mathrm{CH}_{2}\left(\mathrm{CH}_{2} \mathrm{CH}_{2}\right)_{2} \mathrm{~N}\right), 1.41\left(\mathrm{~s}, 9 \mathrm{H}, \mathrm{C}\left(\mathrm{CH}_{3}\right)_{3}\right) ;{ }^{13} \mathrm{C} \mathrm{NMR}\left(125 \mathrm{MHz}, \mathrm{CDCl}_{3}\right) \delta 172.1,170.7,136.5,129.7,128.5$, $127.1,82.3,73.1,64.7,54.9,53.4,49.0,46.7,38.3,28.1,25.8,23.9$; IR (thin film, $\mathrm{NaCl}$ ) 3363, 1359, 1161, 1109, $1053 \mathrm{~cm}^{-1}$; HRMS (ESI, H) $\mathrm{m} / z$ calc'd for $\mathrm{C}_{23} \mathrm{H}_{38} \mathrm{~N}_{3} \mathrm{O}_{6} \mathrm{~S}^{+}(\mathrm{M}+\mathrm{H})^{+} 484.2476$, found 484.2474. [ $[\alpha]_{D}{ }^{25}$ $+21.4\left(\right.$ c 1.00, $\left.\mathrm{CHCl}_{3}\right)$.<smiles>CCCCOC(=O)C(Cc1ccccc1)NC(=O)[C@H](CO)NCCS(=O)(=O)N1CCCCC1</smiles>

tert-butyl (2-(piperidin-1-ylsulfonyl)ethyl)-L-seryl-L-phenylalaninate (4et): (pale yellow oil); ${ }^{1} \mathrm{H}-\mathrm{NMR}$ $\left(500 \mathrm{MHz}, \mathrm{CDCl}_{3}\right)(\mathrm{ppm}) \delta 7.64(\mathrm{~d}, 1 \mathrm{H}, J=8.5 \mathrm{~Hz}, \mathrm{NHCO}), 7.32-7.14(\mathrm{~m}, 5 \mathrm{H}, \mathrm{ArH}), 4.77-4.70(\mathrm{~m}, 1 \mathrm{H}$, $\left.\mathrm{CHNHCH}_{2} \mathrm{CH}_{2} \mathrm{SO}_{2}\right), 3.72-3.61\left(\mathrm{~m}, 2 \mathrm{H}, \mathrm{CH} \mathrm{CH}_{2}\right), 3.26-2.95\left(\mathrm{~m}, 11 \mathrm{H}, \mathrm{CH}_{2}\left(\mathrm{CH}_{2} \mathrm{CH}_{2}\right)_{2} \mathrm{~N}, \mathrm{NHCH}_{2} \mathrm{CH}_{2} \mathrm{SO}_{2}\right.$, $\left.\mathrm{CHCH}_{2} \mathrm{Ph}\right), 2.24\left(\mathrm{br}, 1 \mathrm{H}, \mathrm{CH}_{2} \mathrm{NH}\right), 2.14(\mathrm{br}, 1 \mathrm{H}, \mathrm{OH}), 1.70-1.53\left(\mathrm{~m}, 6 \mathrm{H}, \mathrm{CH}_{2}\left(\mathrm{CH}_{2} \mathrm{CH}_{2}\right)_{2} \mathrm{~N}\right), 1.44(\mathrm{~s}, 9 \mathrm{H}$, $\left.\mathrm{C}\left(\mathrm{CH}_{3}\right)_{3}\right) ;{ }^{13} \mathrm{C}$ NMR $\left(125 \mathrm{MHz}, \mathrm{CDCl}_{3}\right) \delta 172.0,170.7,136.4,129.6,128.6,127.2,82.4,63.8,63.1,53.3$, 49.0, 46.7, 42.6, 38.2, 28.1, 25.8, 23.9; IR (thin film, NaCl) 3649, 3352, 1163, 1057, $1031 \mathrm{~cm}^{-1}$; HRMS (ESI, H) $m / z$ calc'd for $\mathrm{C}_{23} \mathrm{H}_{38} \mathrm{~N}_{3} \mathrm{O}_{6} \mathrm{~S}^{+}(\mathrm{M}+\mathrm{H})^{+} 484.2476$, found 484.2479. $[\alpha]_{\mathrm{D}}{ }^{25}+13.0\left(c 1.00, \mathrm{CHCl}_{3}\right)$. 


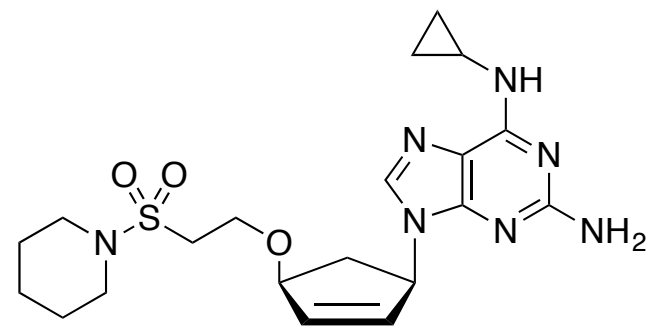

\section{Reaction conditions}

1e $(0.8 \mathrm{mmol})$ in DMF $(0.8 \mathrm{~mL}), 2 \mathrm{u}(0.96 \mathrm{mmol}), \mathrm{DMF}(1.6 \mathrm{~mL}),-20^{\circ} \mathrm{C}, 21 \mathrm{~h}, \mathrm{AgOAc} / \mathrm{dppe}(48.0 \mu \mathrm{mol})$, KHMDS $(64 \mu \mathrm{mol})$.

$N^{6}$-cyclopropyl-9-((1R,4S)-4-((2-(piperidin-1-ylsulfonyl)ethoxy)methyl)cyclopent-2-en-1-yl)-9H-purine-

2,6-diamine (3eu): (white foam, $\mathrm{CH}_{2} \mathrm{Cl}_{2} / \mathrm{MeOH}=20 / 1,85 \%$ yield, $319.1 \mathrm{mg}$ with DMF); ${ }^{1} \mathrm{H}-\mathrm{NMR}$ (500 $\left.\mathrm{MHz}, \mathrm{CDCl}_{3}\right)$ (ppm) (with DMF) $\delta 8.02$ (s, 1H,DMF), 7.52 (s, 1H, ArH), 6.15-6.10 (m, 1H, $\left.\mathrm{OCH}_{2} \mathrm{CHCH}=\mathrm{CHCHCHN}\right)$, 5.92-5.96 (m, $\left.1 \mathrm{H}, \mathrm{OCH}_{2} \mathrm{CHCH}=\mathrm{CHCHCHN}\right), 5.64(1 \mathrm{H}, \mathrm{br}, \operatorname{ArNHCH})$, 5.58-5.50 (m, $\left.1 \mathrm{H}, \mathrm{OCH}_{2} \mathrm{CHCH}=\mathrm{CHCHCHN}\right), 4.77\left(\mathrm{~s}, 2 \mathrm{H}, \mathrm{ArNH}_{2}\right), 3.88-3.78\left(\mathrm{~m}, 2 \mathrm{H}, \mathrm{SO}_{2} \mathrm{CH}_{2} \mathrm{CH}_{2} \mathrm{O}\right), 3.54-3.46(\mathrm{~m}, 2 \mathrm{H}$, $\left.\mathrm{SO}_{2} \mathrm{CH}_{2} \mathrm{CH}_{2} \mathrm{O}\right), 3.26-2.98\left(\mathrm{~m}, 8 \mathrm{H}, \mathrm{OCH}_{2} \mathrm{CHCH}=\mathrm{CHCHCHN}, \mathrm{CH}_{2}\left(\mathrm{CH}_{2} \mathrm{CH}_{2}\right)_{2} \mathrm{~N}, \operatorname{ArNHCH}\left(\mathrm{CH}_{2}\right)_{2}\right), 2.96(\mathrm{~s}, 3 \mathrm{H}$, DMF), 2.84 (s, 3H, DMF), 2.83-2.73 (m, $\left.1 \mathrm{H}, \mathrm{OCH}_{2} \mathrm{CHCH}_{2} \mathrm{CHCHN}\right), 1.70-1.50\left(\mathrm{~m}, 7 \mathrm{H}, \mathrm{OCH}_{2} \mathrm{CHCH}_{2} \mathrm{CHCHN}\right.$, $\left.\mathrm{CH}_{2}\left(\mathrm{CH}_{2} \mathrm{CH}_{2}\right)_{2} \mathrm{~N}\right), 0.90-0.80\left(\mathrm{~m}, 2 \mathrm{H}, \operatorname{ArNHCH}\left(\mathrm{CH}_{2}\right)_{2}\right), 0.65-0.55\left(\mathrm{~m}, 2 \mathrm{H}, \operatorname{ArNHCH}\left(\mathrm{CH}_{2}\right)_{2}\right) ;{ }^{13} \mathrm{C} \mathrm{NMR}(125$ $\mathrm{MHz}, \mathrm{CDCl}_{3}$ ) (with DMF) $\delta 162.6(\mathrm{DMF}), 160.0,156.4,151.2,137.9,135.8,130.4,115.0,74.3,65.0,58.7$, 49.3, 46.6, 45.5, 36.6(DMF), 35.5, 31.6(DMF), 25.7, 23.9, 23.8, 7.6;IR (KBr) 3342, 1670, 1356, 1053, 1020 $\mathrm{cm}^{-1}$; HRMS (ESI, H) $\mathrm{m} / z$ calc'd for $\mathrm{C}_{21} \mathrm{H}_{32} \mathrm{~N}_{7} \mathrm{O}_{3} \mathrm{~S}(\mathrm{M}+\mathrm{H})^{+}$462.2282, found 462.2278. [ $\left.\alpha\right]_{\mathrm{D}}{ }^{25}-59.0$ (c 1.00, $\left.\mathrm{CHCl}_{3}\right)$.

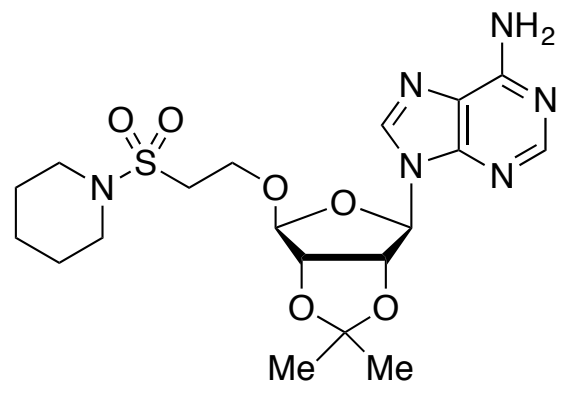

\section{$\underline{\text { Reaction conditions }}$}

1e $(0.8 \mathrm{mmol})$ in DMF $(0.8 \mathrm{~mL}), 2 \mathrm{v}(0.96 \mathrm{mmol}), \operatorname{DMF}(1.6 \mathrm{~mL}),-20^{\circ} \mathrm{C}, 24 \mathrm{~h}$, AgOAc/dppe $(48.0 \mu \mathrm{mol})$, 
KHMDS (64 $\mu \mathrm{mol})$.

9-((3aR,4R,6R,6aR)-2,2-dimethyl-6-((2-(piperidin-1-ylsulfonyl)ethoxy)methyl)tetrahydrofuro[3,4-

d][1,3]dioxol-4-yl)-9H-purin-6-amine (3ev): (white foam, $\mathrm{CH}_{2} \mathrm{Cl}_{2} / \mathrm{MeOH}=20 / 1,63 \%$ yield, $266.6 \mathrm{mg}$ with DMF); ${ }^{1} \mathrm{H}-\mathrm{NMR}\left(500 \mathrm{MHz}, \mathrm{CDCl}_{3}\right.$ ) (ppm) (with DMF) $\delta 8.37$ (s, $\left.1 \mathrm{H}, \mathrm{ArH}\right), 8.04(\mathrm{~s}, 1 \mathrm{H}, \mathrm{ArH}), 8.02(\mathrm{~s}, 1 \mathrm{H}$, DMF), $6.16(\mathrm{dd}, 1 \mathrm{H}, J=4.0 \mathrm{~Hz}, \mathrm{OCHAr}), 5.78\left(\mathrm{br}, 2 \mathrm{H}, \mathrm{NH}_{2}\right.$ ), $5.43\left(\mathrm{dd}, 1 \mathrm{H}, J=2.5,12.5 \mathrm{~Hz}, \mathrm{HOCH}_{2} \mathrm{CHO}\right.$ ), 5.05 (dd, $1 \mathrm{H}, J=2.5,6.0 \mathrm{~Hz}, \mathrm{COCHCHNAr}) ; 4.48\left(\mathrm{~m}, 1 \mathrm{H}, \mathrm{OCHCHCH}{ }_{2} \mathrm{OH}\right), 3.90-3.60\left(\mathrm{~m}, 4 \mathrm{H}, \mathrm{SO}_{2} \mathrm{CH}_{2} \mathrm{CH}_{2} \mathrm{OCH}_{2}\right.$ ), $3.43\left(\mathrm{t}, 4 \mathrm{H}, J=5.0 \mathrm{~Hz}, \mathrm{CH}_{2}\left(\mathrm{CH}_{2} \mathrm{CH}_{2}\right)_{2} \mathrm{~N}\right), 3.05-2.98\left(\mathrm{~m}, 2 \mathrm{H}, \mathrm{SO}_{2} \mathrm{CH}_{2} \mathrm{CH}_{2} \mathrm{O}\right), 2.96(\mathrm{~s}, 3 \mathrm{H}, \mathrm{DMF}), 2.89(\mathrm{~d}, 3 \mathrm{H}$, DMF), 1.68-1.45 (m, 9H, $\left.\mathrm{CH}_{2}\left(\mathrm{CH}_{2} \mathrm{CH}_{2}\right)_{2} \mathrm{~N}, \mathrm{C}\left(\mathrm{CH}_{3}\right)_{2}\right), 1.40\left(\mathrm{~s}, 3 \mathrm{H}, \mathrm{C}\left(\mathrm{CH}_{3}\right)_{2}\right) ;{ }^{13} \mathrm{C} \mathrm{NMR}\left(125 \mathrm{MHz}, \mathrm{CDCl}_{3}\right) \delta$ 162.6 (DMF), 155.6, 153.3, 149.7, 139.8, 120.2, 114.4, 91.5, 86.0, 84.6, 81.9, 71.6, 65.1, 48.6, 46.5, 36.6 (DMF), 31.5 (DMF), 27.3, 25.7, 25.4, 23.8; IR (KBr) 3329, 1361, 1161, 1055, $1028 \mathrm{~cm}^{-1}$; HRMS (ESI, H) $\mathrm{m} / \mathrm{z}$ calc'd for $\mathrm{C}_{20} \mathrm{H}_{31} \mathrm{~N}_{6} \mathrm{O}_{6} \mathrm{~S}^{+}(\mathrm{M}+\mathrm{H})^{+} 438.2020$, found 438.2021. $[\alpha]_{D}{ }^{25}-43.8$ (c 1.00, $\mathrm{CHCl}_{3}$ ).

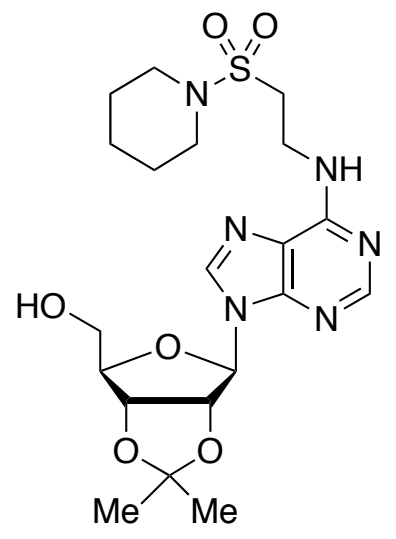

((3aR,4R,6R,6aR)-2,2-dimethyl-6-(6-((2-(piperidin-1-ylsulfonyl)ethyl)amino)-9H-purin-9-

yl)tetrahydrofuro[3,4-d][1,3]dioxol-4-yl)methanol (4ev): (white foam); ${ }^{1} \mathrm{H}-\mathrm{NMR}\left(500 \mathrm{MHz}, \mathrm{CDCl}_{3}\right)(\mathrm{ppm})$ (with DMF) $\delta 8.34$ (s, 1H, ArH), 8.02 (s, 1H, DMF), 7.83 (dd, 1H, J = 2.5 Hz, ArH), 6.69 (br, 1H, NH), 5.86 (dd, $1 \mathrm{H}, J=5.0 \mathrm{~Hz}, \mathrm{OCHAr}$ ), $5.20\left(\mathrm{t}, 1 \mathrm{H}, J=5.0 \mathrm{~Hz}, \mathrm{HOCH}_{2} \mathrm{CHO}\right.$ ), 5.11 (d, $1 \mathrm{H}, J=5.5 \mathrm{~Hz}, \mathrm{COCHCHNAr}$ ), 4.54 (s, $\left.1 \mathrm{H}, \mathrm{OCHCHCH}{ }_{2} \mathrm{OH}\right), 4.13\left(\mathrm{~s}, 2 \mathrm{H}, \mathrm{SO}_{2} \mathrm{CH}_{2} \mathrm{CH}_{2} \mathrm{~N}\right), 3.98\left(\mathrm{~m}, 1 \mathrm{H}, \mathrm{CH}_{2} \mathrm{OH}\right), 3.85-3.75\left(\mathrm{~m}, 1 \mathrm{H}, \mathrm{CH}_{2} \mathrm{OH}\right)$, 3.33$3.20\left(\mathrm{~m}, 6 \mathrm{H}, \mathrm{CH}_{2}\left(\mathrm{CH}_{2} \mathrm{CH}_{2}\right)_{2} \mathrm{~N}, \mathrm{SO}_{2} \mathrm{CH}_{2} \mathrm{CH}_{2} \mathrm{~N}\right), 2.96$ (s, 3H, DMF), 2.88 (s, 3H, DMF), 1.98 (br, 1H, OH), 1.70$1.50\left(\mathrm{~m}, 9 \mathrm{H}, \mathrm{CH}_{2}\left(\mathrm{CH}_{2} \mathrm{CH}_{2}\right)_{2} \mathrm{~N}, \mathrm{C}\left(\mathrm{CH}_{3}\right)_{2}\right), 1.38\left(\mathrm{~s}, 3 \mathrm{H}, \mathrm{C}\left(\mathrm{CH}_{3}\right)_{2}\right) ;{ }^{13} \mathrm{C} \mathrm{NMR}\left(125 \mathrm{MHz}, \mathrm{CDCl}_{3}\right)$ (with DMF) $\delta$ 162.7 (DMF), 154.9, 152.6, 140.2, 114.1, 94.4, 86.2, 83.2, 81.8, 63.6, 48.5, 46.7, 46.7, 36.6 (DMF), 35.3, 31.6 (DMF), 27.8, 25.7, 25.4, 23.9; IR (KBr) 3354, 3219, 1161, 1053, $1028 \mathrm{~cm}^{-1}$; HRMS (ESI, H) $\mathrm{m} / z$ calc'd

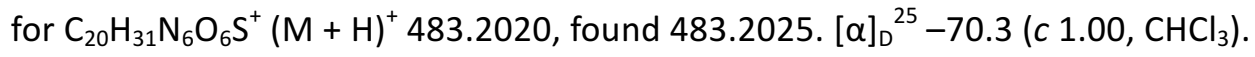




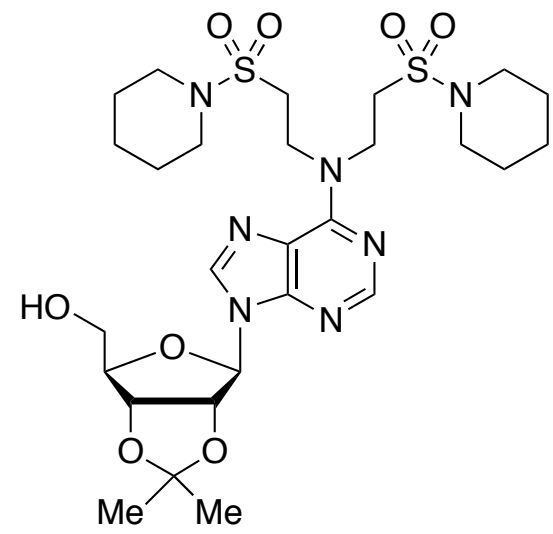

((3aR,4R,6R,6aR)-6-(6-(bis(2-(piperidin-1-ylsulfonyl)ethyl)amino)-9H-purin-9-yl)-2,2-

dimethyltetrahydrofuro[3,4-d][1,3]dioxol-4-yl)methanol (SI-1): (white foam); ${ }^{1} \mathrm{H}-\mathrm{NMR}\left(500 \mathrm{MHz}, \mathrm{CDCl}_{3}\right.$ ) (ppm) (with DMF, DCM) $\delta 8.33(\mathrm{t}, 1 \mathrm{H}, J=6.5 \mathrm{~Hz}, \mathrm{ArH}), 8.02$ (s, 1H, DMF), 7.80 (s, 1H, ArH), 5.84 (d, $1 \mathrm{H}, J=$ $5.0 \mathrm{~Hz}, \mathrm{OCHAr}$ ), 5.30 (s, 2H, DCM), 5.28-5.20 (m, 1H, $\mathrm{HOCH}_{2} \mathrm{CHO}$ ), 5.15-5.08 (m, 1H, COCHCHNAr), 4.70$4.10\left(\mathrm{~m}, 5 \mathrm{H}, \mathrm{OCHCHCH} \mathrm{OH}_{2}\left(\mathrm{SO}_{2} \mathrm{CH}_{2} \mathrm{CH}_{2}\right)_{2} \mathrm{~N}\right), 3.96\left(\mathrm{dd}, 1 \mathrm{H}, \mathrm{J}=12.5 \mathrm{~Hz}, \mathrm{CH}_{2} \mathrm{OH}\right), 3.85-3.75(\mathrm{~m}, 1 \mathrm{H}, \mathrm{CH} \mathrm{OH} \mathrm{OH}$, 3.50-3.20 (m, $\left.12 \mathrm{H},\left(\mathrm{CH}_{2}\left(\mathrm{CH}_{2} \mathrm{CH}_{2}\right)_{2} \mathrm{NSO}_{2} \mathrm{CH}_{2} \mathrm{CH}_{2}\right)_{2} \mathrm{~N}\right), 1.80-1.50\left(\mathrm{~m}, 16 \mathrm{H},\left(\mathrm{CH}_{2}\left(\mathrm{CH}_{2} \mathrm{CH}_{2}\right)_{2} \mathrm{NSO}_{2} \mathrm{CH}_{2} \mathrm{CH}_{2}\right)_{2} \mathrm{~N}, \mathrm{OH}\right.$, $\left.\mathrm{C}\left(\mathrm{CH}_{3}\right)_{2}\right), 1.83\left(\mathrm{~s}, 3 \mathrm{H}, \mathrm{C}\left(\mathrm{CH}_{3}\right)_{2}\right) ;{ }^{13} \mathrm{C} \mathrm{NMR}\left(125 \mathrm{MHz}, \mathrm{CDCl}_{3}\right.$ ) (with DMF, DCM) $\delta 162.6$ (DMF), 154.0, 152.0, 149.5, 139.6, 121.6, 114.1, 94.4, 86.1, 82.8, 81.8, 63.6, 53.6 (DCM), 46.0, 46.0, 36.6 (DMF), 31.5 (DMF), 27.8, 25.7, 25.4, 23.9; IR (KBr) 3524, 1163, 1109, 1053, $1026 \mathrm{~cm}^{-1}$; HRMS (ESI, H) $\mathrm{m} / \mathrm{z}$ calc'd for

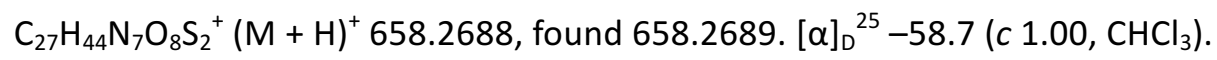

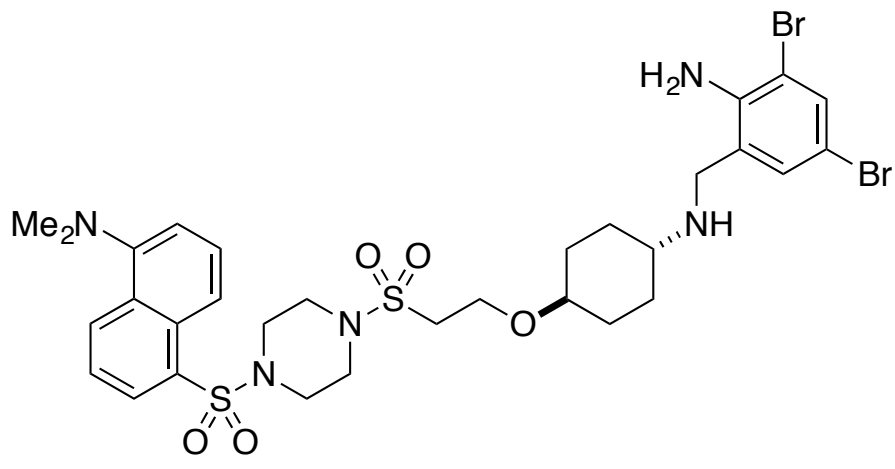

\section{$\underline{\text { Reaction conditions }}$}

1 ( $(0.8 \mathrm{mmol})$ in mixture of DMF and THF (1.6mL, DMF/THF=1:1), $2 \mathrm{~s}(0.96 \mathrm{mmol}), \mathrm{DMF}(1.6 \mathrm{~mL}),-20{ }^{\circ} \mathrm{C}$, $17 \mathrm{~h}, \mathrm{AgOAc} / \mathrm{dppe}(48.0 \mu \mathrm{mol}), \operatorname{KHMDS}(64 \mu \mathrm{mol})$.

5-((4-((2-(((1r,4r)-4-((2-amino-3,5-dibromobenzyl)amino)cyclohexyl)oxy)ethyl)sulfonyl)piperazin-1- 
yl)sulfonyl)- $\mathbf{N}, \boldsymbol{N}$-dimethylnaphthalen-1-amine (3ls): (light green foam, $\mathrm{CH}_{2} \mathrm{Cl}_{2}: \mathrm{MeOH}=50: 1,80 \%$ yield, $526.5 \mathrm{mg}$ with $\left.\mathrm{CH}_{2} \mathrm{Cl}_{2}\right)$; ${ }^{1} \mathrm{H}-\mathrm{NMR}\left(500 \mathrm{MHz}, \mathrm{CDCl}_{3}\right)(\mathrm{ppm})$ (with $\left.\mathrm{CH}_{2} \mathrm{Cl}_{2}\right) \delta 8.35$ (d, $1 \mathrm{H}, J=8.5 \mathrm{~Hz}, \mathrm{ArH}$ ), $8.33(\mathrm{~d}, 1 \mathrm{H}, J=8.5 \mathrm{~Hz}, \operatorname{ArH}) 8.18(\mathrm{dd}, 1 \mathrm{H}, J=1.0,7.3 \mathrm{~Hz}, \operatorname{ArH}), 7.58-7.50(\mathrm{~m}, 4 \mathrm{H}, \operatorname{ArH}), 7.48(\mathrm{~d}, 1 \mathrm{H}, J=2.5$ $\mathrm{Hz}, \operatorname{ArH}), 7.19(\mathrm{~d}, 1 \mathrm{H}, J=7.5 \mathrm{~Hz}, \operatorname{ArH}), 7.10(\mathrm{~d}, 1 \mathrm{H}, J=2.0 \mathrm{~Hz}, \operatorname{ArH}), 5.33\left(\mathrm{~s}, 2 \mathrm{H}, \mathrm{NCH}_{2} \operatorname{Ar}\right), 3.82-3.72(\mathrm{~m}$, $\left.4 \mathrm{H}, \mathrm{NH}_{2}, \mathrm{SO}_{2} \mathrm{CH}_{2} \mathrm{CH}_{2} \mathrm{O}\right), 3.38-3.20\left(\mathrm{~m}, 9 \mathrm{H}, \mathrm{SO}_{2}\left(\mathrm{CH}_{2} \mathrm{CH}_{2}\right)_{2} \mathrm{SO}_{2}, \mathrm{OCH}\left(\mathrm{CH}_{2} \mathrm{CH}_{2}\right)_{2} \mathrm{CHN}\right), 3.15(\mathrm{t}, 2 \mathrm{H}, J=6.5 \mathrm{~Hz}$, $\left.\mathrm{SO}_{2} \mathrm{CH}_{2} \mathrm{CH}_{2} \mathrm{O}\right), 2.89\left(\mathrm{~s}, 6 \mathrm{H}, \mathrm{N}\left(\mathrm{CH}_{3}\right)_{2}\right), 2.50-2.40\left(\mathrm{~m}, 1 \mathrm{H}, \mathrm{OCH}\left(\mathrm{CH}_{2} \mathrm{CH}_{2}\right)_{2} \mathrm{CHN}\right), 1.99(\mathrm{~d}, 4 \mathrm{H}, J=11.0 \mathrm{~Hz}$, $\left.\mathrm{OCH}\left(\mathrm{CH}_{2} \mathrm{CH}_{2}\right)_{2} \mathrm{CHN}\right), 1.30-1.17\left(\mathrm{~m}, 2 \mathrm{H}, \mathrm{OCH}\left(\mathrm{CH}_{2} \mathrm{CH}_{2}\right)_{2} \mathrm{CHN}\right), 1.15-1.05\left(\mathrm{~m}, 2 \mathrm{H}, \mathrm{OCH}\left(\mathrm{CH}_{2} \mathrm{CH}_{2}\right)_{2} \mathrm{CHN}\right), 0.94$ (br, $1 \mathrm{H}, \mathrm{NH}) ;{ }^{13} \mathrm{C}$ NMR $\left(125 \mathrm{MHz}, \mathrm{CDCl}_{3}\right) \delta 152.0,144.0,133.3,132.3,131.4,131.2,130.8,130.4,130.2$, $128.4,126.9,123.3,119.4,115.5,110.5,108.4,78.5,61.7,55.6,53.6\left(\mathrm{CH}_{2} \mathrm{CL}_{2}\right), 51.3,50.7,45.8,45.5$, 45.3, 31.1, 30.5; IR (KBr) 3441, 2933, 1145, 1084, $1059 \mathrm{~cm}^{-1}$; HRMS (ESI, H) $\mathrm{m} / \mathrm{z}$ calc'd for $\mathrm{C}_{31} \mathrm{H}_{42} \mathrm{Br}_{2} \mathrm{~N}_{5} \mathrm{O}_{5} \mathrm{~S}_{2}^{+}(\mathrm{M}+\mathrm{H})^{+}$786.0989, found 786.0994.<smiles>CNc1cccc2c(S(=O)(=O)N3CCN(S(=O)(=O)CCN(Cc4cc(Br)cc(Br)c4N)C4CCC(O)CC4)CC3)cccc12</smiles>

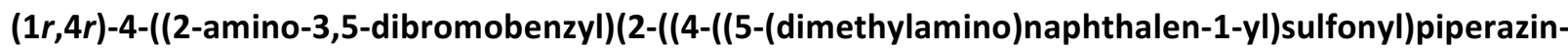

1-yl)sulfonyl)ethyl)amino)cyclohexan-1-ol (4ls): (light green foam); ${ }^{1} \mathrm{H}-\mathrm{NMR}\left(500 \mathrm{MHz}^{\mathrm{CDCl}} \mathrm{Cl}_{3}\right.$ (ppm) (with DMF) $\delta 8.59(\mathrm{~d}, 1 \mathrm{H}, J=8.5 \mathrm{~Hz}, \operatorname{ArH}), 8.33(\mathrm{~d}, 1 \mathrm{H}, J=8.5 \mathrm{~Hz}, \operatorname{ArH}), 8.19$ (dd, $1 \mathrm{H}, J=1.0,7.3 \mathrm{~Hz}, \operatorname{ArH}$ ), $8.02(\mathrm{~s}, 1 \mathrm{H}, \mathrm{DMF}), 7.58-7.50(\mathrm{~m}, 2 \mathrm{H}, \mathrm{ArH}), 8.47(\mathrm{~d}, 1 \mathrm{H}, J=2.0 \mathrm{~Hz}, \operatorname{ArH}), 7.20(\mathrm{~d}, 1 \mathrm{H}, J=7.5 \mathrm{~Hz}, \operatorname{ArH}), 7.04$ $(\mathrm{d}, 1 \mathrm{H}, J=2.0 \mathrm{~Hz}, \mathrm{ArH}), 5.18\left(\mathrm{~s}, 2 \mathrm{H}, \mathrm{ArCH}_{2} \mathrm{~N}\right), 3.65-3.48\left(\mathrm{~m}, 3 \mathrm{H}, \mathrm{ArNH}_{2}, \mathrm{OCH}\left(\mathrm{CH}_{2} \mathrm{CH}_{2}\right)_{2} \mathrm{CHN}\right), 3.30-3.15(\mathrm{~m}$, $\left.8 \mathrm{H}, \mathrm{N}\left(\mathrm{CH}_{2} \mathrm{CH}_{2}\right)_{2} \mathrm{~N}\right), 3.00$ (s, 3H, DMF), 2.92-2.77 (m, $\left.10 \mathrm{H}, \mathrm{N}\left(\mathrm{CH}_{3}\right)_{2}, \mathrm{SO}_{2} \mathrm{CH}_{2} \mathrm{CH}_{2} \mathrm{~N}\right), 2.88(\mathrm{~s}, 3 \mathrm{H}, \mathrm{DMF}), 2.55-$ $2.45\left(\mathrm{~m}, 1 \mathrm{H}, \quad \mathrm{OCH}\left(\mathrm{CH}_{2} \mathrm{CH}_{2}\right)_{2} \mathrm{CHN}\right), 2.08-1.98\left(\mathrm{~m}, 2 \mathrm{H}, \quad \mathrm{OCH}\left(\mathrm{CH}_{2} \mathrm{CH}_{2}\right)_{2} \mathrm{CHN}\right), 1.88-1.76(\mathrm{~m}, 2 \mathrm{H}$, $\left.\mathrm{OCH}\left(\mathrm{CH}_{2} \mathrm{CH}_{2}\right)_{2} \mathrm{CHN}\right), 1.45-1.32\left(\mathrm{~m}, 3 \mathrm{H}, \mathrm{OH}, \mathrm{OCH}\left(\mathrm{CH}_{2} \mathrm{CH}_{2}\right)_{2} \mathrm{CHN}\right), 1.31-1.18\left(\mathrm{~m}, 2 \mathrm{H}, \mathrm{OCH}\left(\mathrm{CH}_{2} \mathrm{CH}_{2}\right)_{2} \mathrm{CHN}\right) ;{ }^{13} \mathrm{C}$ NMR (125 MHz, CDCl ${ }_{3}$ ) (with DMF) $\delta 162.5$ (DMF), 151.9, 143.7, 133.8, 132.2, 131.8, 131.2, 130.8, 130.3, 130.1, 128.4, 124.7, 123.2, 119.2, 115.4, 110.4, 108.5, 70.2, 59.6, 55.3, 48.5, 45.5, 45.4, 45.2, 44.2, 36.5(DMF), 34.5, 31.4 (DMF), 25.9; IR (KBr) 3655, 1610, 1230, 1147, $1060 \mathrm{~cm}^{-1}$; HRMS (ESI, H) m/z calc'd for $\mathrm{C}_{31} \mathrm{H}_{42} \mathrm{Br}_{2} \mathrm{~N}_{5} \mathrm{O}_{5} \mathrm{~S}_{2}^{+}(\mathrm{M}+\mathrm{H})^{+} 786.0989$, found 786.0994. 


\section{5-2. Catalytic Chemo- and Regioselective Conjugate Addition Reactions and Characterization of the} Products

Procedure catalytic chemo- and regioselective conjugate addition reactions of amino alcohols

A flame-dried $20 \mathrm{~mL}$ test tube equipped with a magnetic stirring bar and 3-way glass stopcock was charged with metal catalyst $(24.0 \mu \mathrm{mol})$, ligand $(24.1 \mu \mathrm{mol})$ and distilled DMF via syringe. After stirring for 5 minutes, $\beta$-amino alcohol (2) $(0.96 \mathrm{mmol})$ and other amino alcohol (2) $(0.96 \mathrm{mmol})$ were added to the mixture. After stirred at room temperature for about 10 minutes, the resulting mixture was stirred at $-20{ }^{\circ} \mathrm{C}$. Then KHMDS solution $(32.0 \mu \mathrm{l}, 1.0 \mathrm{M}$ in THF, $32 \mu \mathrm{mol})$, which was prepared freshly in Glove Box using KHMDS solid and deoxidized THF, was added followed by the addition of solution of 1 e $(0.8$ $\mathrm{mmol}$ in $0.8 \mathrm{~mL} \mathrm{DMF}$ ). The resulting solution was stirred at the same temperature under Ar. Reaction mixture was quenched by $\mathrm{AcOH}(32 \mu \mathrm{mol})$ in $\mathrm{THF}^{12}$, of which little part was took for ${ }^{1} \mathrm{H} \mathrm{NMR}$ analysis to determine $\mathrm{O} / \mathrm{N}$ product ratio immediately, and then diluted with $\mathrm{CH}_{2} \mathrm{Cl}_{2} / \mathrm{MeOH}$ (4/1). The diluted solution was filtered through silica short column and washed with $\mathrm{CH}_{2} \mathrm{Cl}_{2} / \mathrm{MeOH}$ (4/1) (ca. $80 \mathrm{ml}$ ). After evaporation of the organic solvent under reduced pressure, yield was determined by ${ }^{1} \mathrm{H}-\mathrm{NMR}$ analysis.

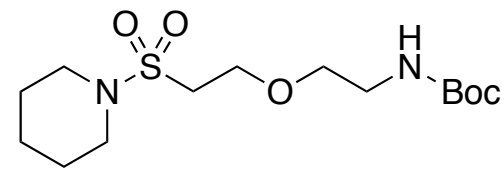

Reaction conditions

1e $(0.8 \mathrm{mmol})$ in DMF (0.8 mL), $2 \mathrm{c}(0.96 \mathrm{mmol}), 2 \mathrm{~d}(0.96 \mathrm{mmol}), \mathrm{DMF}(4.0 \mathrm{~mL}),-20^{\circ} \mathrm{C}, 0.5 \mathrm{~h}, \mathrm{AgOAc} / \mathrm{rac}-$ BINAP $(24.0 \mu \mathrm{mol}), \mathrm{KHMDS}(32 \mu \mathrm{mol})$; Boc protection: $\mathrm{Boc}_{2} \mathrm{O}(2.1 \mathrm{mmol}), \mathrm{Et}_{3} \mathrm{~N}(2.9 \mathrm{mmol}), \mathrm{CH}_{2} \mathrm{Cl}_{2}(10$ $\mathrm{mL}), 4^{\circ} \mathrm{C}$ to $\mathrm{RT}$, overnight.

tert-butyl (2-(2-(piperidin-1-ylsulfonyl)ethoxy)ethyl)carbamate (3ec): (white semisolid, EtOAC/Hexane $=1 / 4$ to $1 / 1,48 \%$ yield for two steps, purity $90 \%, 144.2 \mathrm{mg}$ with $3 \mathrm{ed}$ ).

\section{$\underline{\text { Reaction conditions }}$}

1e $(0.8 \mathrm{mmol})$ in DMF $(0.8 \mathrm{~mL}), 2 \mathrm{c}(0.96 \mathrm{mmol}), 2 \mathrm{~d}(0.96 \mathrm{mmol}), \operatorname{DMF}(4.0 \mathrm{~mL}),-20{ }^{\circ} \mathrm{C}, 0.5 \mathrm{~h}$, AgOAc/dppe $(24.0 \mu \mathrm{mol}), \mathrm{KHMDS}(32 \mu \mathrm{mol}) ; \mathrm{Boc}$ protection: $\mathrm{Boc}_{2} \mathrm{O}(2.1 \mathrm{mmol}), \mathrm{Et}_{3} \mathrm{~N}(2.9 \mathrm{mmol}), \mathrm{CH}_{2} \mathrm{Cl}_{2}$ (10 mL), $4{ }^{\circ} \mathrm{C}$ to $\mathrm{RT}$, overnight.

tert-butyl (2-(2-(piperidin-1-ylsulfonyl)ethoxy)ethyl)carbamate (3ec): (white semisolid, EtOAC/Hexane 
$=1 / 4$ to $1 / 1,60 \%$ yield for two steps, purity $69 \%, 237.1 \mathrm{mg}$ with $3 \mathrm{ed})$.

\section{Reaction conditions}

1e $(0.8 \mathrm{mmol})$ in DMF $(0.8 \mathrm{~mL}), 2 \mathrm{c}(0.96 \mathrm{mmol}), 2 \mathrm{e}(0.96 \mathrm{mmol}), \mathrm{DMF}(4.0 \mathrm{~mL}),-20^{\circ} \mathrm{C}, 0.5 \mathrm{~h}, \mathrm{AgOAc} / \mathrm{rac}-$ BINAP $(24.0 \mu \mathrm{mol}), \operatorname{KHMDS}(32 \mu \mathrm{mol})$; Boc protection: Boc $2 \mathrm{O}(2.1 \mathrm{mmol}), \mathrm{Et}_{3} \mathrm{~N}(2.9 \mathrm{mmol}), \mathrm{CH}_{2} \mathrm{Cl}_{2}(10$ $\mathrm{mL}), 4^{\circ} \mathrm{C}$ to $\mathrm{RT}$, overnight.

tert-butyl (2-(2-(piperidin-1-ylsulfonyl)ethoxy)ethyl)carbamate (3ec): (white semisolid, EtOAC/Hexane $=1 / 4$ to $1 / 1,45 \%$ yield for two steps, purity $94 \%, 129.5 \mathrm{mg}$ with $3 e e)$.

\section{$\underline{\text { Reaction conditions }}$}

1e $(0.8 \mathrm{mmol})$ in DMF $(0.8 \mathrm{~mL}), 2 \mathrm{c}(0.96 \mathrm{mmol}), 2 \mathrm{f}(0.96 \mathrm{mmol}), \mathrm{DMF}(4.0 \mathrm{~mL}),-20^{\circ} \mathrm{C}, 0.5 \mathrm{~h}, \mathrm{AgOAc} / \mathrm{rac}-$ BINAP $(24.0 \mu \mathrm{mol}), \operatorname{KHMDS}(32 \mu \mathrm{mol})$; Boc protection: $\mathrm{Boc}_{2} \mathrm{O}(2.1 \mathrm{mmol}), \mathrm{Et}_{3} \mathrm{~N}(2.9 \mathrm{mmol}), \mathrm{CH}_{2} \mathrm{Cl}_{2}(10$ $\mathrm{mL}), 4{ }^{\circ} \mathrm{C}$ to $\mathrm{RT}$, overnight.

tert-butyl (2-(2-(piperidin-1-ylsulfonyl)ethoxy)ethyl)carbamate (3ec): (white semisolid, EtOAC/Hexane $=1 / 4$ to $1 / 1,44 \%$ yield for two steps, purity $94 \%, 126.9 \mathrm{mg}$ with 3ef).

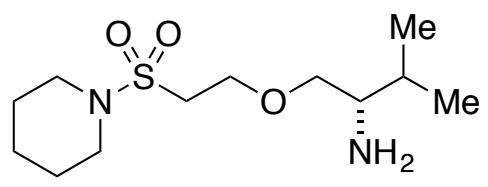

\section{$\underline{\text { Reaction conditions }}$}

1e $(0.8 \mathrm{mmol})$ in DMF $(0.8 \mathrm{~mL}), \mathbf{2 b}(0.96 \mathrm{mmol}), 2 \mathrm{f}(0.96 \mathrm{mmol}), \mathrm{DMF}(4.0 \mathrm{~mL}),-20^{\circ} \mathrm{C}, 2 \mathrm{~h}, \mathrm{AgOAc} / \mathrm{rac}-$ BINAP $(24.0 \mu \mathrm{mol})$, KHMDS (32 $\mu \mathrm{mol})$.

(S)-3-methyl-1-(2-(piperidin-1-ylsulfonyl)ethoxy)butan-2-amine (3eb): (pale yellow oil, $\mathrm{CH}_{2} \mathrm{Cl}_{2} / \mathrm{MeOH}=$ 9/1, 62\% yield, $138.7 \mathrm{mg}$ ).

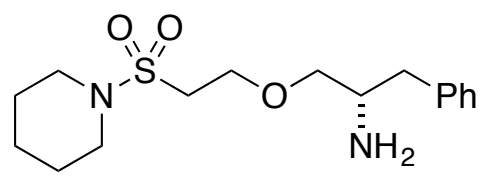

\section{$\underline{\text { Reaction conditions }}$}

1e $(0.8 \mathrm{mmol})$ in DMF $(0.8 \mathrm{~mL}), 2 \mathrm{a}(0.96 \mathrm{mmol}), 2 \mathrm{~g}(0.96 \mathrm{mmol}) \mathrm{DMF}(1.2 \mathrm{~mL}),-20{ }^{\circ} \mathrm{C}, 18 \mathrm{~h}, \mathrm{AgOAc} / \mathrm{rac}-$ BINAP $(24.0 \mu \mathrm{mol})$, KHMDS $(32 \mu \mathrm{mol})$. 
(S)-1-phenyl-3-(2-(piperidin-1-ylsulfonyl)ethoxy)propan-2-amine (3ea): (pale orange oil, $\mathrm{CH}_{2} \mathrm{Cl}_{2} / \mathrm{MeOH}$ $=10 / 1,85 \%$ yield, $221.2 \mathrm{mg}$ ).

\section{$\underline{\text { Reaction conditions }}$}

1e $(0.8 \mathrm{mmol})$ in DMF $(0.8 \mathrm{~mL}), 2 \mathrm{a}(0.96 \mathrm{mmol}), 2 \mathbf{i}(0.96 \mathrm{mmol}) \mathrm{DMF}(1.2 \mathrm{~mL}),-20^{\circ} \mathrm{C}, 30 \mathrm{~h}, \mathrm{AgOAc} / \mathrm{rac}-$

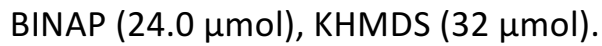

(S)-1-phenyl-3-(2-(piperidin-1-ylsulfonyl)ethoxy)propan-2-amine (3ea): (pale orange oil, $\mathrm{CH}_{2} \mathrm{Cl}_{2} / \mathrm{MeOH}$ $=10 / 1,88 \%$ yield, $230.5 \mathrm{mg}$ ).

\section{5-3. Procedure of catalytic chemo- and regioselective conjugate addition reaction using $2 \mathrm{w}$}

A flame-dried $20 \mathrm{~mL}$ test tube equipped with a magnetic stirring bar and 3-way glass stopcock was charged with AgOAc (7.5 mg, $45 \mu \mathrm{mol})$, rac-BINAP (28.0 mg, $45 \mu \mathrm{mol}), 2$ w $(64.7 \mathrm{mg}, 0.54 \mathrm{mmol})$ and distilled DMF $(0.45 \mathrm{~mL})$ via syringe. After stirred at room temperature for about 10 minutes, the resulting mixture was stirred at $-10^{\circ} \mathrm{C}$. Then KHMDS solution $(50 \mu \mathrm{L}, 1.0 \mathrm{M}$ in THF, $50 \mu \mathrm{mol})$, which was prepared freshly in Glove Box using KHMDS solid and deoxidized THF, was added followed by the addition of solution of $1 \mathrm{e}(0.45 \mathrm{mmol}$ in $0.45 \mathrm{~mL} \mathrm{DMF})$. The resulting solution was stirred at the same temperature under Ar for 9 hours and then diluted with $\mathrm{CH}_{2} \mathrm{Cl}_{2} / \mathrm{MeOH}$ (4/1) solution. The diluted solution was filtered through silica short column and washed with $\mathrm{CH}_{2} \mathrm{Cl}_{2} / \mathrm{MeOH}$ (4/1) (ca. $80 \mathrm{ml}$ ). After evaporation of the organic solvent under reduced pressure, yield of $\mathrm{N}$-adduct was determined by ${ }^{1} \mathrm{H}$ NMR analysis and then crude mixture was dried under reduced pressure. To a stirred solution of crude mixture in dry $\mathrm{CH}_{2} \mathrm{Cl}_{2}(10 \mathrm{~mL})$ was added $\mathrm{Et}_{3} \mathrm{~N}(115 \mu \mathrm{L}, 0.81 \mathrm{mmol})$ and $\mathrm{Boc}_{2} \mathrm{O}(135 \mu \mathrm{L}, 0.59 \mathrm{mmol})$ at $4{ }^{\circ} \mathrm{C}$. The reaction mixture was stirred at room temperature for $1 \mathrm{~h}$. The residue was subjected to flash column chromatography (EtOAc only) to obtain 3ew with yield of 55\% (98.3 mg) and 5ew (14.1 mg) with the yield of $8 \%$.

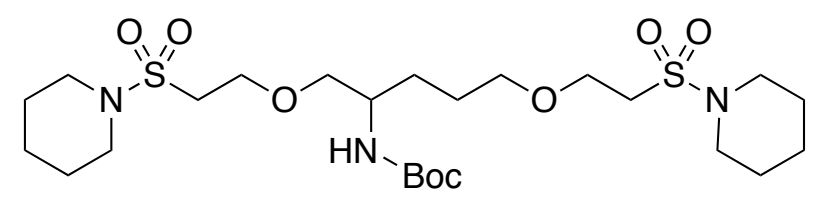


tert-butyl (1,5-bis(2-(piperidin-1-ylsulfonyl)ethoxy)pentan-2-yl)carbamate (6ew): (pale yellow oil); ${ }^{1} \mathrm{H}$ $\operatorname{NMR}\left(500 \mathrm{MHz}, \mathrm{CDCl}_{3}\right)(\mathrm{ppm}) \delta 4.77(\mathrm{~d}, 1 \mathrm{H}, J=8.0 \mathrm{~Hz}, \mathrm{NH}), 3.88-3.68\left(\mathrm{~m}, 5 \mathrm{H}, \mathrm{CH}_{2} \mathrm{OCH}_{2} \mathrm{CH}_{2} \mathrm{CH}_{2} \mathrm{CH}\right.$, $\mathrm{CH}_{2} \mathrm{OCH}_{2} \mathrm{CHN}$ ), 3.52-3.42 (m, $4 \mathrm{H}, \mathrm{CH}_{2} \mathrm{OCH}_{2} \mathrm{CH}_{2} \mathrm{CH}_{2} \mathrm{CH}, \mathrm{CH}_{2} \mathrm{OCH}_{2} \mathrm{CHN}$ ), $3.23\left(\mathrm{t}, 8 \mathrm{H}, \mathrm{CH}_{2}\left(\mathrm{CH}_{2} \mathrm{CH}_{2}\right)_{2} \mathrm{NSO}_{2}\right.$, $\left.\mathrm{CH}_{2}\left(\mathrm{CH}_{2} \mathrm{CH}_{2}\right)_{2} \mathrm{NSO}_{2}\right), 3.16\left(\mathrm{dd}, 4 \mathrm{H}, J=6.0,12.5 \mathrm{~Hz}, \mathrm{SO}_{2} \mathrm{CH}_{2} \mathrm{CH}_{2} \mathrm{O}, \mathrm{SO}_{2} \mathrm{CH}_{2} \mathrm{CH}_{2} \mathrm{O}\right), 1.70-1.40(\mathrm{~m}, 25 \mathrm{H}$, $\left.\mathrm{CH}_{2}\left(\mathrm{CH}_{2} \mathrm{CH}_{2}\right)_{2} \mathrm{NSO}_{2}, \mathrm{CH}_{2}\left(\mathrm{CH}_{2} \mathrm{CH}_{2}\right)_{2} \mathrm{NSO}_{2}, \mathrm{OCH}_{2} \mathrm{CH}_{2} \mathrm{CH}_{2} \mathrm{CHN}, \mathrm{C}\left(\mathrm{CH}_{3}\right)_{3}\right) ;{ }^{13} \mathrm{C} \mathrm{NMR}\left(125 \mathrm{MHz}, \mathrm{CDCl}_{3}\right) \delta 155.8$, $79.4,73.4,71.1,65.0,64.5,50.1,49.6,49.3,46.8,46.6,46.6,28.9,28.5,26.3,25.8,24.0,23.9$; IR (thin film, $\mathrm{NaCl}$ ) 1699, 1163, 1112, 1053, $1026 \mathrm{~cm}^{-1}$; HRMS (ESI, H) $\mathrm{m} / z$ calc'd for $\mathrm{C}_{24} \mathrm{H}_{48} 3_{2} \mathrm{O}_{8} \mathrm{~S}_{2}^{+}(\mathrm{M}+\mathrm{H})^{+}$ 570.2877 , found 570.2872 .

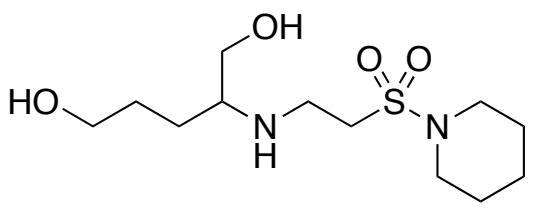

2-((2-(piperidin-1-ylsulfonyl)ethyl)amino)pentane-1,5-diol (4ew): (white solid); ${ }^{1} \mathrm{H}-\mathrm{NMR}$ (500 MHz, $\left.\mathrm{CDCl}_{3}\right)(\mathrm{ppm}) \delta 3.70-3.58\left(\mathrm{~m}, 3 \mathrm{H}, \mathrm{HOCH}{ }_{2} \mathrm{CH}, \mathrm{HOCH}_{2} \mathrm{CH}_{2} \mathrm{CH}_{2}\right), 3.46(\mathrm{dd}, 1 \mathrm{H}, J=6.0,11.0 \mathrm{~Hz}$, $\left.\mathrm{HOCH}_{2} \mathrm{CH}_{2} \mathrm{CH}_{2}\right), 3.23\left(\mathrm{t}, 4 \mathrm{H}, \mathrm{J}=5.5 \mathrm{~Hz}, \mathrm{~N}\left(\mathrm{CH}_{2} \mathrm{CH}_{2}\right)_{2} \mathrm{CH}_{2}\right), 3.18-3.02\left(\mathrm{~m}, 4 \mathrm{H}, \mathrm{NCH}_{2} \mathrm{CH}_{2} \mathrm{SO}_{2}\right), 2.90-2.10(\mathrm{~m}, 4 \mathrm{H}$, $\mathrm{HNCH}, \mathrm{OH}, \mathrm{OH}), 1.73-1.50\left(\mathrm{~m}, 10 \mathrm{H}, \mathrm{N}\left(\mathrm{CH}_{2} \mathrm{CH}_{2}\right)_{2} \mathrm{CH}_{2}, \mathrm{HOCH}_{2} \mathrm{CH}_{2} \mathrm{CH}_{2} \mathrm{CHN}\right) ;{ }^{13} \mathrm{C} \mathrm{NMR}\left(125 \mathrm{MHz}, \mathrm{CDCl}_{3}\right) \delta$ 63.2, 62.8, 58.9, 48.9, 46.7, 40.7, 29.6, 29.1, 25.7, 23.8; IR (KBr) 3377, 3323, 1157, 1055, $1026 \mathrm{~cm}^{-1}$; HRMS (ESI, H) $m / z$ calc'd for $\mathrm{C}_{12} \mathrm{H}_{27} \mathrm{~N}_{2} \mathrm{O}_{4} \mathrm{~S}^{+}(\mathrm{M}+\mathrm{H})^{+} 295.1686$, found 295.1689.

Isolated yield: 3ew 53\% (156.5 mg), 5ew 9\% (26.0 mg), 6ew 12\% (51.9 mg).

\section{$\underline{\text { Reaction conditions }}$}

1e $(0.75 \mathrm{mmol})$ in DMF $(0.75 \mathrm{~mL}), 2 \mathrm{w}(0.9 \mathrm{mmol}), \operatorname{DMF}(0.75 \mathrm{~mL}),-10{ }^{\circ} \mathrm{C}, 9 \mathrm{~h}, \mathrm{AgOAc} / \mathrm{dppe}(75.0 \mu \mathrm{mol})$, KHMDS (82.5 $\mu \mathrm{mol}) ;$ Boc protection: $\mathrm{Boc}_{2} \mathrm{O}(1.0 \mathrm{mmol}), \mathrm{Et}_{3} \mathrm{~N}(1.4 \mathrm{mmol}), \mathrm{CH}_{2} \mathrm{Cl}_{2}(10 \mathrm{~mL}), 0{ }^{\circ} \mathrm{C}$ to $\mathrm{RT}, 1 \mathrm{~h}$.

Isolated yield: 3ew 7\% (31.8 mg), 5ew 14\% (60.0 mg), 6ew 25\% (155.3 mg).

\section{Reaction conditions}

1e $(1.1 \mathrm{mmol})$ in DMF (1.1 mL), 2w (1.3 mmol), DMF $(1.1 \mathrm{~mL}),-10{ }^{\circ} \mathrm{C}, 9 \mathrm{~h}, \mathrm{KHMDS}(121.0 \mu \mathrm{mol})$; Boc protection: $\mathrm{Boc}_{2} \mathrm{O}(1.5 \mathrm{mmol}), \mathrm{Et}_{3} \mathrm{~N}(2.0 \mathrm{mmol}), \mathrm{CH}_{2} \mathrm{Cl}_{2}(10 \mathrm{~mL}), 0{ }^{\circ} \mathrm{C}$ to $\mathrm{RT}, 1 \mathrm{~h}$. 
<smiles>CNc1cccc2c(S(=O)(=O)N3CCN(S(=O)(=O)CCOCC(CCCO)NC(=O)OC(C)(C)C)CC3)cccc12</smiles>

\section{Reaction conditions}

1) $(0.58 \mathrm{mmol})$ in mixture of DMF and THF (1.2 mL, DMF : THF=1:1), $2 \mathbf{w}(0.7 \mathrm{mmol}), \mathrm{DMF}(0.6 \mathrm{~mL}),-$ $10{ }^{\circ} \mathrm{C}, 9 \mathrm{~h}, \mathrm{AgOAc} / \mathrm{rac}-\mathrm{BINAP}(58.0 \mu \mathrm{mol}), \mathrm{KHMDS}(63.8 \mu \mathrm{mol})$; Boc protection: $\mathrm{Boc}_{2} \mathrm{O}(0.8 \mathrm{mmol}), \mathrm{Et}_{3} \mathrm{~N}$ (1.1 mmol), $\mathrm{CH}_{2} \mathrm{Cl}_{2}(10 \mathrm{~mL}), 0{ }^{\circ} \mathrm{C}$ to $\mathrm{RT}, 1 \mathrm{~h}$.

tert-butyl (1-(2-((4-((5-(dimethylamino)naphthalen-1-yl)sulfonyl)piperazin-1-yl)sulfonyl)ethoxy)-5hydroxypentan-2-yl)carbamate (3/w): (light green foam, EtOAc:Hexane = 8:1, 68\%, 246.9 mg); ${ }^{1} \mathrm{H}-\mathrm{NMR}$ $\left(500 \mathrm{MHz}_{\mathrm{CDCl}}\right)(\mathrm{ppm}) \delta 8.59(\mathrm{~d}, 1 \mathrm{H}, J=8.5 \mathrm{~Hz}, \mathrm{ArH}), 8.34$ (d, $\left.1 \mathrm{H}, J=8.5 \mathrm{~Hz}, \operatorname{ArH}\right), 8.18(\mathrm{dd}, 1 \mathrm{H}, J=1.5$, $7.3 \mathrm{~Hz}, \operatorname{ArH}), 7.60-7.52(\mathrm{~m}, 2 \mathrm{H}, \operatorname{ArH}), 7.20(\mathrm{~d}, 1 \mathrm{H}, J=7.5 \mathrm{~Hz}, \operatorname{ArH}), 4.76(\mathrm{~d}, 1 \mathrm{H}, J=8.0 \mathrm{~Hz}, \mathrm{NH}), 3.82-3.62$ (m, $\left.5 \mathrm{H}, \mathrm{CH}_{2} \mathrm{OH}, \mathrm{CH}_{2} \mathrm{OCH}_{2} \mathrm{CHN}\right), 3.46-3.20\left(\mathrm{~m}, 9 \mathrm{H}, \mathrm{CH}_{2} \mathrm{OCH}_{2} \mathrm{CHN}, \mathrm{SO}_{2} \mathrm{~N}\left(\mathrm{CH}_{2} \mathrm{CH}_{2}\right)_{2} \mathrm{SO}_{2}\right), 3.16(\mathrm{t}, 2 \mathrm{H}, J=5.5$ Hz. $\left.\mathrm{SO}_{2} \mathrm{CH}_{2} \mathrm{CH}_{2} \mathrm{O}\right), 2.90\left(\mathrm{~s}, 6 \mathrm{H}, \mathrm{N}\left(\mathrm{CH}_{3}\right)_{2}\right), 1.94(\mathrm{br}, 1 \mathrm{H}, \mathrm{OH}), 1.73-1.35\left(\mathrm{~m}, 13 \mathrm{H}, \mathrm{NHCHCH} \mathrm{CH}_{2} \mathrm{CH}_{2} \mathrm{OH}\right.$, $\left.\mathrm{C}\left(\mathrm{CH}_{3}\right)_{3}\right) ;{ }^{13} \mathrm{C} \mathrm{NMR}\left(125 \mathrm{MHz}, \mathrm{CDCl}_{3}\right) \delta 155.9,152.1,132.3,131.3,130.8,130.4,130.3,128.5,123.3$, 119.3, 115.6, 79.6, 73.6, 64.8, 62.5, 50.4, 50.1, 45.9, 45.6, 45.3, 29.0, 28.9, 28.6; IR (KBr) 3371, 2935, 1697, 1161, $1084 \mathrm{~cm}^{-1}$; HRMS (ESI, H) $\mathrm{m} / z$ calc'd for $\mathrm{C}_{28} \mathrm{H}_{45} \mathrm{~N}_{4} \mathrm{O}_{8} \mathrm{~S}_{2}{ }^{+}(\mathrm{M}+\mathrm{H})^{+} 629.2673$, found 629.2662.

\section{Synthesis of 3ew and 5ew}<smiles>NC(CCC(=O)O)C(=O)O</smiles>

dl-glutamic acid<smiles>COC(=O)CCC(NC(=O)OCc1ccccc1)C(=O)OC</smiles>

SI-2

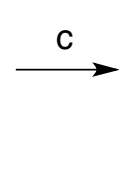<smiles>CC(C)(C)OC(=O)NC(CO)CCCO</smiles>

SI-3

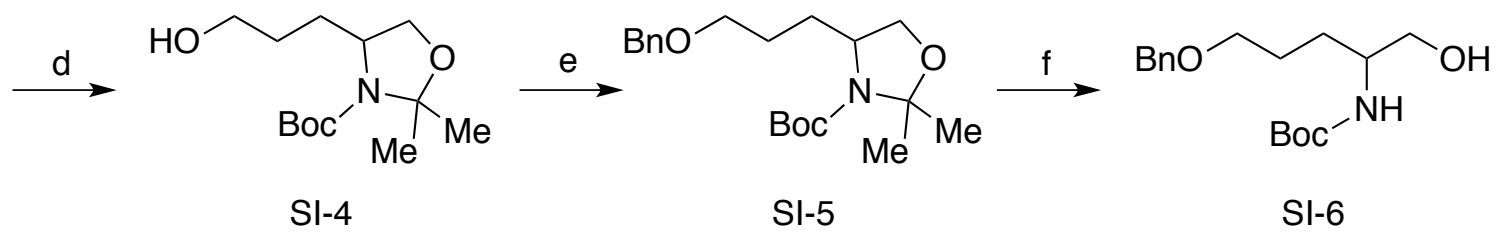

\section{Conditions:}

a. $\mathrm{SOCl}_{2}, \mathrm{MeOH}, 0^{\circ} \mathrm{C}$ to $\mathrm{RT}$, overnight, quant. ${ }^{8}$

b. $\mathrm{Boc}_{2} \mathrm{O}, \mathrm{Et}_{3} \mathrm{~N}, \mathrm{CH}_{2} \mathrm{Cl}_{2}, 0{ }^{\circ} \mathrm{C}$ to RT, overnight, $85 \%$ of two steps. 
C. $\mathrm{NaBH} 4, \mathrm{THF}: \mathrm{MeOH}=5: 1,0{ }^{\circ} \mathrm{C}, 24 \mathrm{~h}, 90 \% .{ }^{13}$

d. $\mathrm{Et}_{2} \mathrm{O} \cdot \mathrm{BF}_{3}$ cat., acetone:2,2-NMP $=10: 3, \mathrm{RT}, 24 \mathrm{~h}, 85 \%$ as two rotamers. ${ }^{13}$

e. $\mathrm{NaH}, \mathrm{BnBr}$, TBAl cat., $\mathrm{RT}, 18 \mathrm{~h}, 67 \%$ as two rotamers. ${ }^{14}$

f. Montmorillonite K10 clay, MeOH, RT, 21h, 37\%, SI-5 47\% recovery. ${ }^{15}$

\section{Procedure:}

e. $\mathrm{NaH}$ (60\% dispersion in oil, $300 \mathrm{mg}, 7.5 \mathrm{mmol}$ ) was added in one portion to a stirred solution of SI-4 $(974.7 \mathrm{mg}, 3.8 \mathrm{mmol})$ in $\operatorname{dry} \operatorname{THF}(25 \mathrm{~mL})$ at $0{ }^{\circ} \mathrm{C}$ under Ar. The mixture was stirred at $0{ }^{\circ} \mathrm{C}$ for $1 \mathrm{~h}$ and then benzyl bromide $(1.7 \mathrm{ml}, 14.3 \mathrm{mmol})$ was added dropwise, TBAI $(50 \mathrm{mg}, 0.14 \mathrm{mmol}$ ) was added in one portion and the mixture was then allowed to warm to room temperature $18 \mathrm{~h}$. Water $(10 \mathrm{~mL})$ was added carefully followed by $\mathrm{Et}_{2} \mathrm{O}(20 \mathrm{~mL})$. The separated aqueous phase was extracted with $\mathrm{Et}_{2} \mathrm{O}(2 \times 20 \mathrm{~mL})$ and the combined organic phase was dried $\left(\mathrm{Na}_{2} \mathrm{SO}_{4}\right)$ and concentrated in vacuum. The residue was purified by silica gel column chromatography (Hexane : EtOAc $=9: 1$ ) to give SI-5 as colorless oil with yield of $67 \%$ ( $875.9 \mathrm{mg})$.

f. To the solution of SI-5 $(875.9 \mathrm{mg}$ ) and $\mathrm{MeOH}(10 \mathrm{~mL})$ was added Montmorillonite K10 clay (175 mg. $20 \% \mathrm{w} / \mathrm{w})$, then stirred at room temperature for $21 \mathrm{~h}$. Then the mixture was filtered through a short plug of celite and wash with EtOAc $(20 \mathrm{~mL})$. After evaporation of the organic solvent under reduced pressure, the crude mixture was purified by silica gel column chromatography (Hexane : EtOAc =1:1) to give SI-6 with yield of $37 \%$ ( $287.7 \mathrm{mg}$ )as colorless oil. And the recovery of SI-5 was $47 \%$ (411.7 $\mathrm{mg}$ ).

dimethyl (tert-butoxycarbonyl)glutamate (SI-2): CAS Registry Number: 861658-15-3

tert-butyl (1,5-dihydroxypentan-2-yl)carbamate (SI-3): CAS Registry Number: 859174-76-8

tert-butyl 4-(3-hydroxypropyl)-2,2-dimethyloxazolidine-3-carboxylate (SI-4): CAS Registry Number: 1822571-55-0

tert-butyl 4-(3-(benzyloxy)propyl)-2,2-dimethyloxazolidine-3-carboxylate (SI-5): (two rotamers) (colorless oil); ${ }^{1} \mathrm{H}-\mathrm{NMR}\left(500 \mathrm{MHz}, \mathrm{CDCl}_{3}\right)(\mathrm{ppm}) \delta$ 7.37-7.25 (m, 5H, $\left.\mathrm{ArH}\right), 4.50\left(\mathrm{~s}, 2 \mathrm{H}, \mathrm{OCH}_{2} \mathrm{Ph}\right), 3.96-3.88$ (m, $1.5 \mathrm{H}, \mathrm{COCH}_{2} \mathrm{CHNC}$ ), 3.80 (br, 0.5H, $\mathrm{COCH}_{2} \mathrm{CHNC}$ ), 3.75-3.70 (d, $1 \mathrm{H}, J=7.0 \mathrm{~Hz}, \mathrm{COCH}_{2} \mathrm{CHNC}$ ), 3.55-3.45 (m, $\left.2 \mathrm{H}, \mathrm{CH}_{2} \mathrm{OCH}_{2} \mathrm{Ph}\right), 1.85-1.33\left(\mathrm{~m}, 19 \mathrm{H}, \mathrm{C}\left(\mathrm{CH}_{3}\right)_{2}, \mathrm{C}\left(\mathrm{CH}_{3}\right)_{3}, \mathrm{CH}_{2} \mathrm{CH}_{2} \mathrm{CH}_{2} \mathrm{OCH}_{2} \mathrm{Ph}\right) ;{ }^{13} \mathrm{C}$ NMR $\left(125 \mathrm{MHz}, \mathrm{CDCl}_{3}\right)$ $\delta 152.4,152.0,138.7,138.7,128.5,128.5,127.8,127.8,127.7,127.7,93.8,93.3,80.1,79.6,73.0,73.0$, 
70.3, 70.3, 67.2, 66.9, 57.7, 57.4, 30.7, 29.7, 28.6, 27.7, 26.9, 26.7, 24.7, 23.4; IR (thin film, $\mathrm{NaCl}$ ) 1739, 1693, 1207, 1176, $1087 \mathrm{~cm}^{-1}$; HRMS (ESI, H) $\mathrm{m} / \mathrm{z}$ calc'd for $\mathrm{C}_{20} \mathrm{H}_{32} \mathrm{NO}_{4}{ }^{+}(\mathrm{M}+\mathrm{H})^{+} 350.2326$, found 350.2325 .

tert-butyl (5-(benzyloxy)-1-hydroxypentan-2-yl)carbamate (SI-6): (colorless oil); ${ }^{1} \mathrm{H}-\mathrm{NMR}$ (500 MHz, $\left.\mathrm{CDCl}_{3}\right)(\mathrm{ppm}) \delta$ 7.38-7.26 (m, 5H, $\left.\mathrm{ArH}\right), 4.49\left(\mathrm{~s}, 2 \mathrm{H}, \mathrm{OCH}_{2} \mathrm{Ph}\right), 3.66-3.46\left(\mathrm{~m}, 5 \mathrm{H}, \mathrm{HOCH}_{2} \mathrm{CHN} \mathrm{CH}_{2} \mathrm{OCH}_{2} \mathrm{Ph}\right)$, 2.84 (br, $1 \mathrm{H}, \mathrm{OH}), 1.76-1.38\left(\mathrm{~m}, 13 \mathrm{H}, \mathrm{CH}_{2} \mathrm{CH}_{2} \mathrm{CH}_{2} \mathrm{OCH}_{2} \mathrm{Ph}, \mathrm{C}\left(\mathrm{CH}_{3}\right)_{3}\right) ;{ }^{13} \mathrm{C} \mathrm{NMR}\left(125 \mathrm{MHz}, \mathrm{CDCl}_{3}\right) \delta 156.6$, $138.4,128.5,127.8,127.7,79.6,73.1,70.1,65.9,52.7,28.5,28.3,26.3 ; \operatorname{IR}(\mathrm{KBr}) 3382,1811,1683,1172$, $1080 \mathrm{~cm}^{-1}$; HRMS (ESI, H) $\mathrm{m} / z$ calc'd for $\mathrm{C}_{17} \mathrm{H}_{28} \mathrm{NO}_{4}{ }^{+}(\mathrm{M}+\mathrm{H})^{+} 310.2013$, found 310.2014.

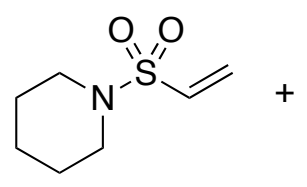

$1 e$<smiles>CC(C)(C)OC(=O)N1C(CCCO)COC1(C)C</smiles>

SI-4<smiles>CC(C)(C)[C@H]1CCCN(S(=O)(=O)CCOCCCC(CO)NC(=O)OCc2ccccc2)C1</smiles>

5ew

\section{Conditions:}

a. KHMDS, DMF, RT, $2 \mathrm{~h}$

b. (+)-CSA, MeOH, RT, 22 h. $^{16}$

\section{Procedure:}

a. A flame-dried $20 \mathrm{~mL}$ test tube equipped with a magnetic stirring bar and 3-way glass stopcock was charged with SI-4 $(0.71 \mathrm{mmol})$. To the test tube was added distilled DMF $(0.8 \mathrm{~mL})$ via syringe. Then KHMDS solution $(140.0 \mu \mathrm{l}, 1.0 \mathrm{M}$ in THF, $20 \mathrm{~mol} \%)$, which was prepared freshly in Glove Box using KHMDS solid and deoxidized THF, was added followed by the addition of solution of sulfonyl compound 1e (150 mg in $0.8 \mathrm{~mL} \mathrm{DMF}, 1.2$ eq.). The resulting solution was stirred at the same temperature for $2 \mathrm{~h}$ under $\mathrm{Ar}$ and diluted with EtOAc. The diluted solution was filtered through silica short column and washed with EtOAc (ca. $80 \mathrm{ml}$ ). Crude was obtained as brown oil after evaporation of the organic solvent under reduced pressure. This crude was used for the next step without purification.

b. To a stirred solution of crude in $\mathrm{MeOH}(4 \mathrm{~mL})$ at room temperature was added (+)-camphorsulfonic acid $\left(2.5 \mathrm{mg}\right.$ ). After $22 \mathrm{~h}$ at room temperature, reaction was quenched with $\mathrm{NaHCO}_{3}$ solid. After evaporation of the organic solvent under reduced pressure, the crude mixture was purified by silica gel 
column chromatography (Hexane : EtOAc $=1: 4)$ to give 5 ew with yield of $29 \%(80.1 \mathrm{mg}$ ) as white solid.<smiles>O=C(O)NC(CO)CCCOCCS(=O)(=O)N1CCCCC1</smiles>

tert-butyl (1-hydroxy-5-(2-(piperidin-1-ylsulfonyl)ethoxy)pentan-2-yl)carbamate (5ew): (white solid); ${ }^{1} \mathrm{H}-\mathrm{NMR}\left(500 \mathrm{MHz}, \mathrm{CDCl}_{3}\right)(\mathrm{ppm}) \delta 4.75(\mathrm{br}, 1 \mathrm{H}, \mathrm{NH}), 3.80\left(\mathrm{t}, 2 \mathrm{H}, J=6.5 \mathrm{~Hz}, \mathrm{SO}_{2} \mathrm{CH}_{2} \mathrm{CH}_{2} \mathrm{O}\right), 3.70-3.52(\mathrm{~m}$, $3 \mathrm{H}, \mathrm{NCHCH} \mathrm{OH}_{2}, 3.48\left(\mathrm{t}, 2 \mathrm{H}, J=6.0 \mathrm{~Hz}, \mathrm{SO}_{2} \mathrm{CH}_{2} \mathrm{CH}_{2} \mathrm{OCH}\right.$ ), $3.23\left(\mathrm{t}, 4 \mathrm{H}, J=5.5 \mathrm{~Hz}, \mathrm{CH}_{2}\left(\mathrm{CH}_{2} \mathrm{CH}_{2}\right){ }_{2} \mathrm{~N}\right.$ ), 3.17 (t, $\left.2 \mathrm{H}, J=6.5 \mathrm{~Hz}, \mathrm{SO}_{2} \mathrm{CH}_{2}\right), 2.32(\mathrm{br}, 1 \mathrm{H}, \mathrm{OH}), 1.72-1.40\left(\mathrm{~m}, 19 \mathrm{H}, \mathrm{CH}_{2}\left(\mathrm{CH}_{2} \mathrm{CH}_{2}\right)_{2} \mathrm{~N}, \mathrm{C}\left(\mathrm{CH}_{3}\right)_{3}\right.$, $\left.\mathrm{CH}_{2} \mathrm{OCH}_{2} \mathrm{CH}_{2} \mathrm{CH}_{2} \mathrm{CHN}\right) ;{ }^{13} \mathrm{C}$ NMR $\left(125 \mathrm{MHz}, \mathrm{CDCl}_{3}\right) \delta 156.6,79.8,71.0,66.0,64.5,52.7,49.5,46.6,28.5$, 28.2, 26.2, 25.8, 24.0; IR (KBr) 3323, 1680, 1159, 1116, $1053 \mathrm{~cm}^{-1}$; HRMS (ESI, H) $\mathrm{m} / \mathrm{z}$ calc'd for $\mathrm{C}_{17} \mathrm{H}_{35} \mathrm{~N}_{2} \mathrm{O}_{6} \mathrm{~S}^{+}(\mathrm{M}+\mathrm{H})^{+}$395.2210, found 395.2208.

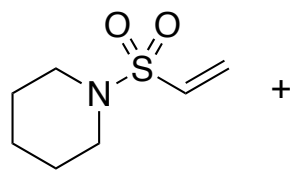

$1 e$<smiles>CC(C)(C)OC(=O)NC(CO)CCCOc1ccccc1</smiles>

SI-6

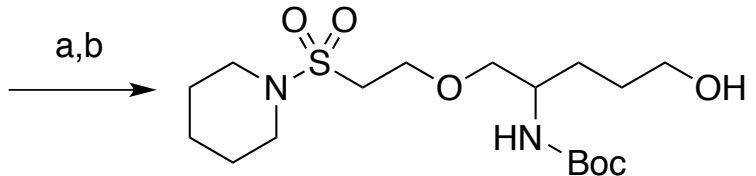

3ew

\section{Conditions:}

a) KHMDS, DMF, $-20^{\circ} \mathrm{C}, 4 \mathrm{~h}$;

b) $\mathrm{Pd} / \mathrm{C}, \mathrm{H}_{2}$, EtOH, RT, overnight.

\section{Procedure:}

a) A flame-dried $20 \mathrm{~mL}$ test tube equipped with a magnetic stirring bar and 3-way glass stopcock was charged with SI-6 $(0.68 \mathrm{mmol})$. To the test tube was added distilled DMF $(0.8 \mathrm{~mL})$ via syringe. After stirred at room temperature for about 10 minutes, the resulting mixture was stirred at $-20{ }^{\circ} \mathrm{C}$. Then KHMDS solution ( $68.0 \mu \mathrm{l}, 1.0 \mathrm{M}$ in THF, $10 \mathrm{~mol} \%)$, which was prepared freshly in Glove Box using KHMDS solid and deoxidized THF, was added followed by the addition of solution of 1e (143 mg in $0.8 \mathrm{~mL} \mathrm{DMF}$, 1.2 eq.). The resulting solution was stirred at the same temperature for $4 \mathrm{~h}$ under Ar and diluted with EtOAc. The diluted solution was filtered through silica short column and washed with EtOAc (ca. $80 \mathrm{ml}$ ). Crude was obtained as brown oil after evaporation of the organic solvent under reduced pressure. This crude was used for the next step without purification. 
b) To the mixture of crude, $10 \%$ palladium/carbon $(40 \mathrm{mg})$ and EtOH $(10 \mathrm{~mL})$ was stirred under a balloon of $\mathrm{H}_{2}$ at room temperature overnight. Then the crude mixture was filtered through a short plug of celite and wash with EtOAc $(20 \mathrm{~mL})$. After evaporation of the organic solvent under reduced pressure, the crude mixture was purified by silica gel column chromatography (EtOAc only) to give 3ew with yield of $50 \%(132.0 \mathrm{mg})$ as pale yellow oil.<smiles>O=C(O)NC(CCCO)COCCS(=O)(=O)N1CCCCC1</smiles>

tert-butyl (5-hydroxy-1-(2-(piperidin-1-ylsulfonyl)ethoxy)pentan-2-yl)carbamate (3ew): (pale yellow oil); ${ }^{1} \mathrm{H}-\mathrm{NMR}\left(500 \mathrm{MHz}, \mathrm{CDCl}_{3}\right)(\mathrm{ppm}) \delta 4.81(\mathrm{br}, 1 \mathrm{H}, \mathrm{NH}), 3.82\left(\mathrm{t}, 2 \mathrm{H}, J=6.0 \mathrm{~Hz}, \mathrm{SO}_{2} \mathrm{CH}_{2} \mathrm{CH}_{2} \mathrm{O}\right), 3.77$ (br, $1 \mathrm{H}, \mathrm{NHCH}), 3.72-3.62\left(\mathrm{~m}, 2 \mathrm{H}, \mathrm{HOCH}_{2}\right), 3.53-3.42\left(\mathrm{~m}, 2 \mathrm{H}, \mathrm{SO}_{2} \mathrm{CH}_{2} \mathrm{CH}_{2} \mathrm{OCH}_{2}\right), 3.24(\mathrm{t}, 4 \mathrm{H}, J=5.0 \mathrm{~Hz}$, $\left.\mathrm{CH}_{2}\left(\mathrm{CH}_{2} \mathrm{CH}_{2}\right)_{2} \mathrm{~N}\right), 3.16\left(\mathrm{t}, 2 \mathrm{H}, J=6.0 \mathrm{~Hz}, \mathrm{SO}_{2} \mathrm{CH}_{2} \mathrm{CH}_{2} \mathrm{O}\right), 1.77(\mathrm{br}, 1 \mathrm{H}, \mathrm{OH}), 1.71-1.50(\mathrm{~m}, 10 \mathrm{H}$, $\mathrm{CH}_{2}\left(\mathrm{CH}_{2} \mathrm{CH}_{2}\right)_{2} \mathrm{~N}, \mathrm{HOCH}_{2} \mathrm{CH}_{2} \mathrm{CH}_{2} \mathrm{CHN}$ ), 1.44 (s, 9H, C(CH3) $\left.)_{3}\right) ;{ }^{13} \mathrm{CNMR}\left(125 \mathrm{MHz}, \mathrm{CDCl}_{3}\right) \delta$ 155.9, 79.5, 73.3, 65.0, 62.8, 50.1, 49.3, 46.6, 29.0, 28.9, 28.5, 25.8, 23.9; IR (thin film, NaCl) 3504, 3373, 1693, 1114, 1053 $\mathrm{cm}^{-1}$; HRMS (ESI, H) $\mathrm{m} / \mathrm{z}$ calc'd for $\mathrm{C}_{17} \mathrm{H}_{35} \mathrm{~N}_{2} \mathrm{O}_{6} \mathrm{~S}^{+}(\mathrm{M}+\mathrm{H})^{+}$395.2210, found 395.2209. 


\section{Proposed Catalytic Cycle}

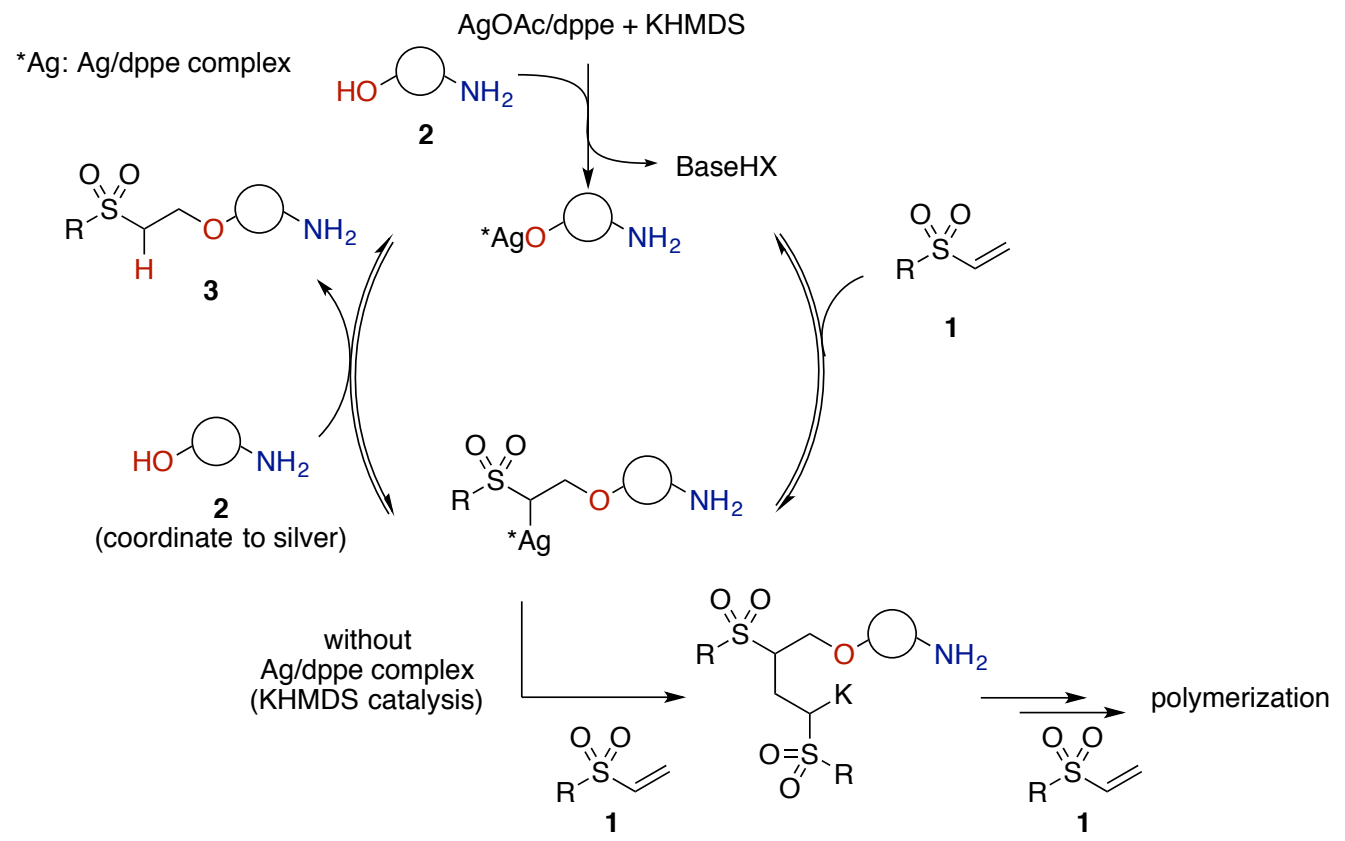

\section{Reference}

1. Carr, R. V. C.; Williams, R. V.; Paquette, L. A. J. Org. Chem. 1983, 48, 4976.

2. Dana, D.; Das, T. K.; Kumar, I.; Davalos, A. R.; Mark, K. J.; Ramai, D.; Chang, E. J.; Talele, T. T.; Kumar, S. Chem. Biol. Drug. Des. 2012, 80, 489.

3. Llamas, T.; Arrayás, R. G.; Carretero, J. C. Org. Lett. 2006, 8, 1795.

4. Janin, Y. L.; Aubertin, A.-M.; Chiaroni, A.; Riche, C.; Monneret, C.; Bisagni, E.; Grierson, D. S. Tetrahedron, 1996, 52, 15157.

5. Esser-Kahn, A. P.; Sottos, N. R.; White, S. R.; Moore, J. S. J. Am. Chem. Soc. 2010, 132, 10266.

6. Thomson, S. P.; Allanson, N. M.; Davies, G. M.; Edwards, P. N.;Kuvshinov, A. Int. Appl. PCT. WO2006123145 (A1), 2006.

7. Panchgalle, S.; Gore, R. G.; Chavan, S. P, Kalkote, U. R. Tetrahedron Asymmetry, 2009, 20, 1767.

8. Jung, Y. C.; Yoon, C. H.; Turos, E.; Yoo, K. S.; Jung, K. W. J. Org. Chem. 2007, 72, 10114.

9. Edwards, M. L.; Stemerick, D. M.; Bitonti, A J.; Dumont, J. A; McCann, P. P.; Bey, P.; Sjoerdsma, A. J. Med. Chem. 1991, 34, 569.

10. Wang, M.; Wang, Y.; Qi, X.; Xia, G.; Tong, K.; Tu, J.; Pittman Jr, C.U.; Zhou, A. Org. Lett. 2012, 14, 3700.

11. Yamashita, Y.; Imaizumi, T.; Guo, X.; Kobayashi, S.; Chem. Asian J. 2011, 6, 2550. 
12. Iwata, M.; Yazaki, R.; Chen, I.; Sureshkumar, D.; Kumagai, N.; Shibasaki, M. J. Am. Chem. Soc. 2011, 133,5554 .

13. Xu, Z.; Zhang, F.; Zhang, L.; Jia, Y. Org. Biomol. Chem. 2011, 9, 2512.

14. Pattenden, G.; Ashweek, N. J.; Baker-Glenn, C. A. G.; Kempson, J.; Walker, G. M.; Yee, J. G. K. Org. Biomol. Chem. 2008, 6, 1478.

15. Shaikh, N. S.; Bhor, S. S.; Gajare, A. S.; Deshpande, V. H.; Wakharkar, R. D. Tetrahedron Letters, 2004, 45, 5395.

16. Albert, B. J.; Sivaramakrishnan, A.; Naka, T.; Koide, K. J. Am. Chem. Soc. 2006, 128, 2792. 


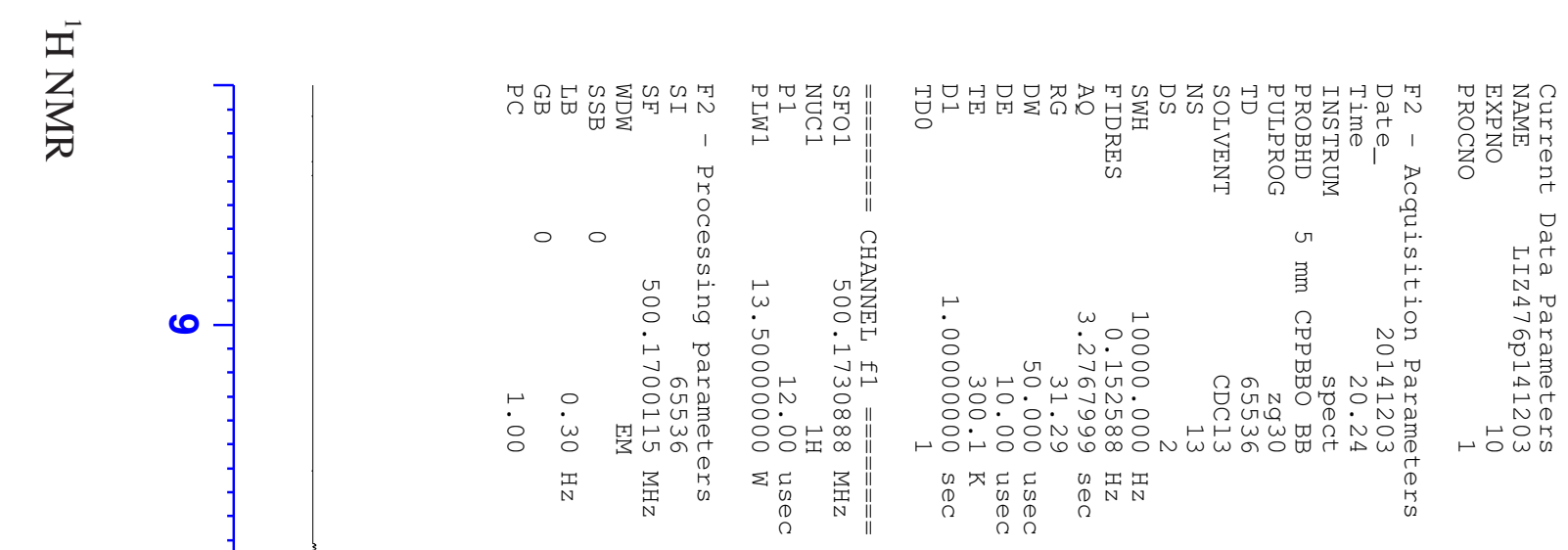

7.914

$\left[\begin{array}{l}7.914 \\ 7.901\end{array}\right.$

7.897

7.895

7.658

7.647

7.643

$F^{7.639}$

2.006

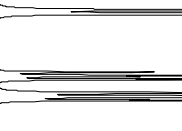

$-7.628$

3.074

$=$

$\frac{}{\frac{1.000}{1.047}}$
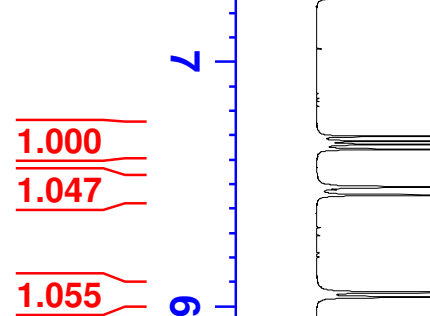

0

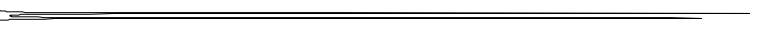

or

$\rightarrow$

\begin{tabular}{l}
0 \\
- \\
- \\
0 \\
$\frac{0}{3}$ \\
\hline
\end{tabular}

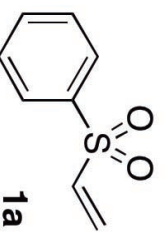




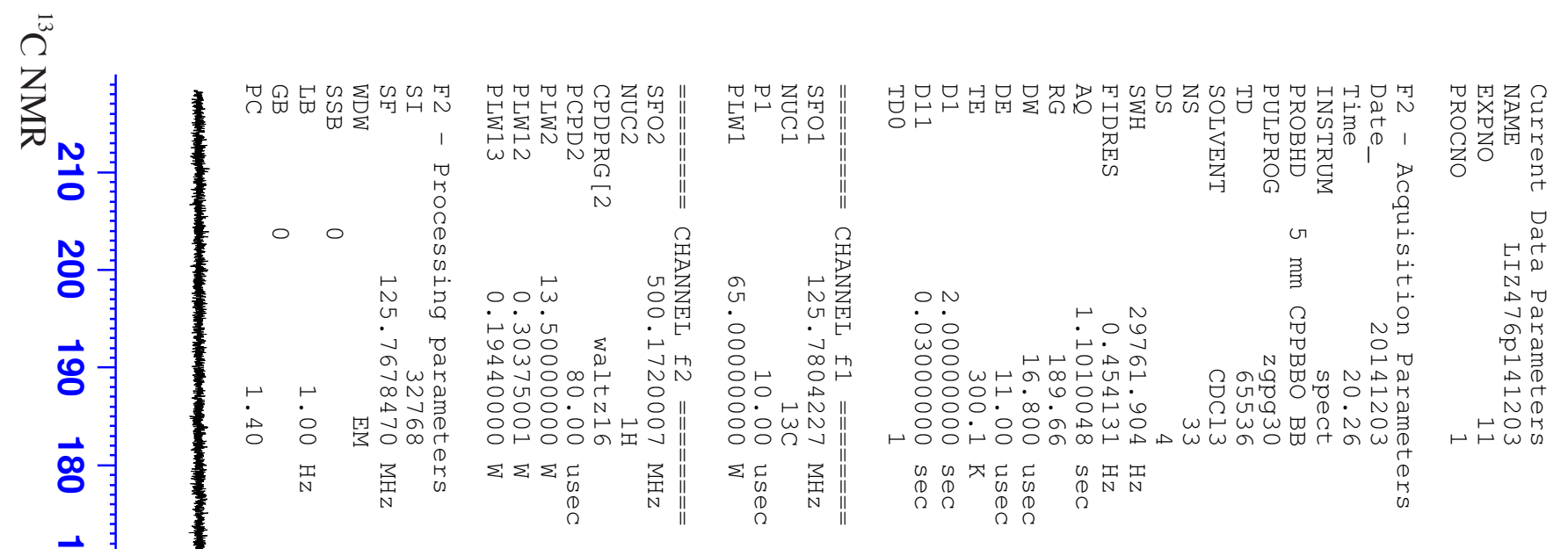

a

ज्

$\overrightarrow{0}$

$\vec{\omega}$

$\overrightarrow{0}$

훙

웅

우

ஜ

g

G

b

$\omega$

ㅇ

$\overrightarrow{0}$

홐

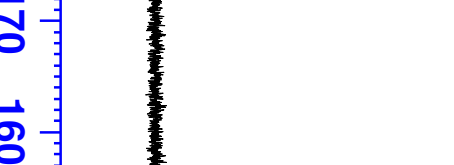

139.613

138.508

$-133.644$

$-129.351$

$-127.938$

127.735

77.269
-77.015

77.015

76.761

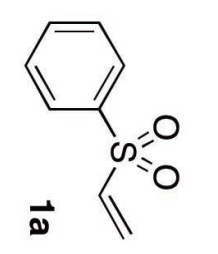

$--0.004$ 


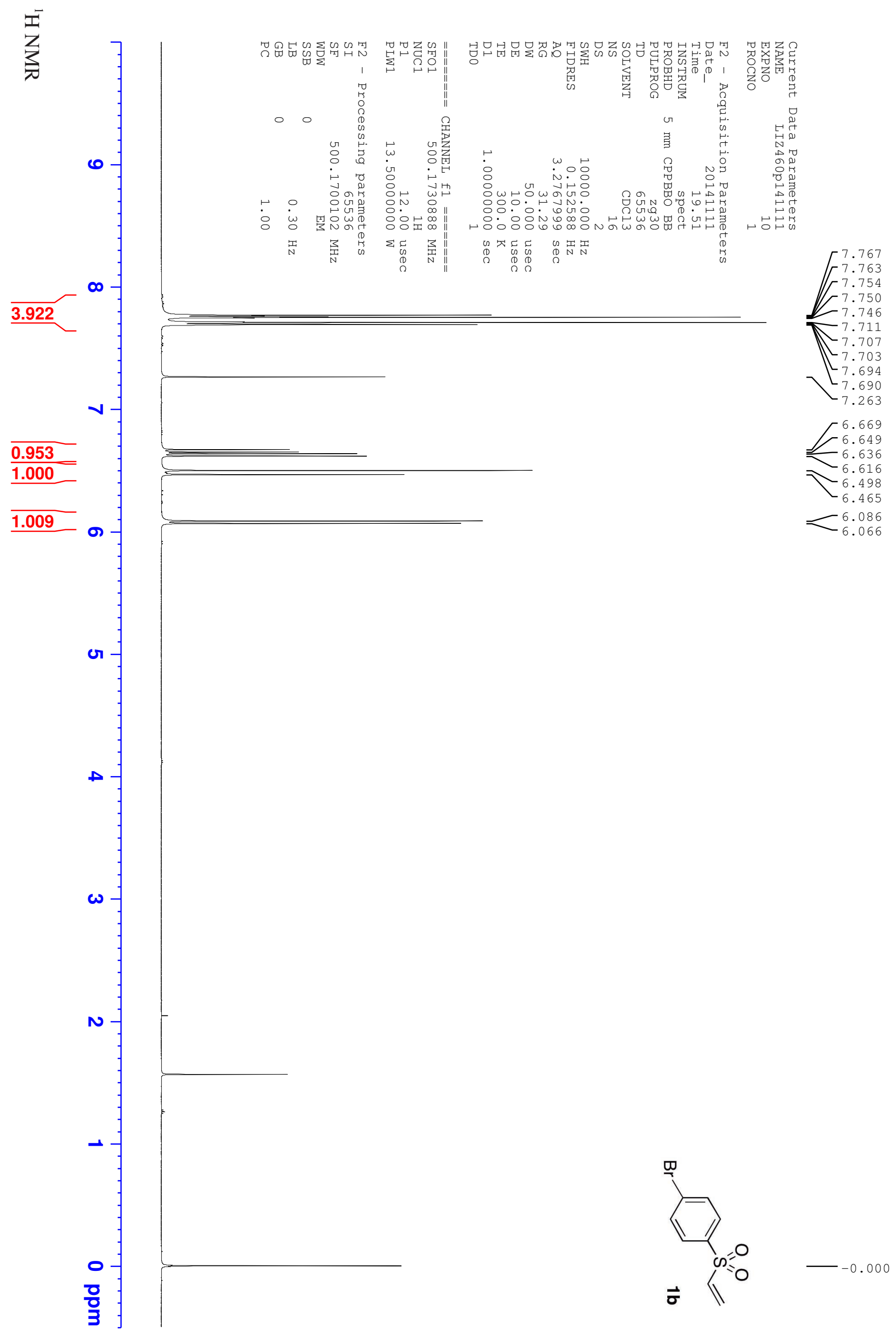




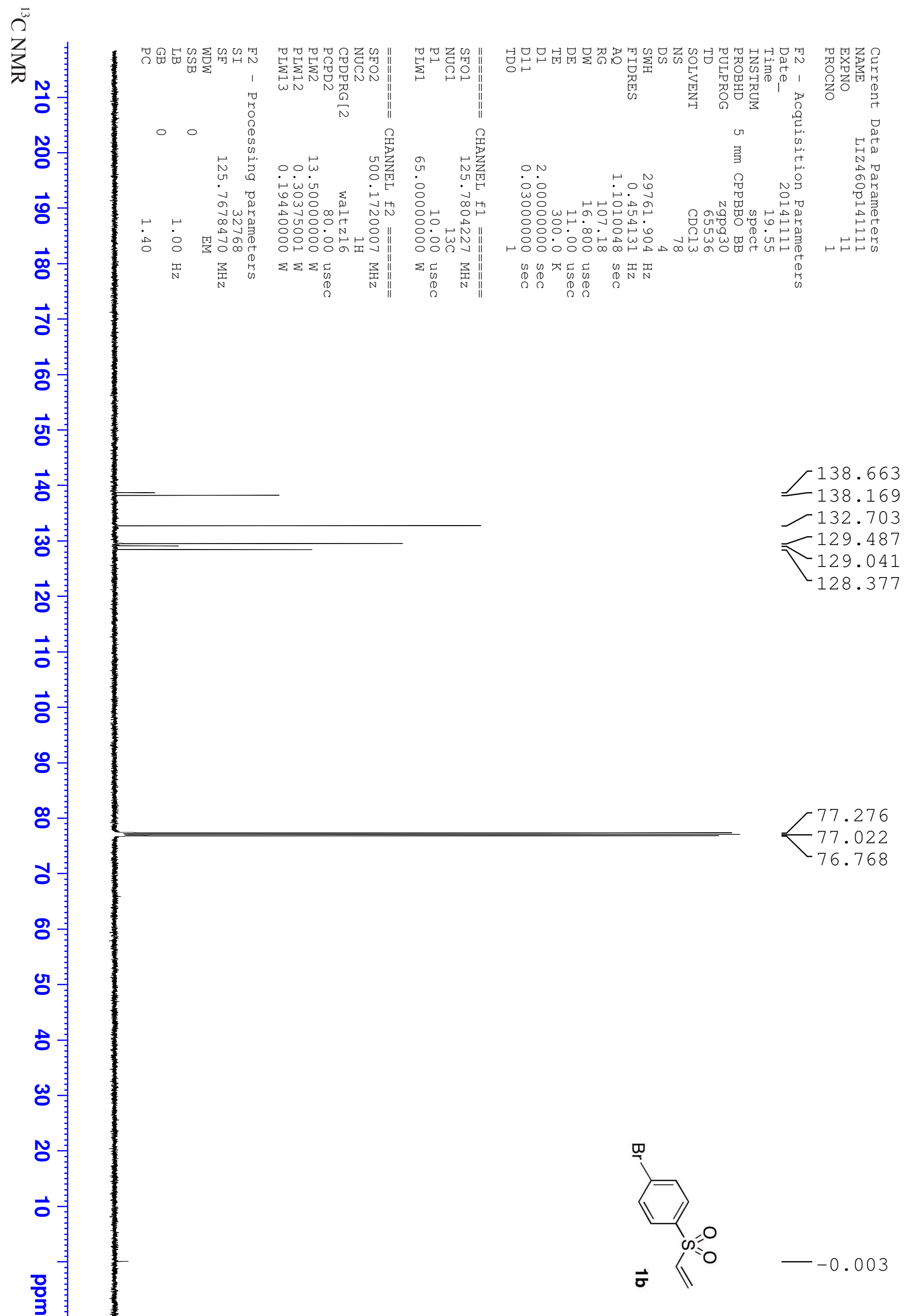




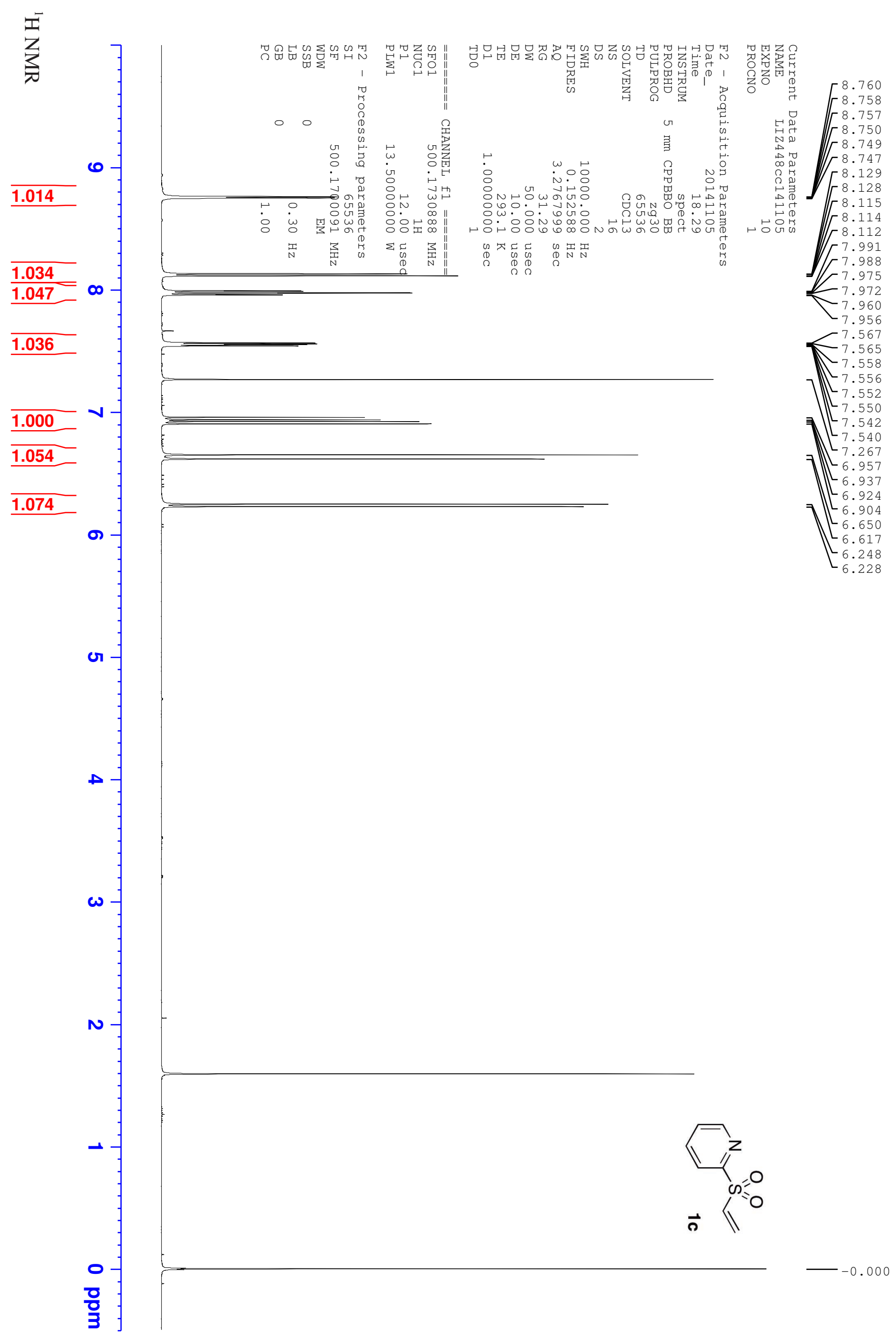




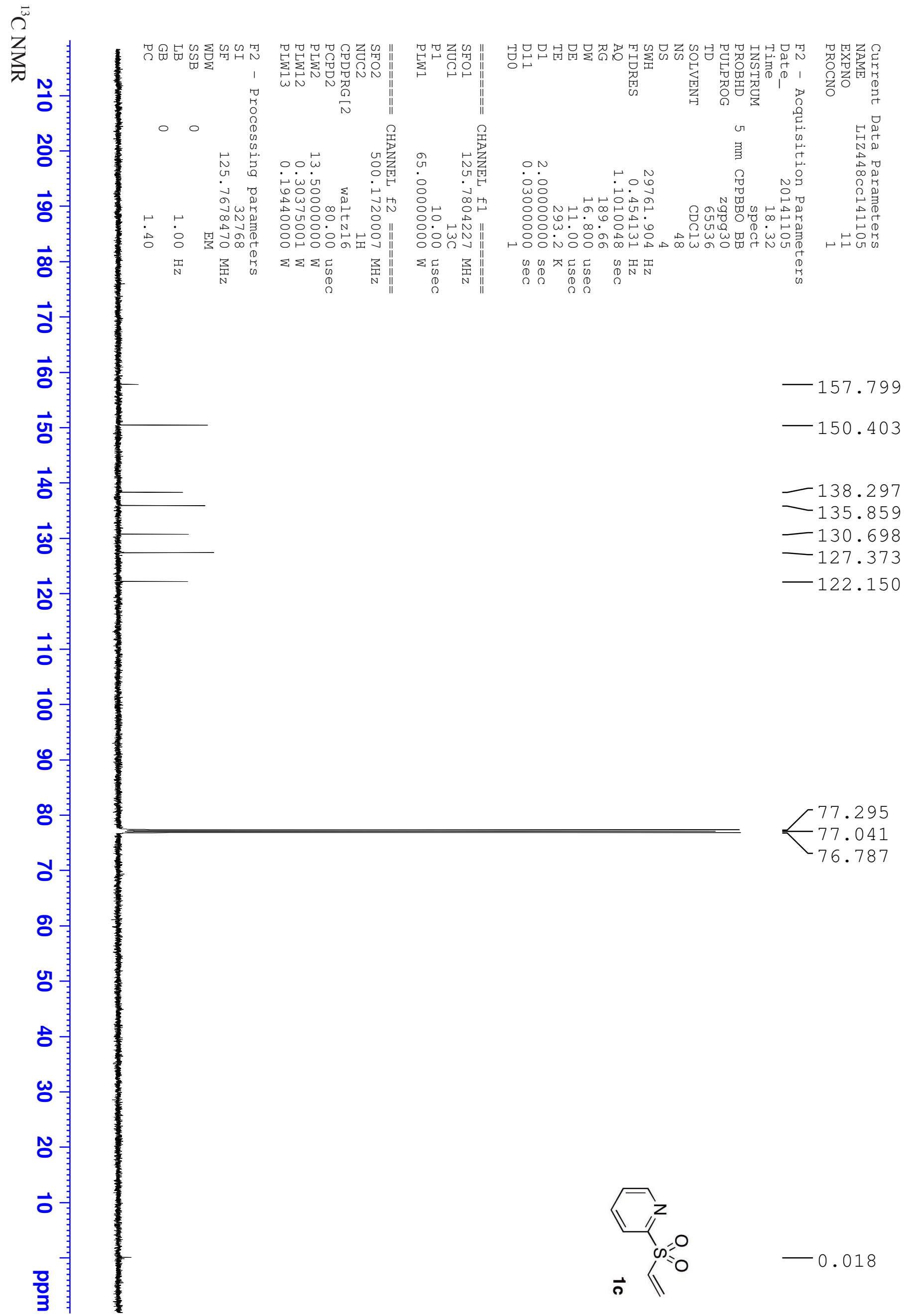



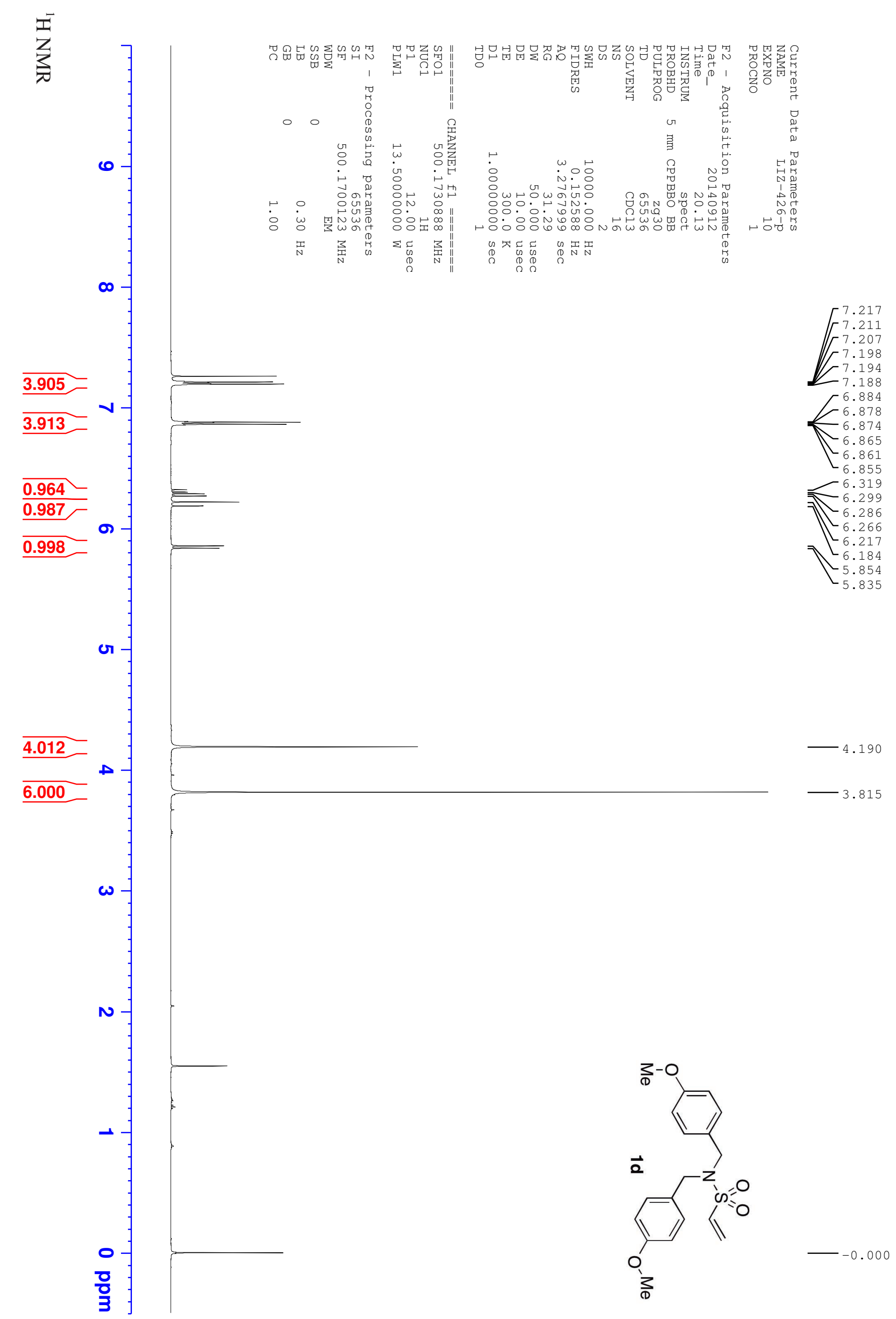


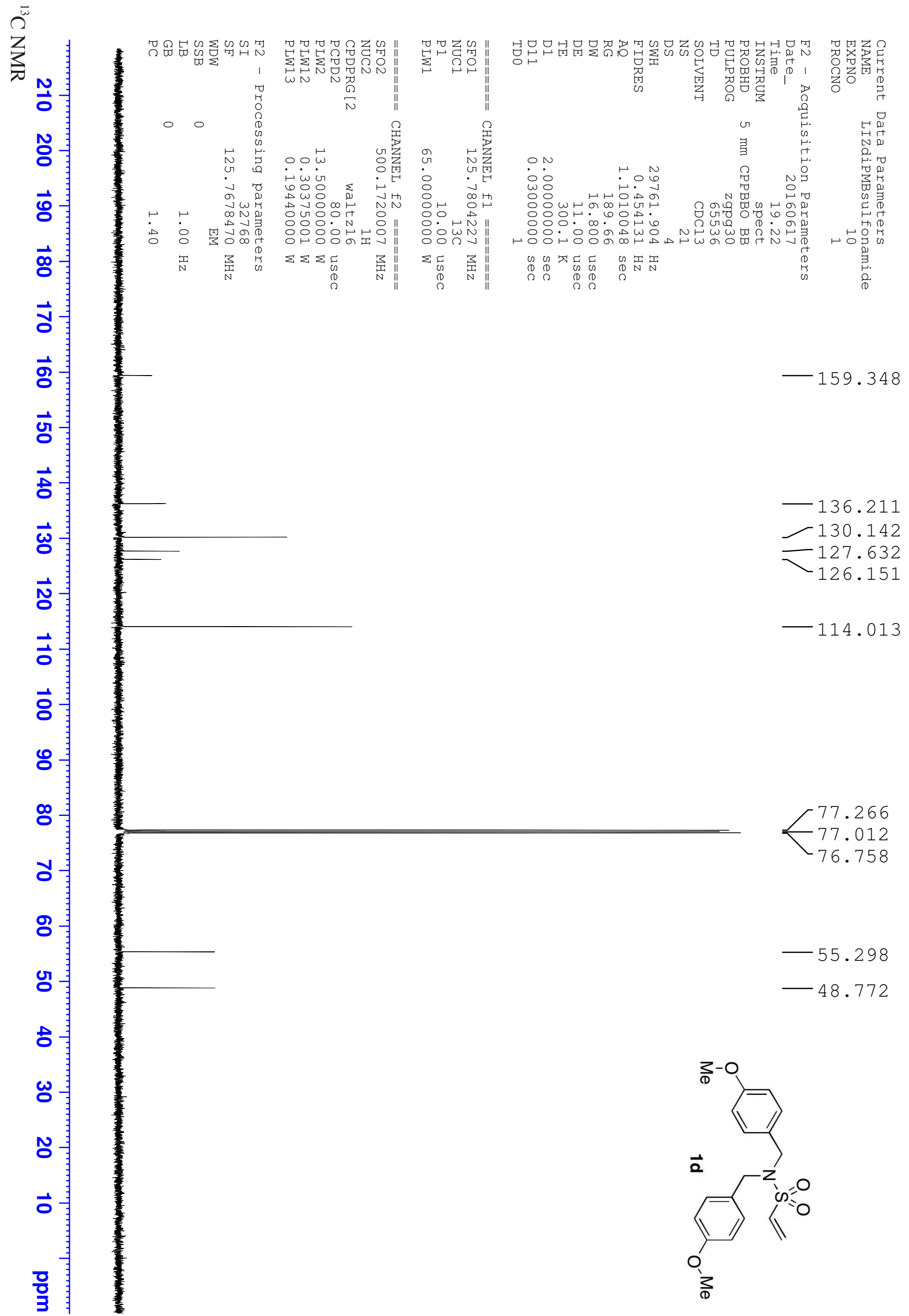




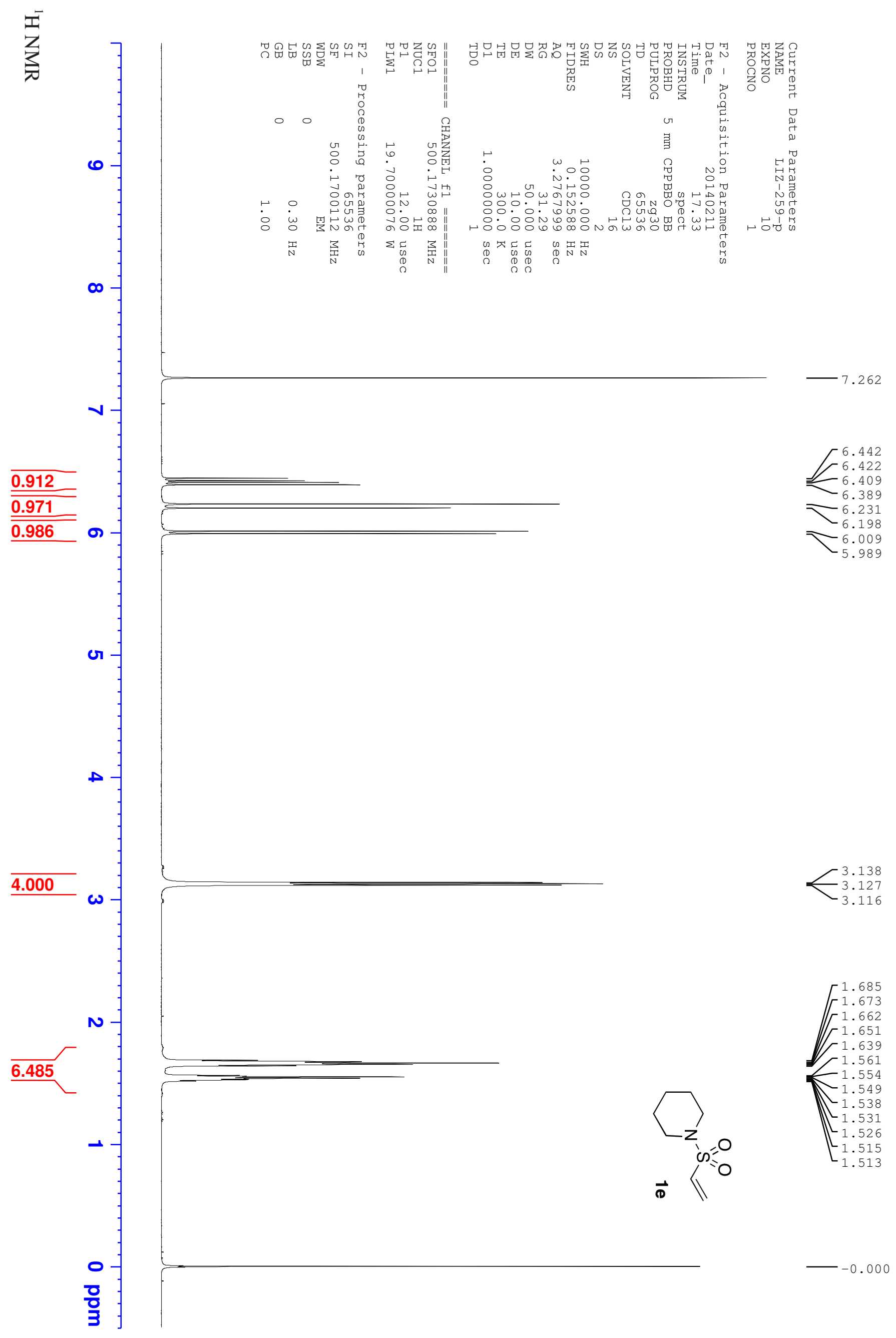




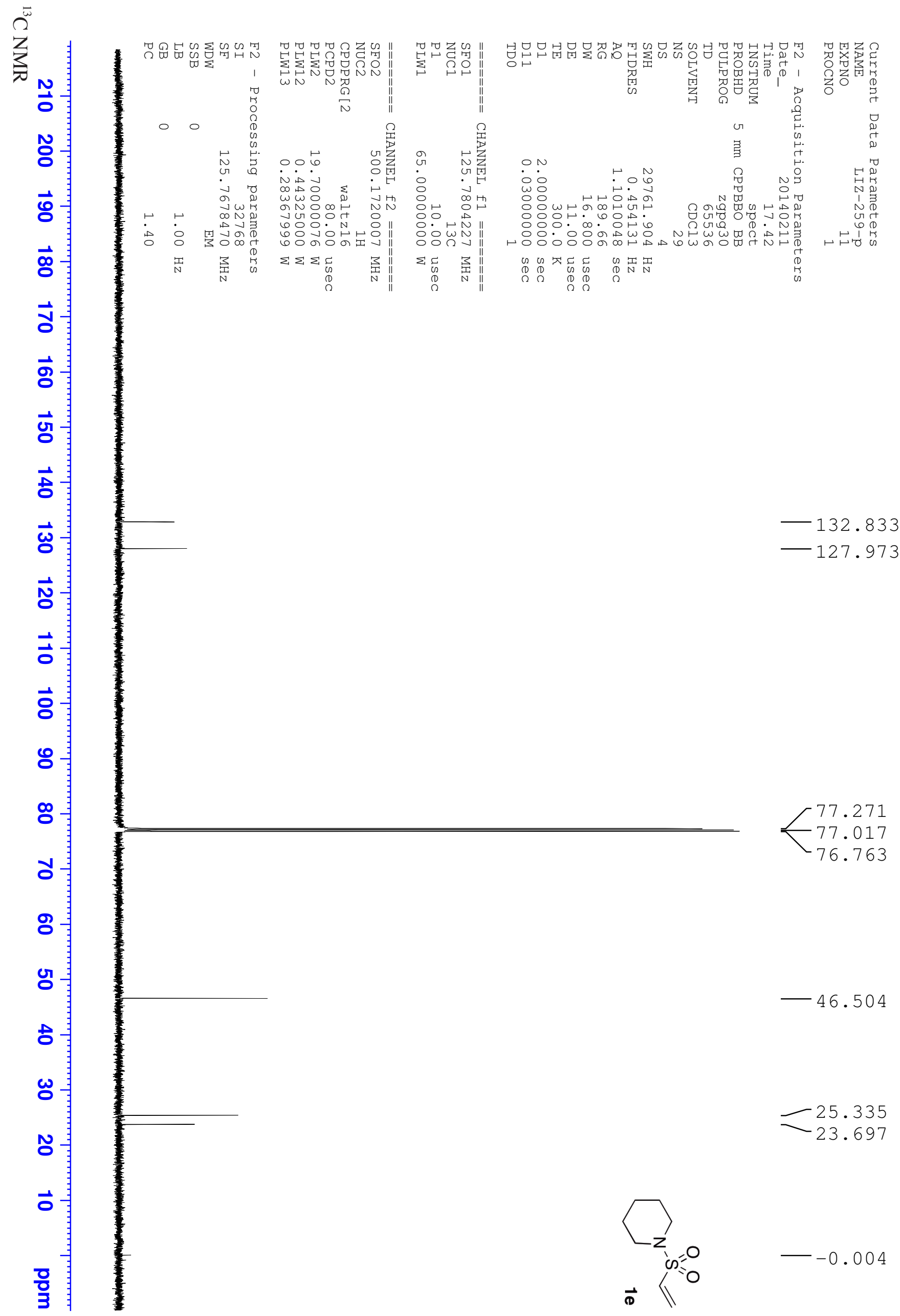




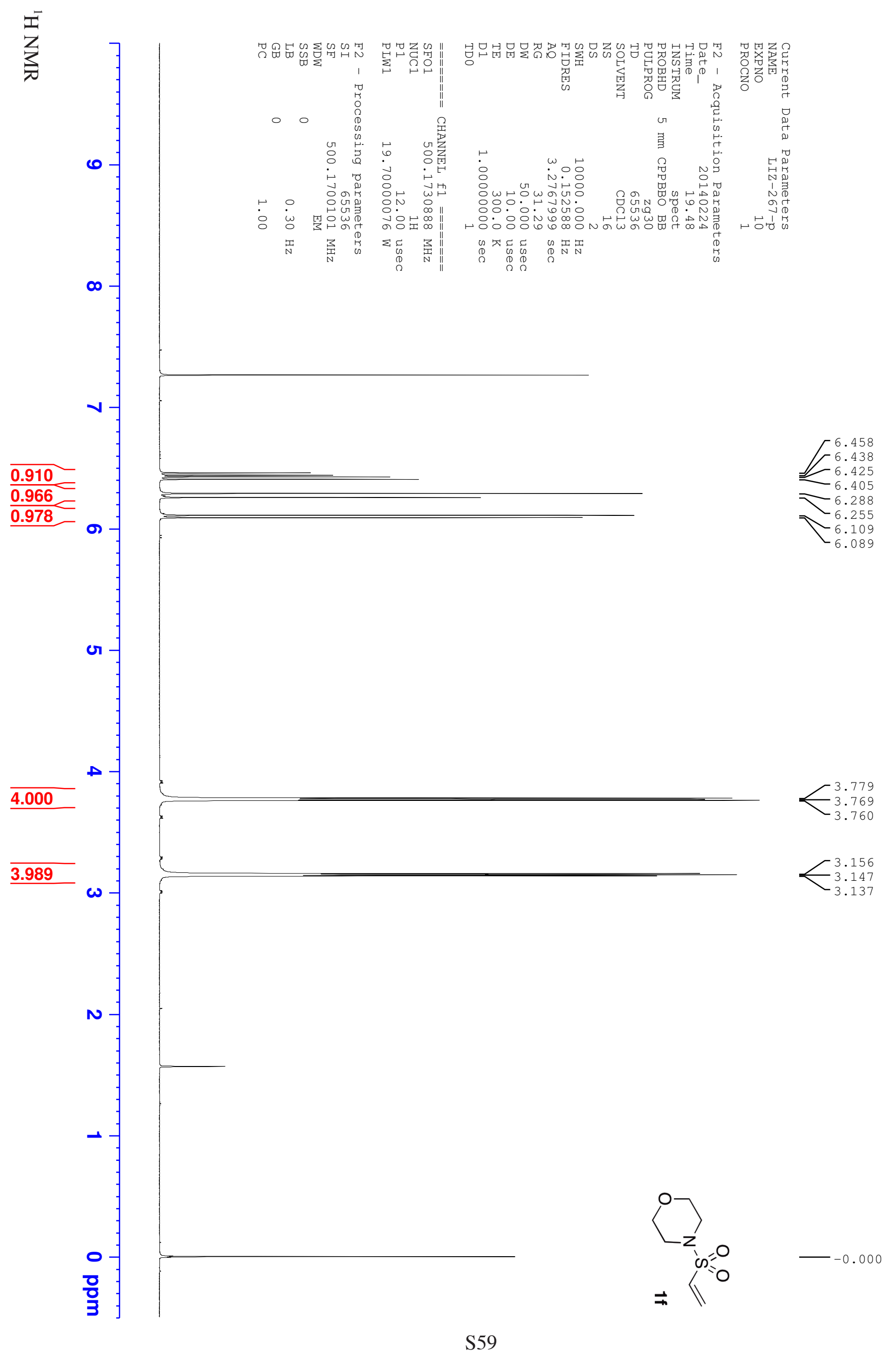




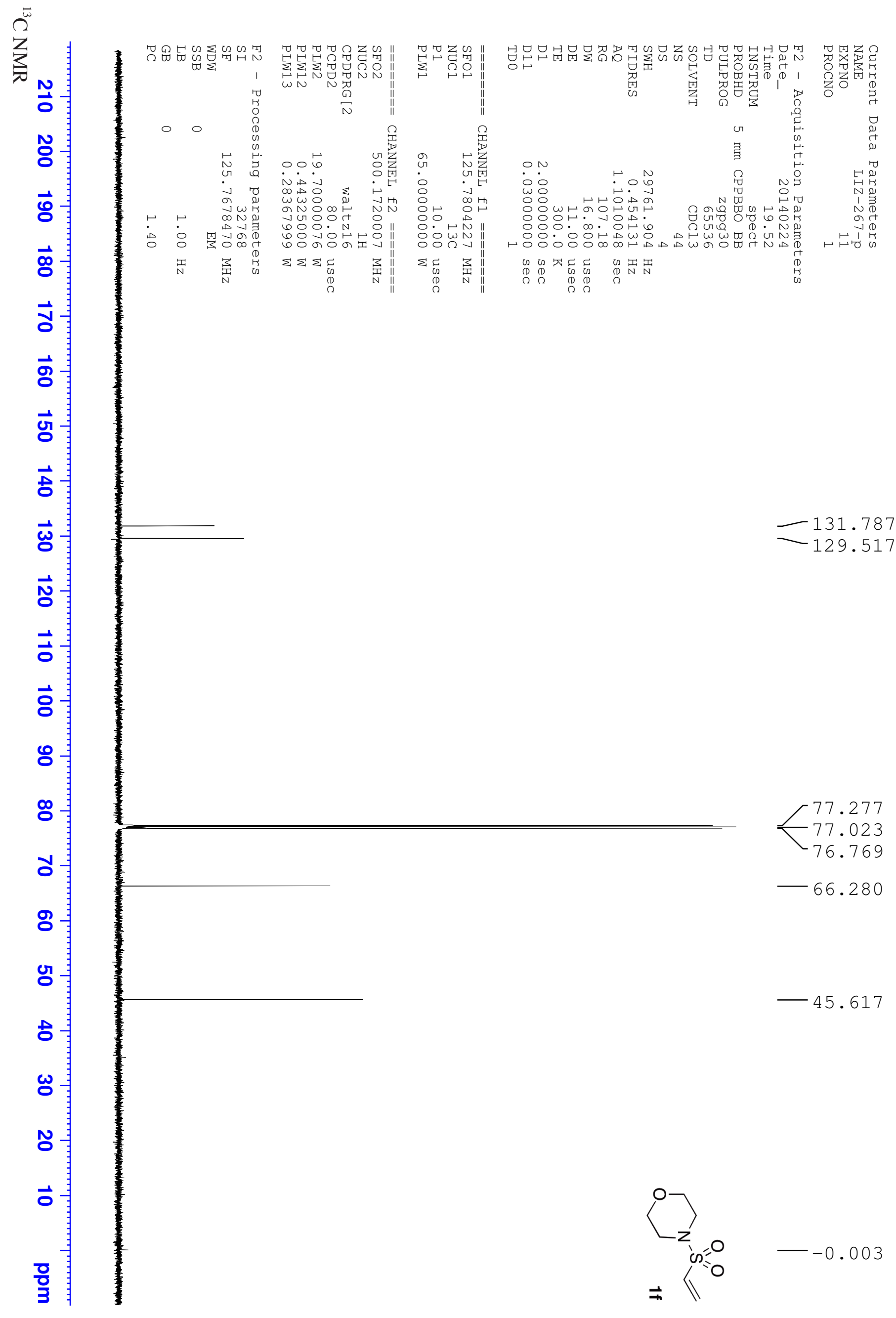



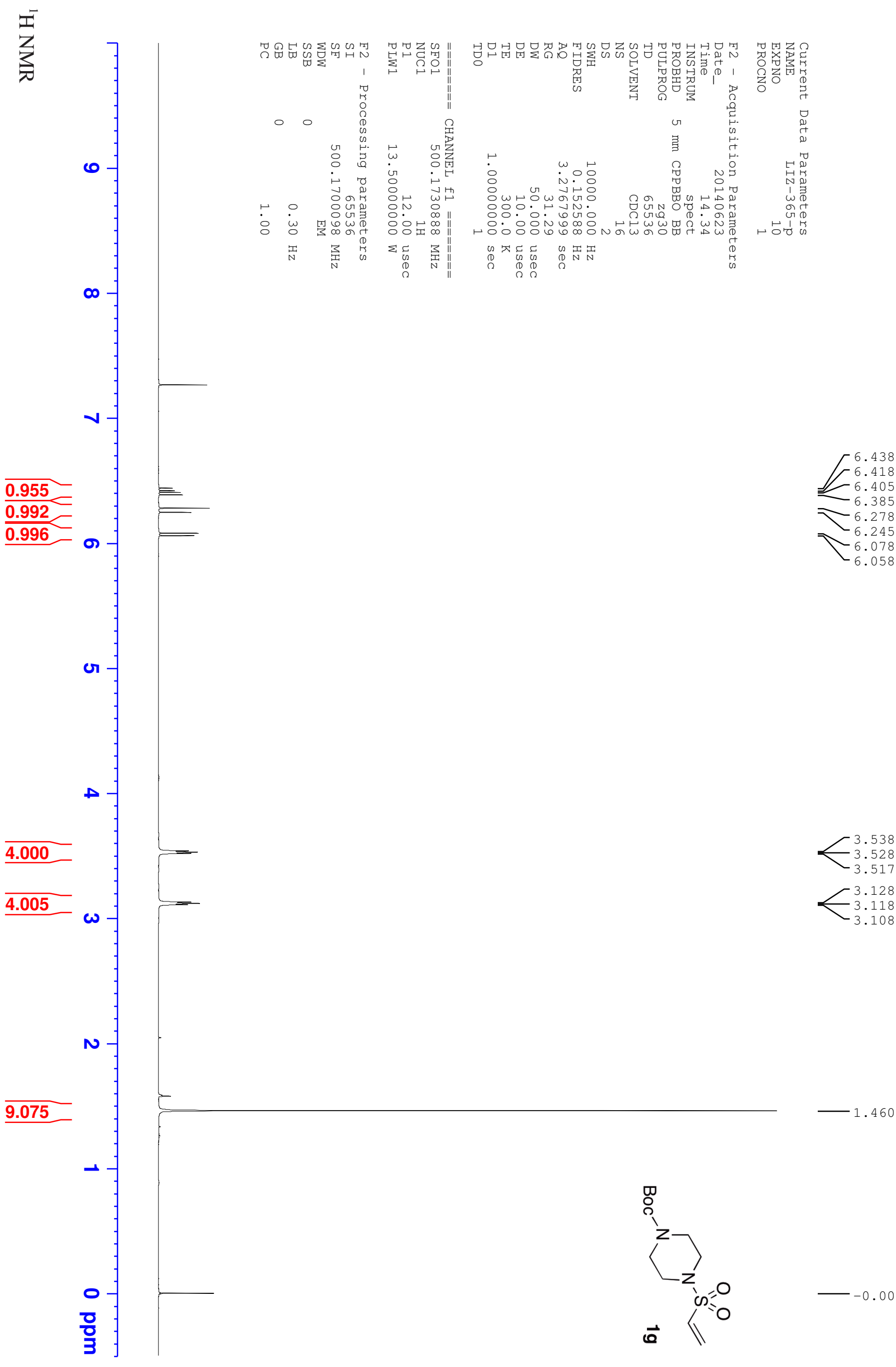

3.528

3.118

$-3.108$

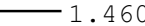

$-0.000$ 


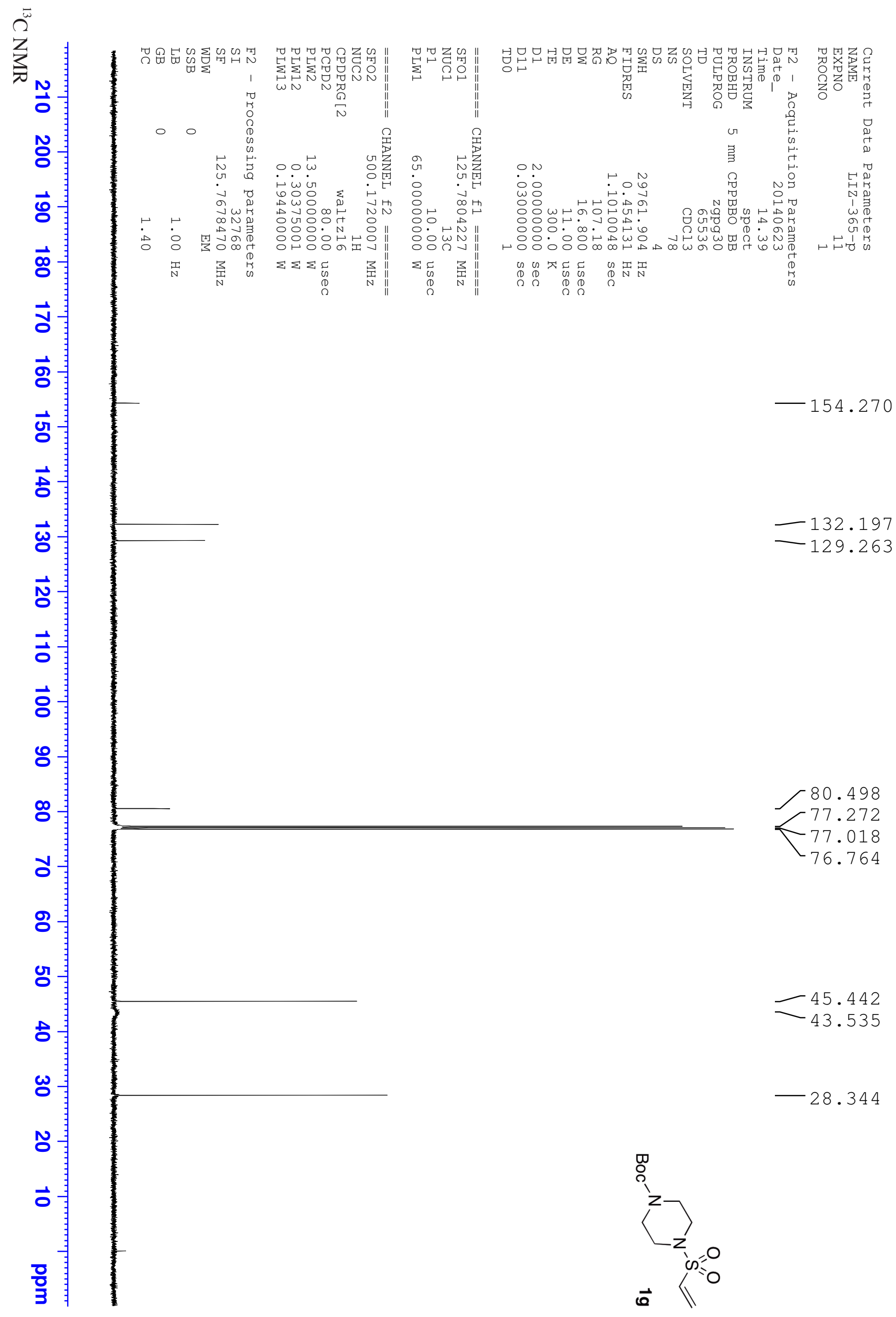




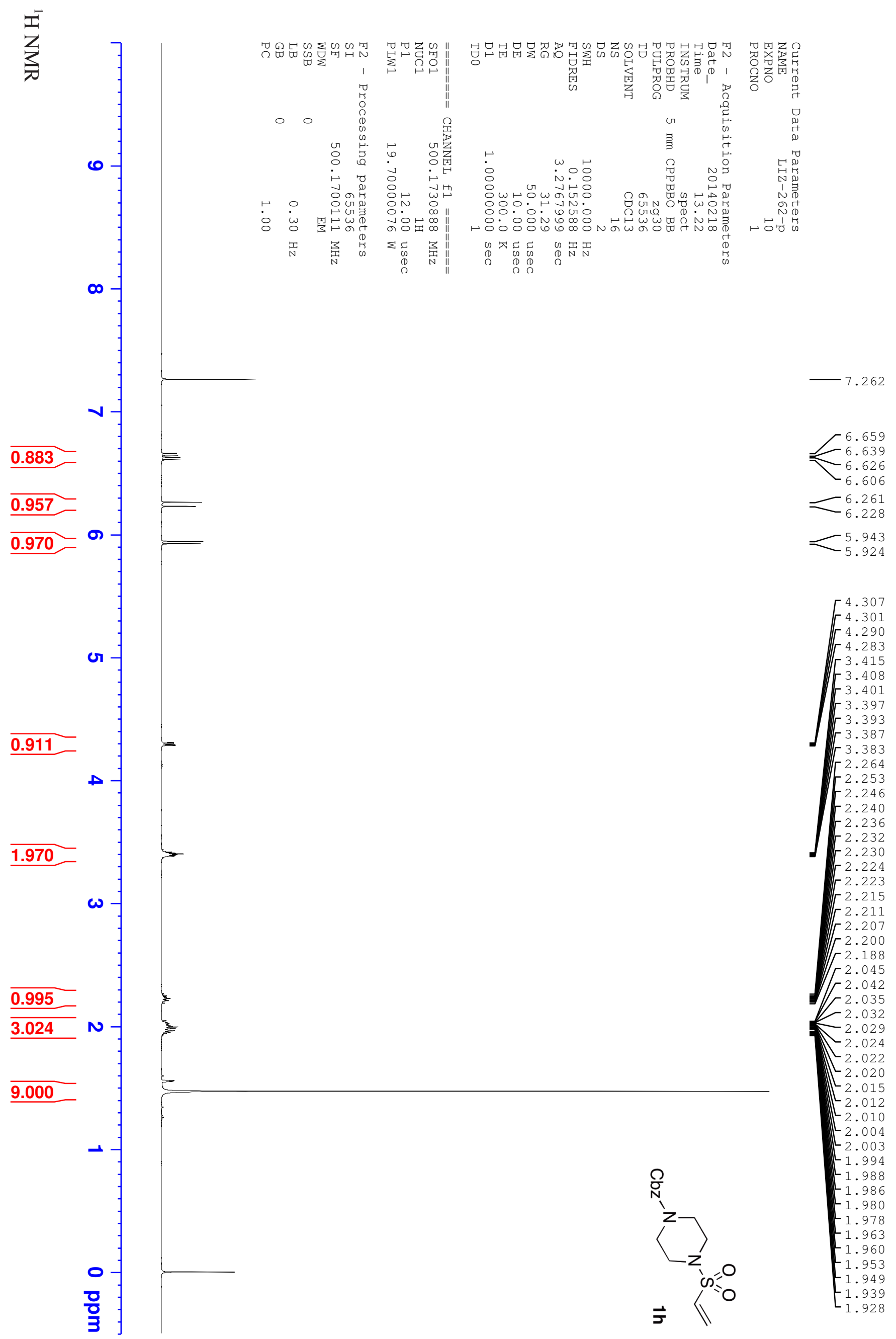




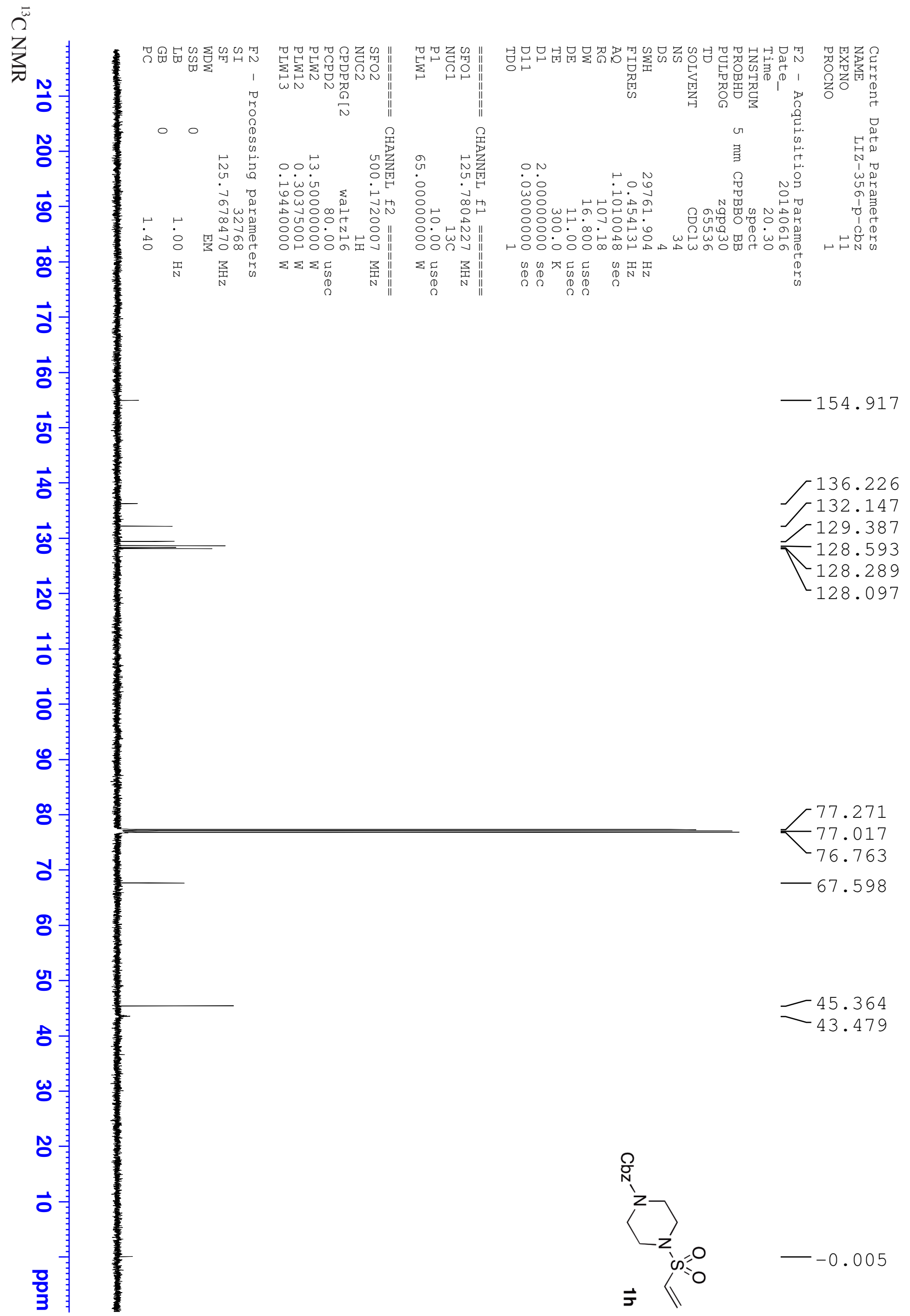




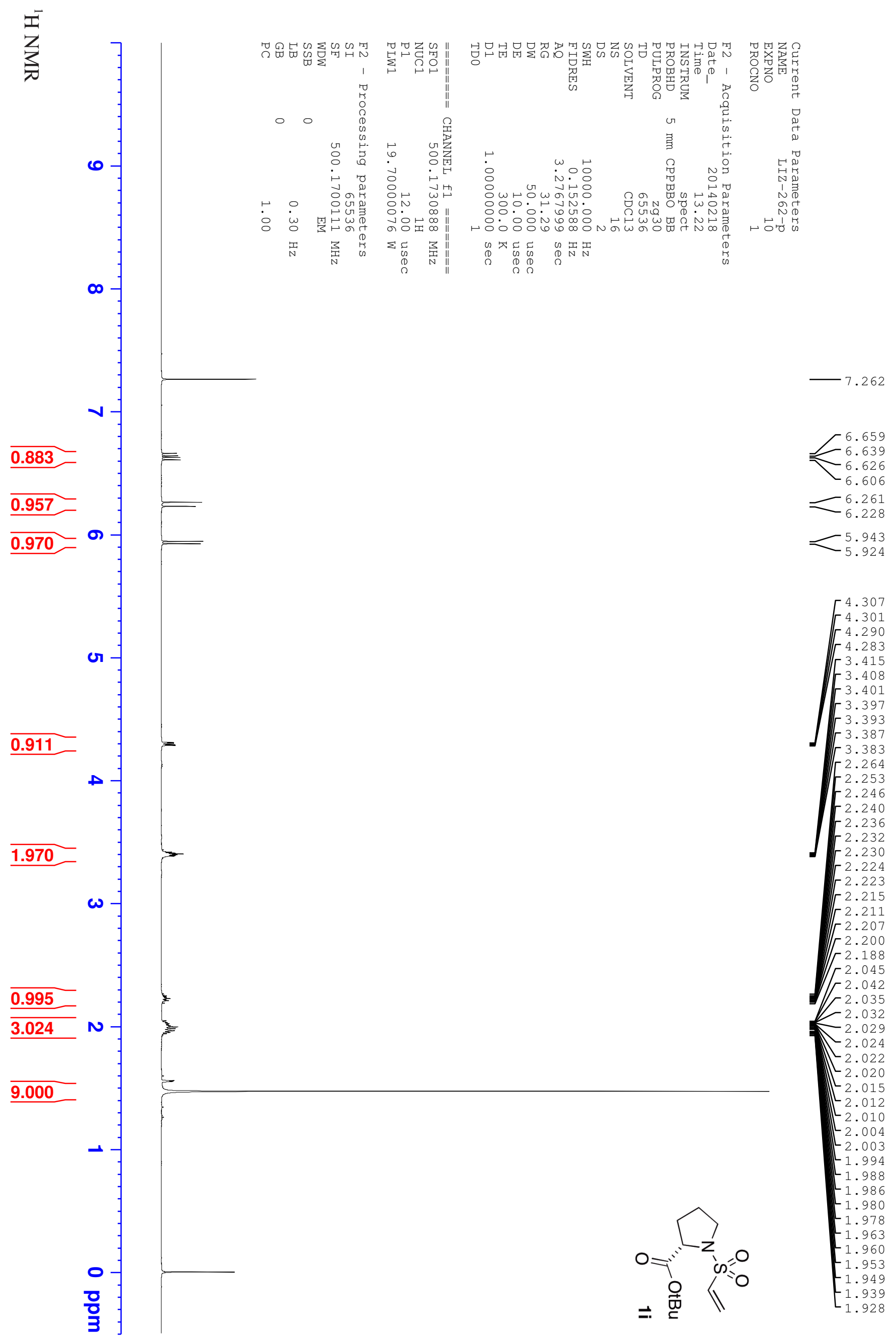




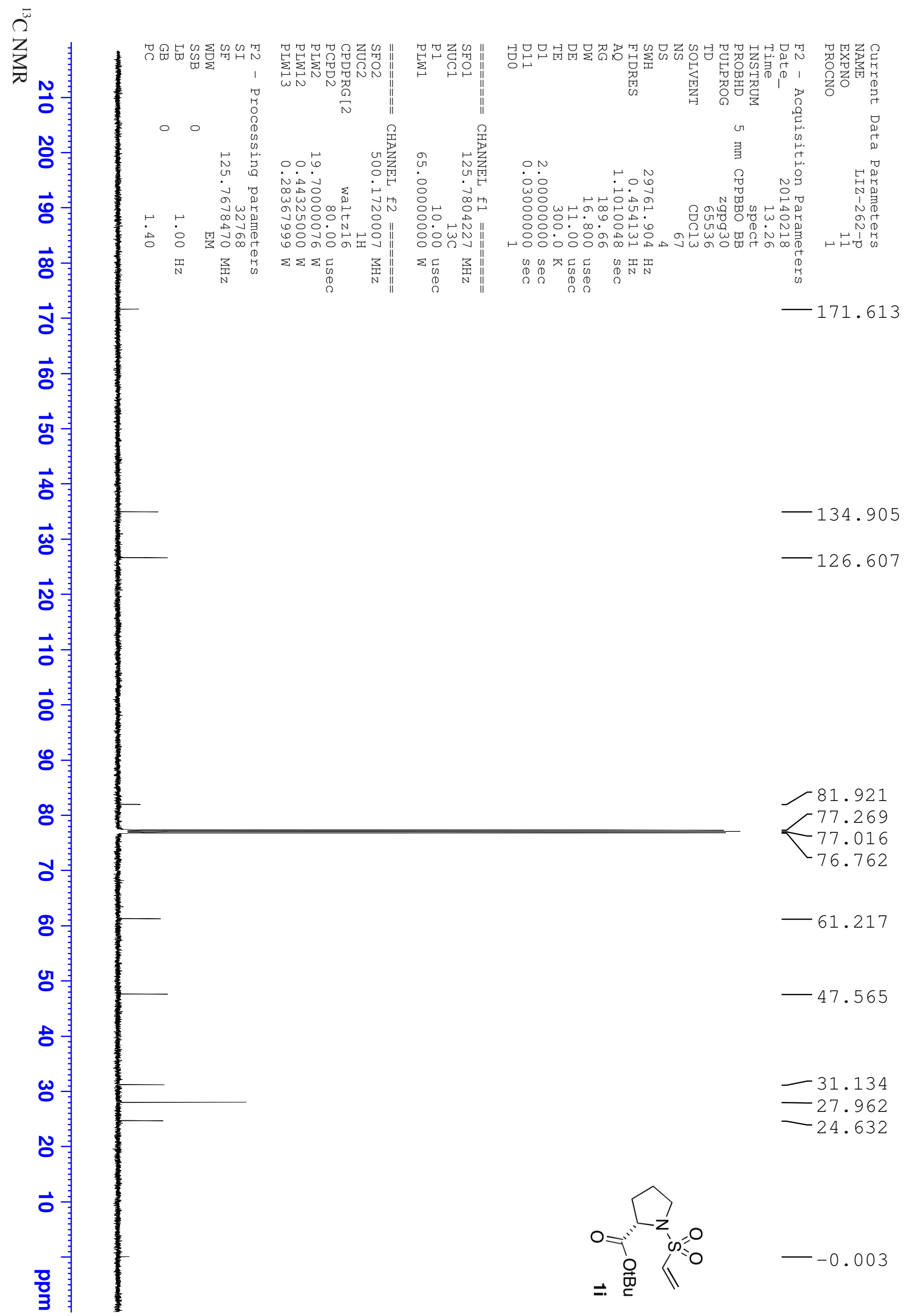




\section{NMR Spectra of New Compounds}

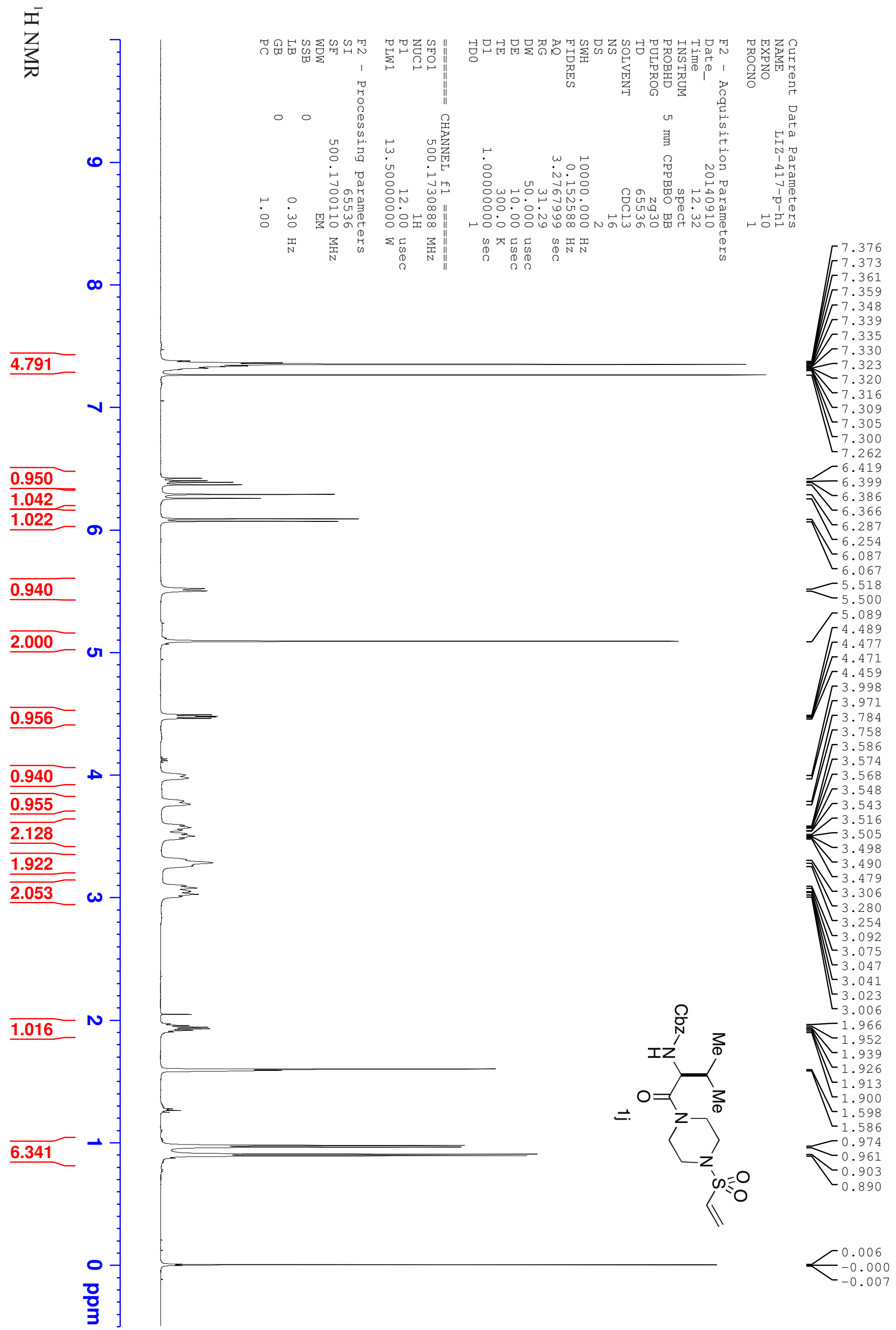




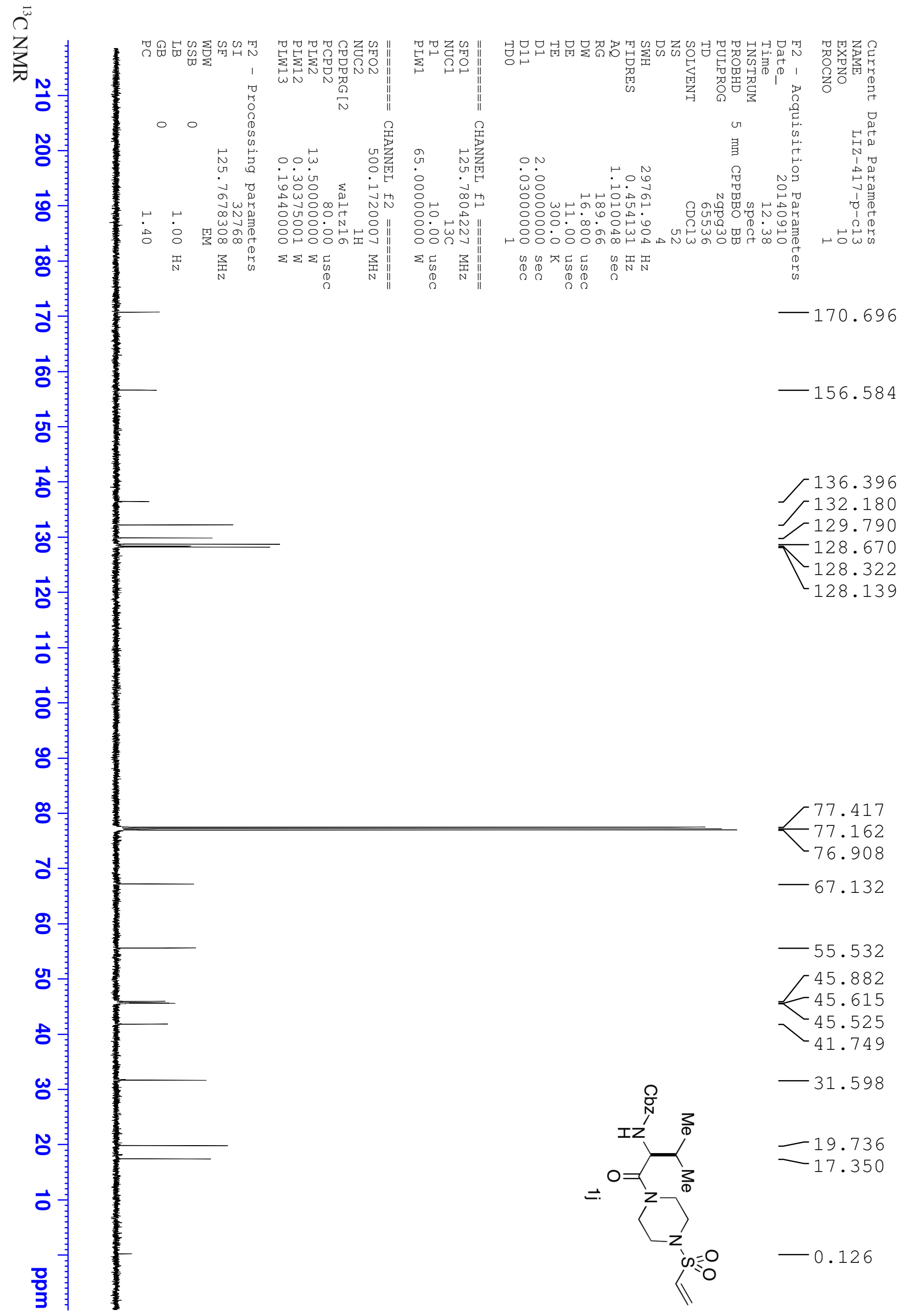




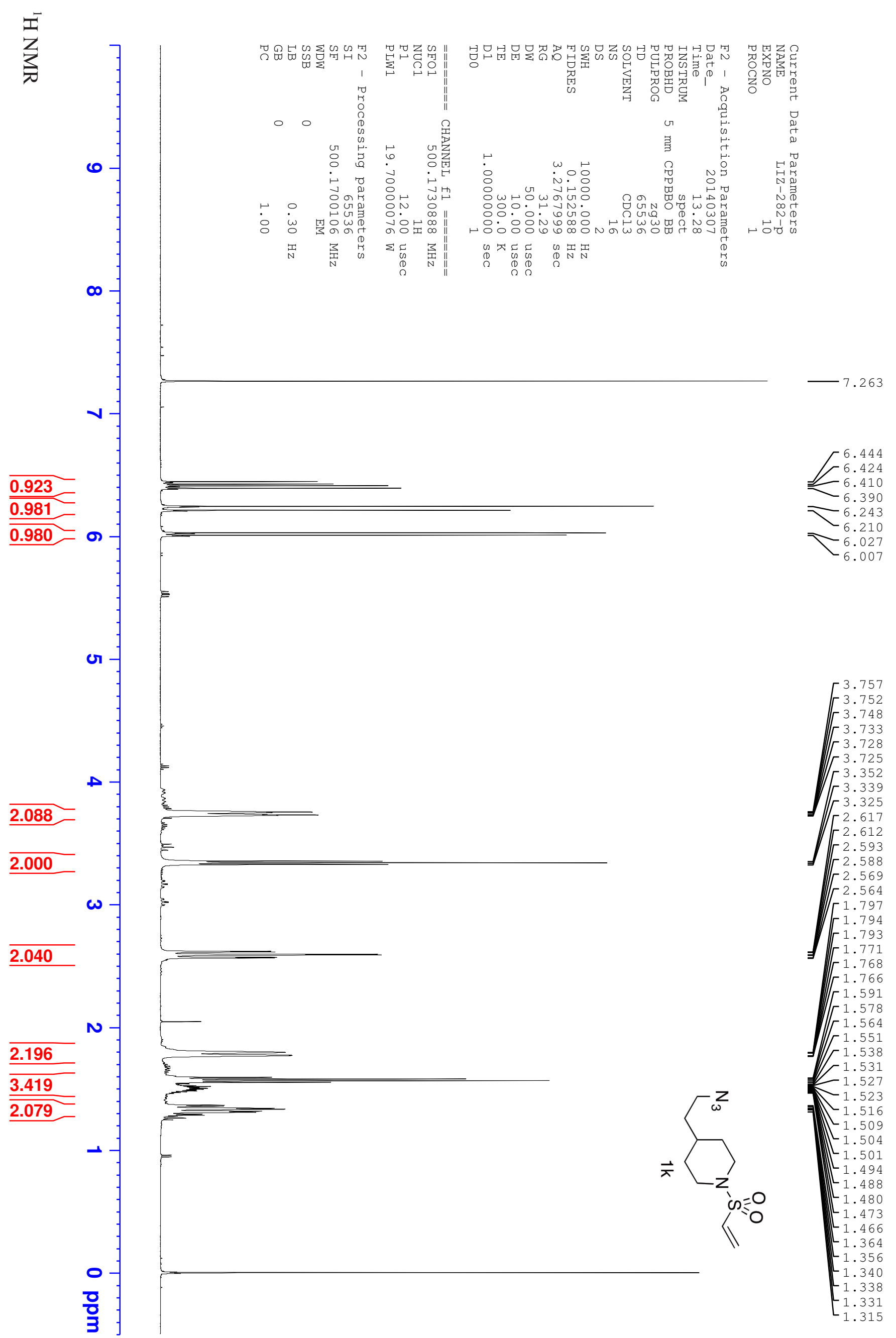




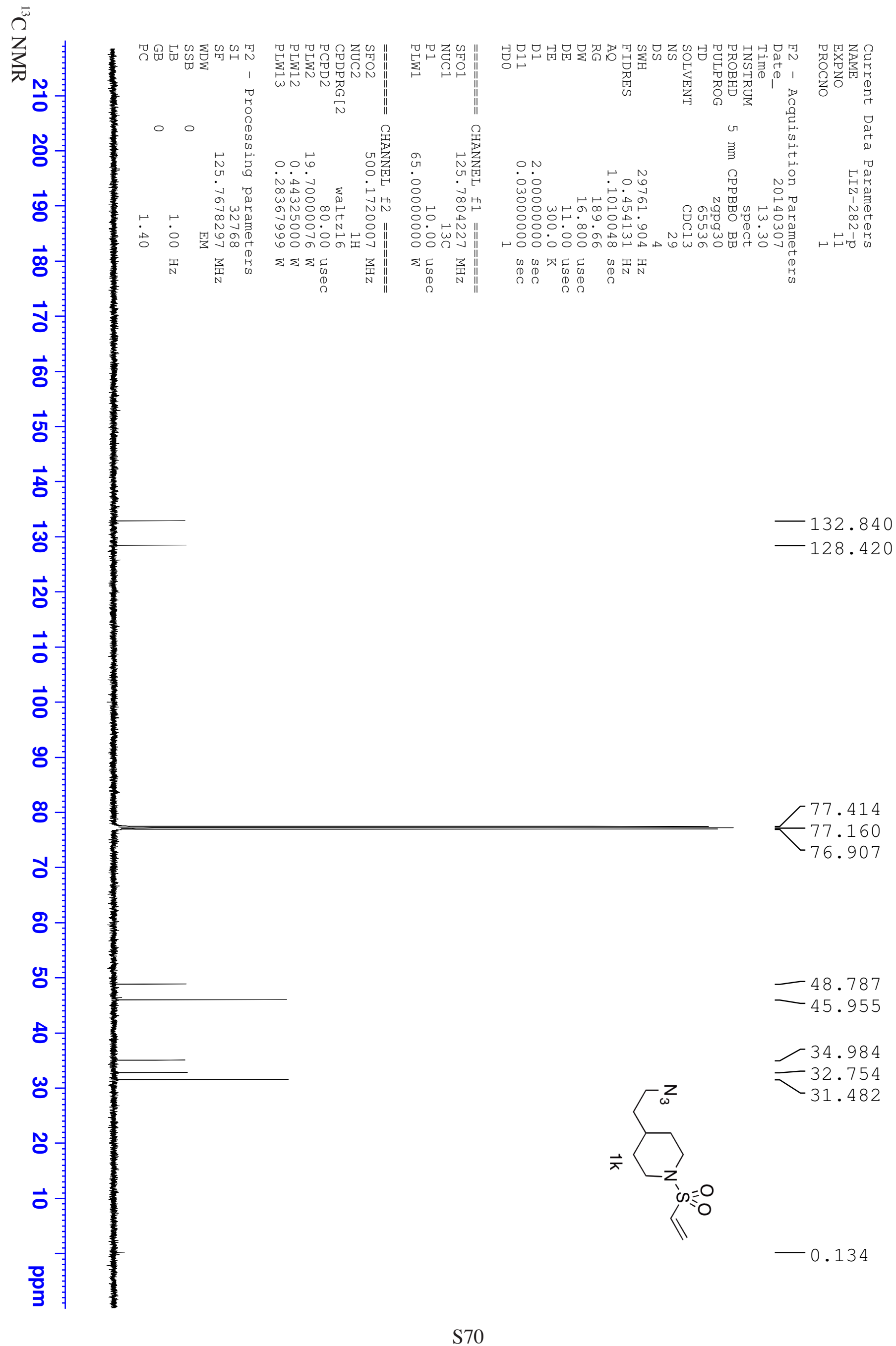




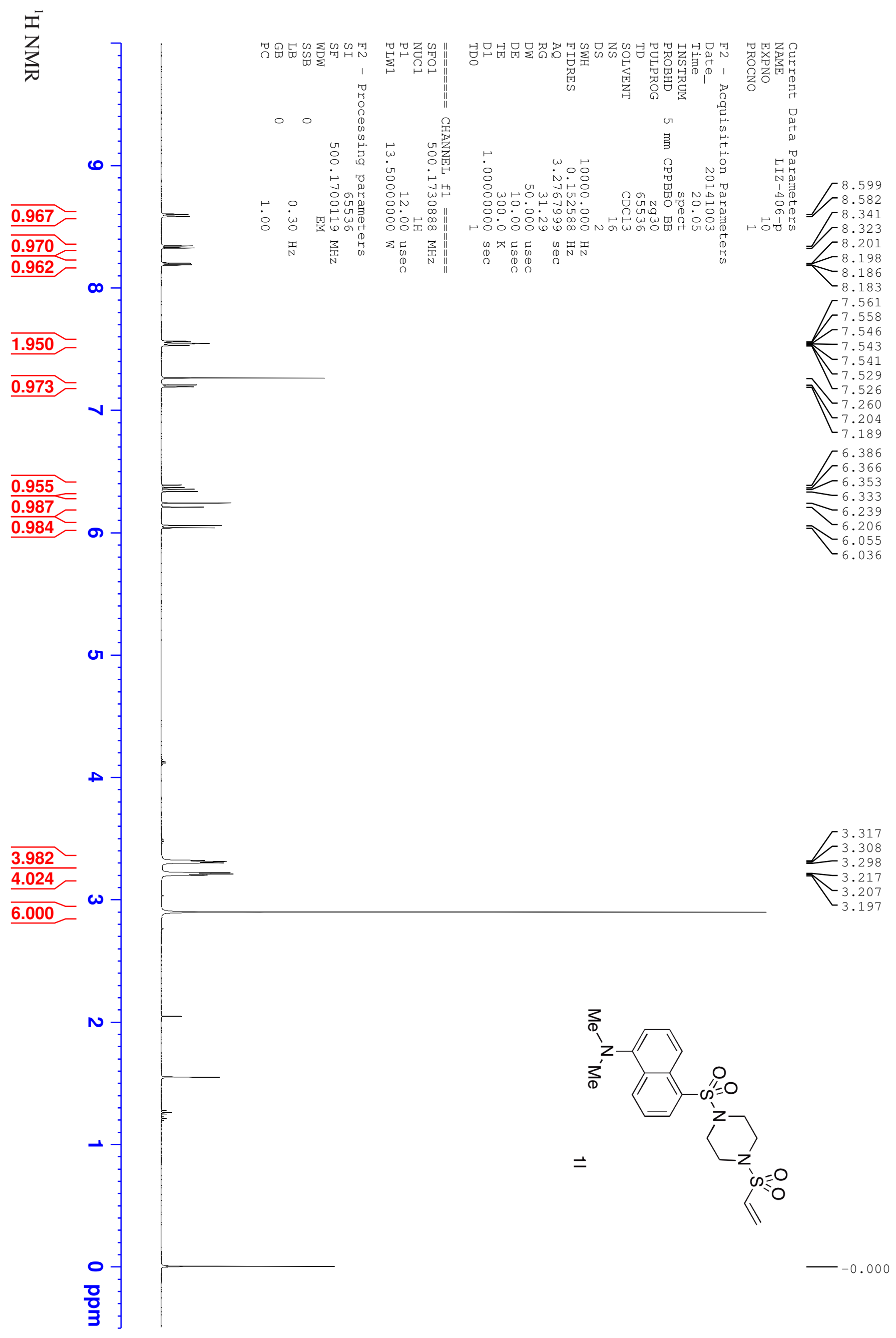




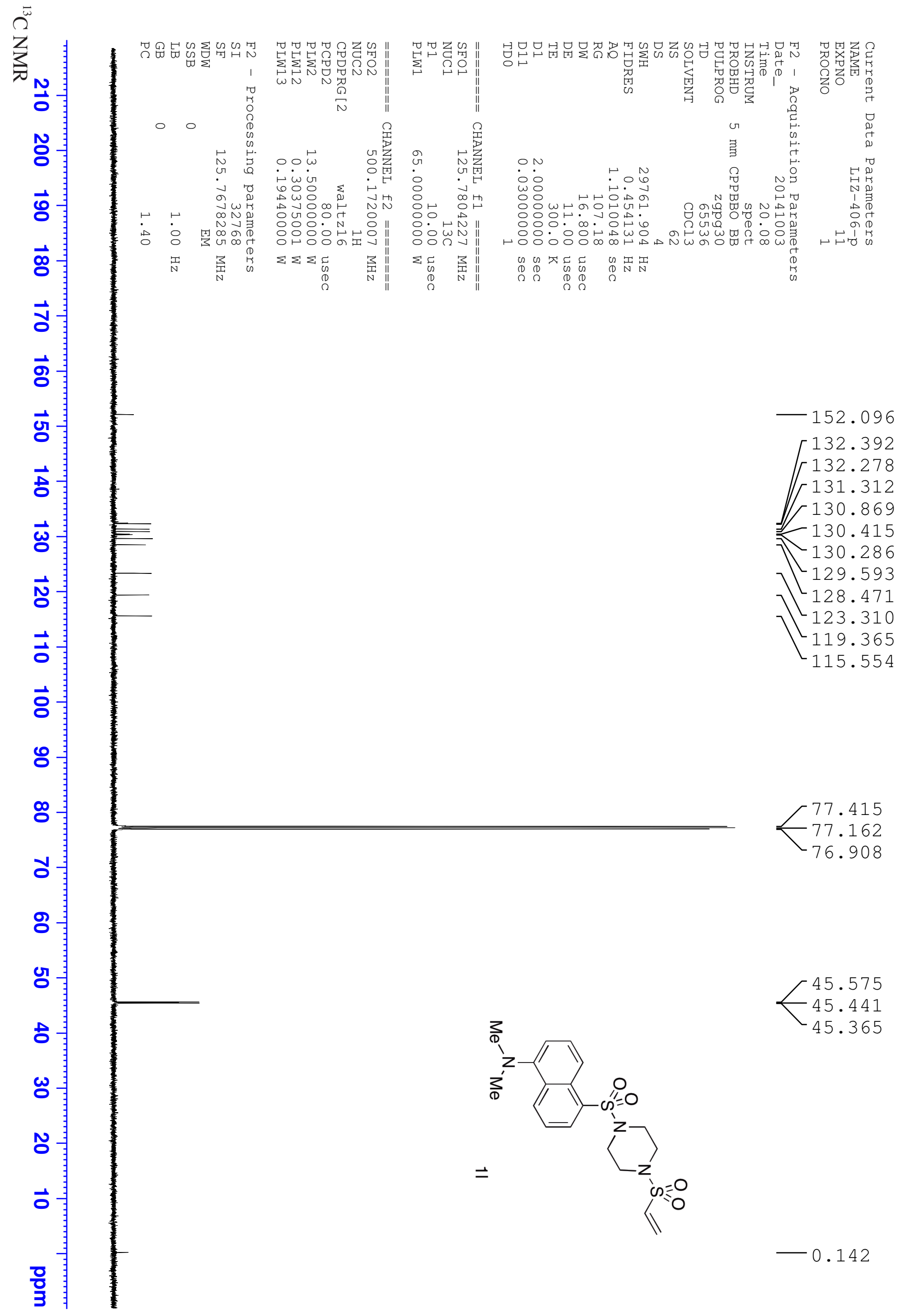


艺

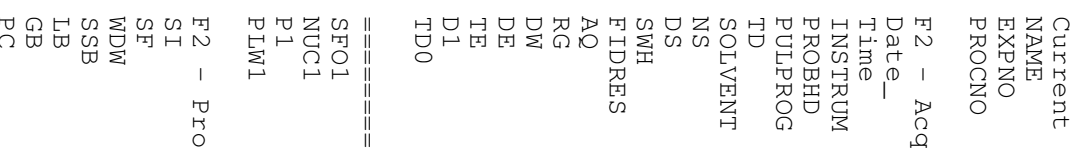

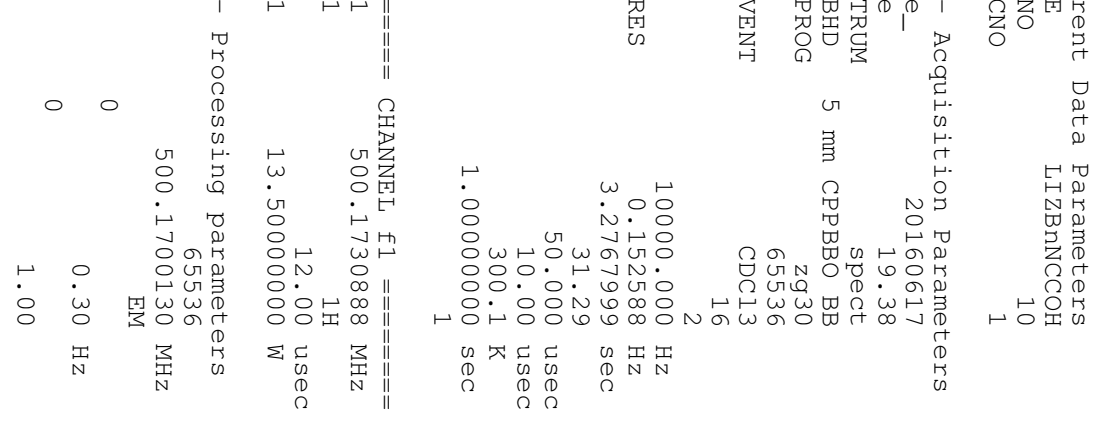

$\infty-$

$\checkmark$

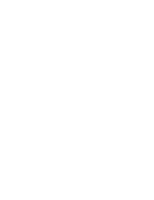

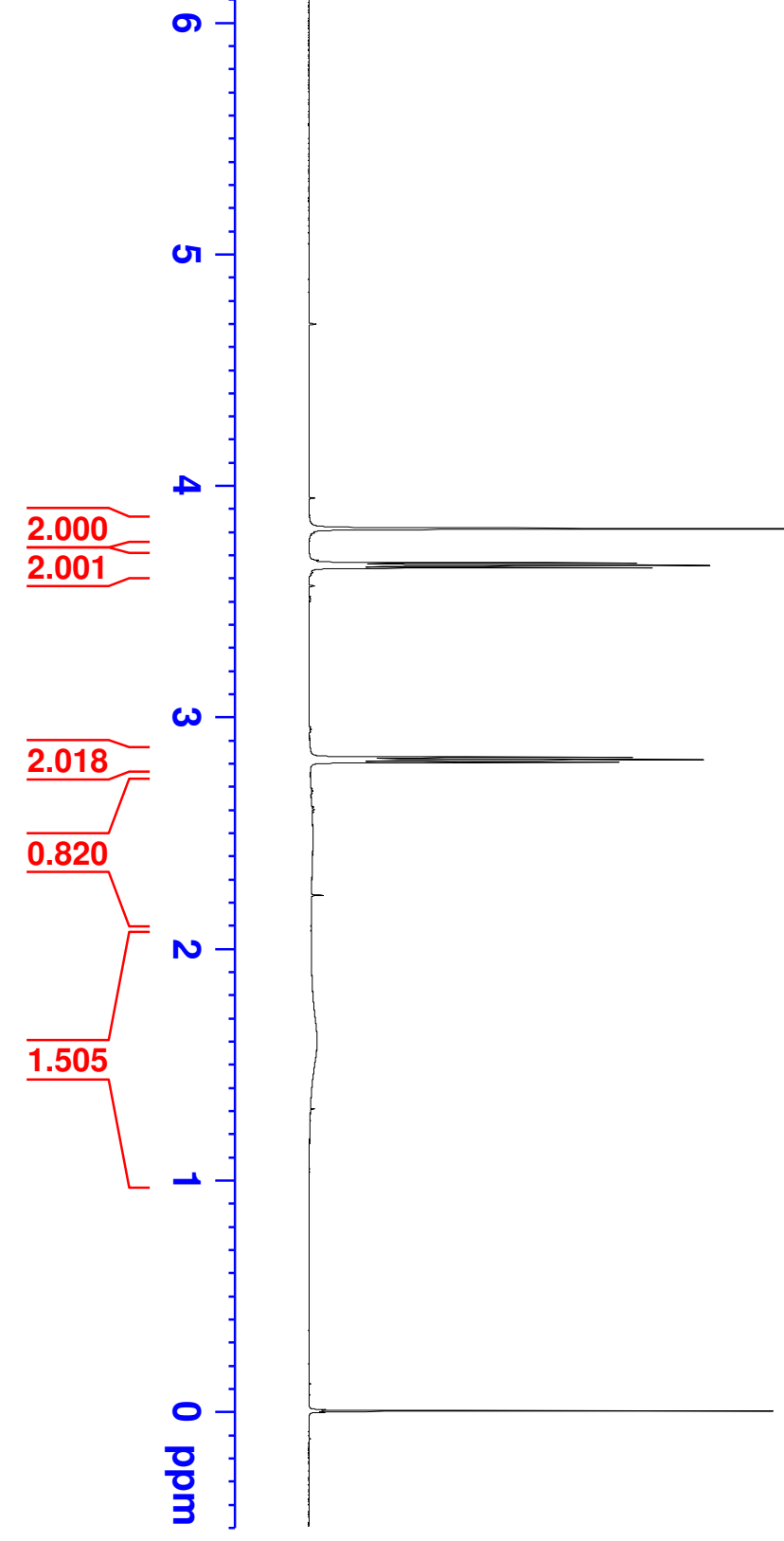

${ }^{7.347}$

7.332

7.319

7.306
-7.273
-7.269

7.269

7.262

7.250

$-7.244$

$-3.811$

3.663

3.653

3.642

2.824

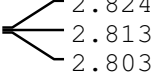

2.803

2.472

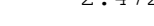

$-1.592$

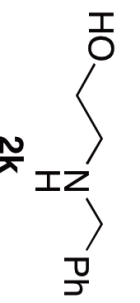




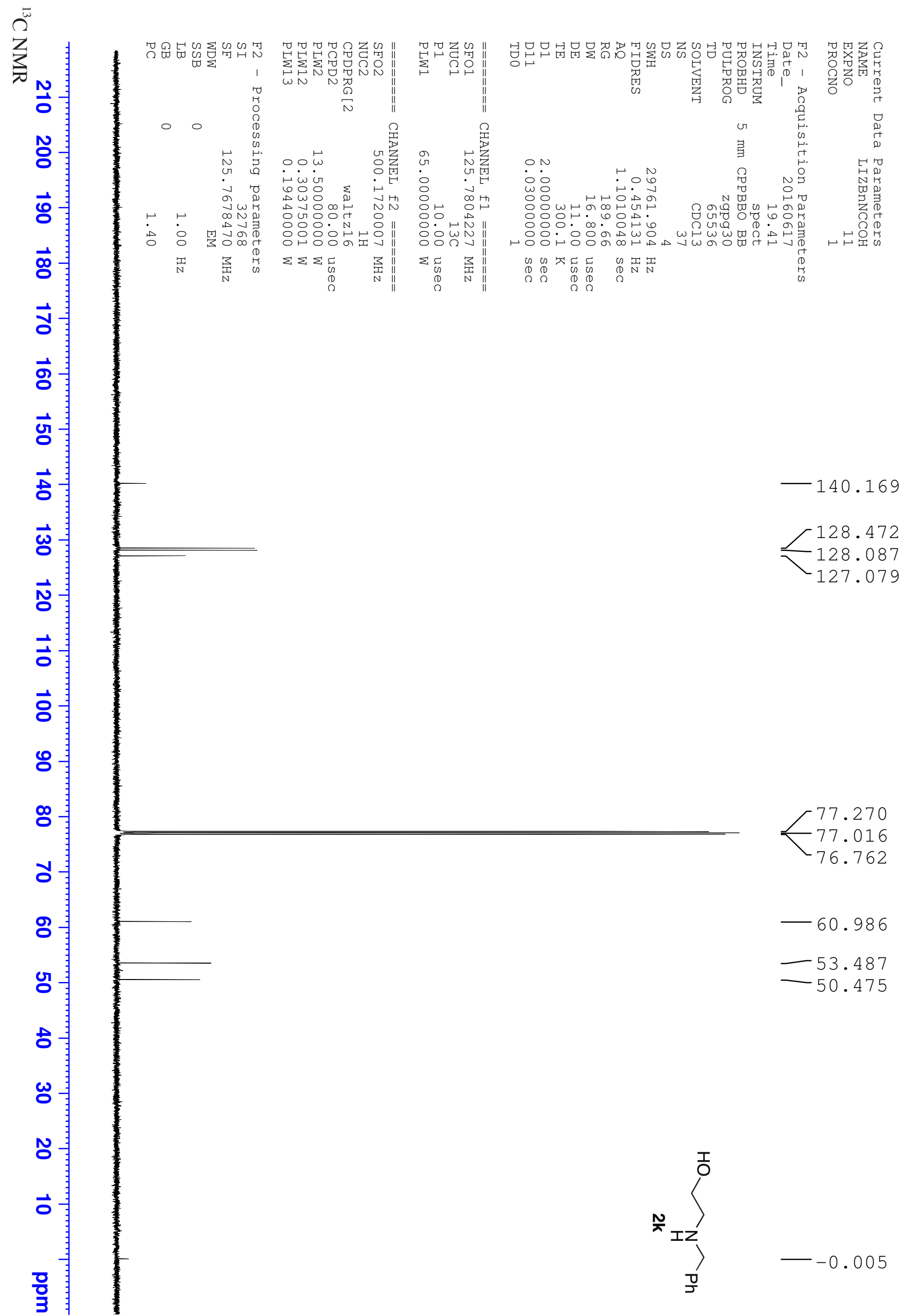


望

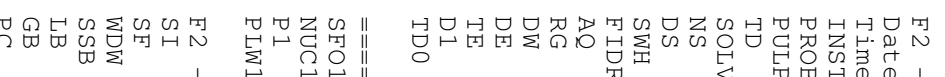

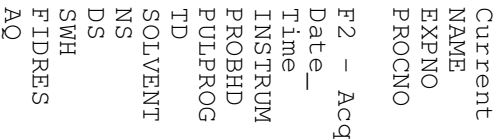

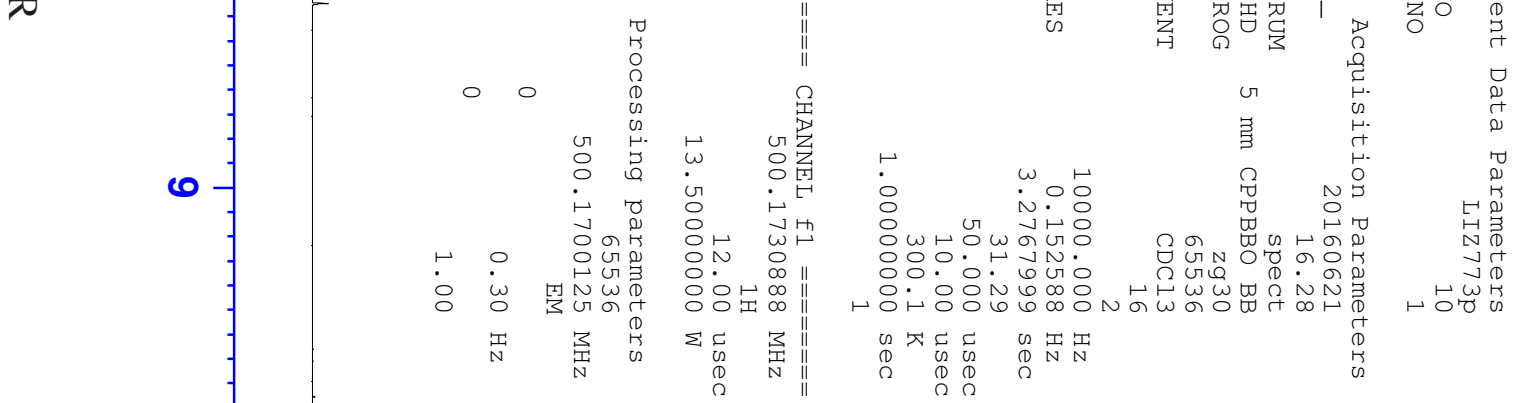

0.923

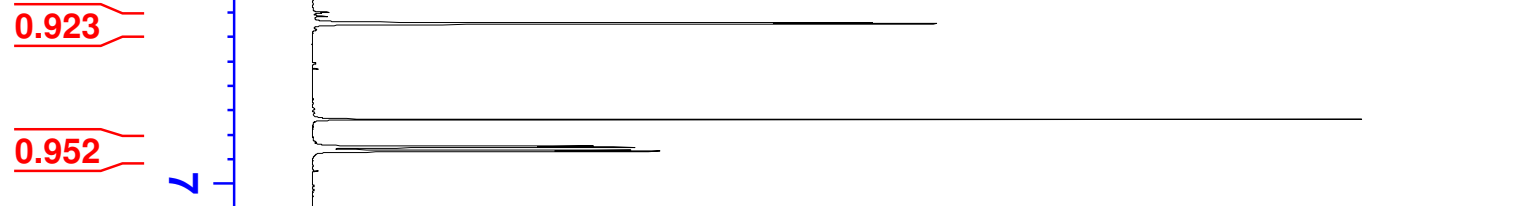

$\underline{0.999}$

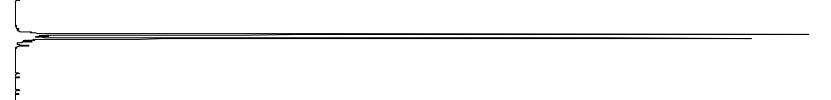

$<7.65$

7.149

7.133

7.129

6.732
-6.716

4.524
$<.512$

$-4.101$

2.065

$\triangle$
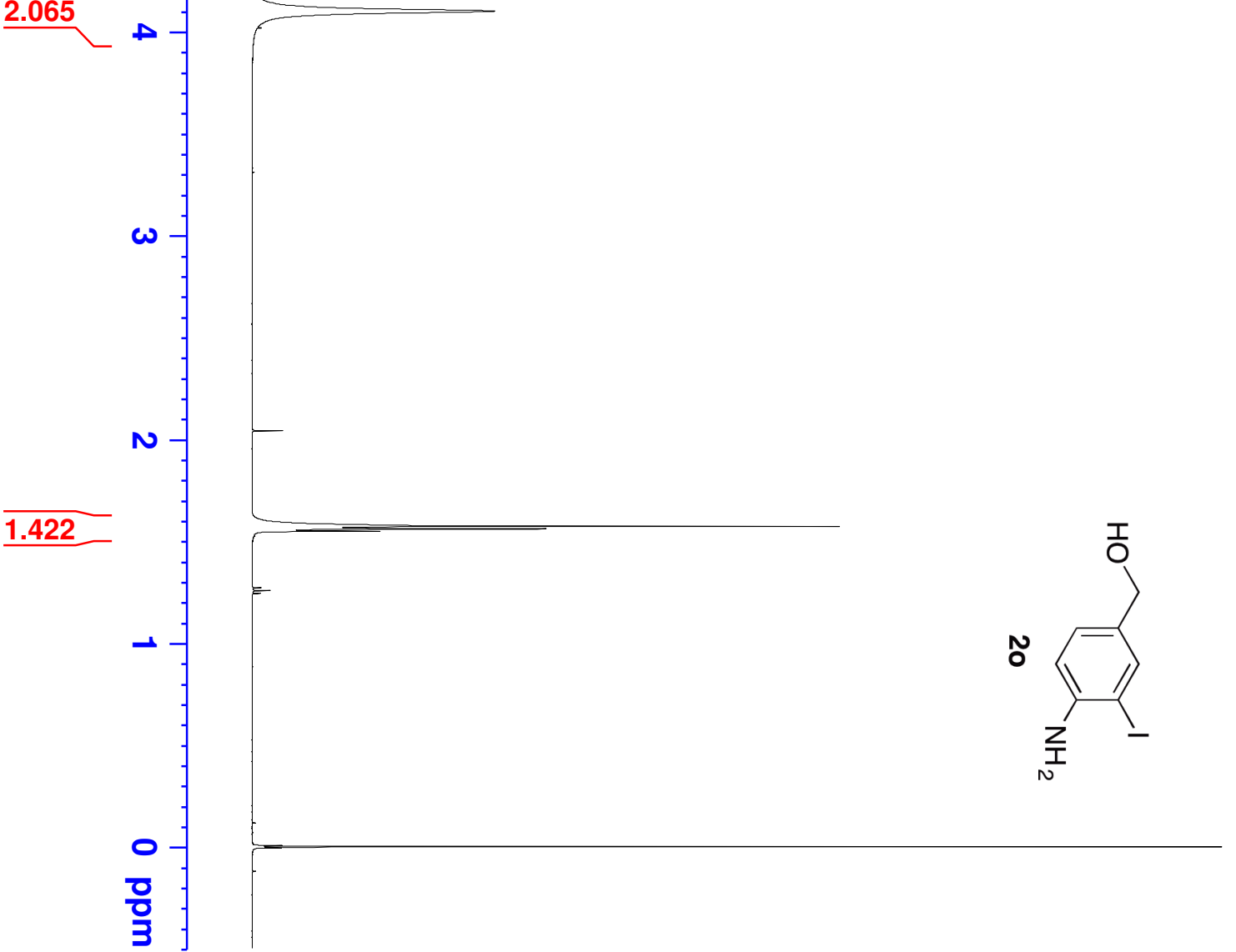

$-1.572$ 


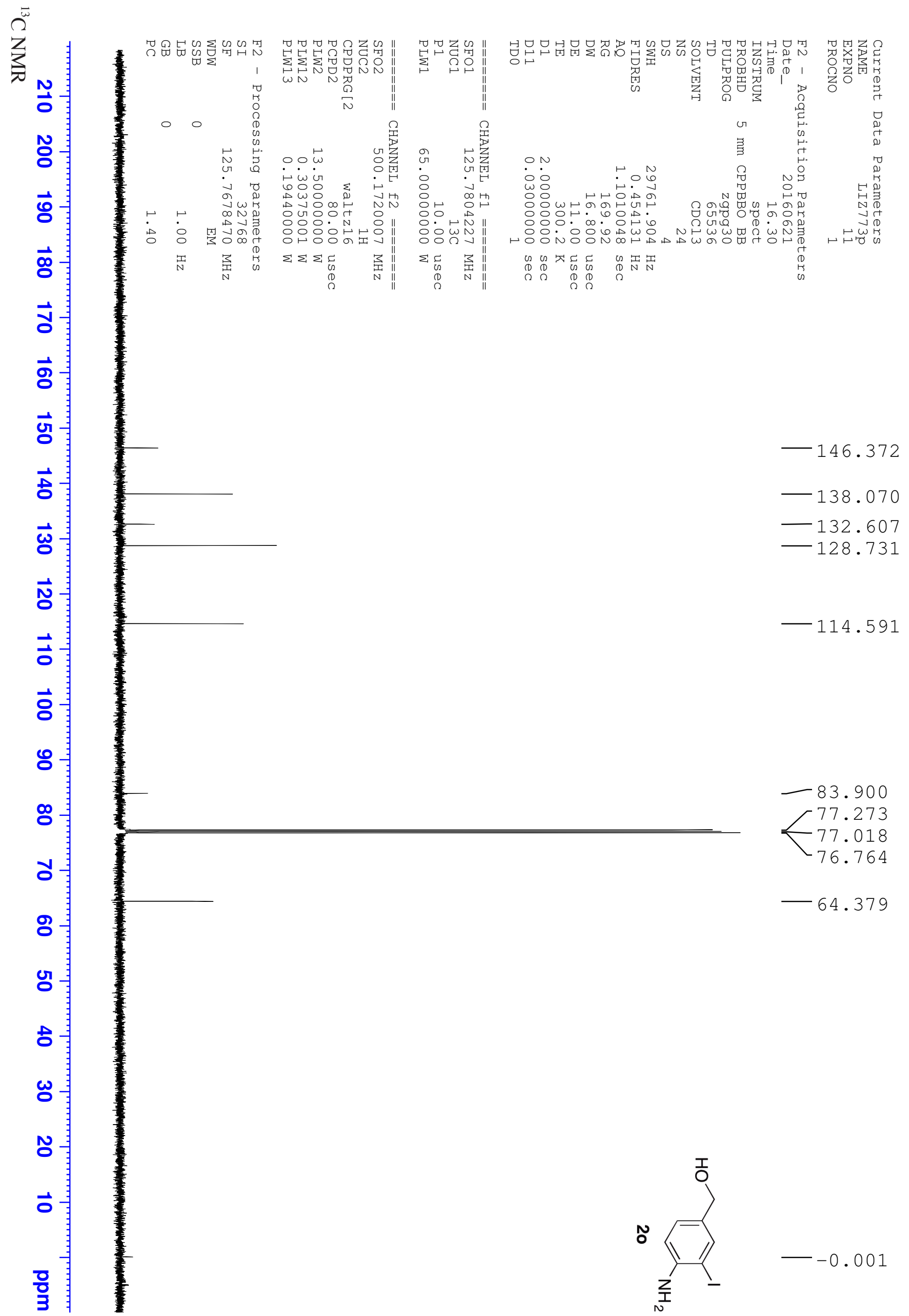




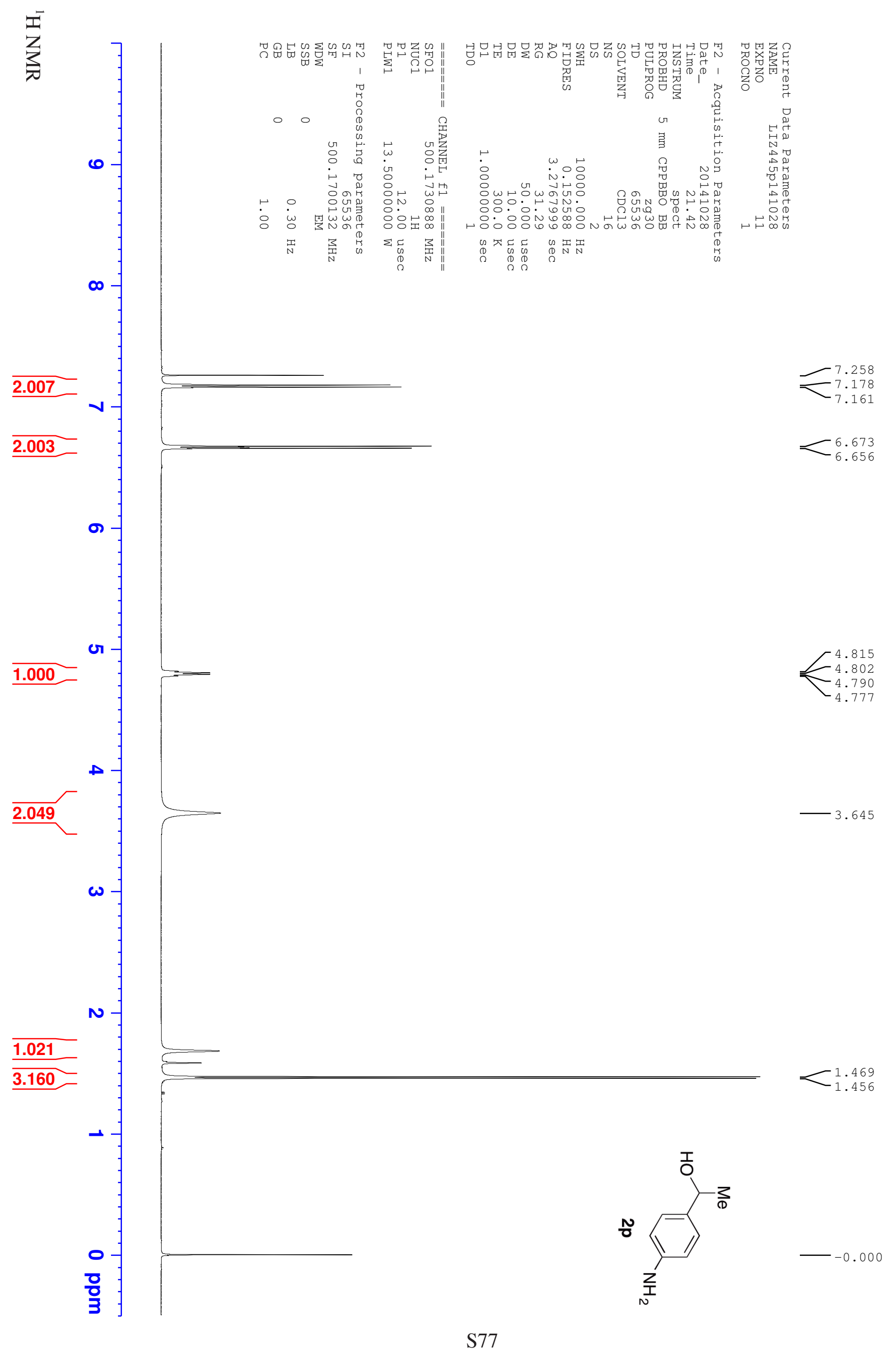




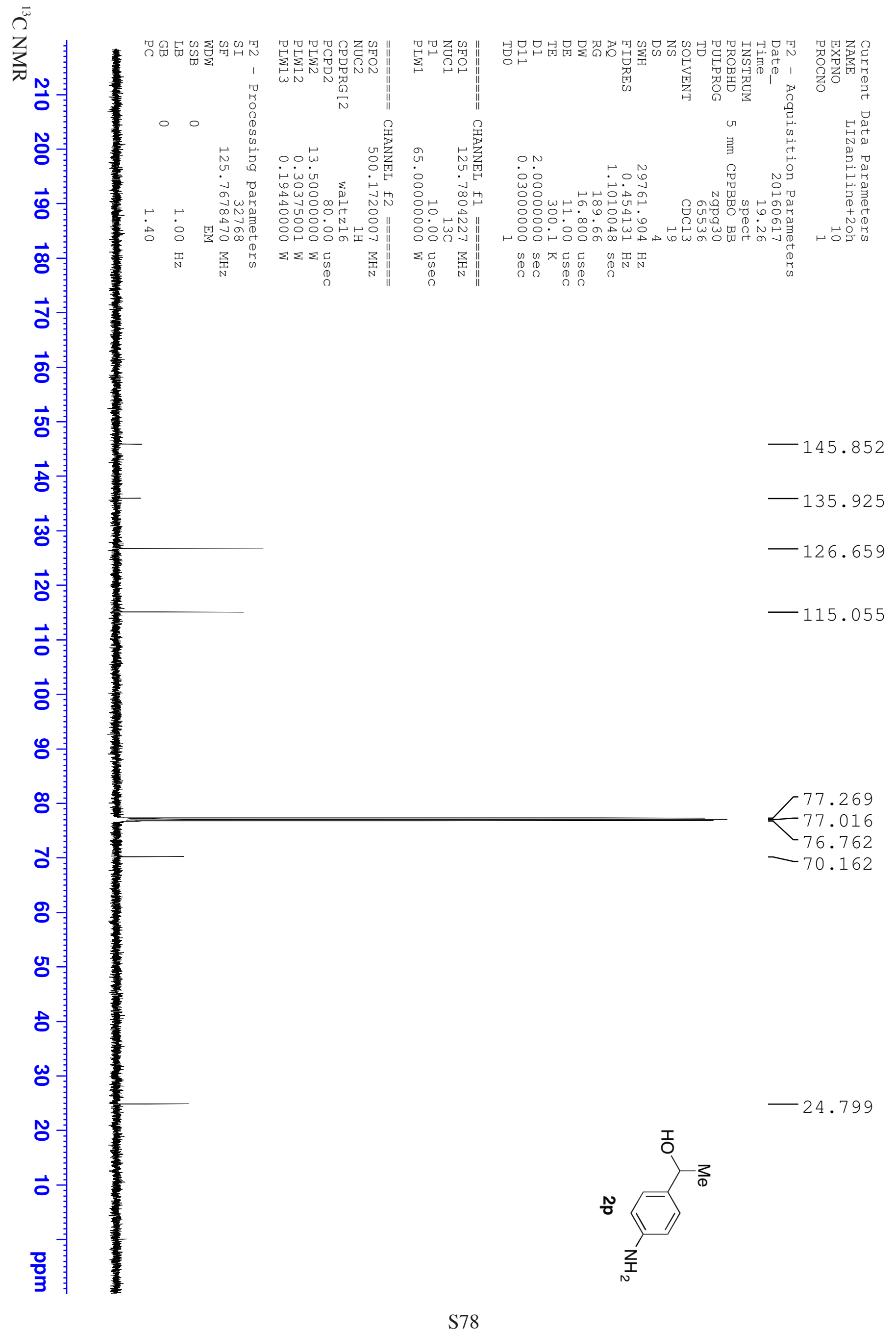




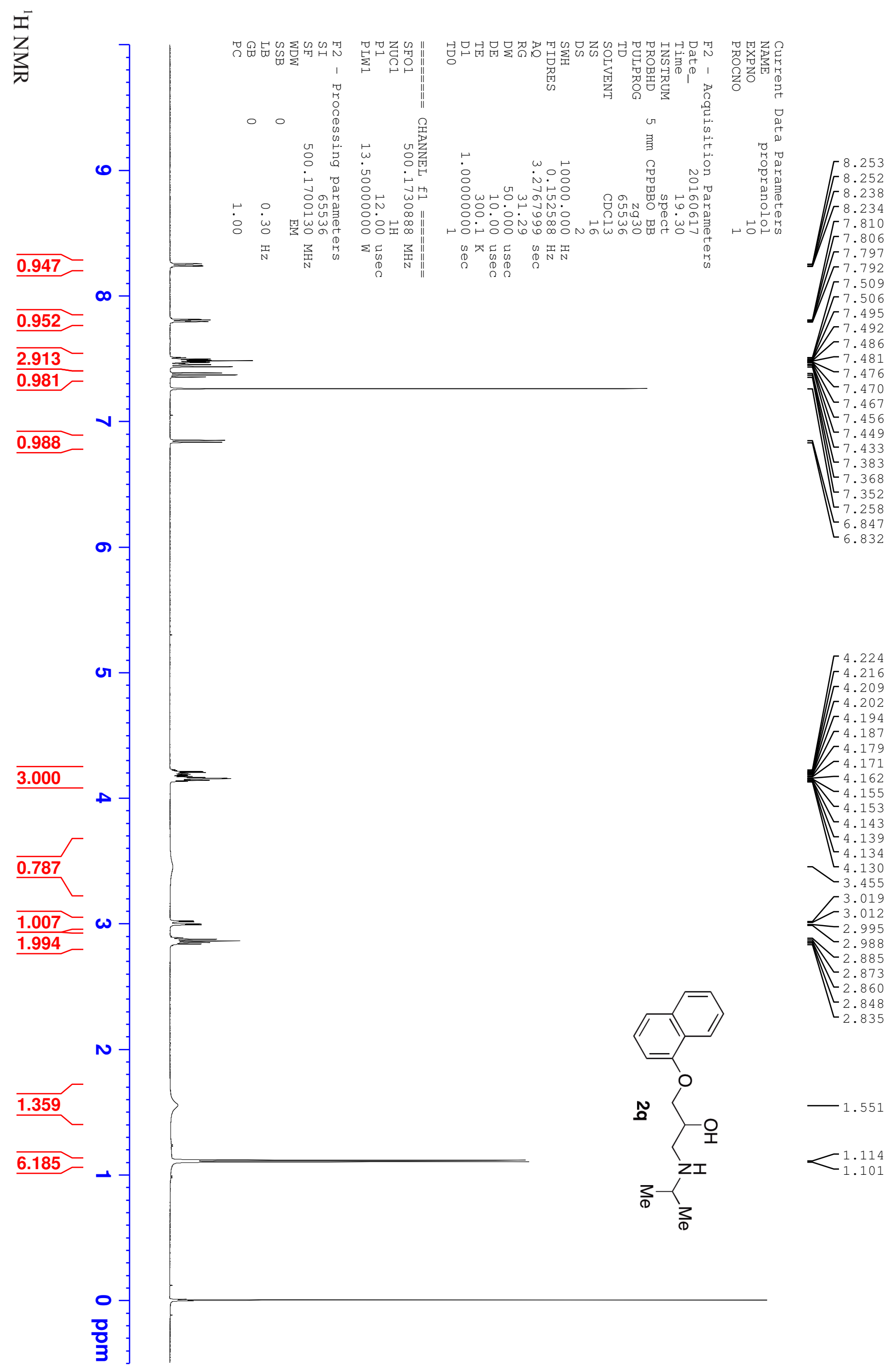




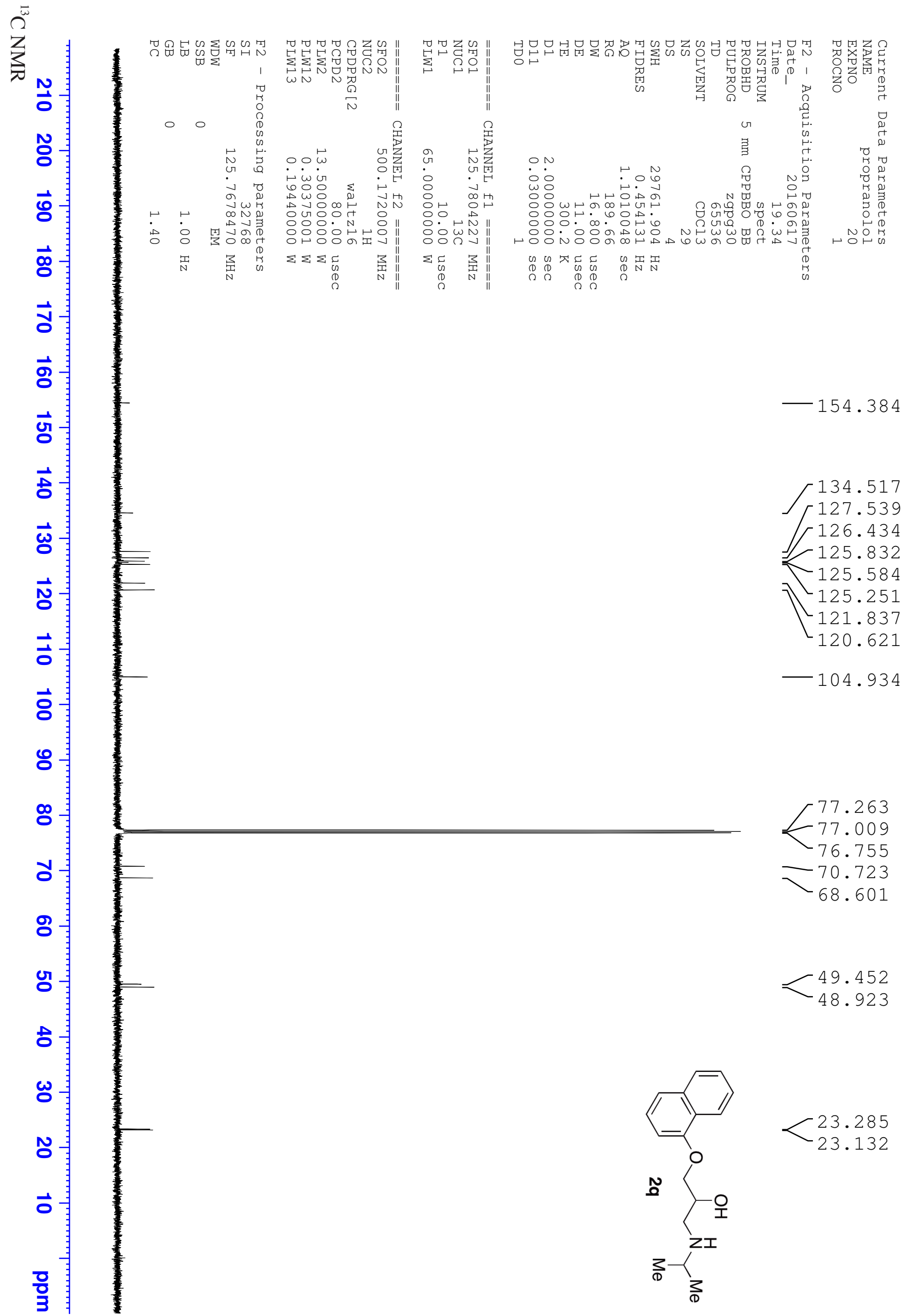




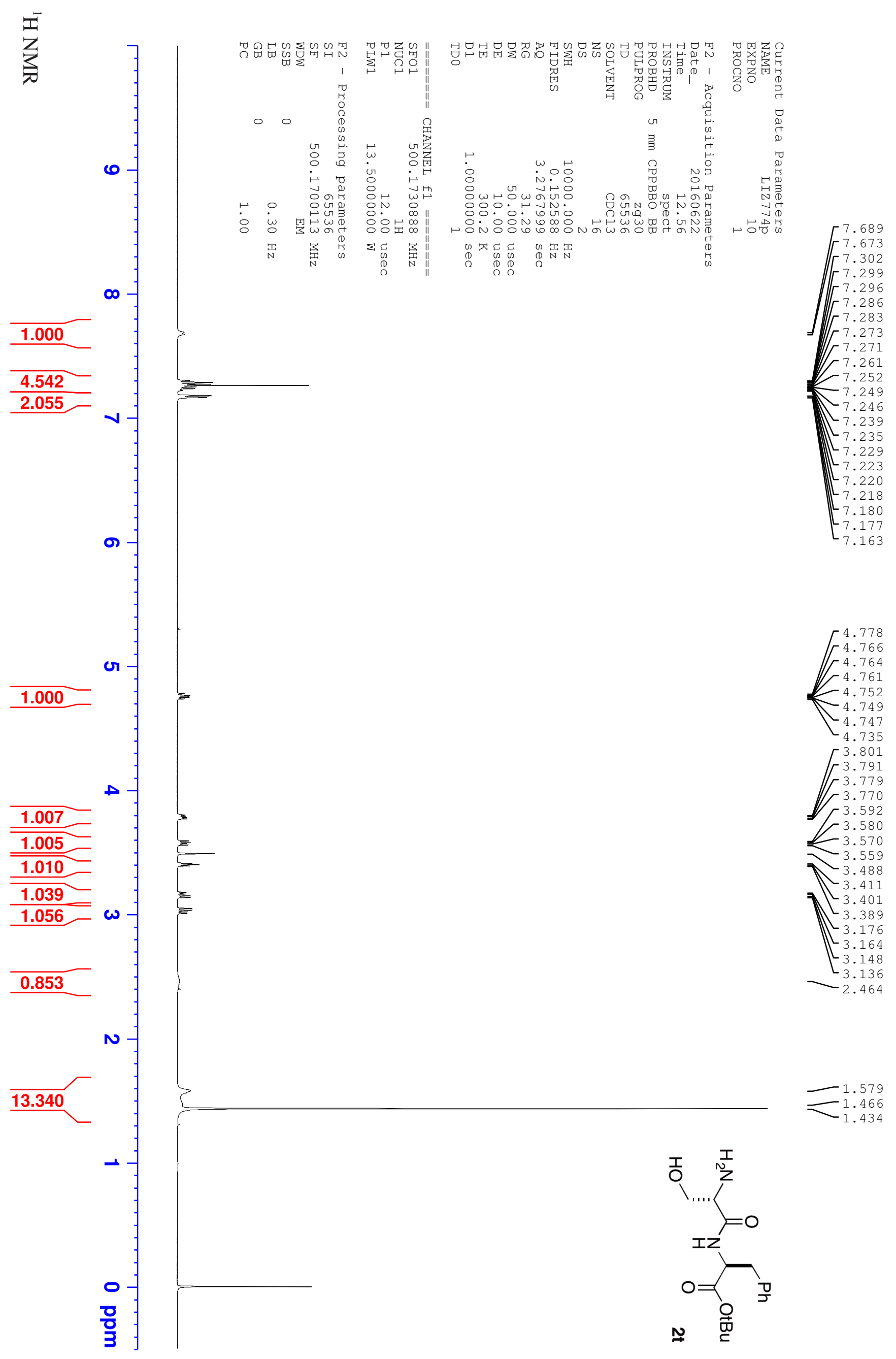




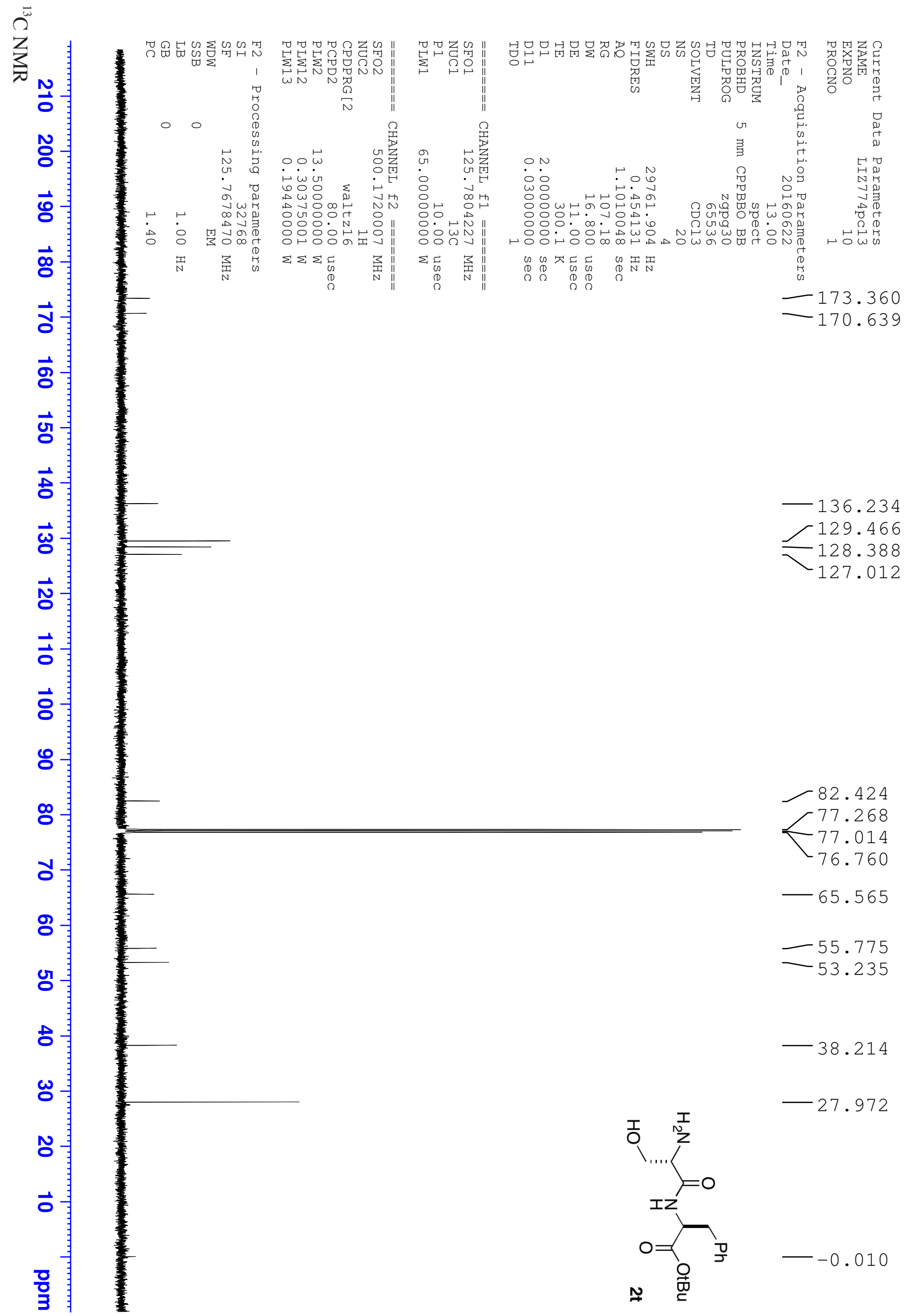




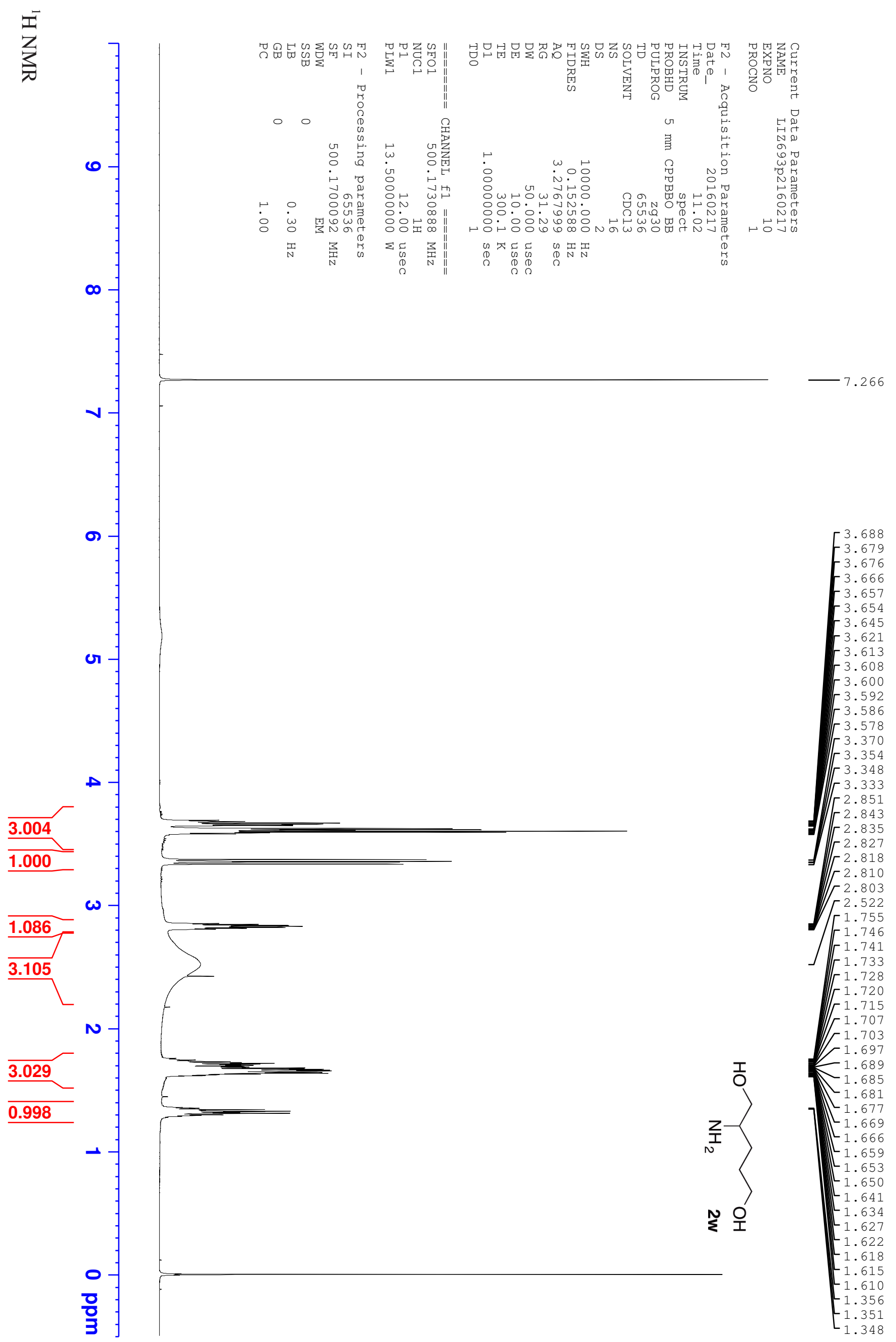




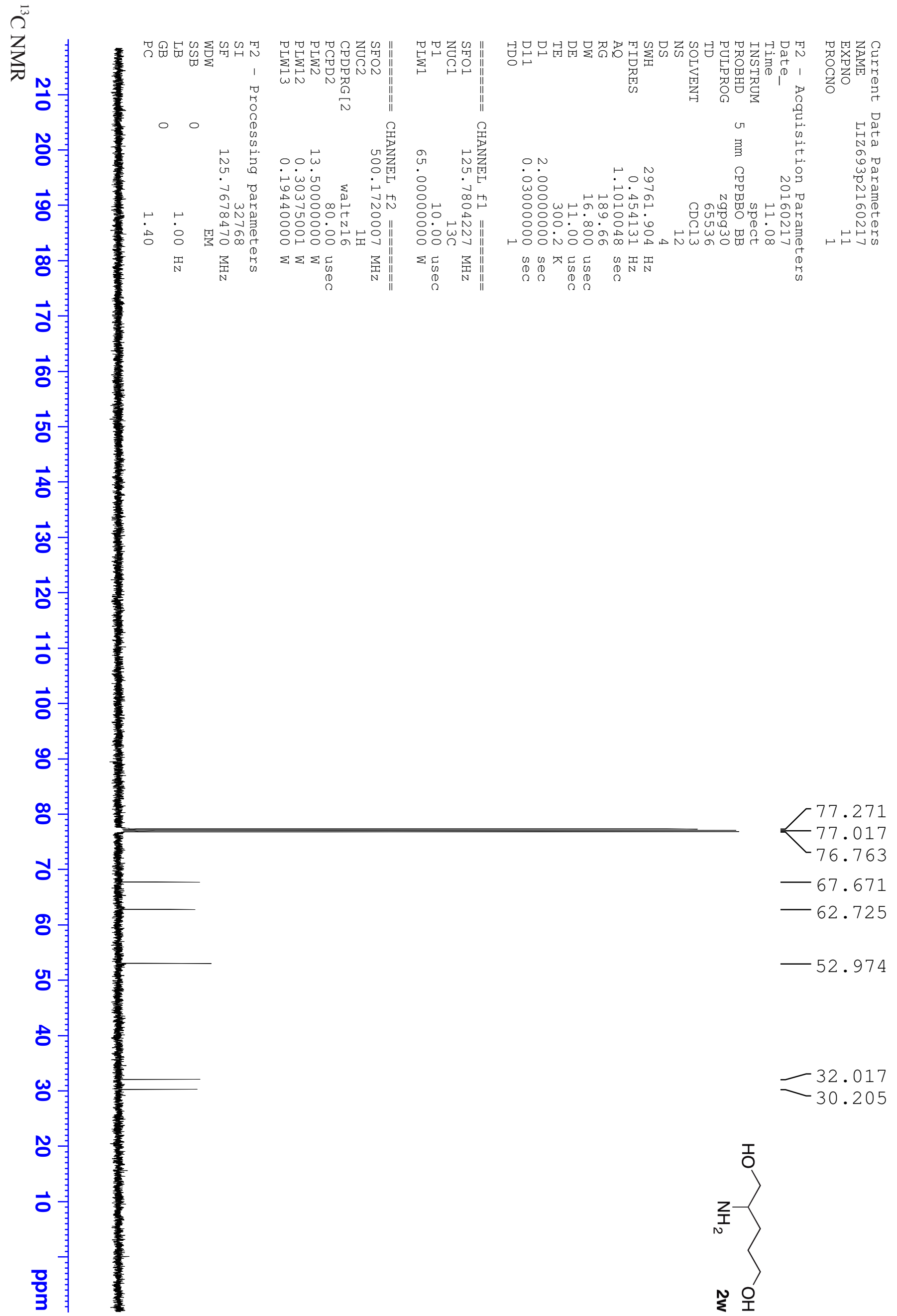




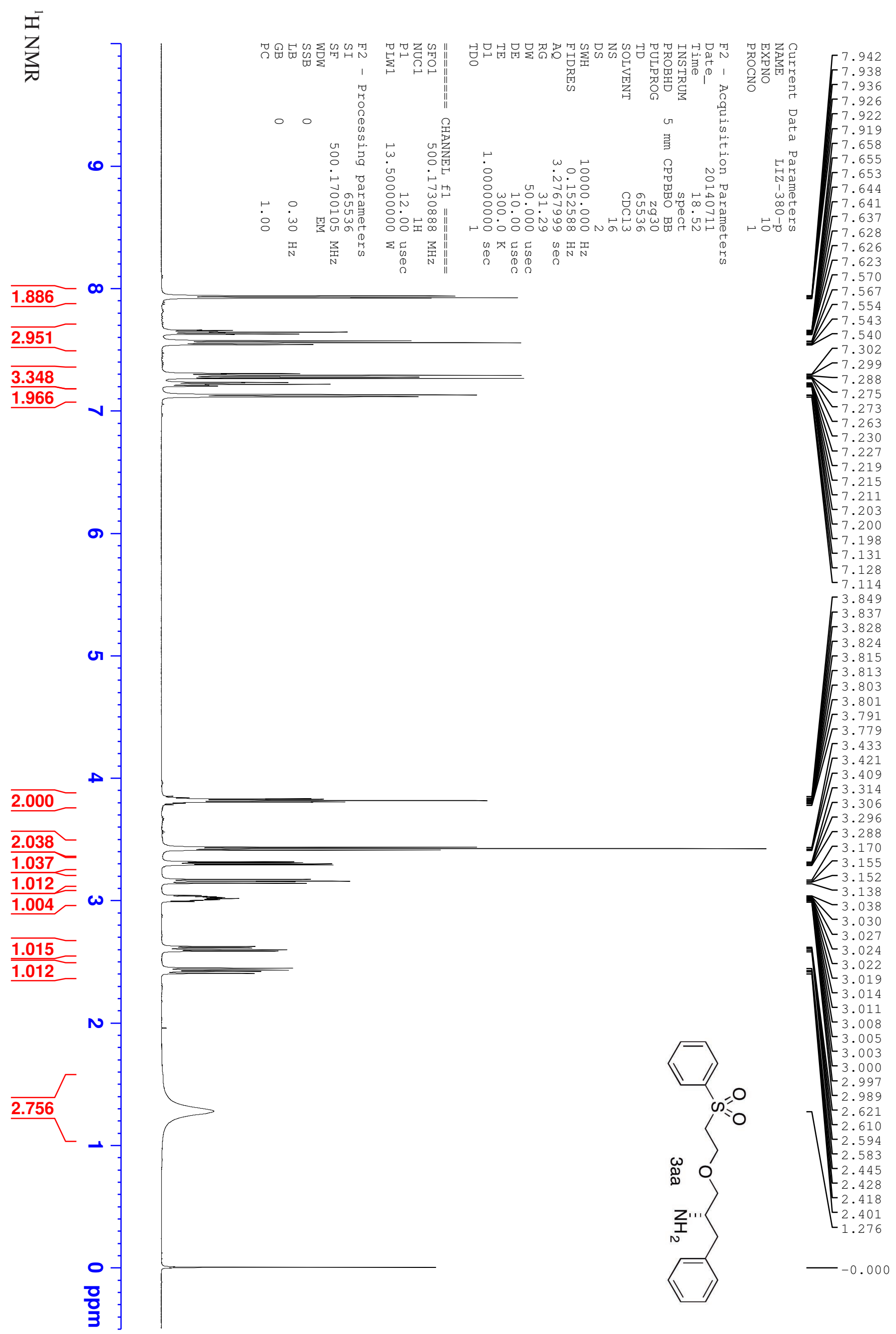




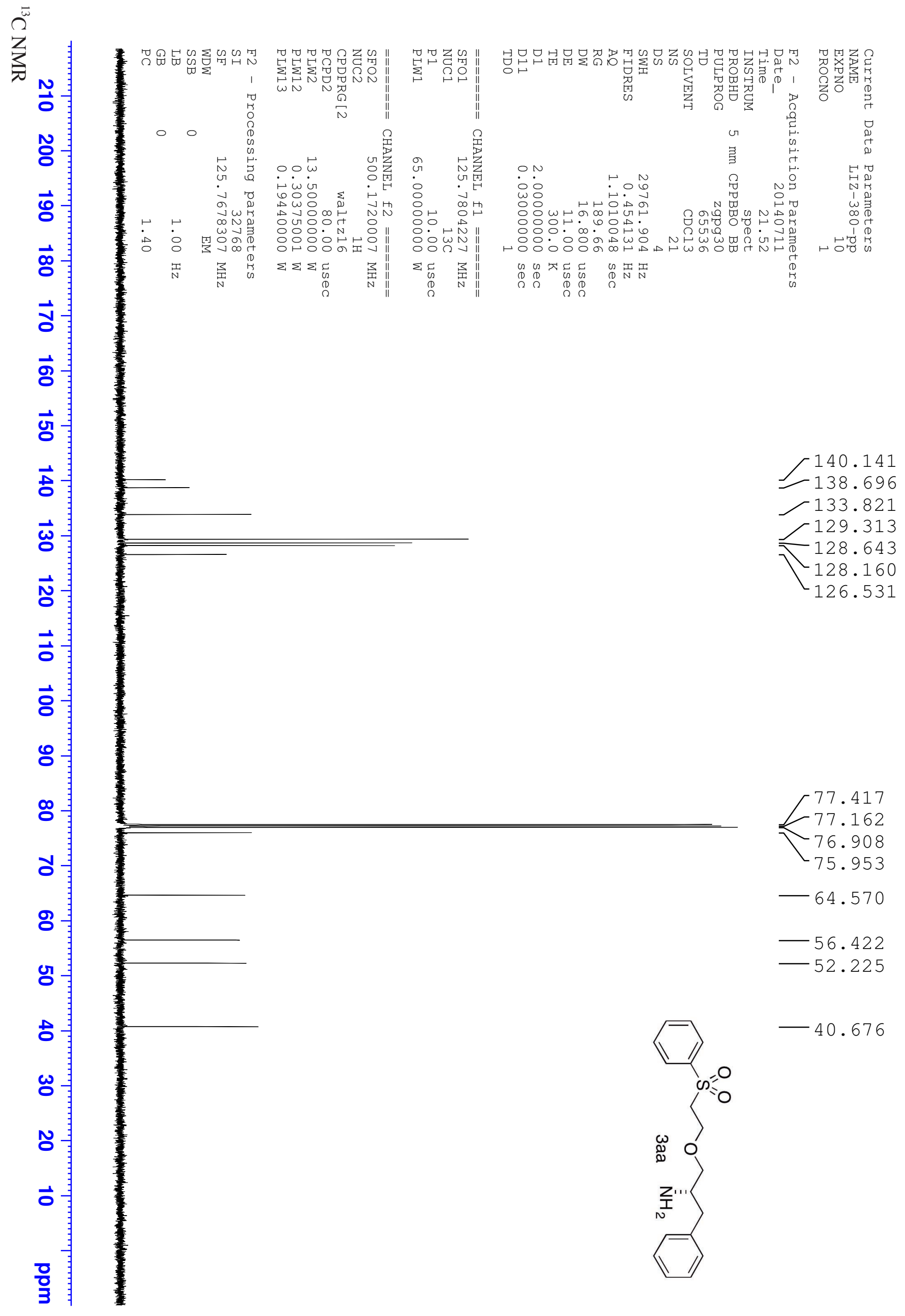




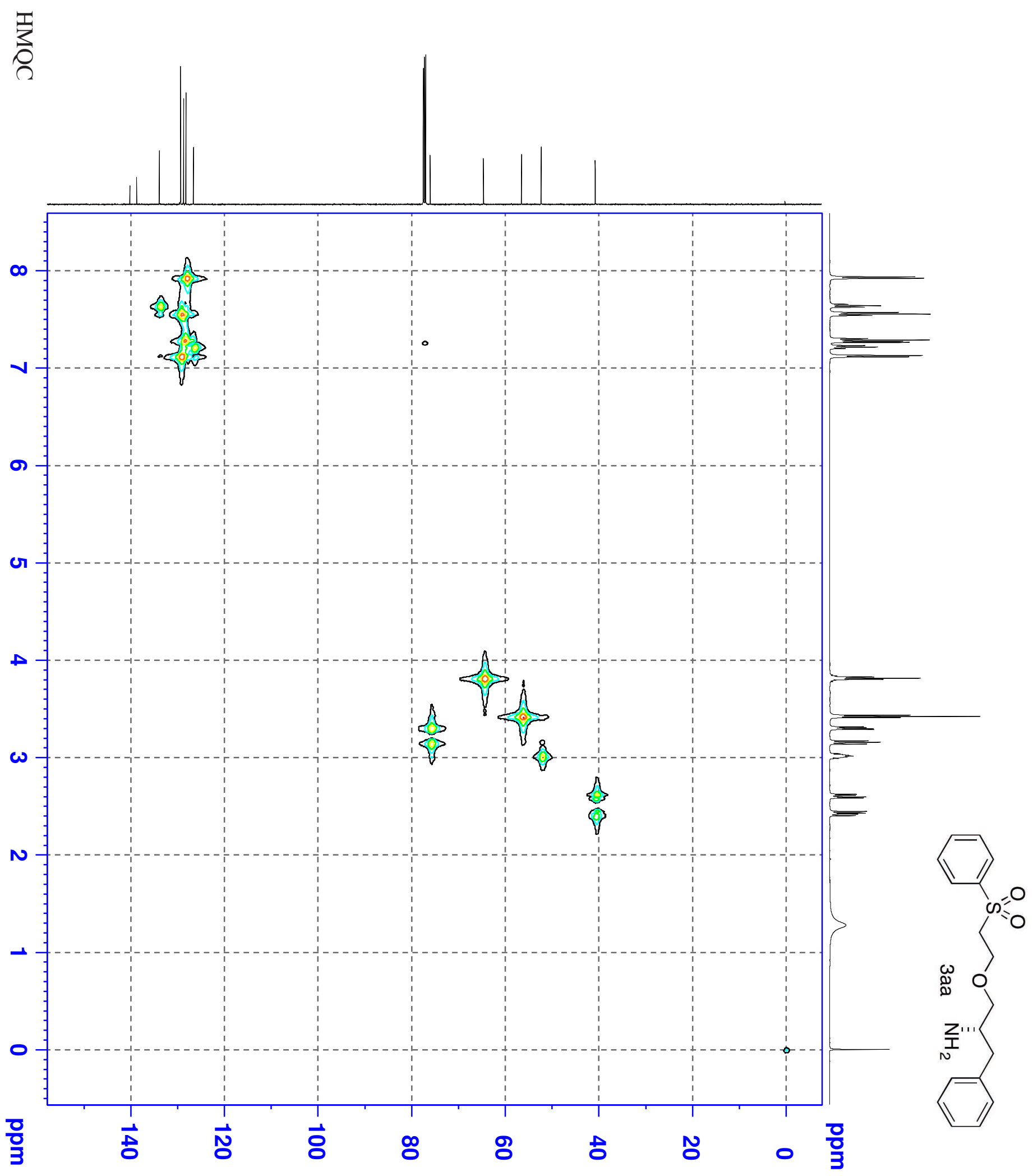

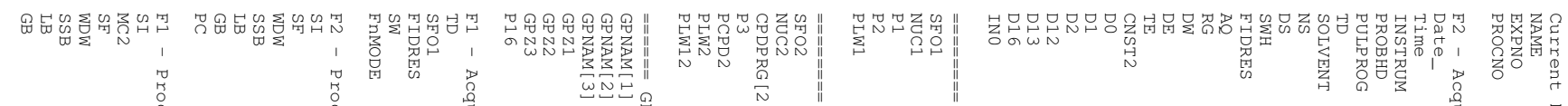

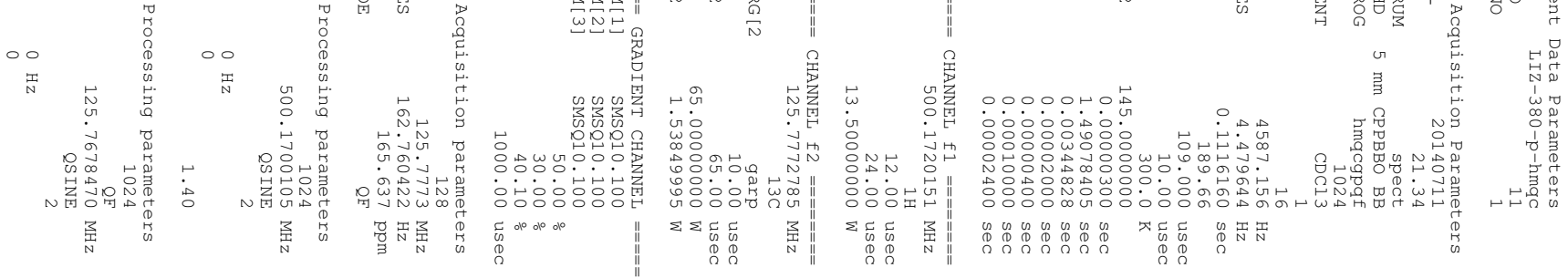




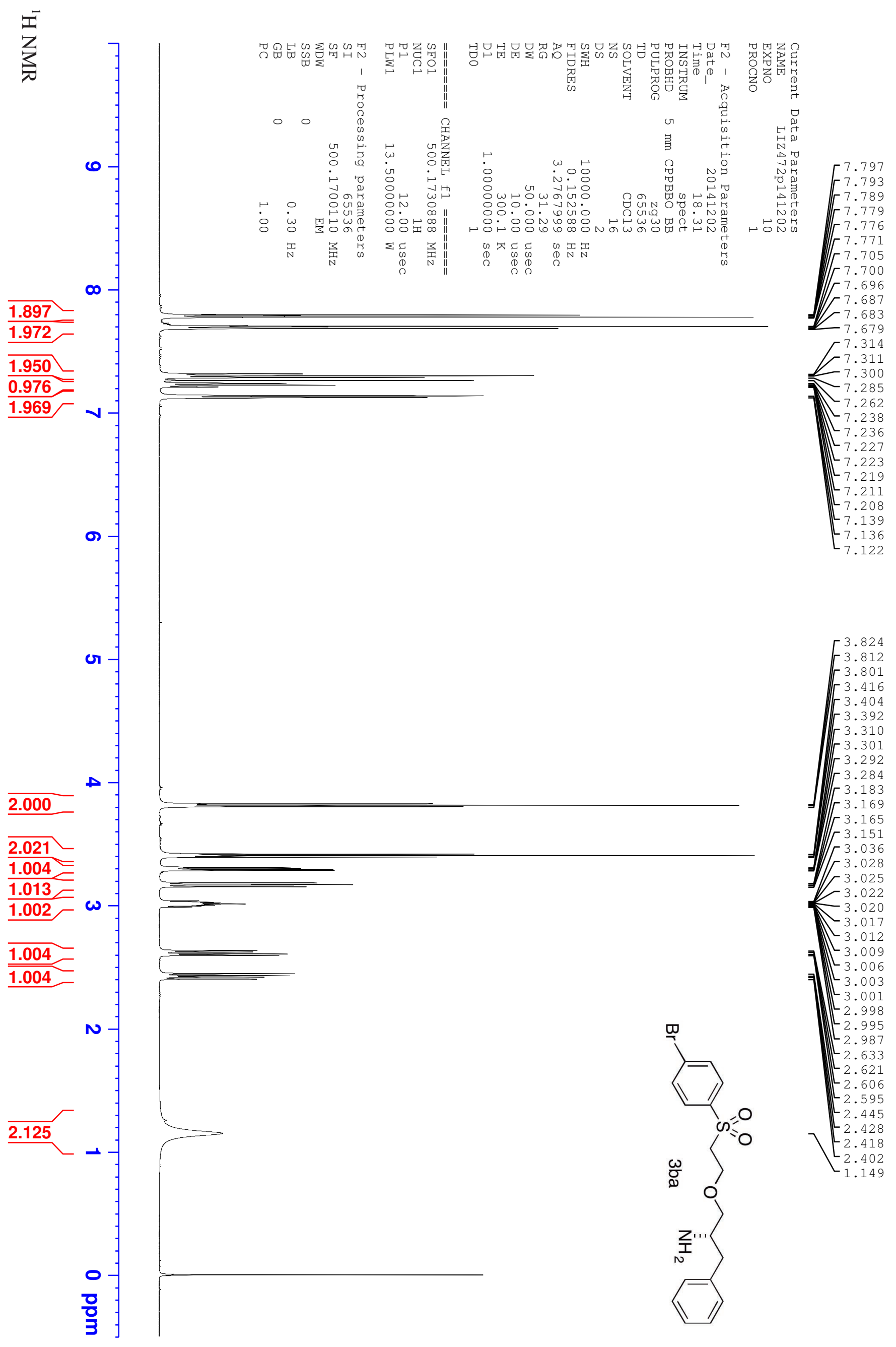




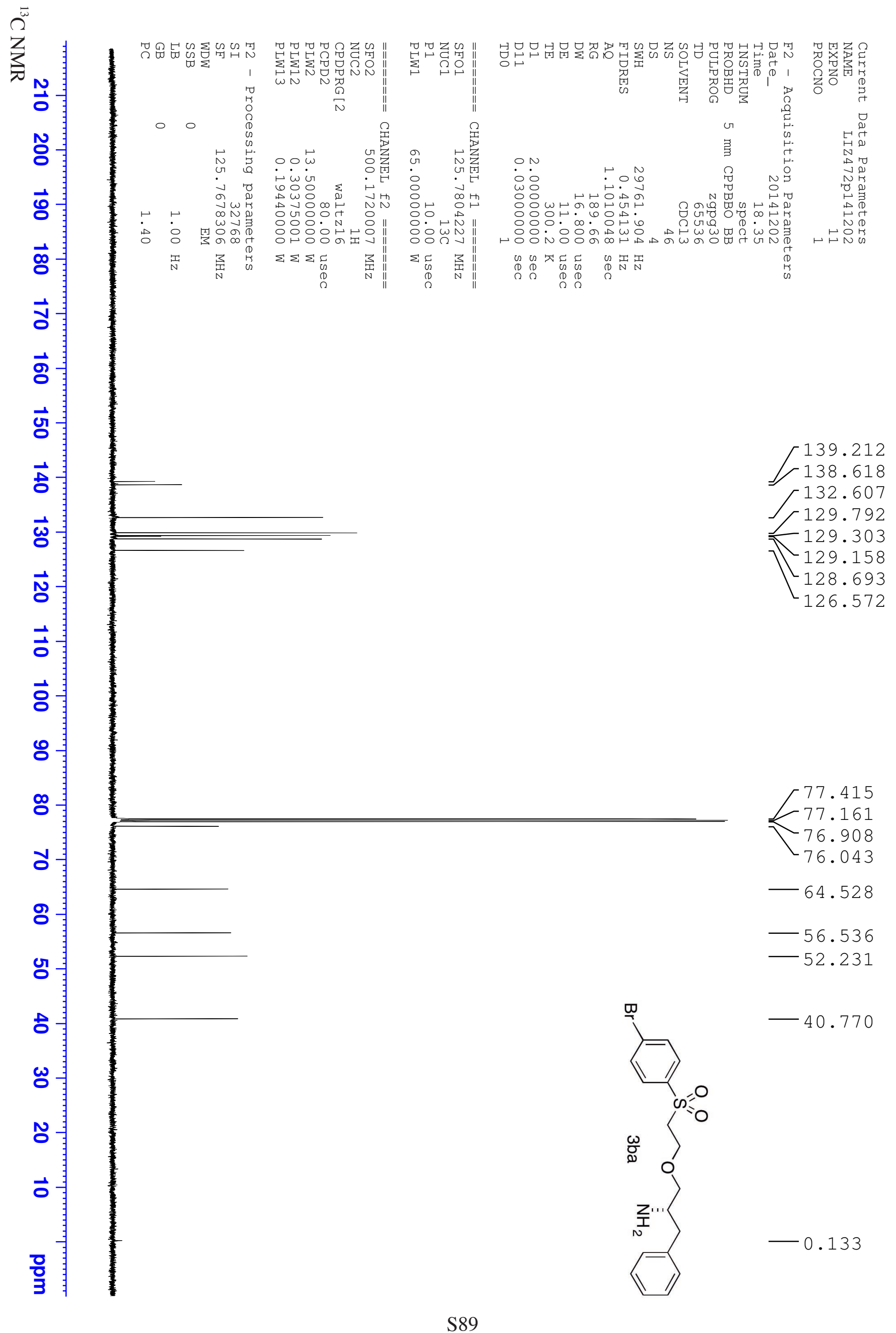




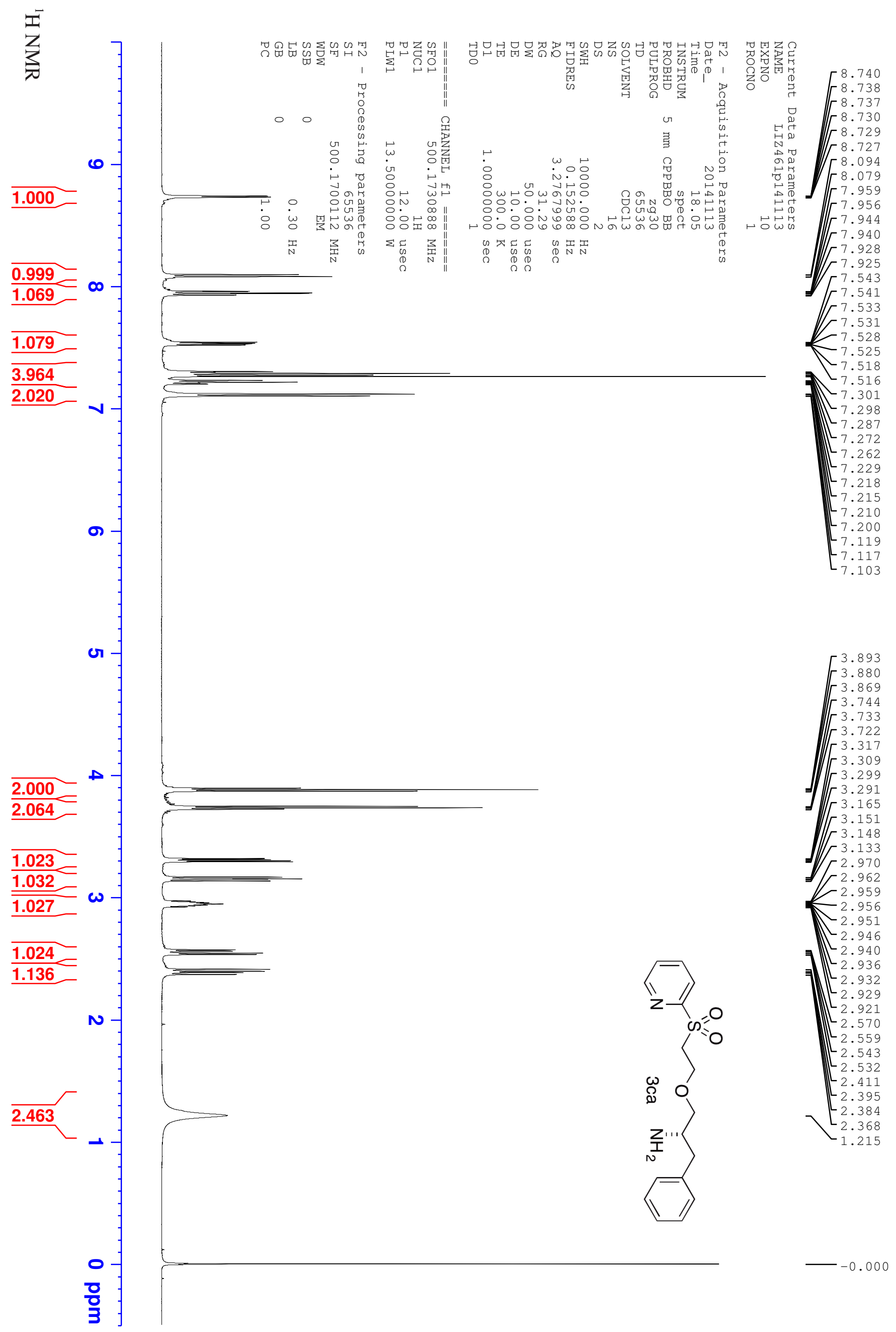




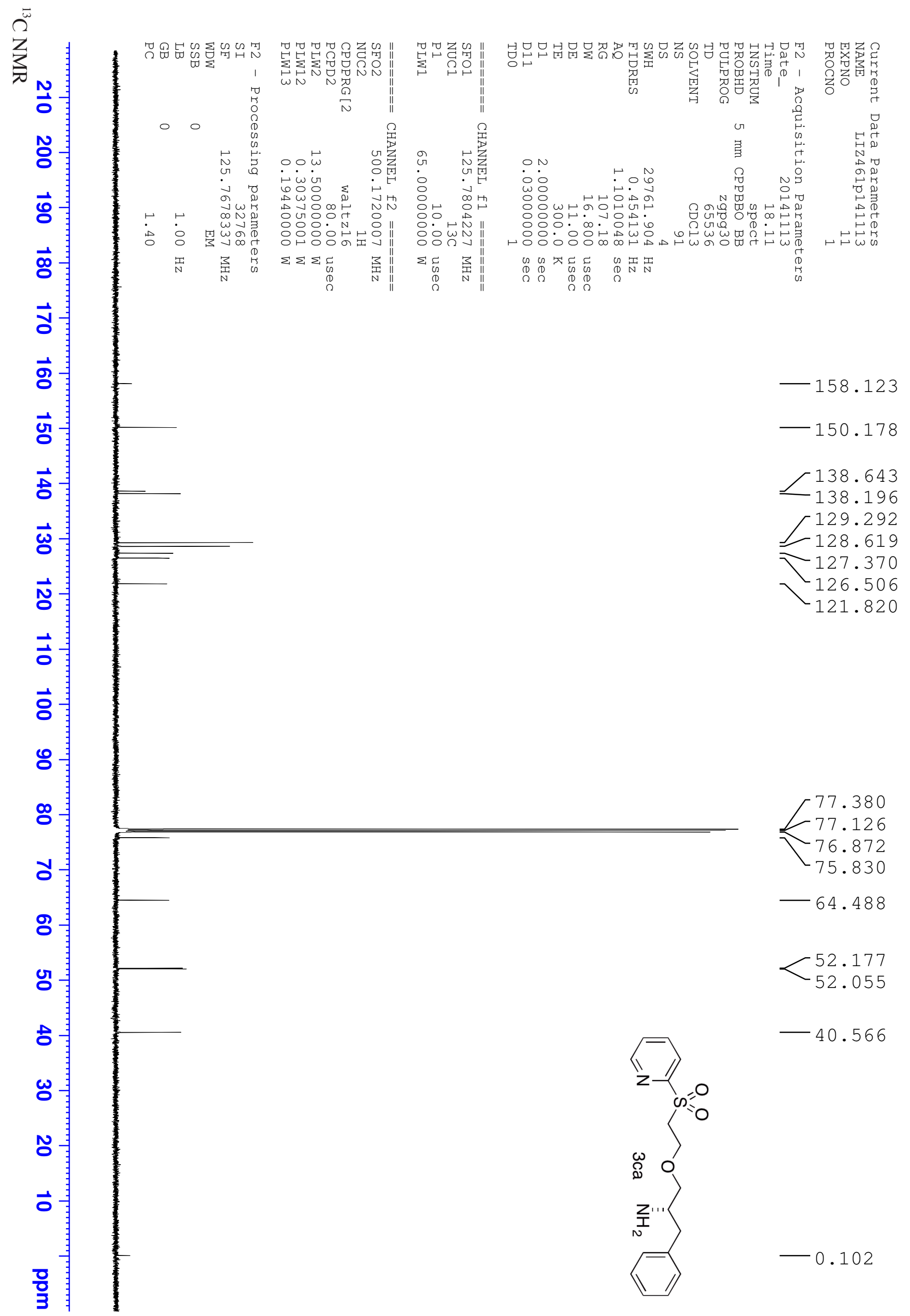




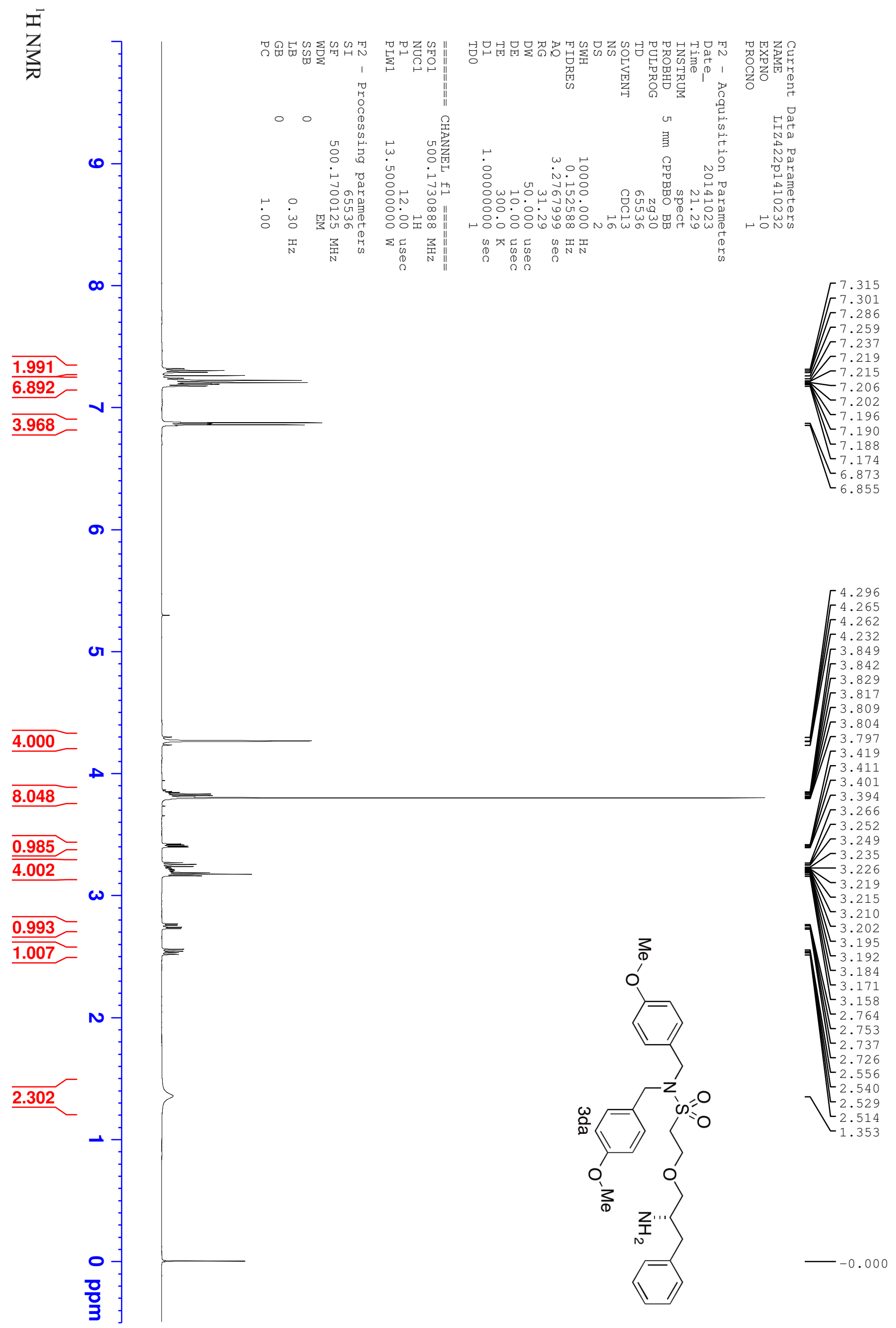




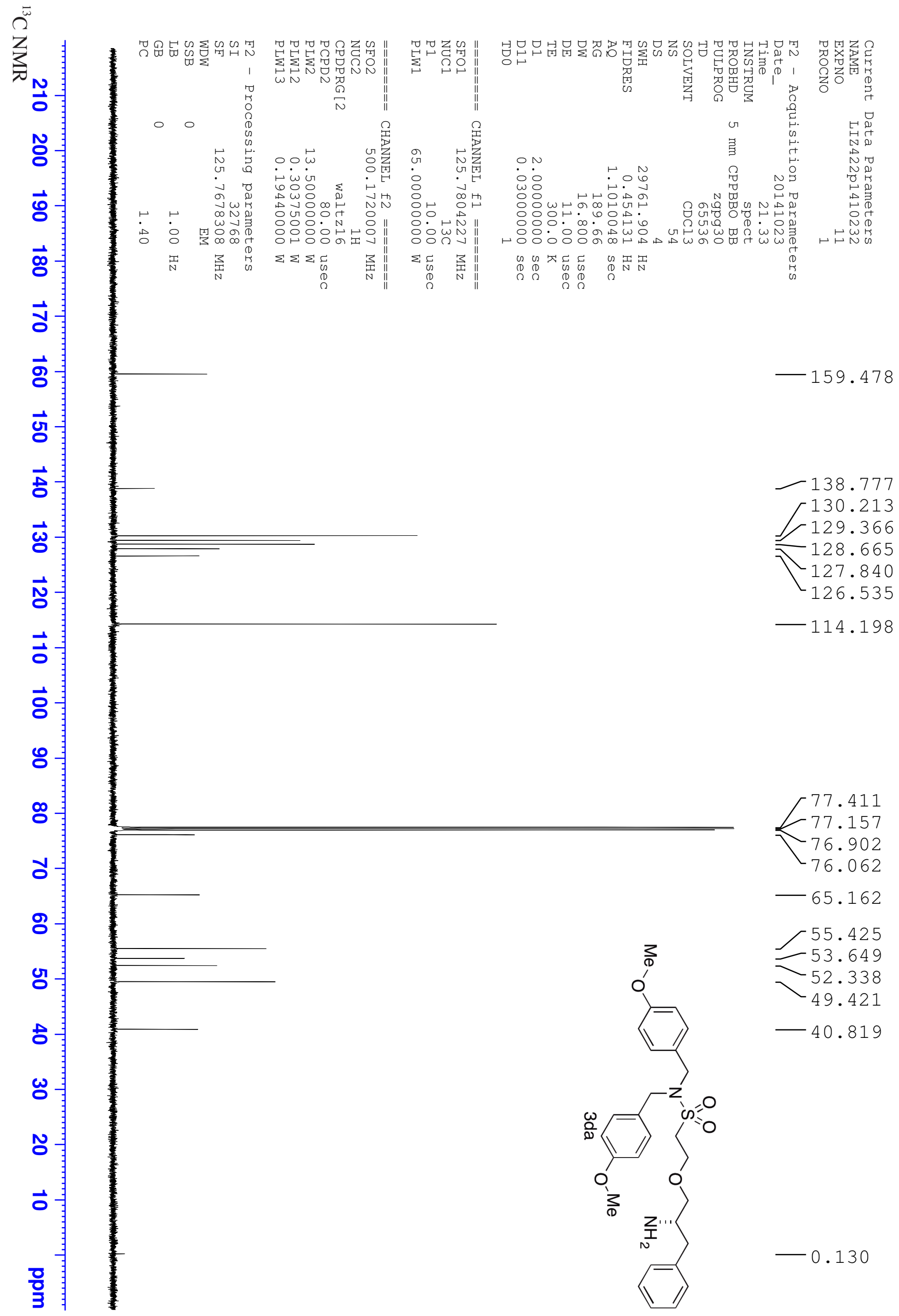




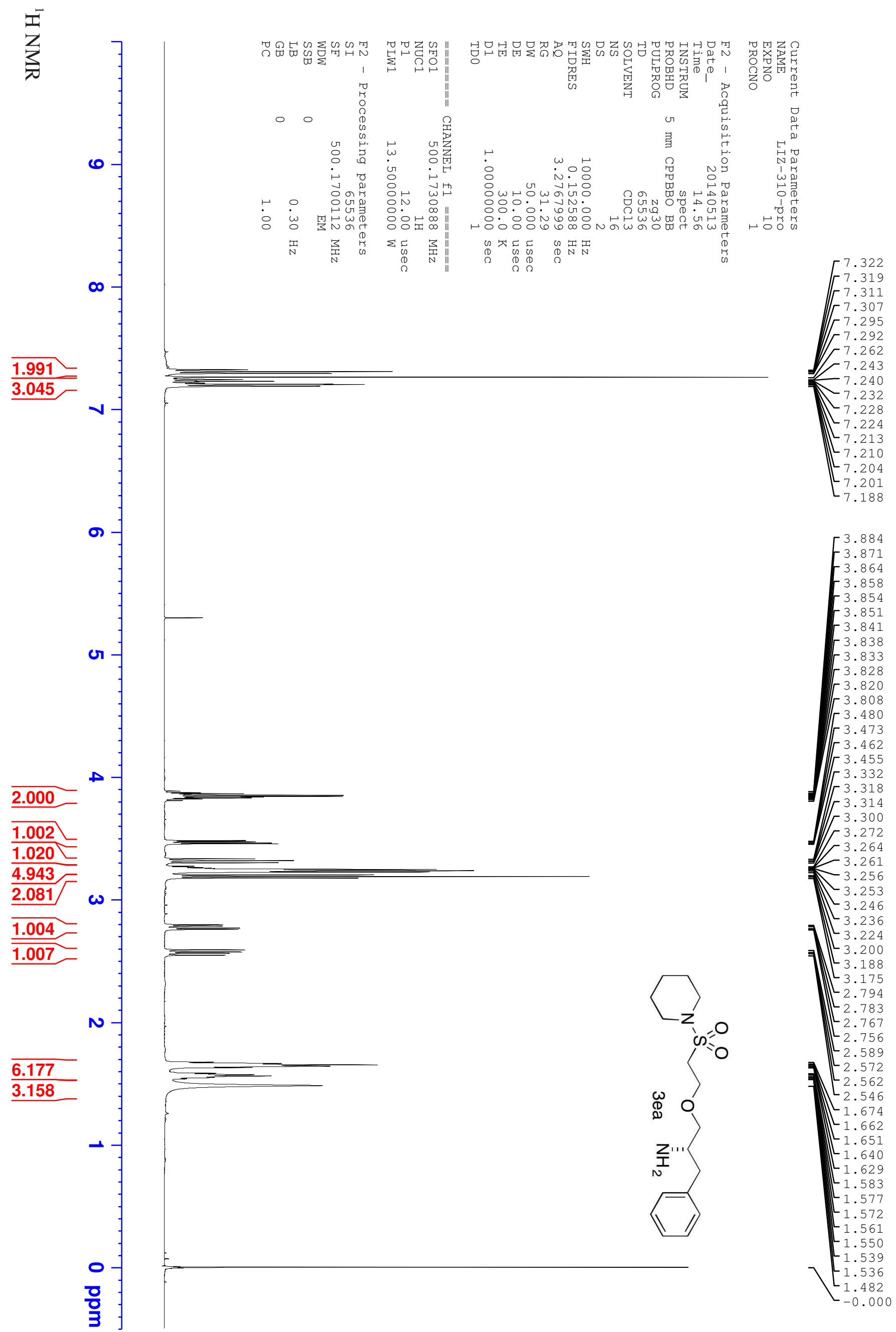




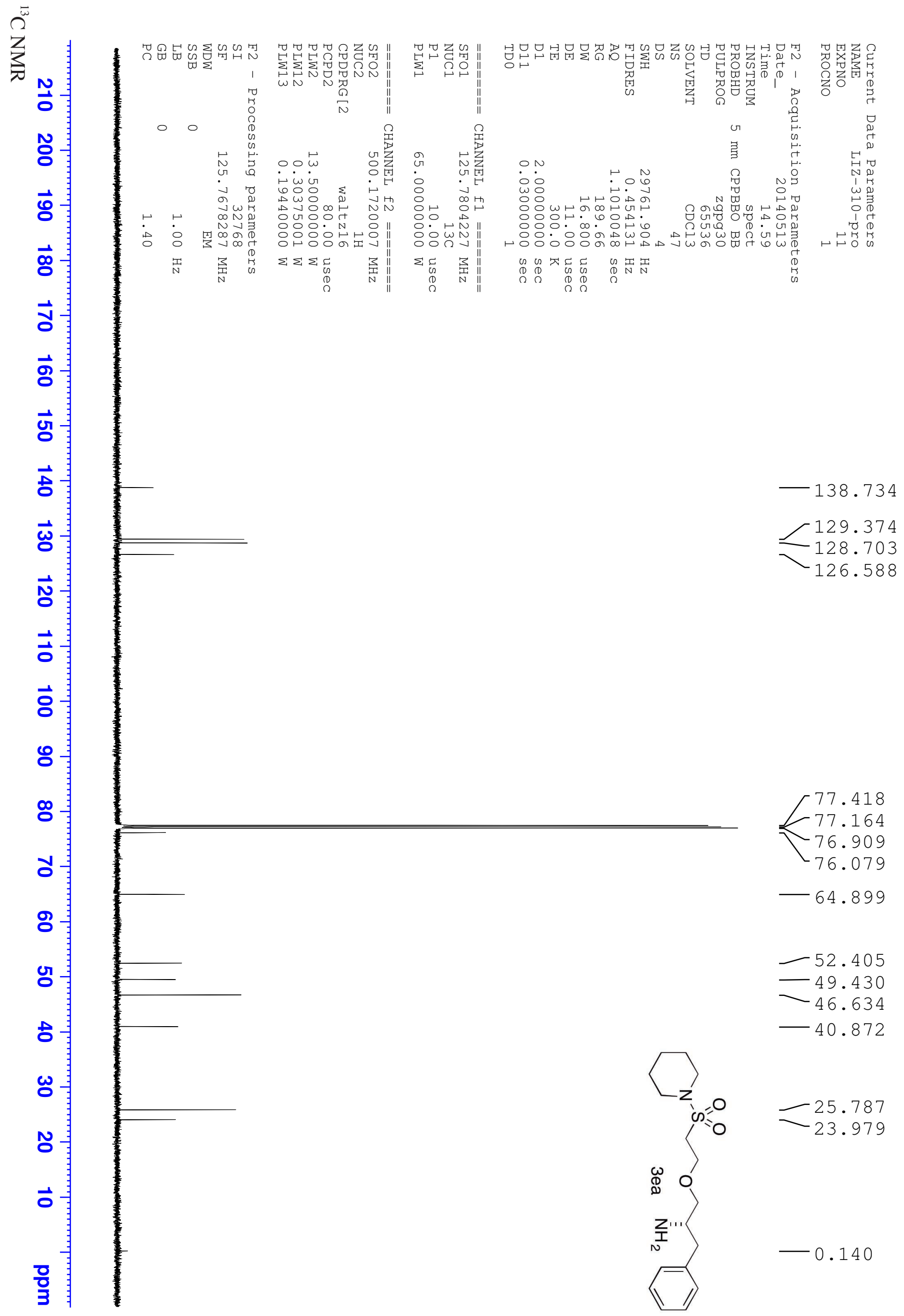




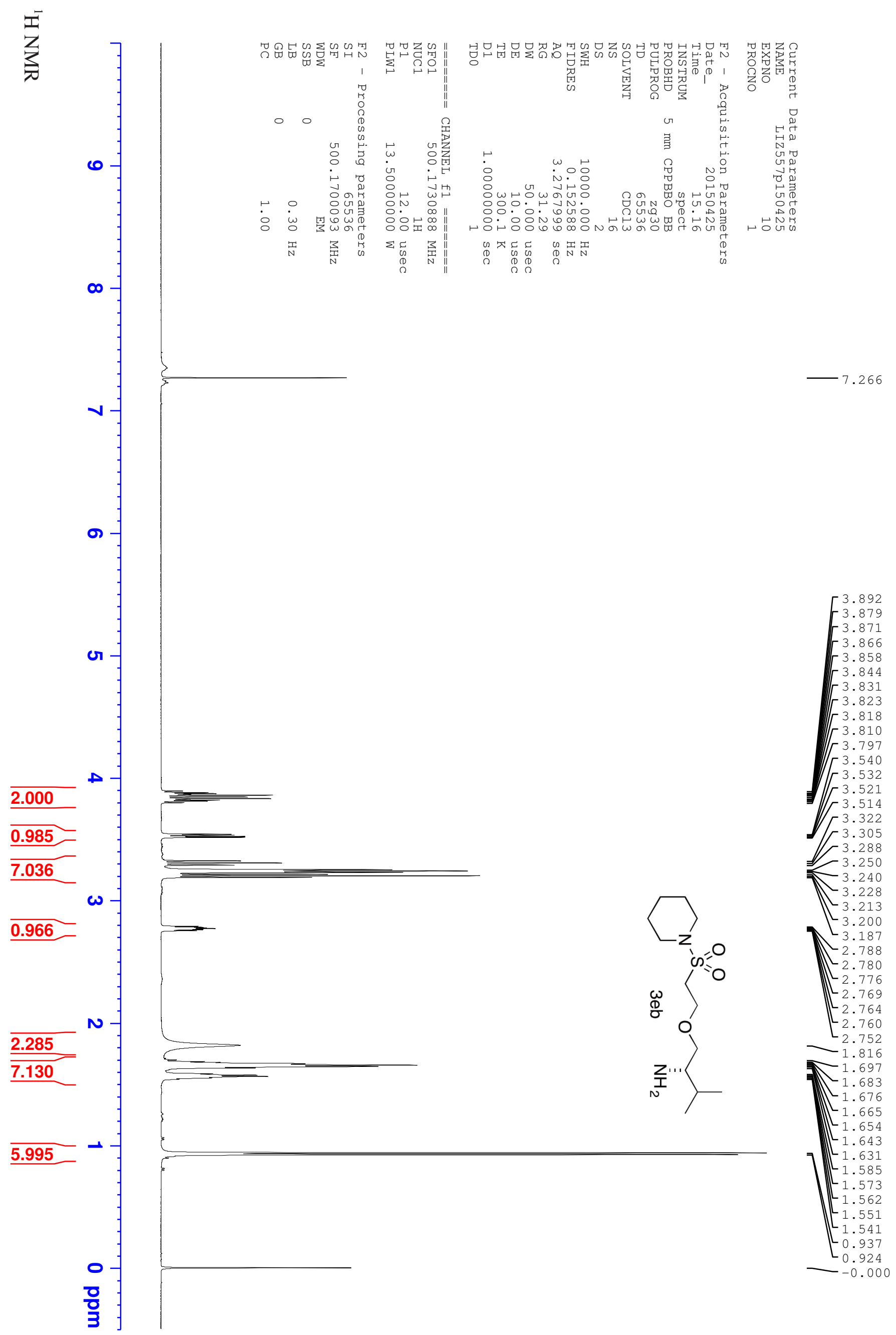




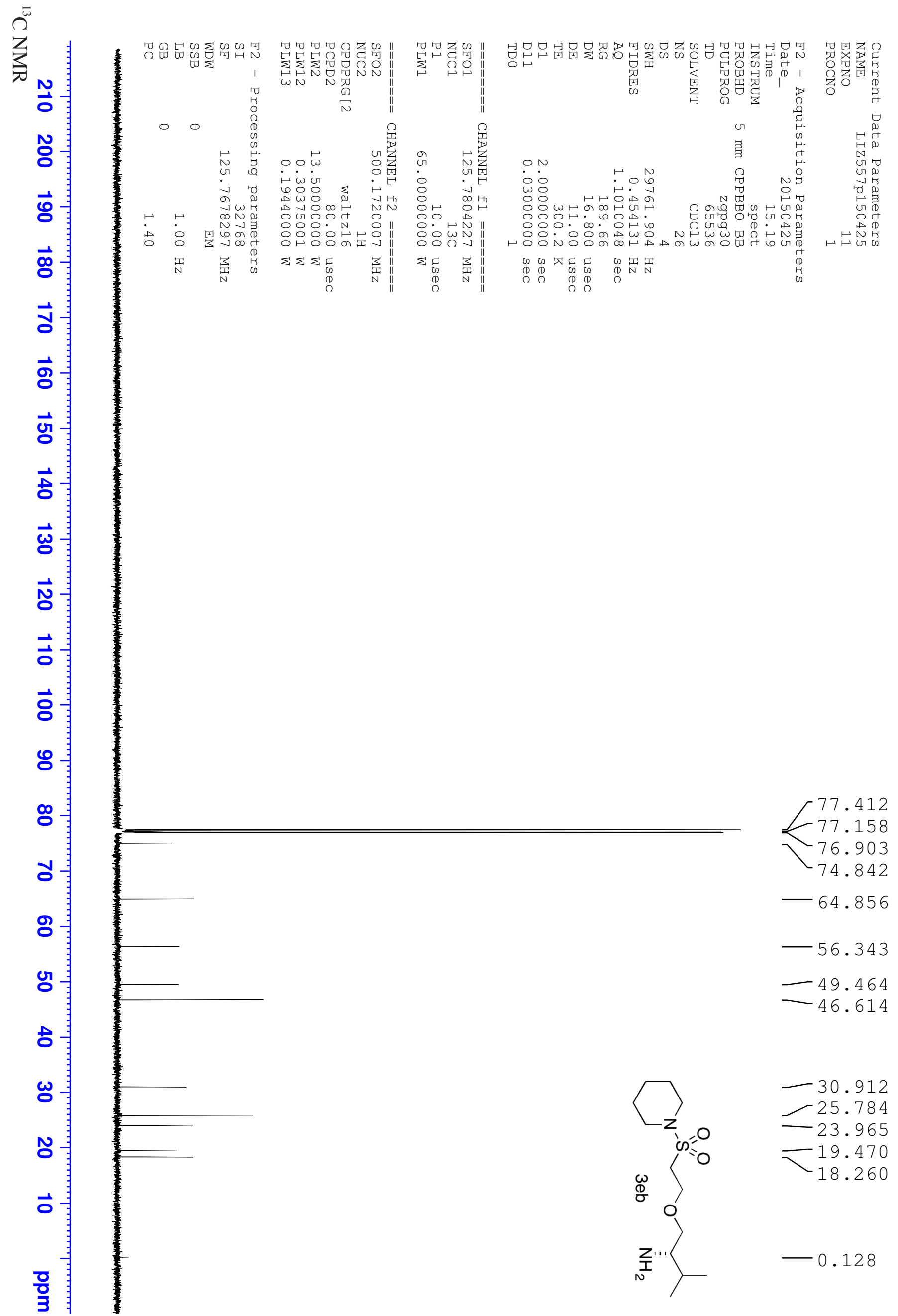




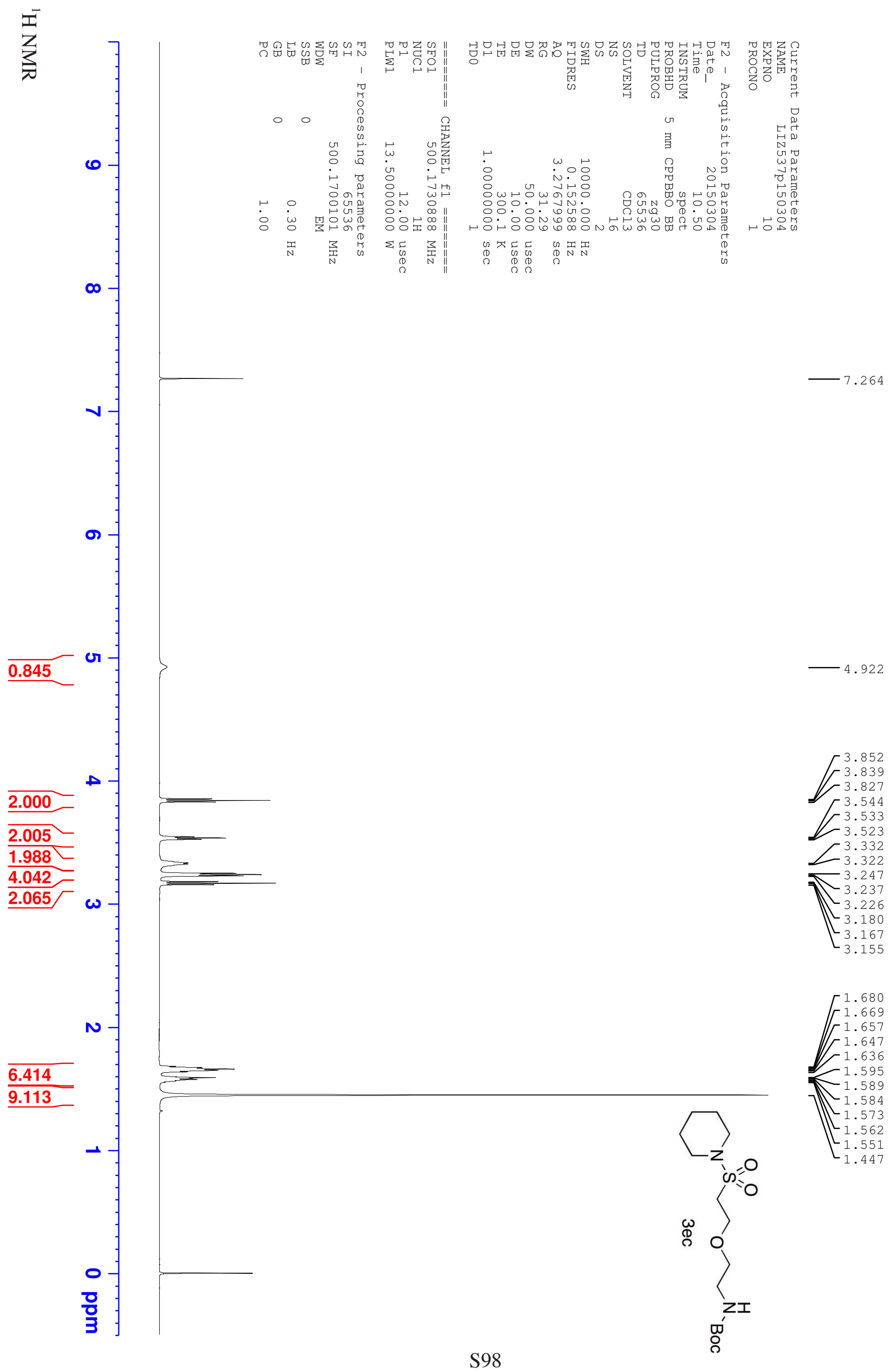




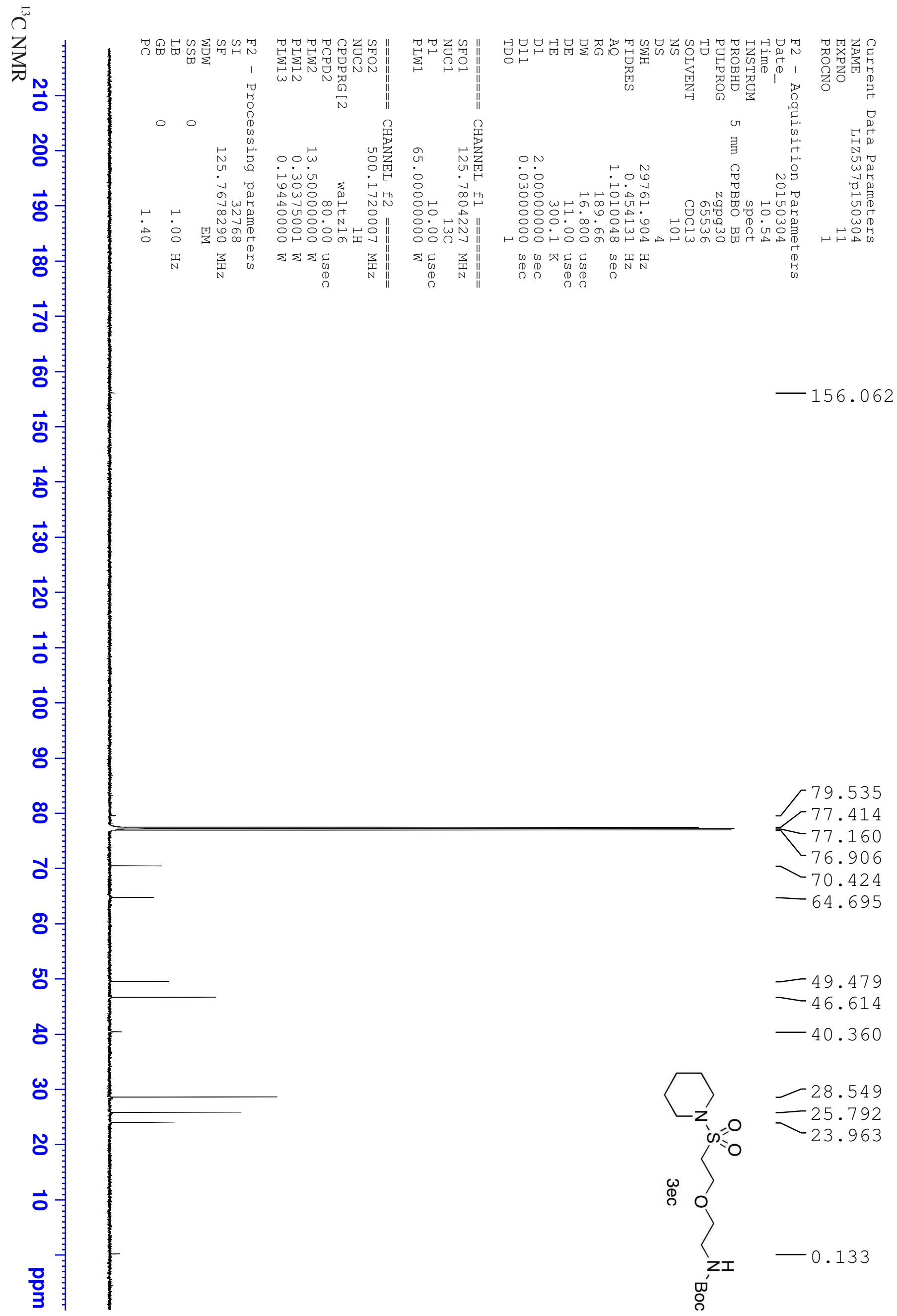




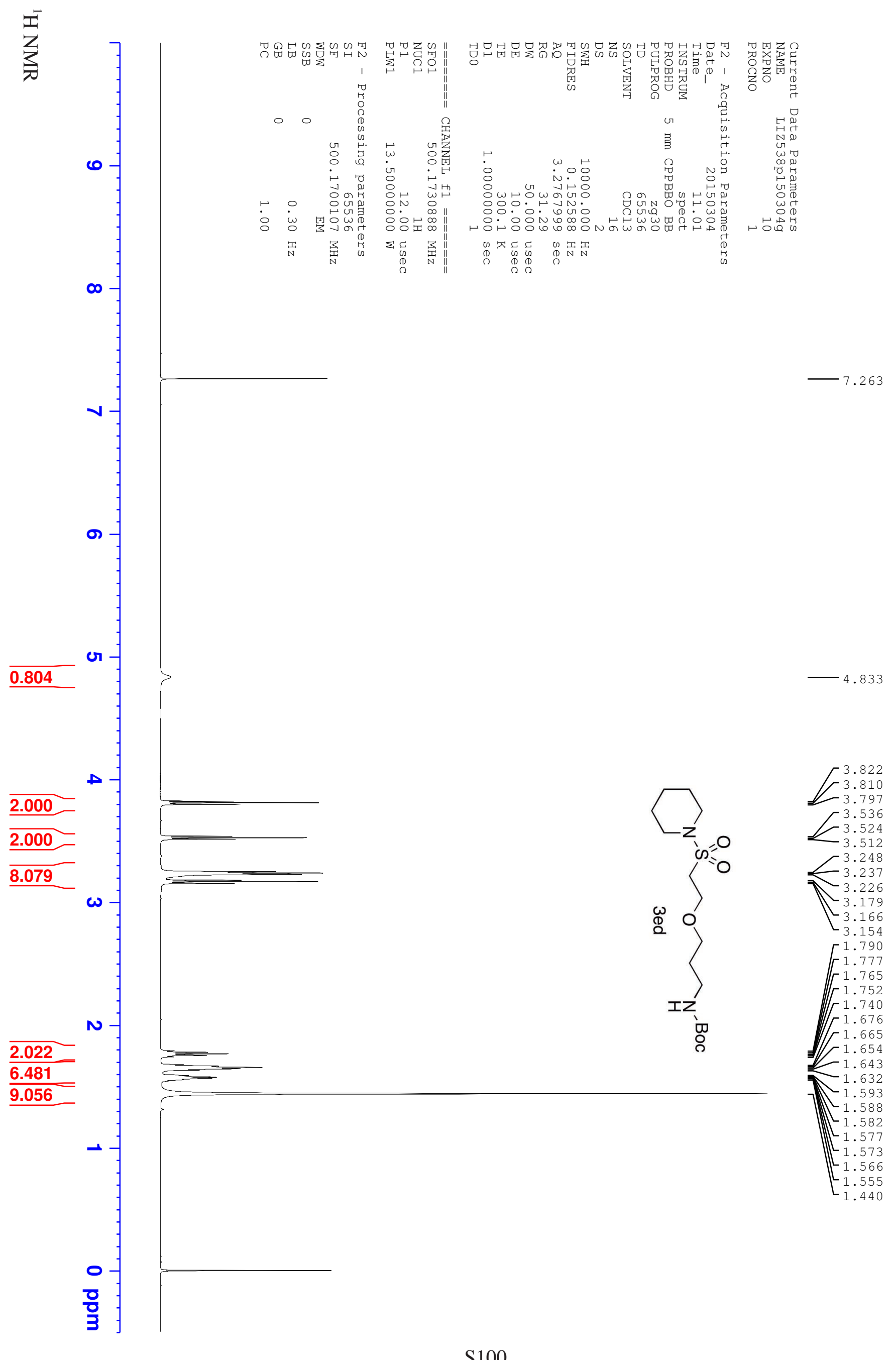




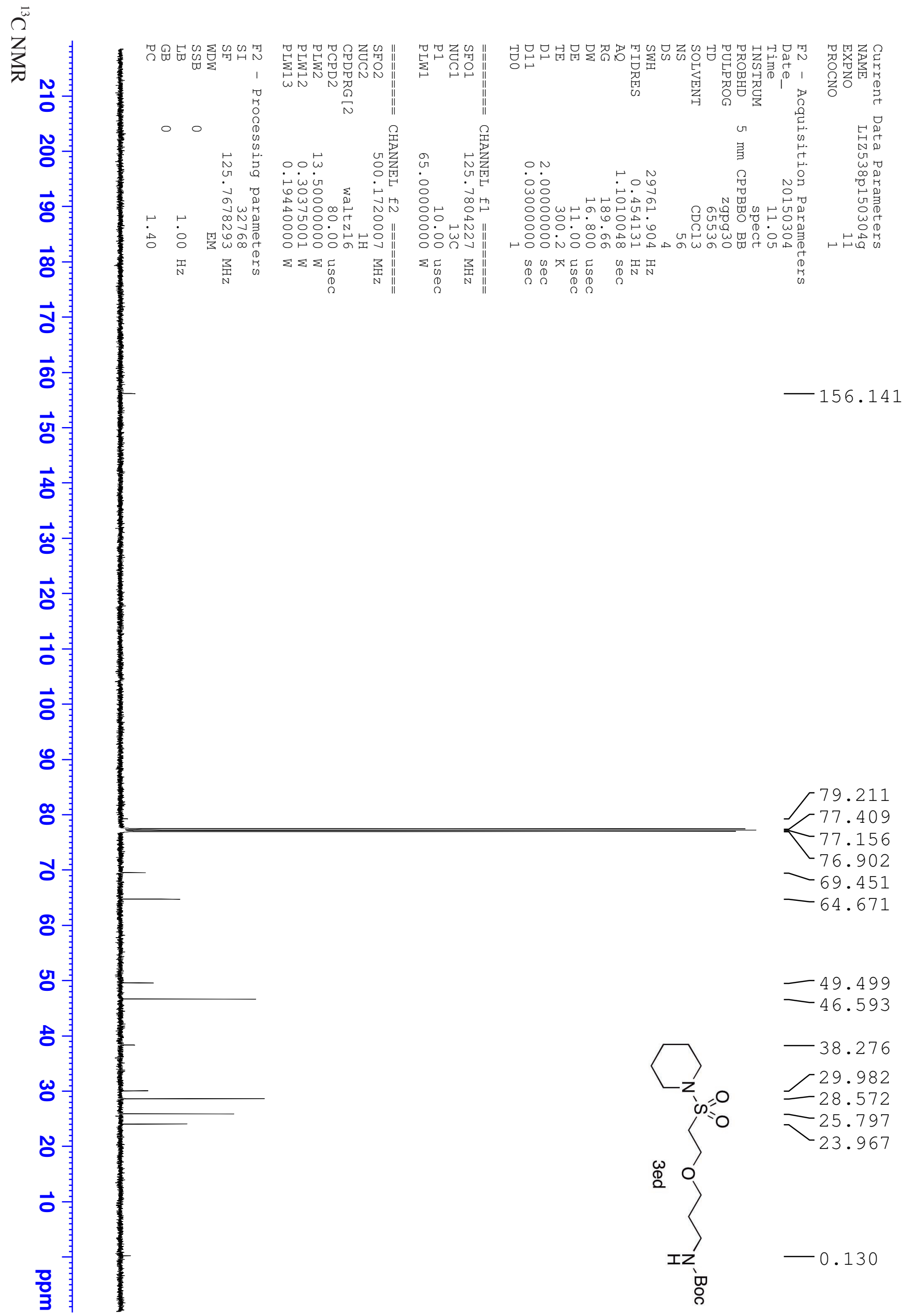




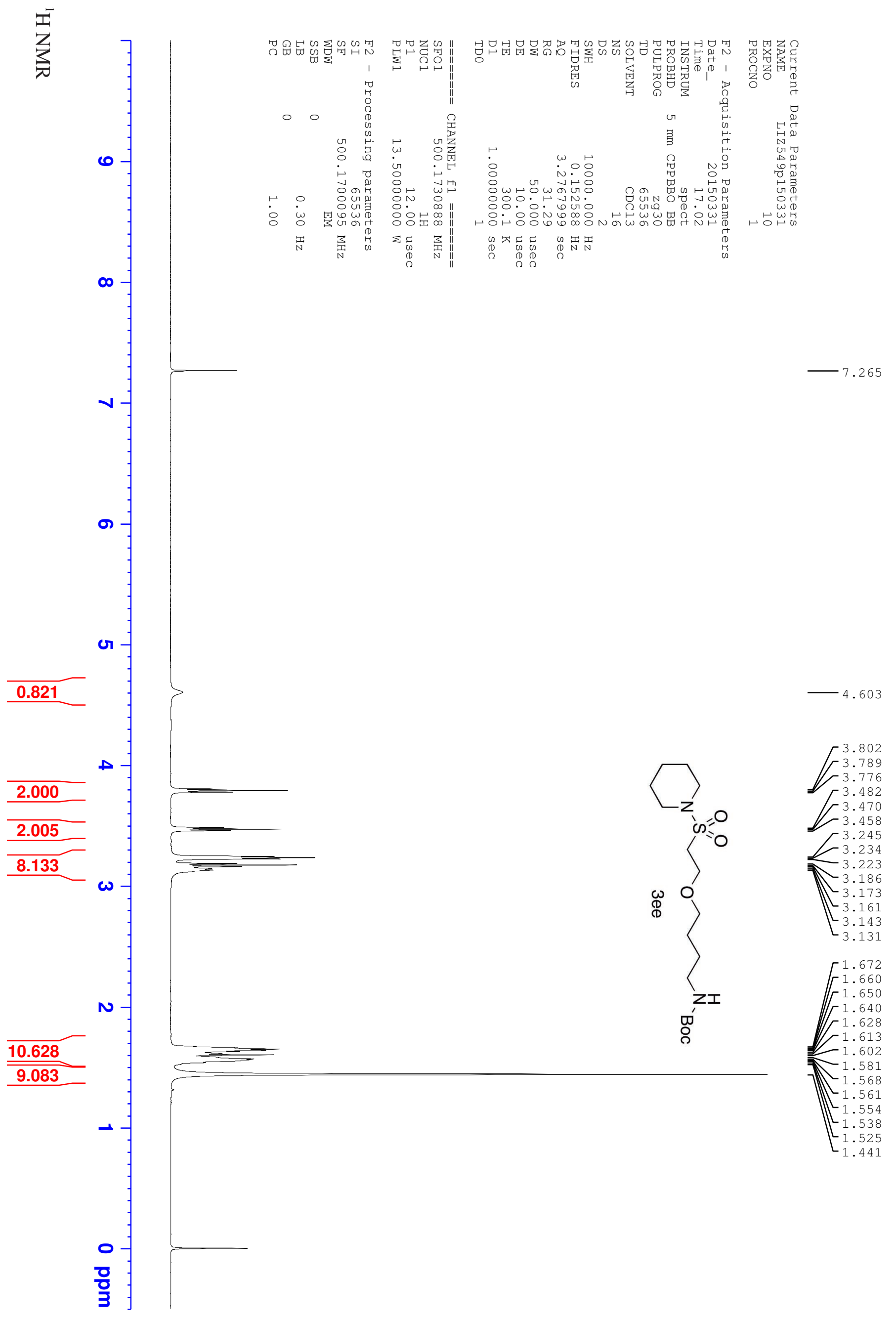




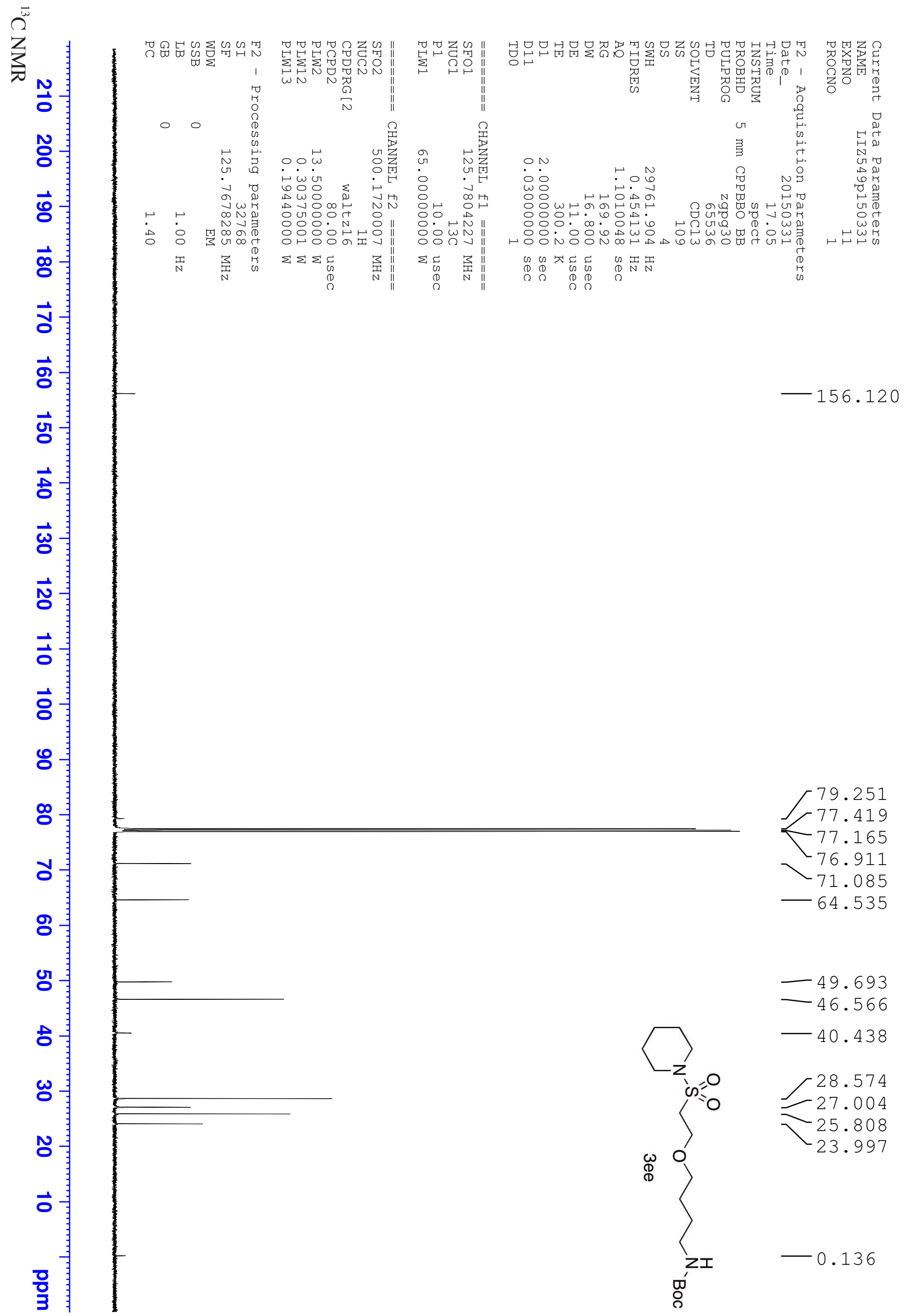


퐁

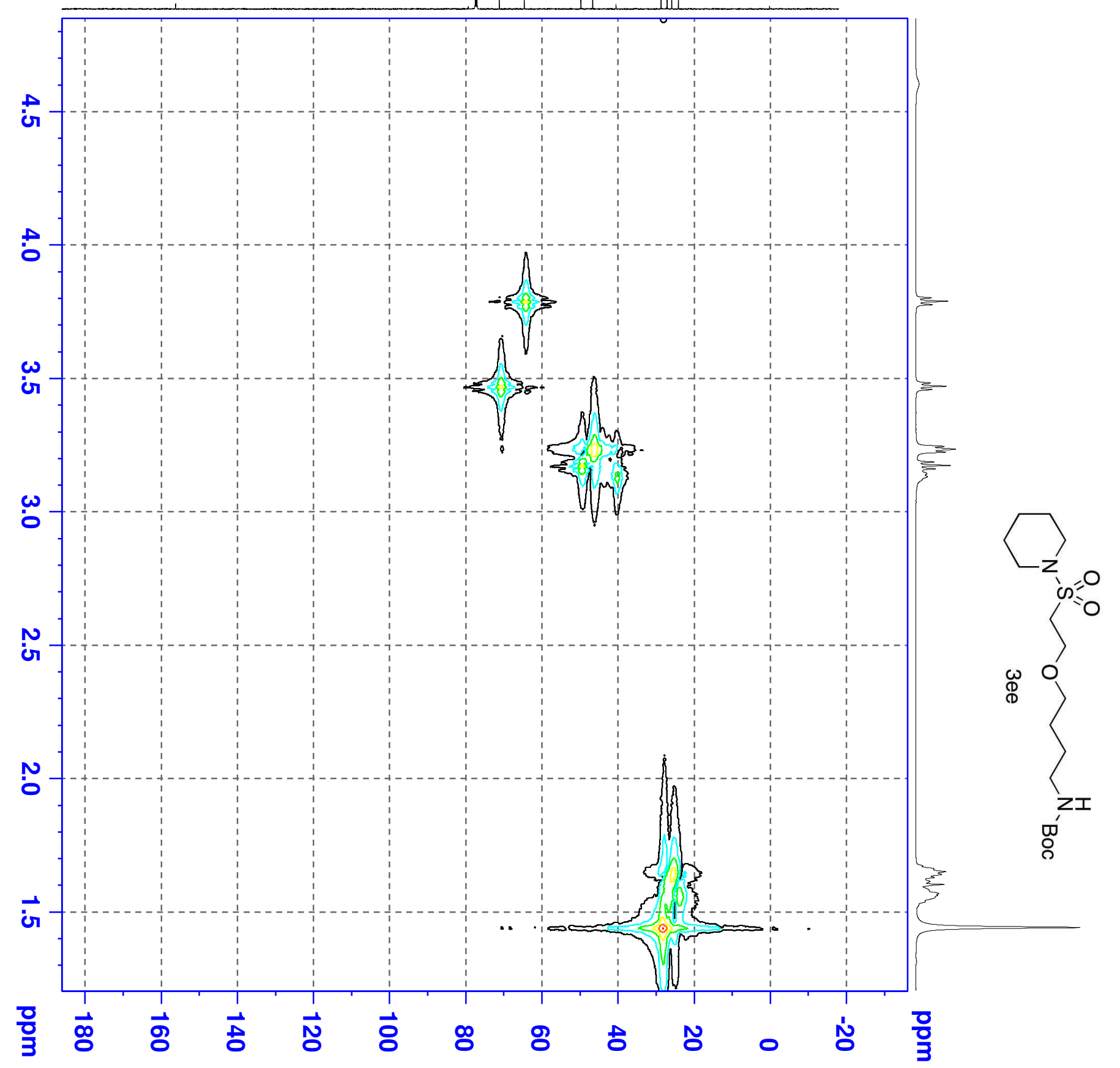

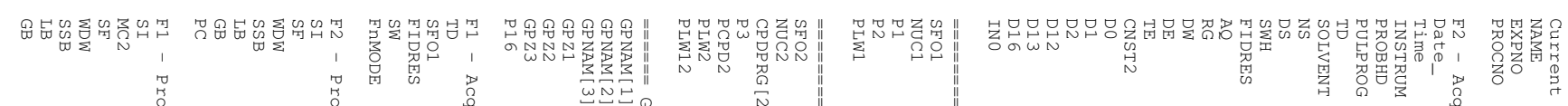

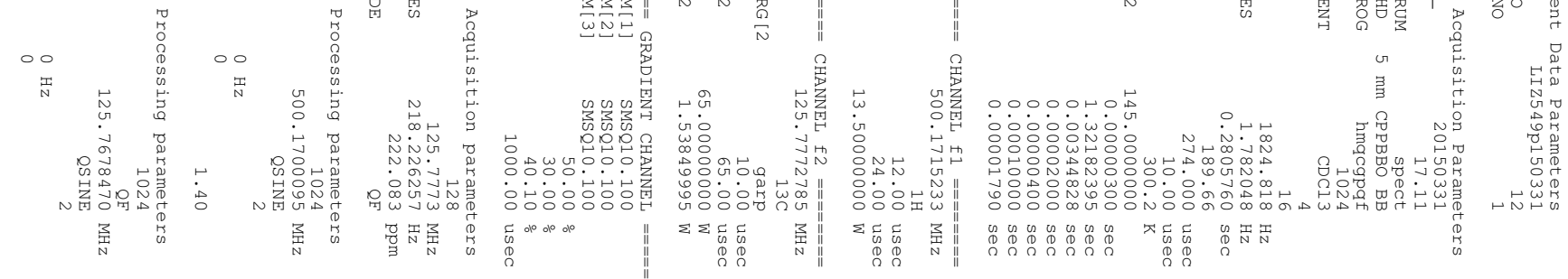




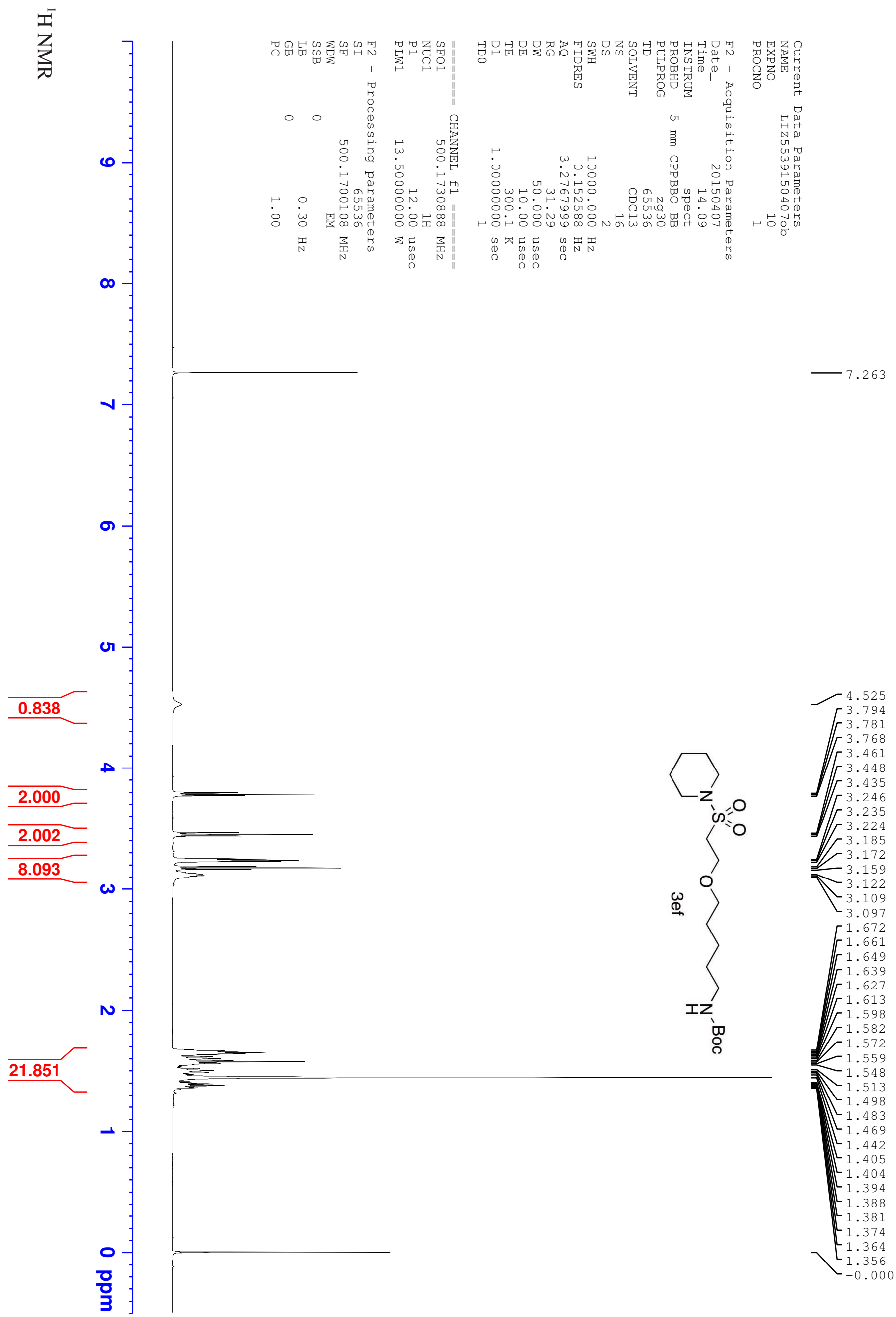




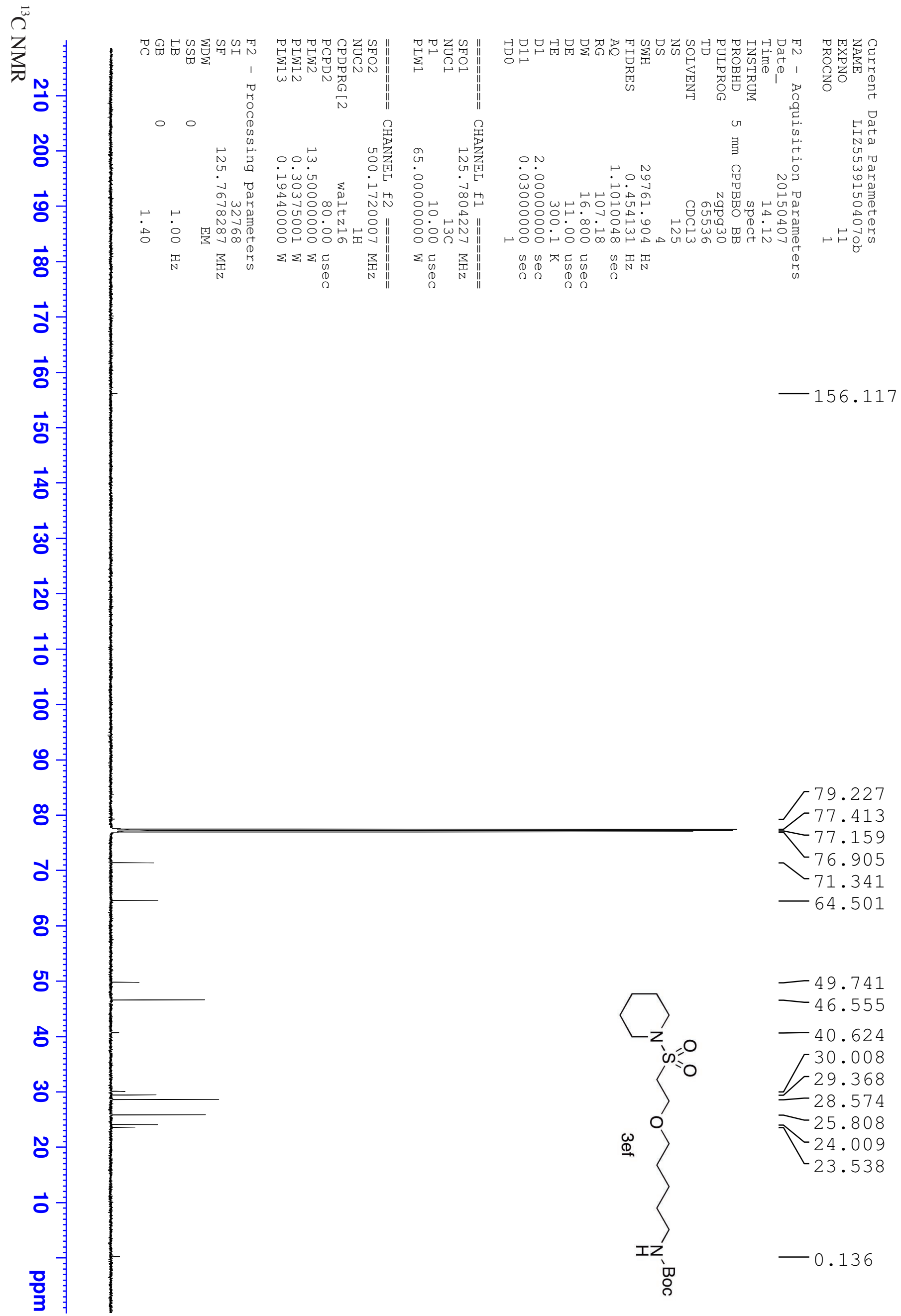




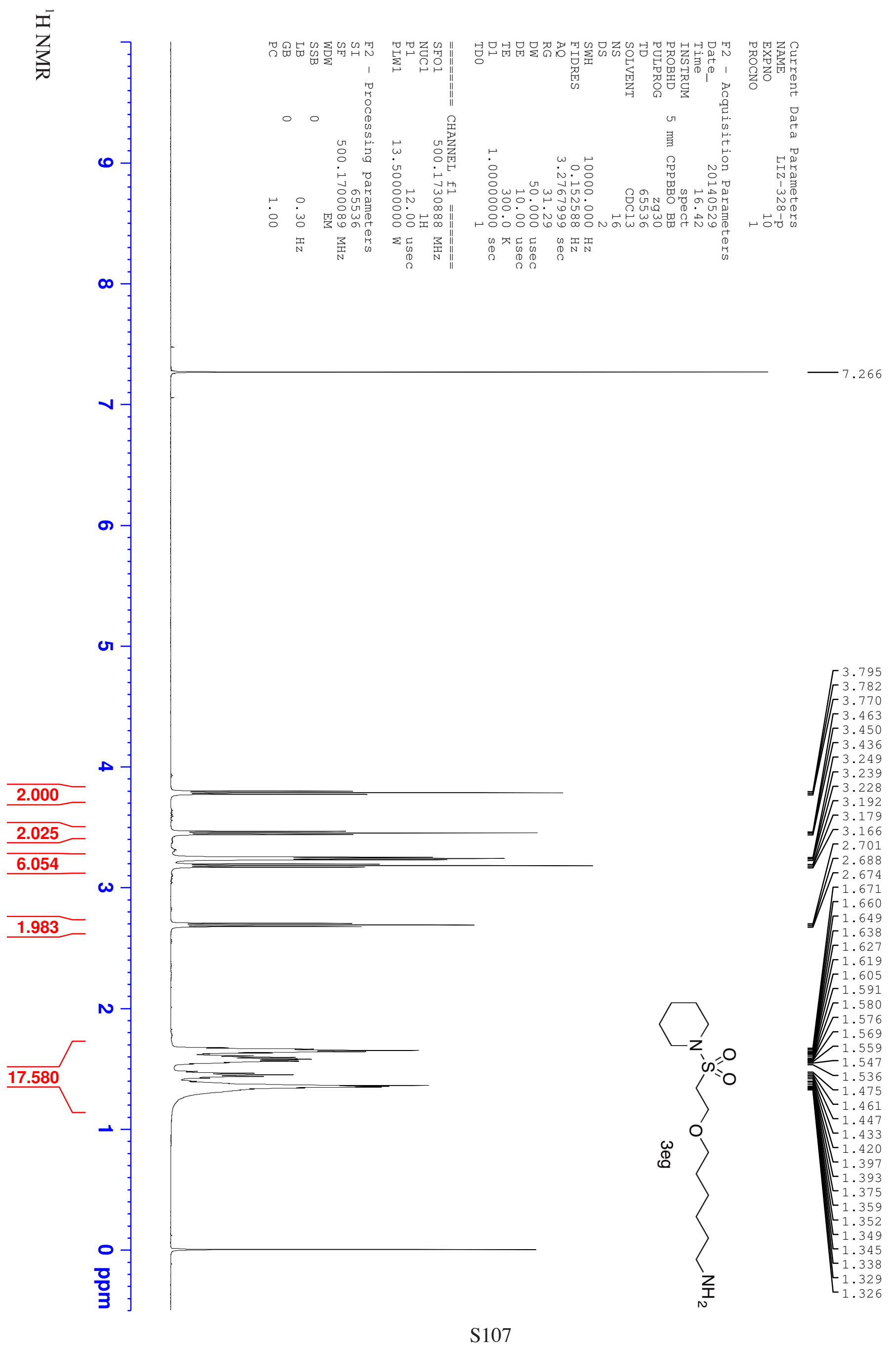




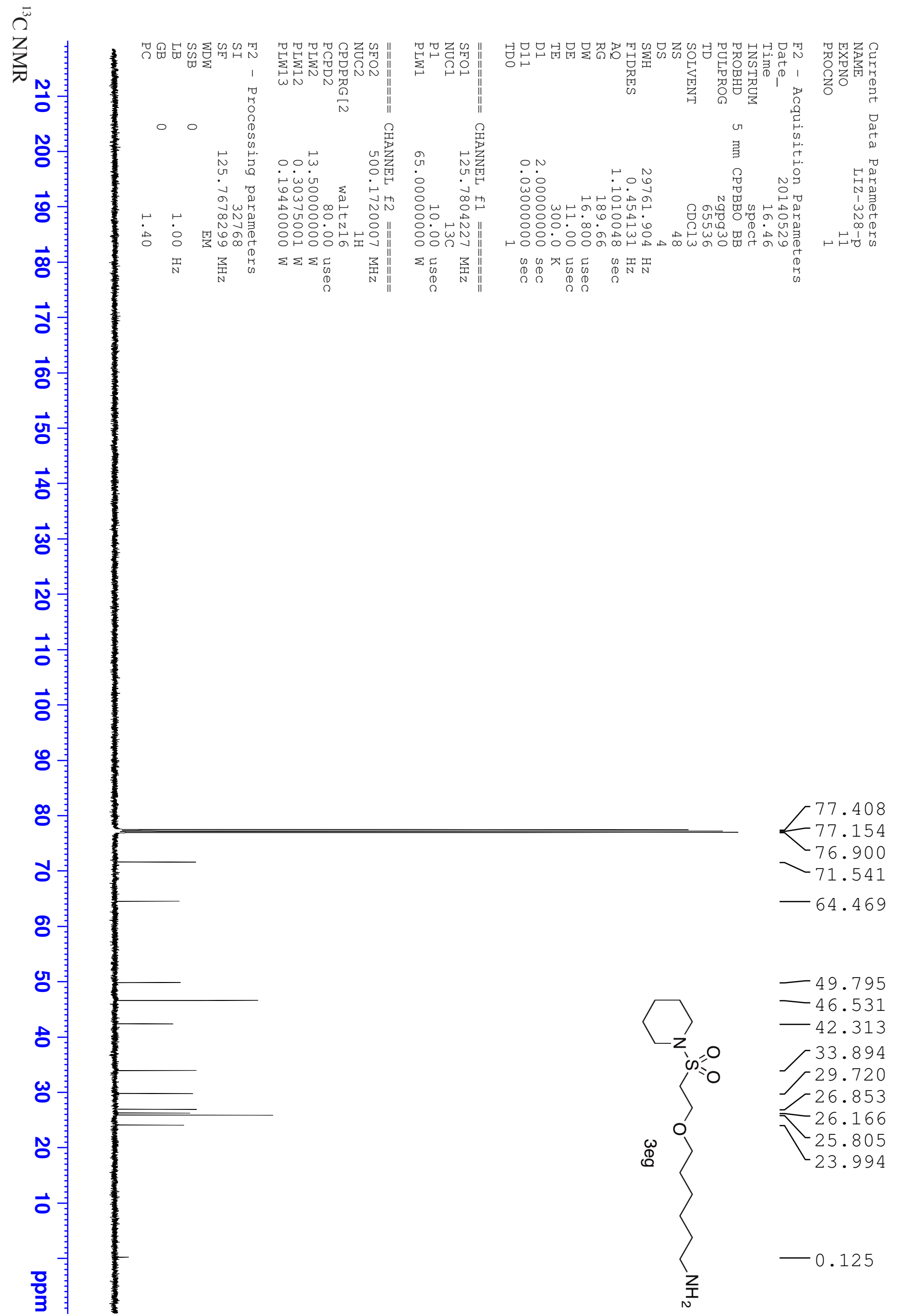




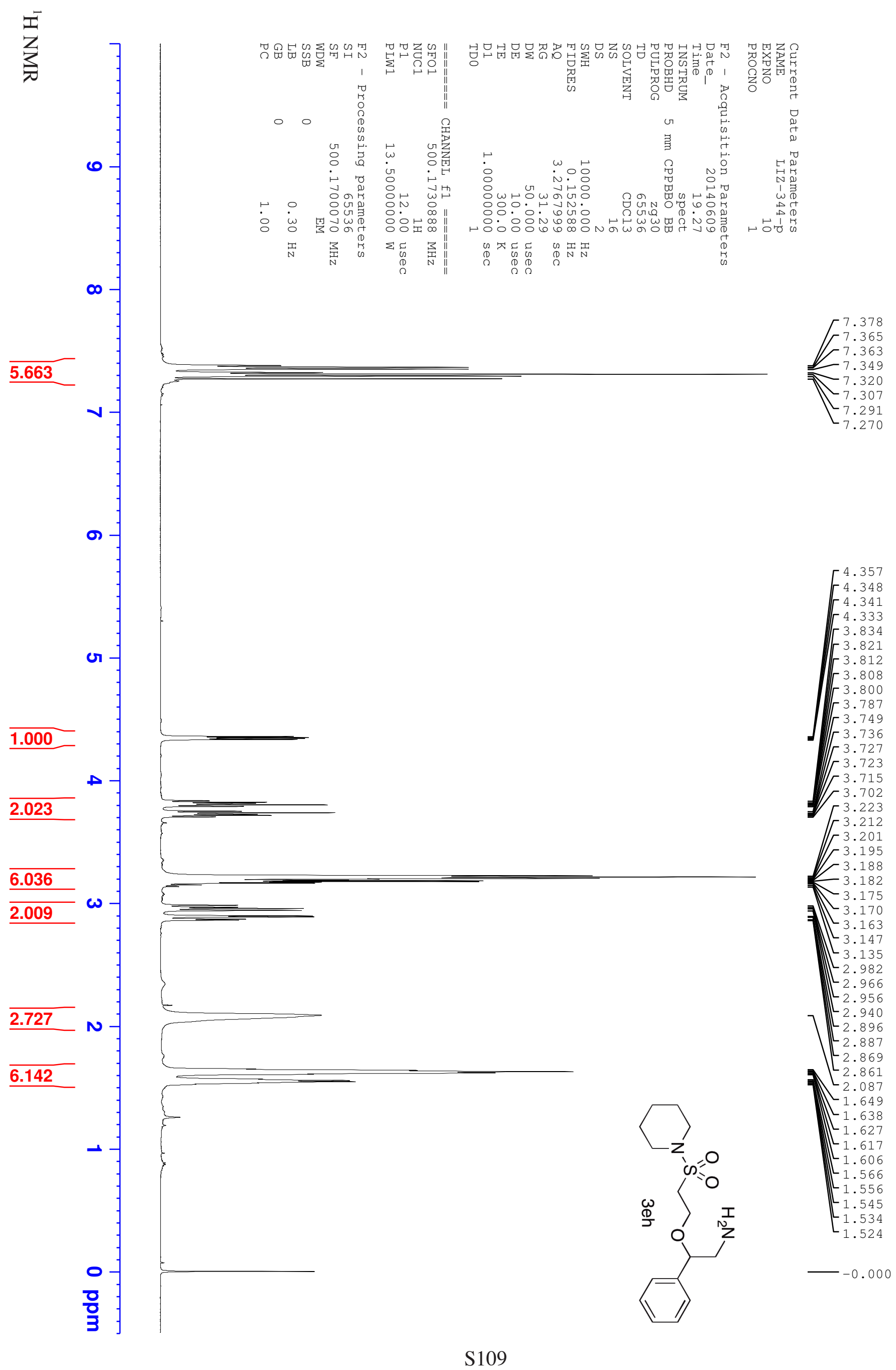




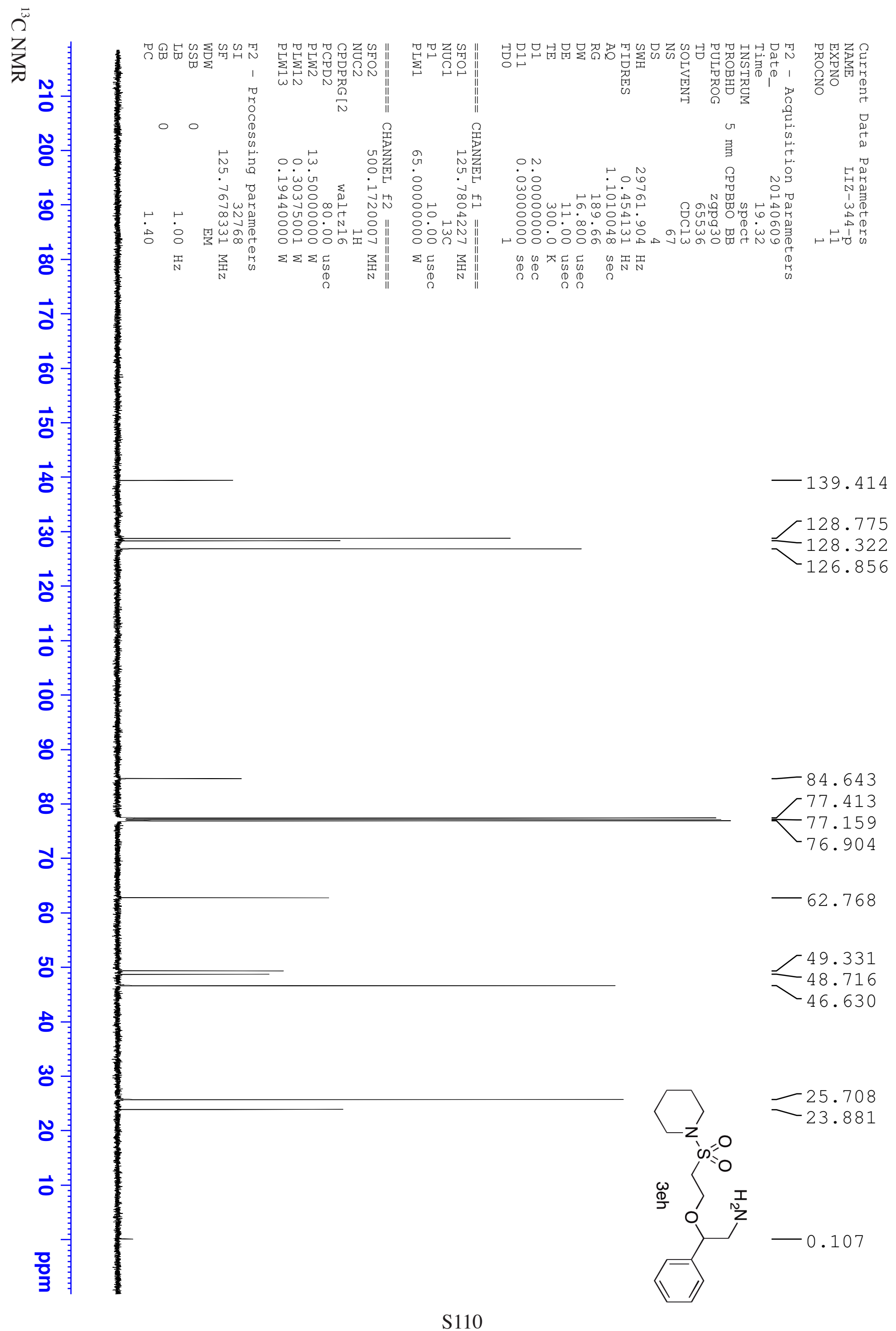




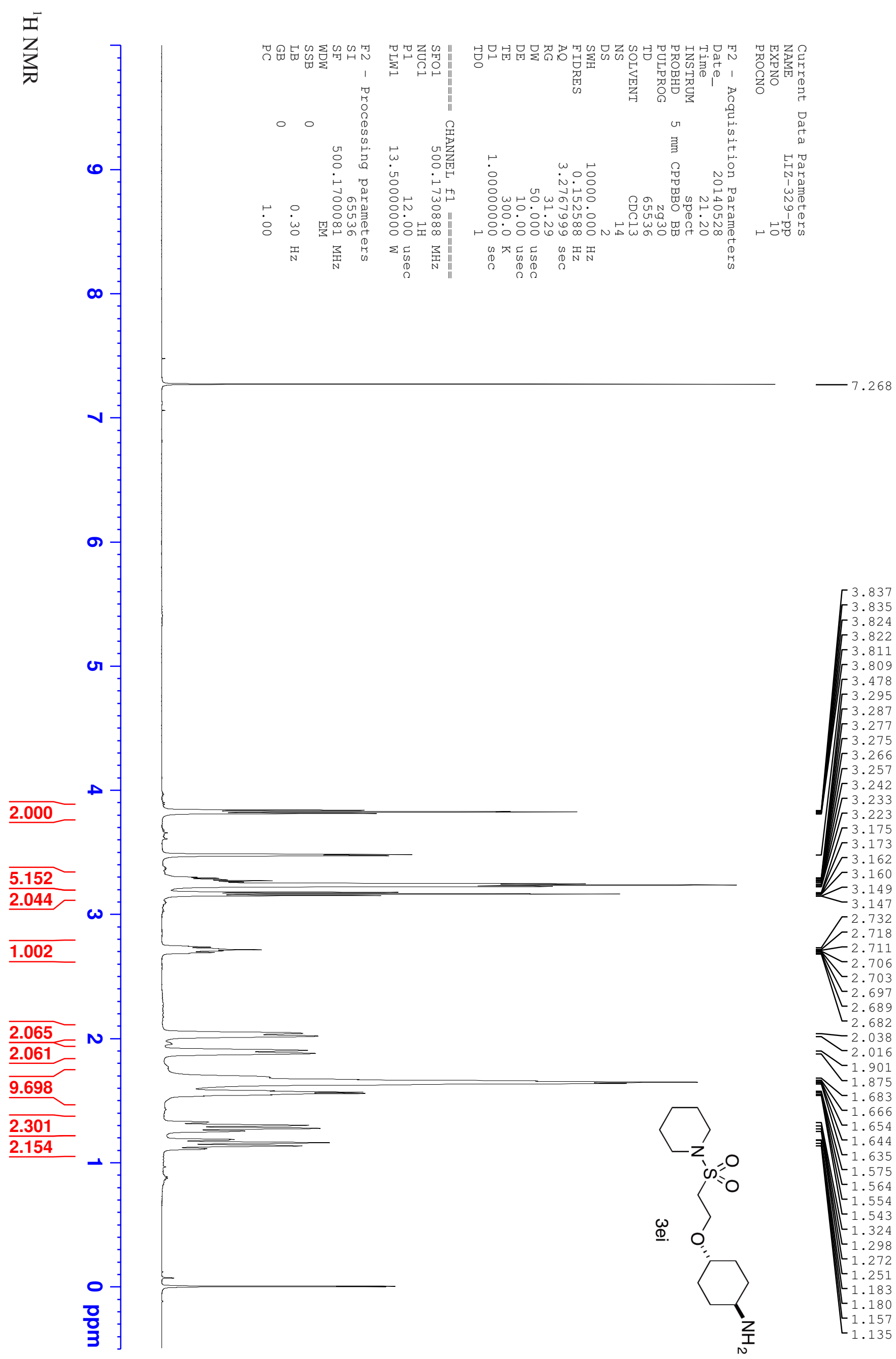




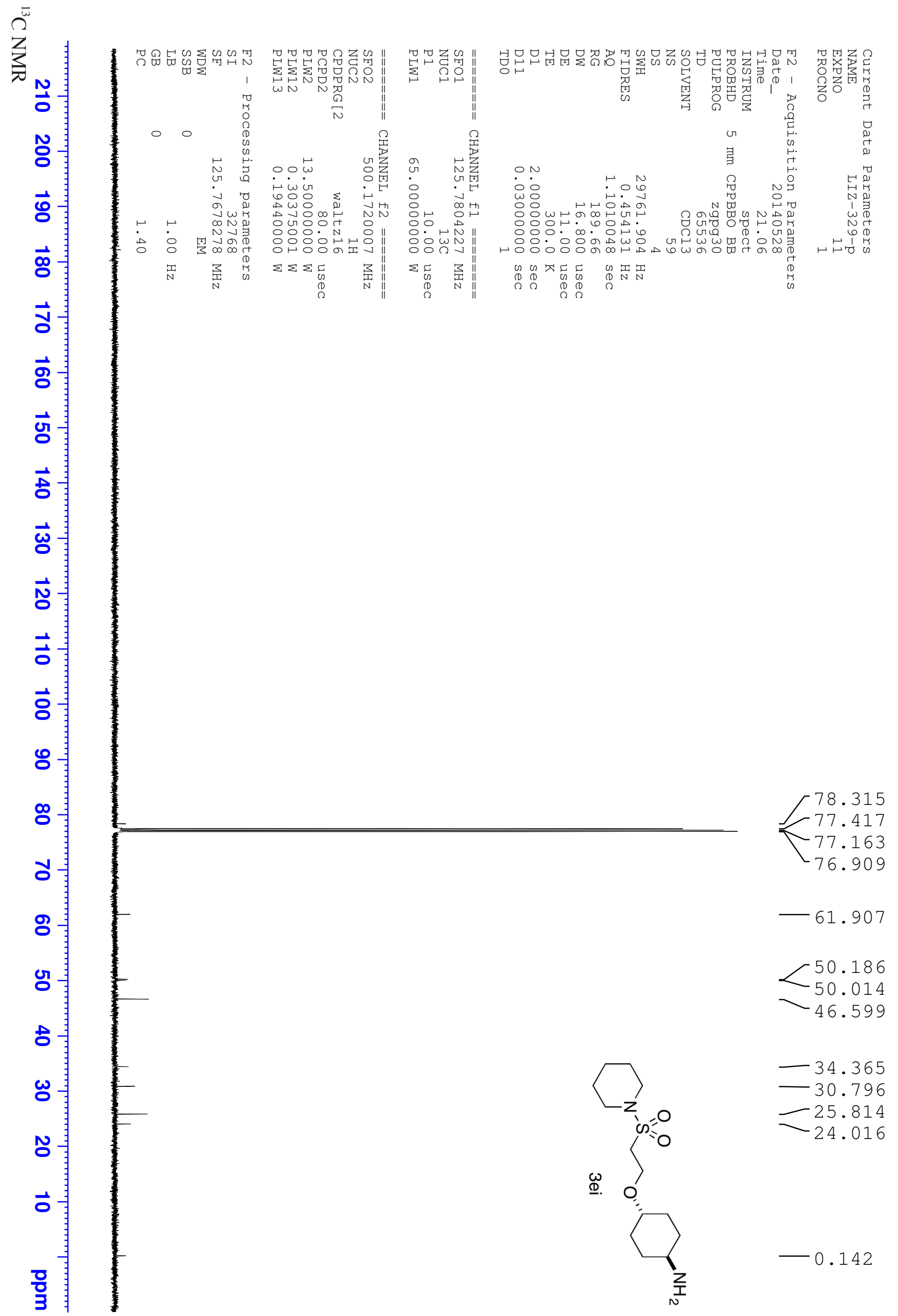




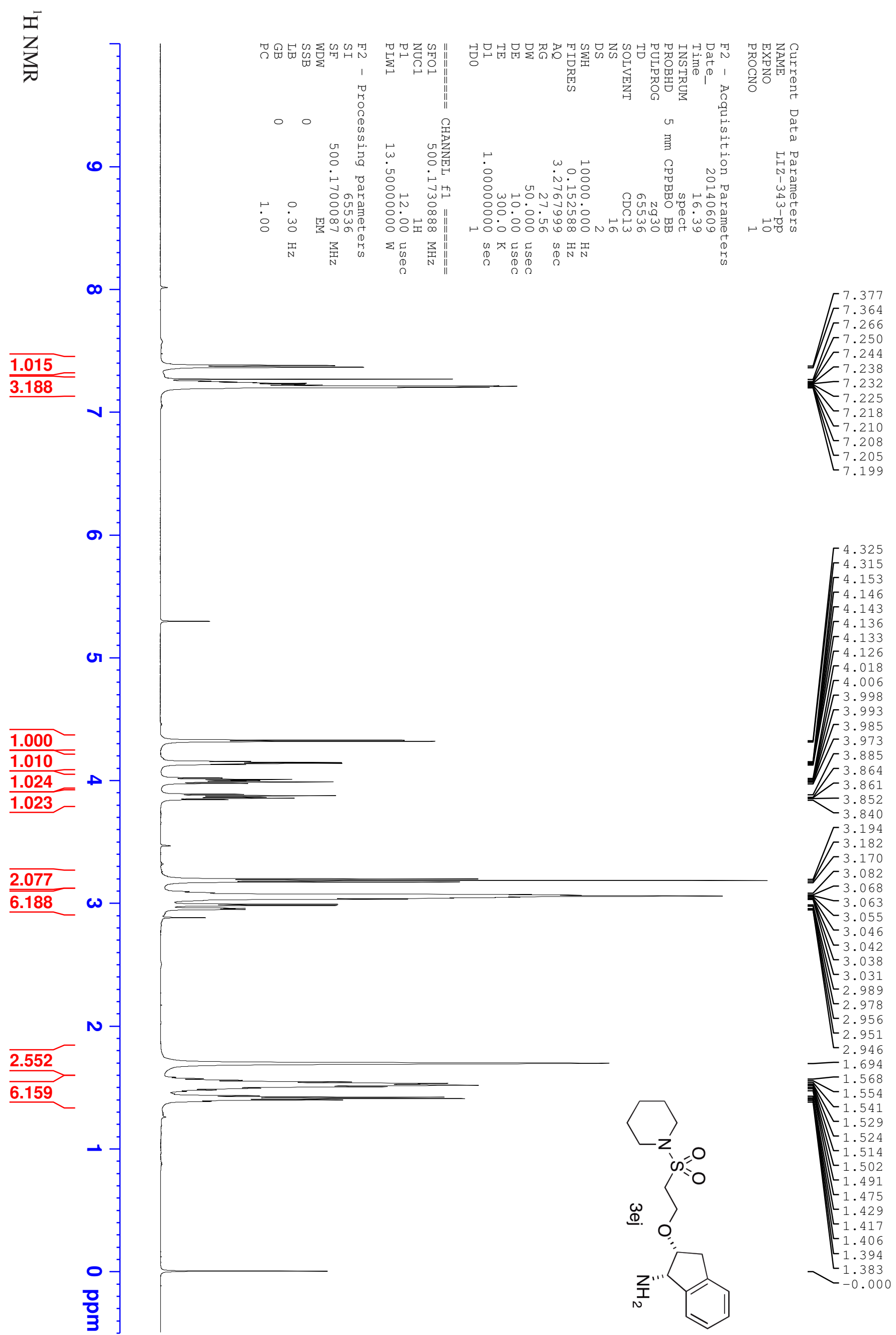




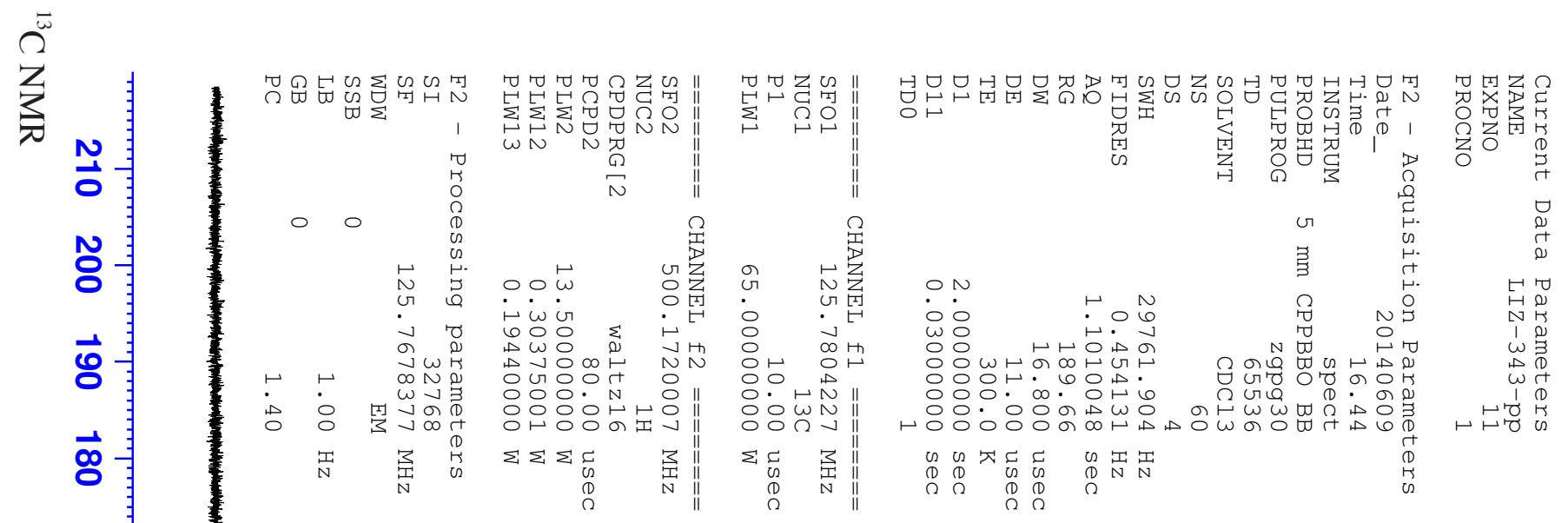

홍

뵹

$\overrightarrow{\mathrm{t}}$

$\vec{\omega}$

$\vec{\nabla}$

$\overrightarrow{0}$

$\overrightarrow{8}$

8

ø

ơ

8

용

요

$\omega$

กั

$\overrightarrow{0}$

○

年

8

a

青: 


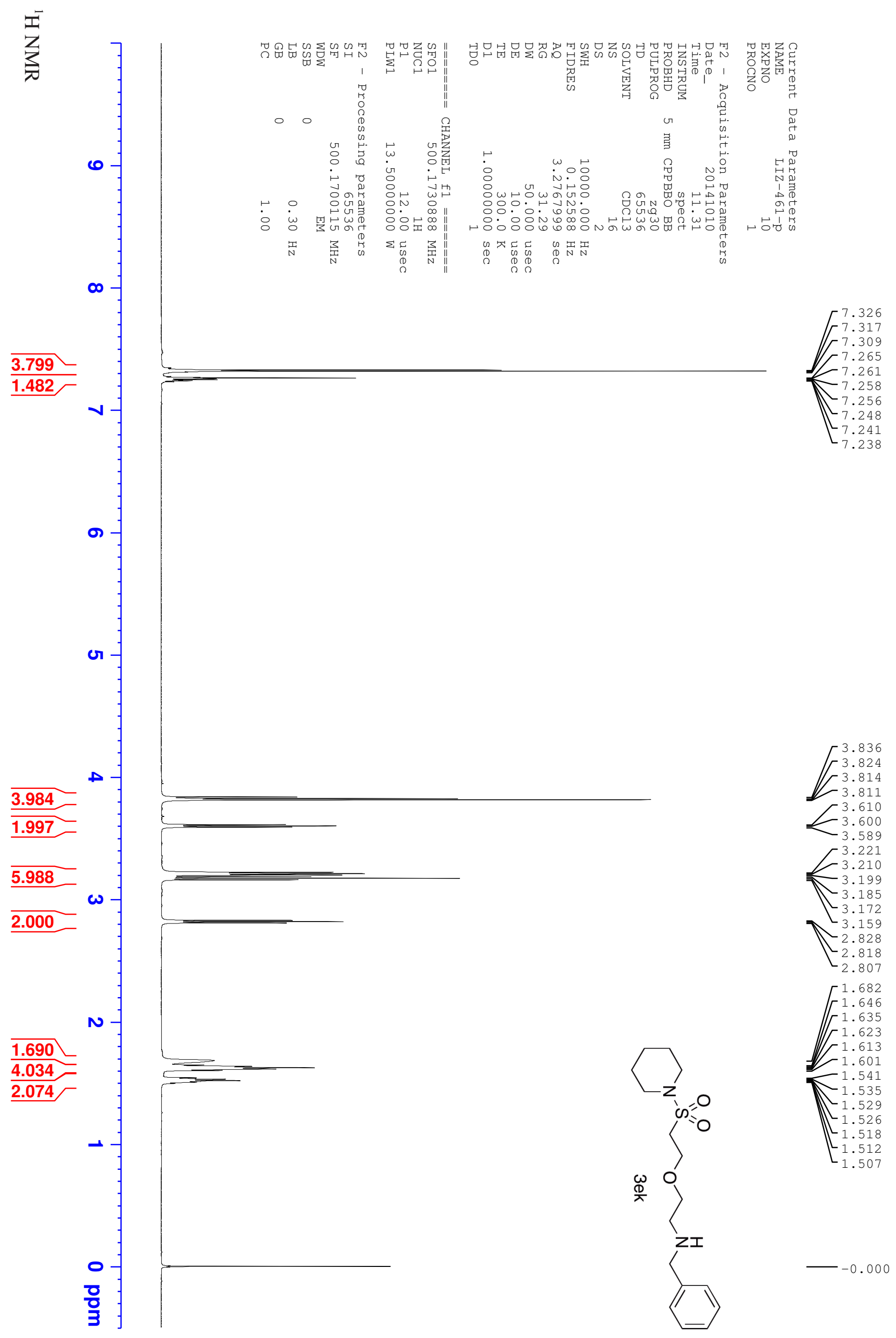




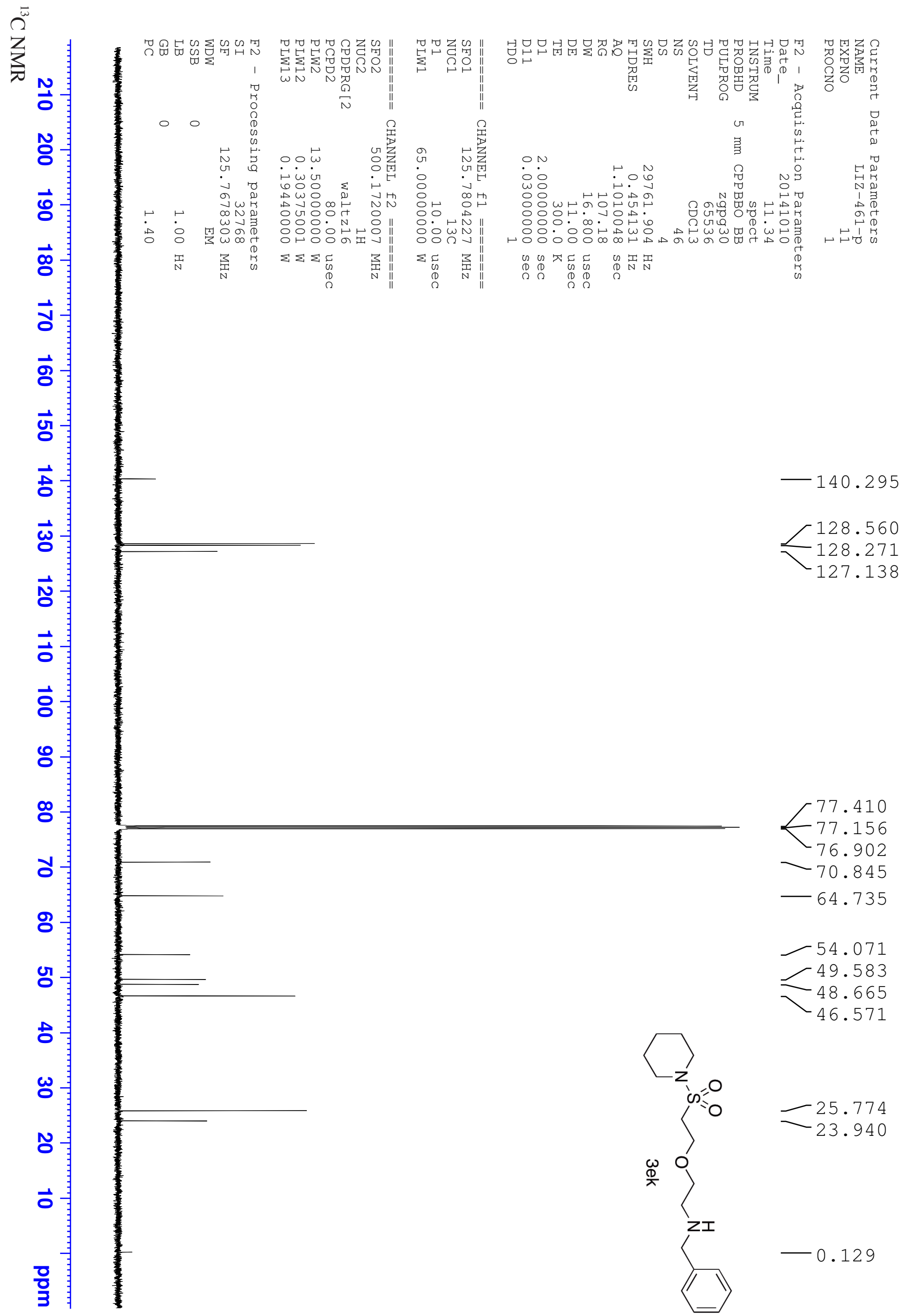




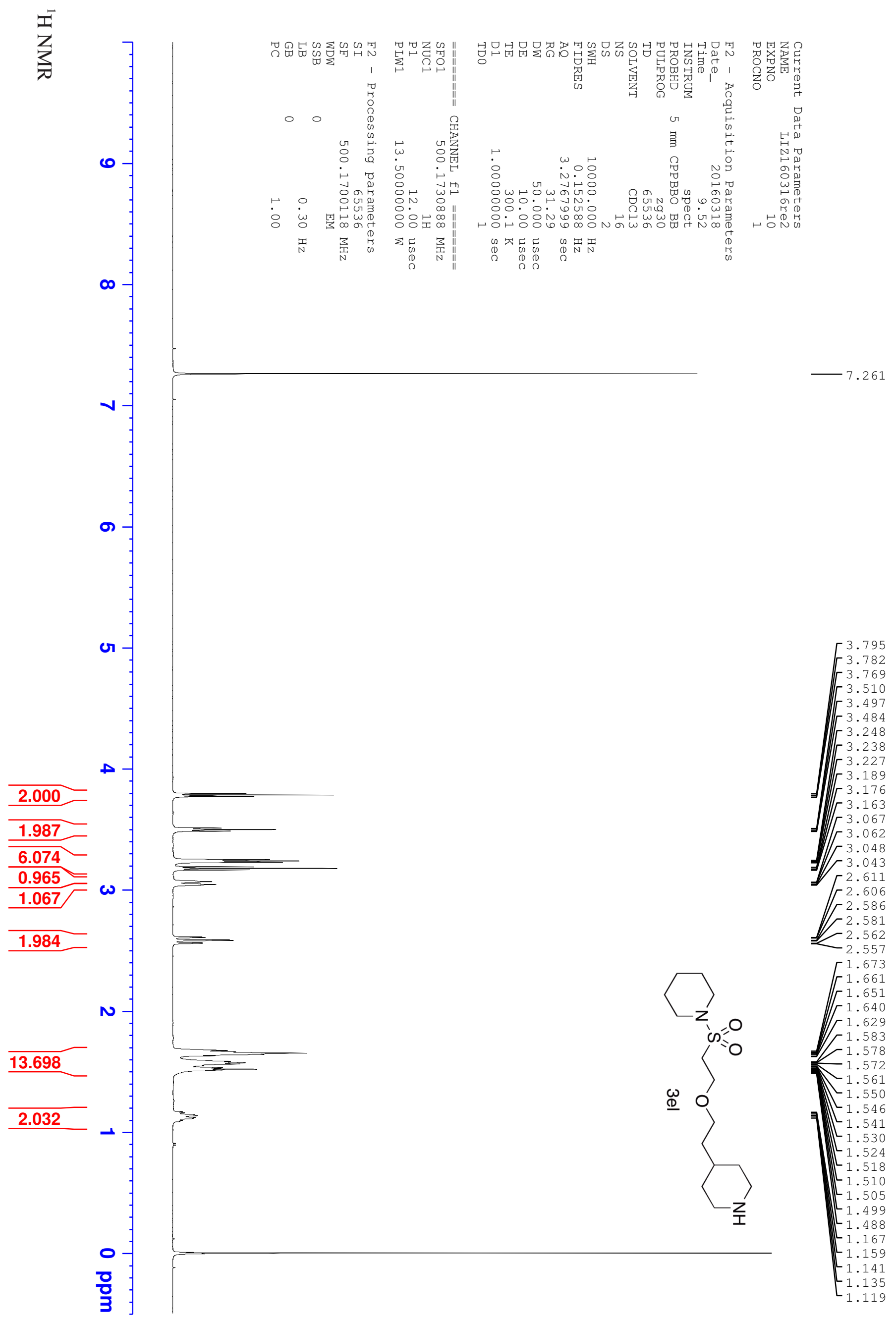




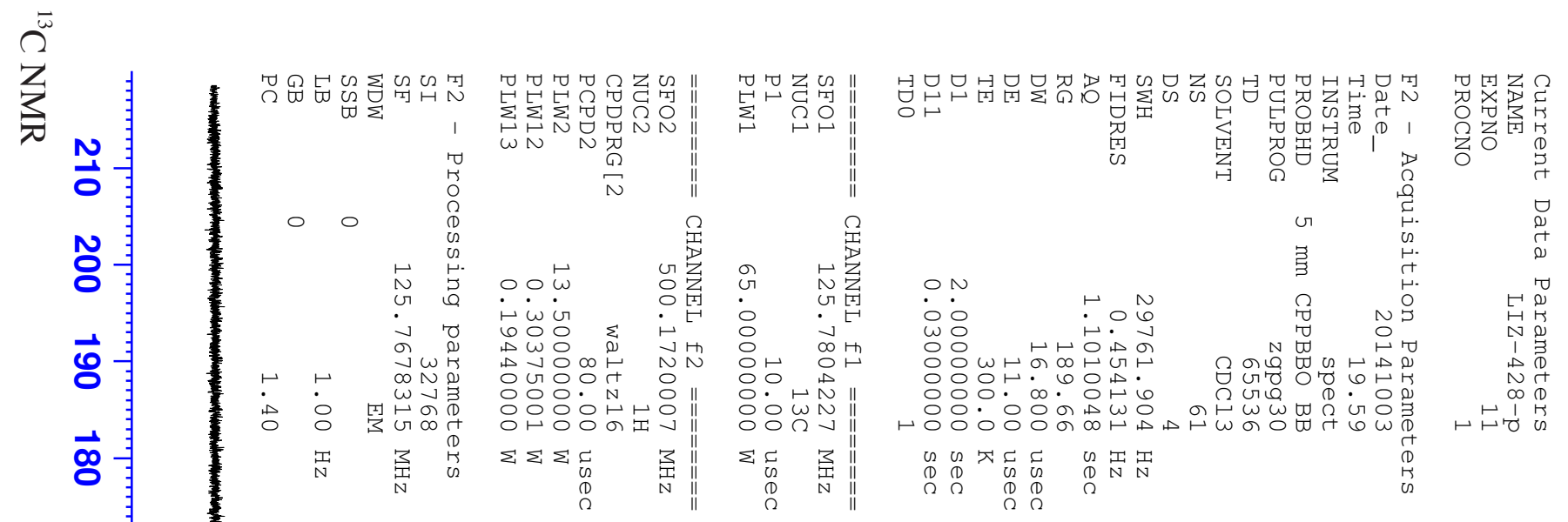

$\vec{\circ}$

ज़

怘

$\vec{\omega}$

$\overrightarrow{\mathrm{N}}$

$\overrightarrow{0}$

웅

อ

ஜ

ऽ

8

잉

뭉

w

ง

$\overrightarrow{0}$

g

衰

. 


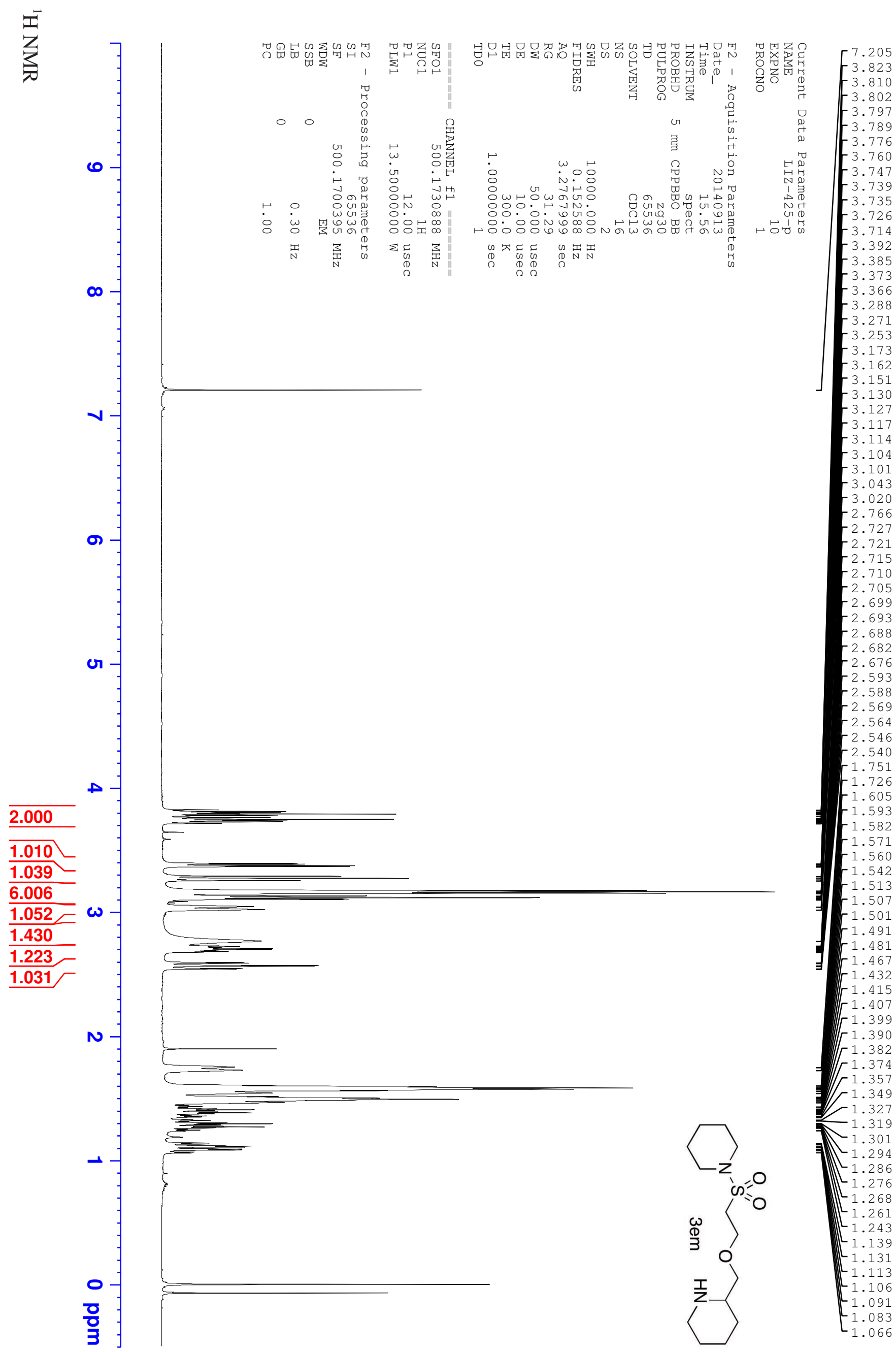




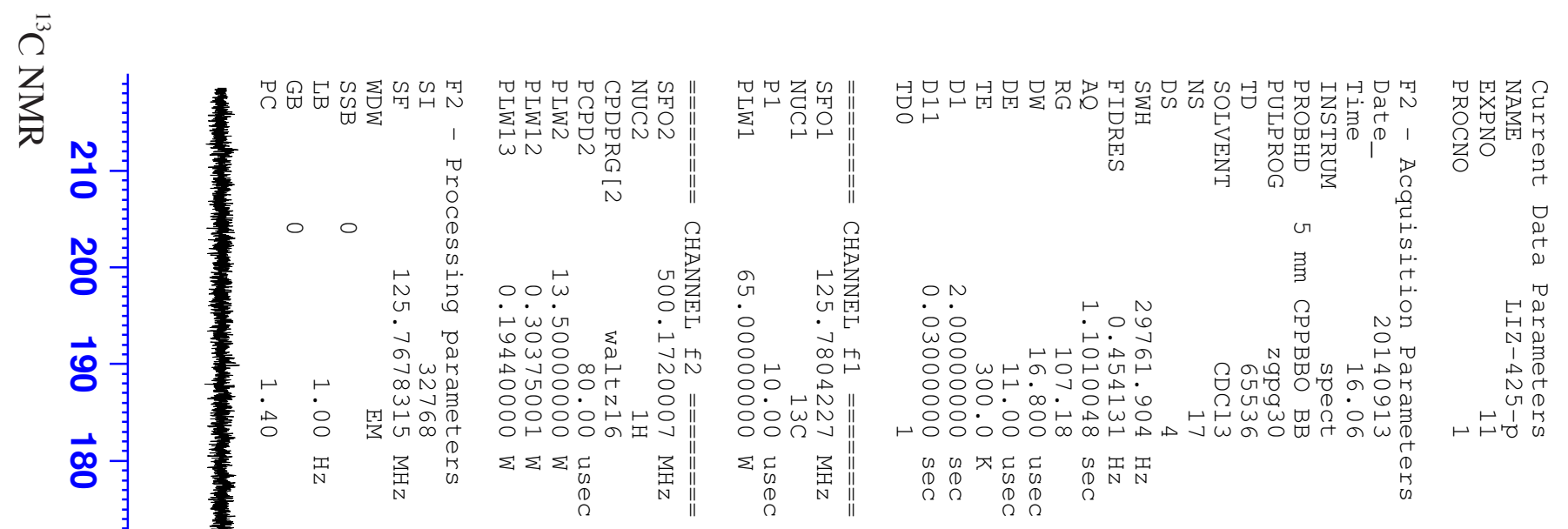

a

$\overrightarrow{\mathrm{g}}$

횡

$\overrightarrow{\mathrm{a}}$

$\overrightarrow{\mathrm{g}}$

$\vec{\Sigma}$

$\vec{\partial}$

$\overrightarrow{8}$

8

ø

77.412

-77.157
-76.903

76.903

75.804

$-64.812$

8

잉

o

o

ก
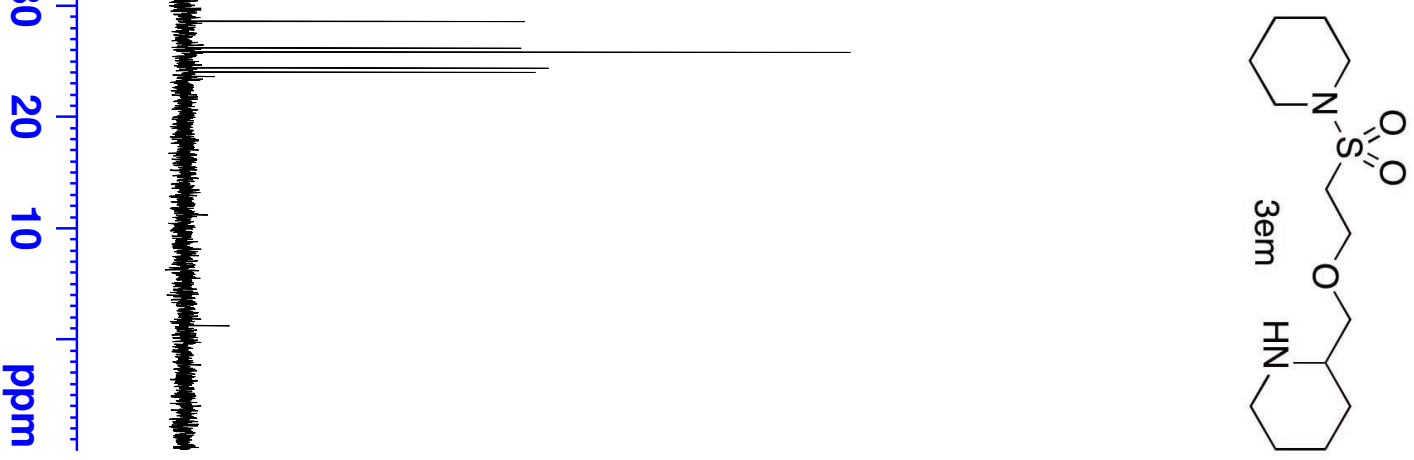

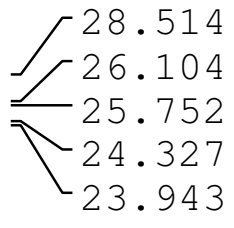

$\vec{o}$ 


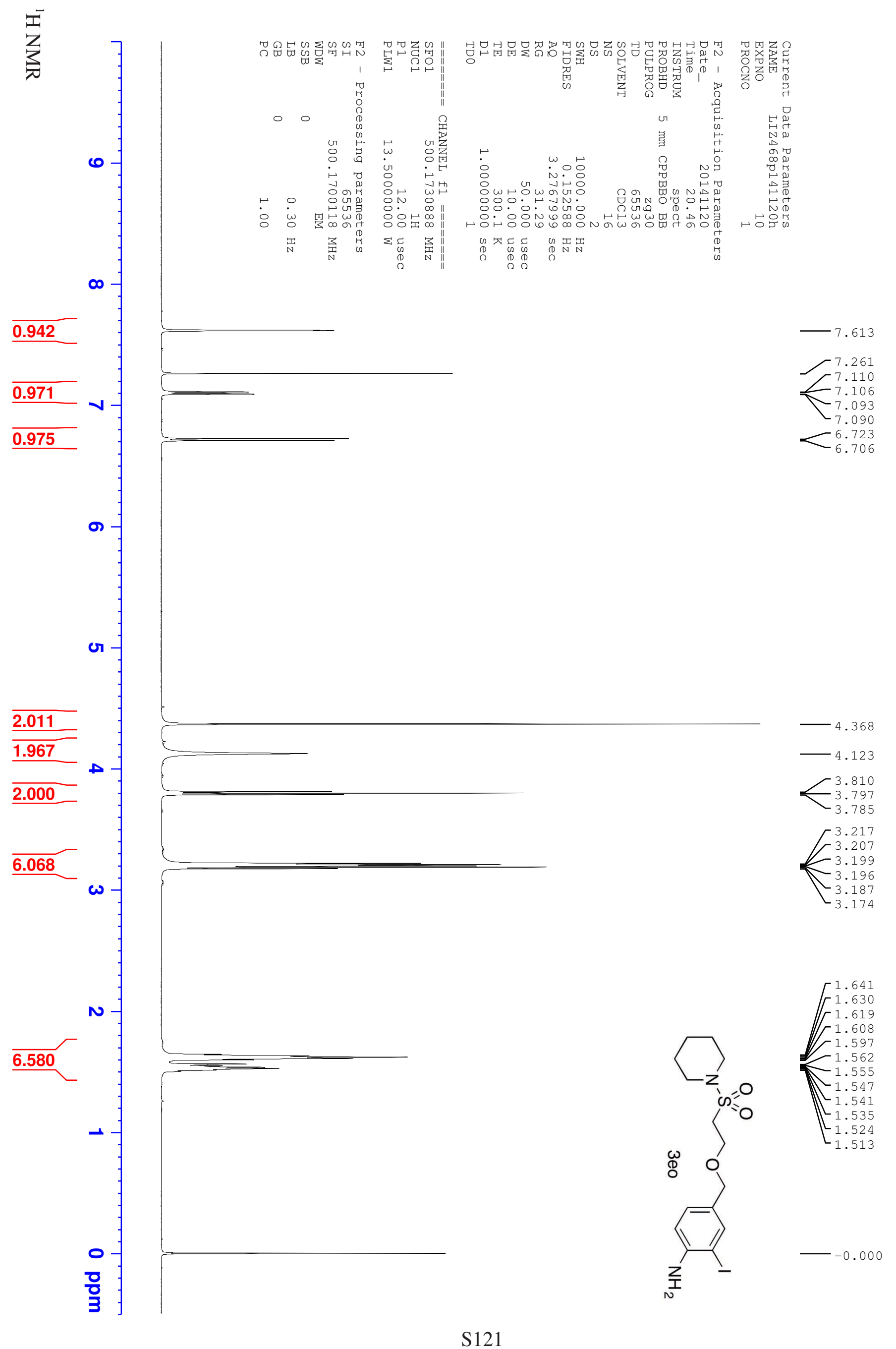


ㄴ

$\overrightarrow{8}$

항

$\overrightarrow{8}$

8

ø

ชั

8

잉

o

$\ddot{o}$

กั

$\overrightarrow{0}$

흘
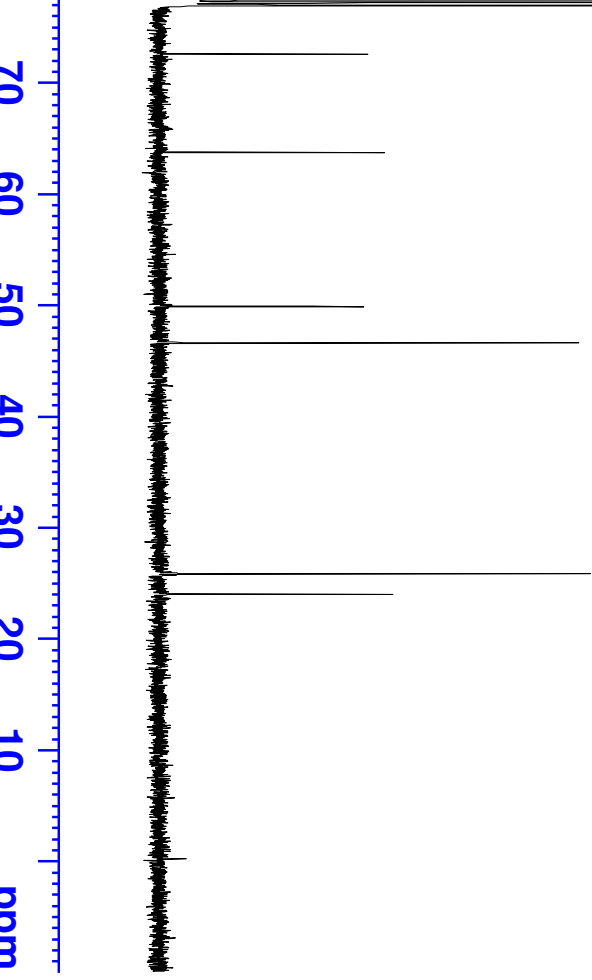

$-83.868$

77.415

$-77.161$

76.907

72.511

$-63.679$

$-49.828$

46.549

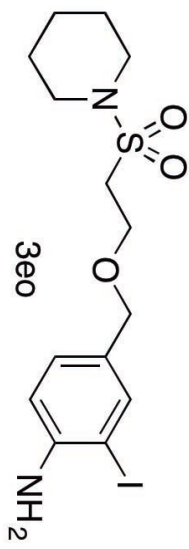

$\omega_{0}=0 \begin{array}{r}25.779 \\ -23.929\end{array}$

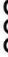




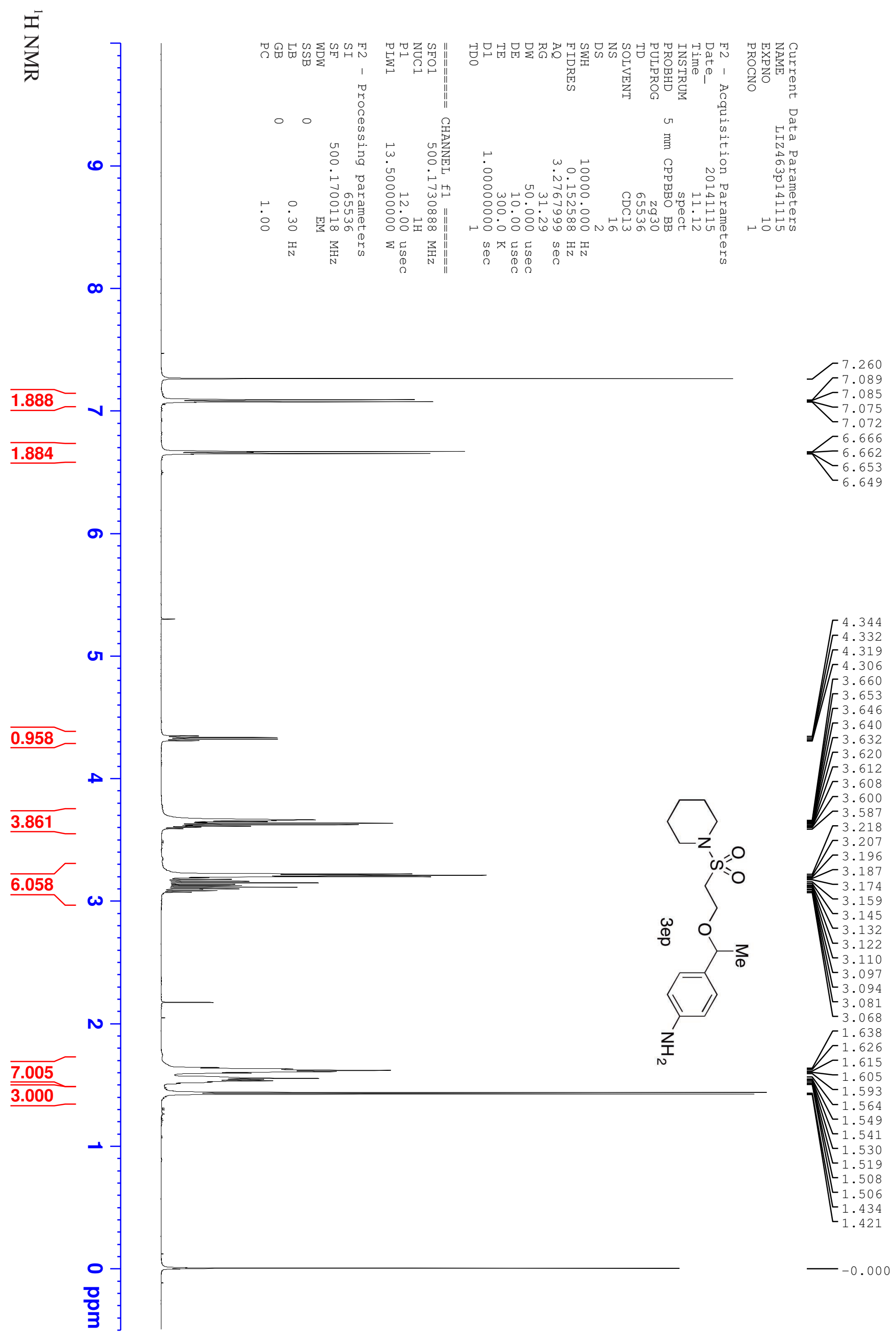




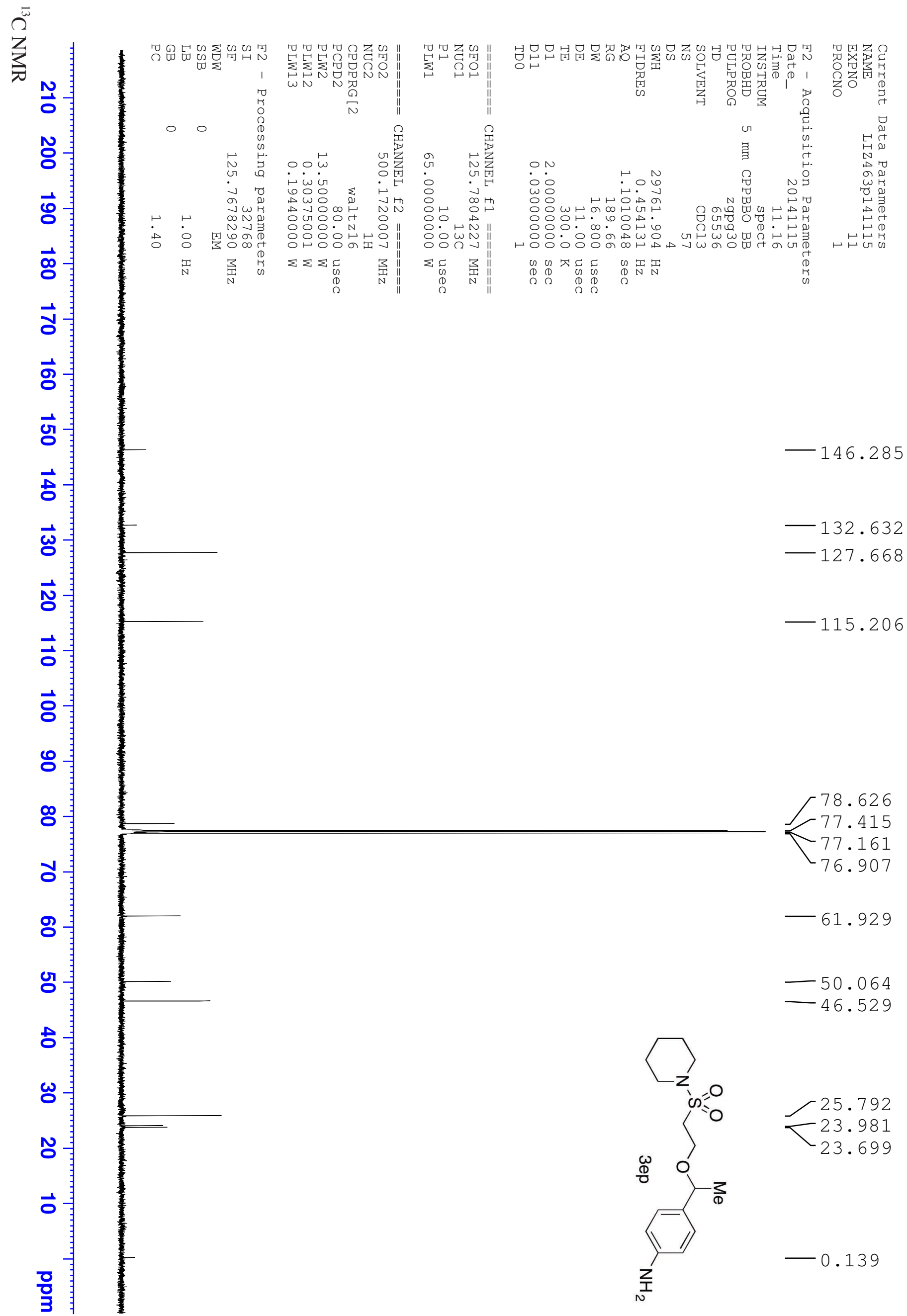




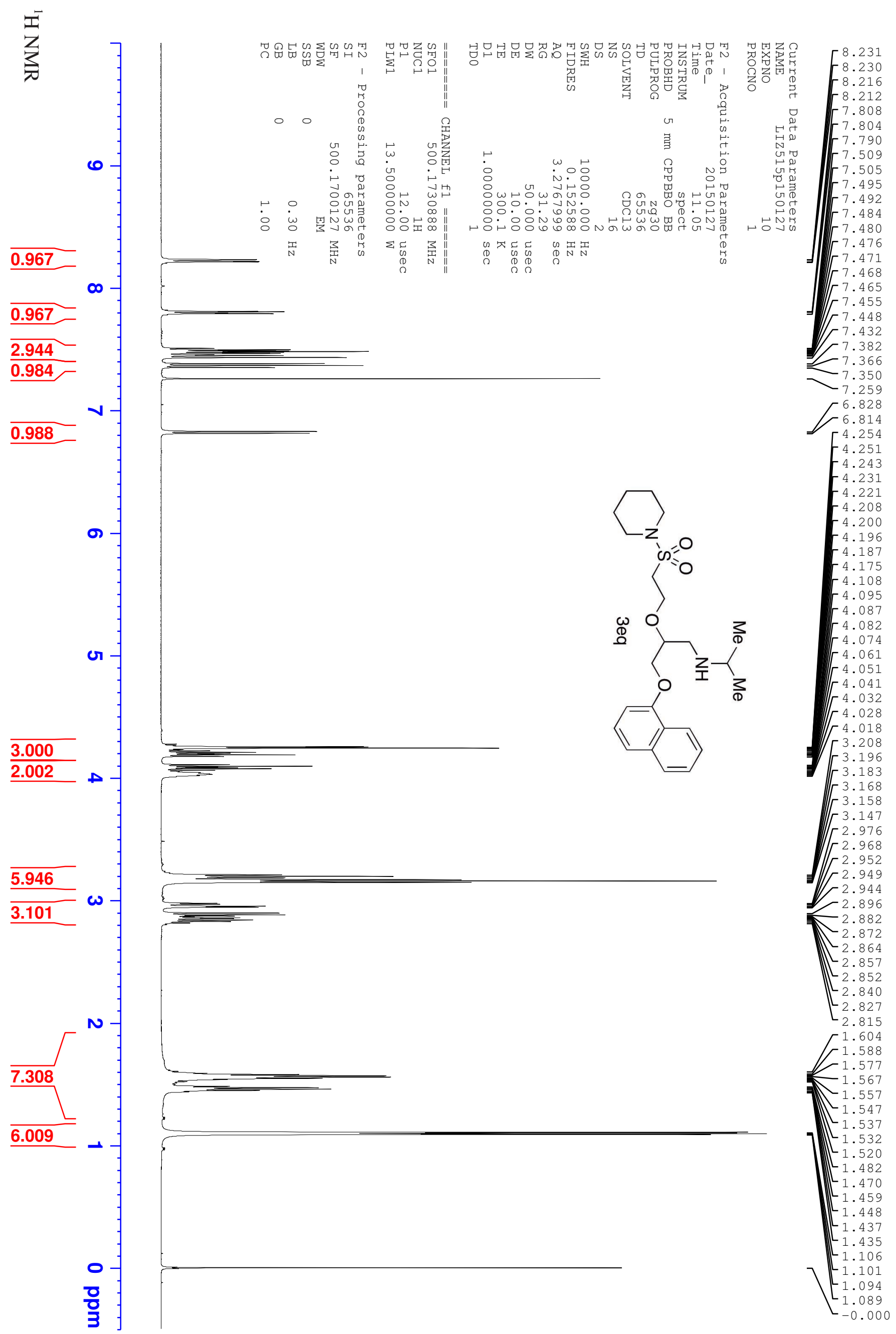




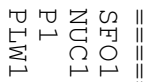

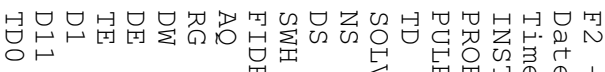

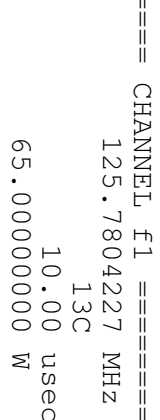

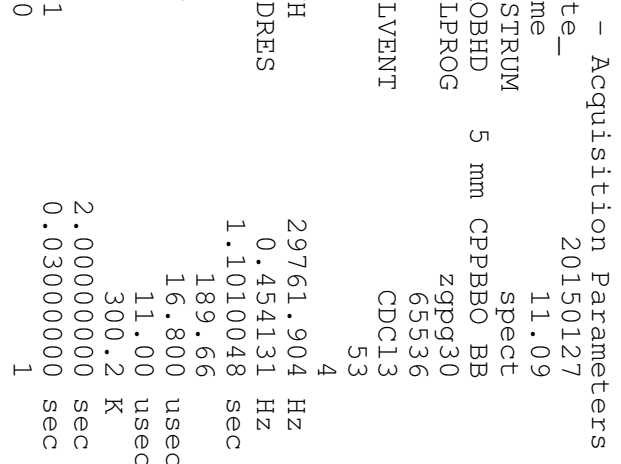

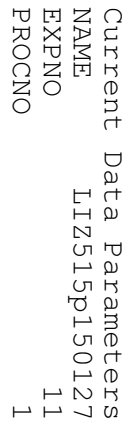

$\overrightarrow{0}$

$\vec{\circ}$

जू

홍

$\vec{\omega}$

$\vec{N}$

$\overrightarrow{8}$

$\overrightarrow{0}$
$\overrightarrow{8}$
8
8

$\overrightarrow{0}$
$\overrightarrow{8}$
8
8

ơ

8

잉

o

$\omega$

ก

$\vec{o}$

흘
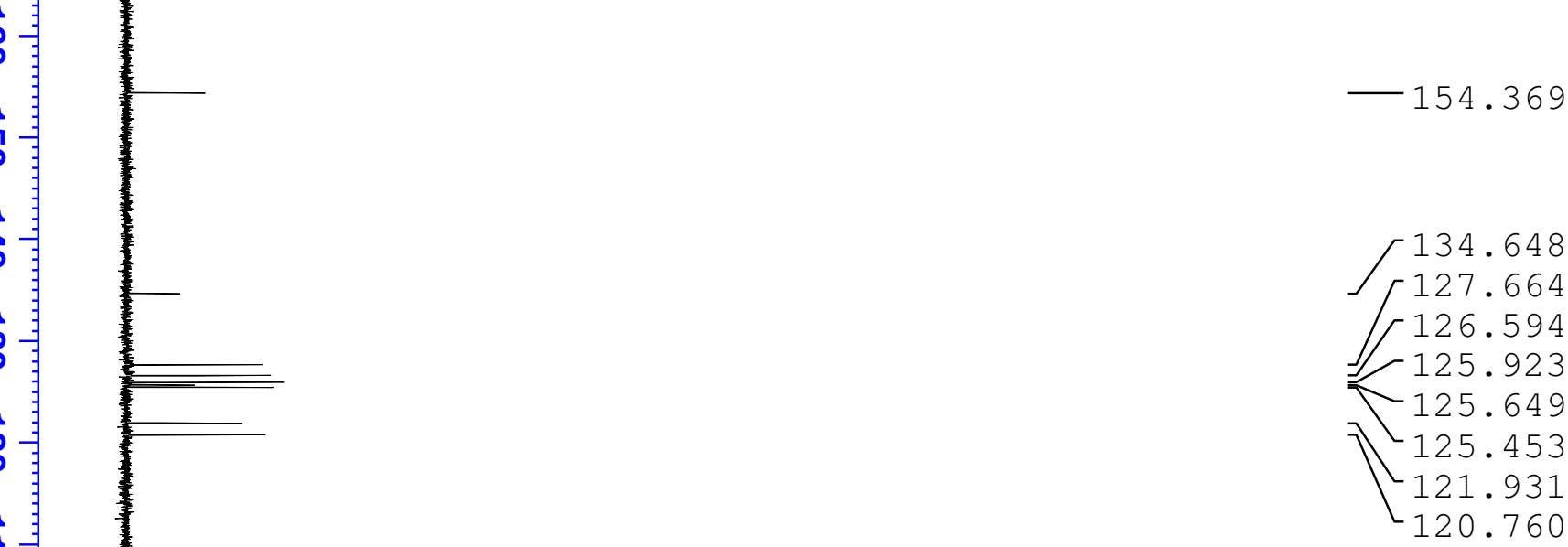

$-104.902$

$\longdiv { 1 }$

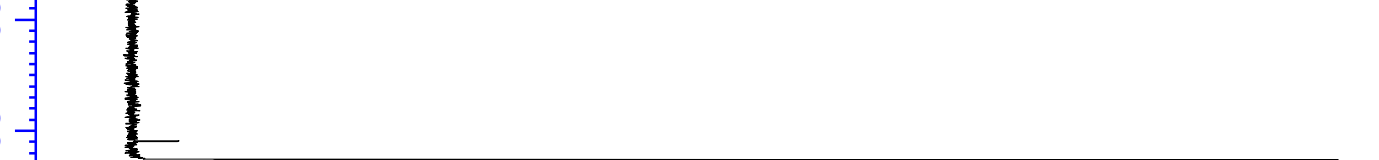

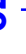
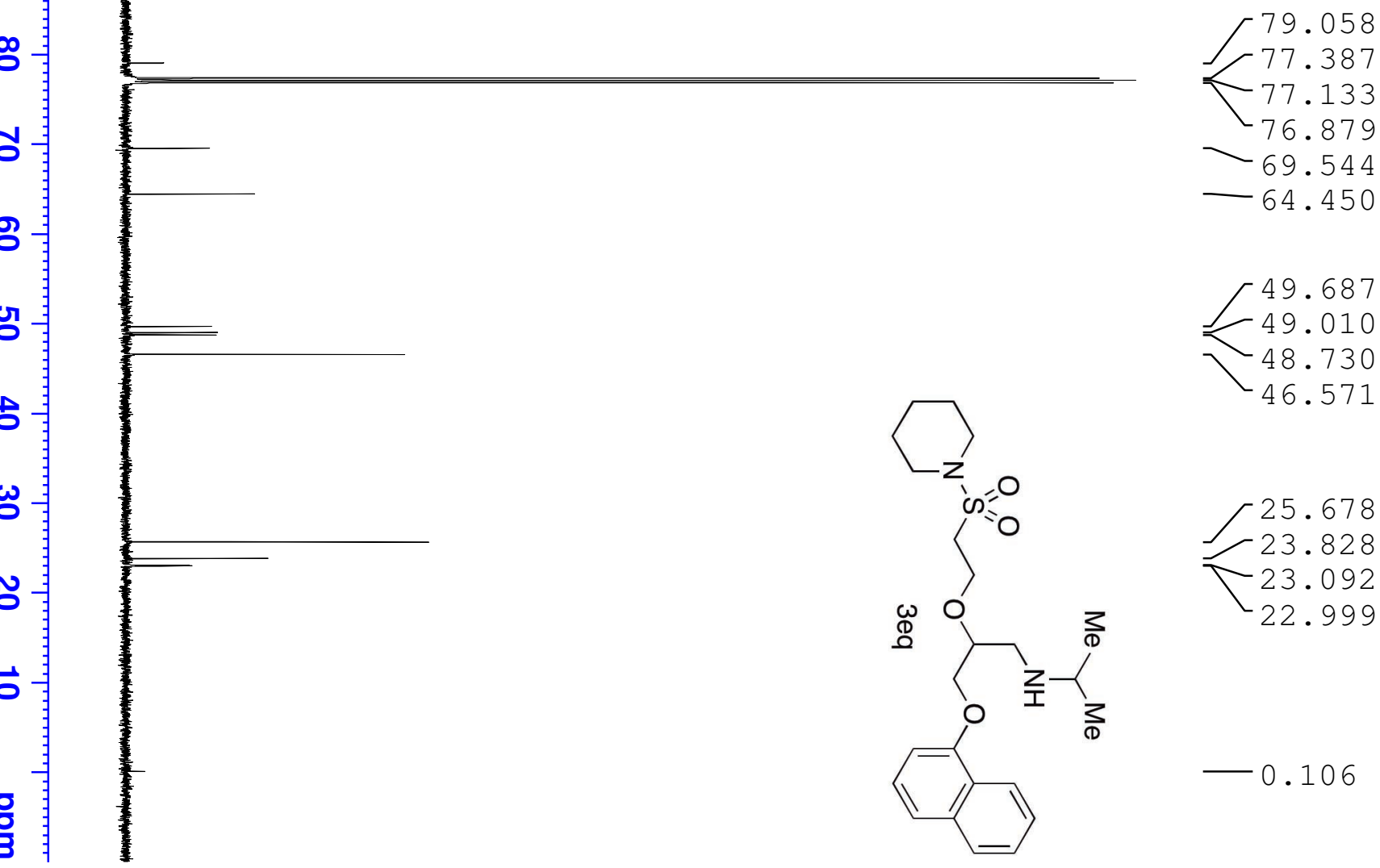

25.678

23.828

23.092

22.999 


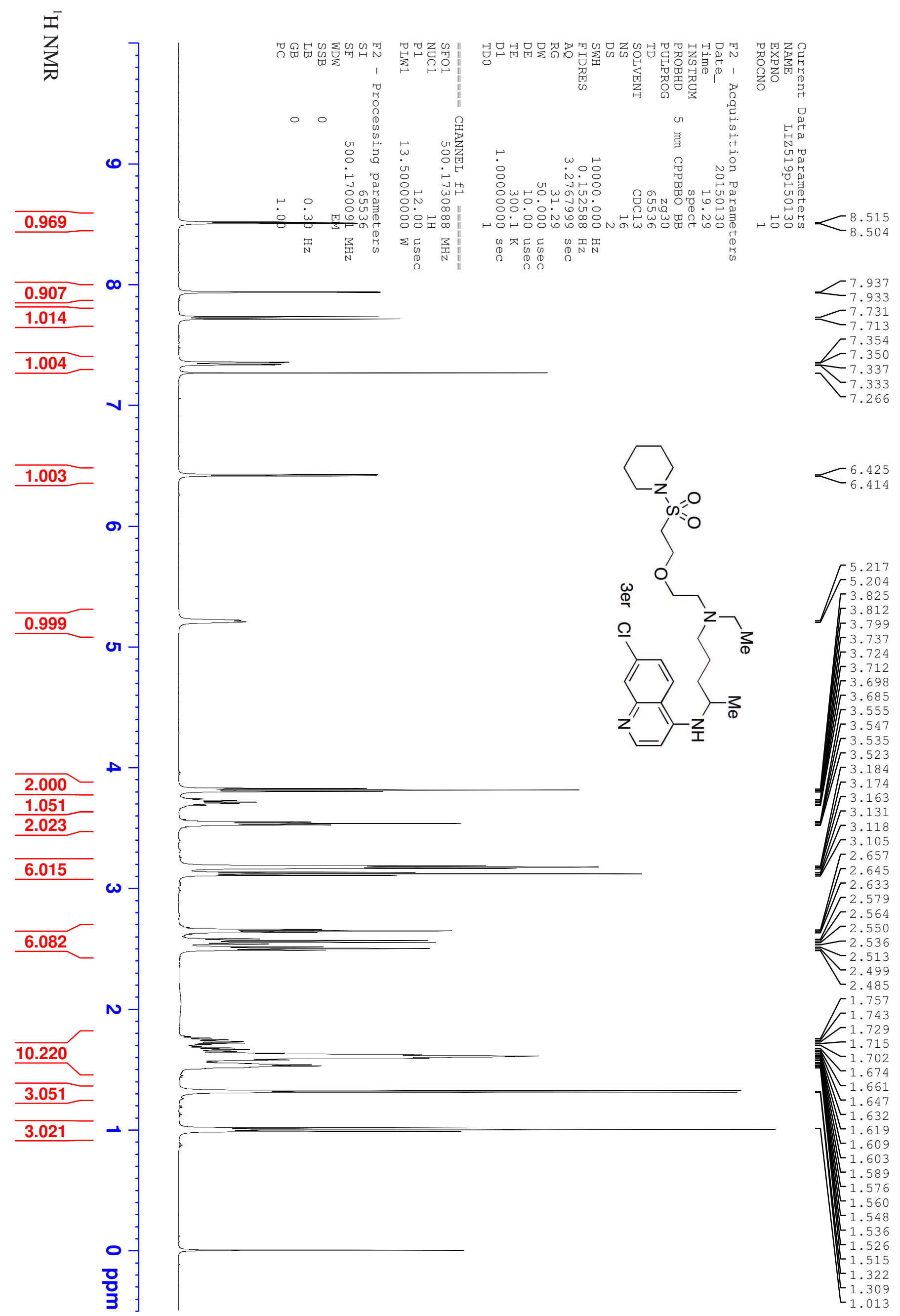




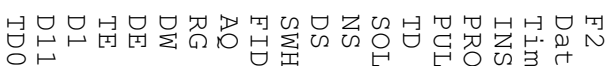
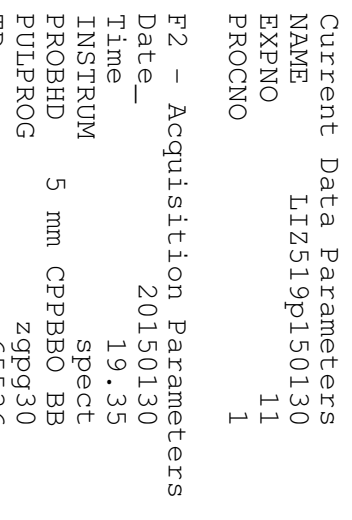

ప

홍

형

152.149

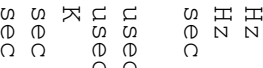

149.561

149.140

$-134.854$

$-128.943$

$-125.087$

$-121.413$

$-117.432$

$\vec{\Delta}$

하

$\overrightarrow{\mathrm{s}}$

$-99.343$

8

$\infty$

ว

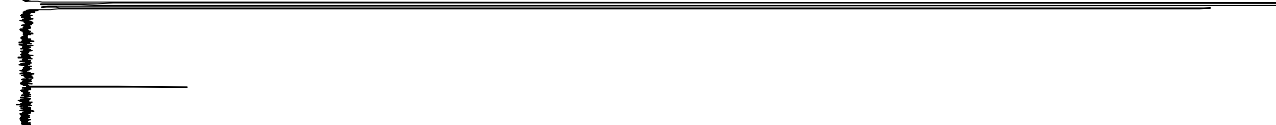

77.389
-77.135

$<77.135$

76.881

$-69.829$

64.772

53.789

52.886

49.354

48.437

$-48.285$

46.542

34.500

25.707

24.016

23.884

20.333

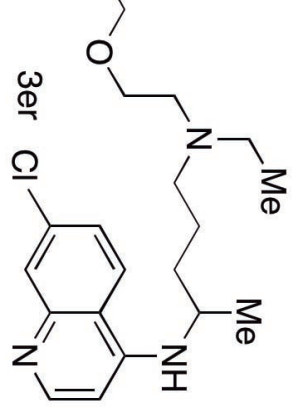

$-11.513$

$\overrightarrow{0}$

흘

$-0.103$ 


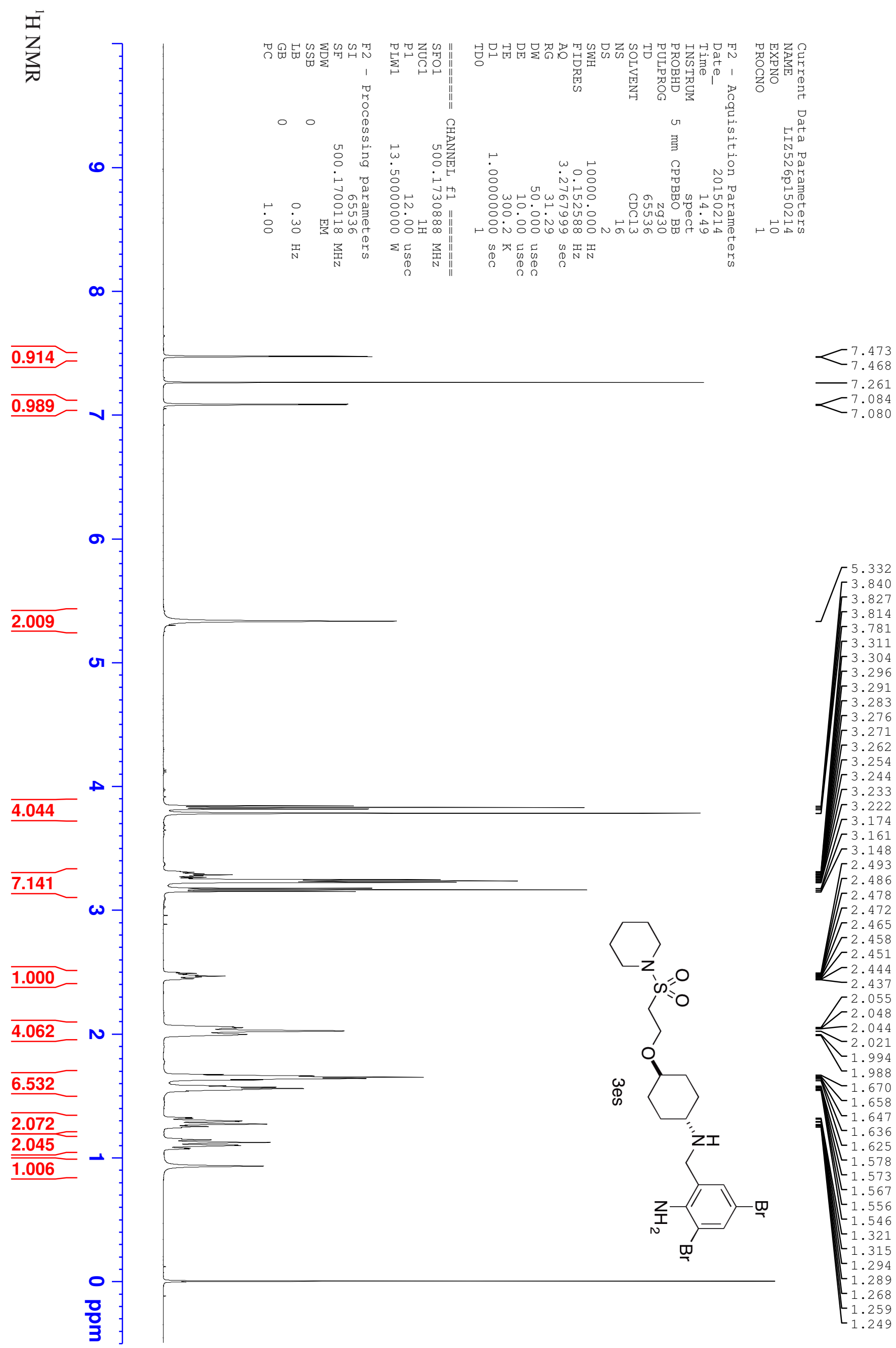




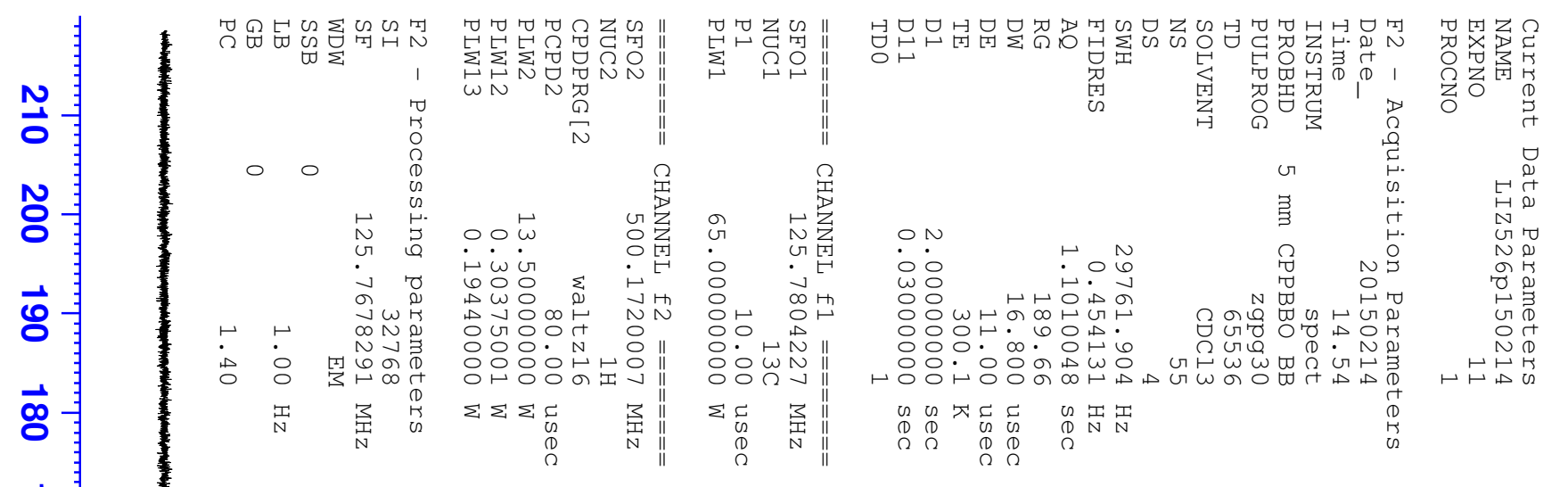

홍

횡

吾

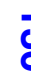

$\vec{\delta}$

$\vec{\tilde{}}$

하

$\overrightarrow{8}$

8

ø

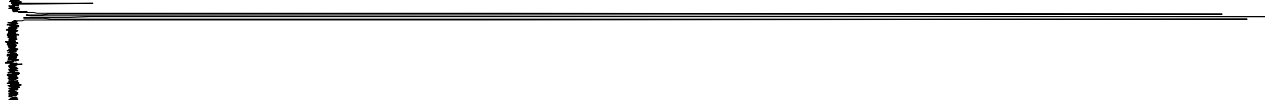

78.334

77.412

77.158

76.905

o

$-61.945$

8

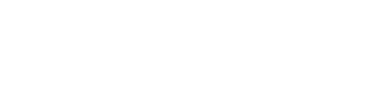

$-55.632$

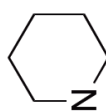

50.770

읭

o

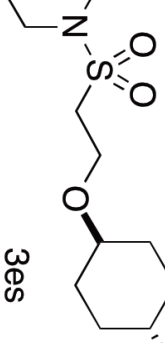

50.132

46.596

$\omega$

ก

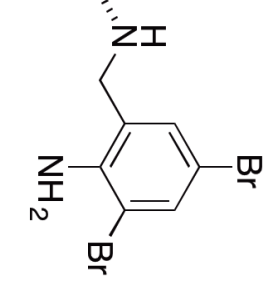

$-0.138$

翋

$\overrightarrow{0}$

음

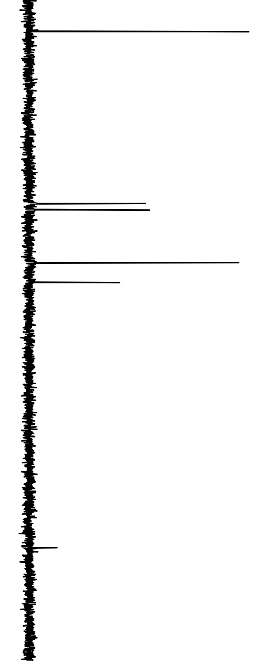



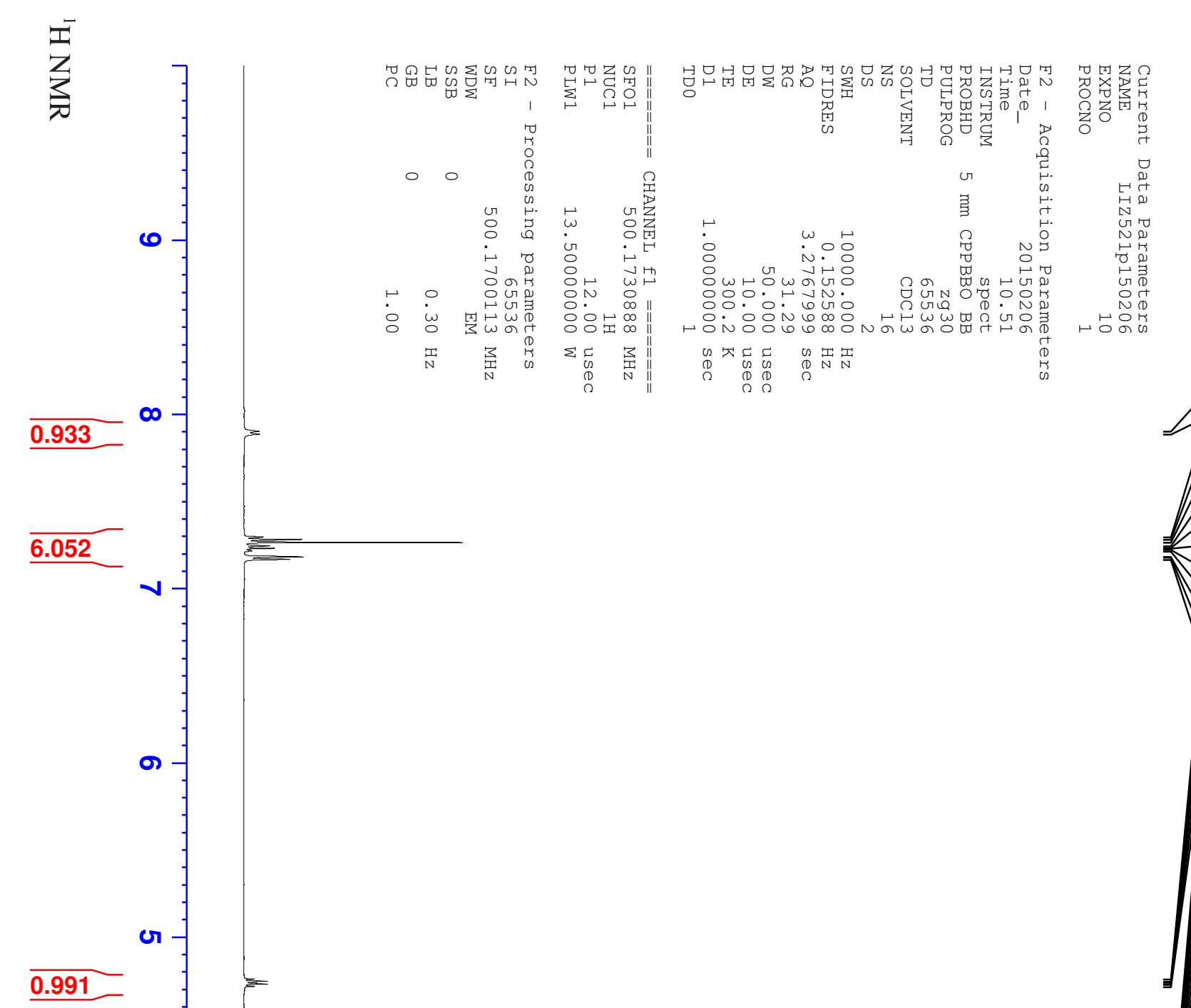

7.900 $-7.883$ 7.292 7.242
-7.233
7.228 7.228 $-7.222$ 7.213 7.181 7.179 4.756 4.743 4.739 4.731 $-4.727$

4.714 3.884 3.871 3.863 3.858 3.850 $-3.845$

3.837

3.837
-3.833
3.824 3.820 3.812 3.799 3.639
-3.630 3.639
-3.630 3.621 3.612
3.606 $r^{3.606}$ 3.594
3.588 3.588
-3.575 3.575 W 4.124 4.125 $\omega$
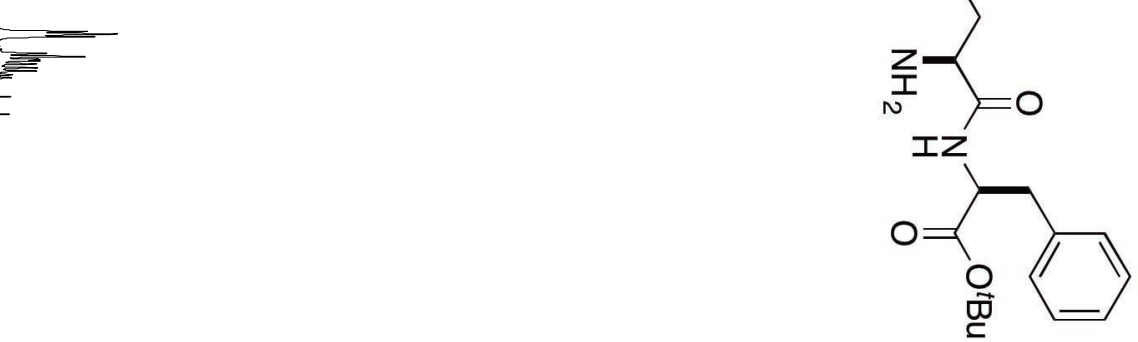

$\mathbf{-}\left[\begin{array}{l}3.533 \\ 3.531 \\ 3.522\end{array}\right.$

$-3.222$

3.211

3.200

3.132

3.131

3.120

3.118

3.108

3.105

$-3.101$

$-3.089$

$-3.072$

3.060
-3.045
3.032

3.032

1.669

1.658

1.647

1.636

1.626

$-1.583$

1.577

$-1.571$

1.561

$-1.550$

$-0.000$

응 


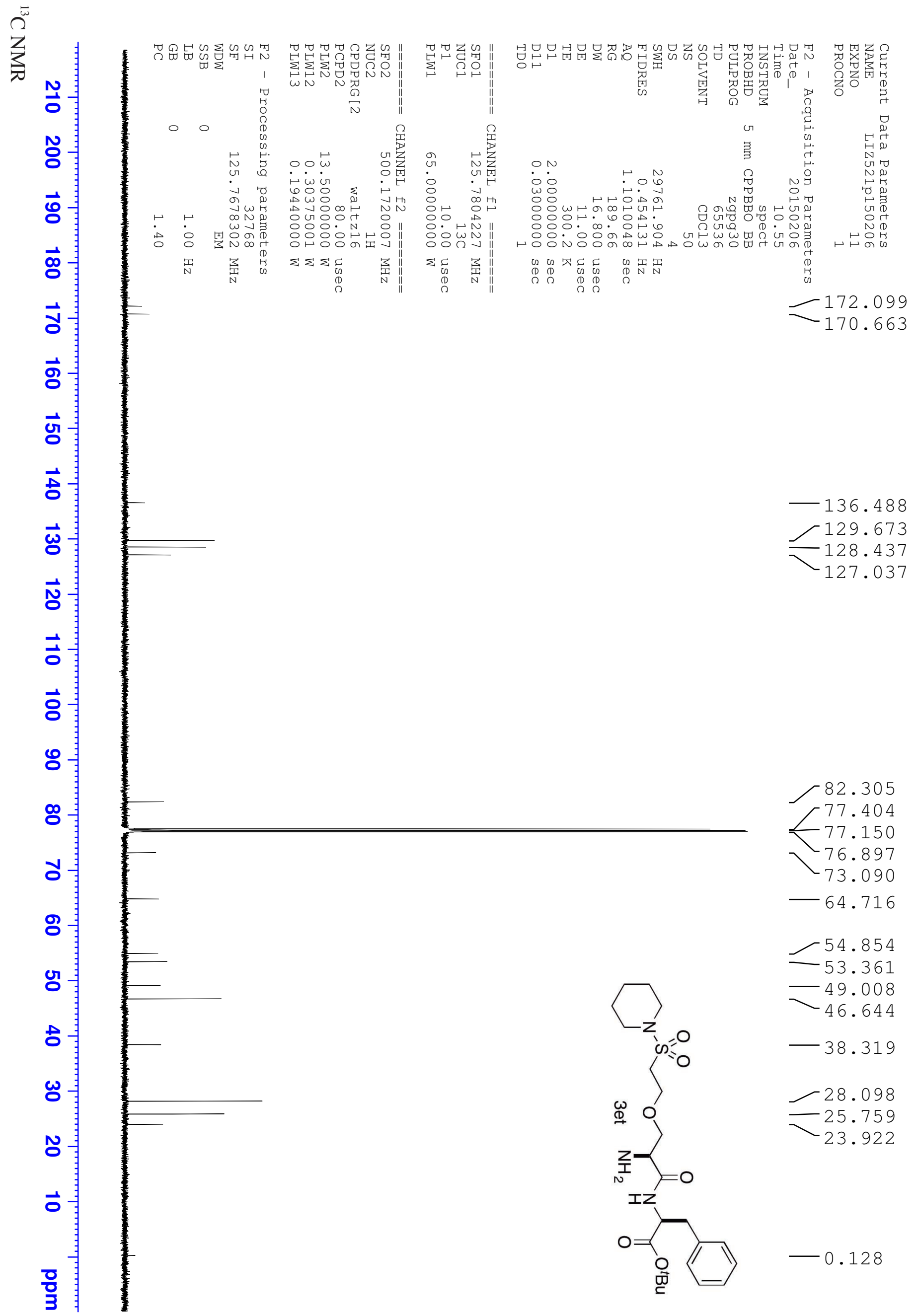




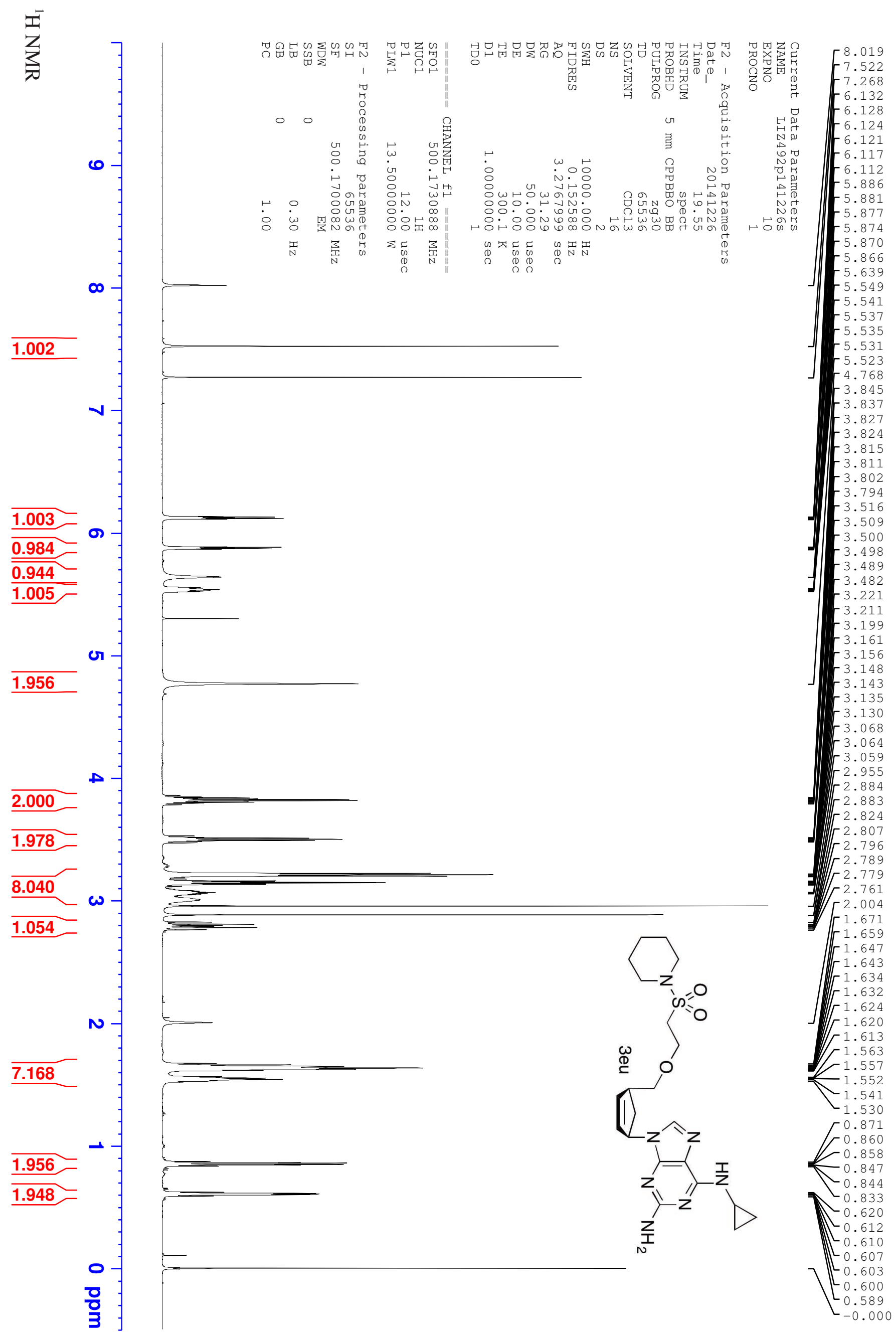




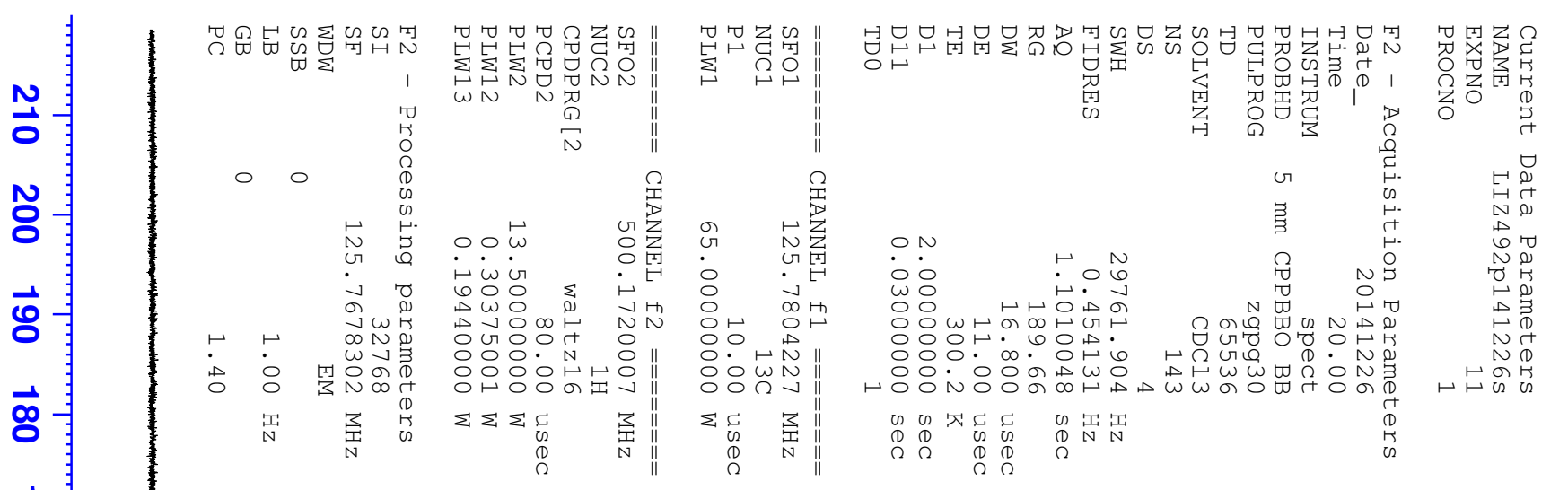

ป

홍

$-162.641$

$-160.063$

$-156.401$

ज

ज

151.153

\section{뭉}

c్

N

$\vec{a}$

용

$\varnothing$

$\checkmark$

8

읭

o

w

N

$\overrightarrow{0}$

밀

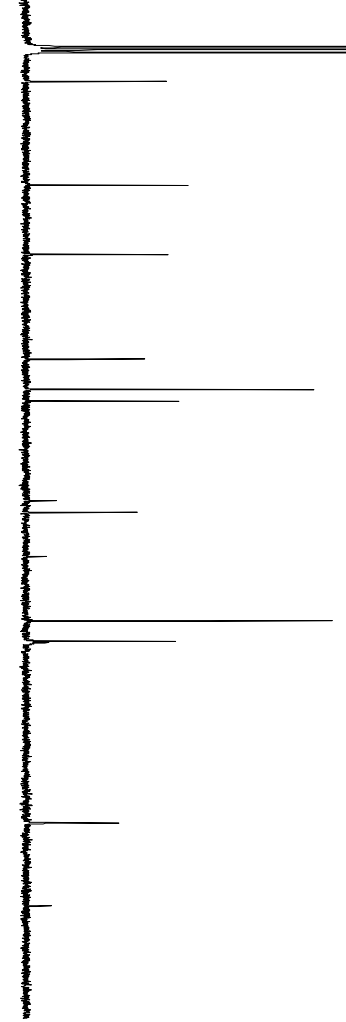




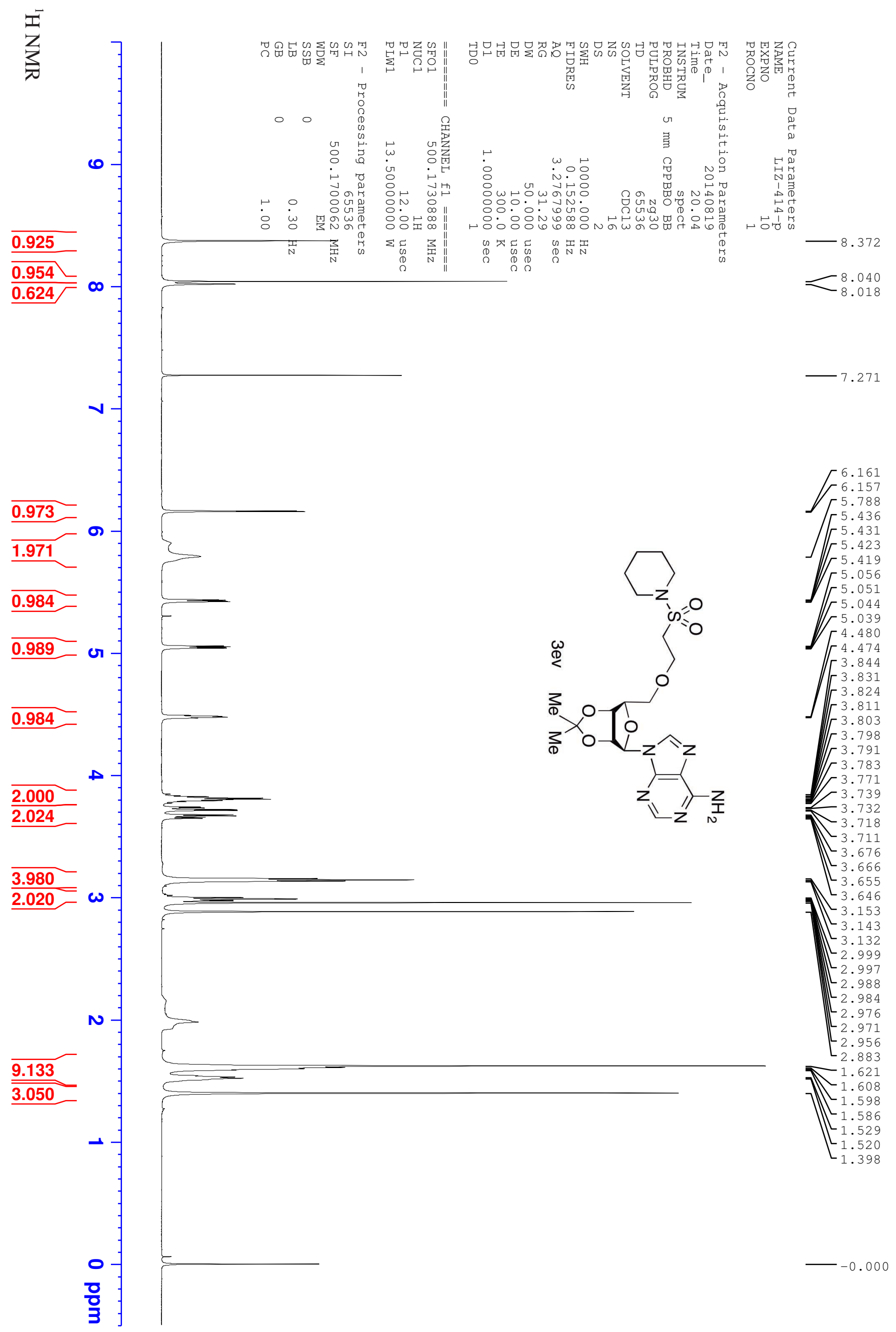



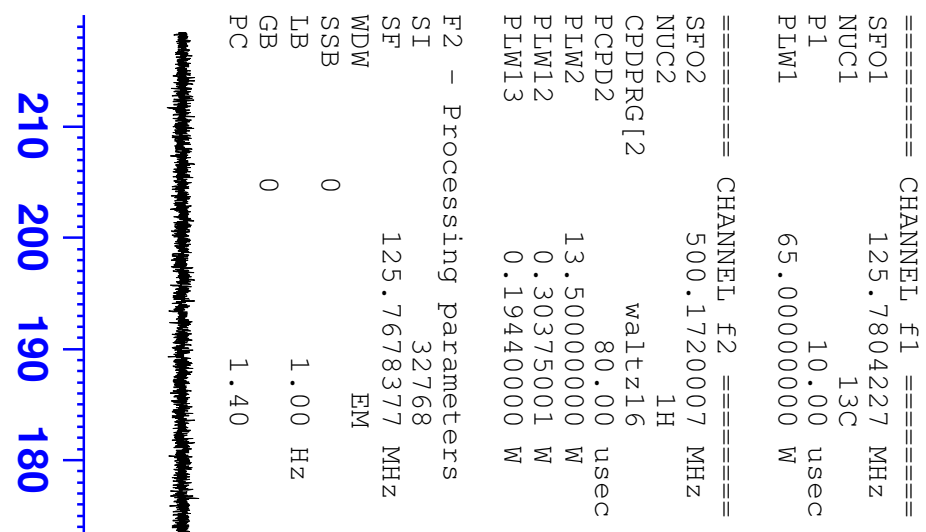

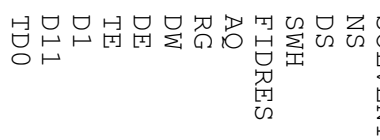

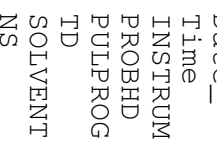

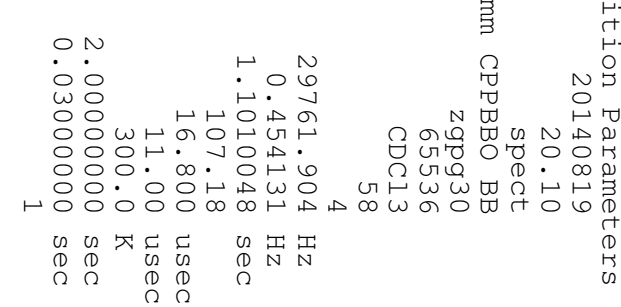

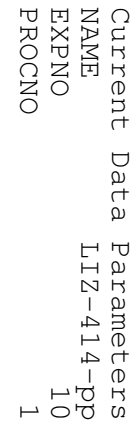

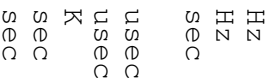

162.617

$-155.750$

$-153.227$

$-149.545$

$\overrightarrow{0}$

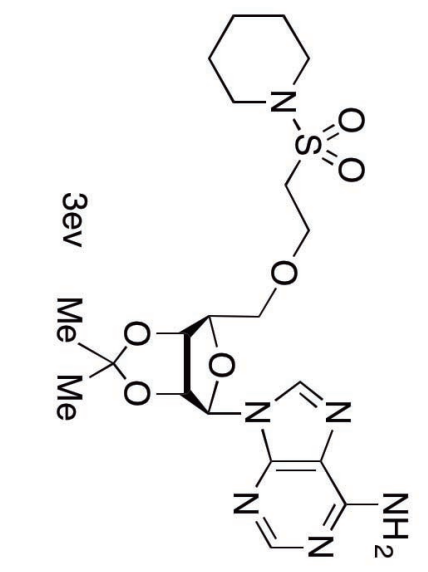

$-139.616$

$-120.022$

$-114.271$

$\overrightarrow{\overrightarrow{0}}$

$\vec{\circ}$

๕

$\stackrel{\infty}{\circ}$

$\omega$

ป઼

8

잉

\&

ఱ

N

o
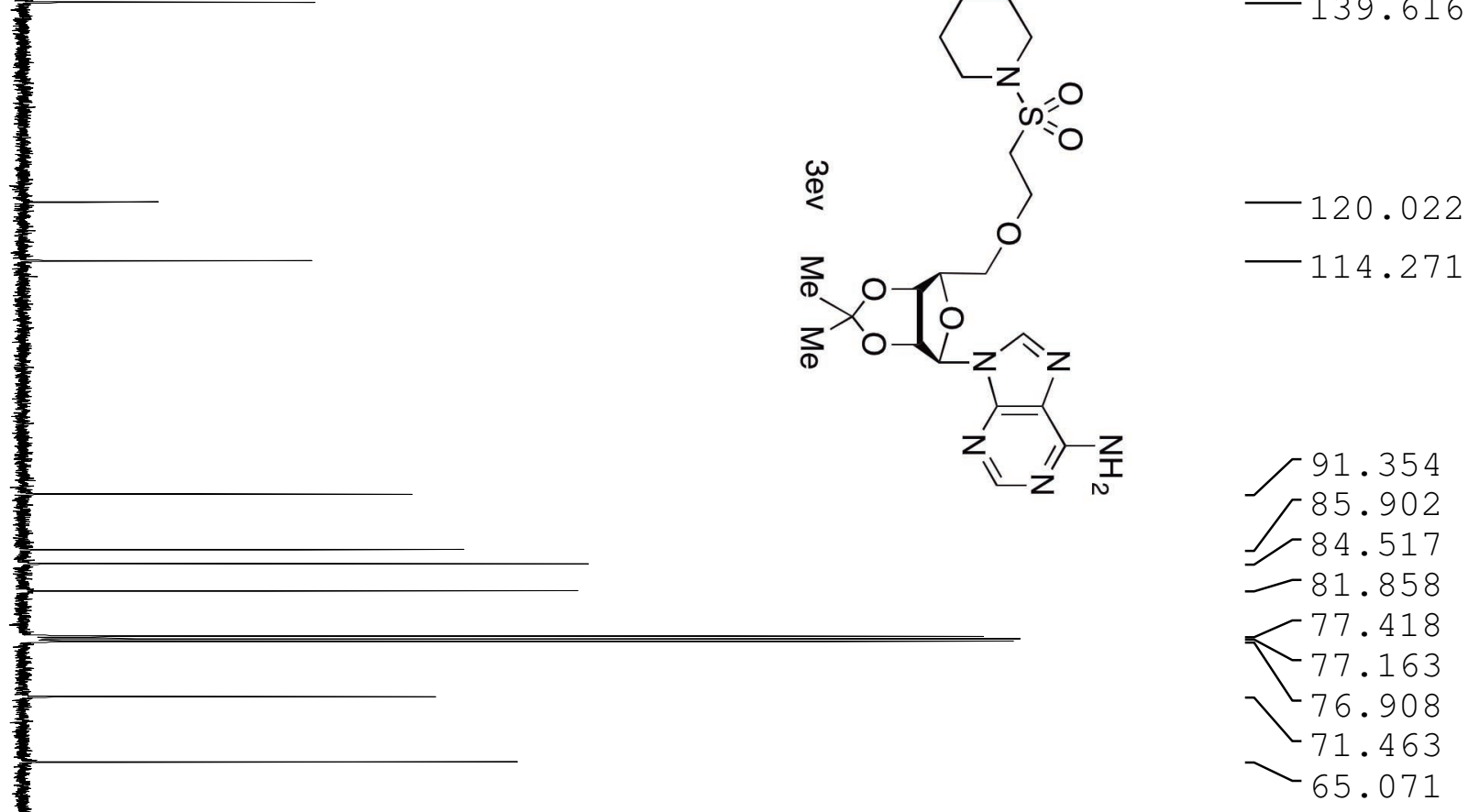

$-48.552$

46.468

$-36.538$

31.488

27.190

25.606

25.380

23.734

$\frac{0}{3}$

$-0.050$ 


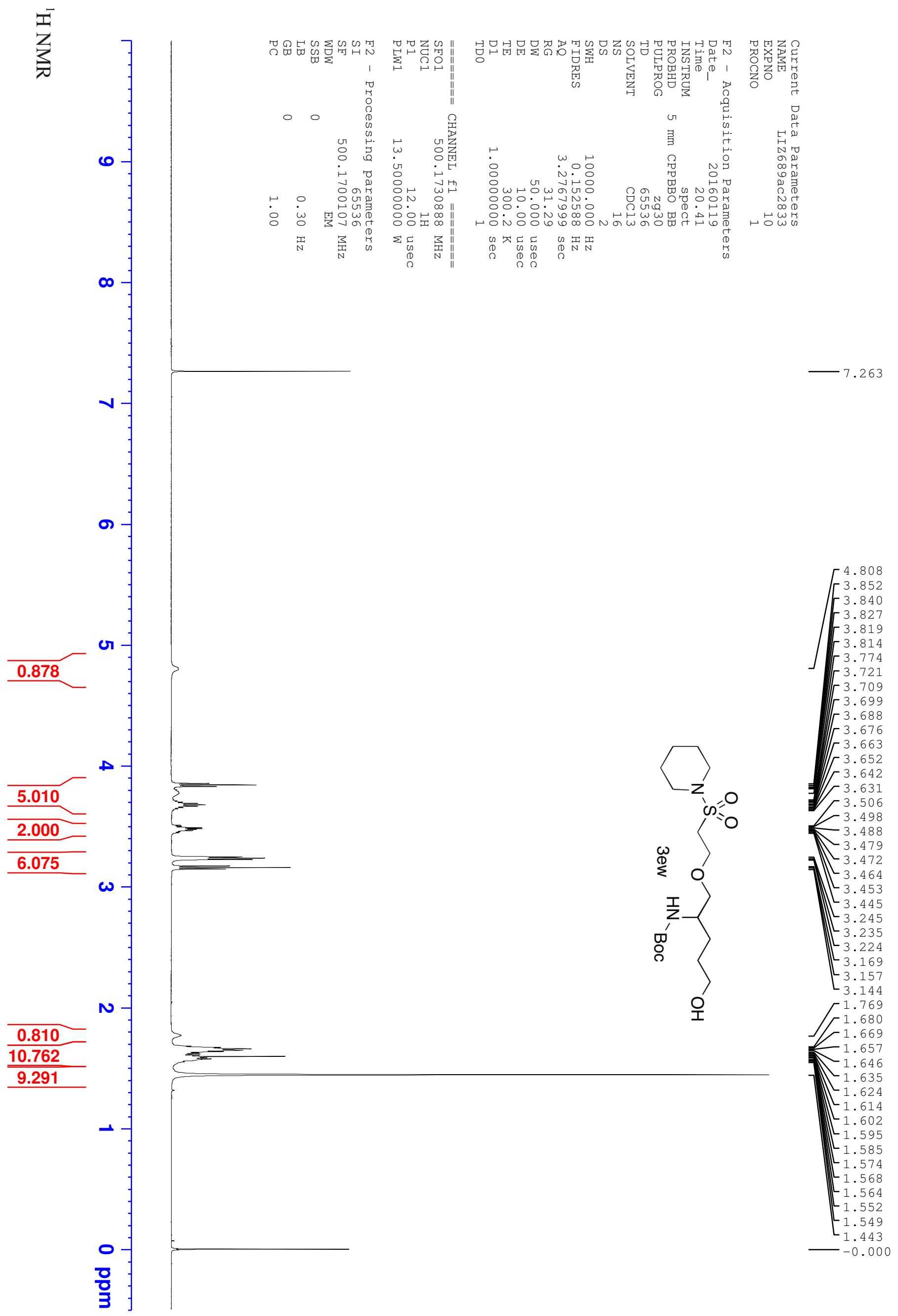




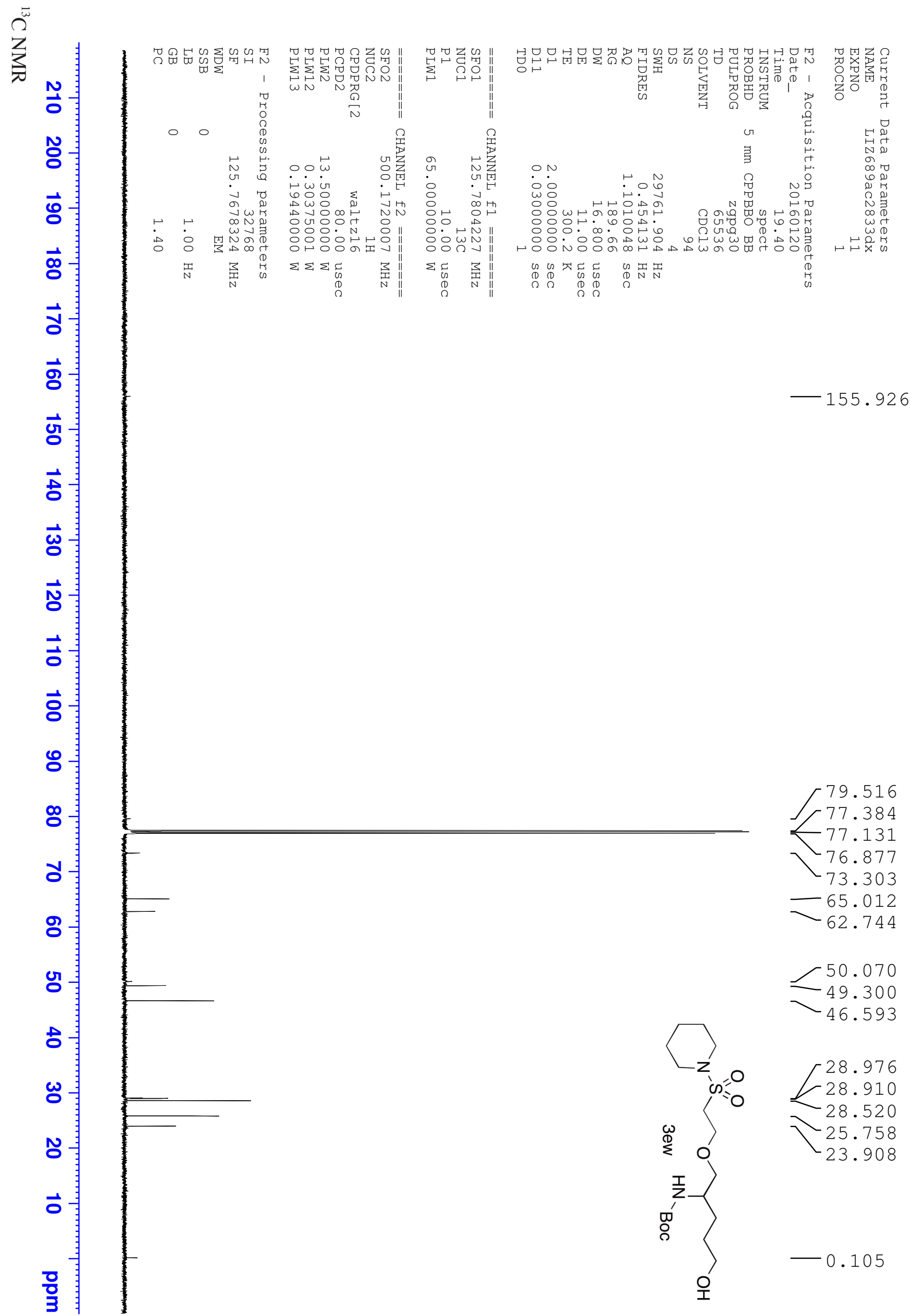



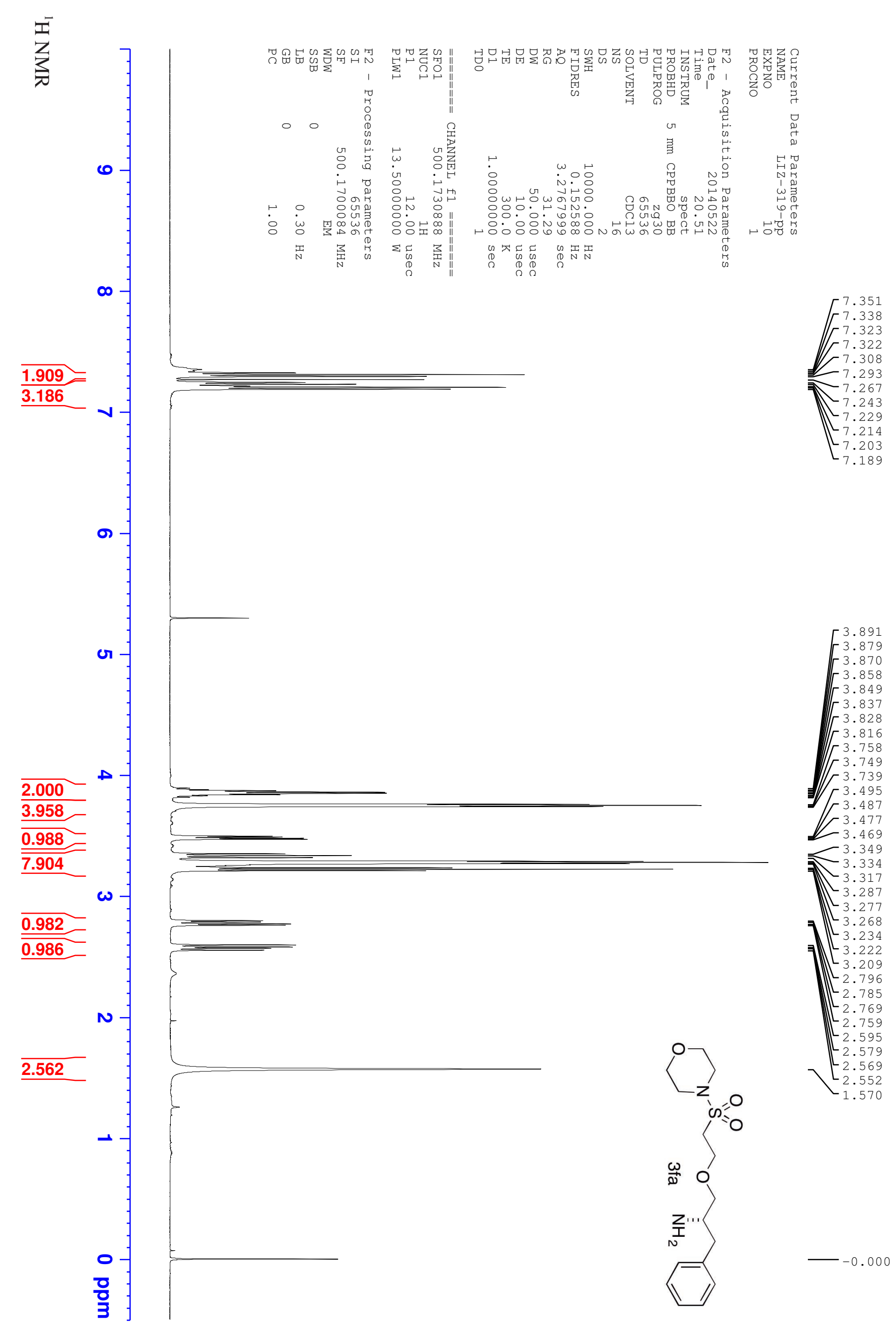


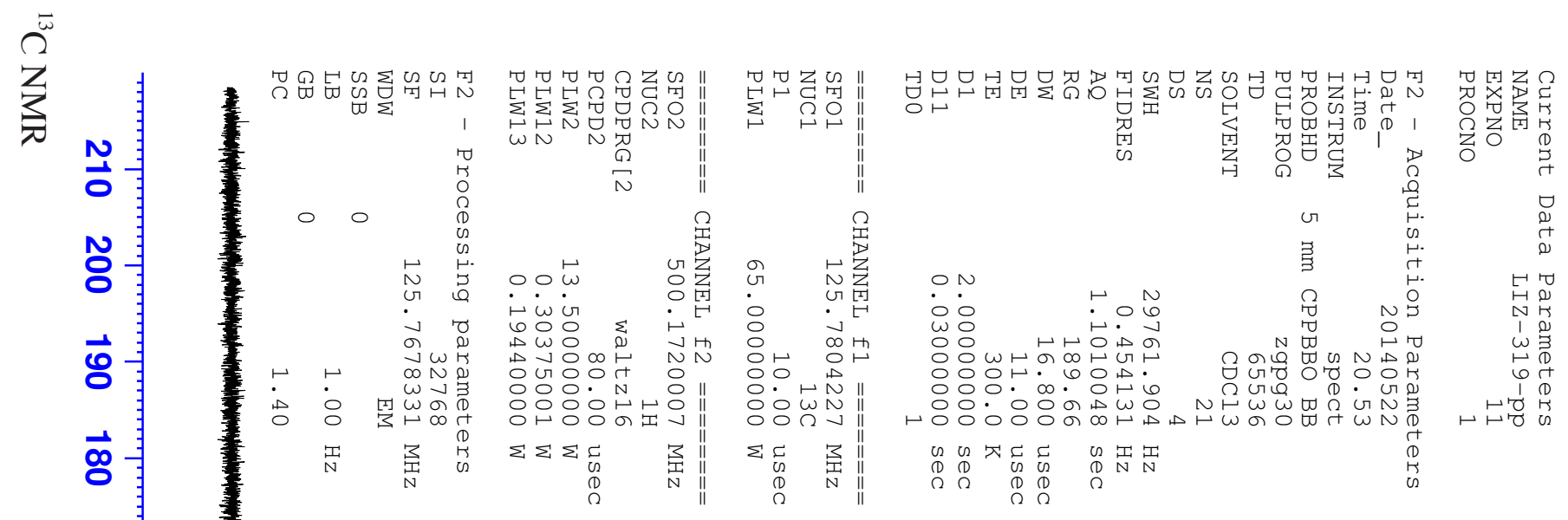

ป

$\overrightarrow{8}$

जे

$\vec{t}$

$\vec{\omega}$

กิ

$\overrightarrow{0}$

$\overrightarrow{8}$

8

ø

o

8

잉

t

$\omega$

ก

$\overrightarrow{0}$

밉

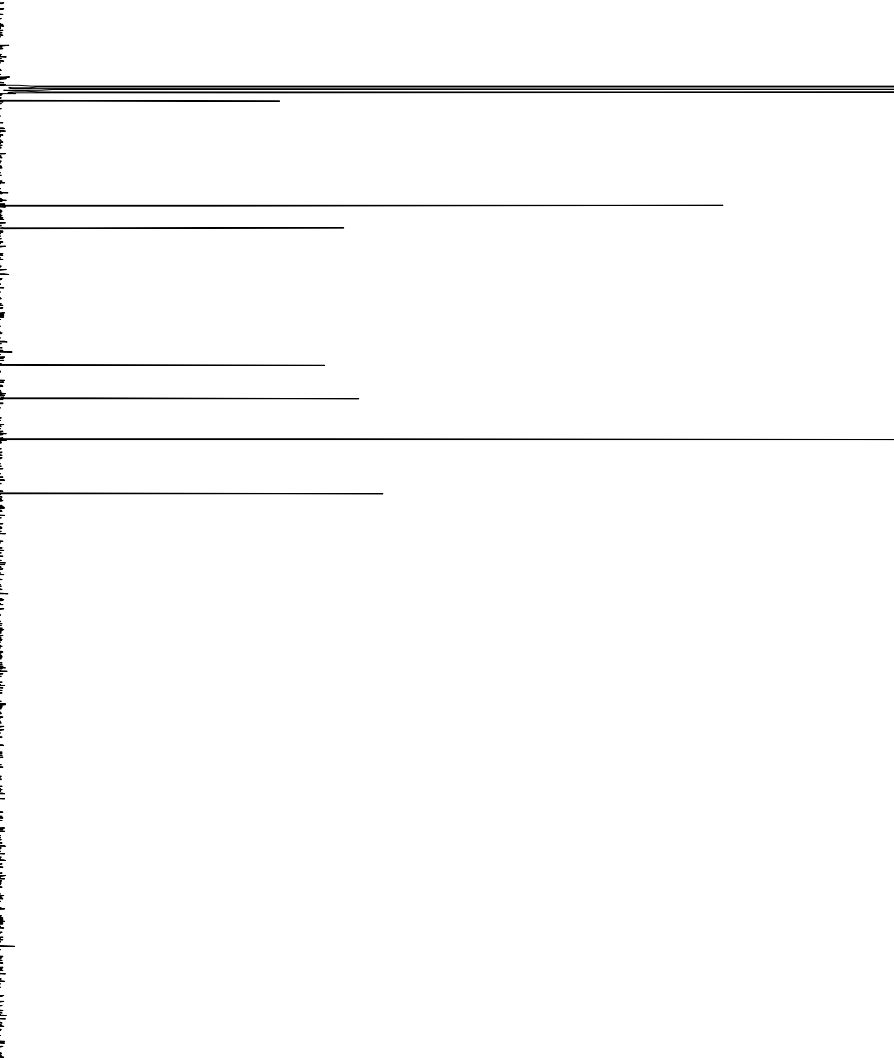

$\begin{array}{r}77.411 \\ -77.157 \\ \hline\end{array}$

77.157

$-76.903$

76.097

-66.693
-64.694

$-52.331$

49.367

$-45.697$

$-40.801$ 


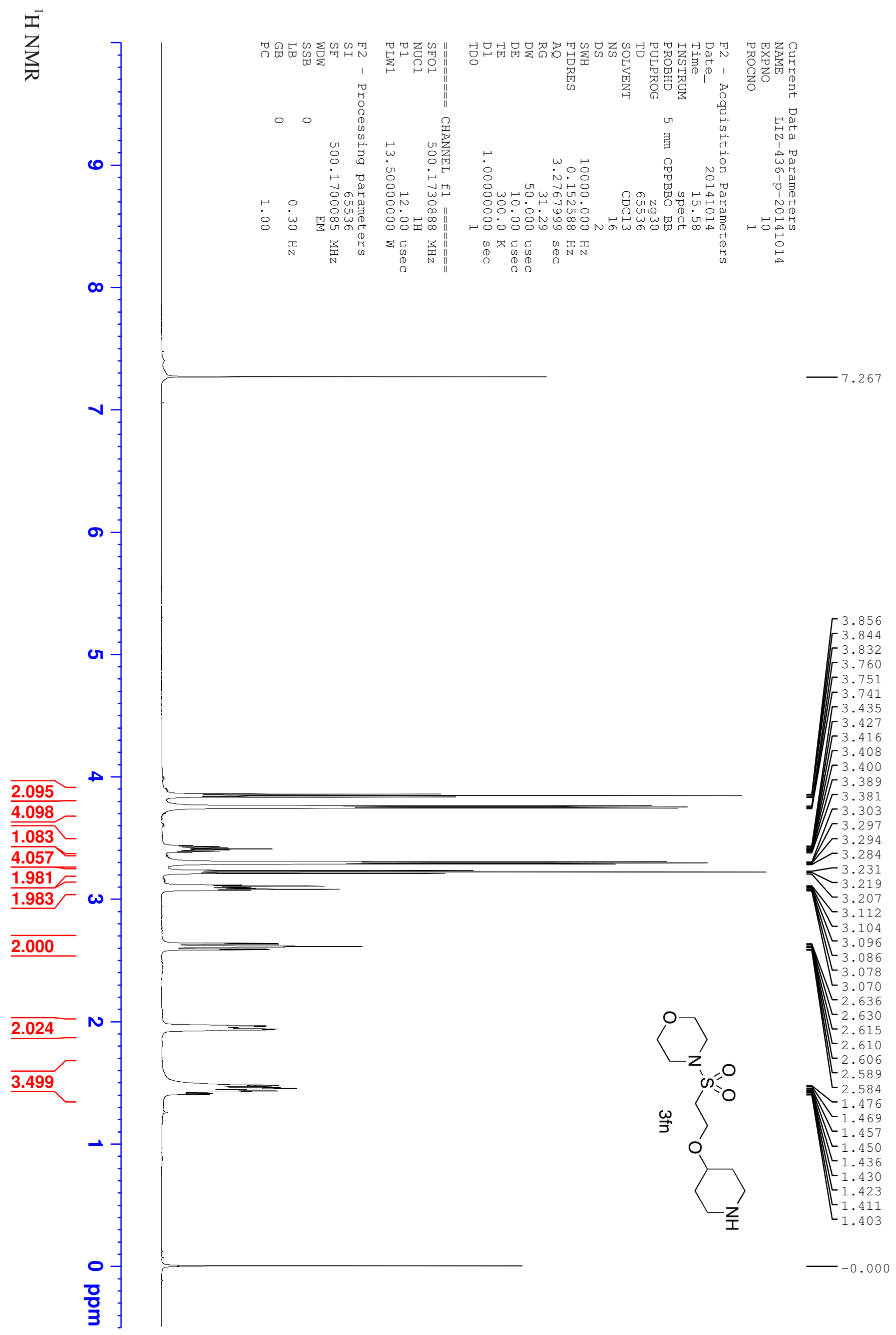




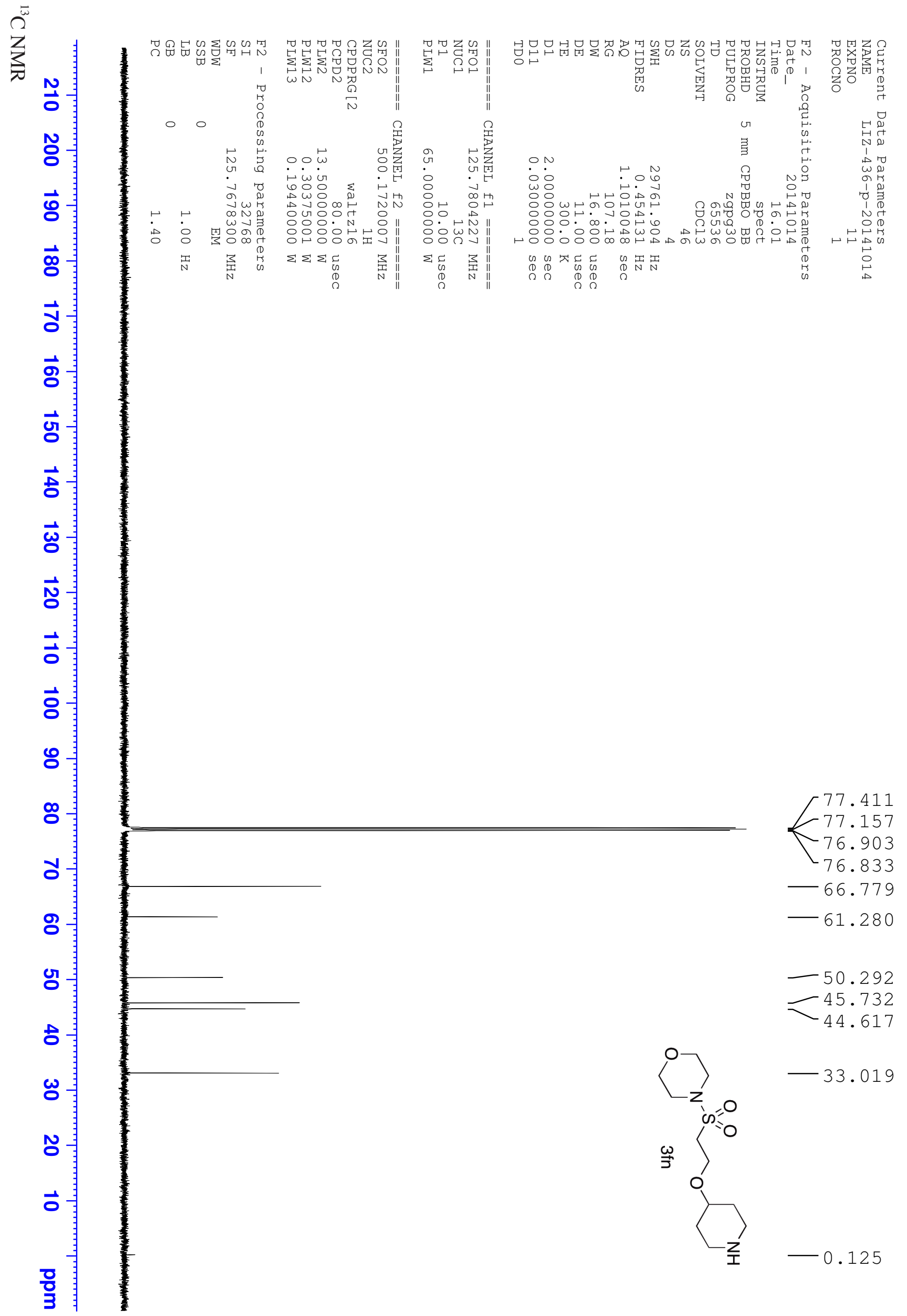




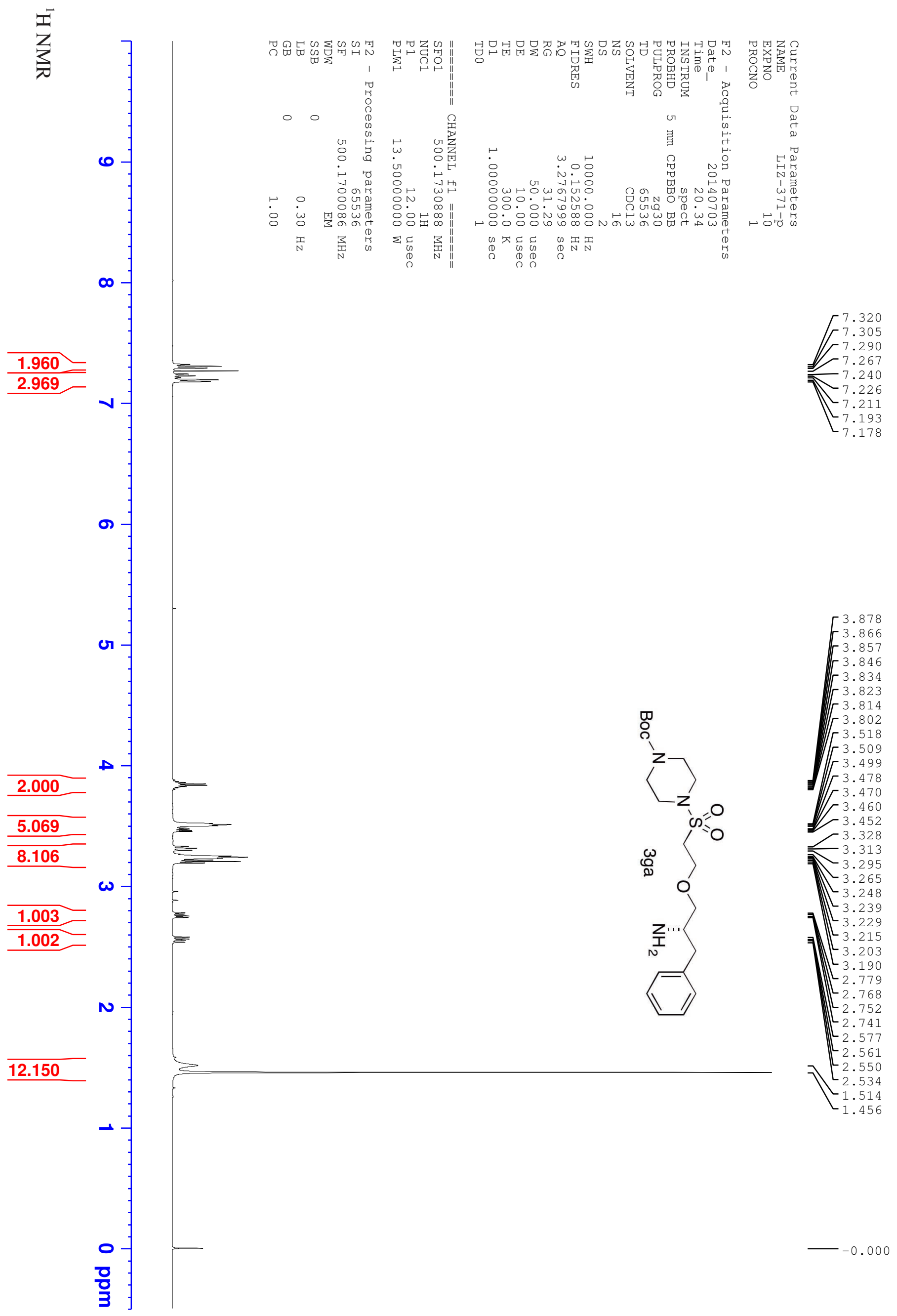




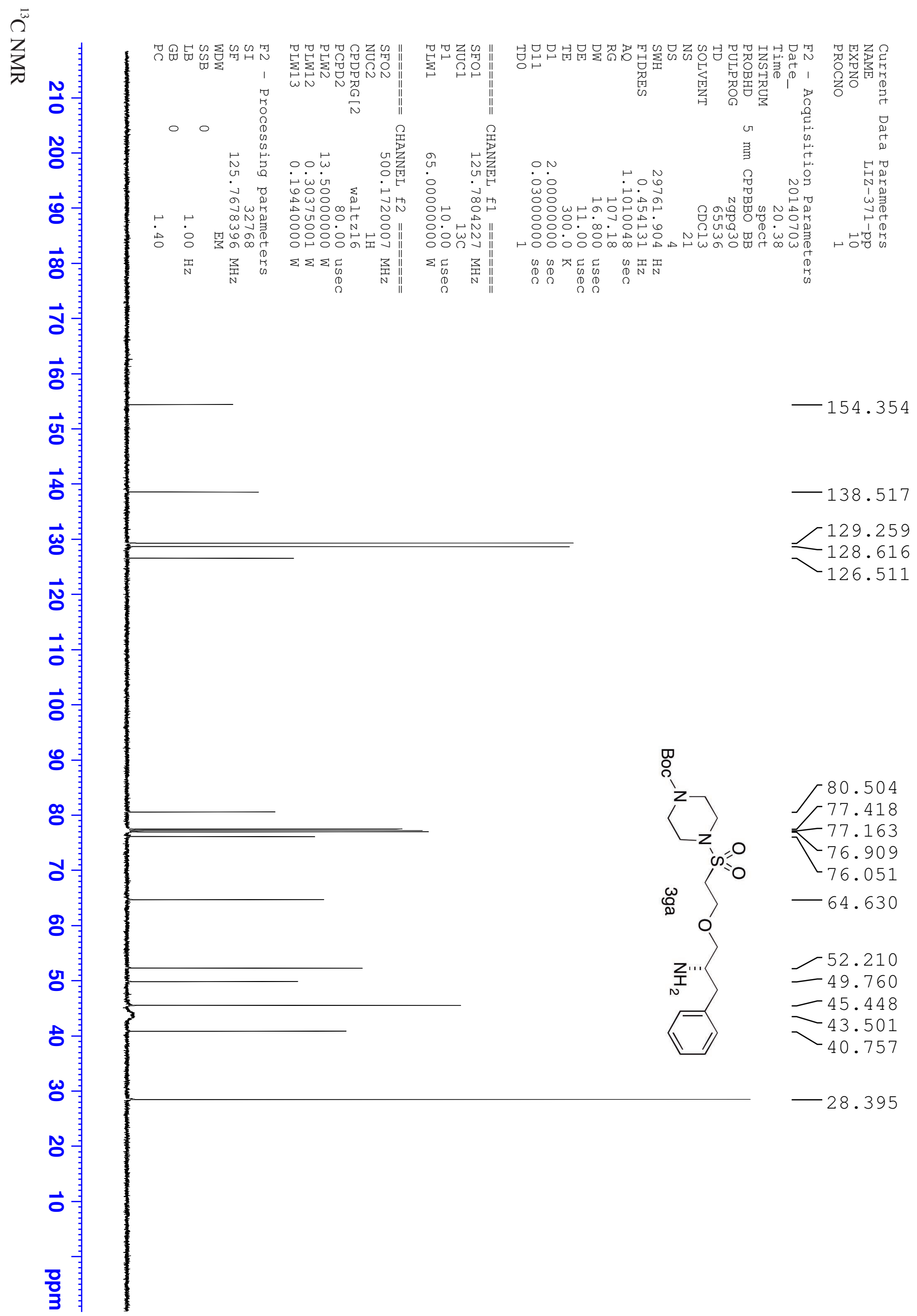




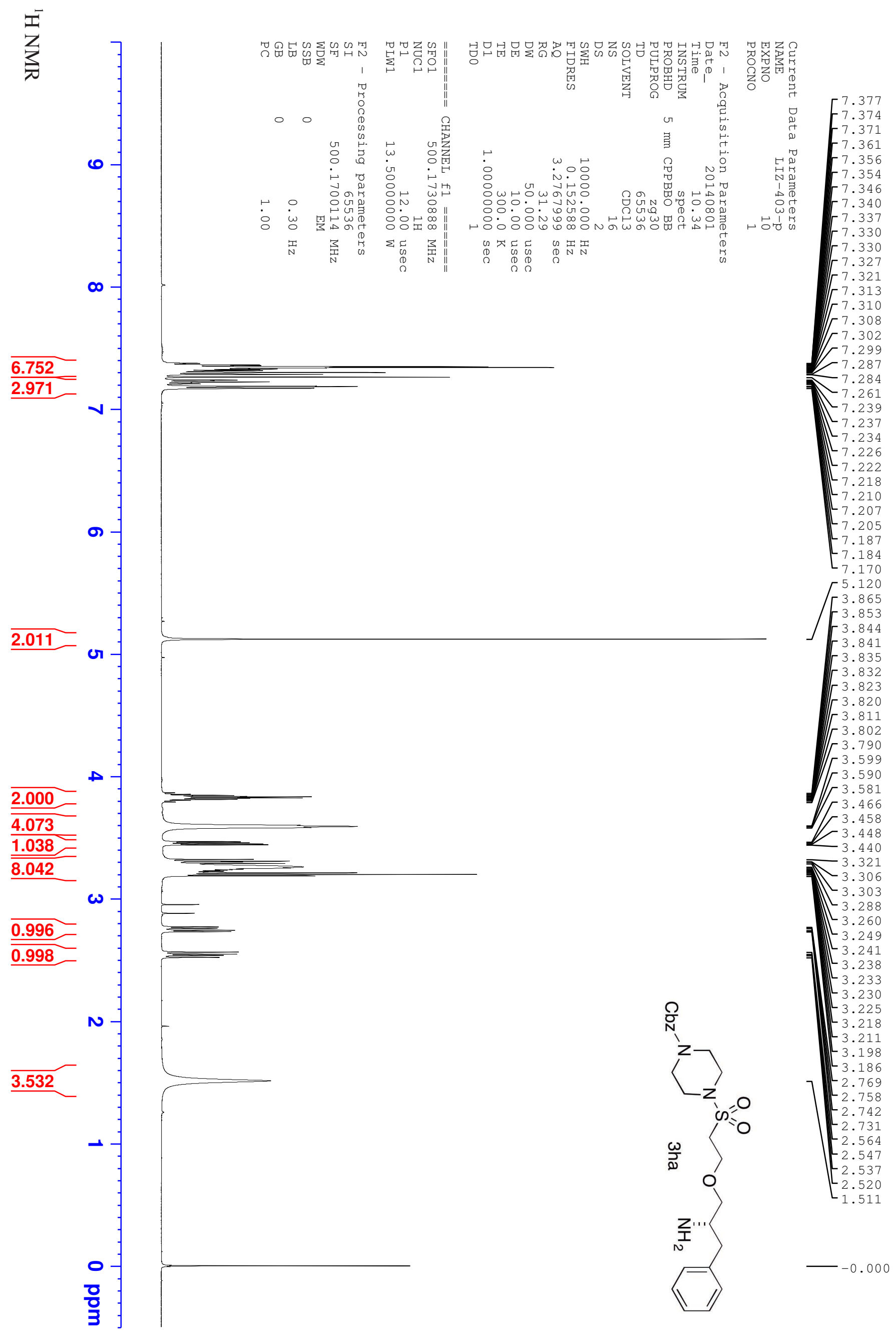




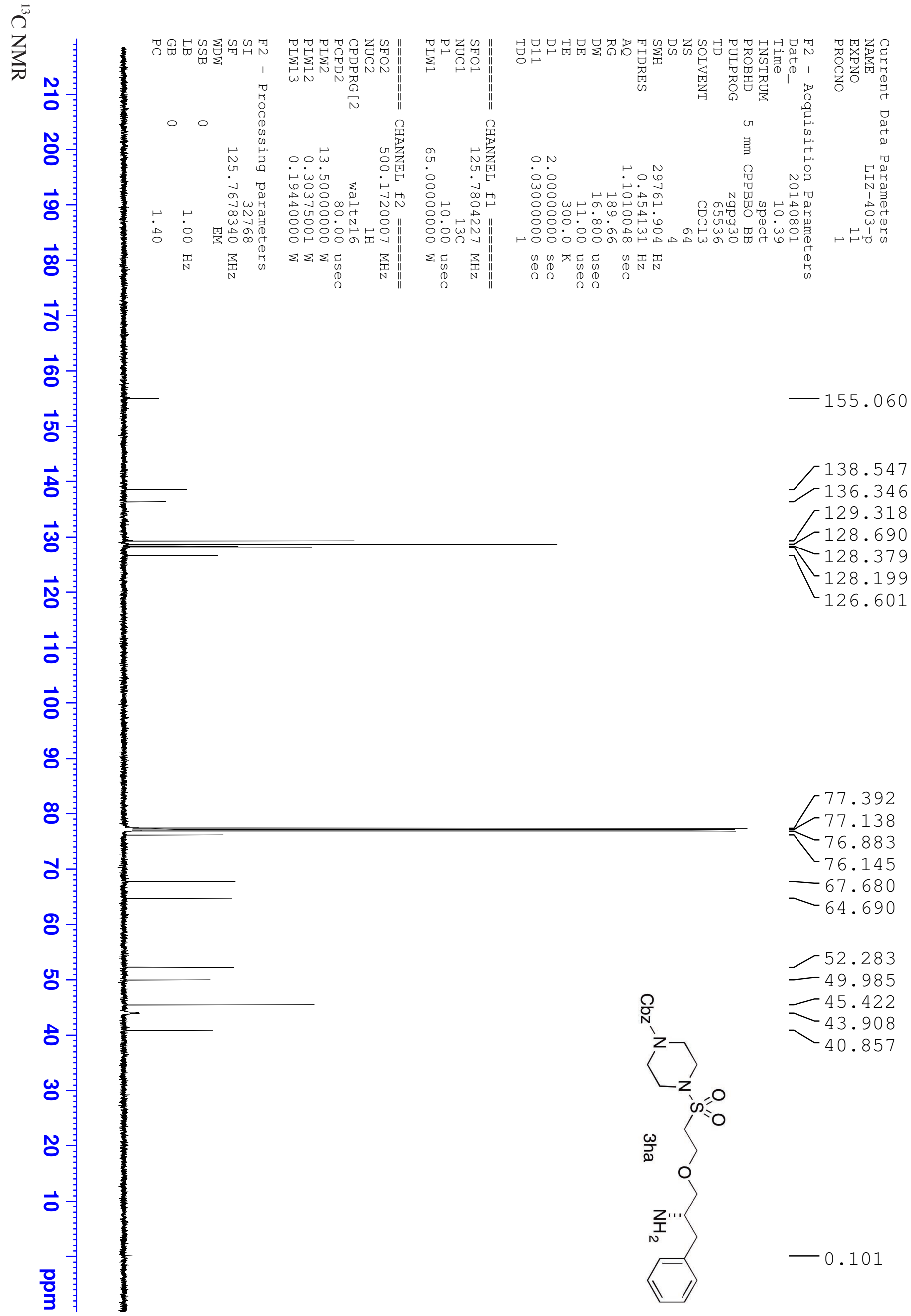




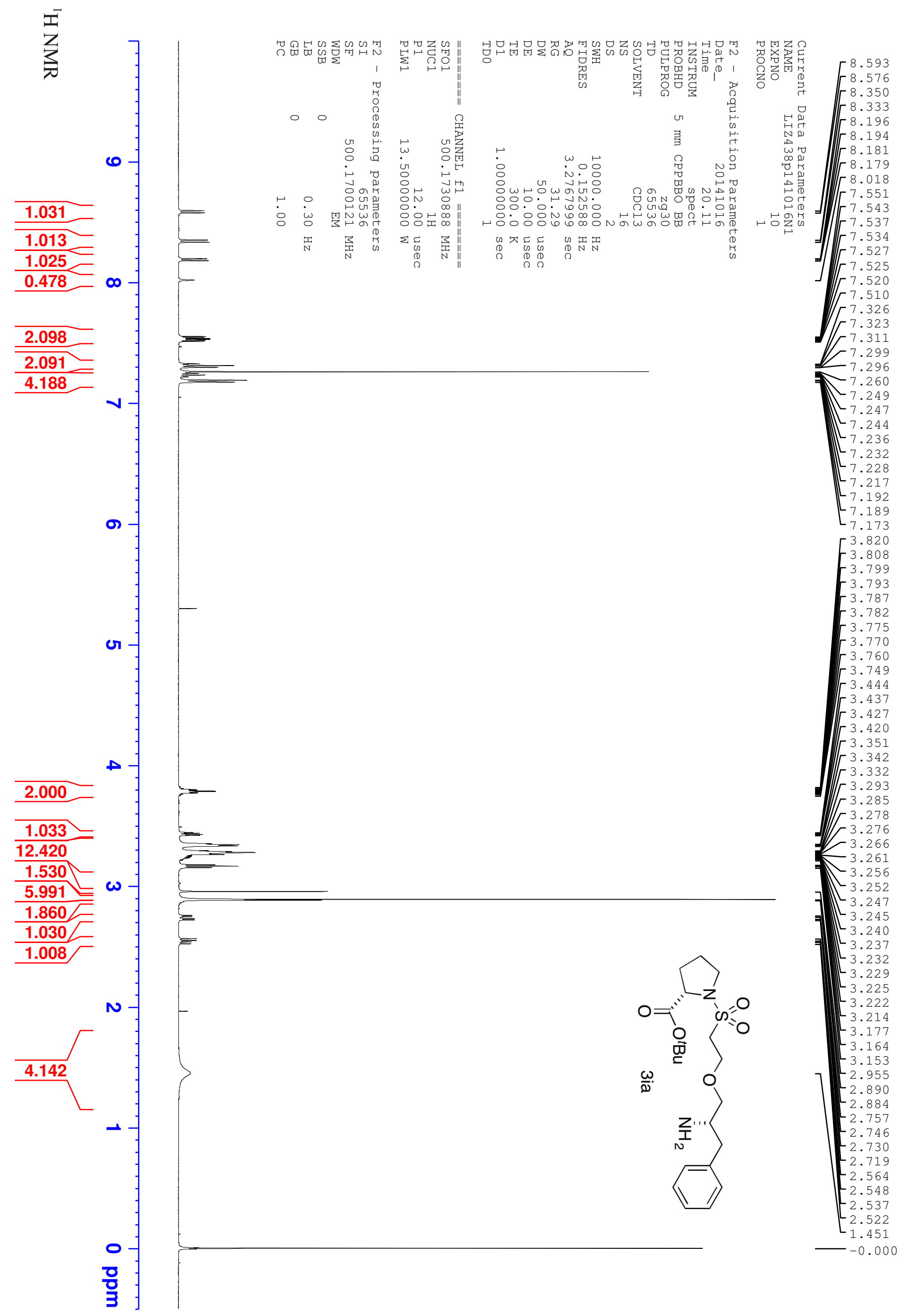




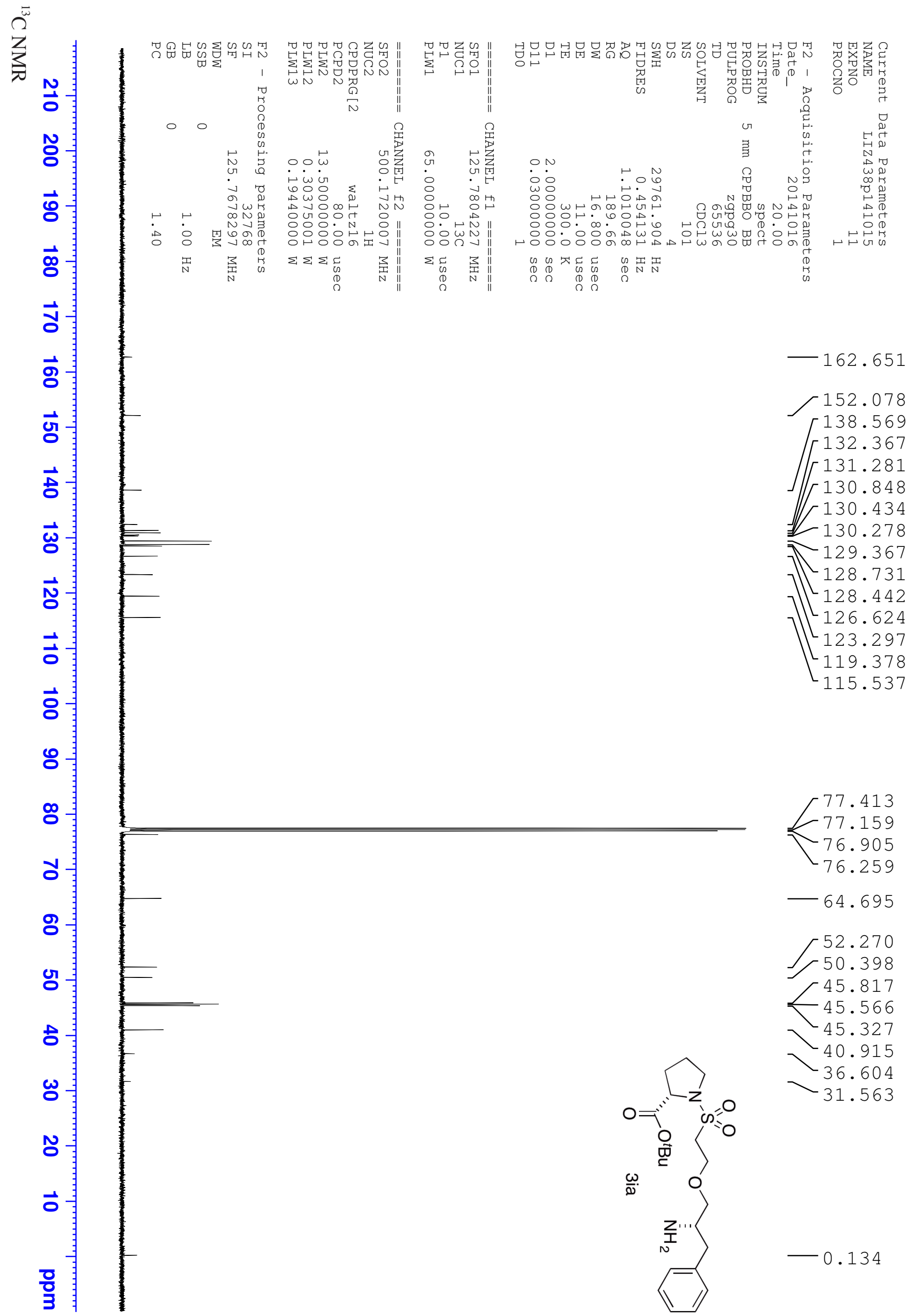



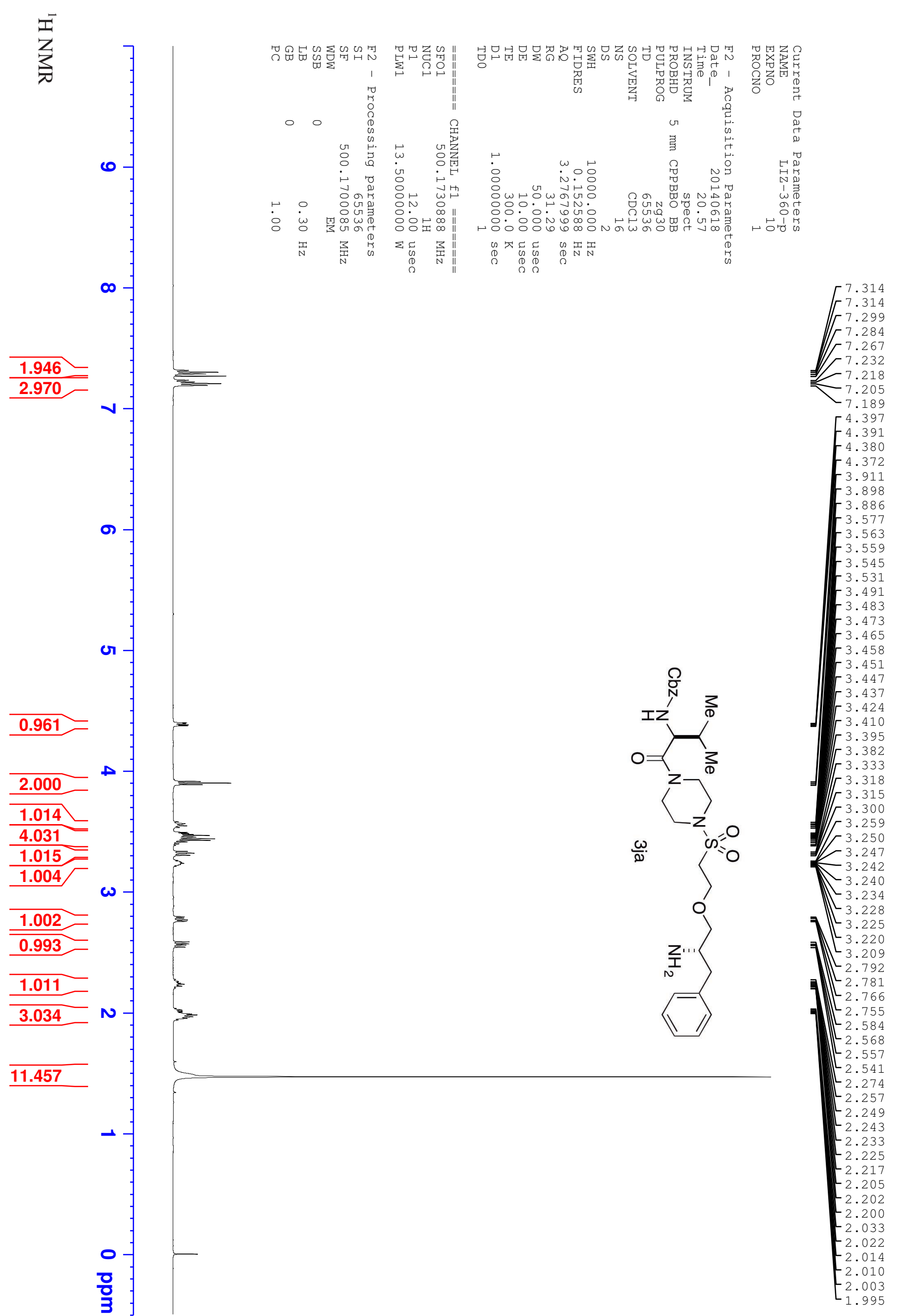


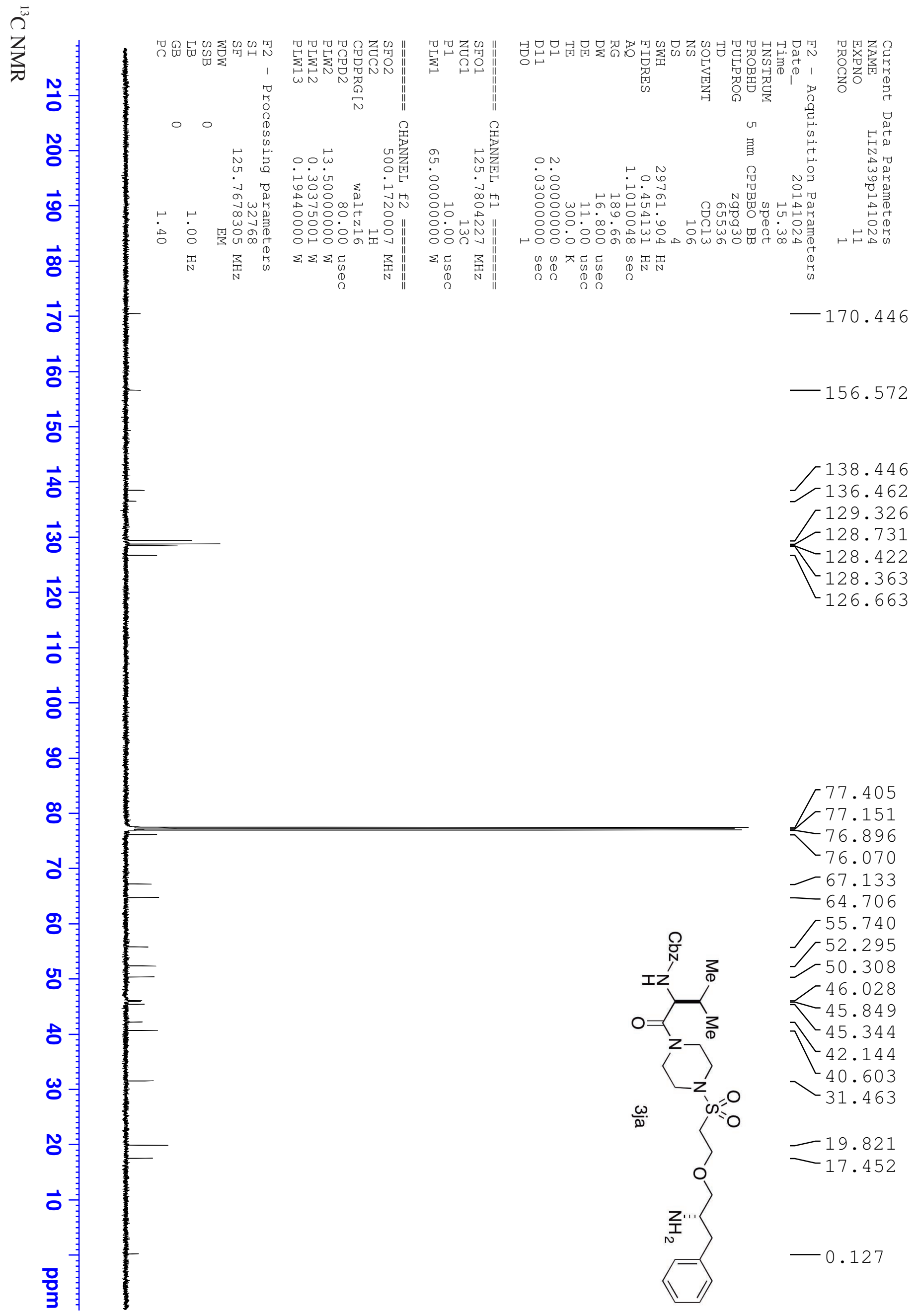




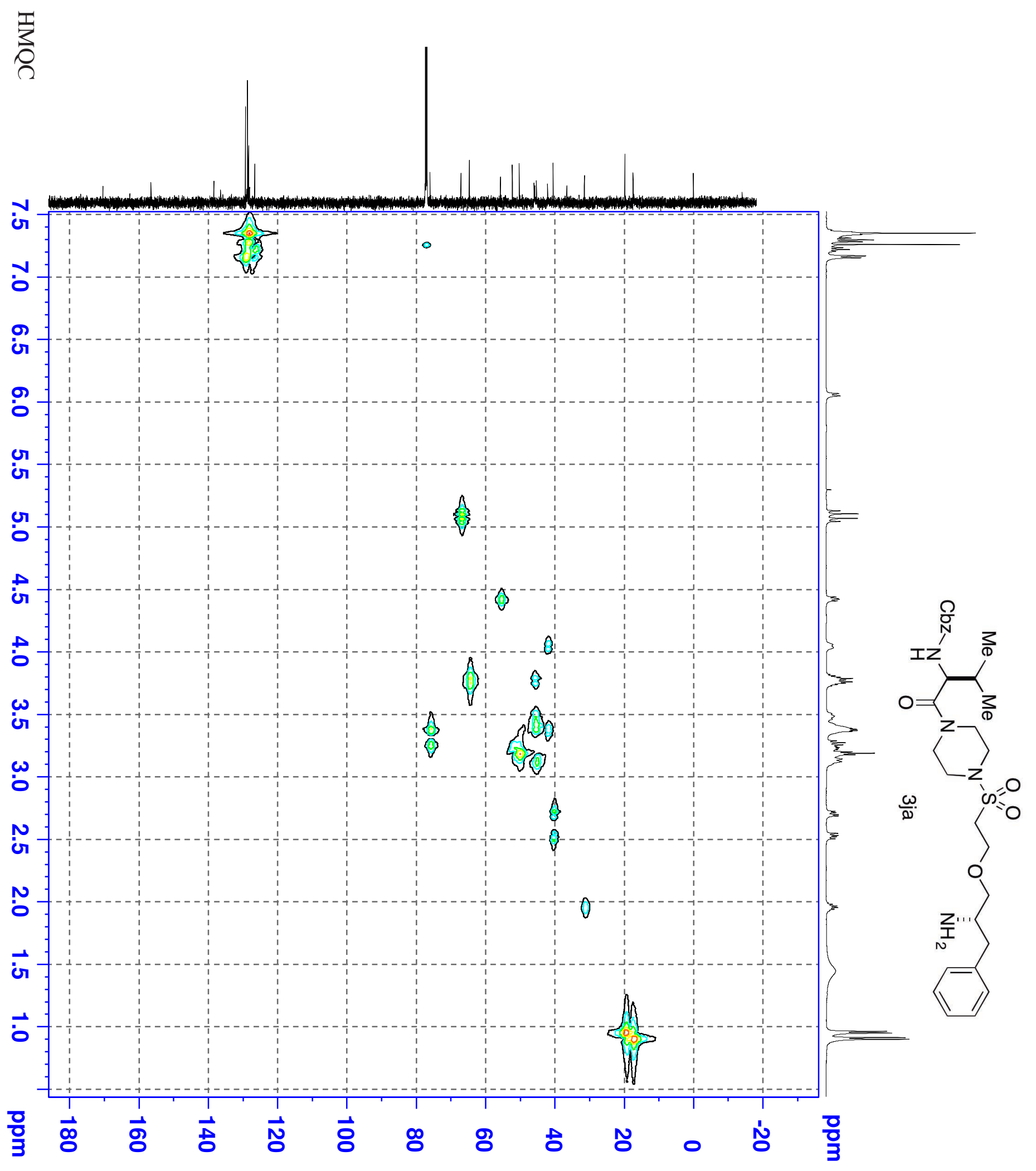

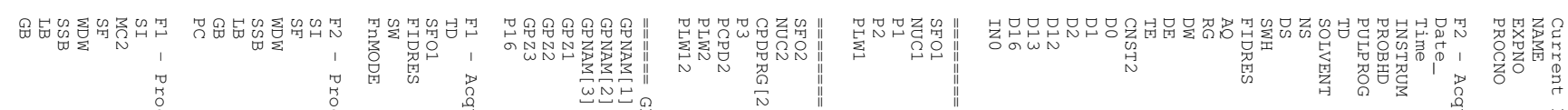

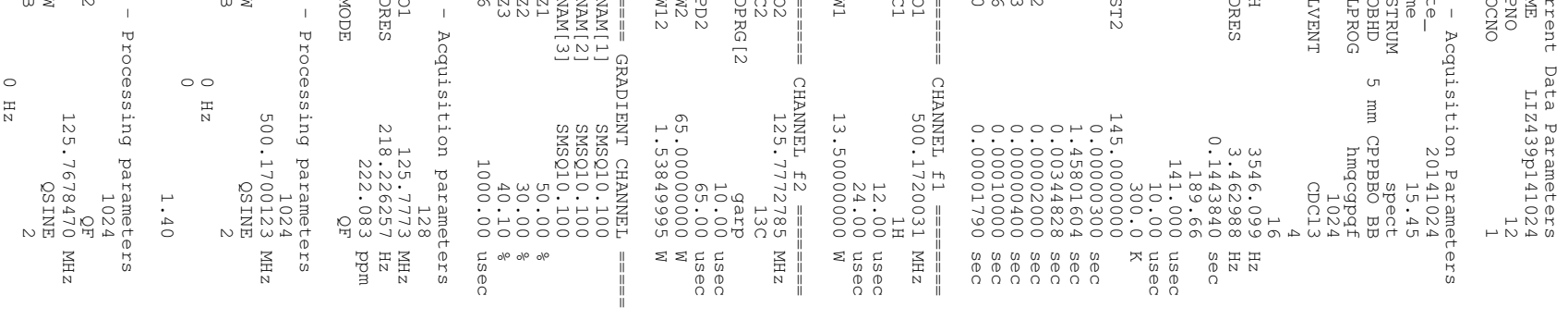




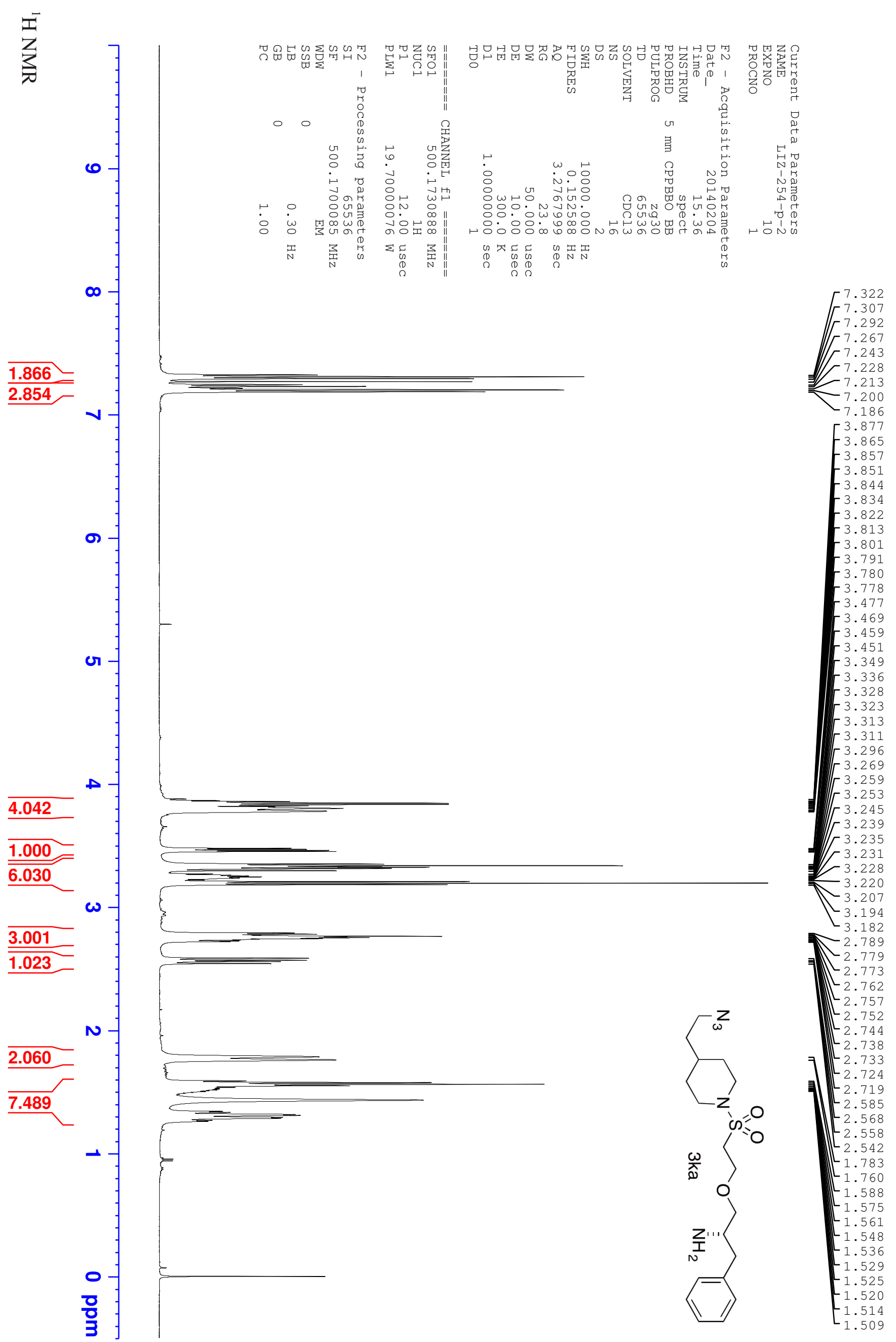




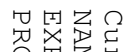
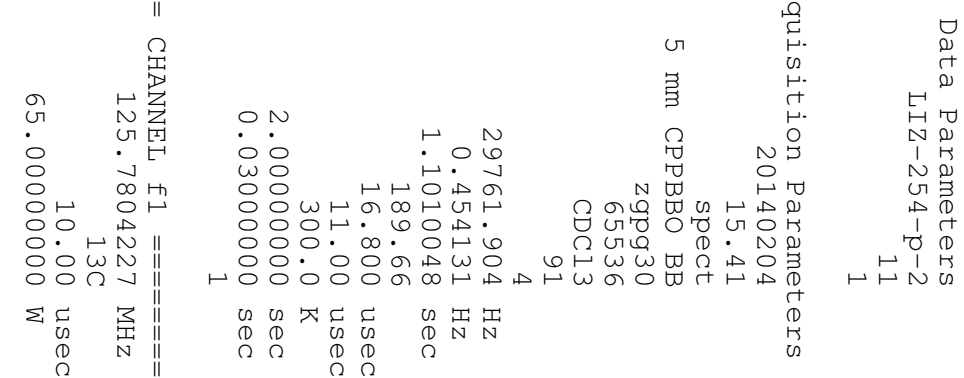

a

$\overrightarrow{8}$

ज़

$\overrightarrow{\mathrm{A}}$

$-138.641$

$\vec{\omega}$

$\vec{\Delta}$

하

$\overrightarrow{8}$

8

ø

129.304

128.644

126.538

๖

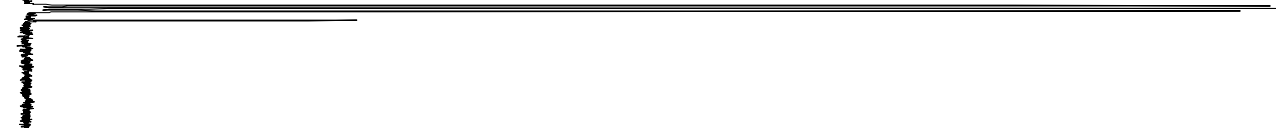

77.394

77.140

76.886

76.049

$-64.807$

8

잉

8)

$\ddot{\sigma}$

ก

$\overrightarrow{0}$

骂

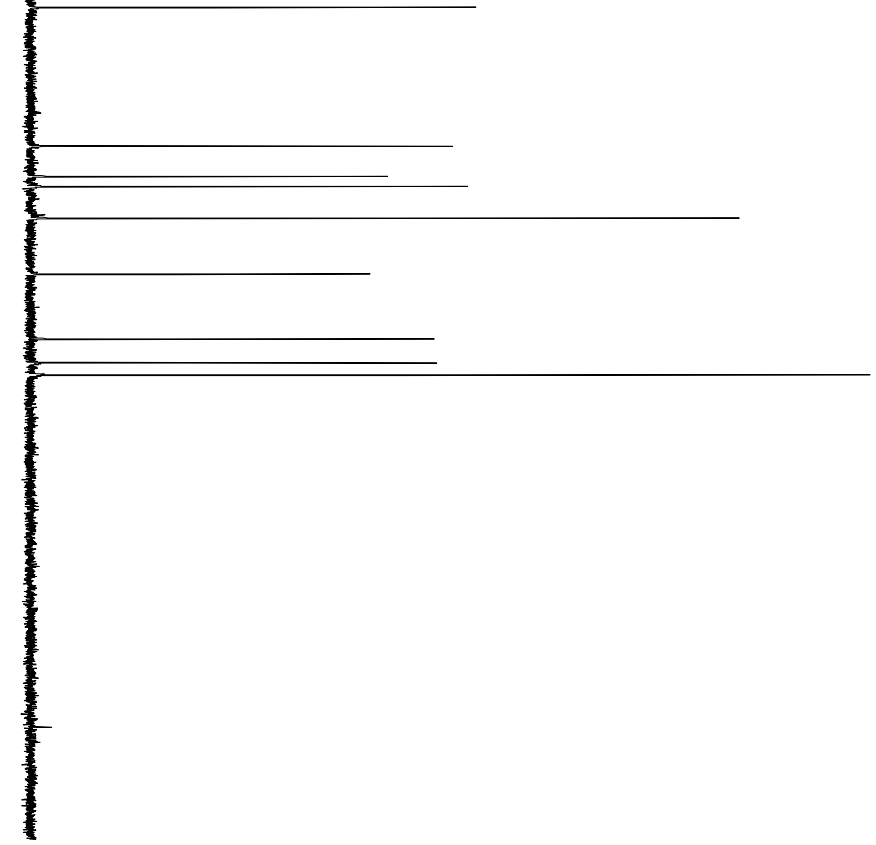

52.326

49.620

48.716

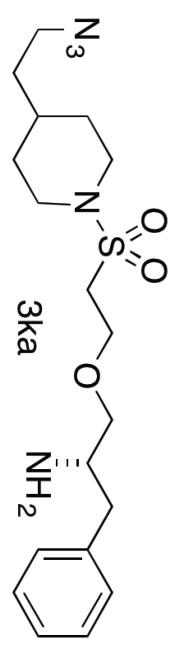

45.860

40.820

$-34.996$

$\bar{\gamma} 32.852$

31.753 


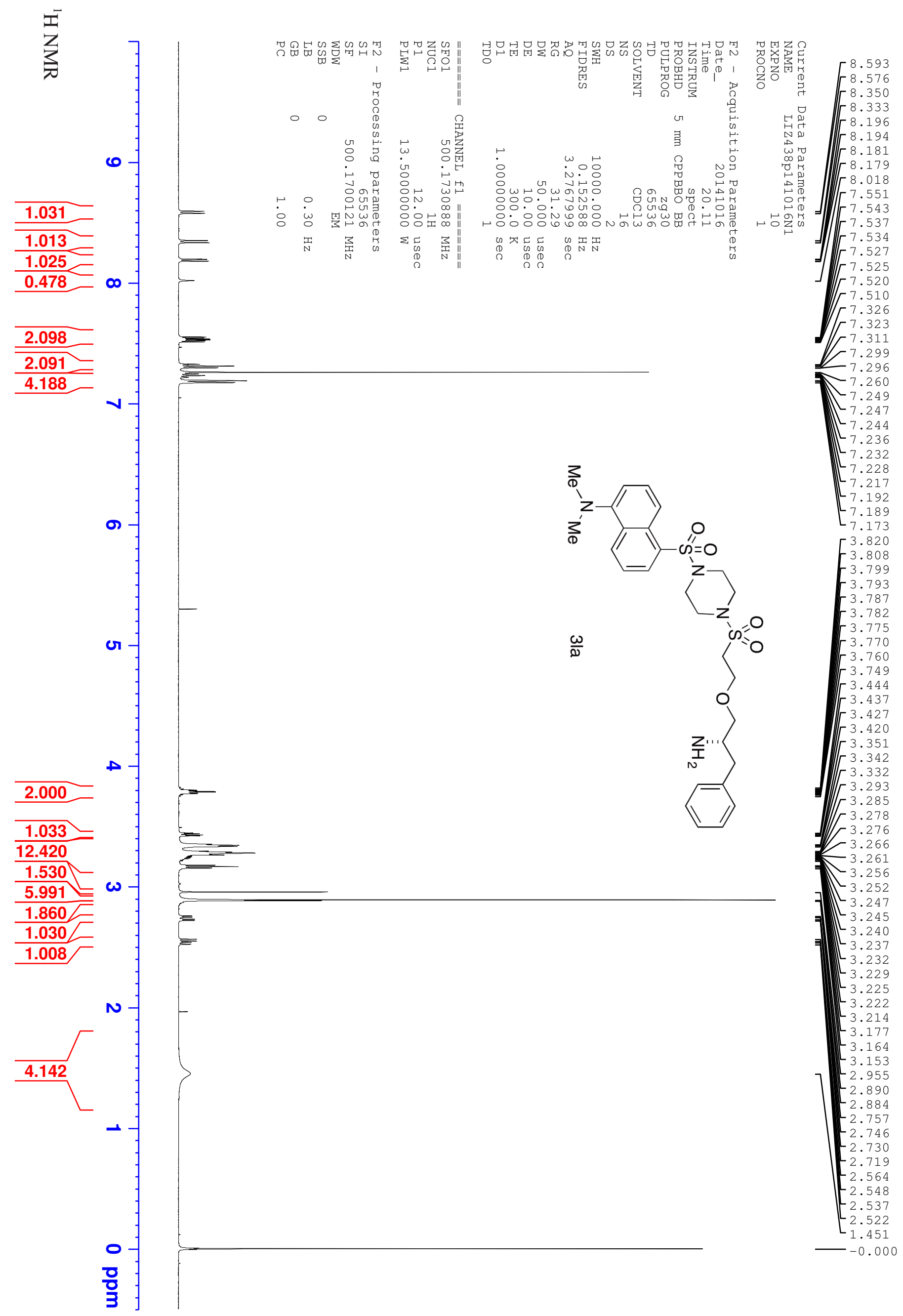




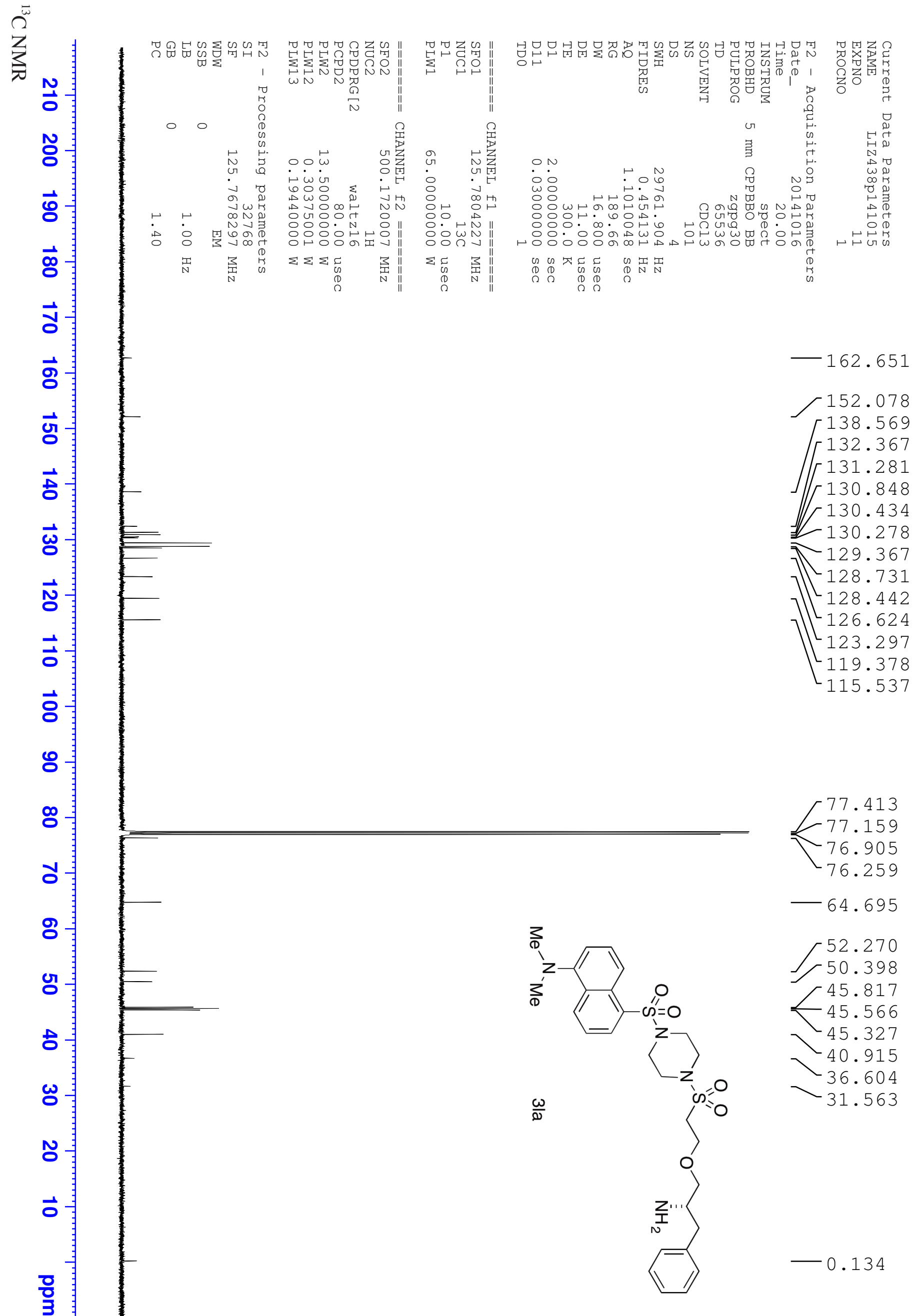




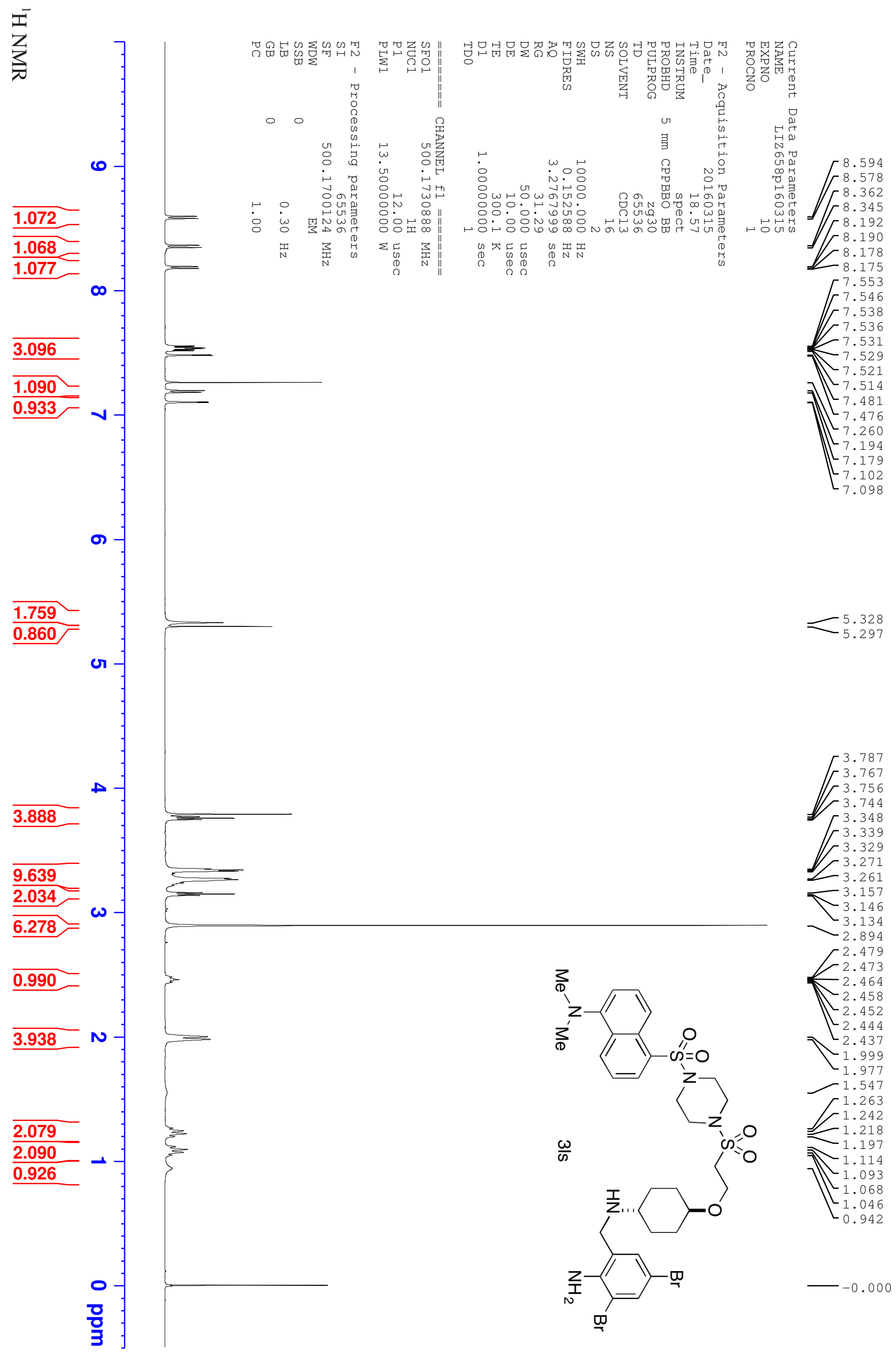




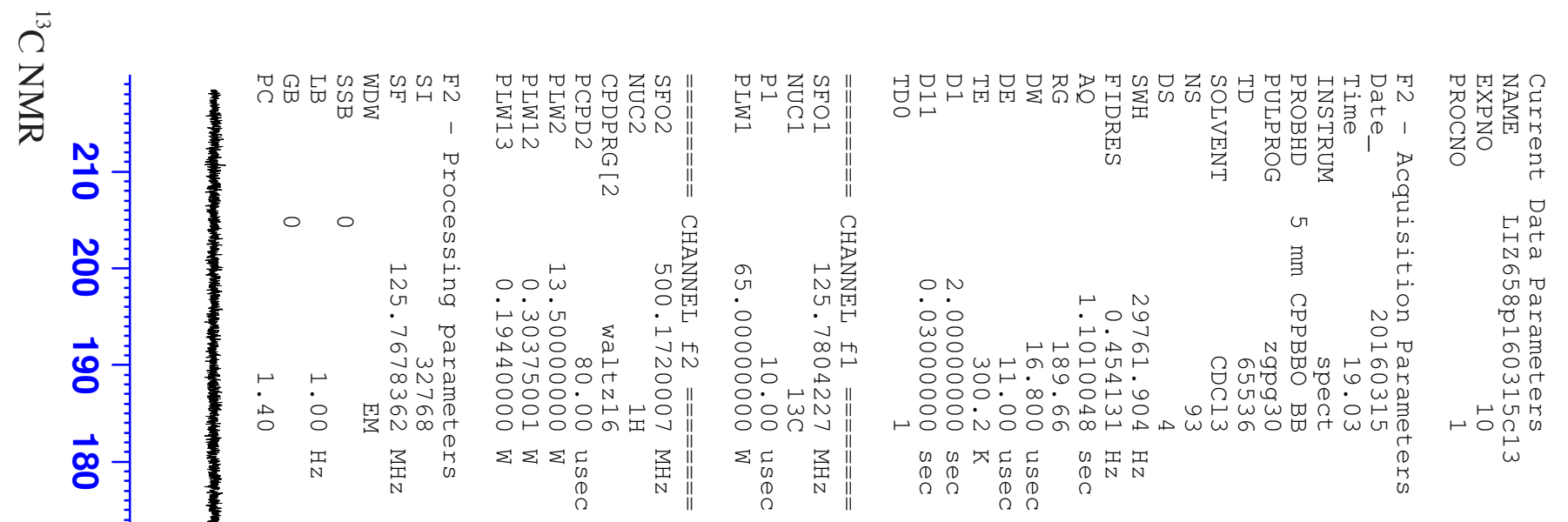

a

홍

जั

古

$\overrightarrow{\mathrm{\omega}}$

$\overrightarrow{\widetilde{\nu}}$

$\vec{\Delta}$

$\overrightarrow{\mathrm{a}}$

8

ø

o

8

잉

t

\%

ก

o

흨

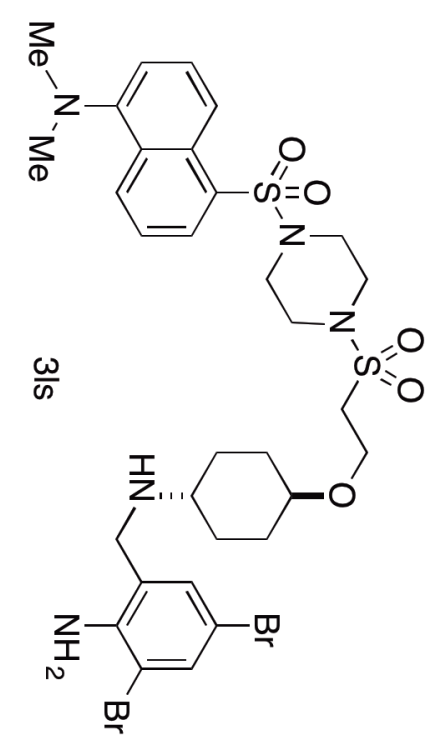

78.466
-77.397
-77.144
76.890

61.721

55.554

53.544

51.299

51.299
-45.831

$\mathbb{}_{45.831}^{45.534}$

45.269

41.049
-30.440

30.440

$-0.102$ 


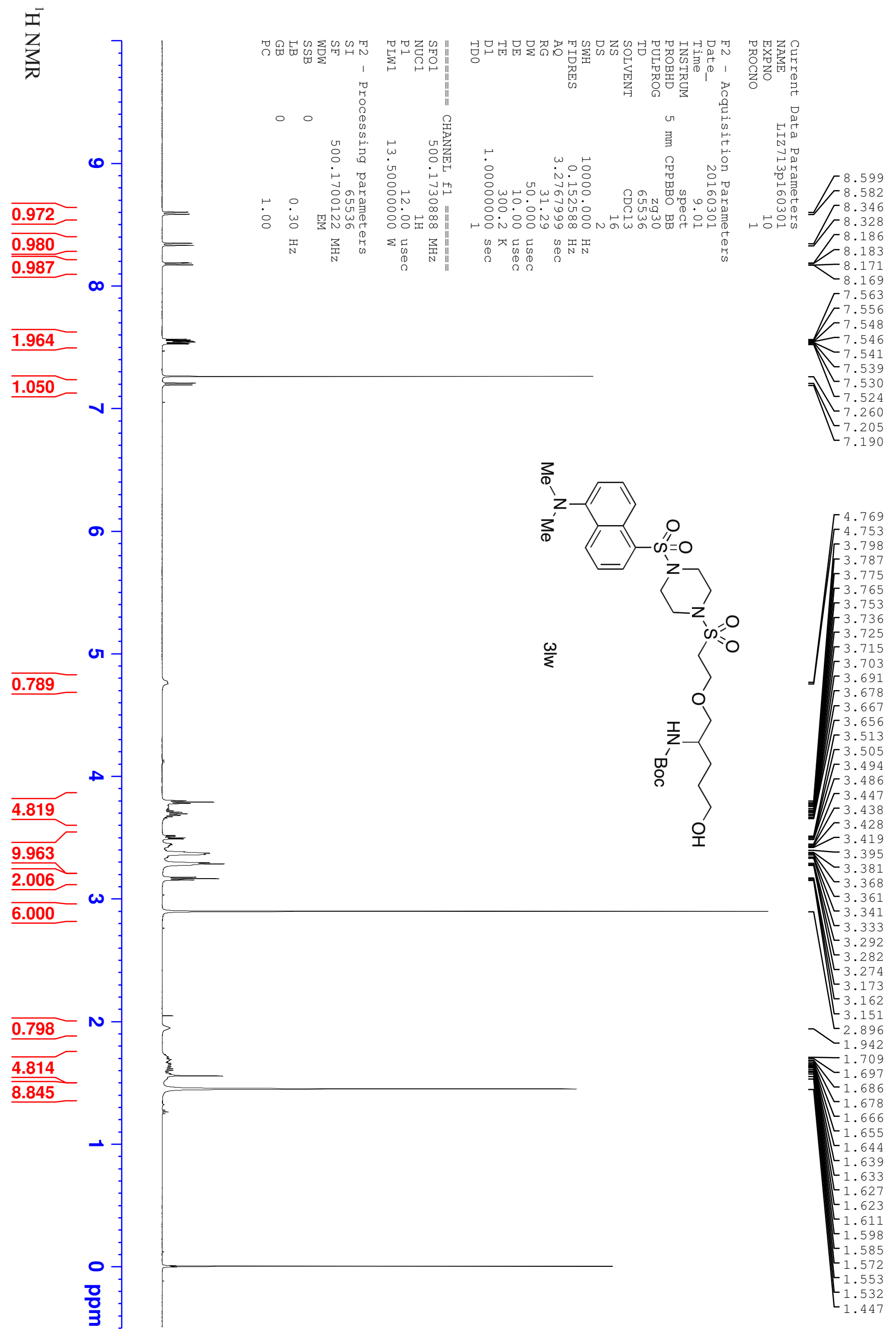



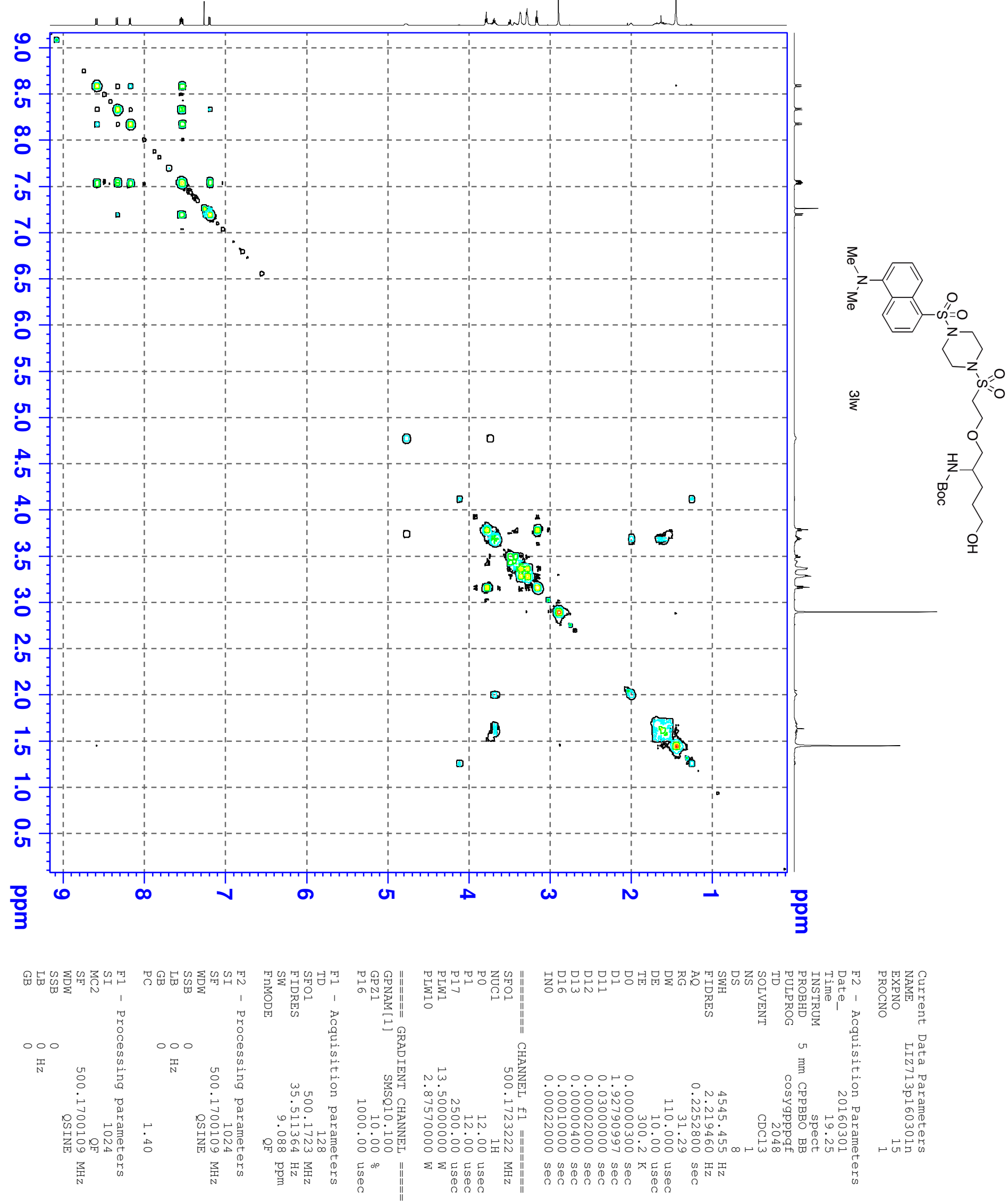
吾
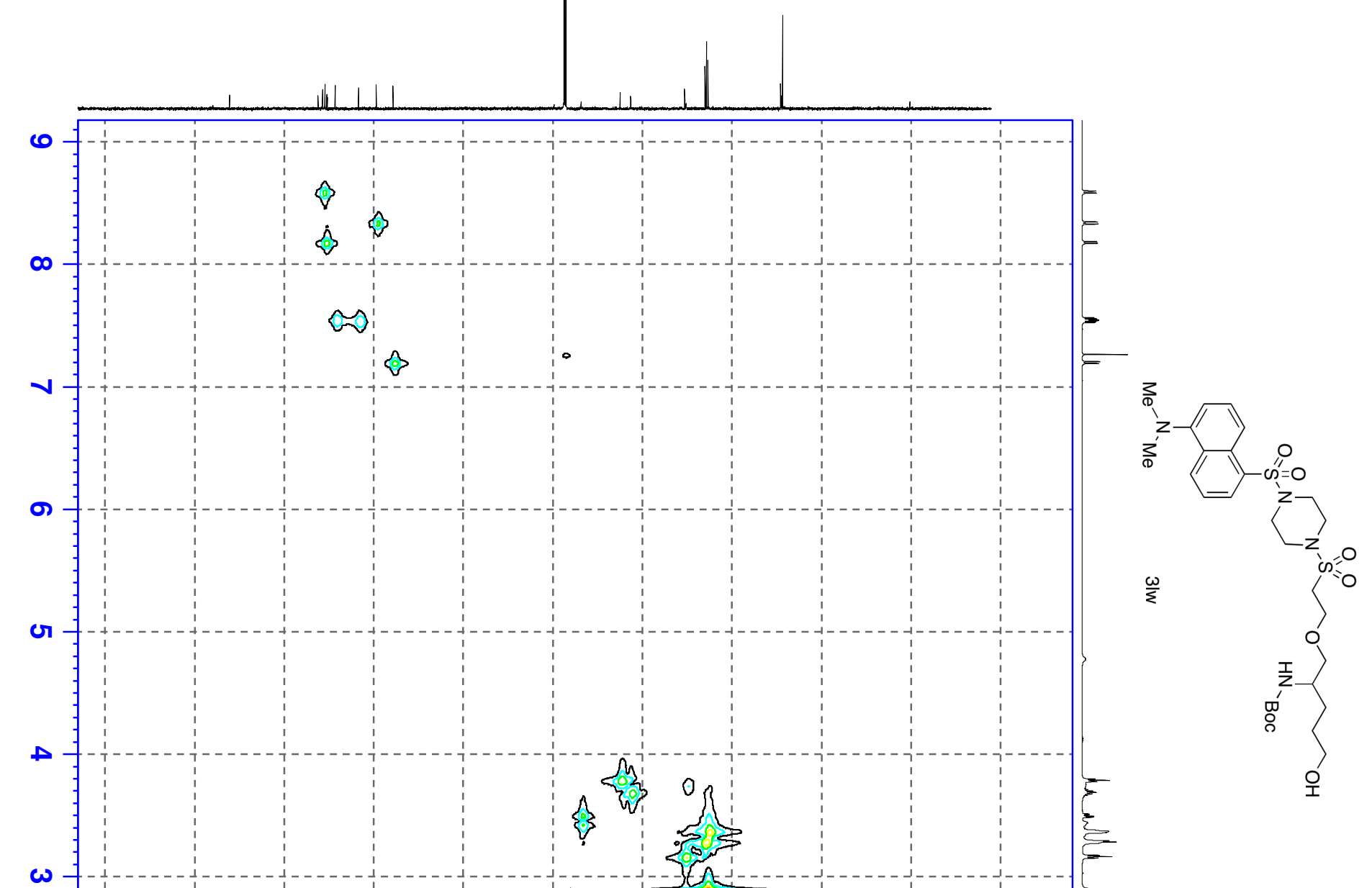

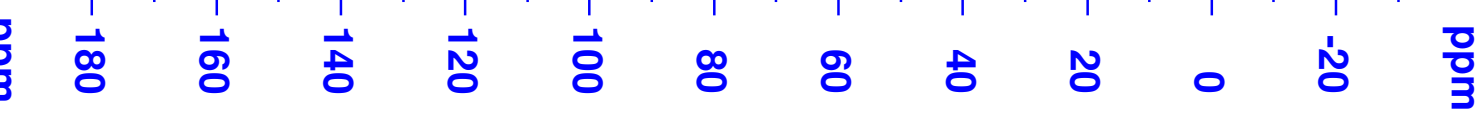

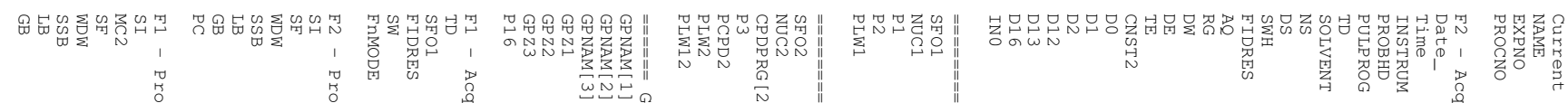

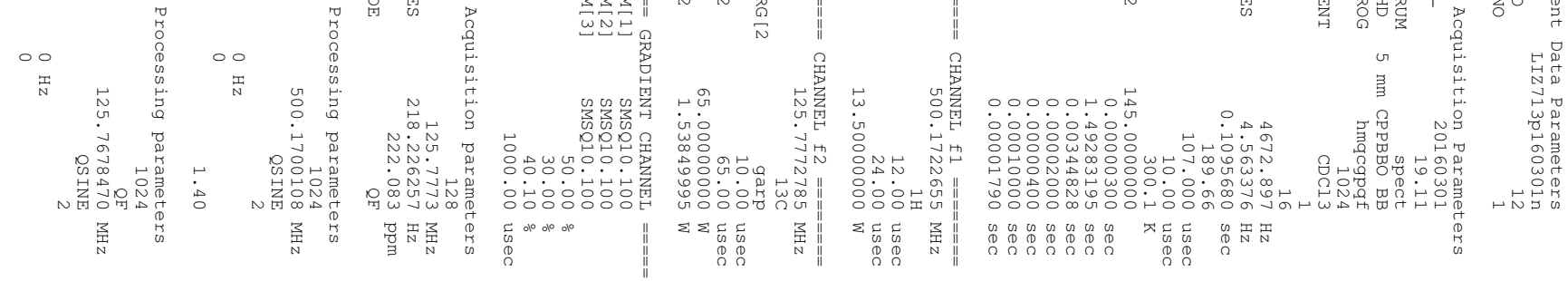


㝻

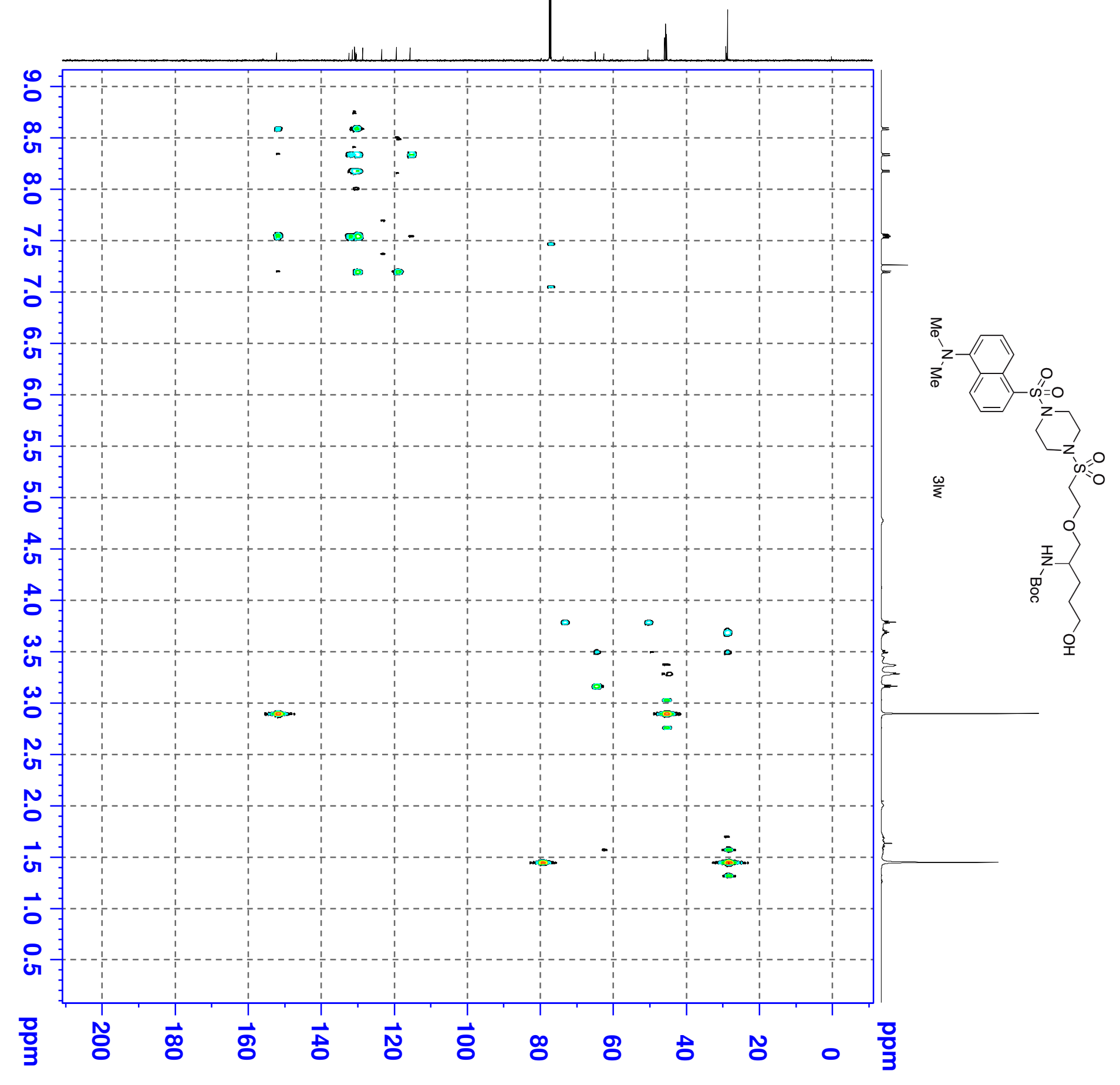

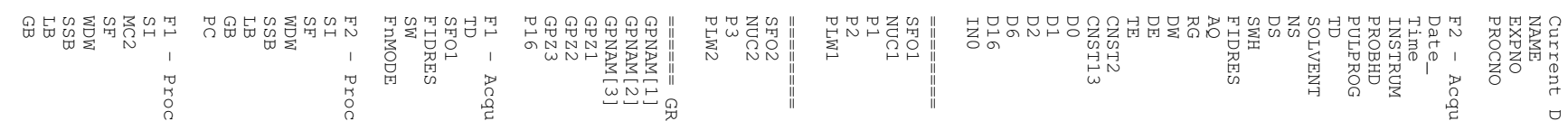

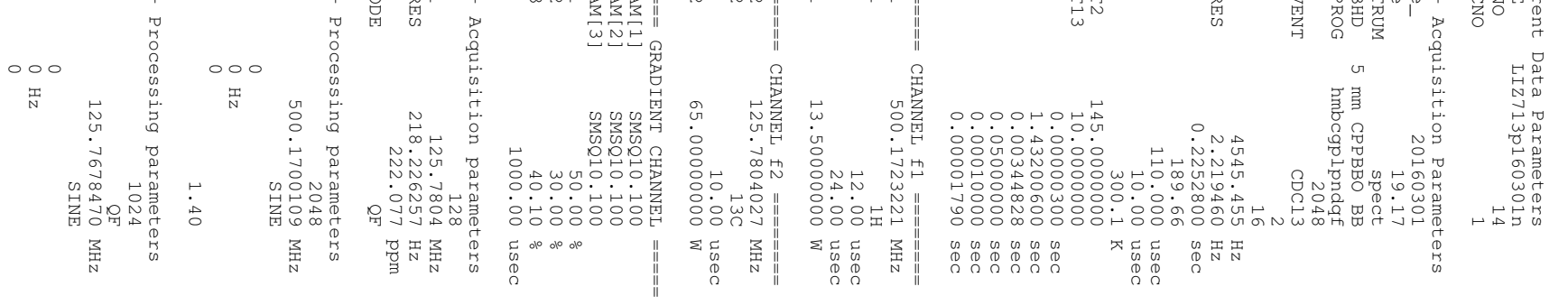



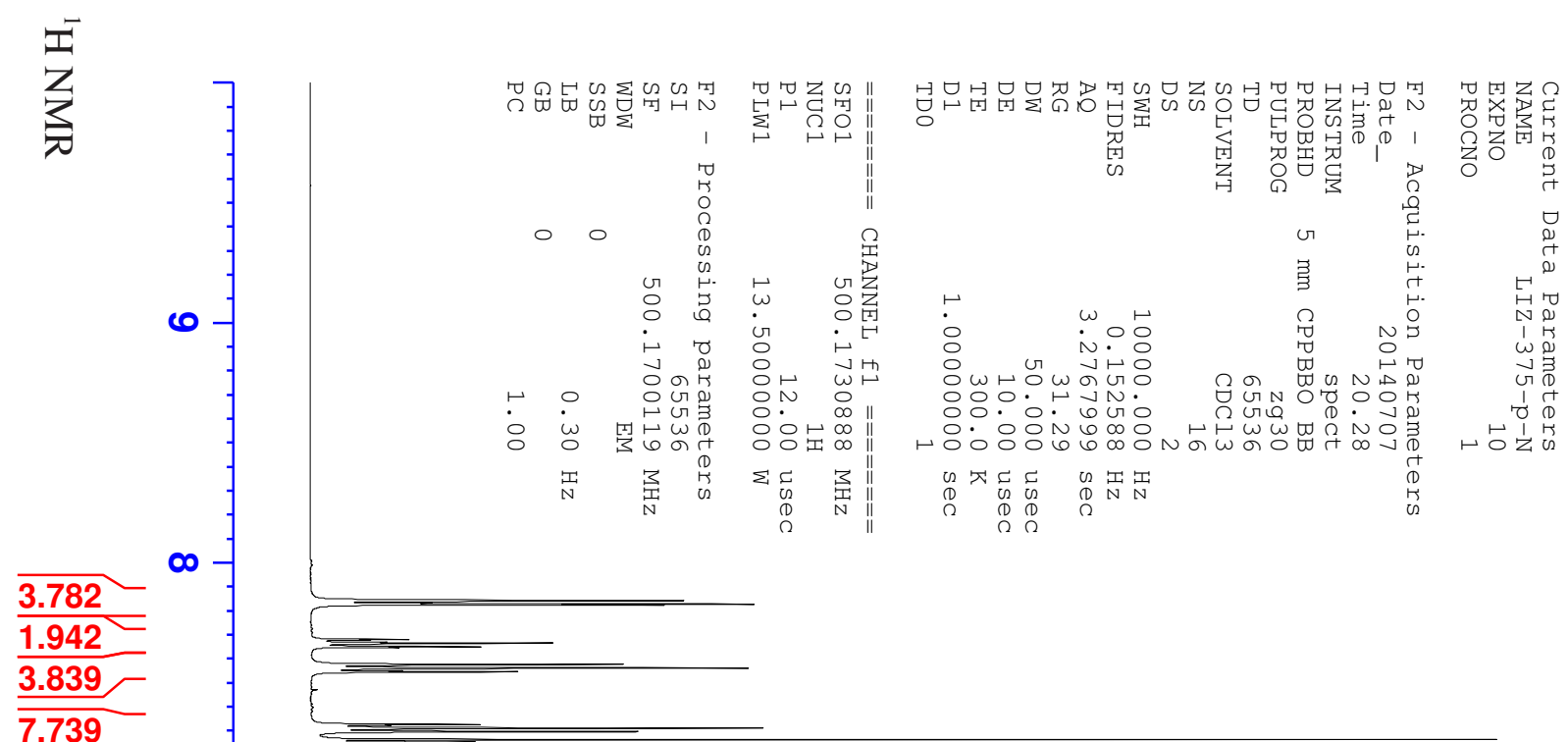

a

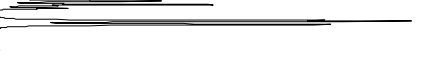

or

2

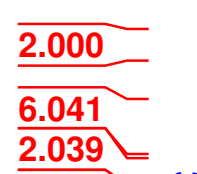

$\frac{2.039}{2.024}=\omega$

$2.023=$

$4.054=$

1.685
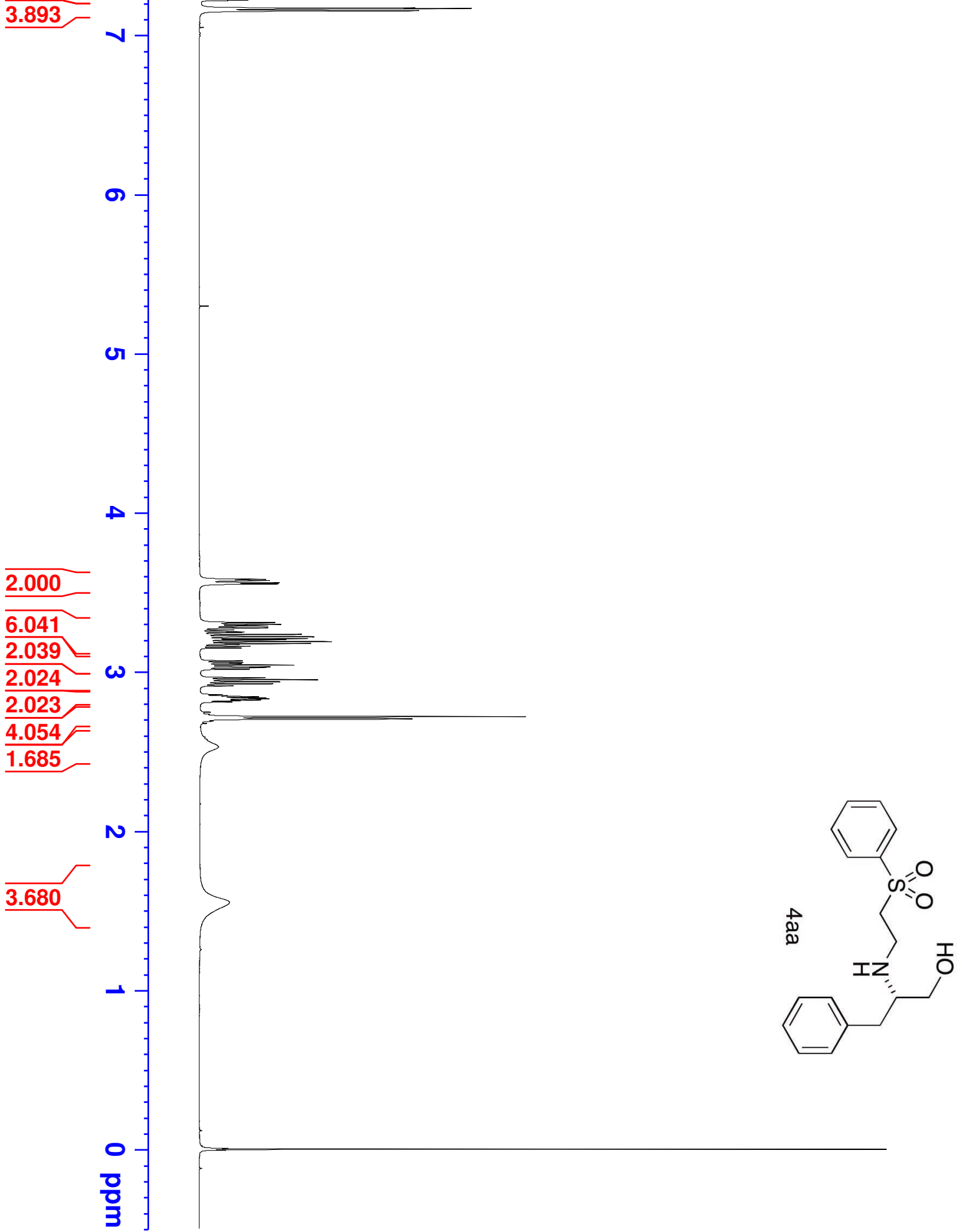


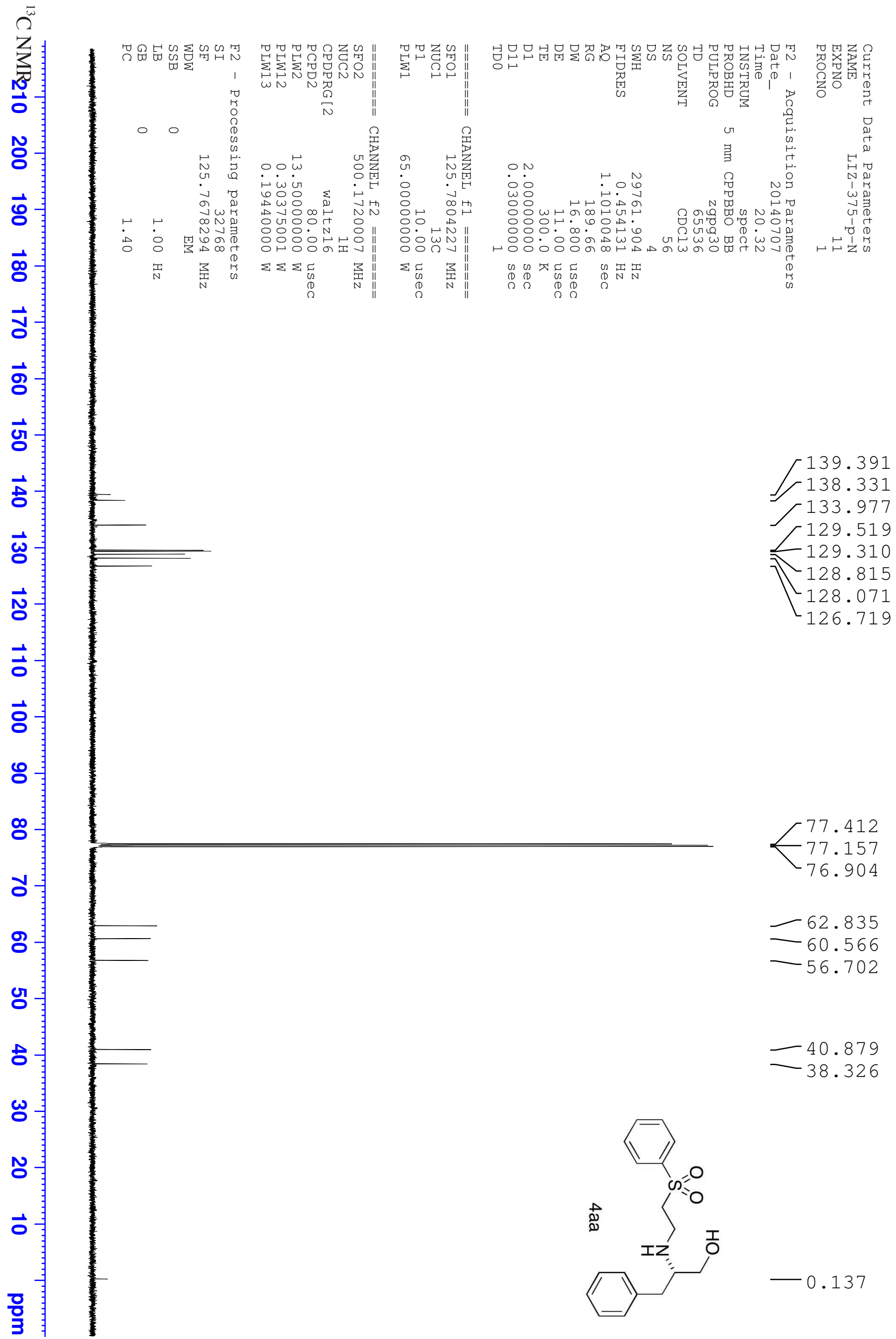




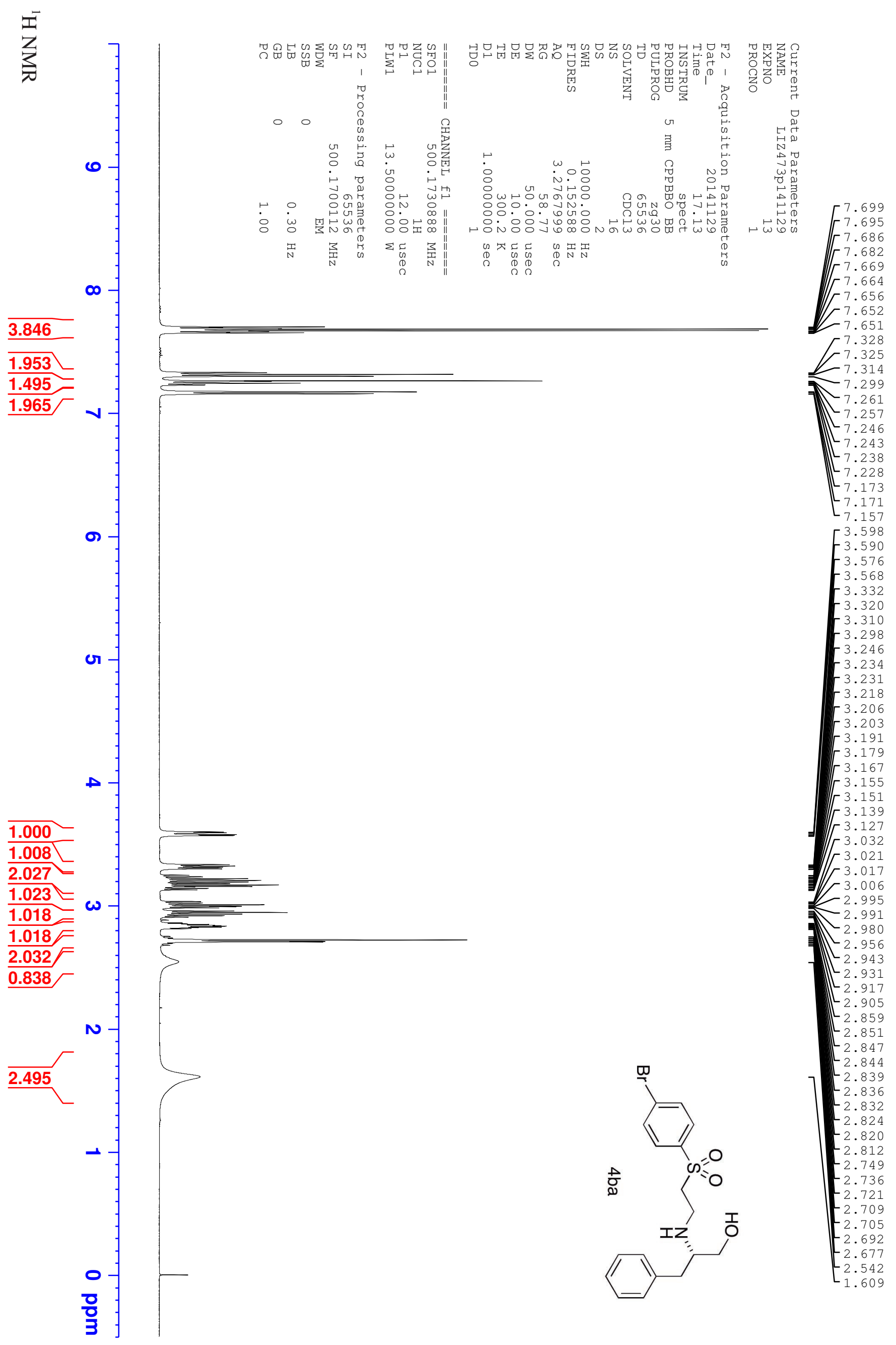



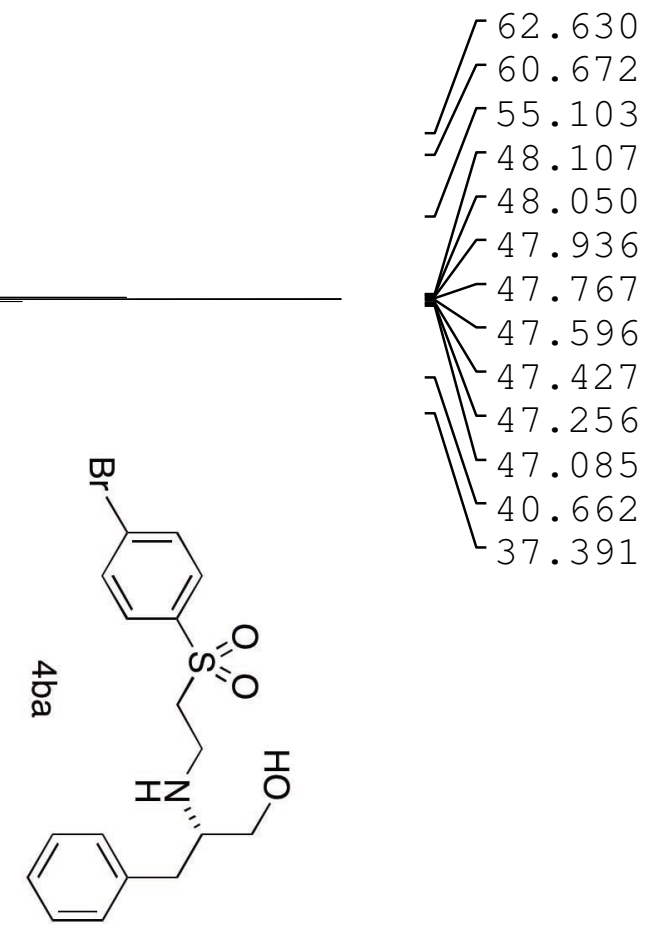


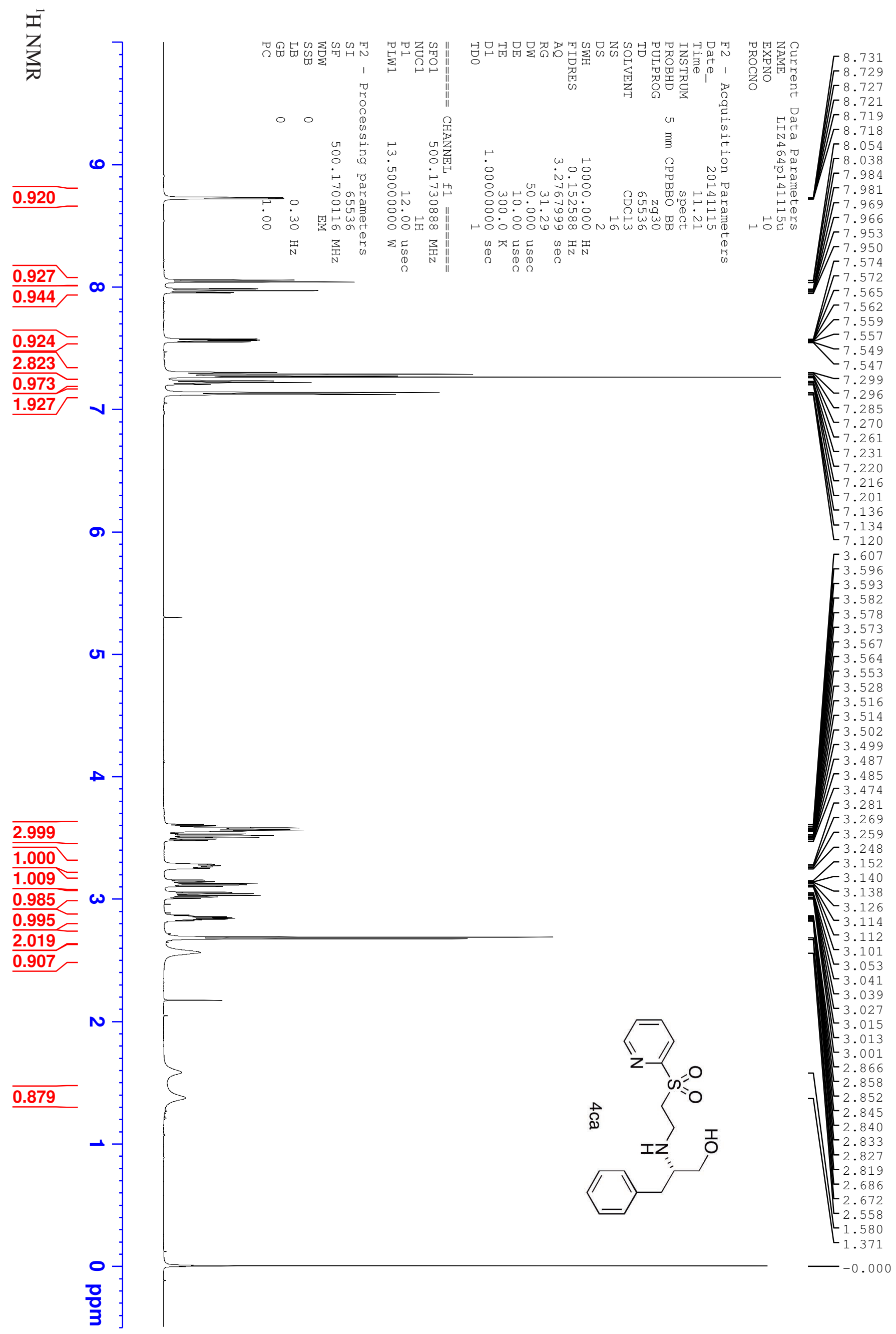




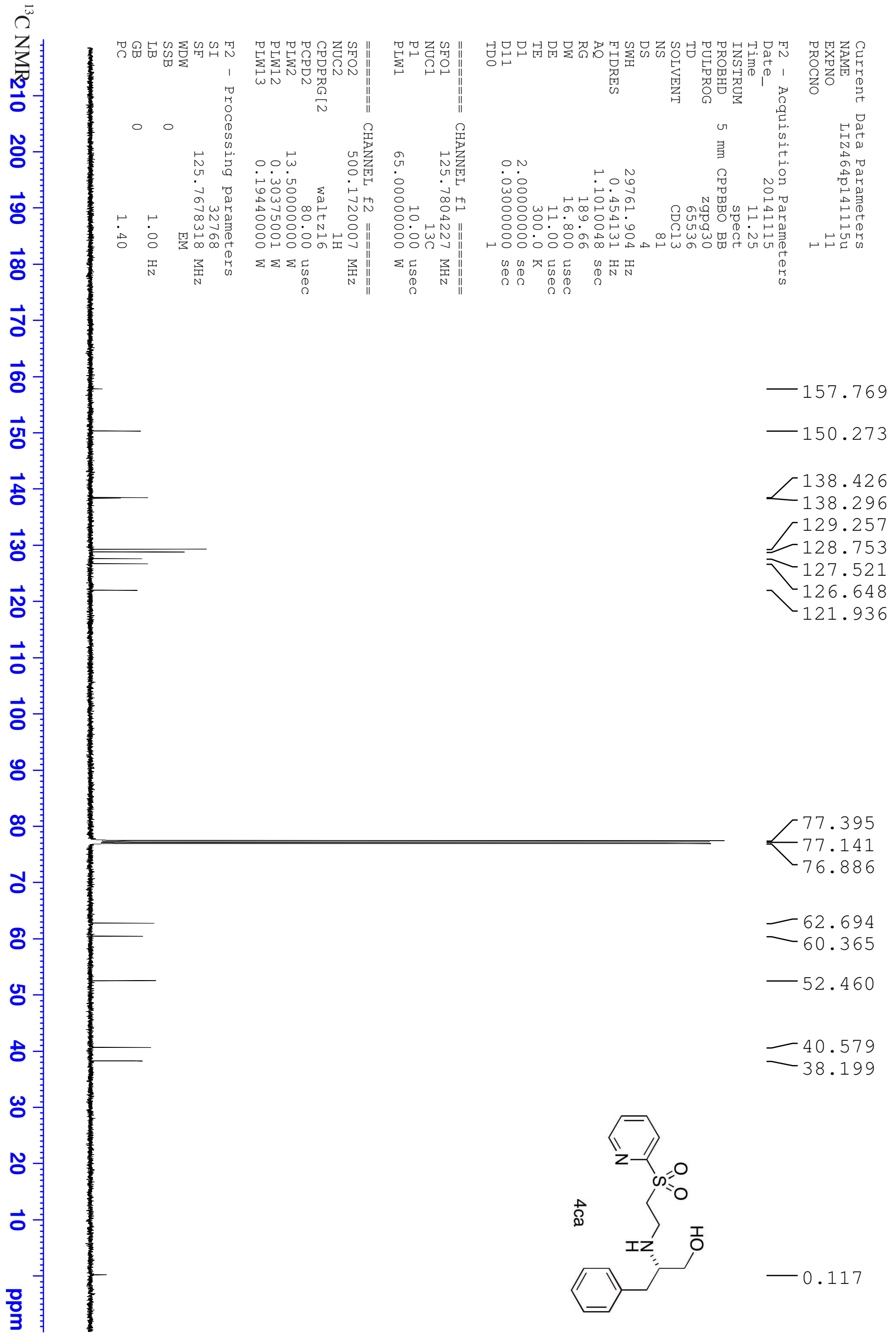




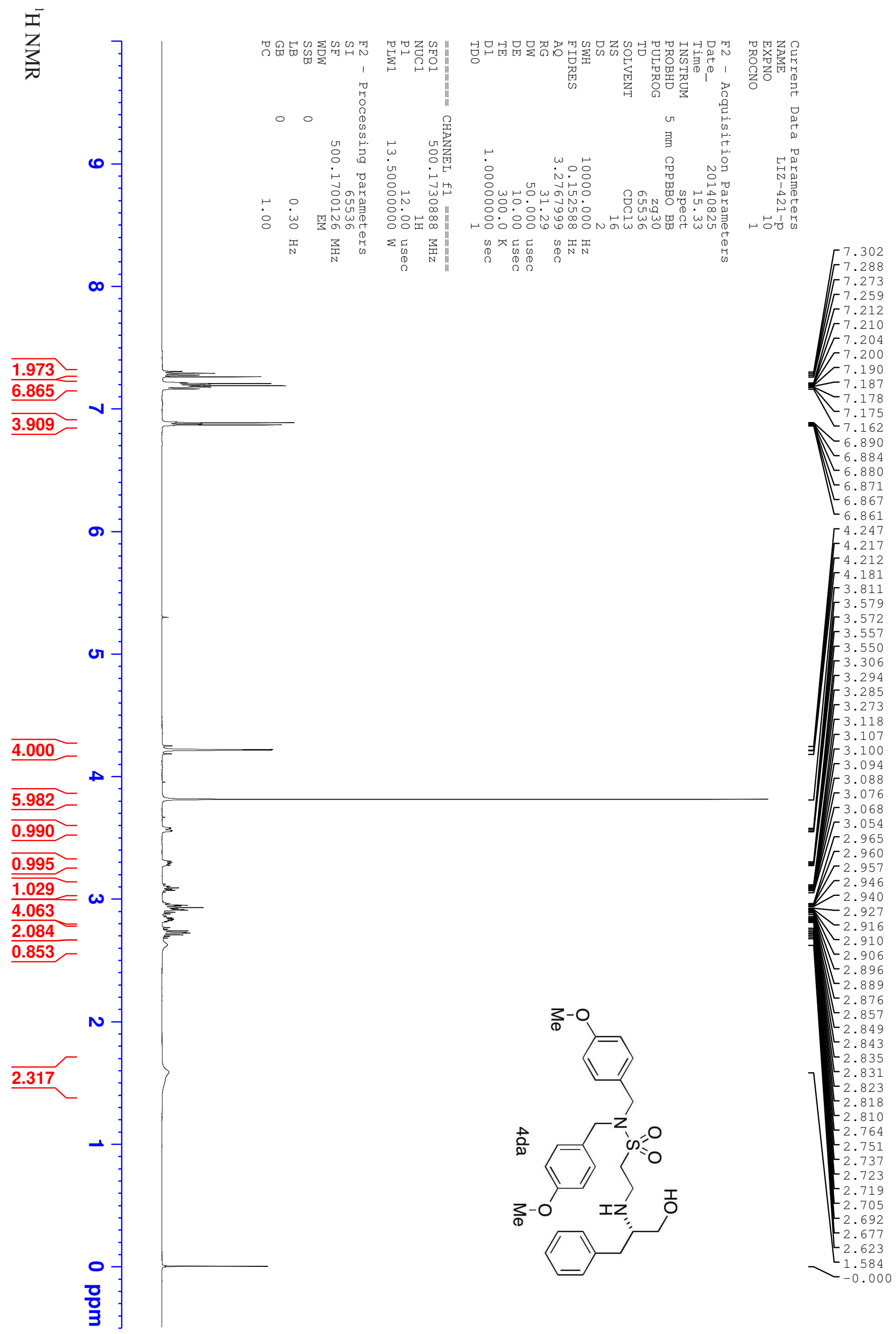




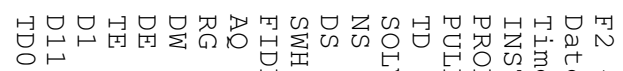

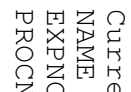

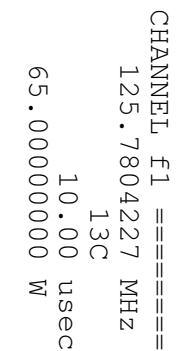

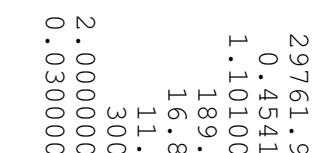

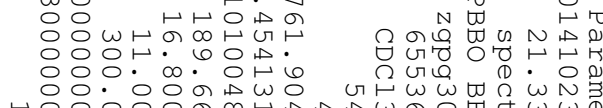

홍

빙

$\vec{t}$

ผั

$\vec{N}$

호

\&े

8

$\infty$

ơ

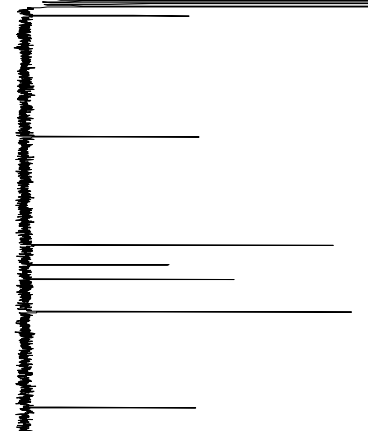

to

w

ㅇ.

$\vec{o}$

밐

守青

$-138.777$

130.213

129.366

128.665

$-127.840$

126.535

$-114.198$

要

.

d-

8

잉

-
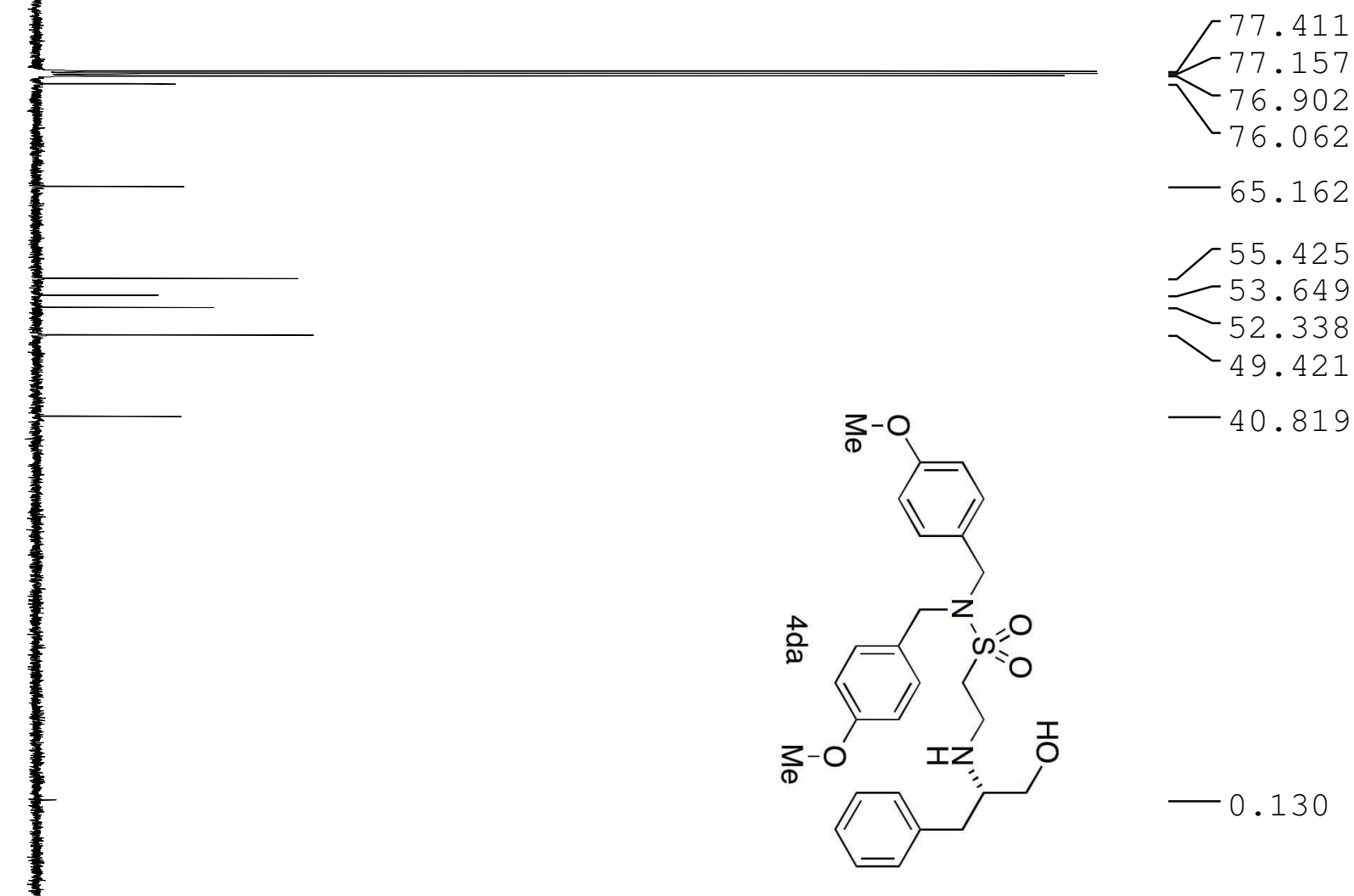

$-40.819$

$-0.130$ 


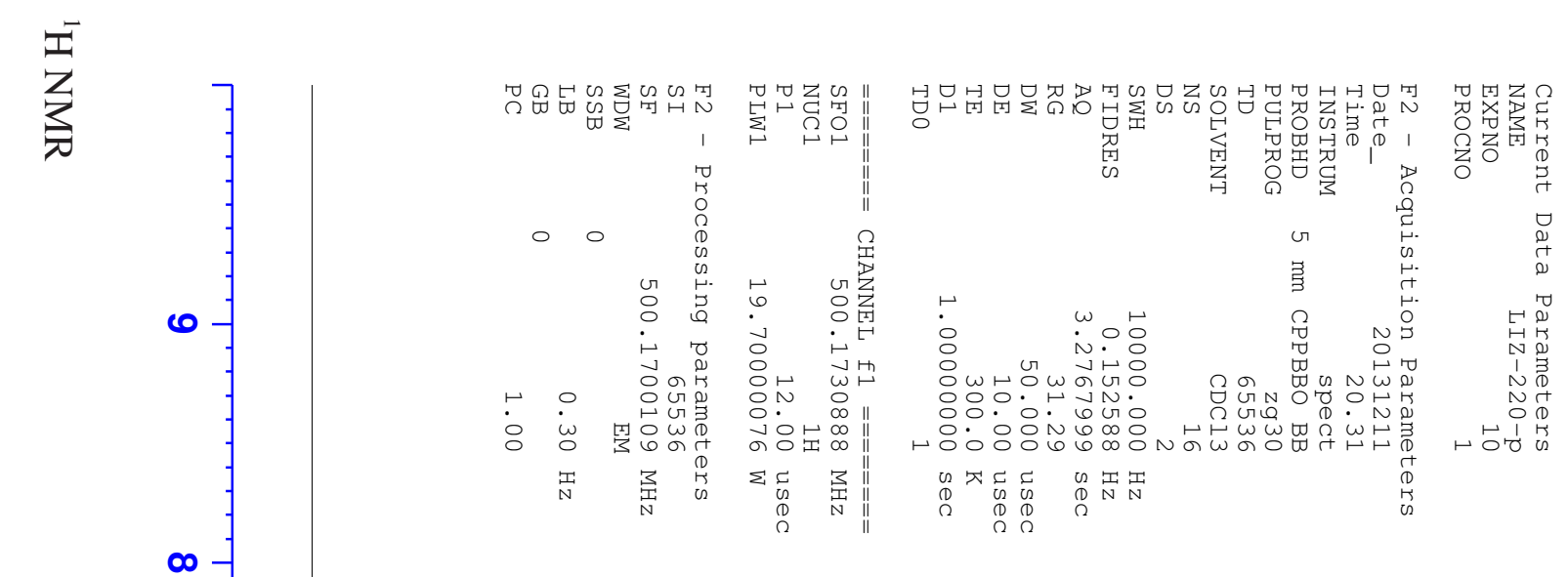

$\left[\begin{array}{r}7.321 \\ 7.318 \\ 7.310\end{array}\right.$

7.310
7.307

7.294

7.292

7.261

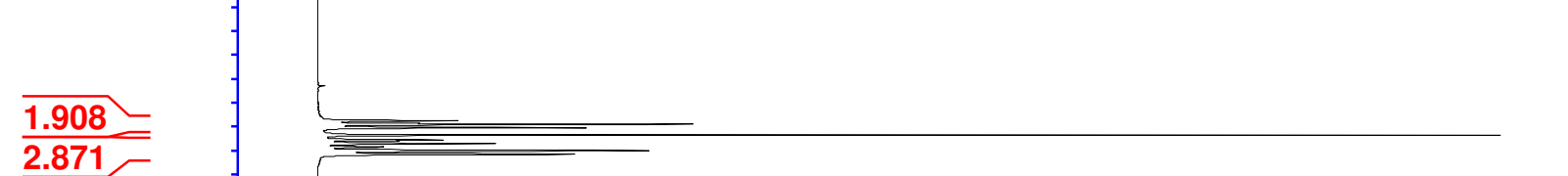

2.871

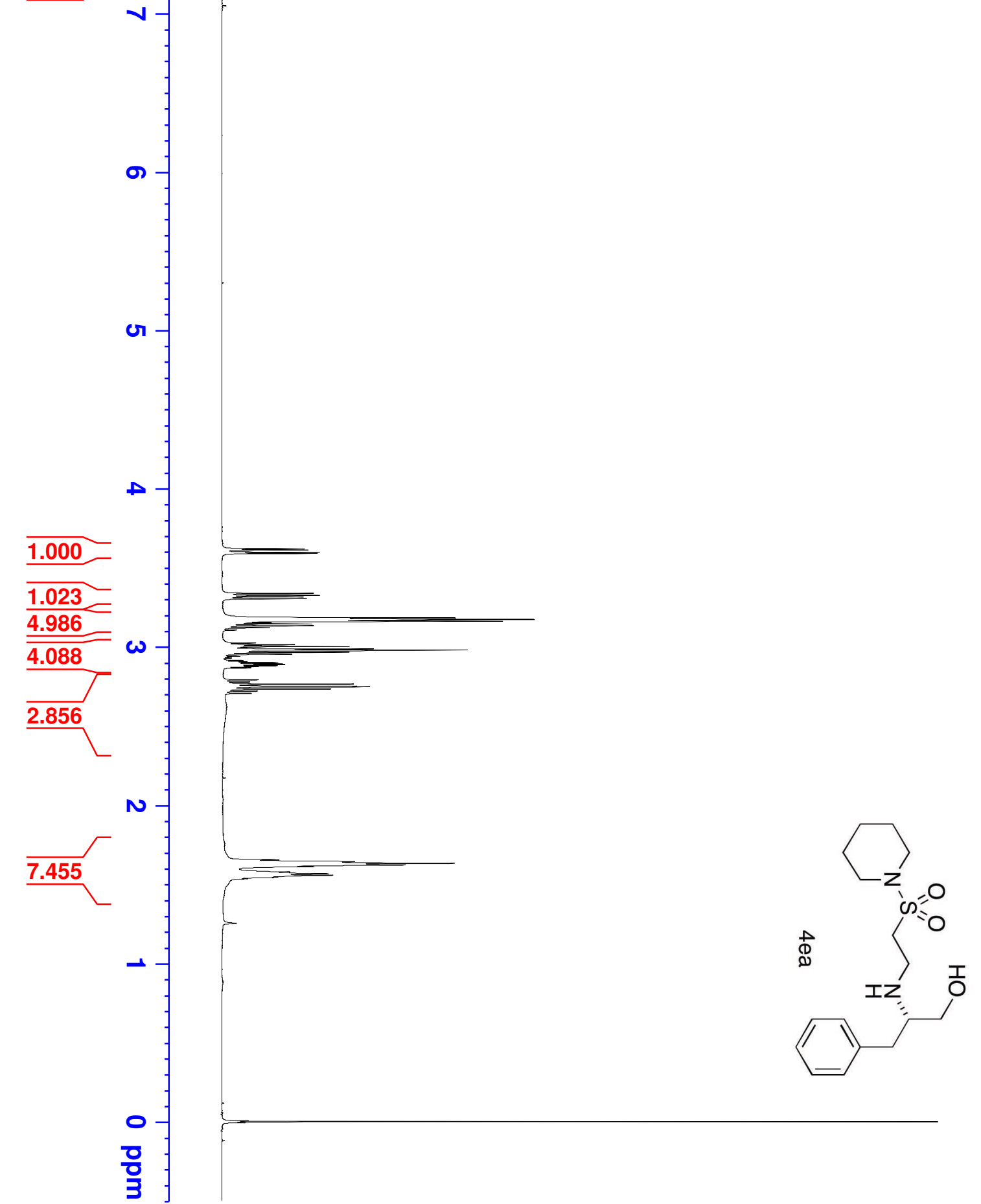

F. 7.241

$-7.238$

$-7.226$

7.222

$-7.211$

7.198

7.181

3.619

$-3.611$

3.597

3.589
-3.337
-3.325

-3.325
-3.316

$-3.316$

$-3.304$

3.183

-3.183
-3.173

3.161

3.152

3.144

3.133
-3.121

3.121

3.105

3.028

$-3.024$

3.013

3.011

3.007
3.001

3.001

2.995
-2.986

2.986

2.984

2.980
$r^{2} .988$

2.978

2.975

2.966
-2.954
-2.940

2.940

2.935

2.913

2.906

2.900

2.892

2.888

2.880

2.874

2.791

-2.778
-2.764
-2.750

2.764
-2.750
-2.747

$-2.747$

$-2.733$

$-2.720$

$-2.706$

2.605

1.654

$-1.643$

$-1.632$

$-1.622$

1.622
1.610
1.578

1.578
1.573 


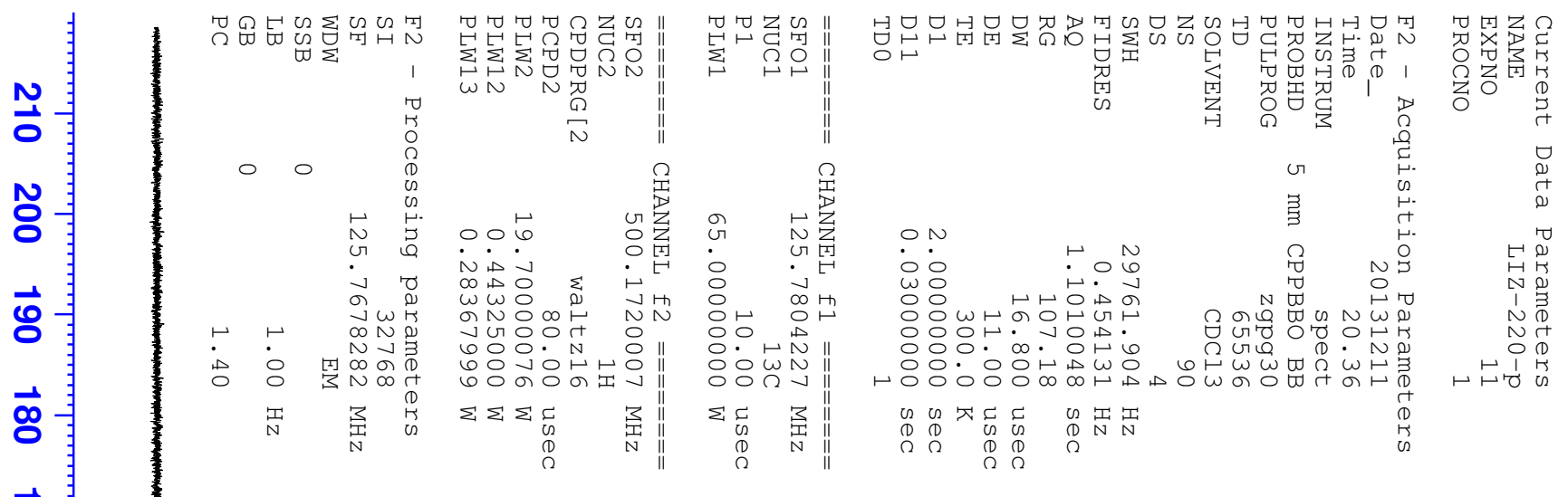

$\overrightarrow{0}$

홍

$\overrightarrow{\mathrm{g}}$

吾

ta

$\vec{\omega}$

నั

ةै

$\overrightarrow{\mathrm{o}}$

8

$\infty$

วั

8

의

혼

8

ผ

ธ

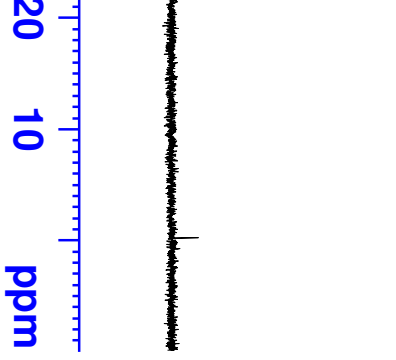

$\gamma_{77.420}$

77.166

76.913

$-62.795$

60.571

$-49.468$

$-46.697$

$-41.280$

$-38.363$

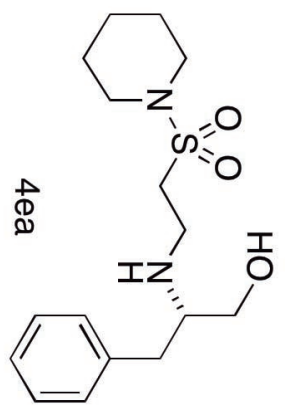

$-25.747$

$-23.902$

$-0.144$ 


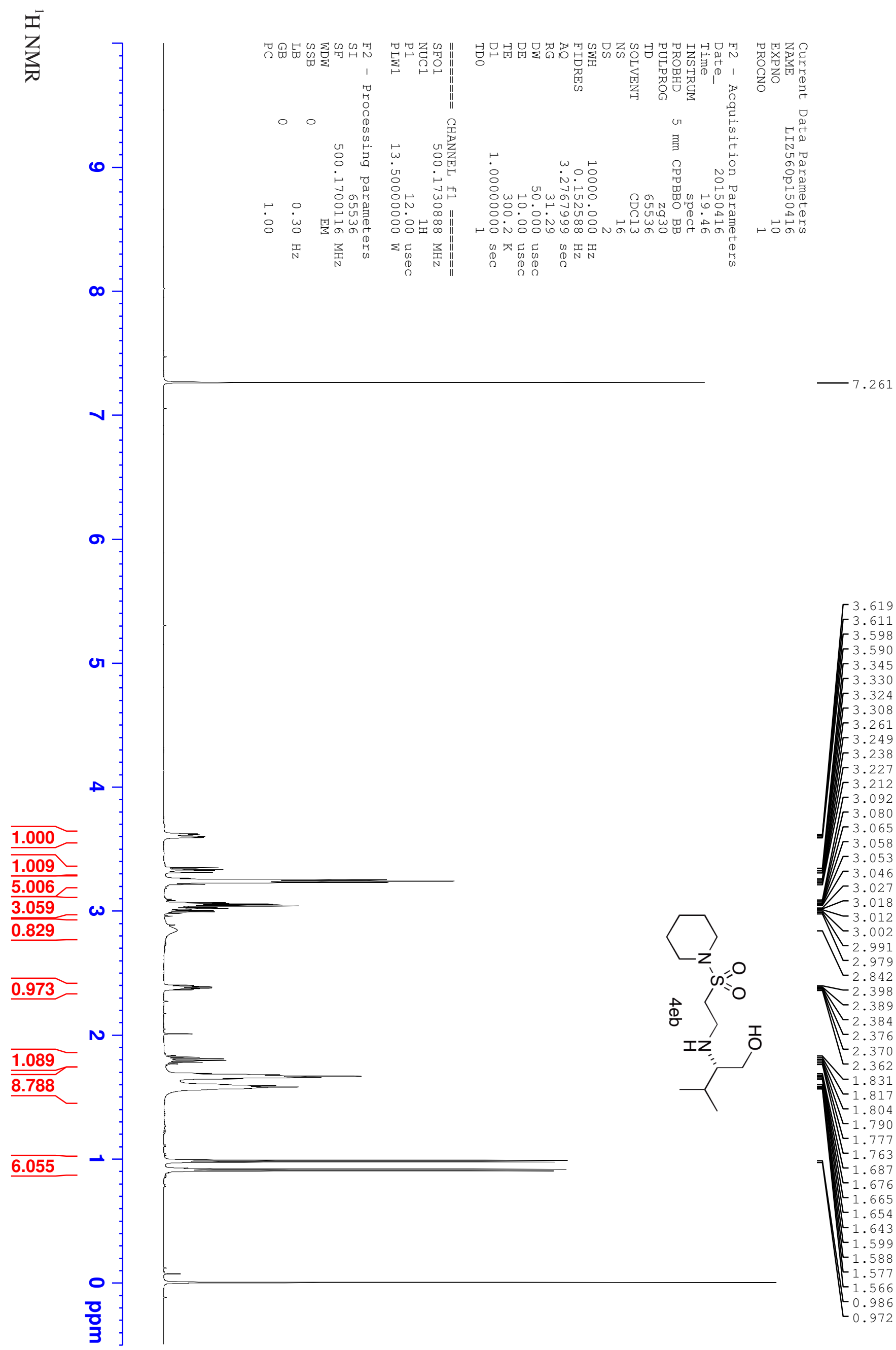




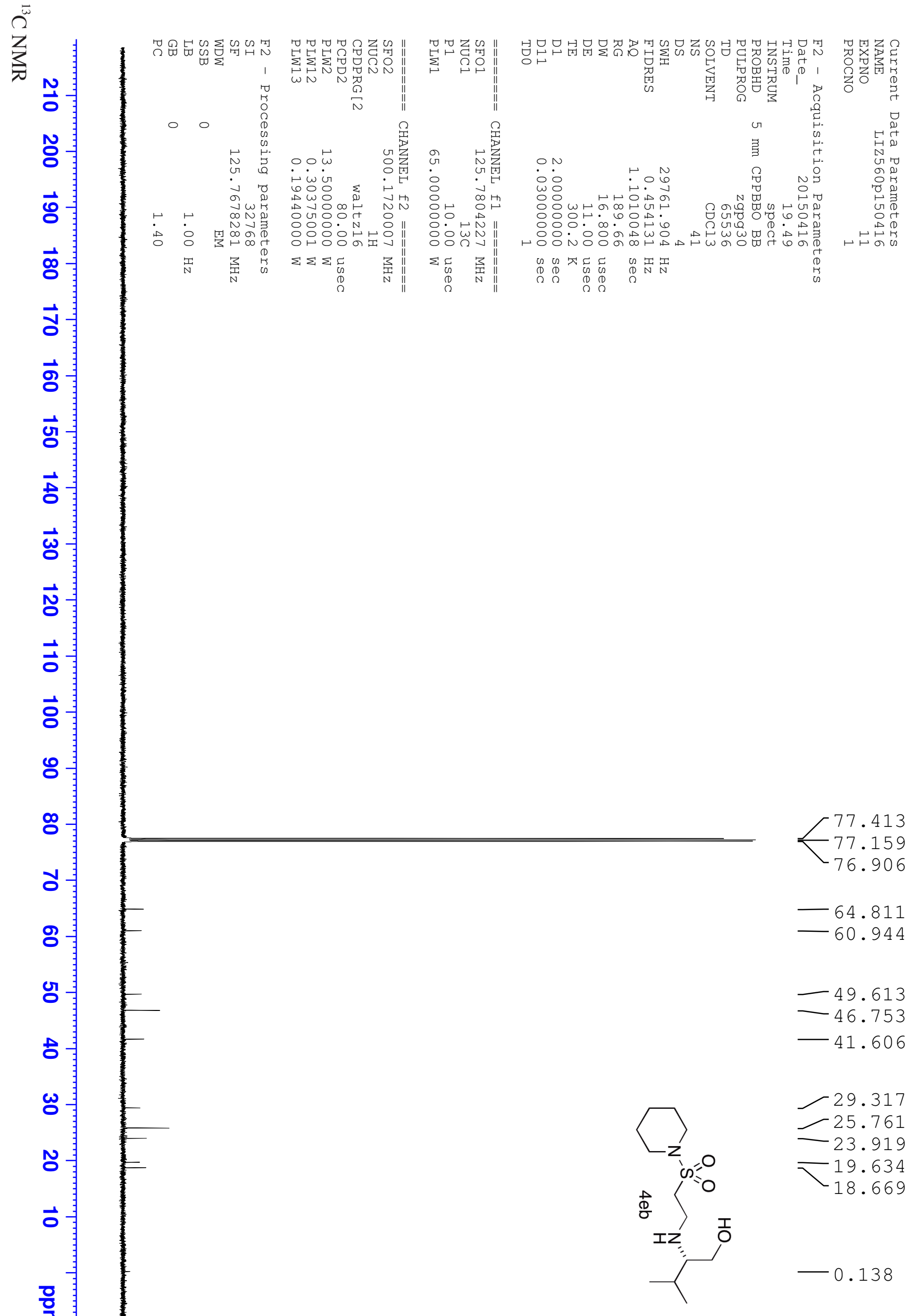




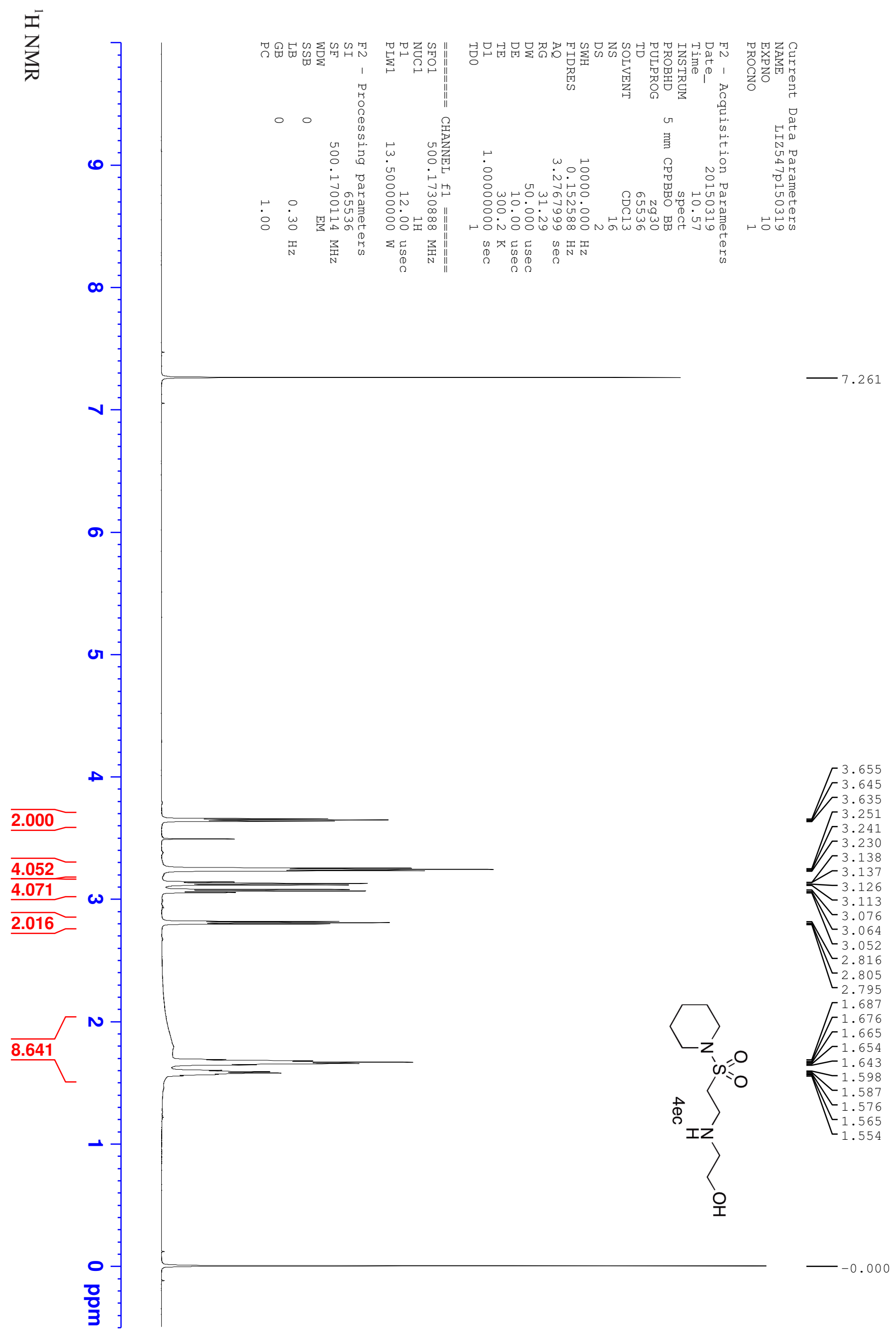


$\overbrace{}^{\bar{\omega}}$

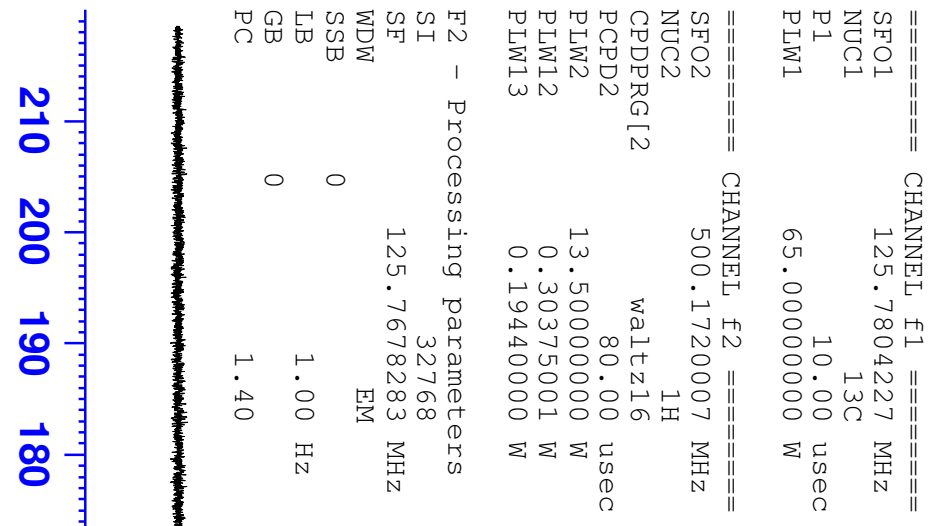

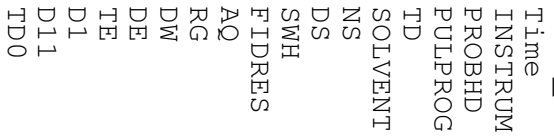

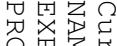

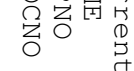

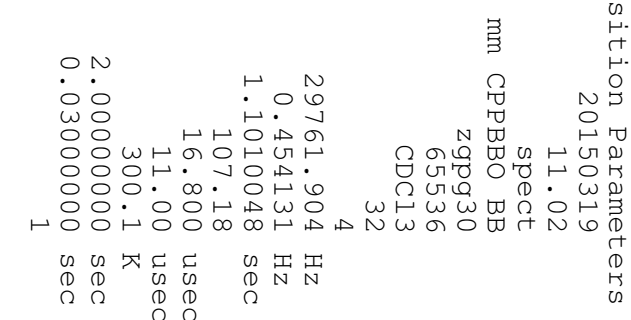

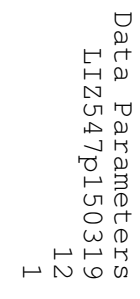

$\overrightarrow{0}$

항

ज़

$\overrightarrow{0}$

$\vec{\omega}$

$\vec{N}$

$\overrightarrow{0}$

웅

ต

$\infty$

Ј

\%

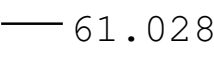

or

o

$-50.853$

$-49.020$

$-46.753$

$\omega$

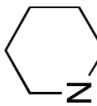

$z=0$

$-25.761$

N

离

总

$\overrightarrow{0}$

量

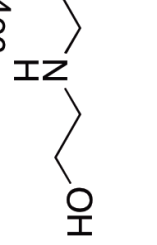




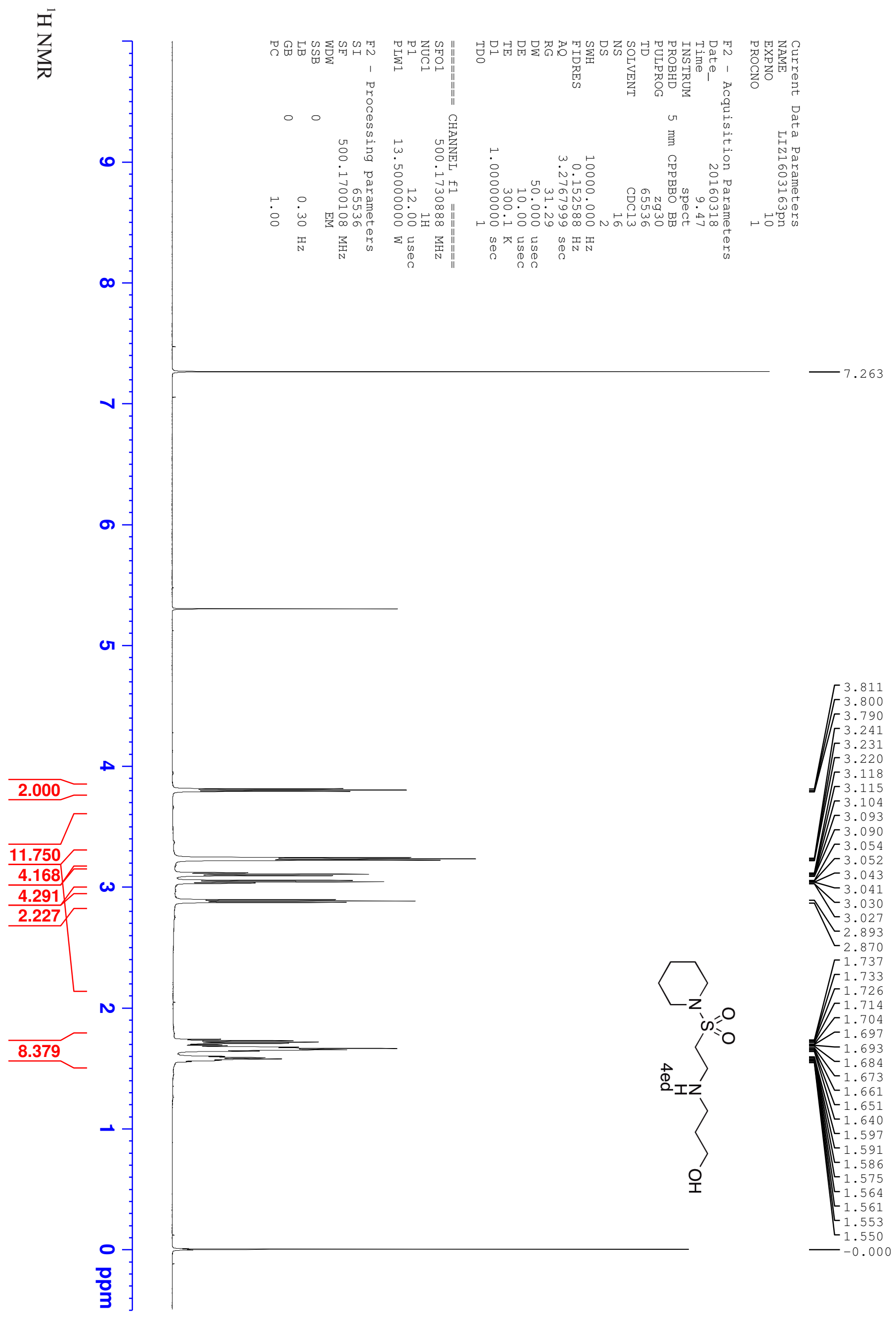



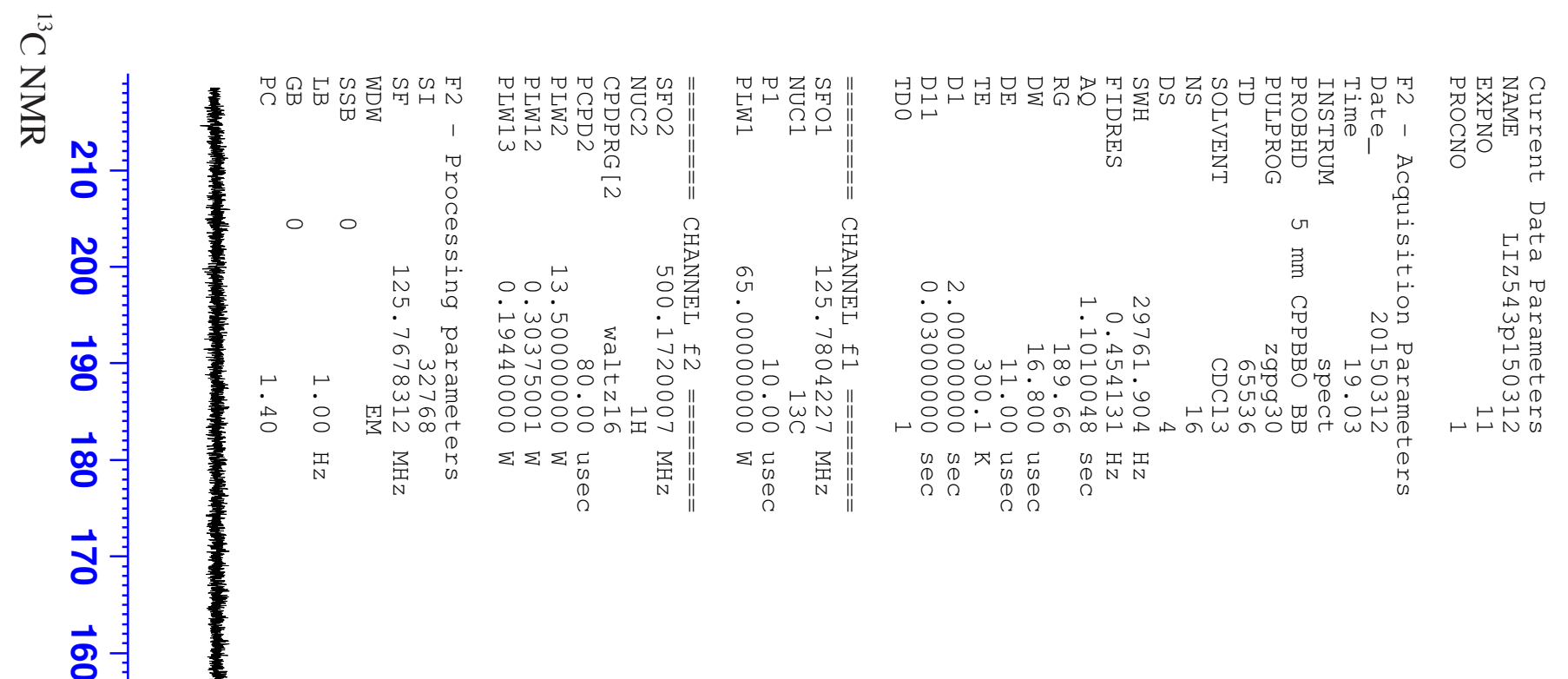


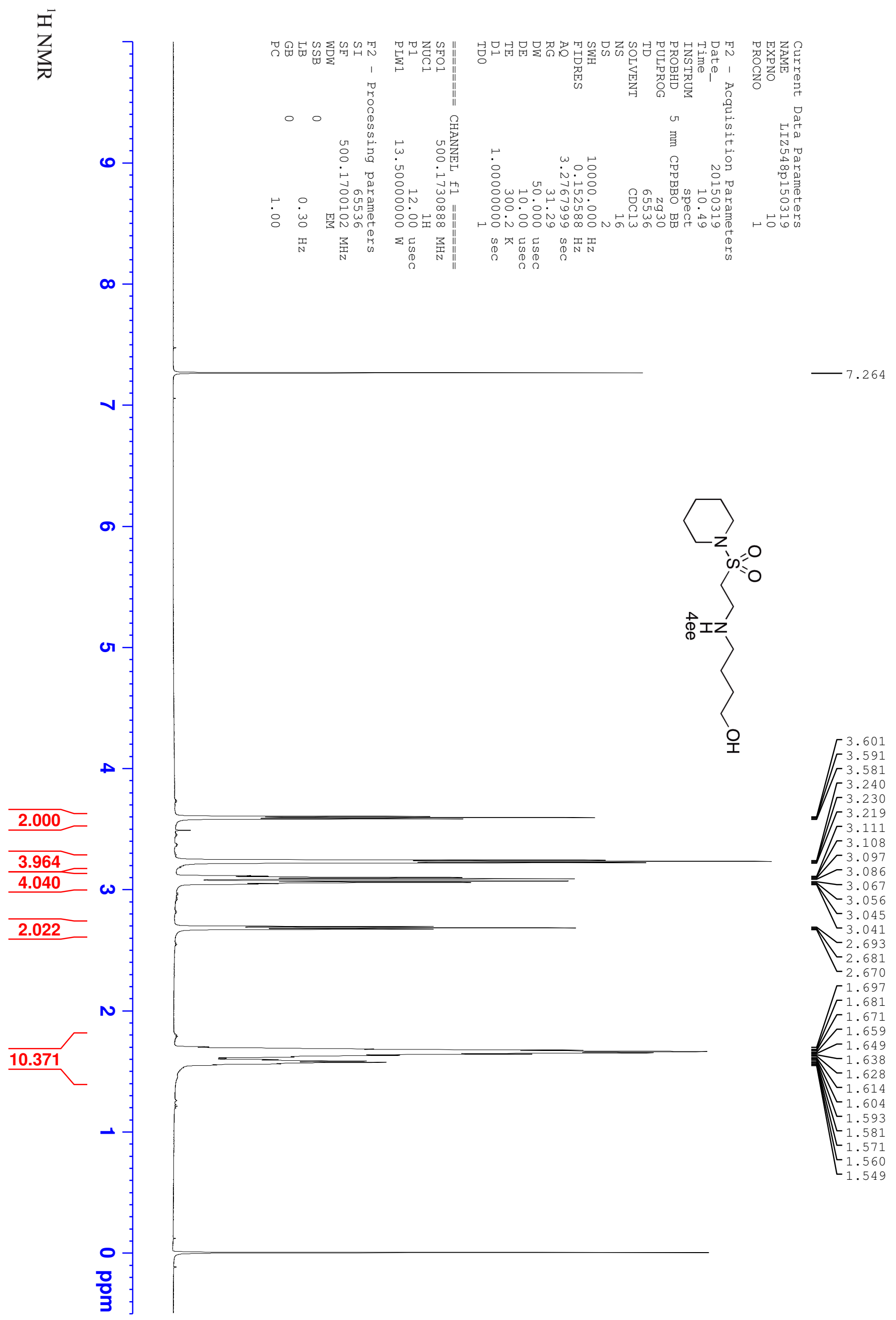




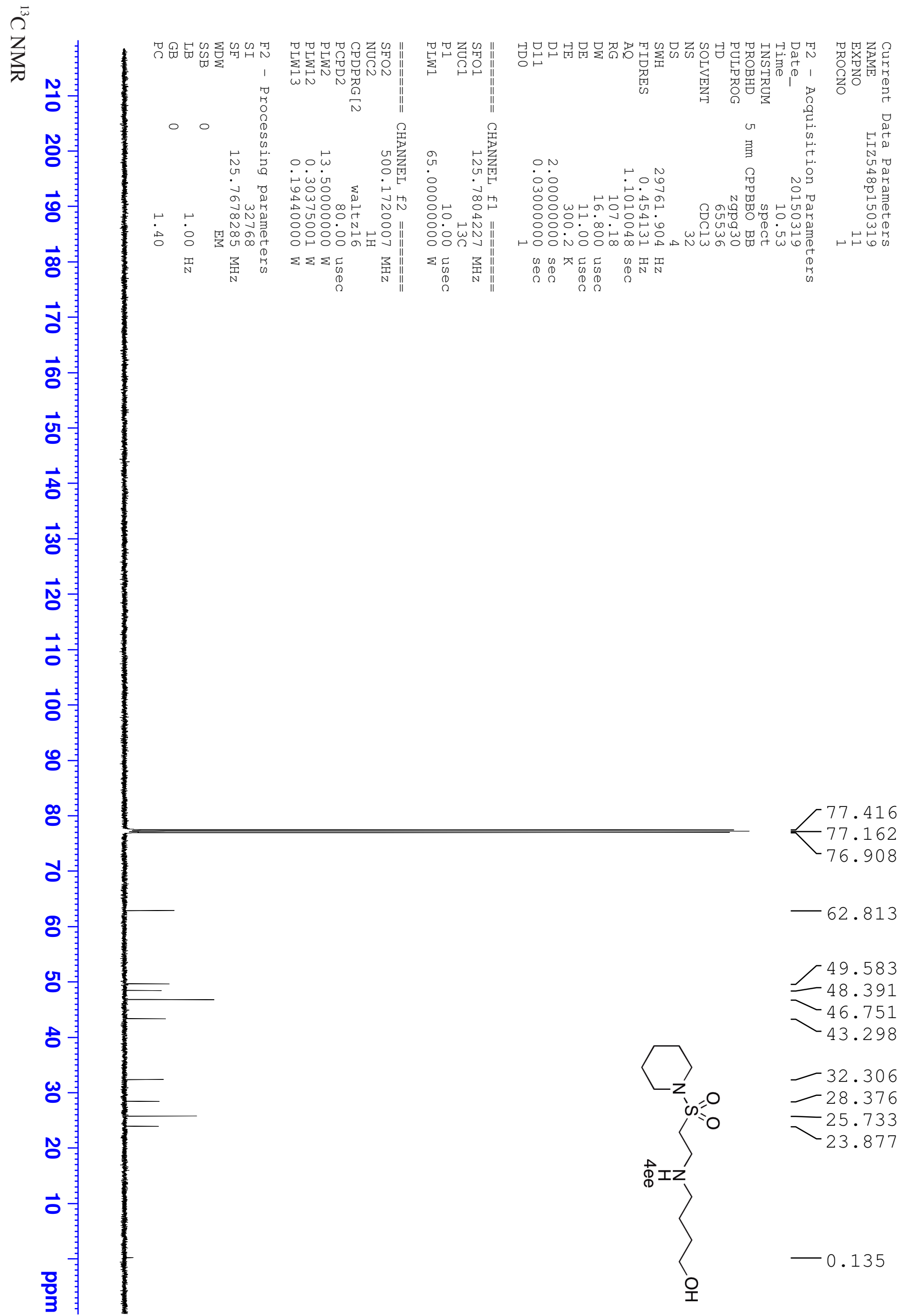




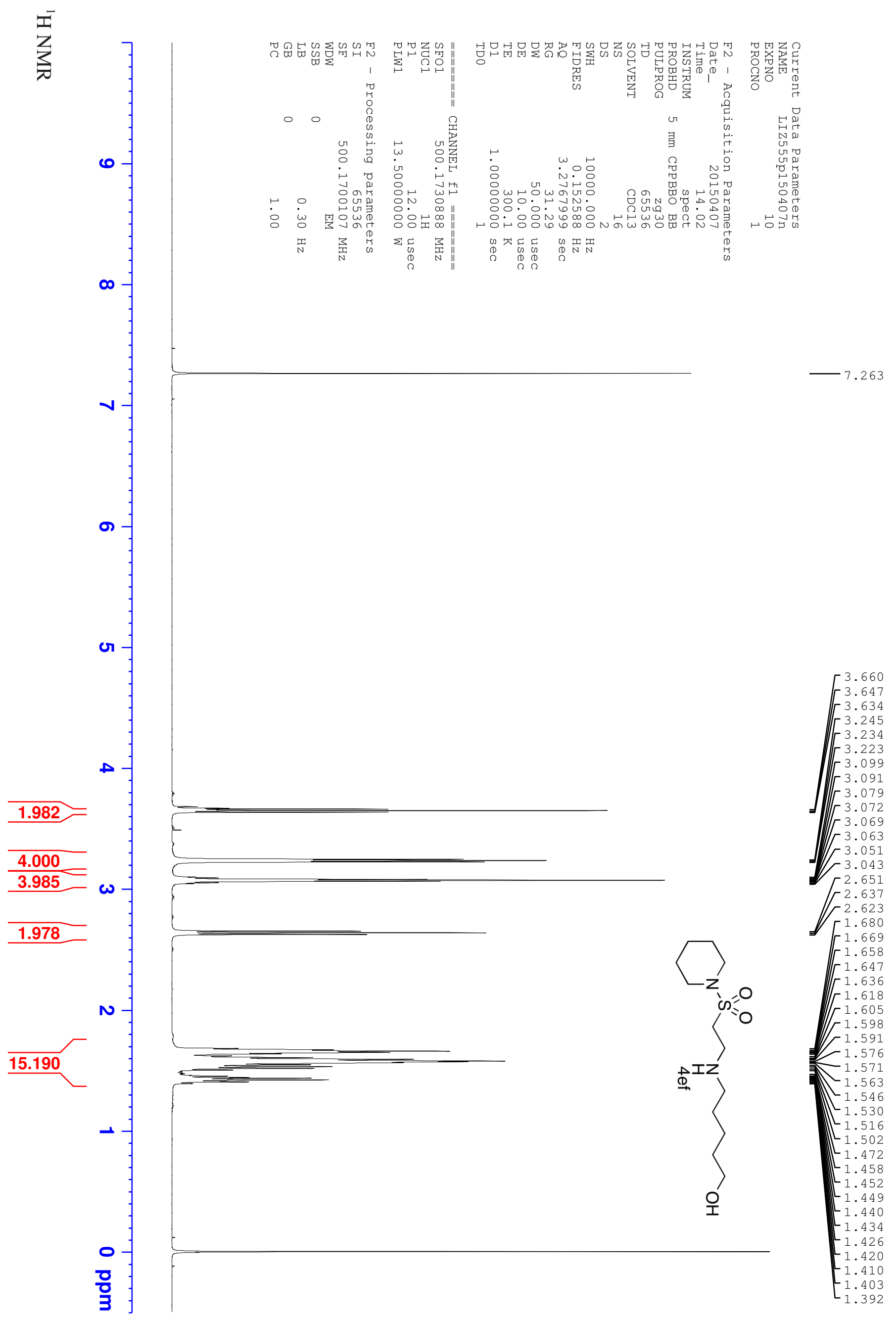




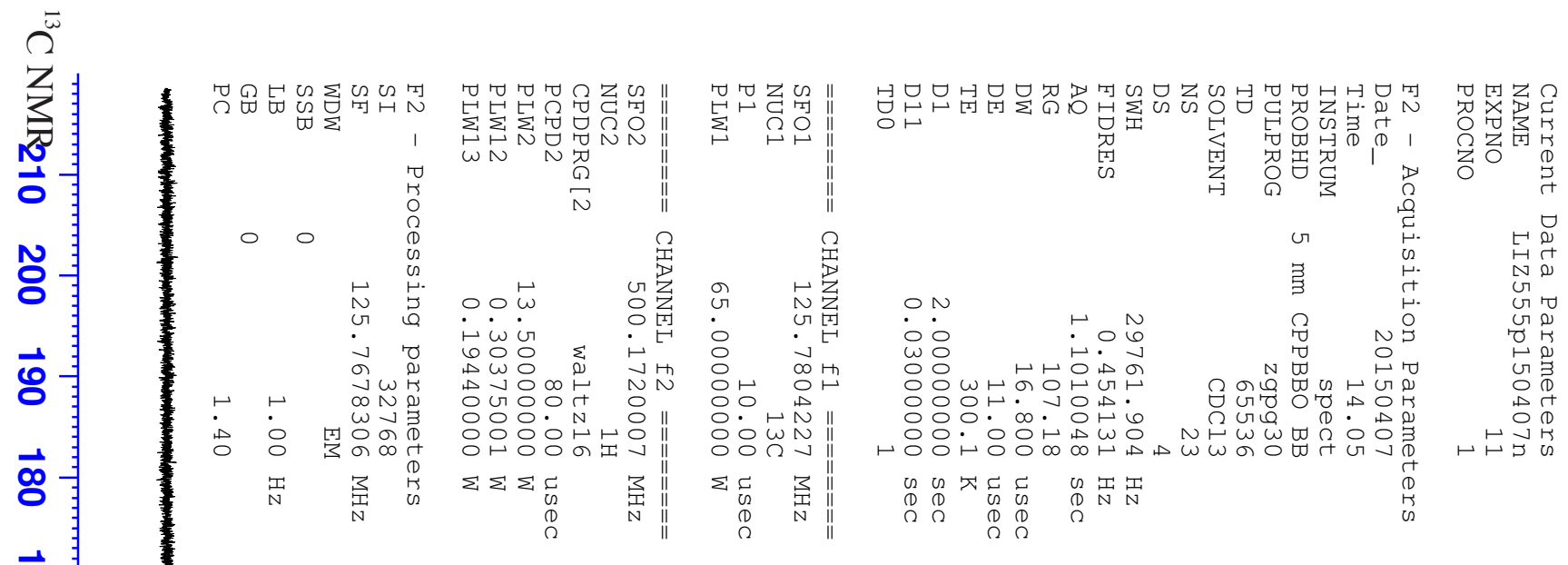

a

$\overrightarrow{\mathrm{g}}$

항

袁

$\vec{\omega}$

$\overrightarrow{\widetilde{v}}$

$\overrightarrow{0}$

$\overrightarrow{8}$

8

ø

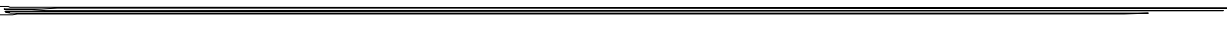

77.398
-77.144

76.890

ป

8

$-62.876$

앙

t

ผ

กั

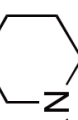

49.643
-48.974
-46.723
-43.704

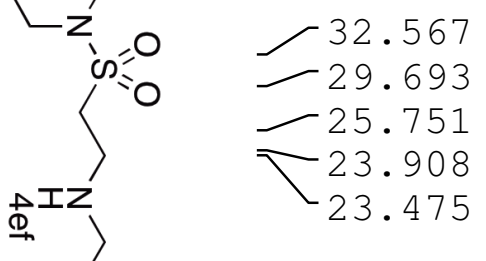

奖 


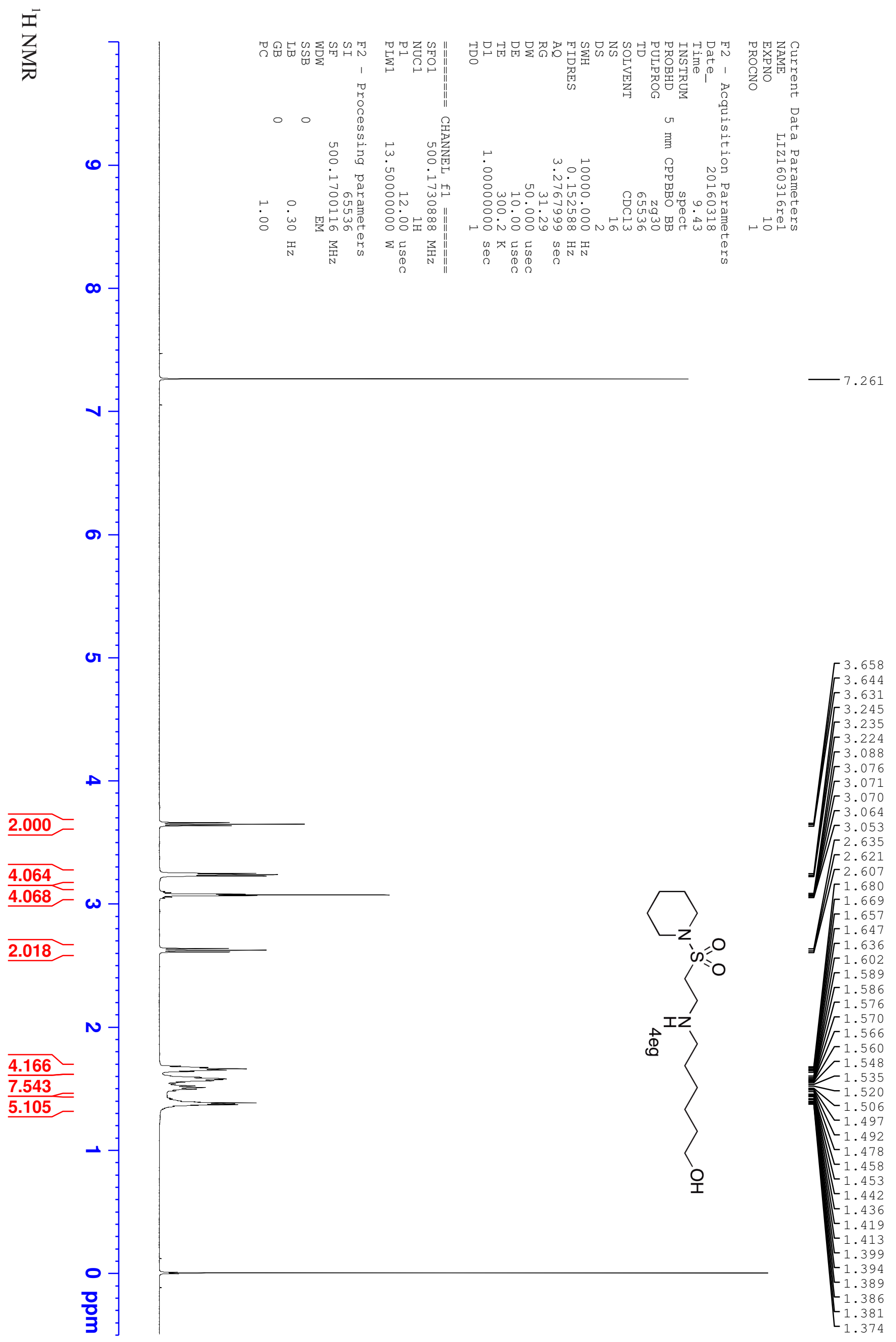




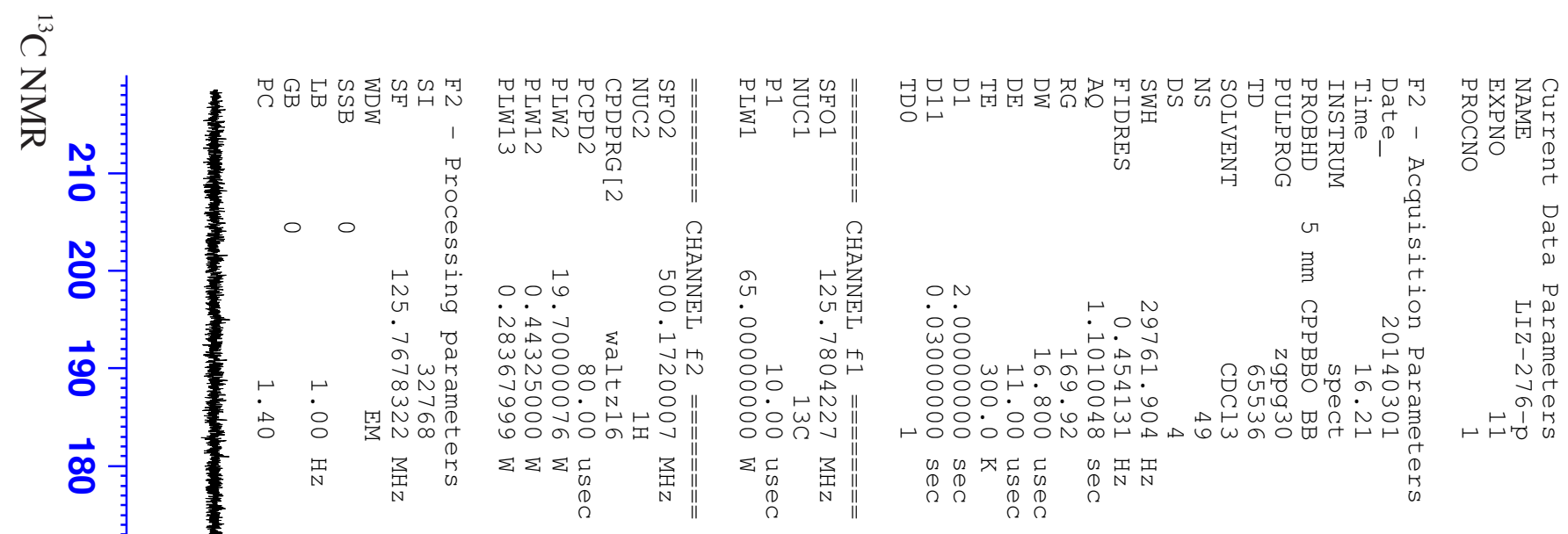

$\vec{\delta}$

$\overrightarrow{\mathrm{g}}$

जे

$\overrightarrow{0}$

$\vec{\omega}$

$\overrightarrow{\text { N }}$

$\overrightarrow{0}$

$\overrightarrow{8}$

8

ø

o

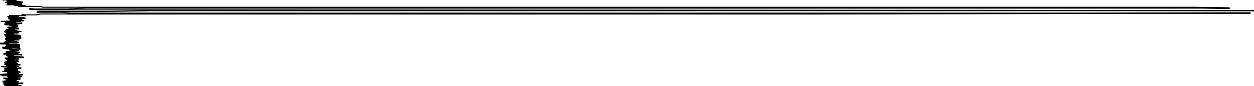

77.410

77.157

76.902

8

62.826

잉

8

$\ddot{\circ}$

ก

$\overrightarrow{0}$

맘

总

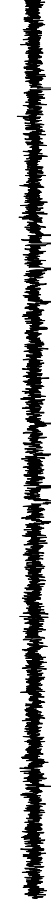

- 


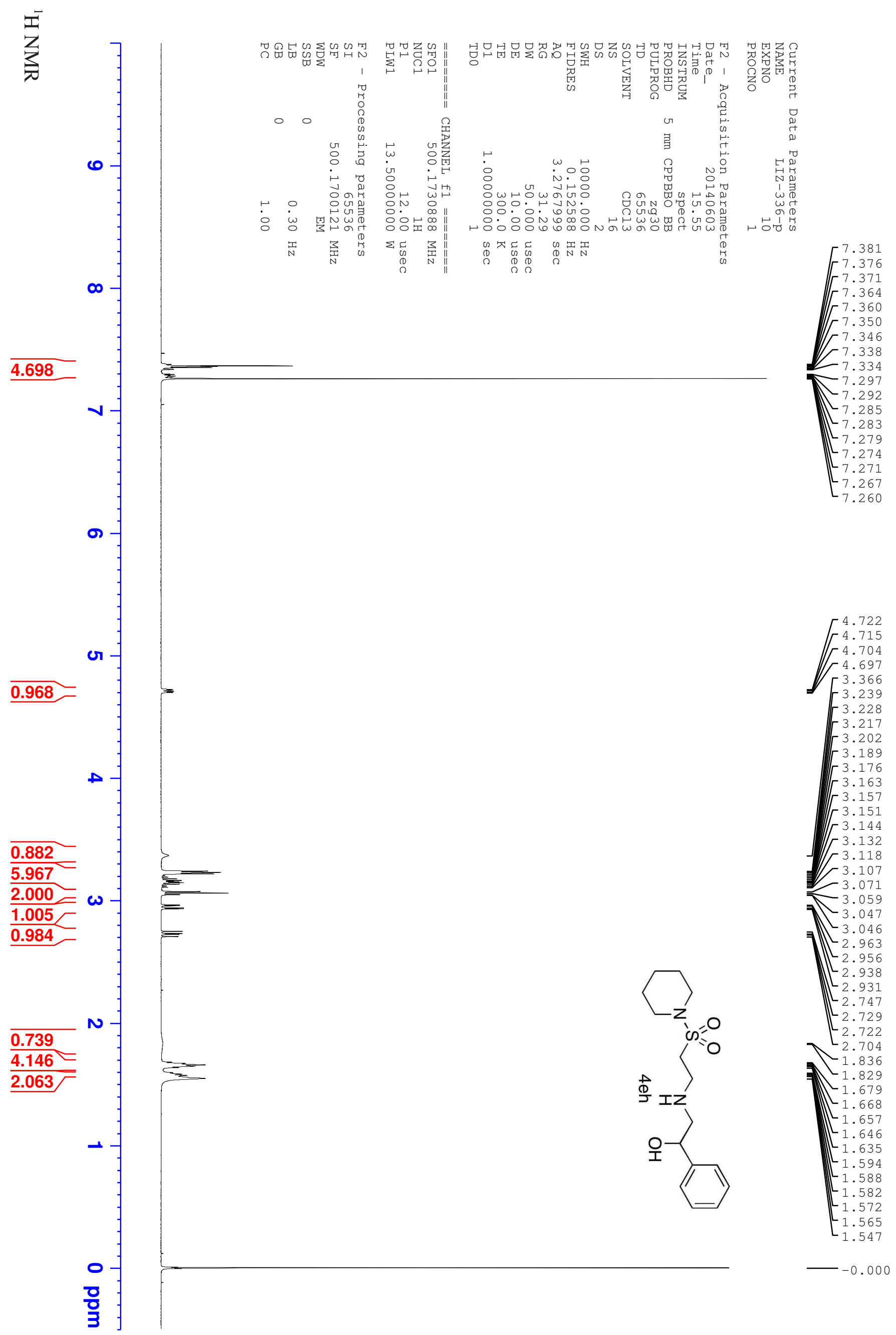



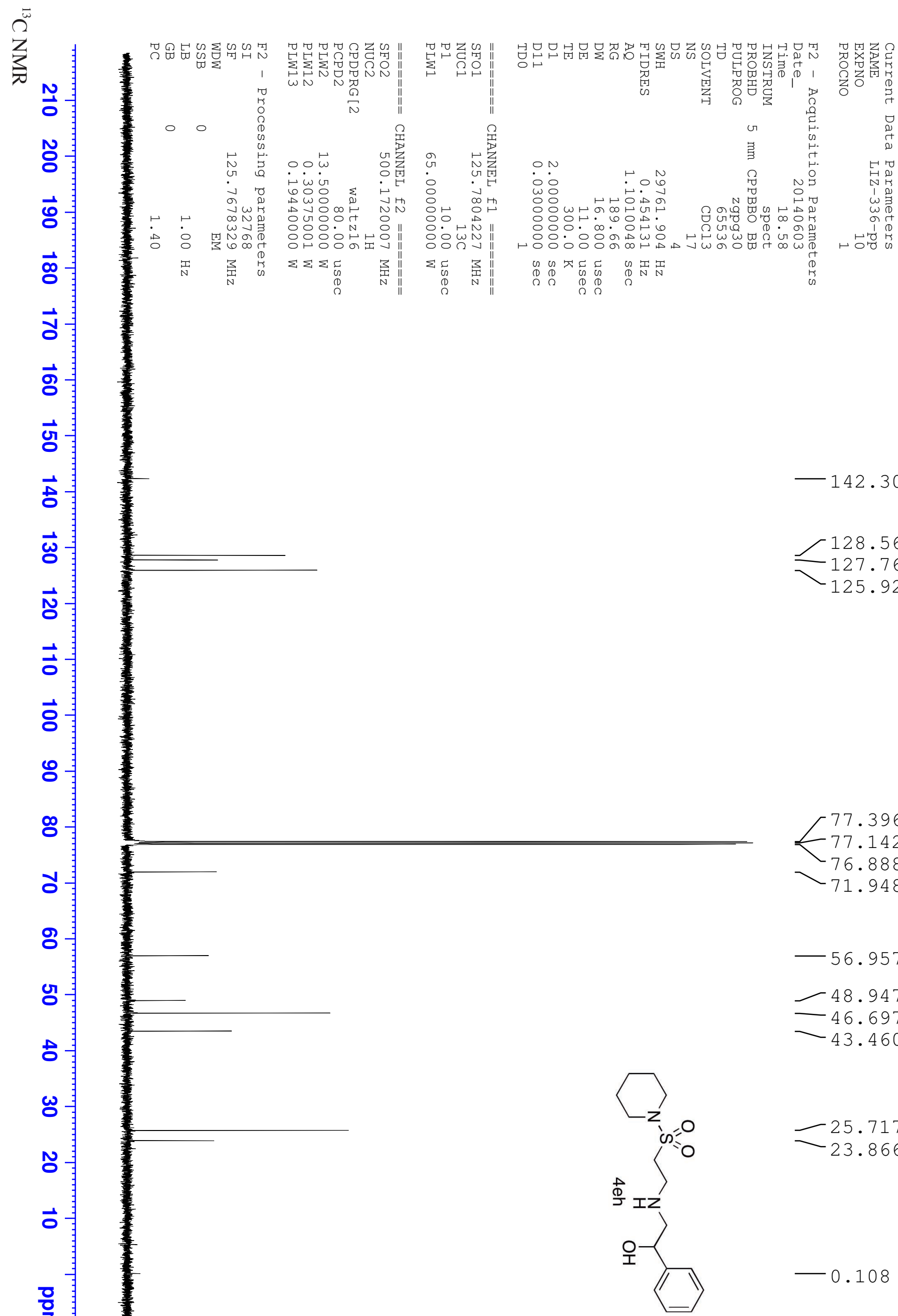


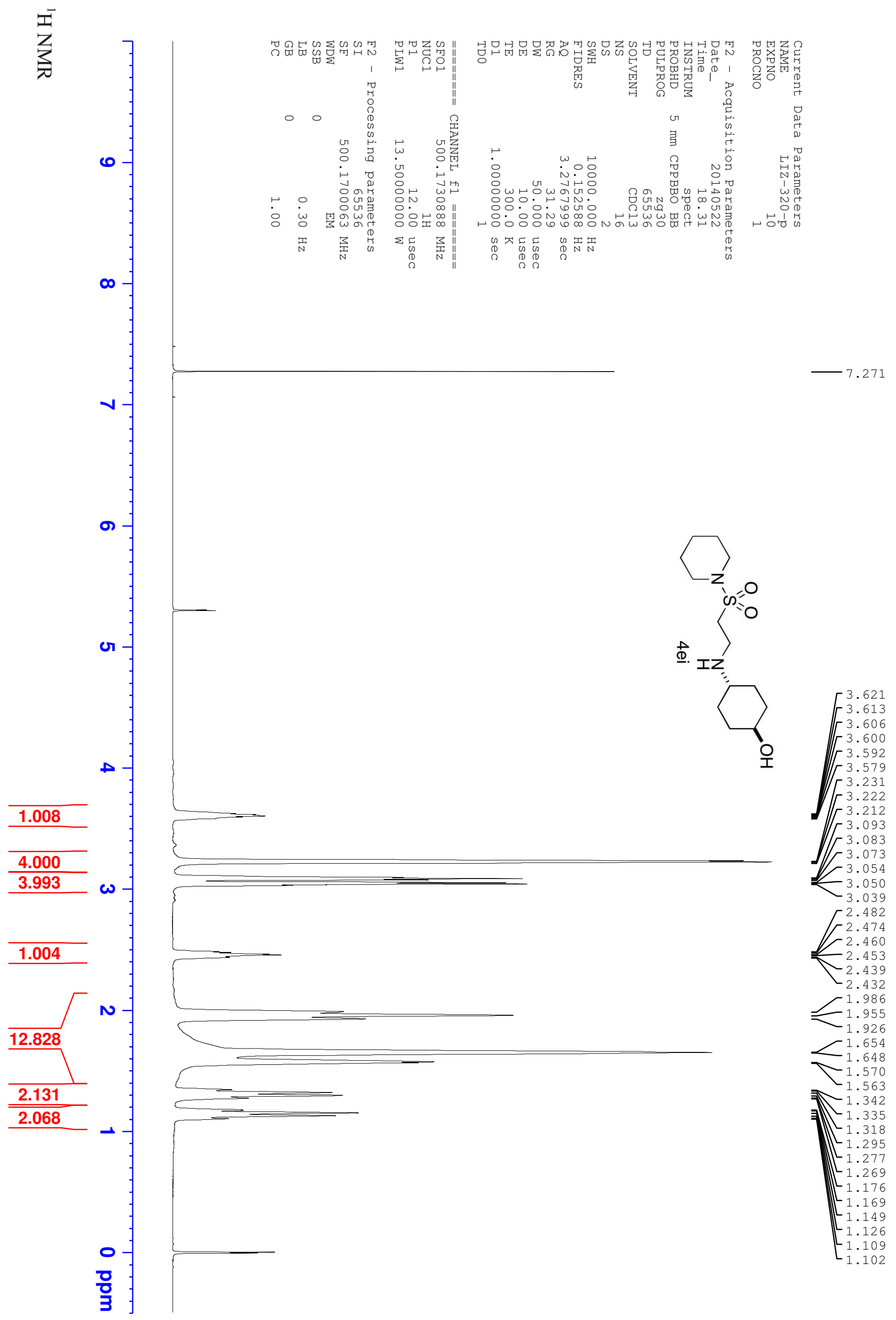




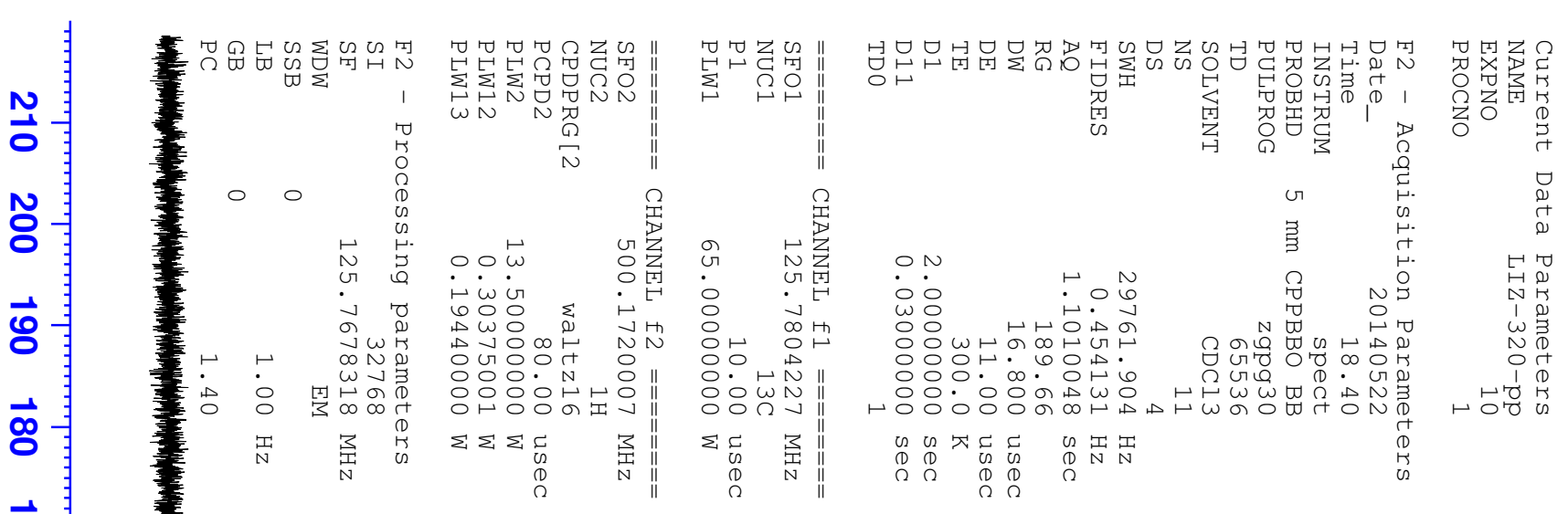

ปี

항

ज्ञ

롱

$\vec{\omega}$

$\vec{N}$

$\overrightarrow{0}$

훙

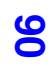

$\infty$

๖

용

당

호

$\omega$

ก

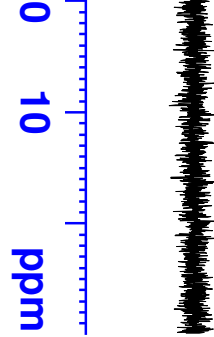

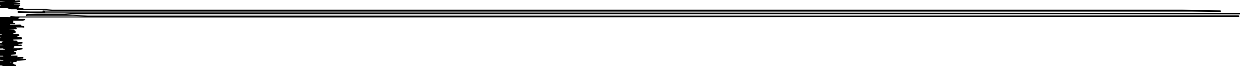

77.414

$\leftarrow 77.160$

76.907

$-70.408$

$-55.809$

$-49.263$

$-46.706$

$-41.103$

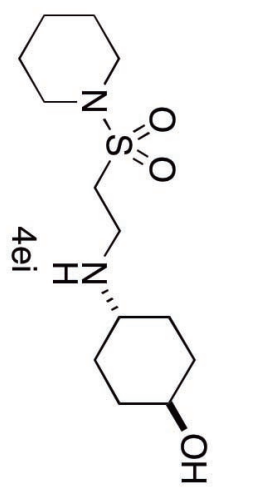

$-34.003$

$-31.190$

$-25.732$

$-23.878$

$-0.109$ 


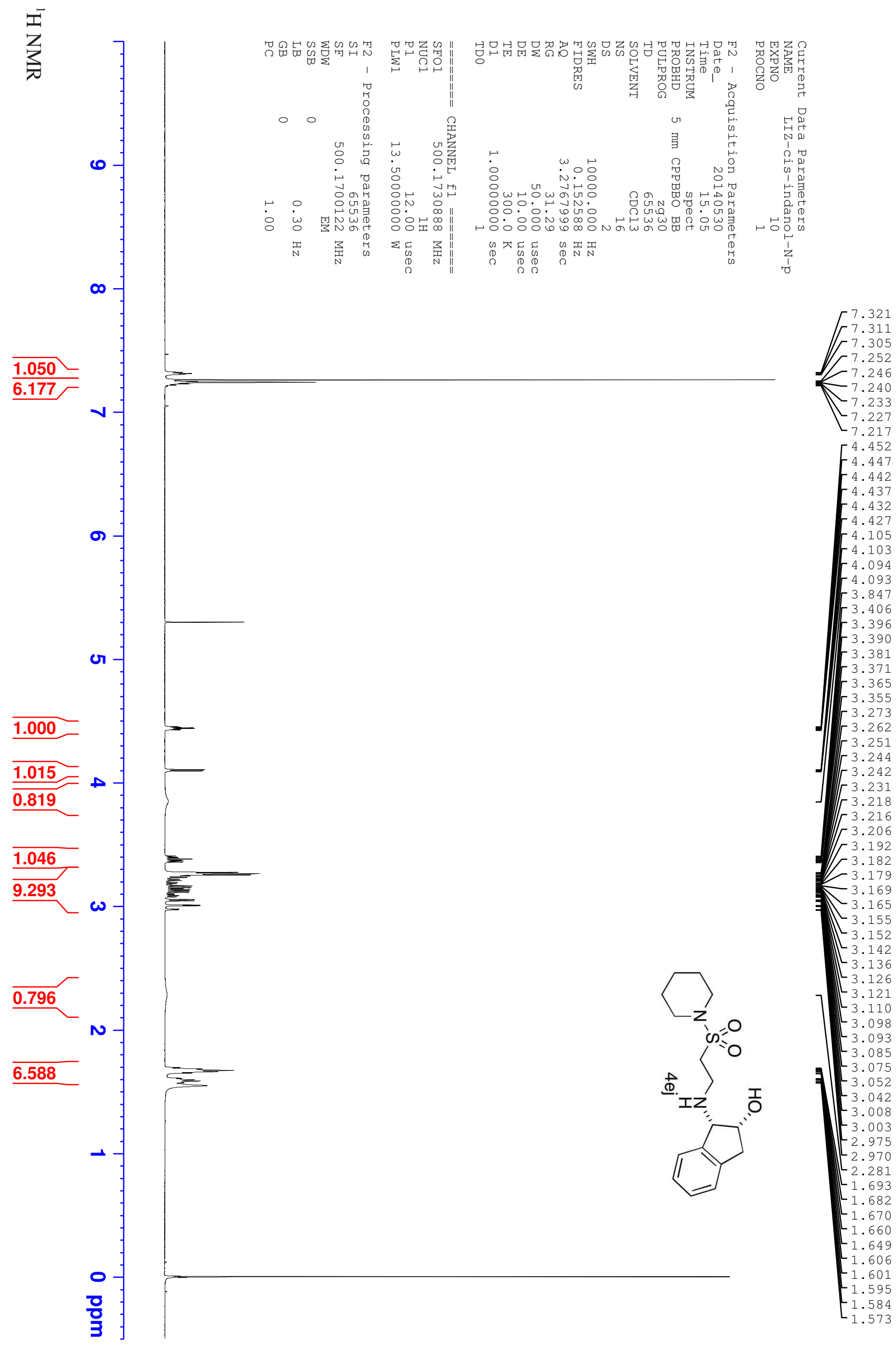


8

잉

b

ผ

ง

ั

o

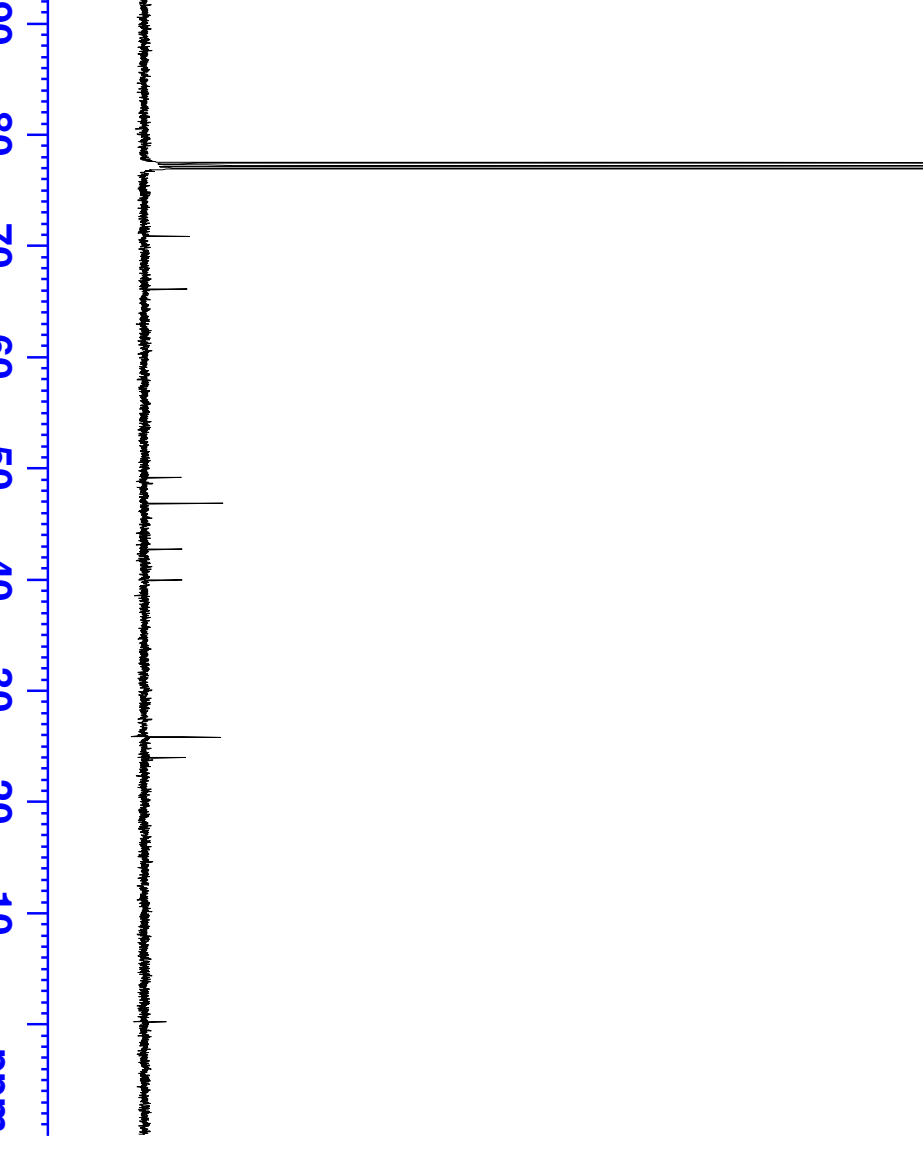

77.416 


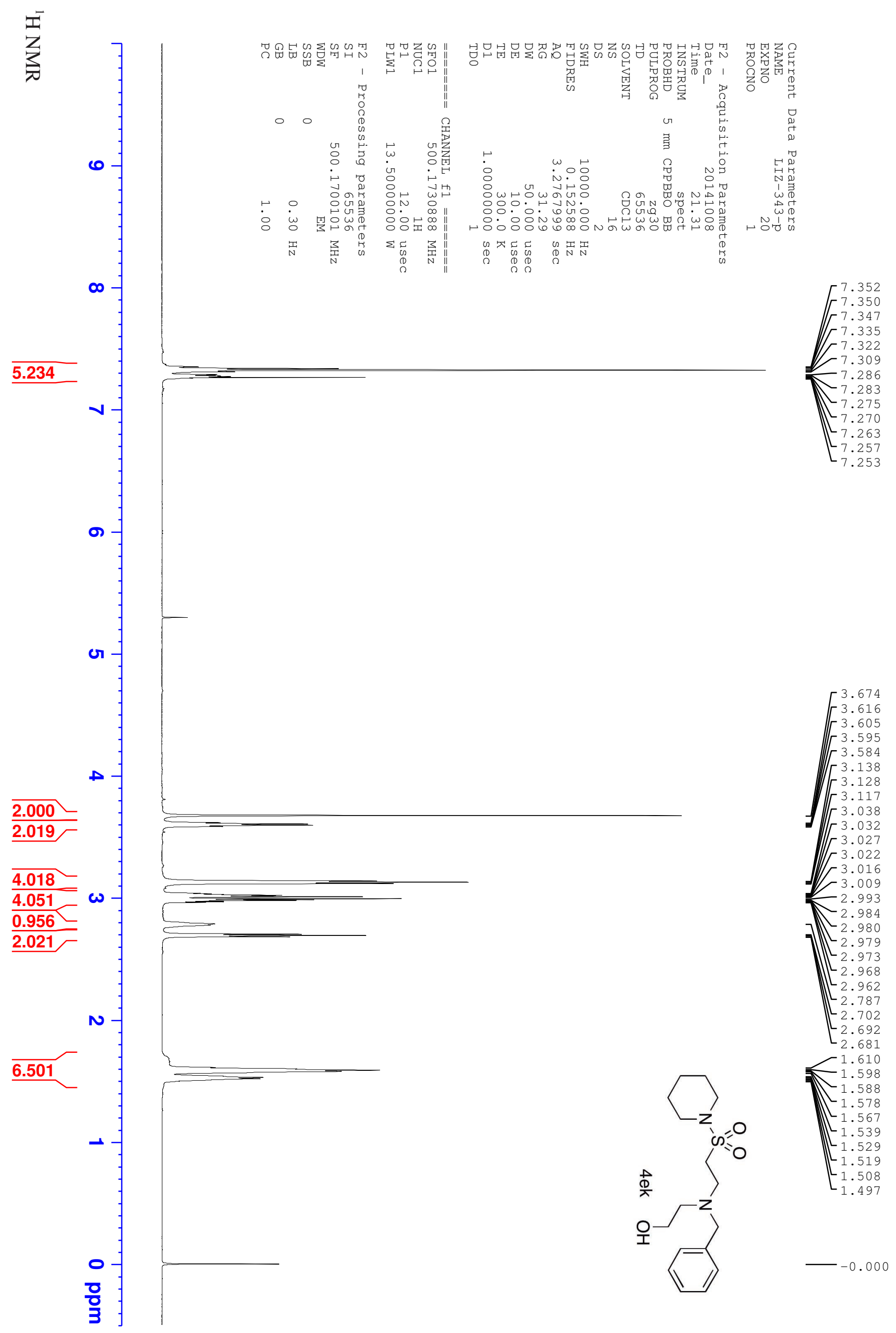




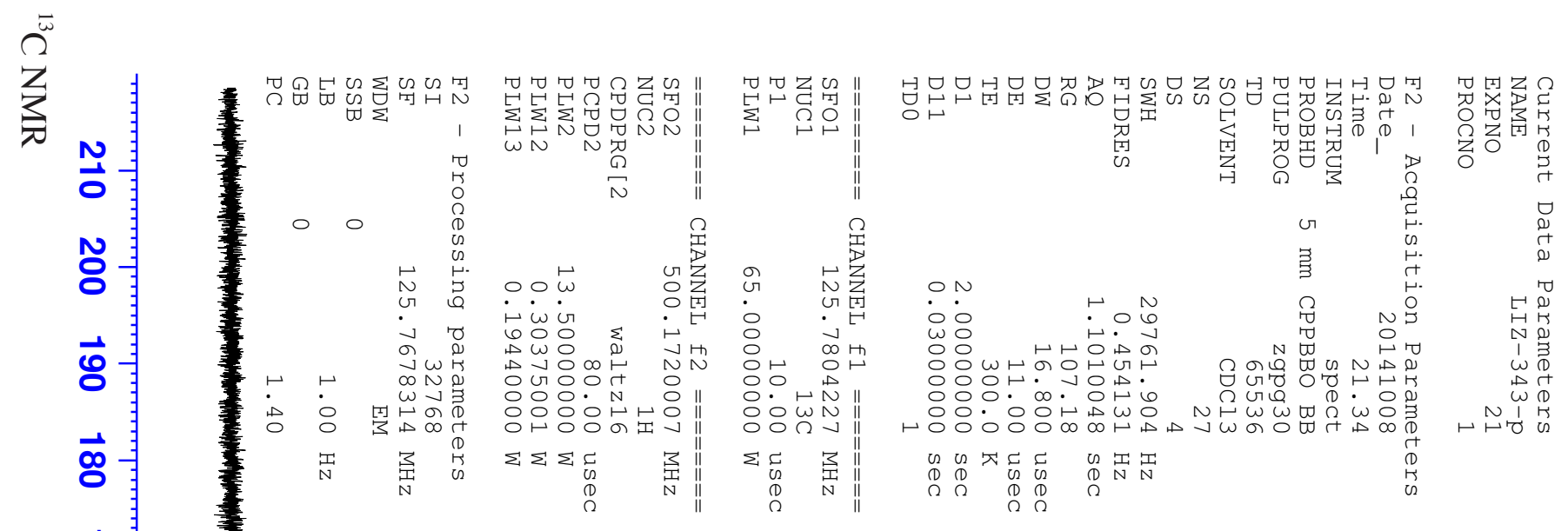

홍

광

$\overrightarrow{\mathbf{a}}$

$\vec{\omega}$

$\vec{\Sigma}$

ذ)

호

$\overrightarrow{\mathrm{s}}$

$-138.229$

$\vec{\omega}$

a

$\overrightarrow{8}$

8

ळ

ป

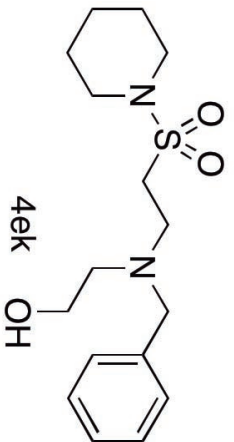

8

II

t

ผ

ก

77.419

77.165

76.911

$\begin{array}{r}59.074 \\ \hline 59.015\end{array}$

59.015

55.968

47.979

46.853

46.621

$-25.707$

$-23.857$

产

$\overrightarrow{0}$ 


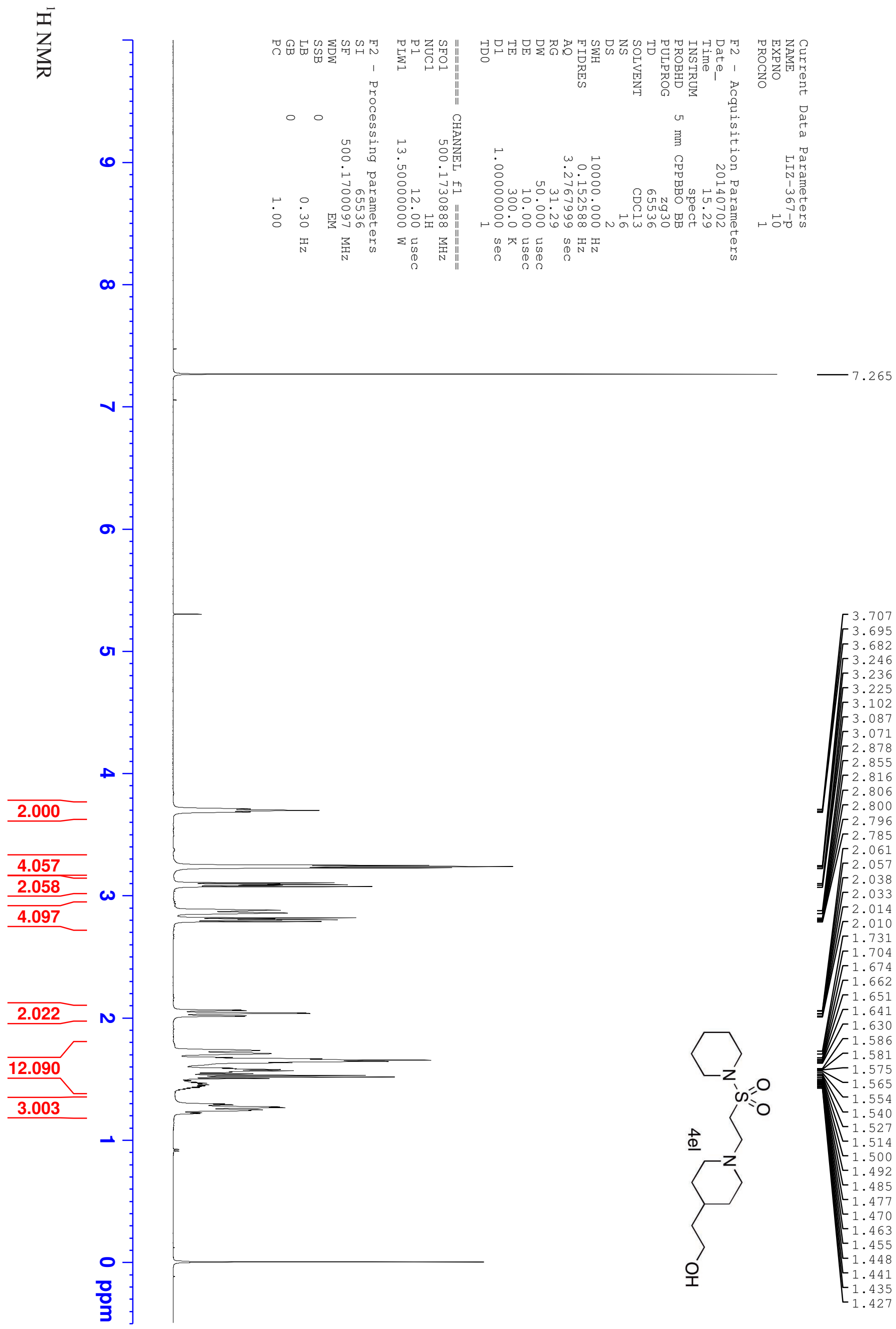




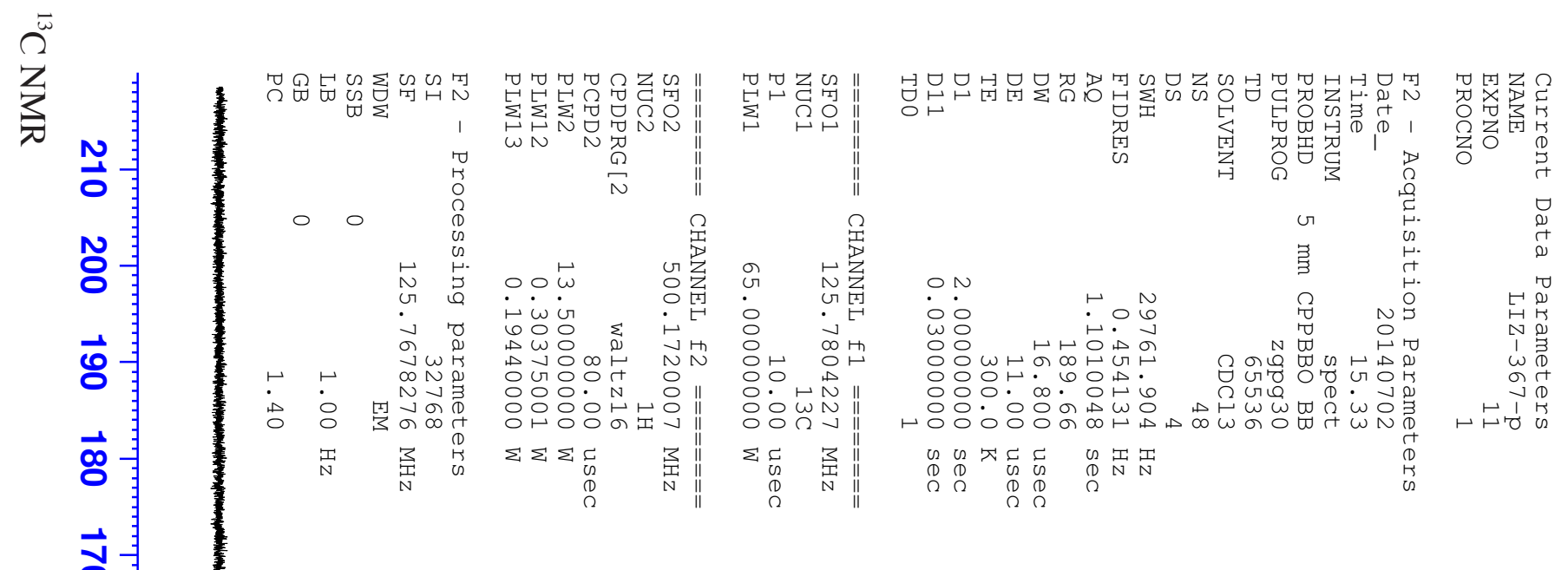

$\vec{\Delta}$

홍

횡

$\overrightarrow{\mathrm{a}}$

డे

茫

$\vec{\Delta}$

$\overrightarrow{\mathrm{o}}$

8

$\infty$

8

잉

호

$\omega$

ก

o

흡
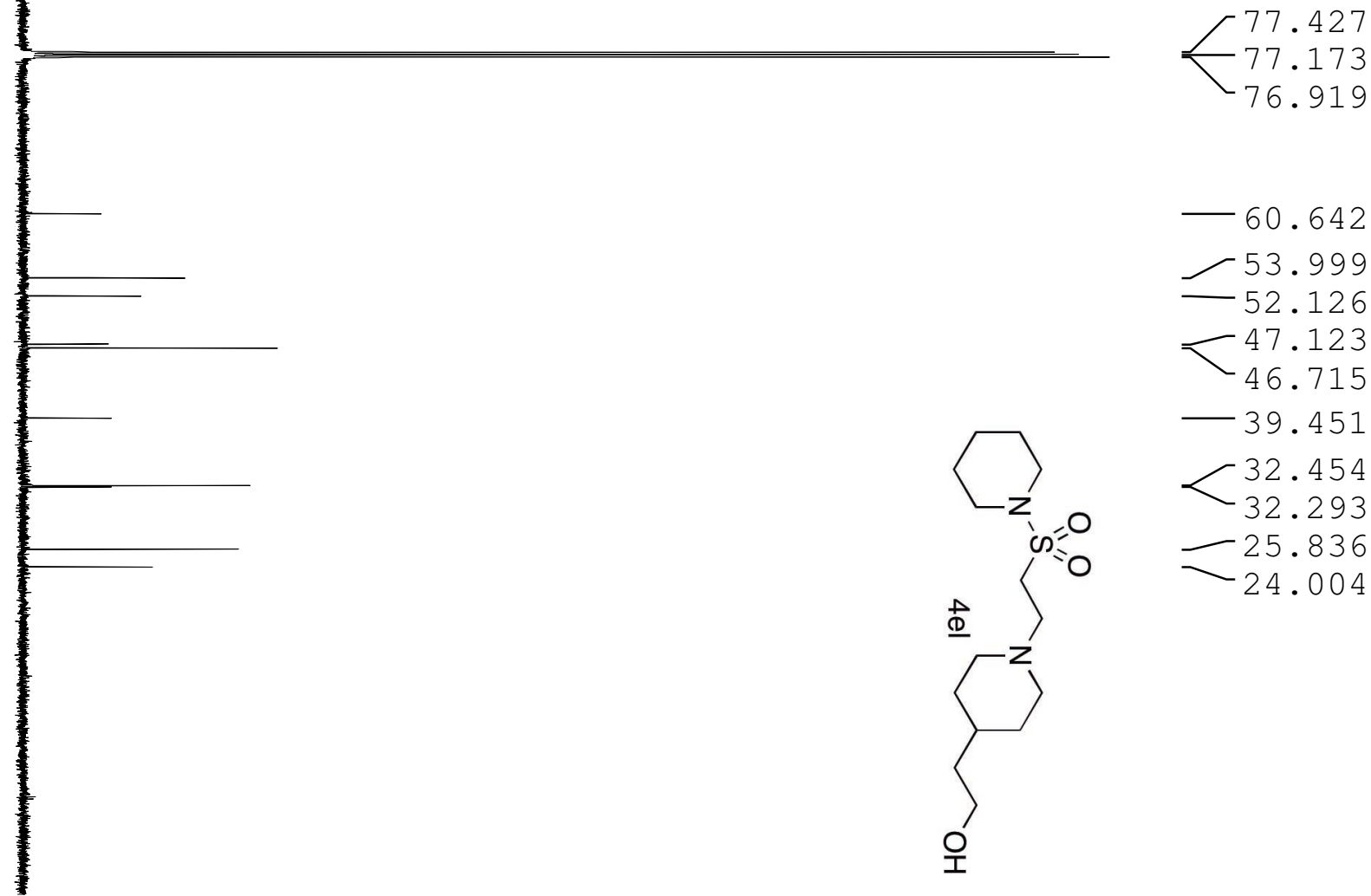

76.919

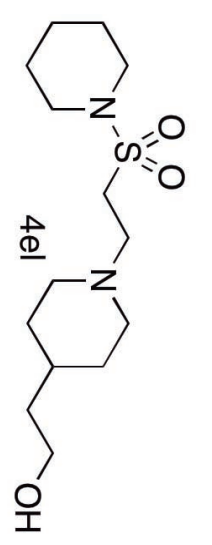

$-39.451$

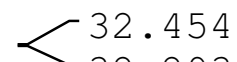

$-32.293$

-25.836
-24.004 


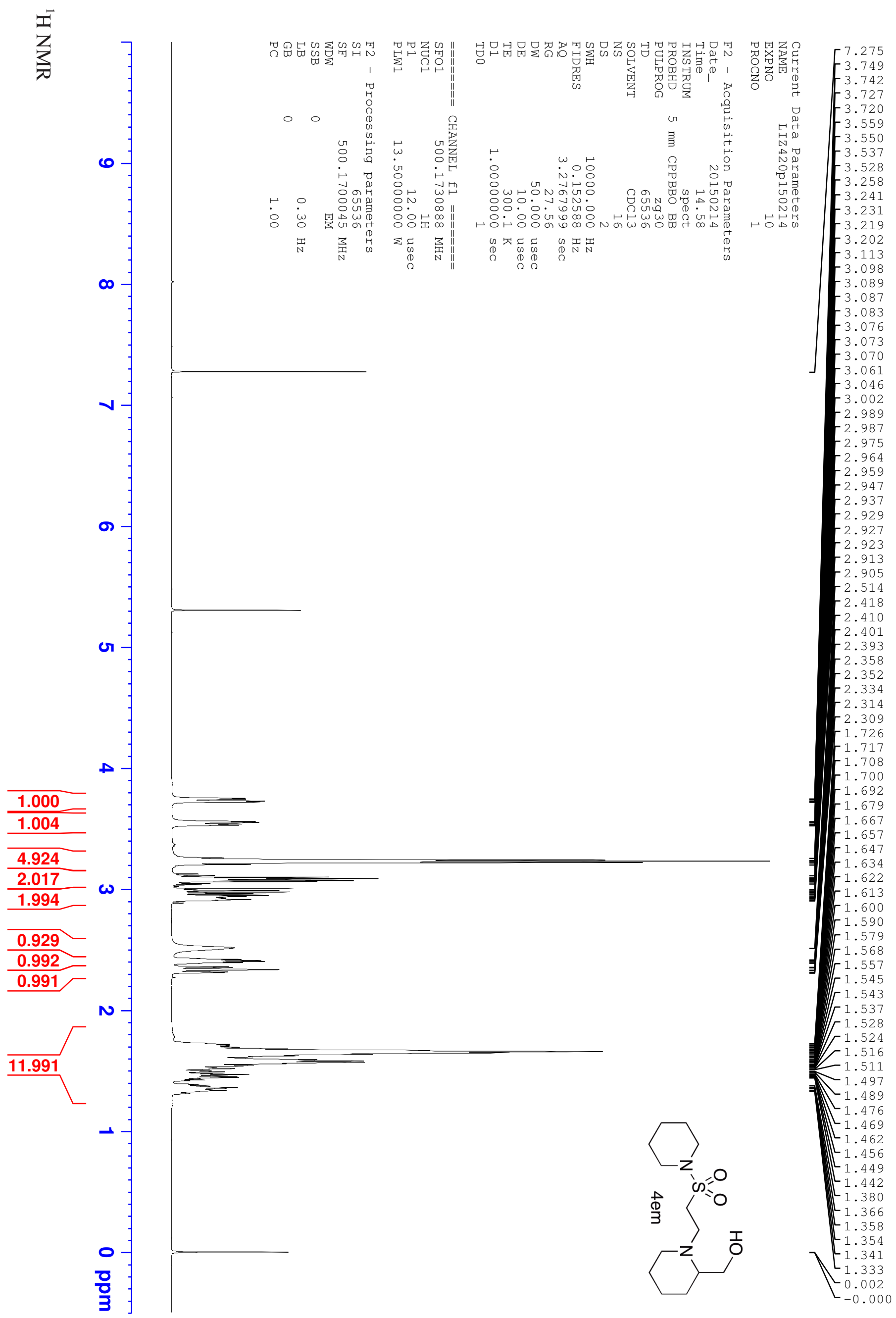




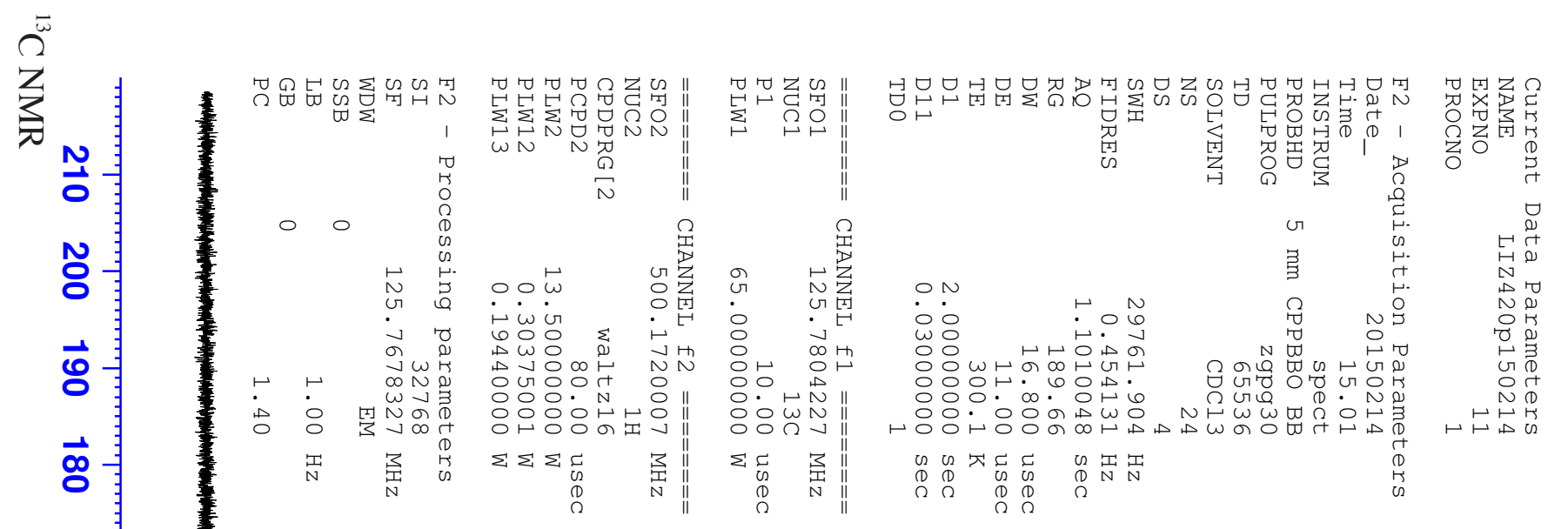

$\vec{d}$

$\overrightarrow{8}$

방

$\overrightarrow{\mathrm{a}}$

$\vec{\omega}$

$\overrightarrow{\text { ๊ }}$

$\vec{a}$

$\overrightarrow{\mathrm{s}}$

8

ø

ò

8

잉

t

$\omega$

กิ

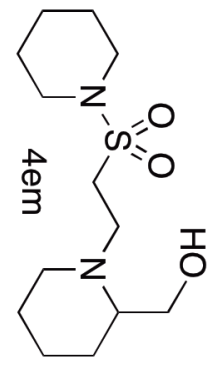

77.410
$\leftarrow 77.156$
76.902

$-62.764$

60.739

$-51.577$

$-47.294$

46.691

46.285

27.289
-25.753

$-24.396$

23.901

23.357

$\overrightarrow{0}$

뫃

등 $\quad 0.103$ 


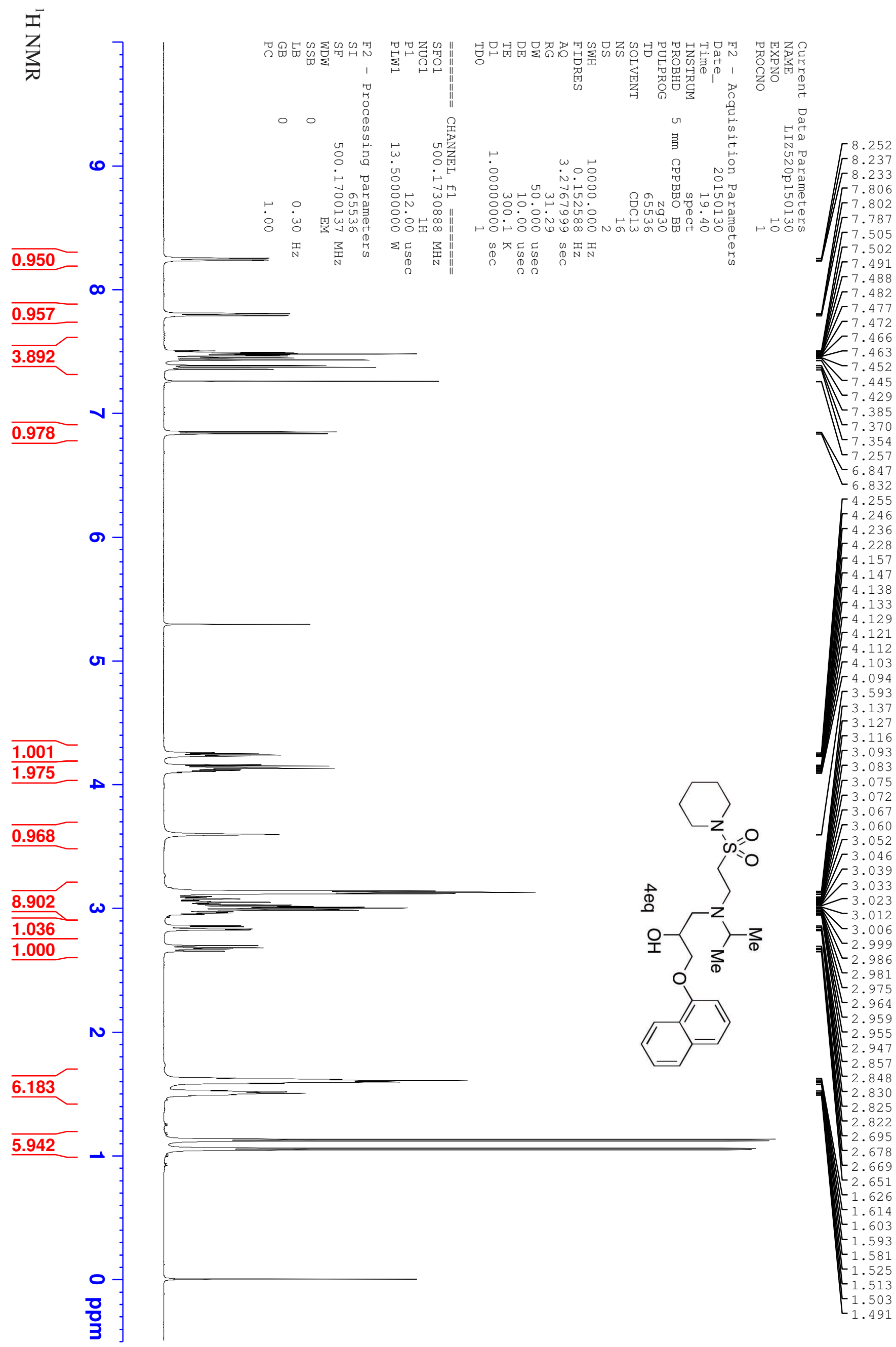




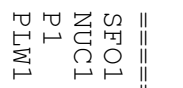

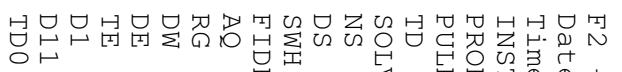

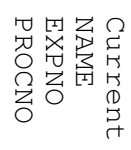

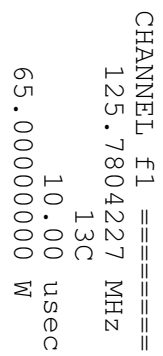

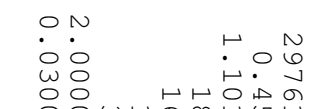

$\begin{array}{lll} & \\ 8 & \end{array}$

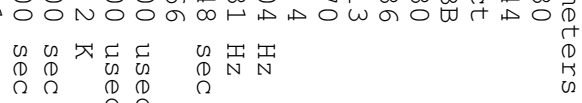

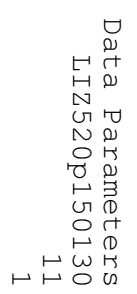

ज़

$\overrightarrow{\vec{A}}$

$\vec{\circ}$

$\vec{\omega}$

$\vec{N}$

ปั่

亲

항

$\mathscr{8}$

\&

ป

g

or

古

w

กิ

ธ

$\overrightarrow{0}$

它青

을

의

$\infty$

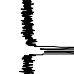

134.651
127.661
126.576
125.992
125.717
125.393
121.965
120.720

$-104.991$

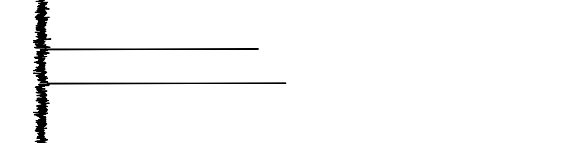

77.415

$-77.160$

76.906

$-70.089$

$-67.012$

8
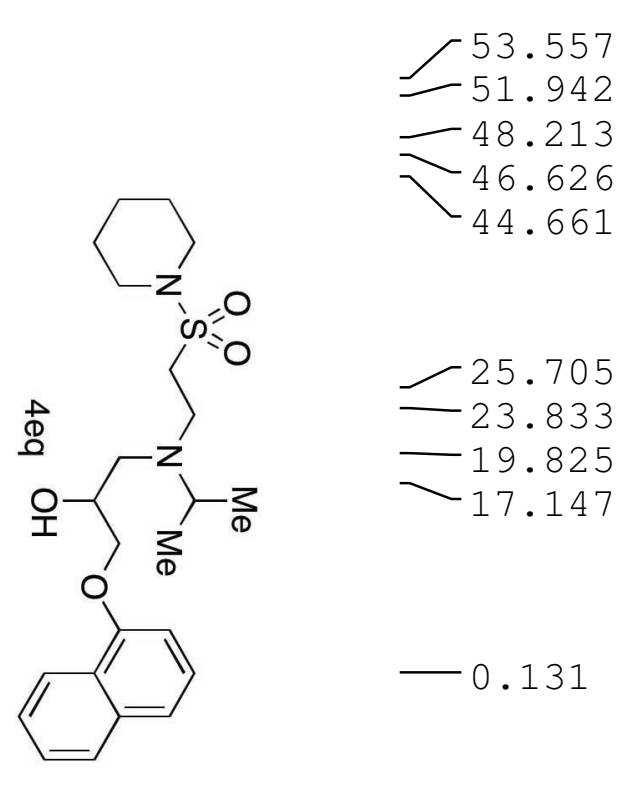

胥

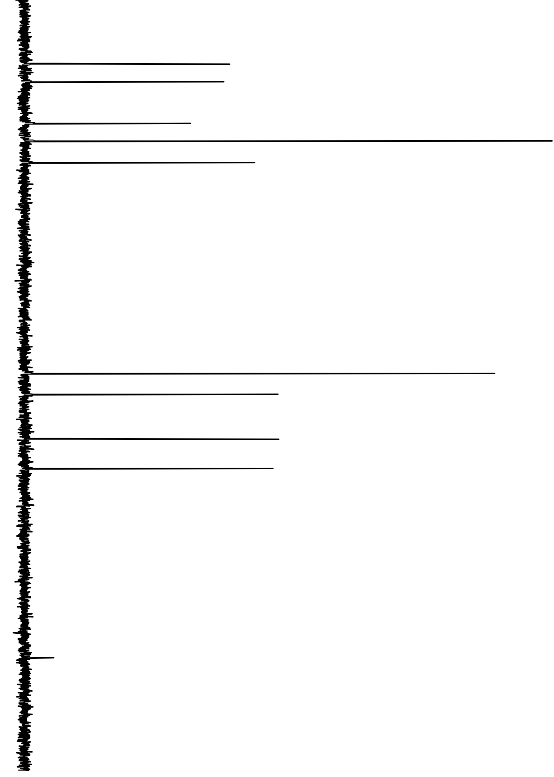

$-25.705$

23.833

19.825

17.147

$-0.131$ 


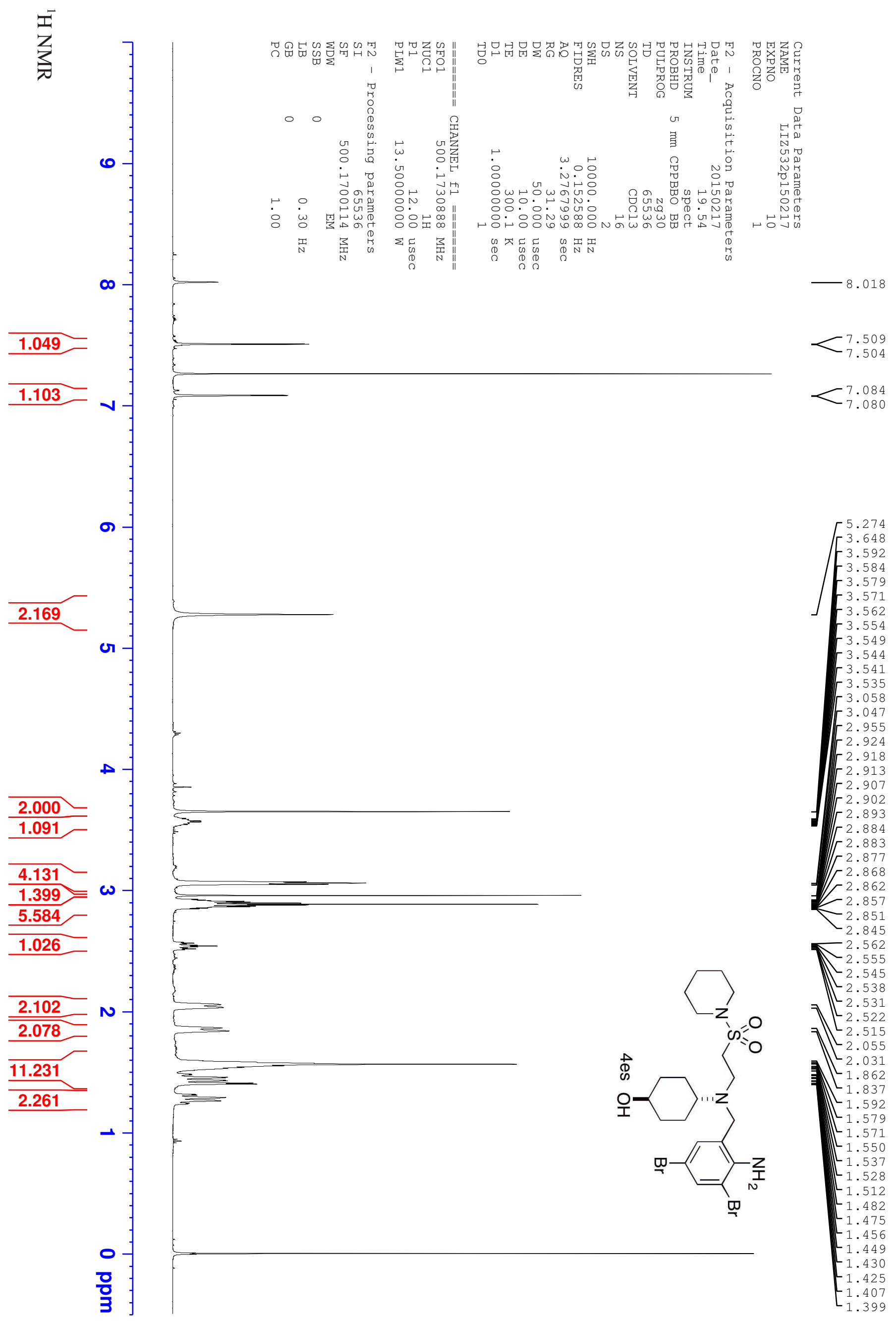




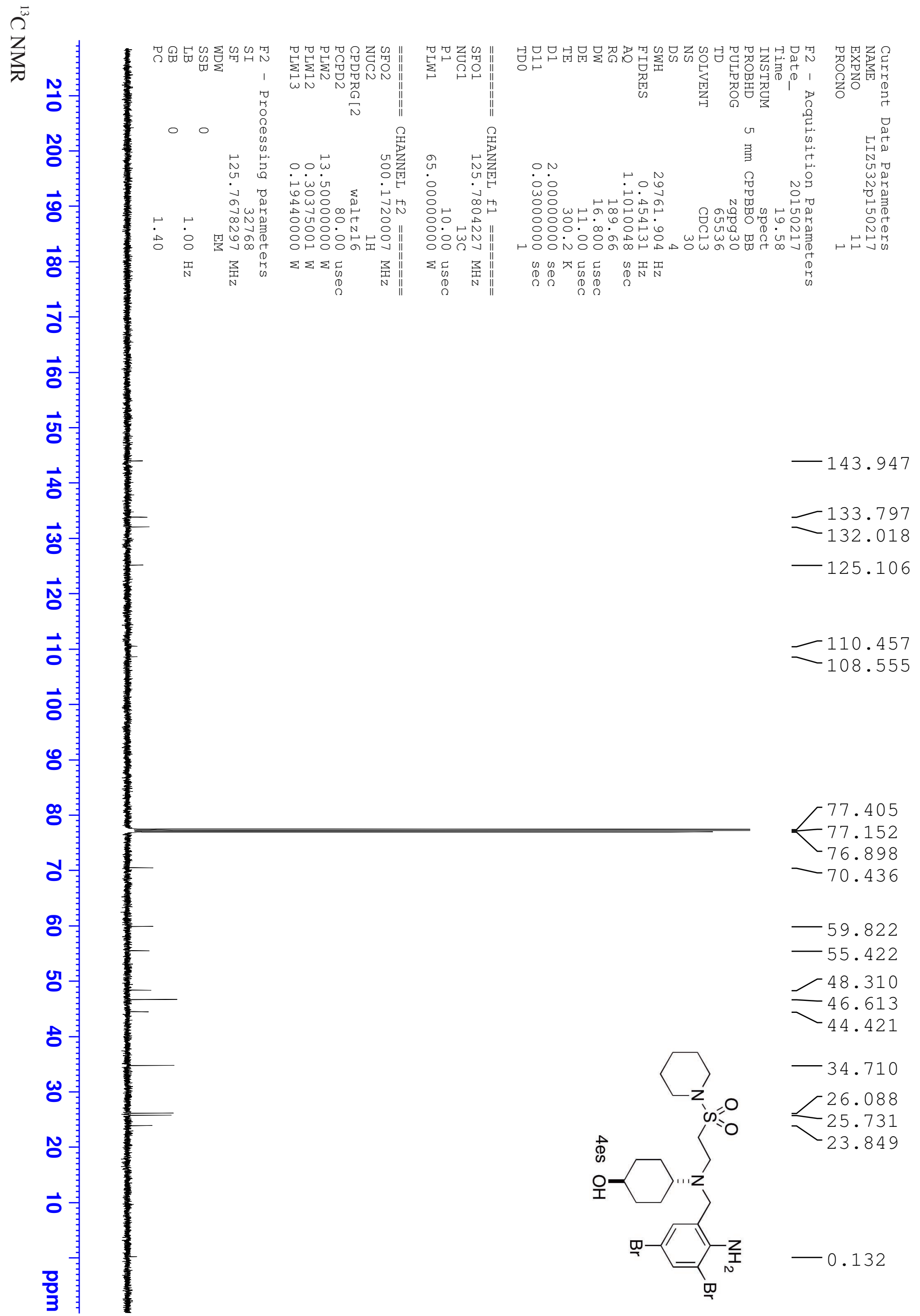




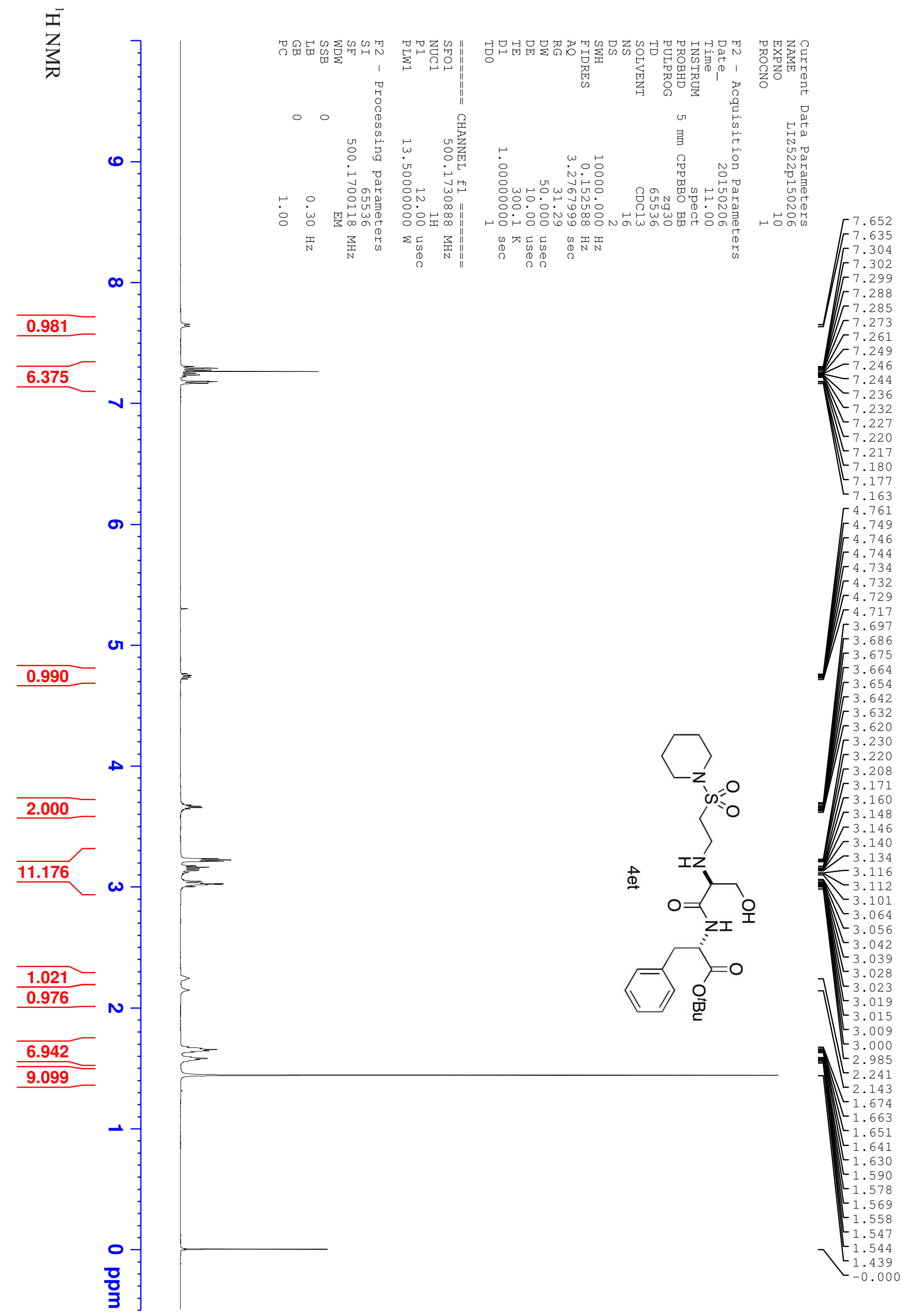




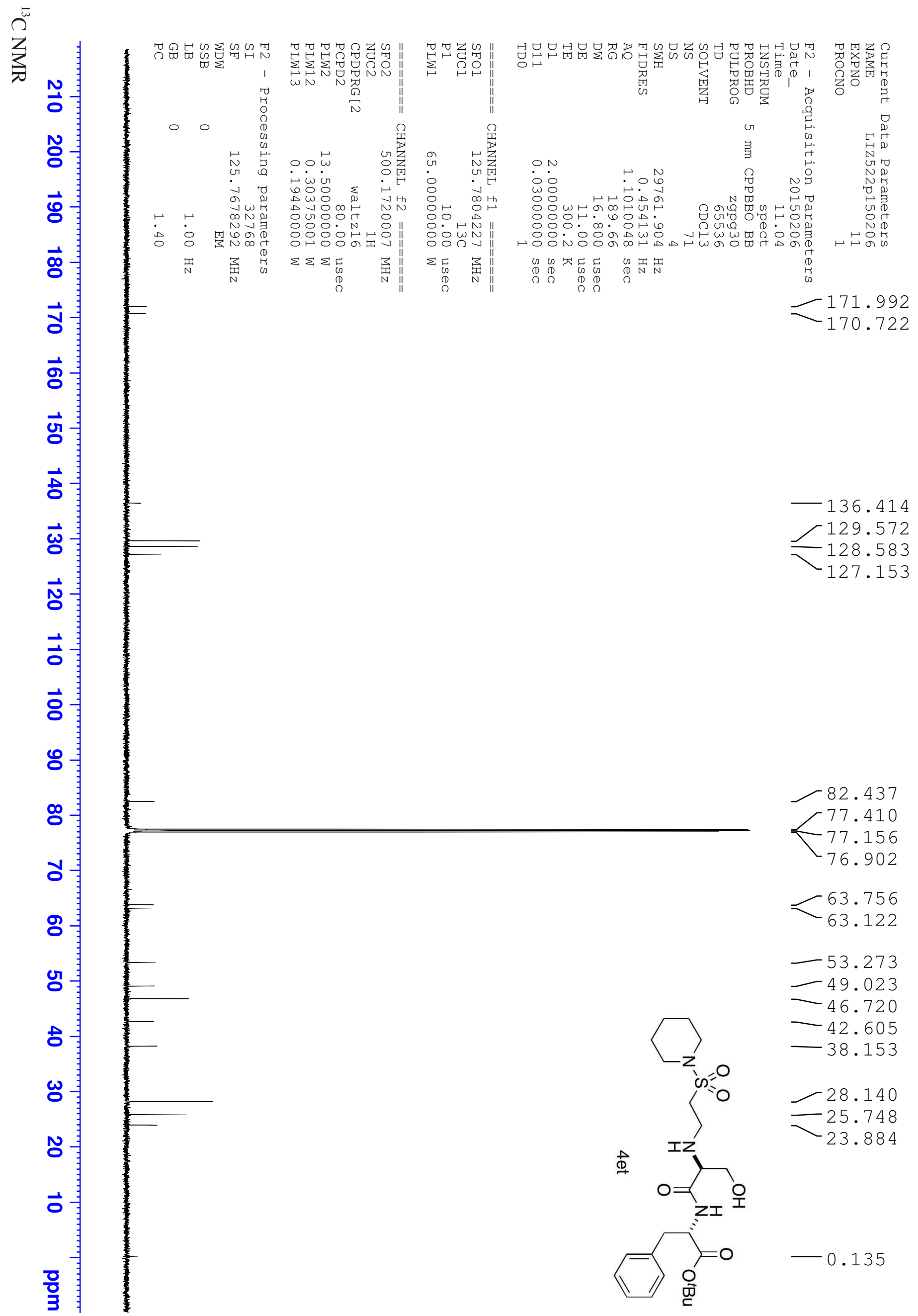




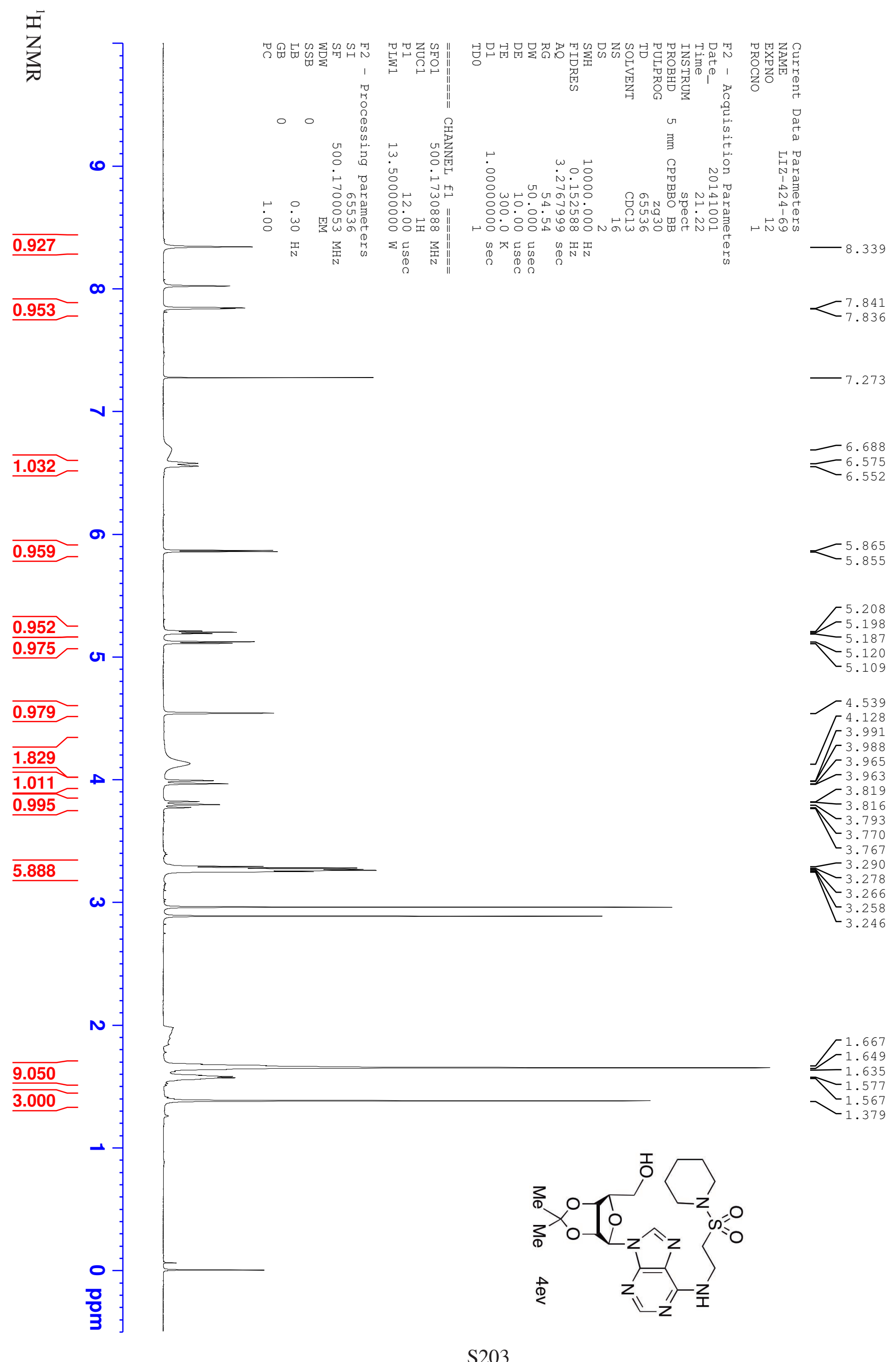




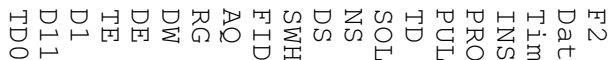

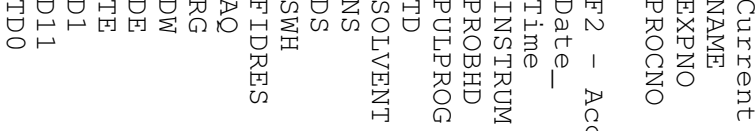

$\overrightarrow{0}$

$\vec{\sigma}$

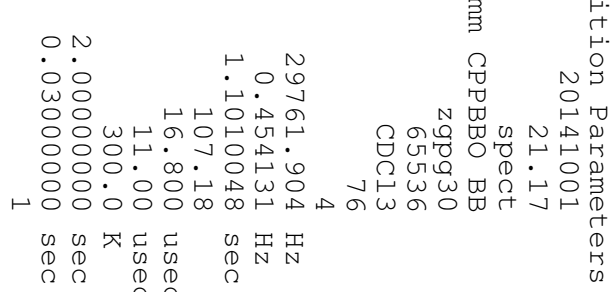

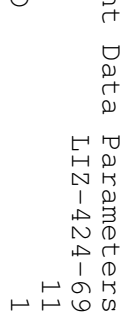

$-162.638$

$-154.842$

ज़

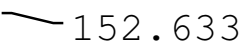

$\vec{f}$

$-140.156$

$\vec{\omega}$

$\vec{N}$

$\overrightarrow{0}$

웅

$\varnothing$

ø

$-114.106$

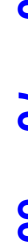

ชั

8

잉

o

ผ

ก
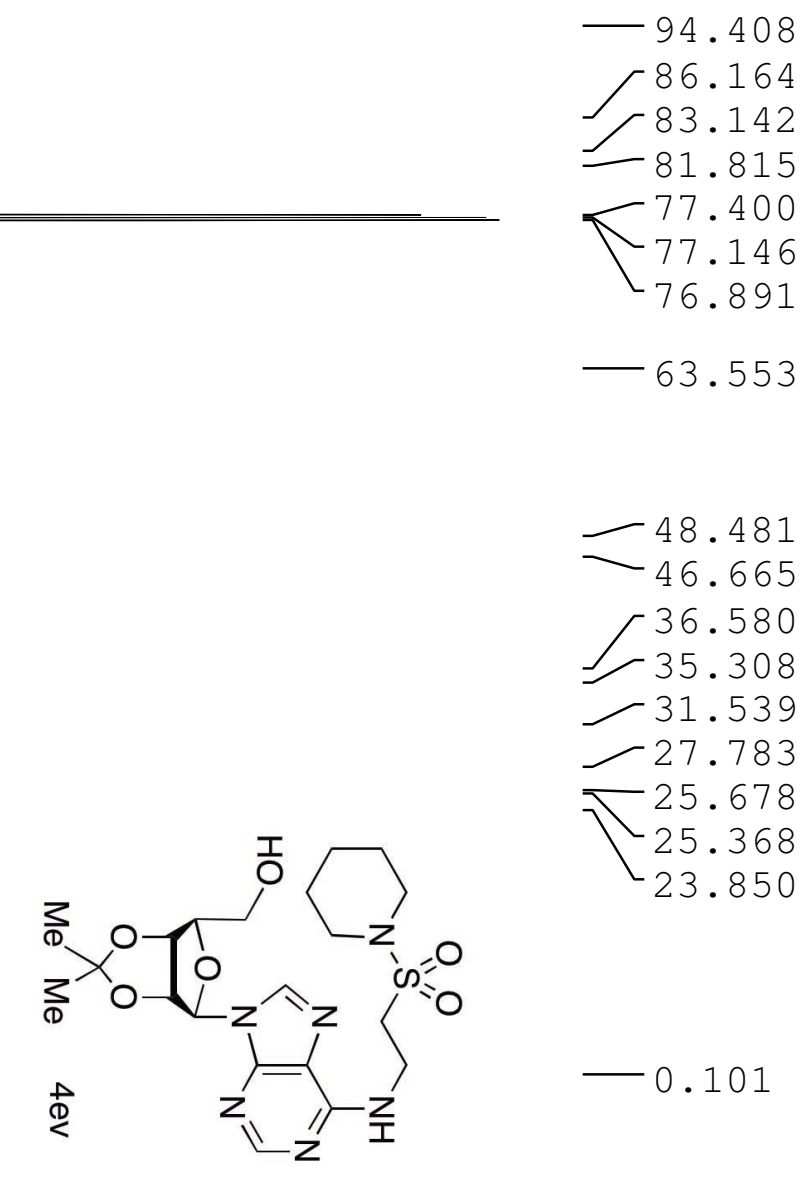

$-48.481$

$-46.665$

36.580

35.308
-31.539

$-31.539$

$-27.783$

$-25.678$

25.368

23.850

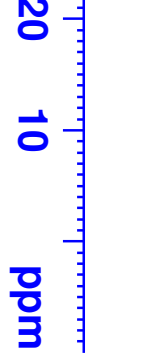

흠

$-0.101$ 
퐁

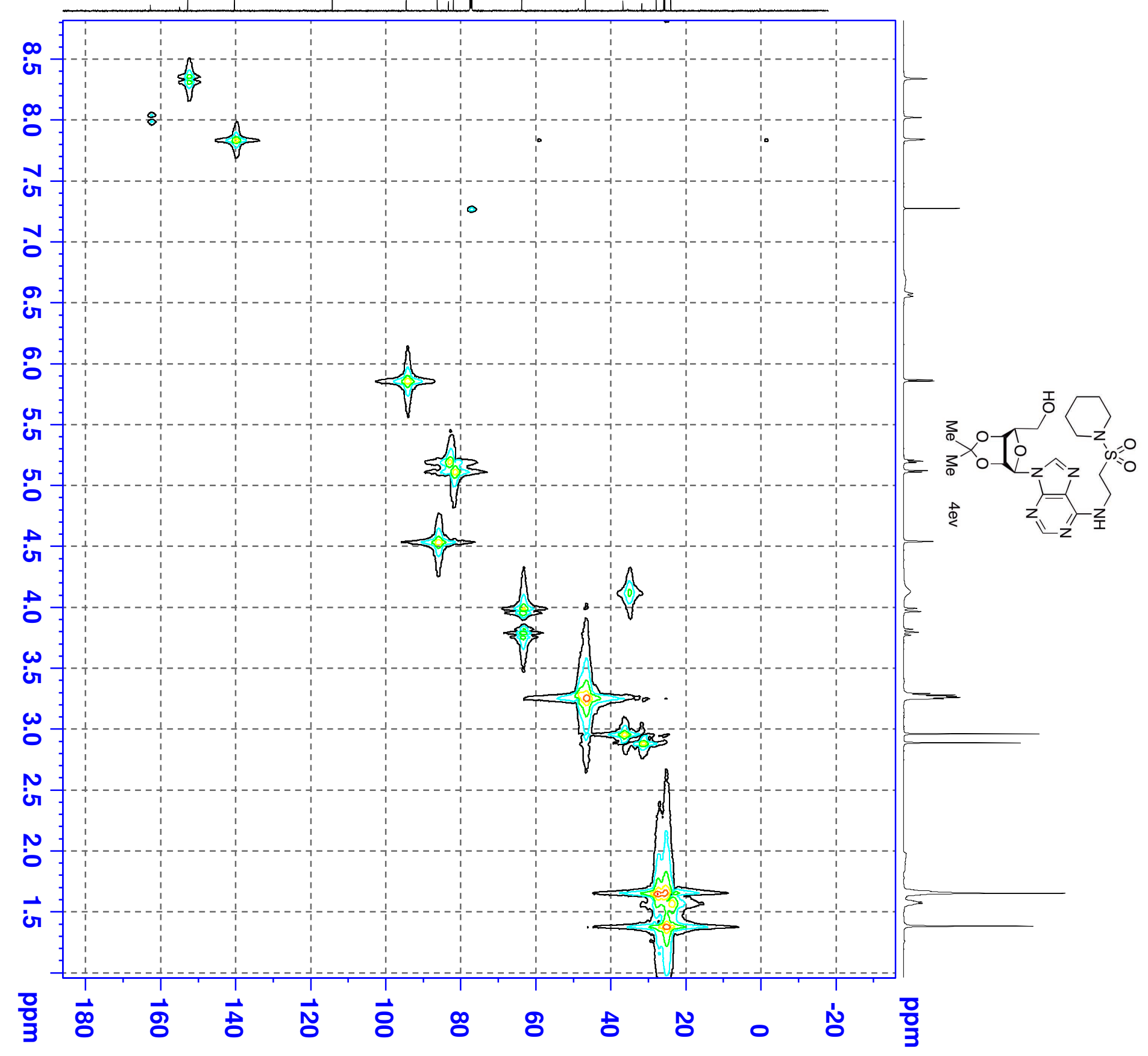

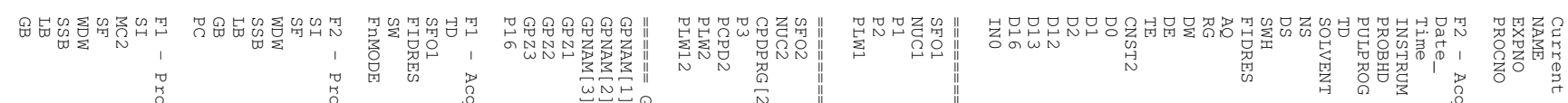

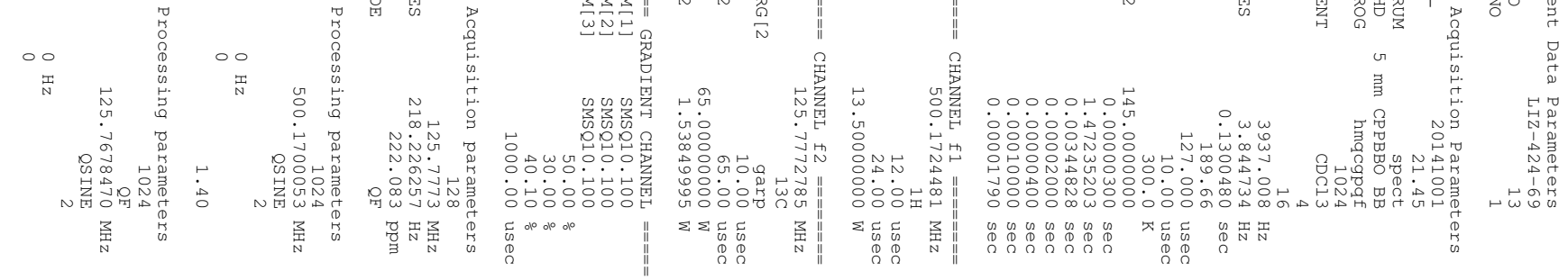




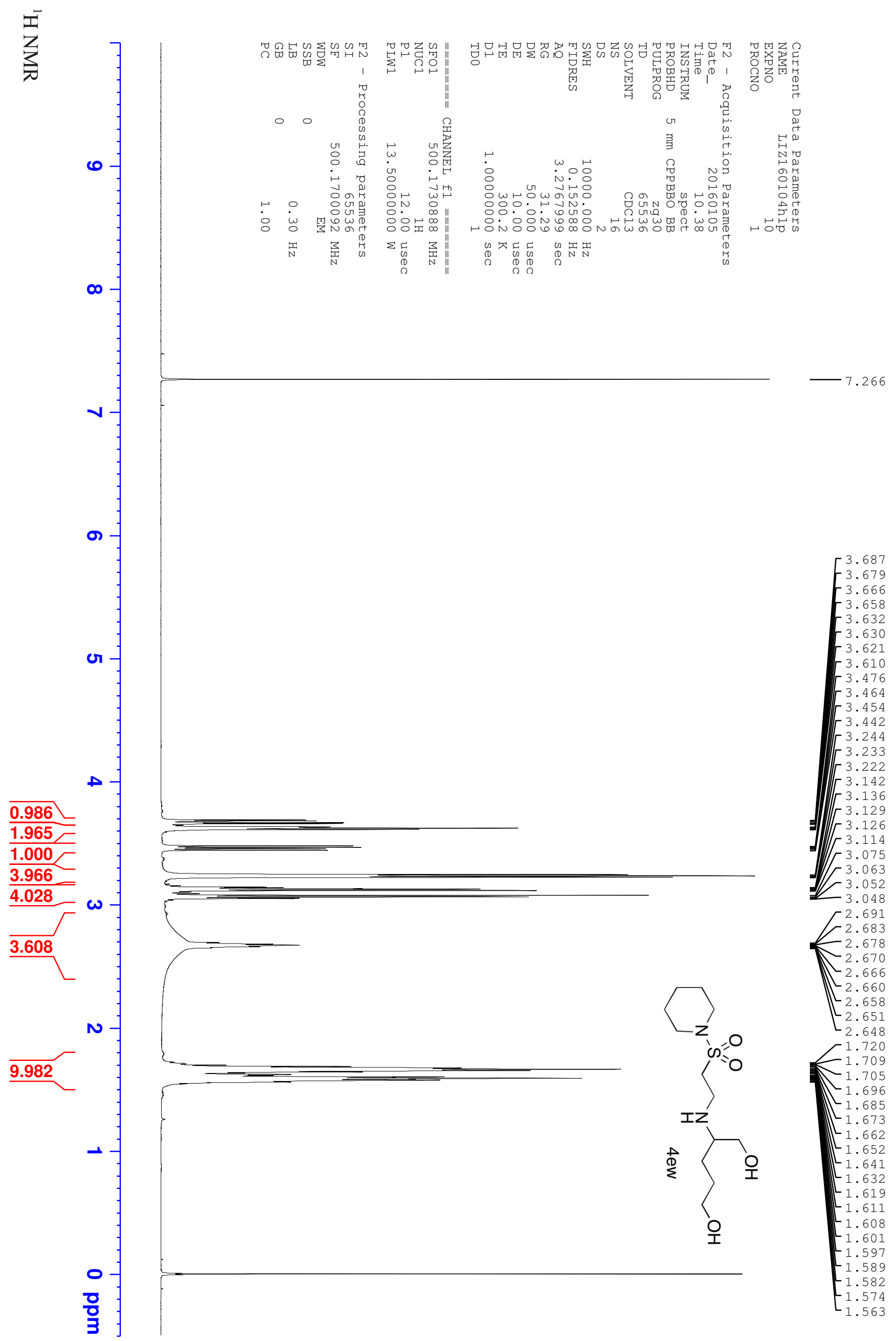




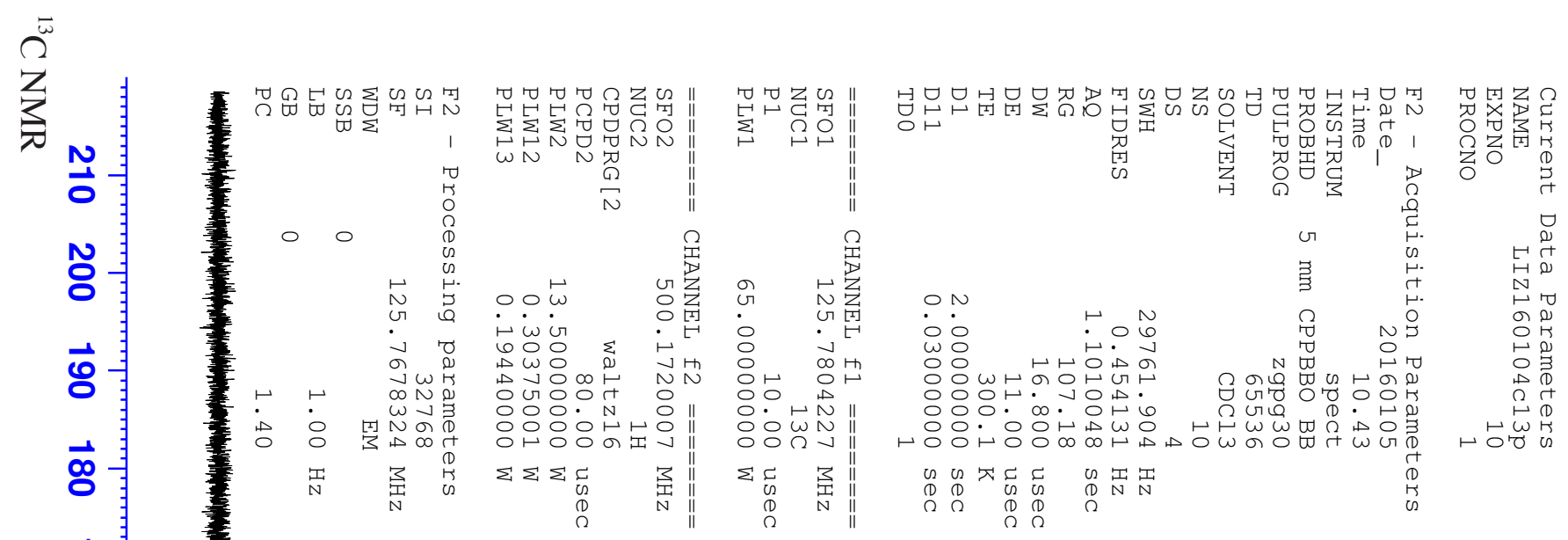

ปั

홍

ज़

$\overrightarrow{0}$

$\vec{\omega}$

$\overrightarrow{\text { N }}$

$\overrightarrow{0}$

$\overrightarrow{8}$

8

ø

ชั

8

잉

\&

ఱ

N

$\overrightarrow{0}$

맙

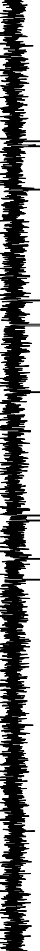

77.415

$-77.161$

76.907

$-63.252$

$-62.832$

$-58.879$

$-48.931$

$-46.705$

$-40.663$

$\langle z$

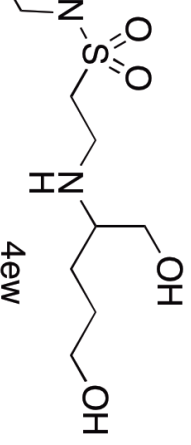

29.598

$\begin{array}{r}29.152 \\ \hline\end{array}$

$\longrightarrow 25.691$

$\checkmark 23.825$ 


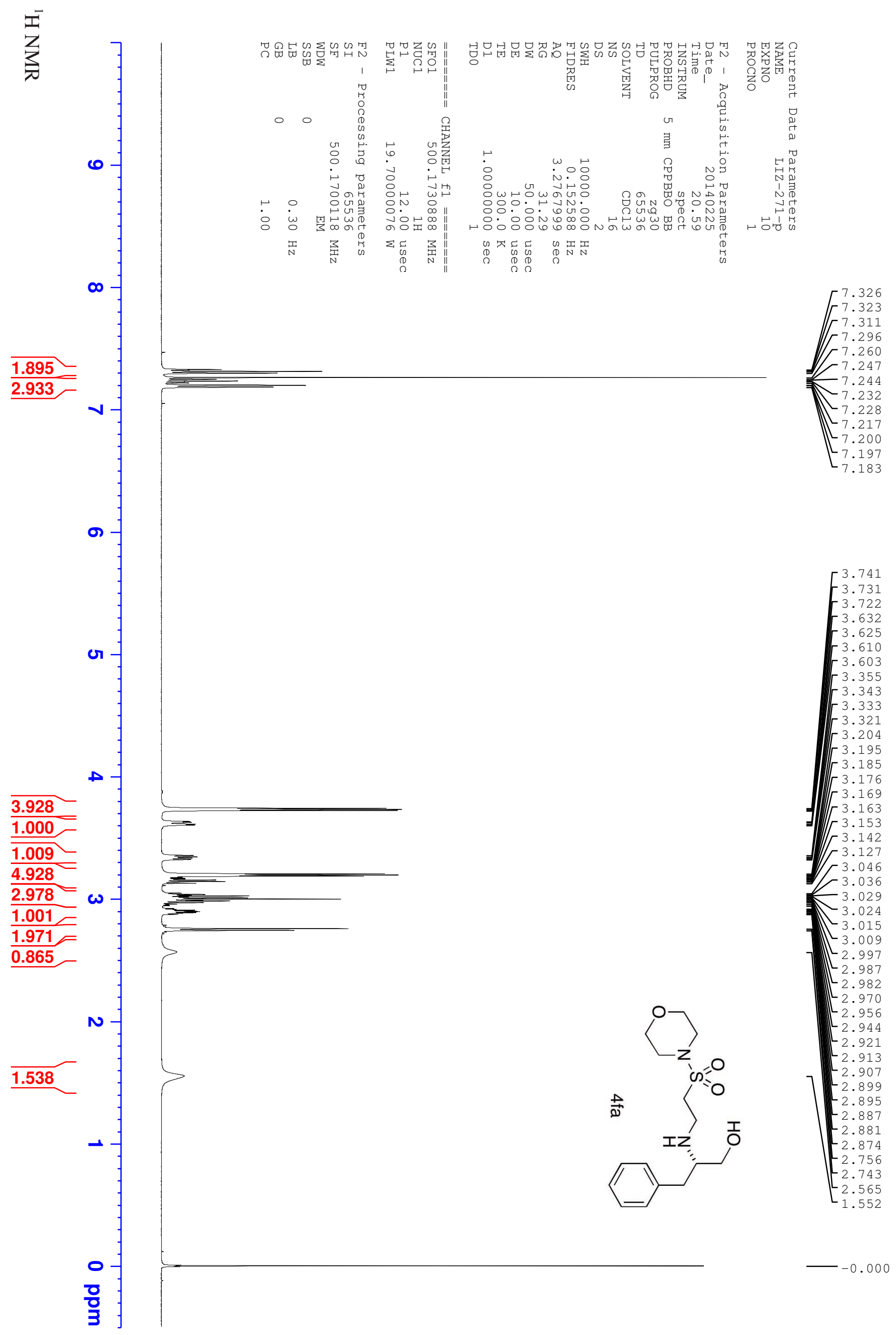




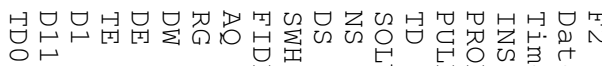

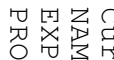

题

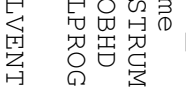

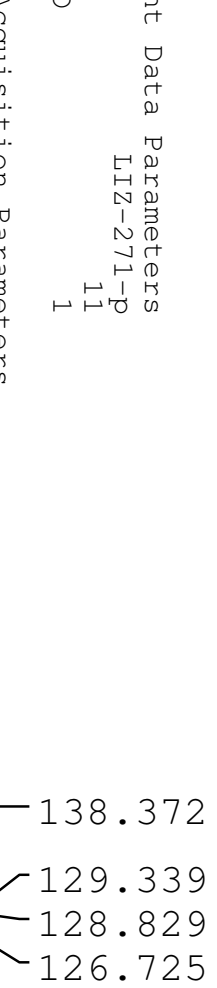




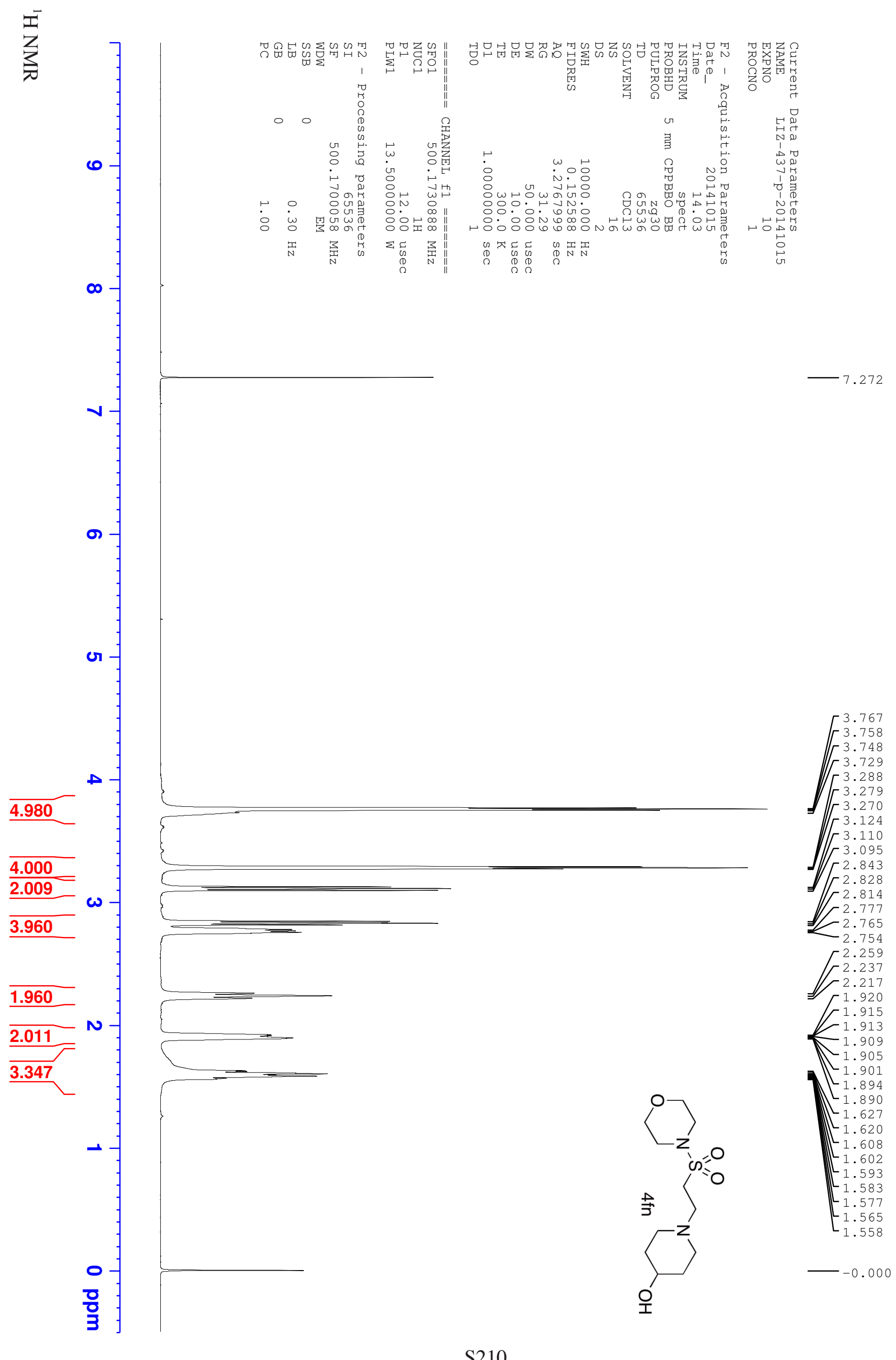


$\overbrace{}^{\bar{\omega}}$

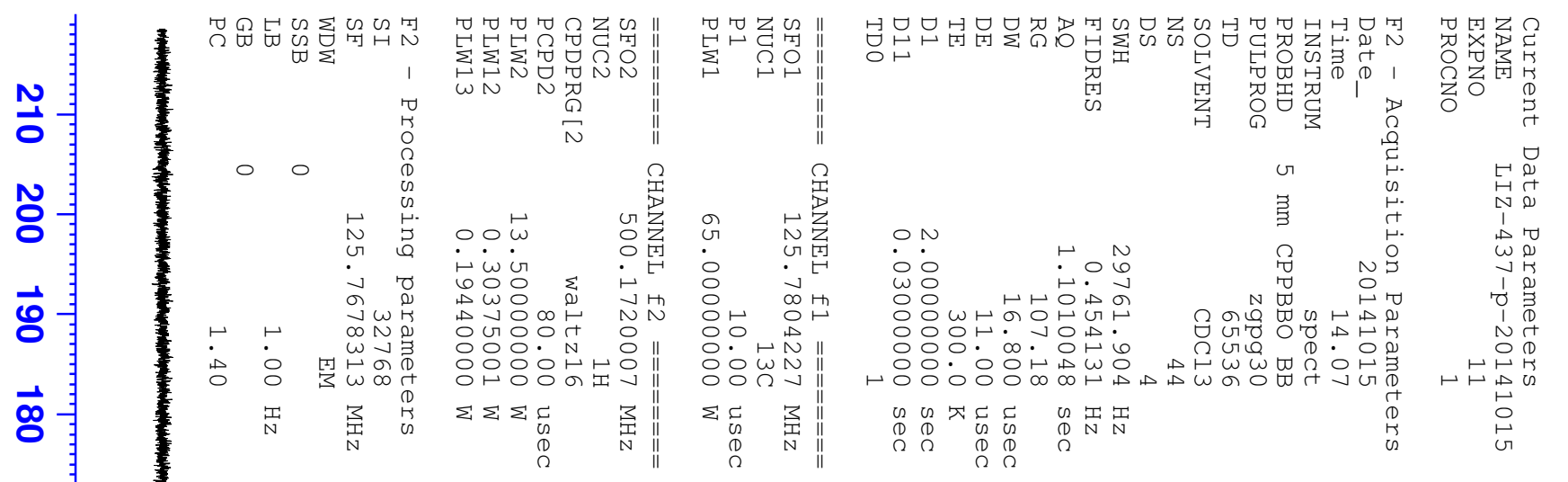

ปे

$\overrightarrow{\mathrm{g}}$

항

$\overrightarrow{\mathrm{a}}$

$\vec{\omega}$

응

항

$\overrightarrow{\mathrm{o}}$

8

ø

ป

8

잉

ㅇ

$\omega$

กิ

o

함

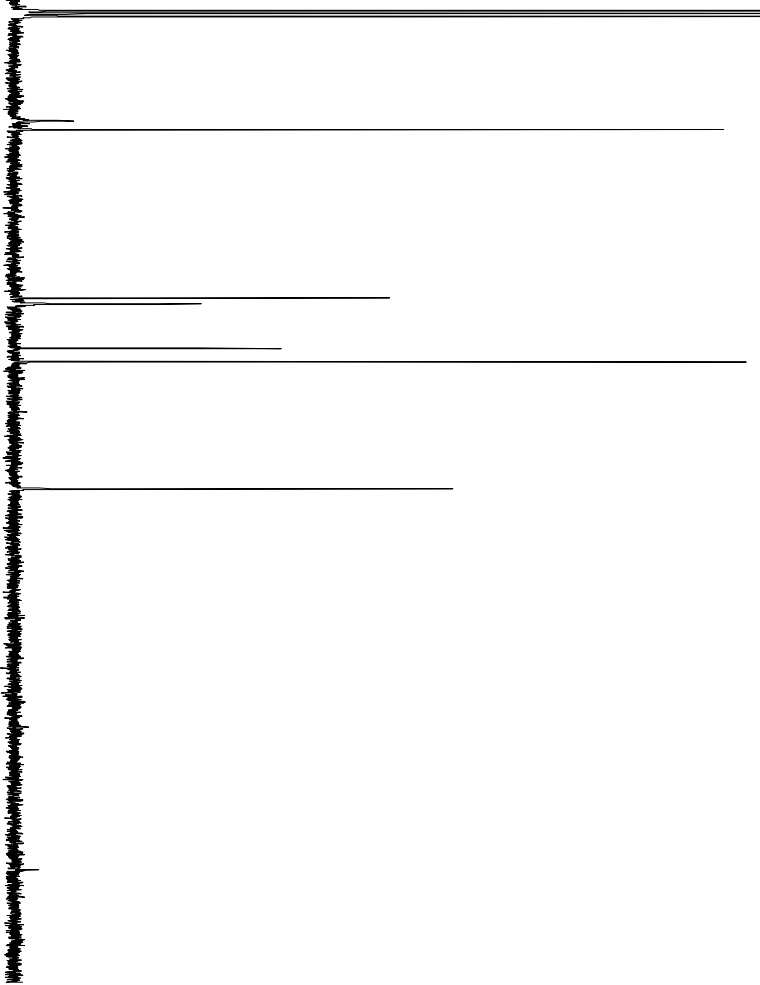

77.413

$-77.159$

76.905

67.495

$-66.732$

51.568

51.036

$-47.046$

45.832

$-34.416$

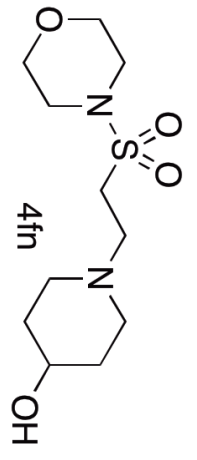

$-0.120$ 


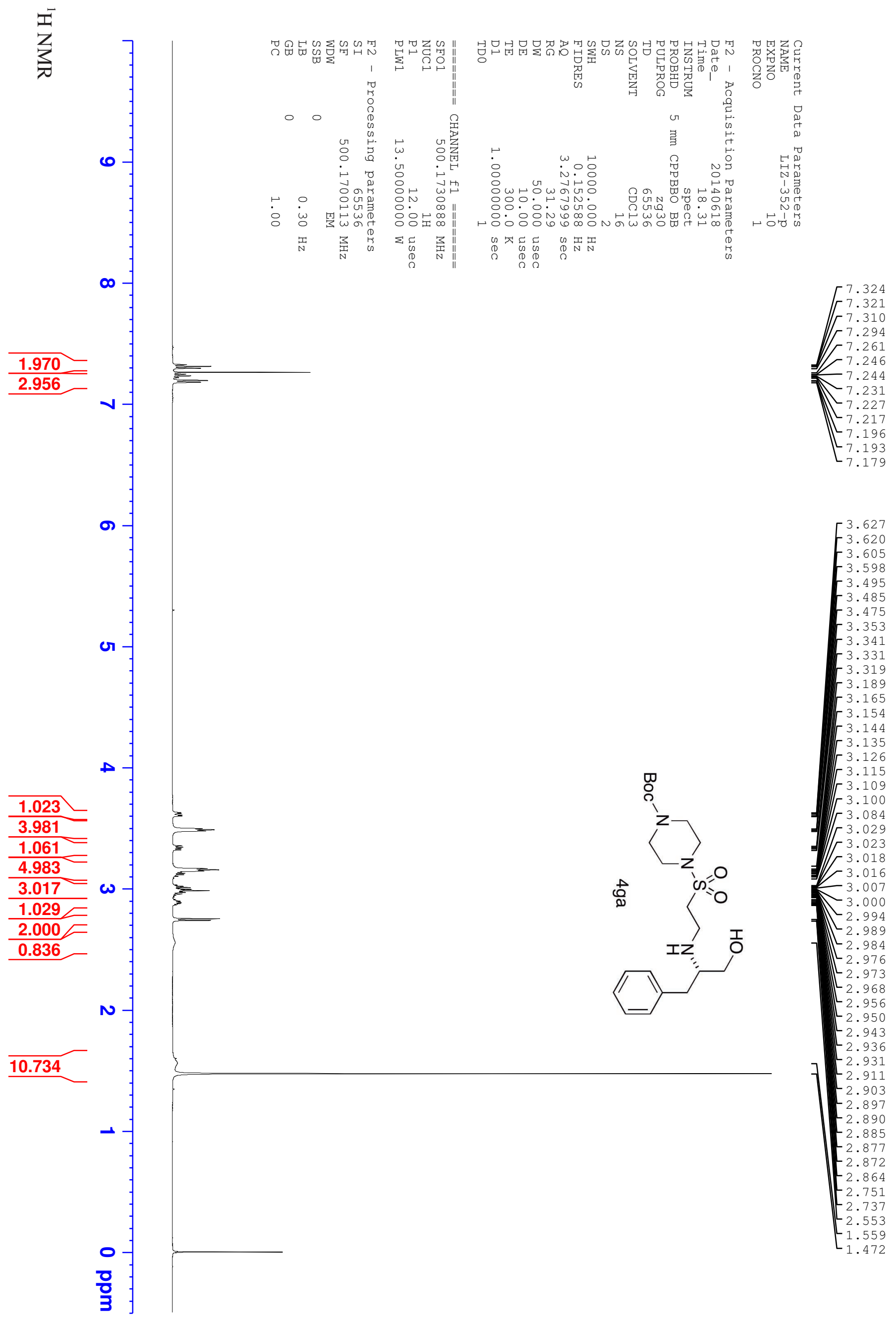




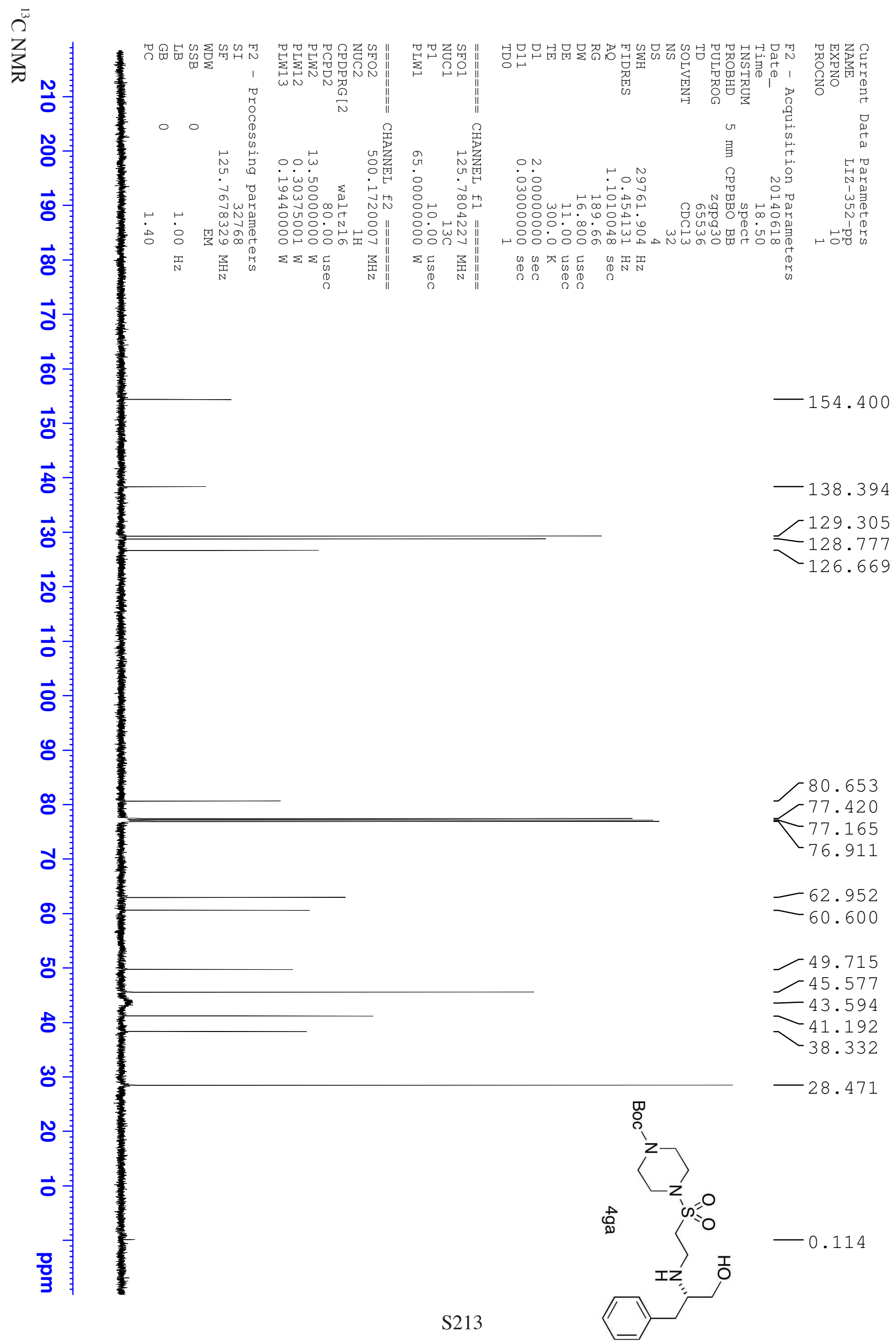




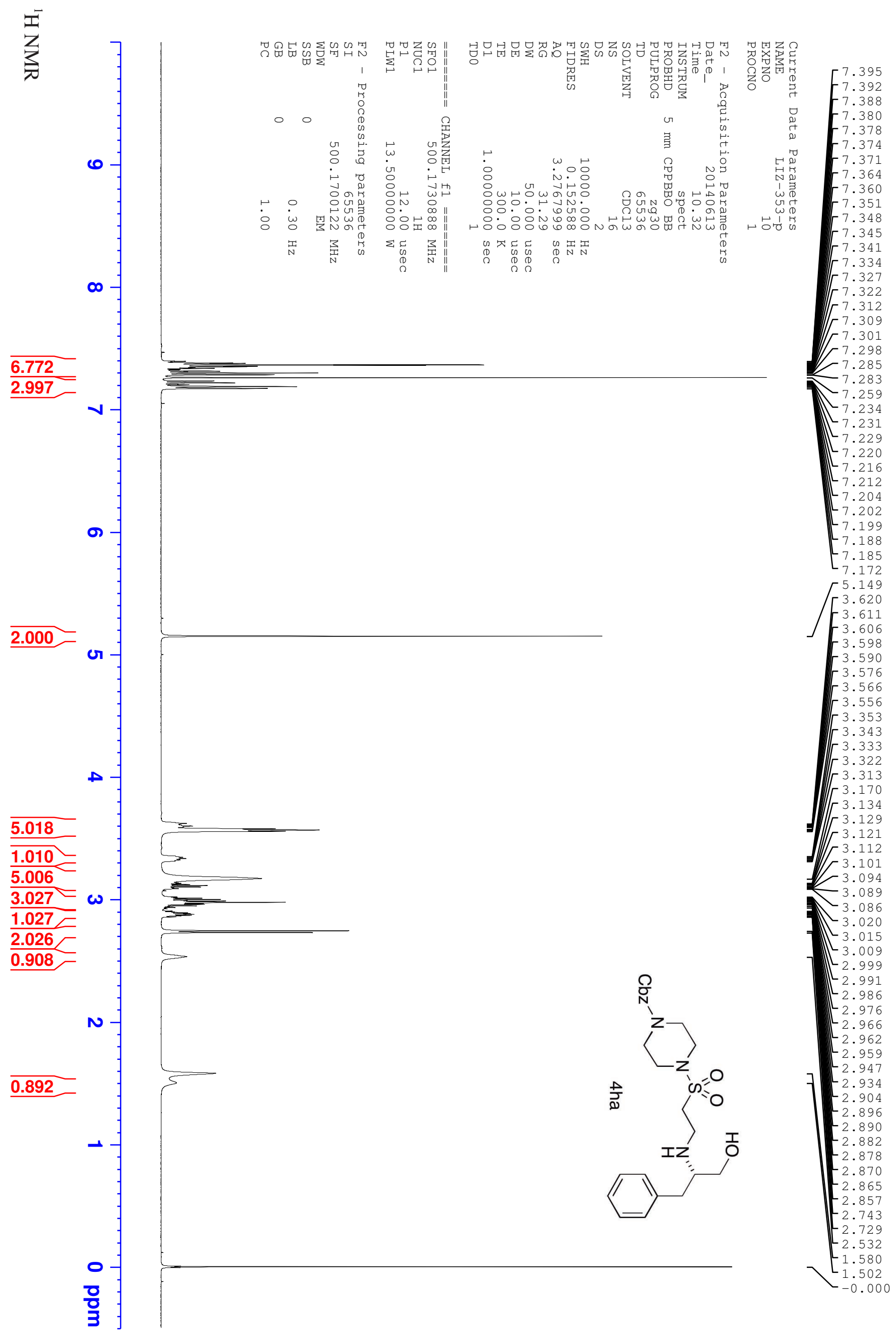




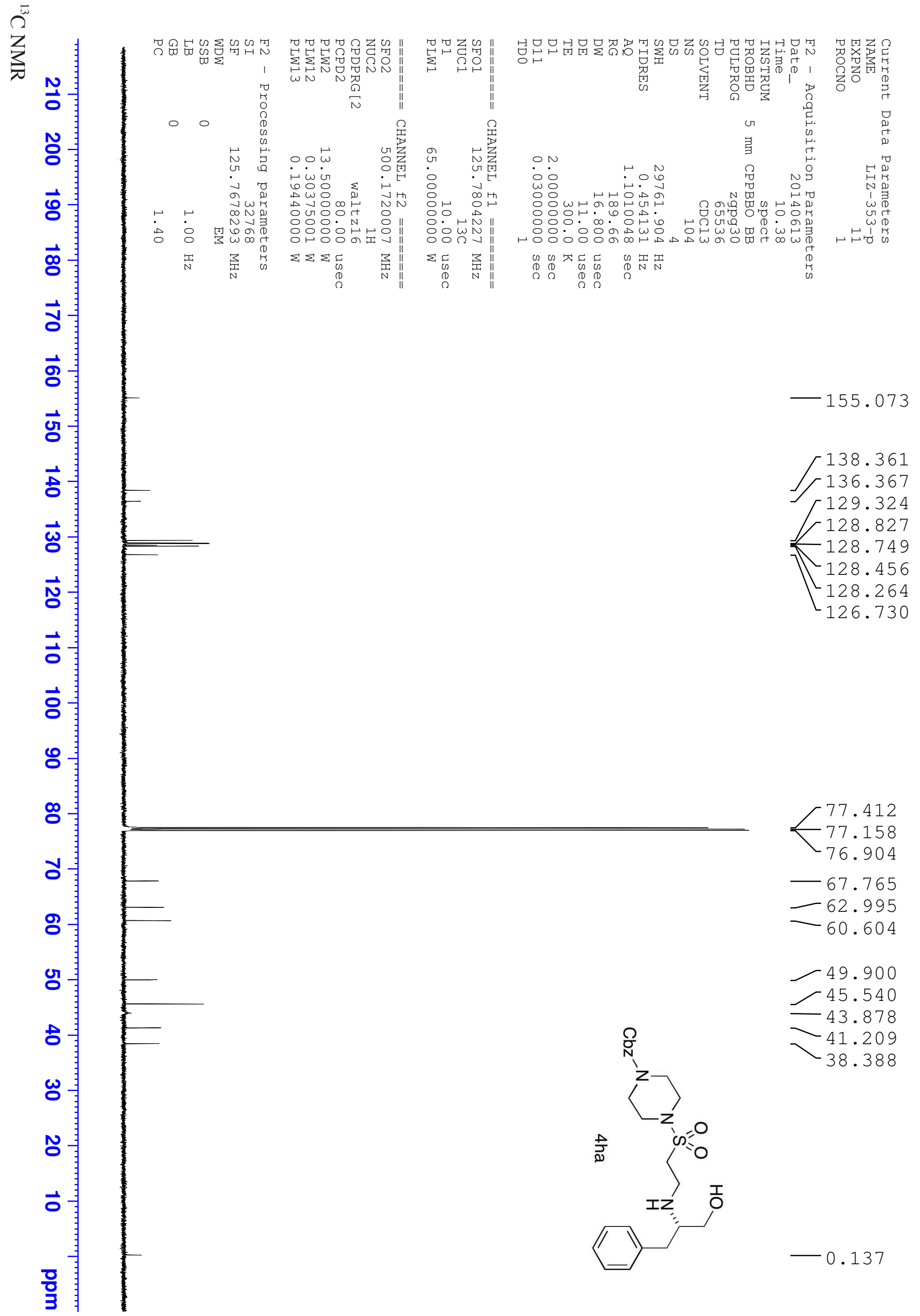




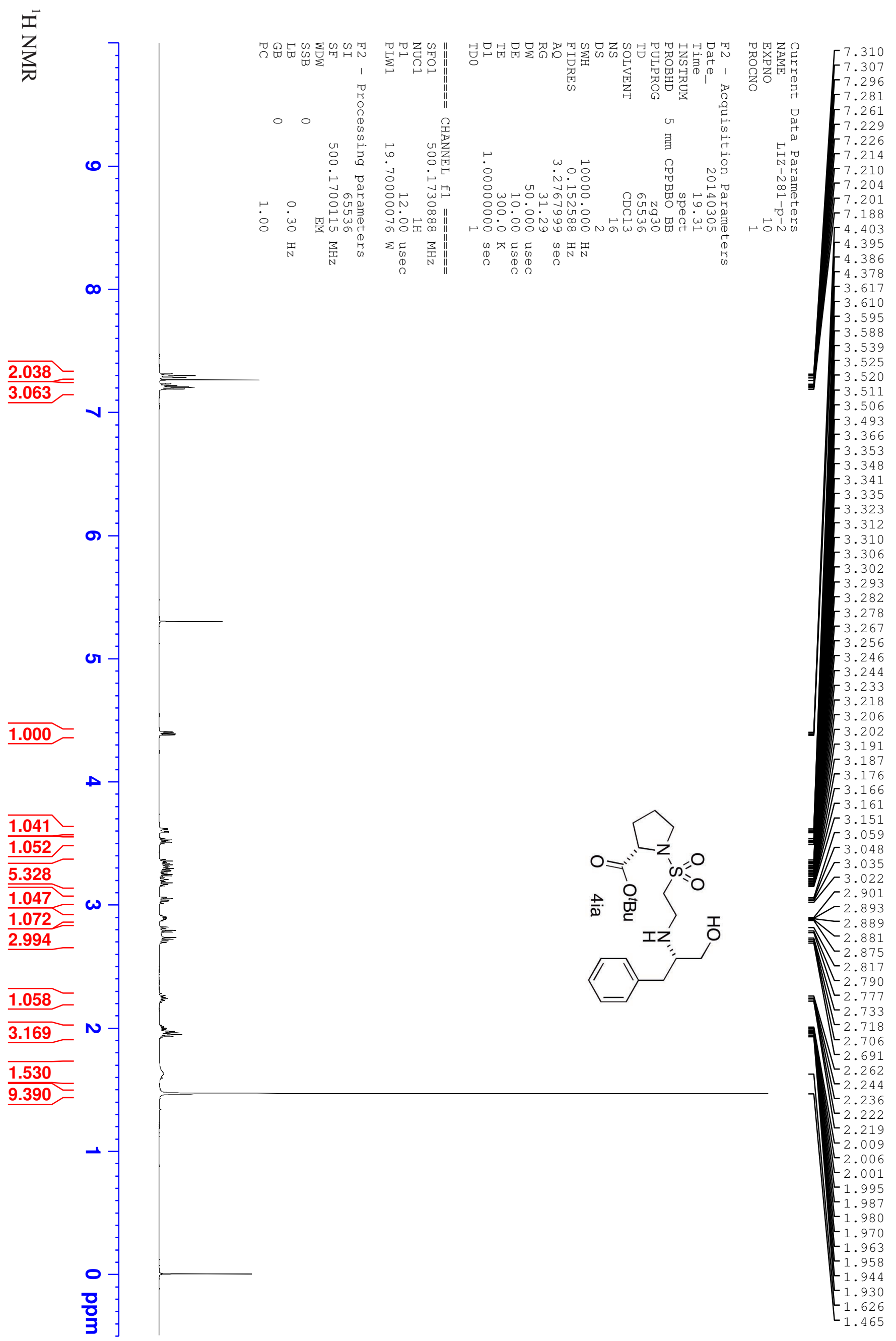




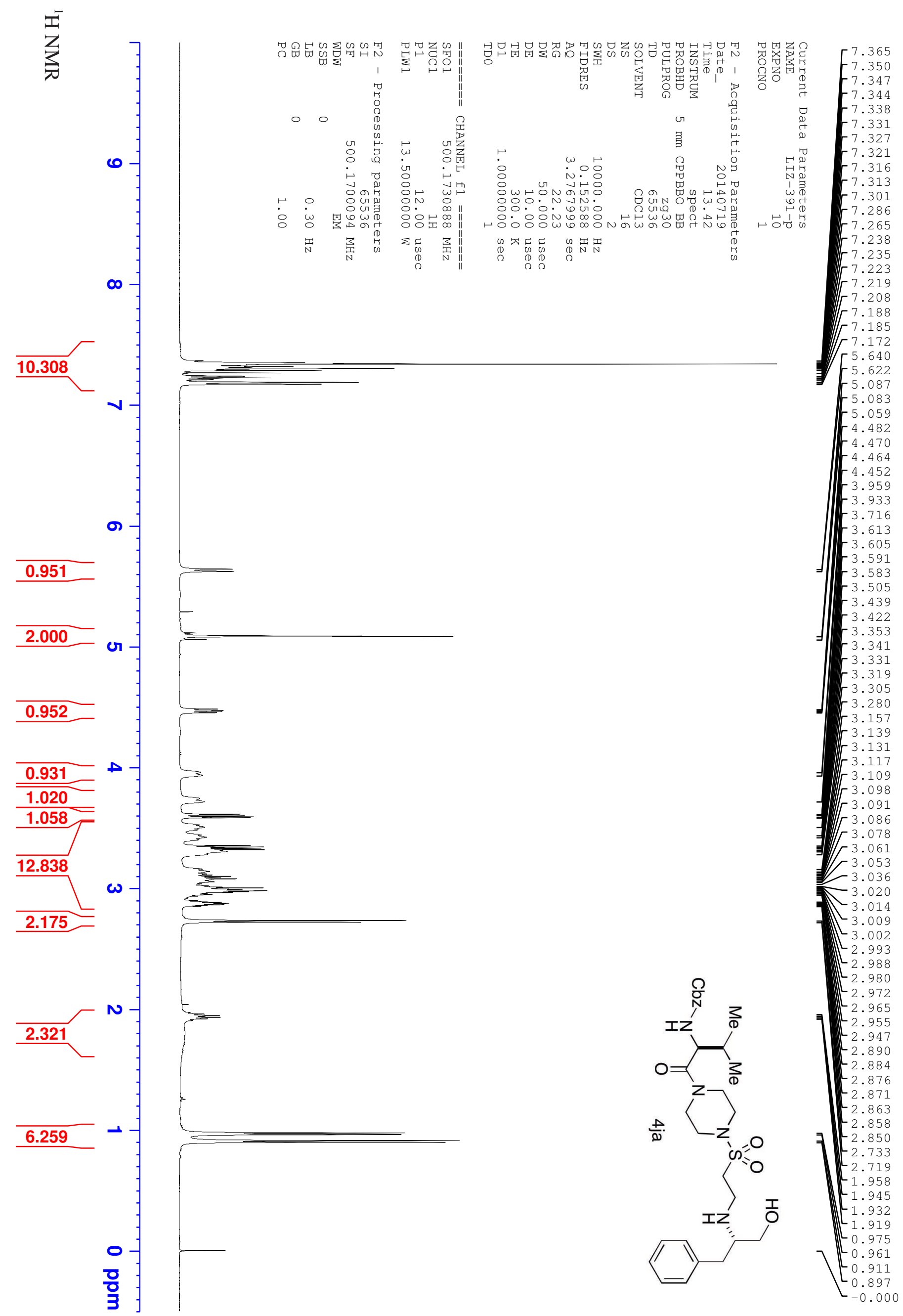




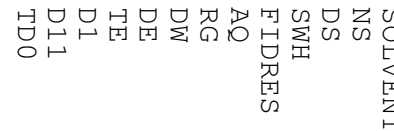

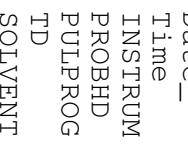

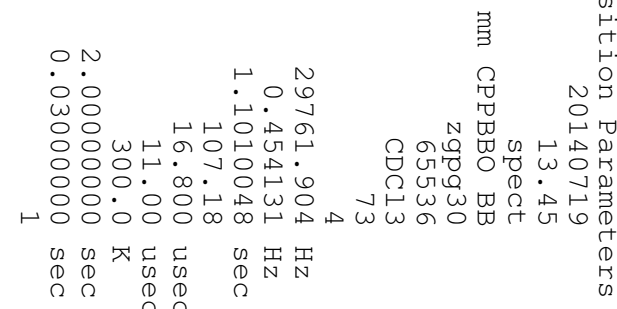

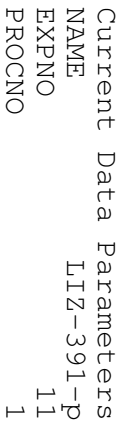
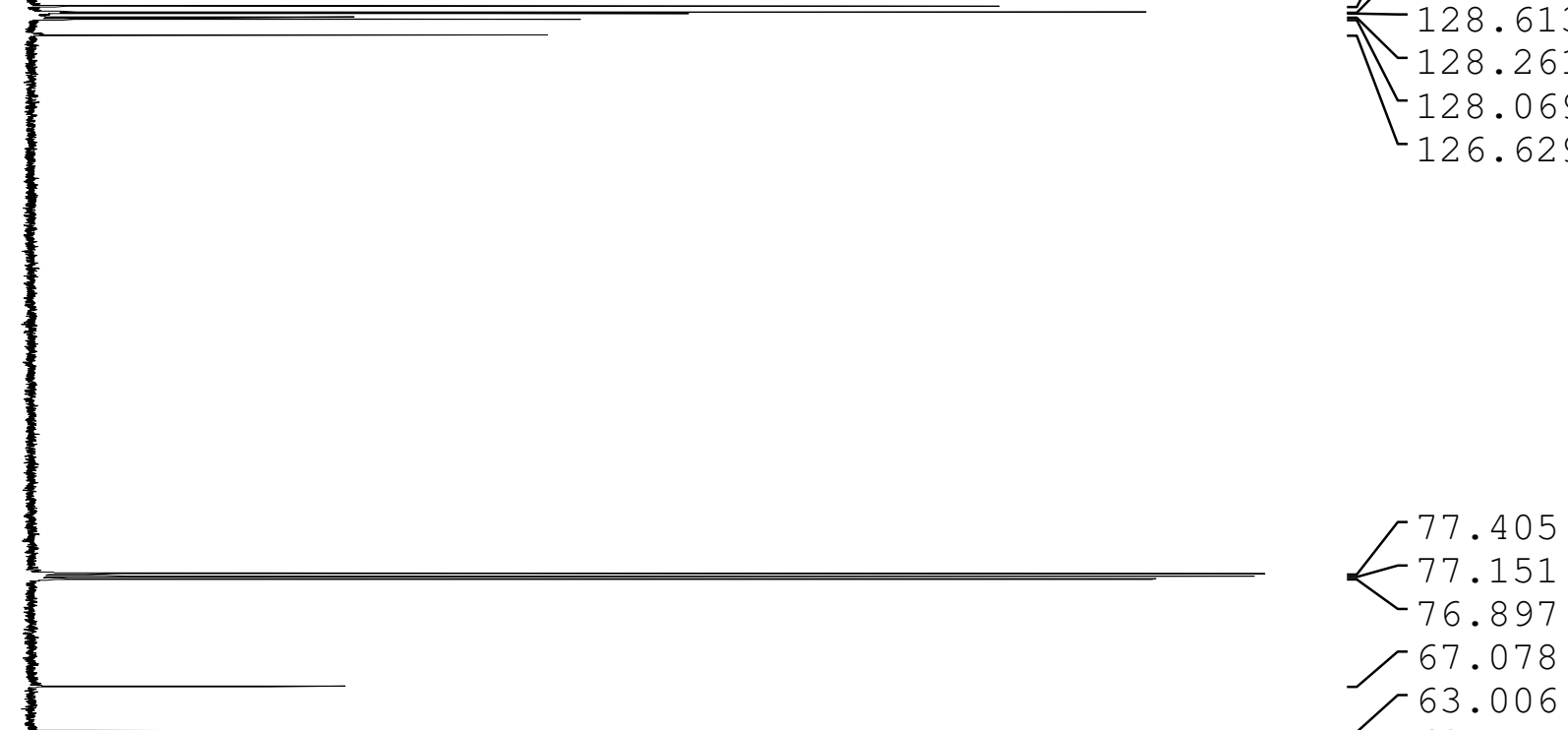

67.078

8

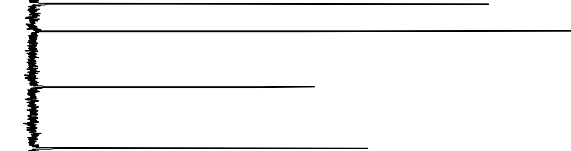

gr

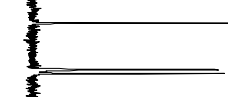

\&

ఱ
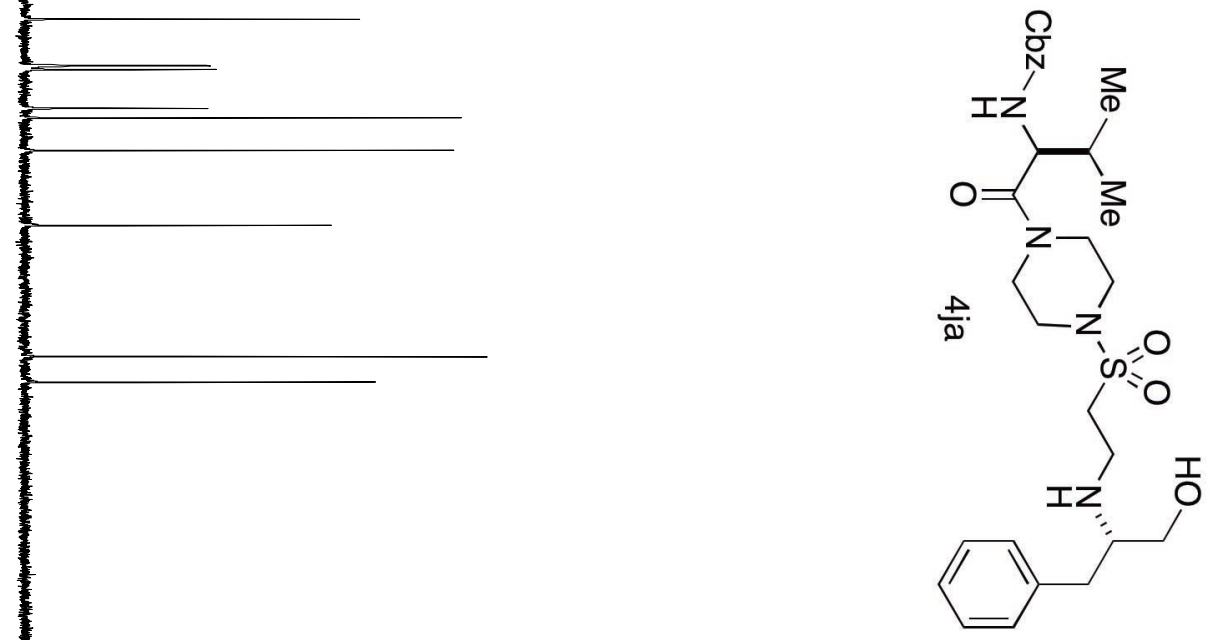

$-128.261$

128.069

126.629

\section{은 \\ $\overrightarrow{0}$}

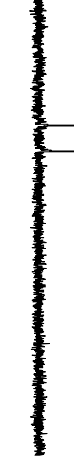

응 국 


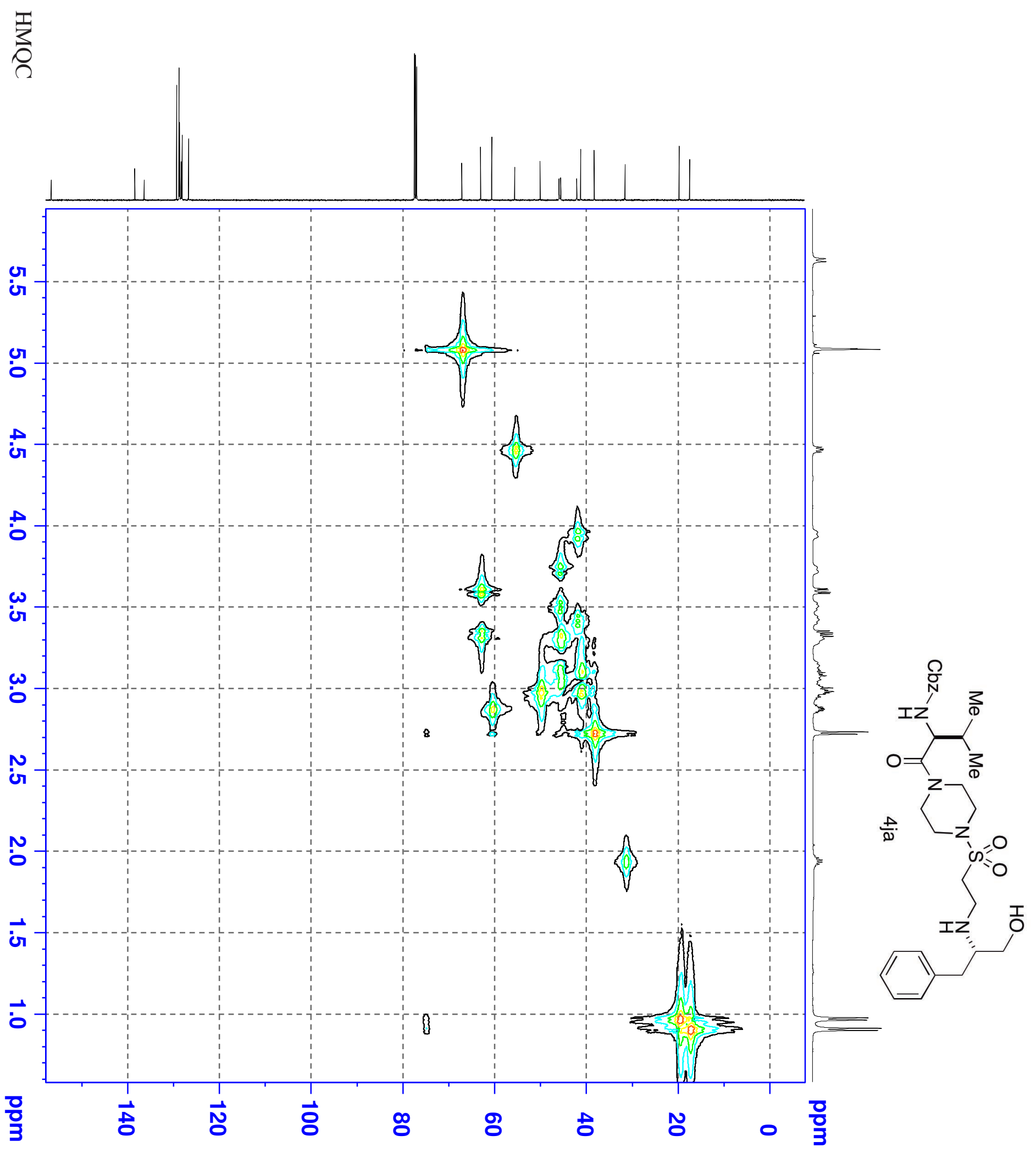

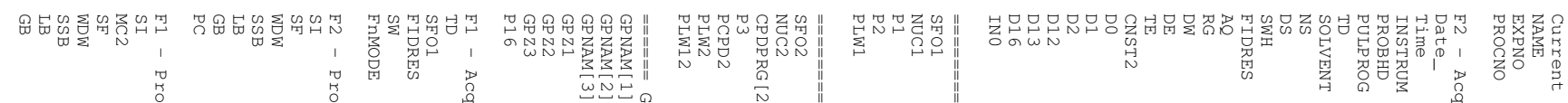

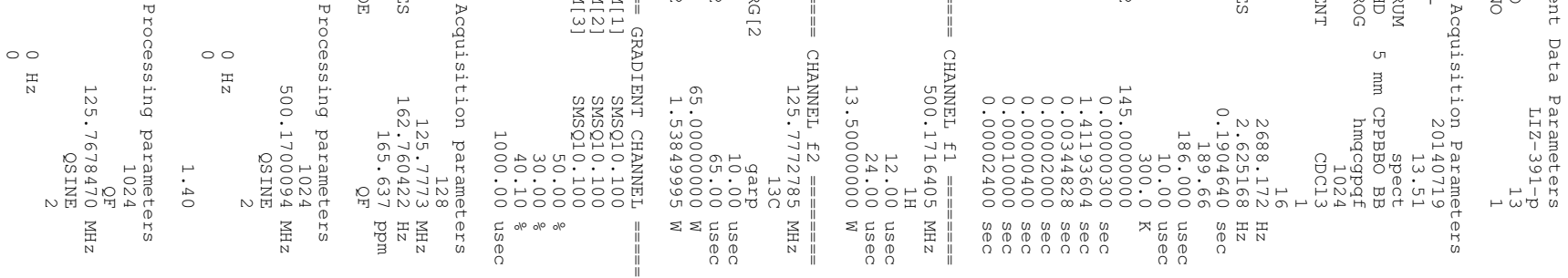




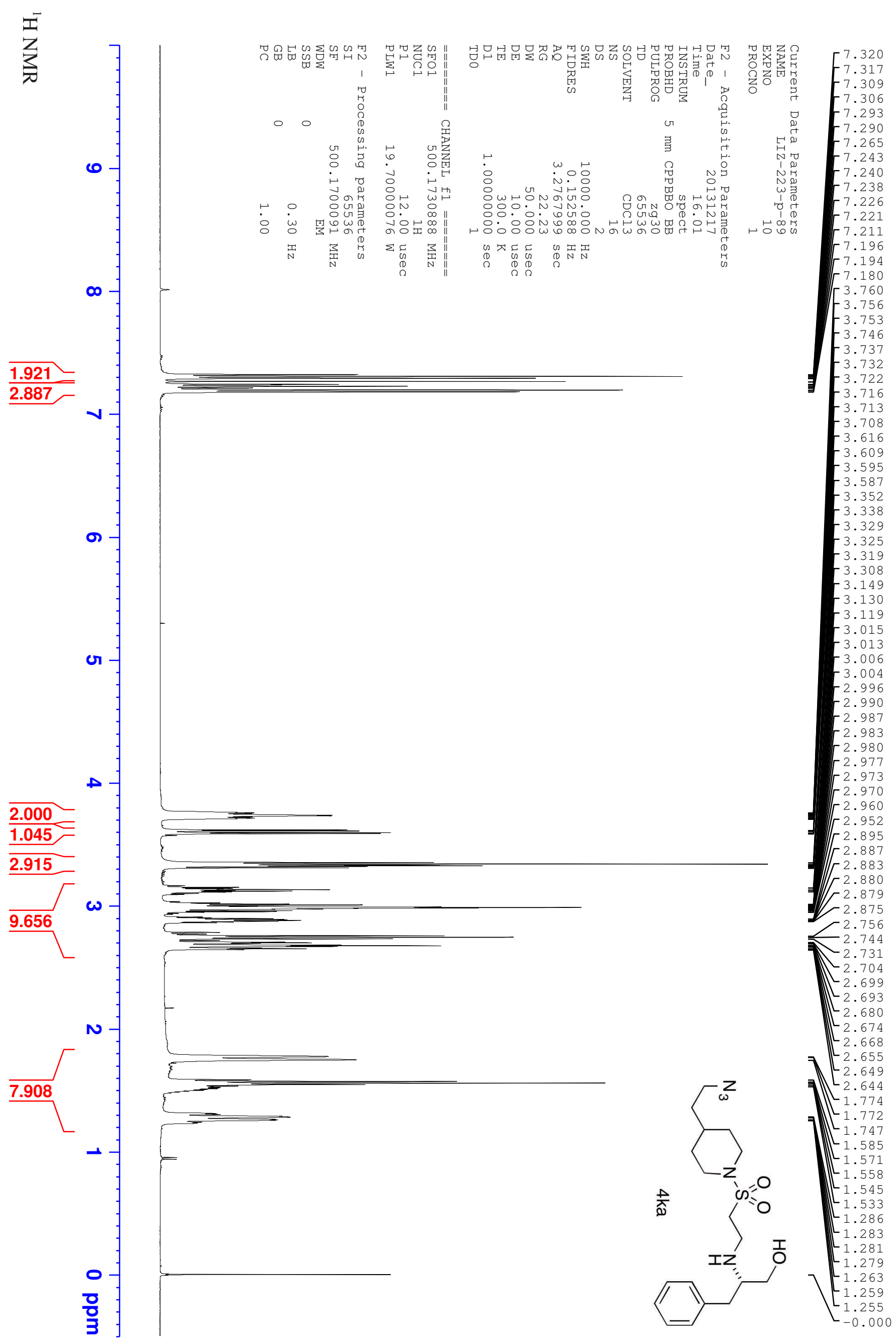




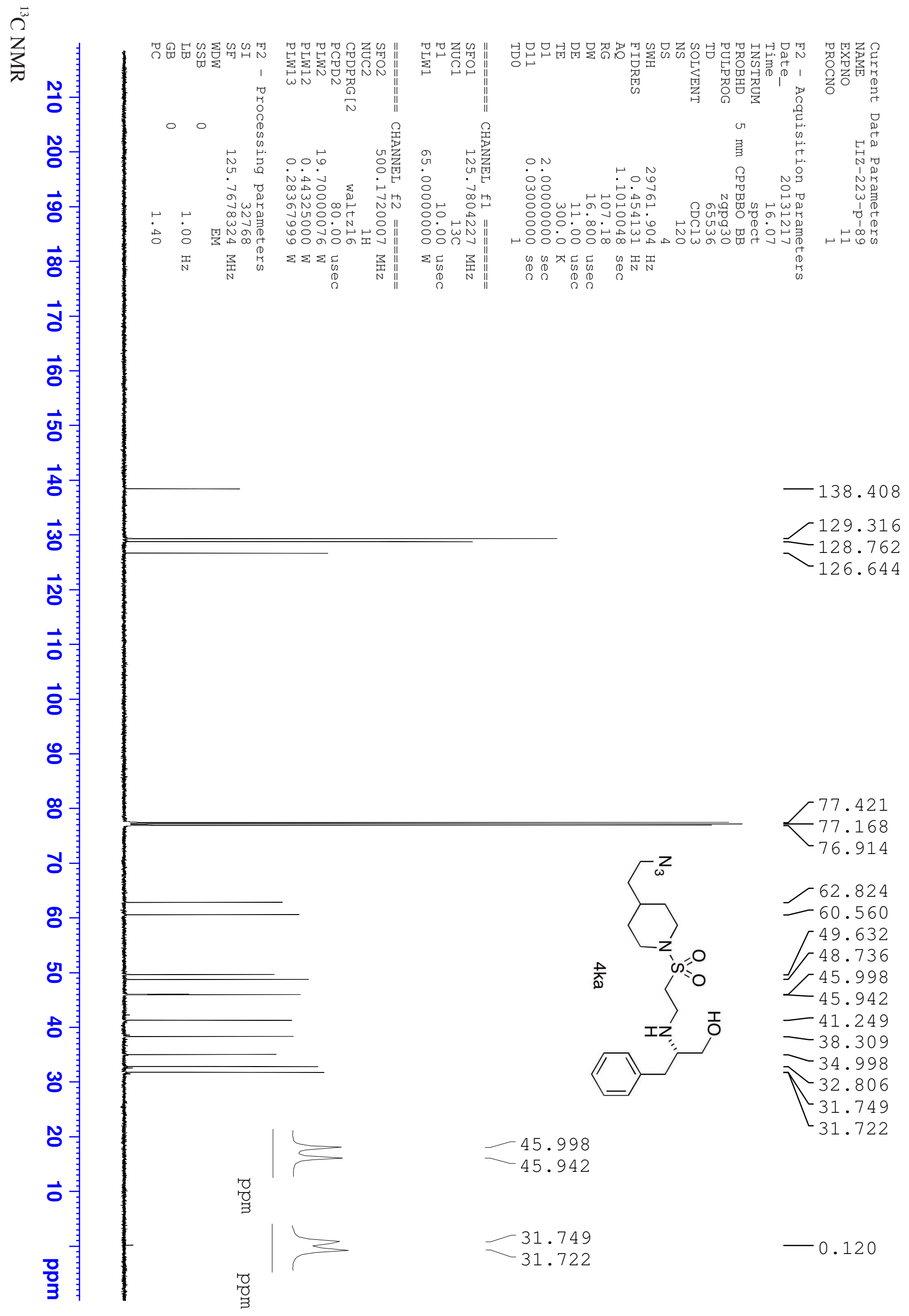




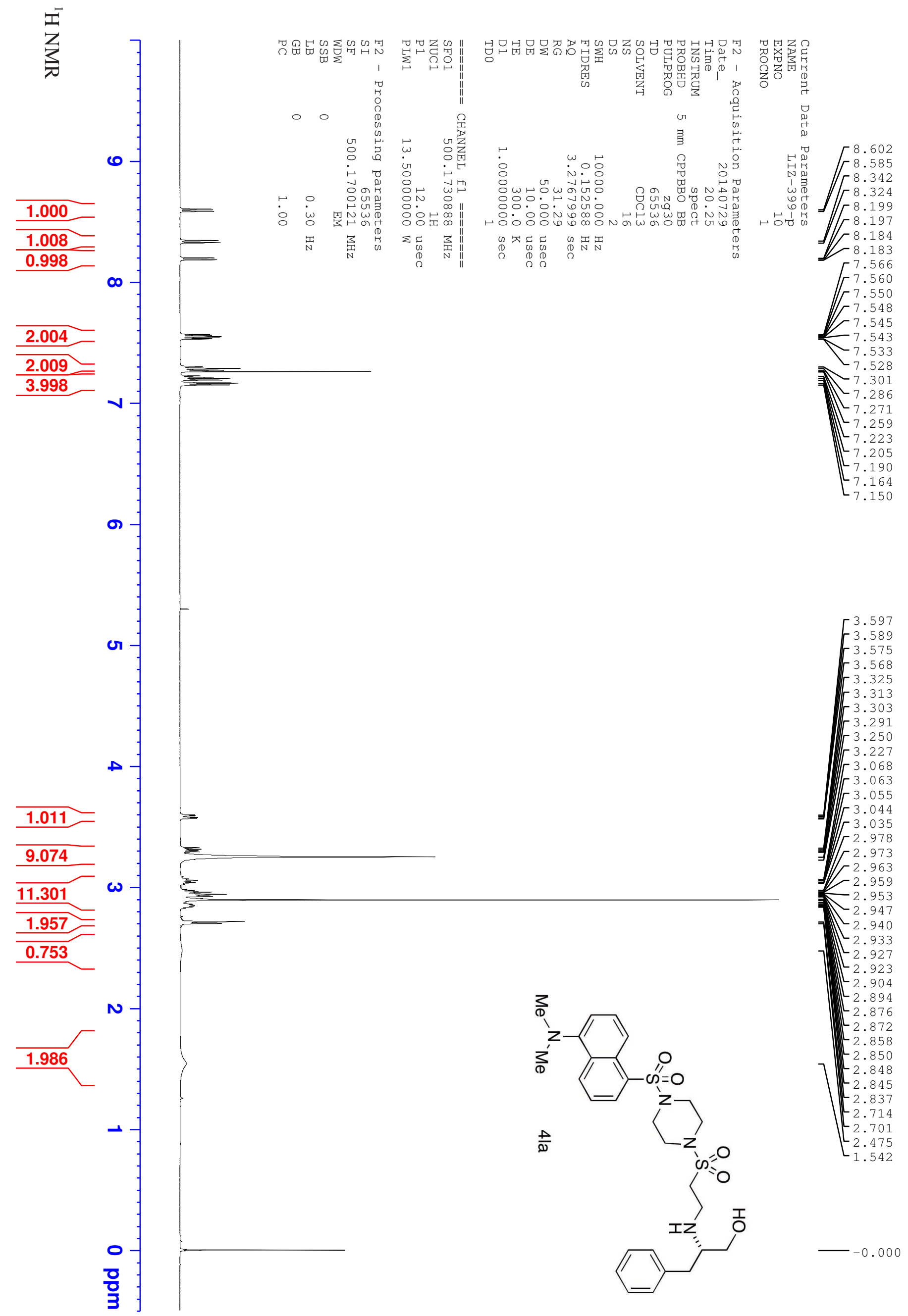




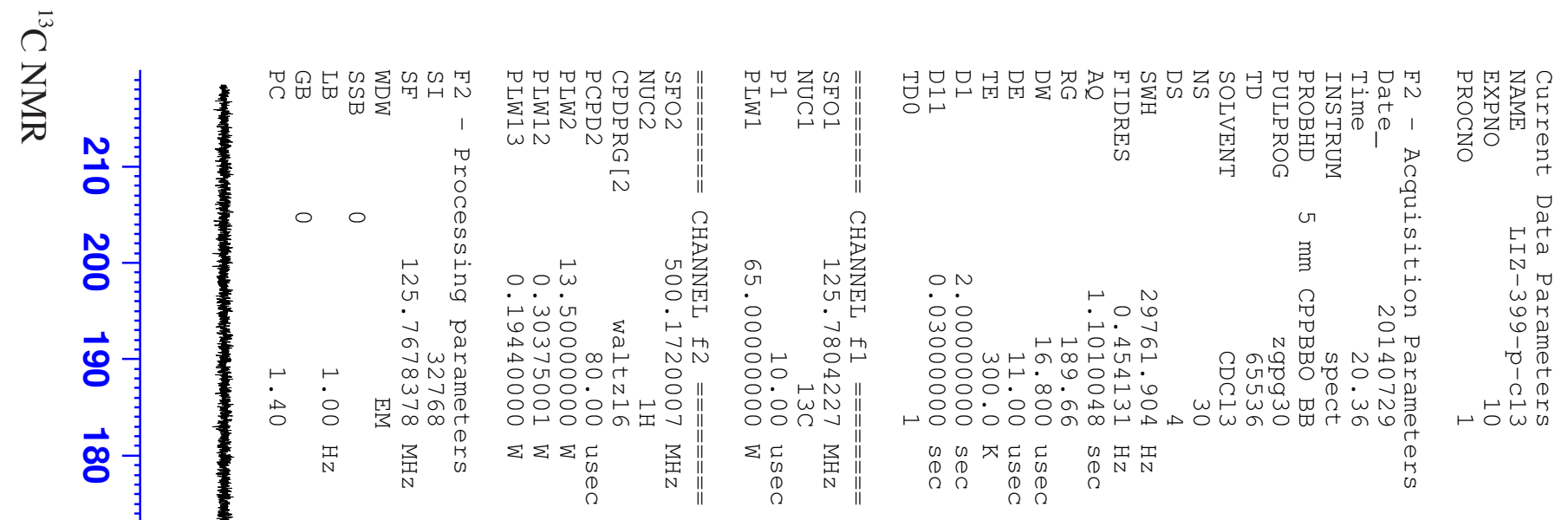

a

몽

8

a

s.

政

홍

8

ø

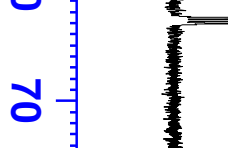

8

잉

a

\&

$\omega$

ก

o

骂 


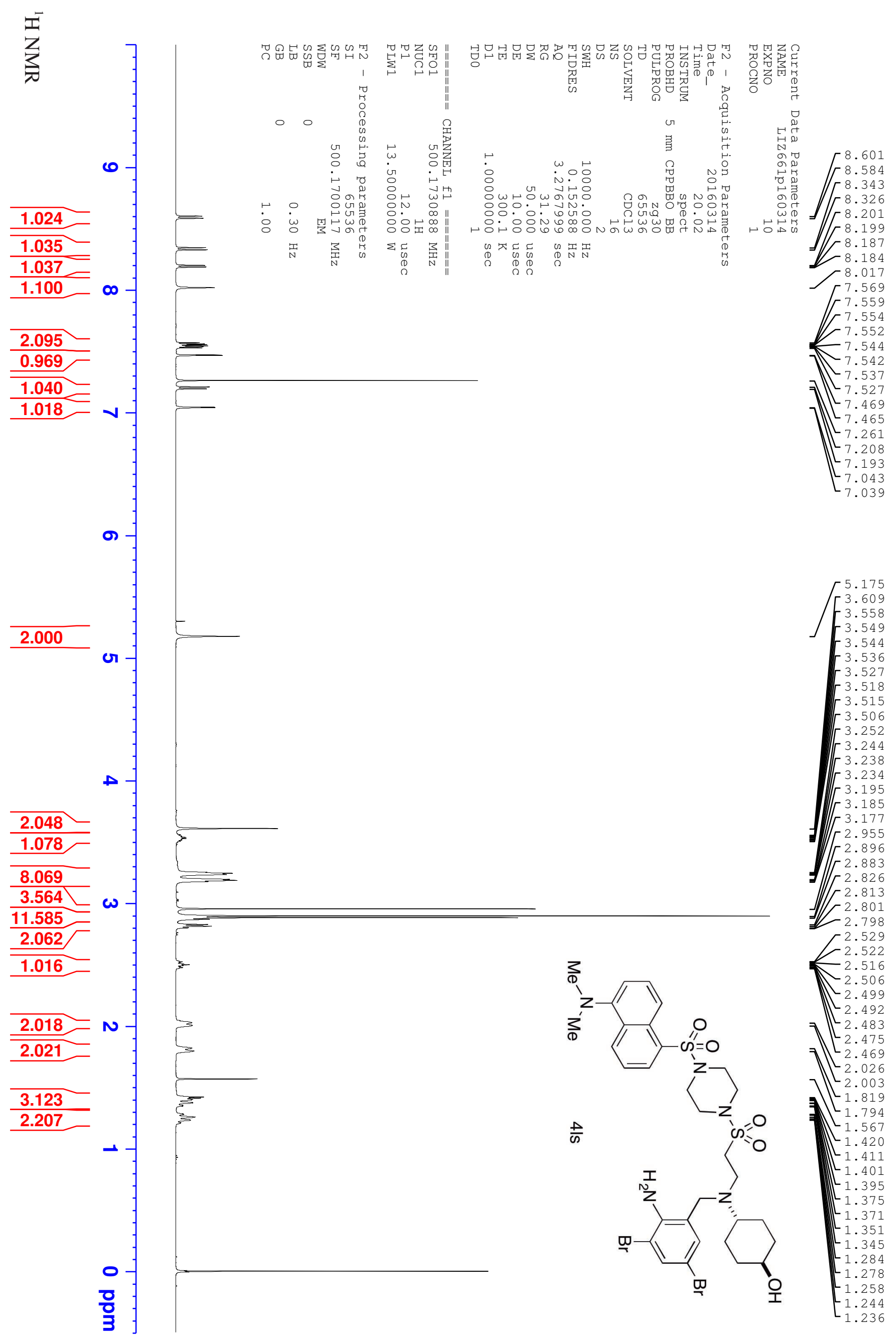




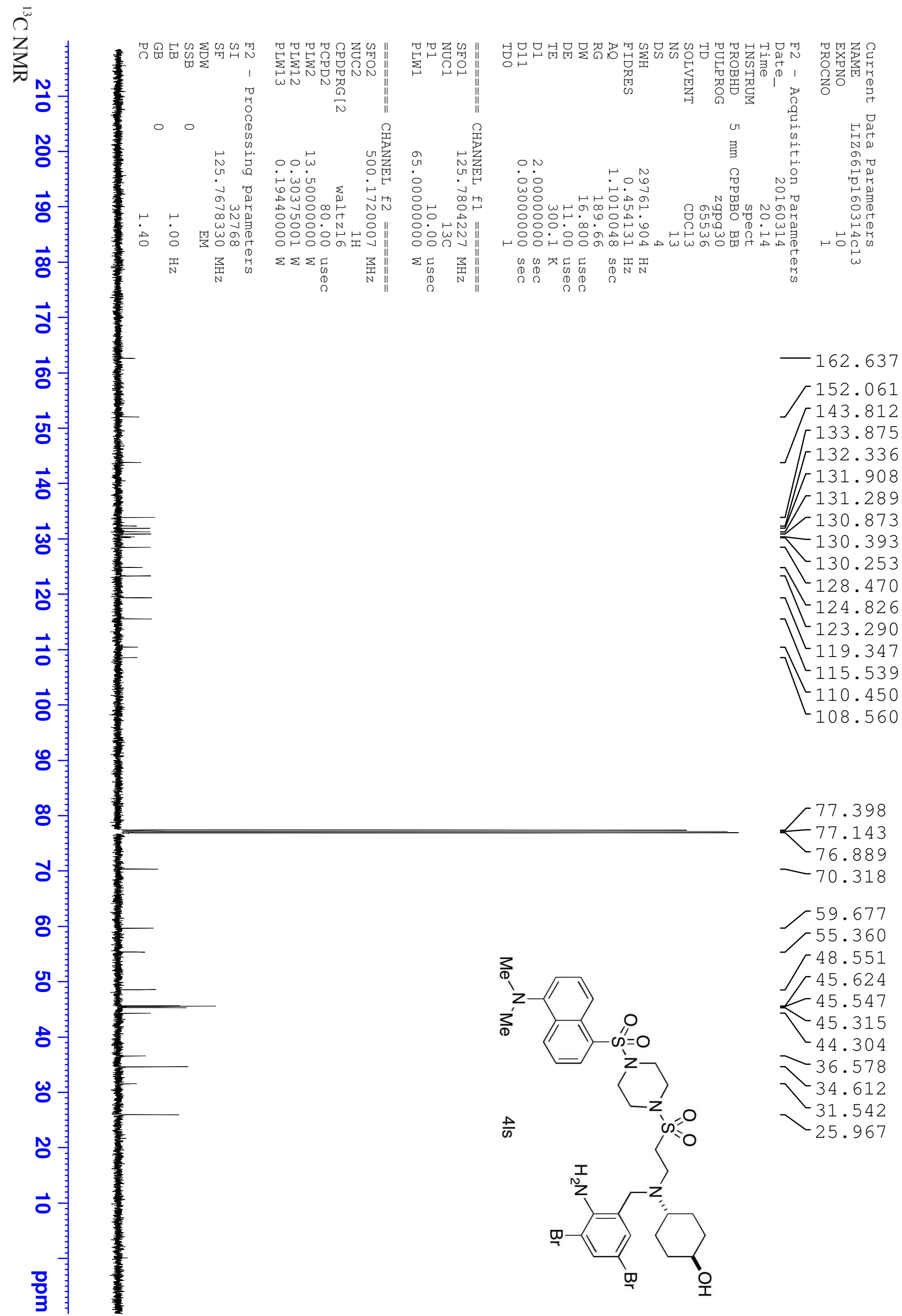




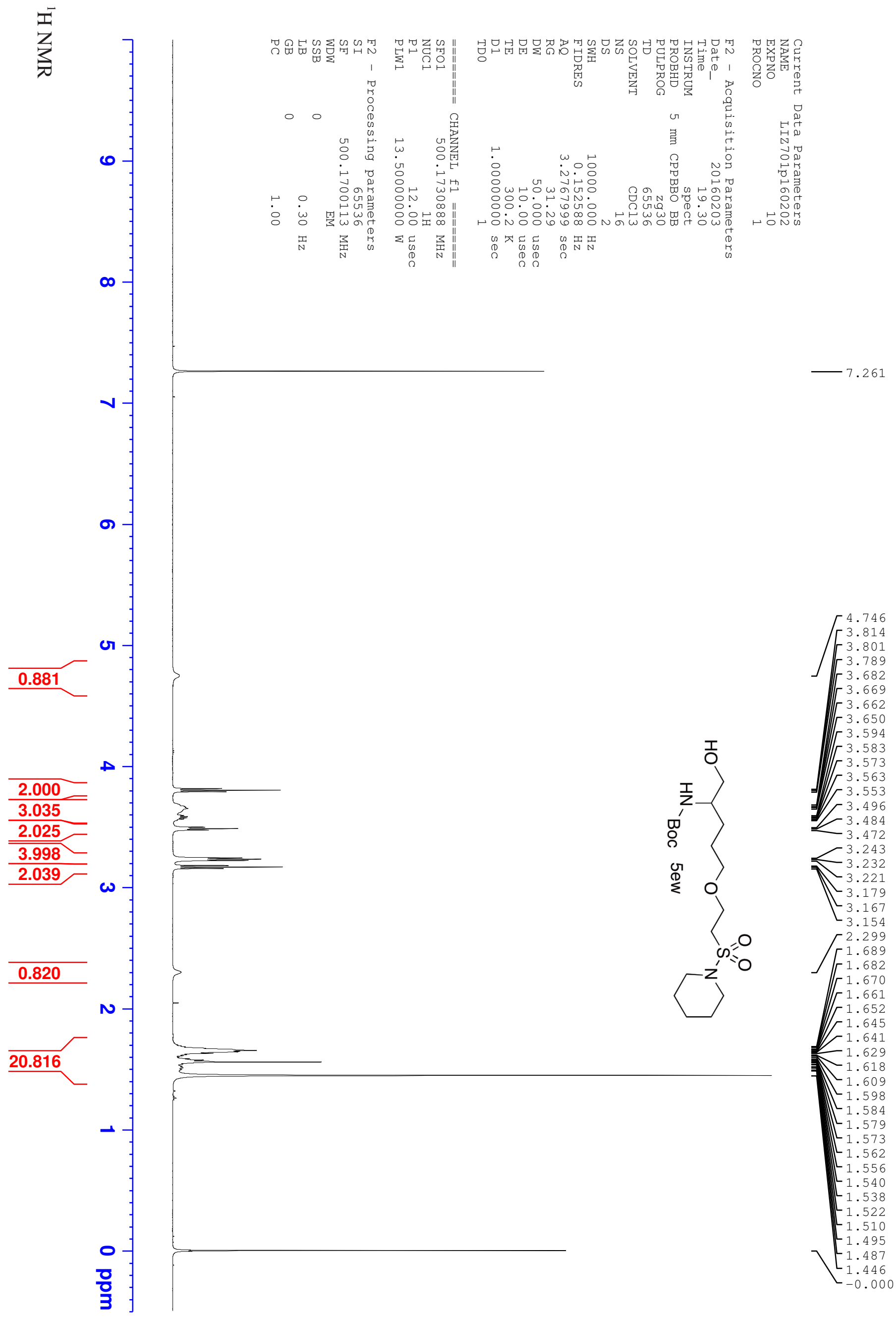




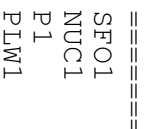

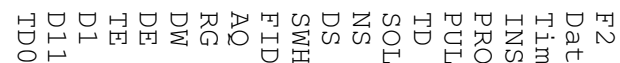

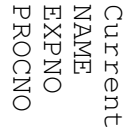
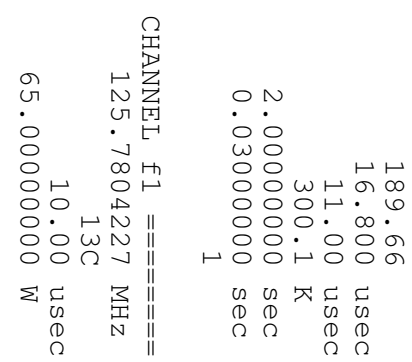

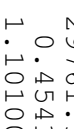

8

8

잉

t

$\omega$

ก

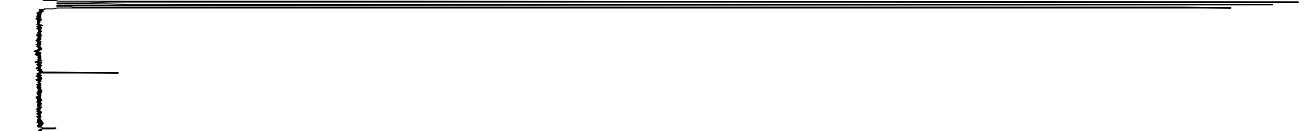

$-156.618$

79.760

77.403

$\checkmark 77.149$

76.895

$-71.032$

$-66.029$

64.514

$-52.652$

$-49.551$

$-46.583$

$\vec{o}$

흡
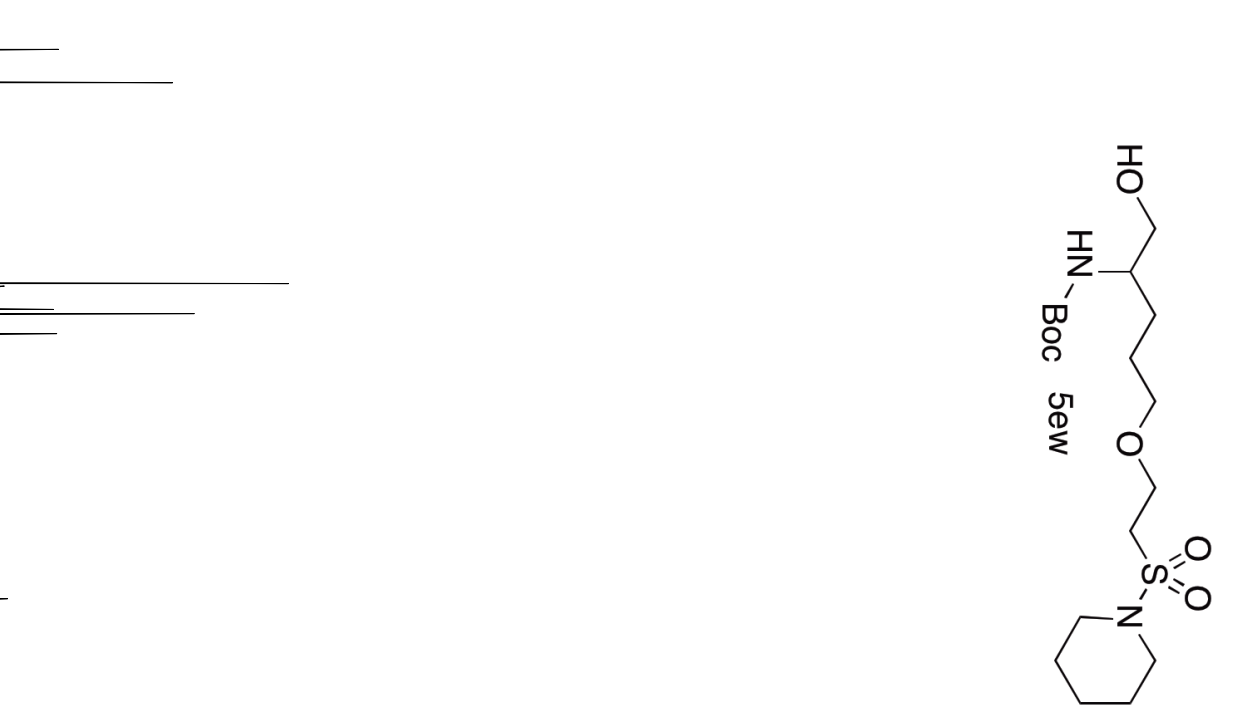

28.521
-28.249

26.197

25.783

23.976 


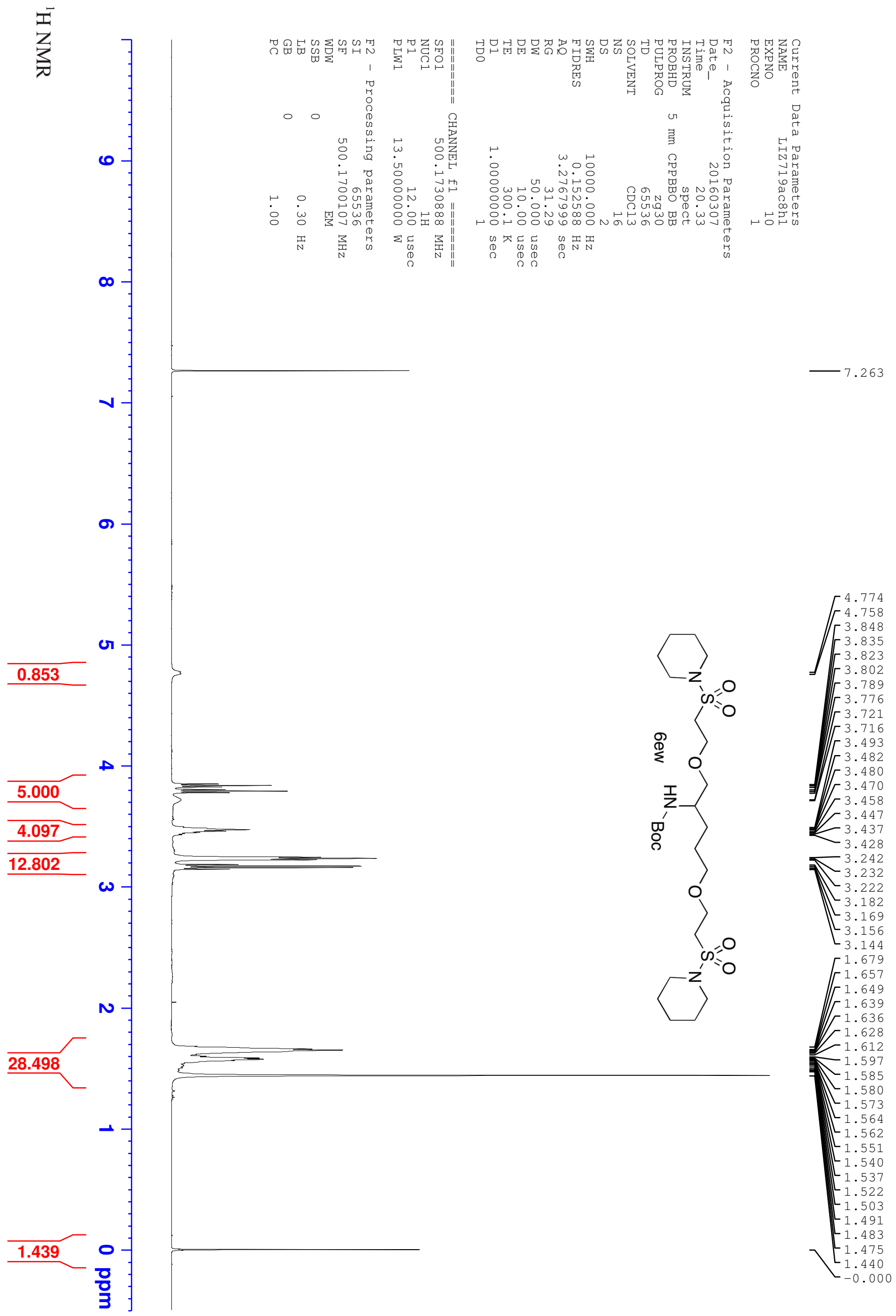




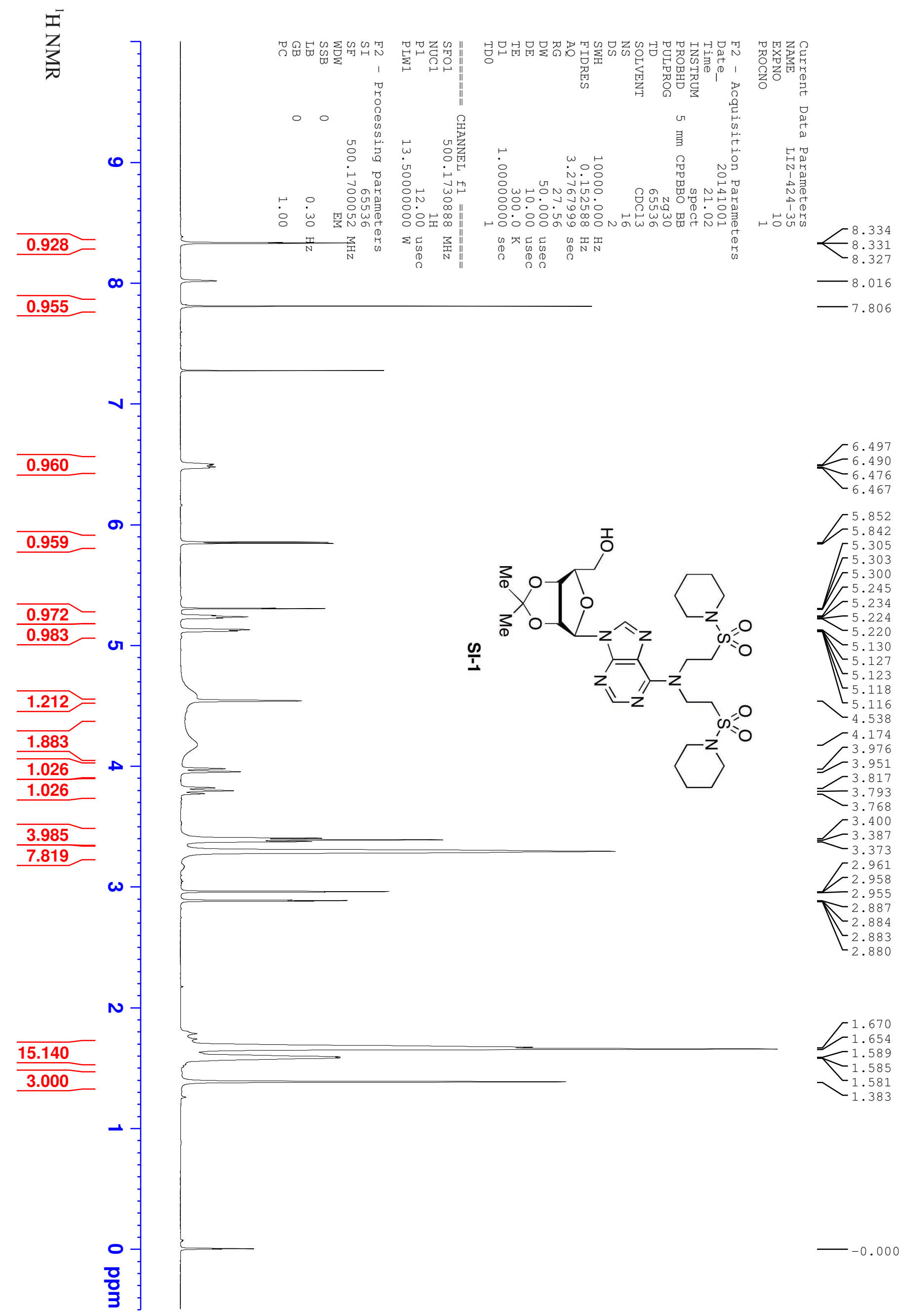



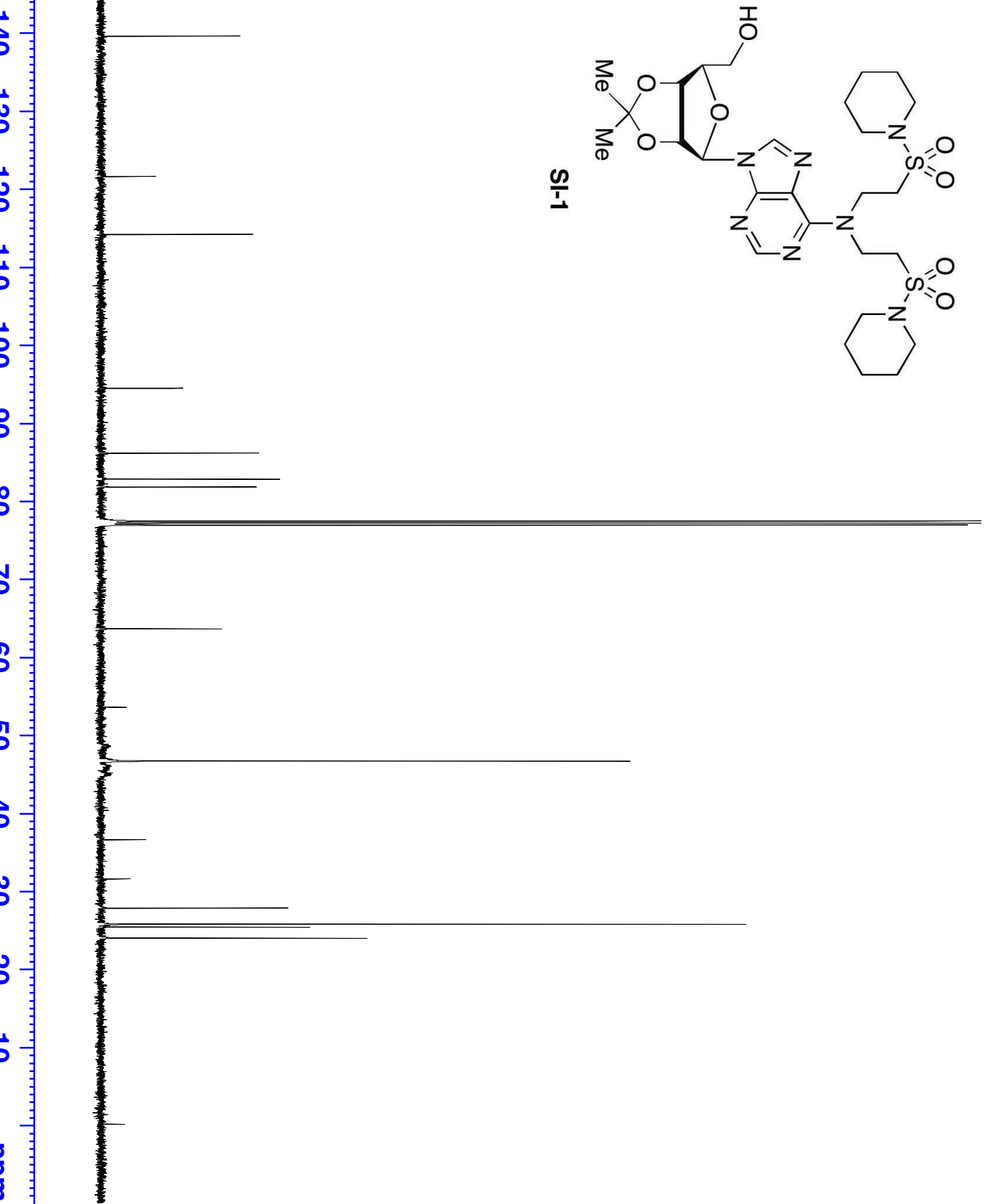
氶

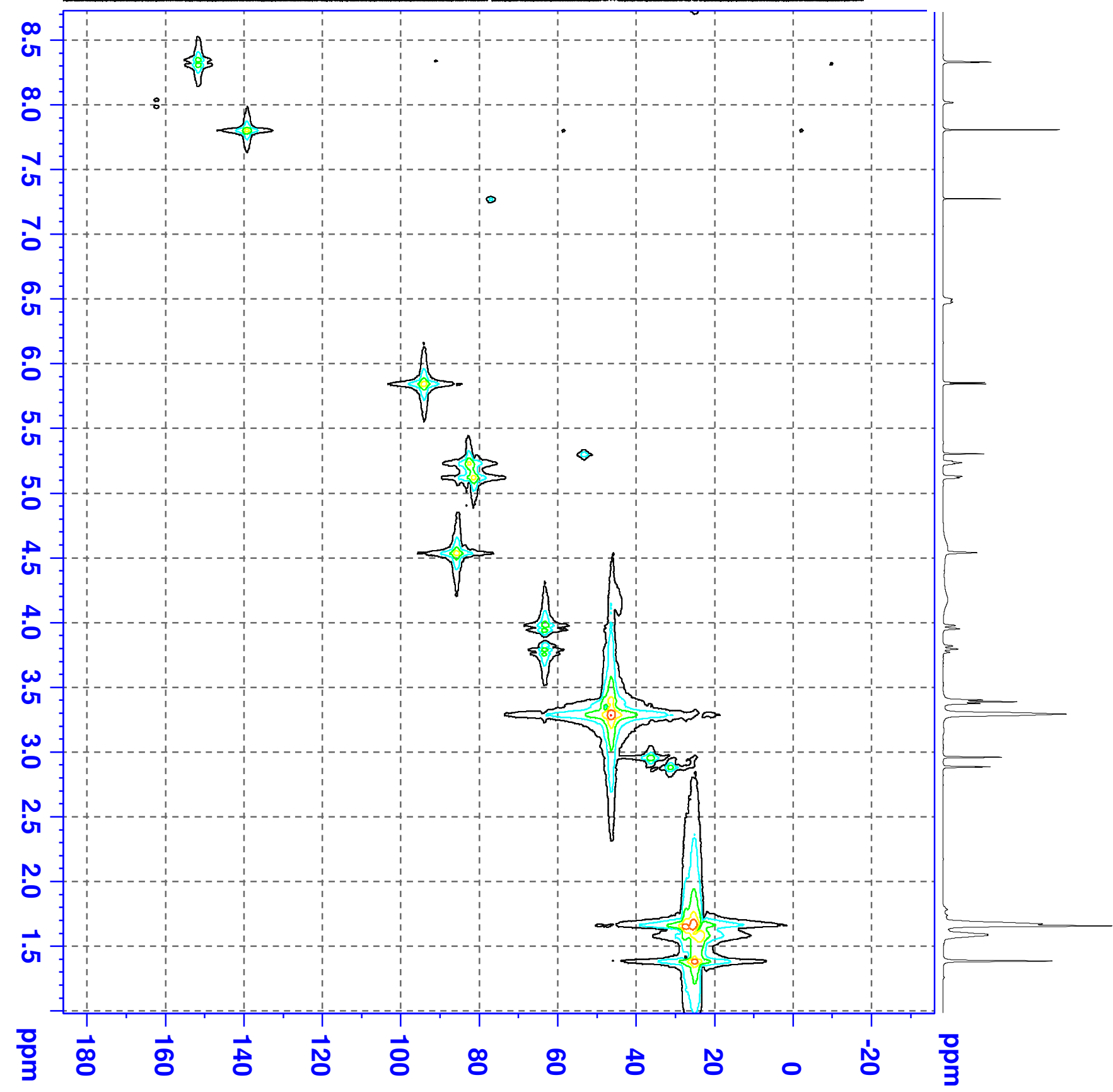

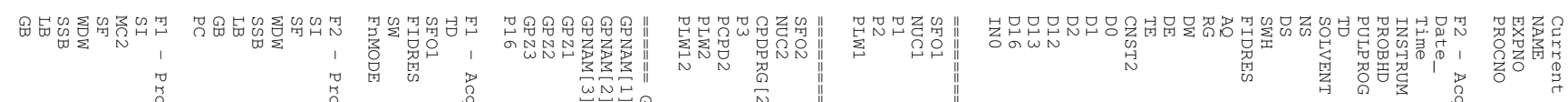

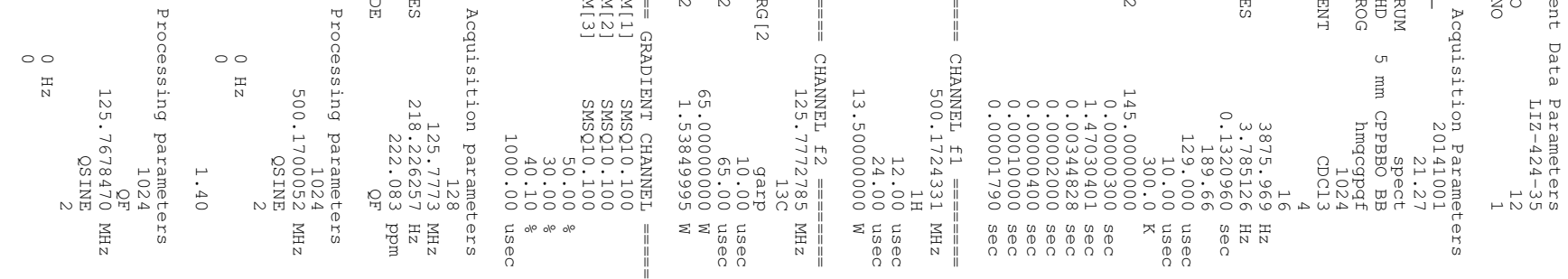




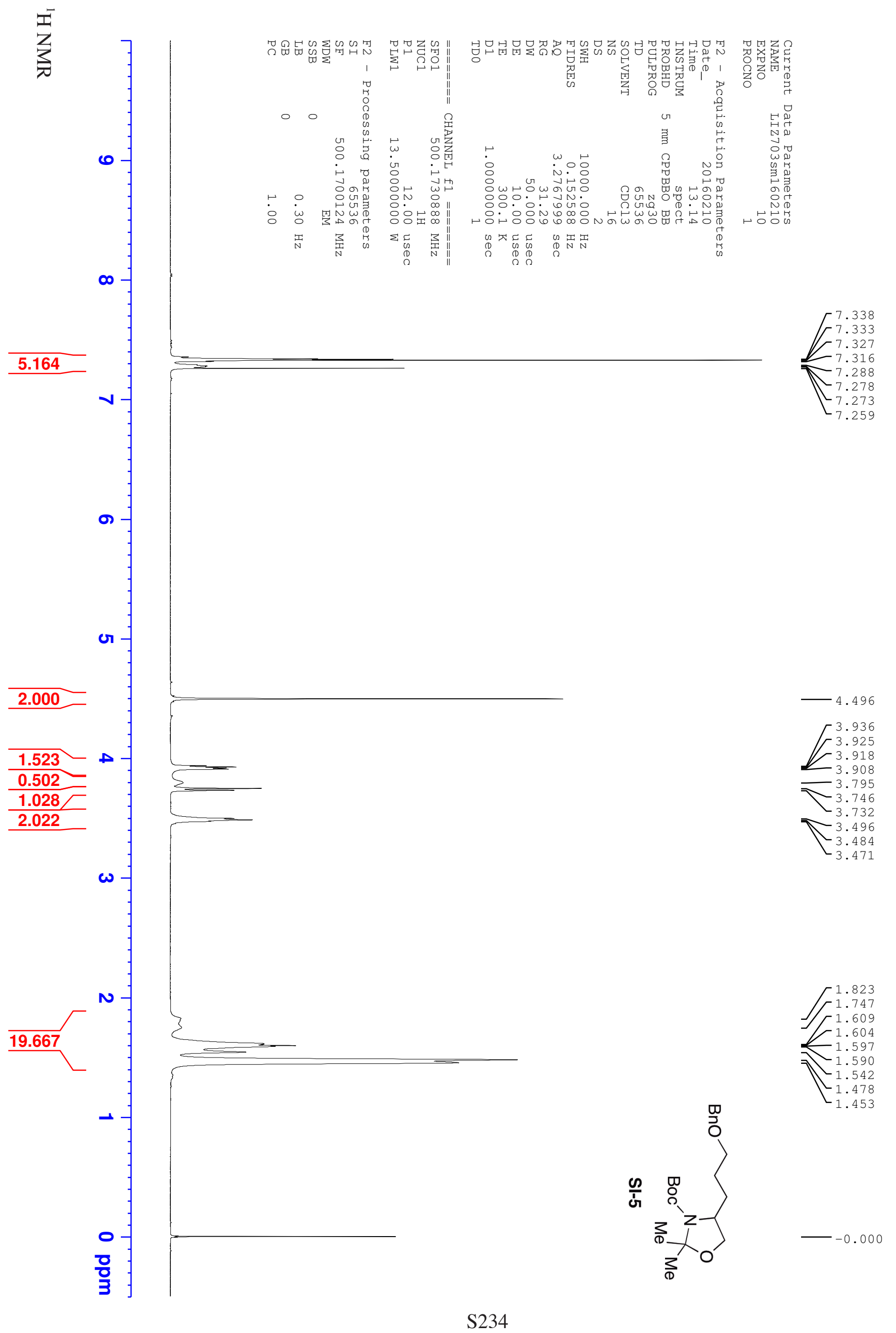




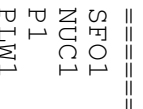

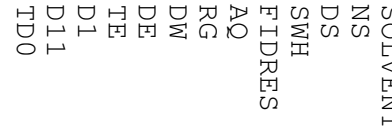

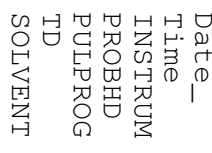

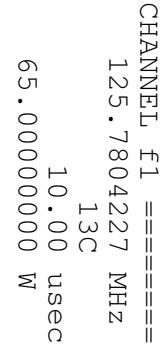

0
$\dot{0}$
$\dot{\omega}:$
0
0
0

$\vdash \circ \stackrel{\circ}{\circ}$

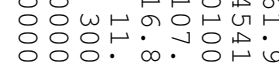

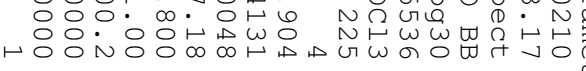

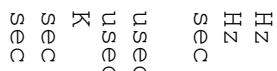

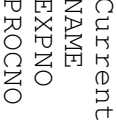

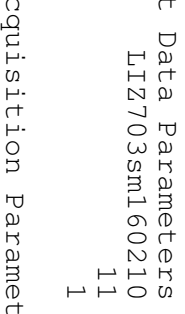

ป̀

$\vec{\circ}$

152.393

152.003

$\vec{\triangleright}$

138.666

$\vec{\omega}$

128.493

127.738

127.657

$\overrightarrow{\mathrm{O}}$

$\overrightarrow{\overrightarrow{0}}$

$\overrightarrow{8}$

๕

曲

๖

근

g

o

号

w

w

93.841

93.345

80.090

79.569

77.417

$-77.162$

$-76.908$

73.022

70.342

67.233

66.927

57.659

57.418

30.667

29.748

28.622

$-27.709$

을

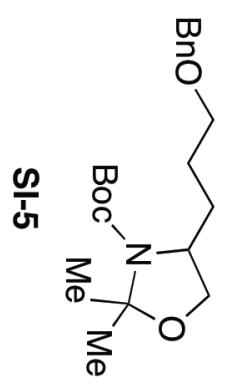

26.920

$-26.694$

24.722

23.418

它青

$-0.140$ 
퐁
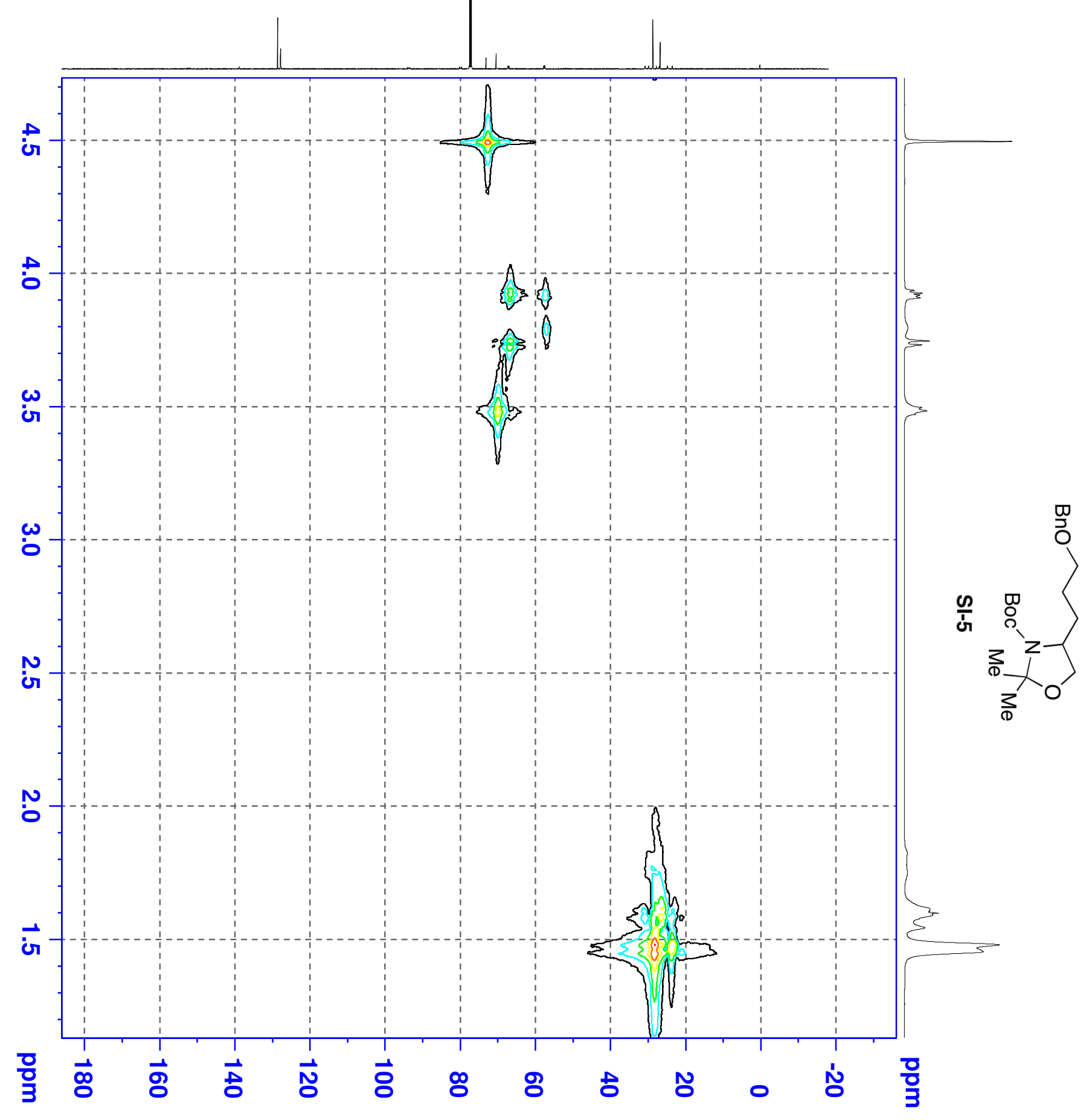

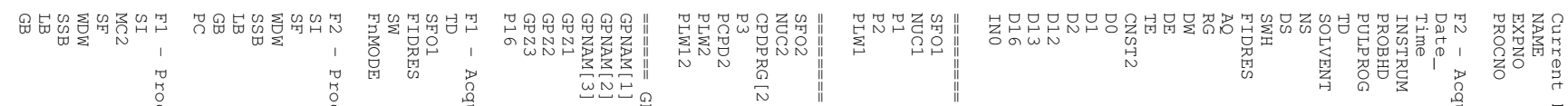

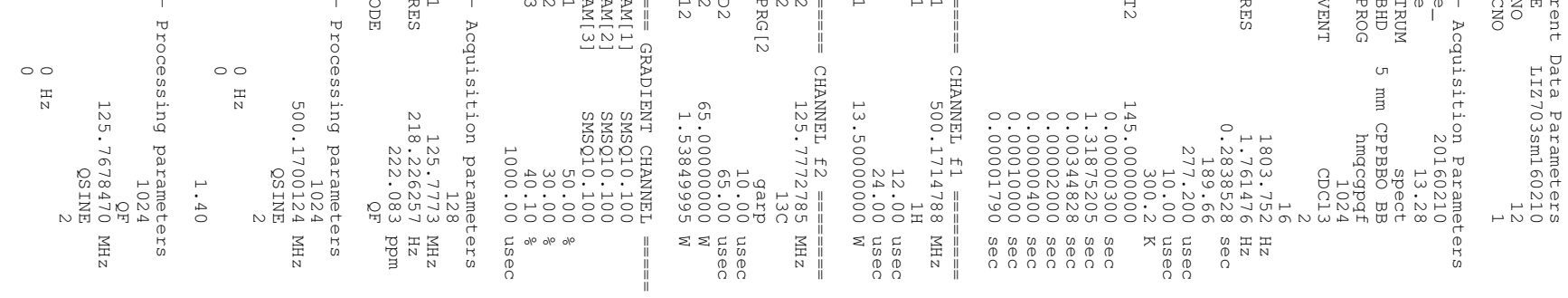




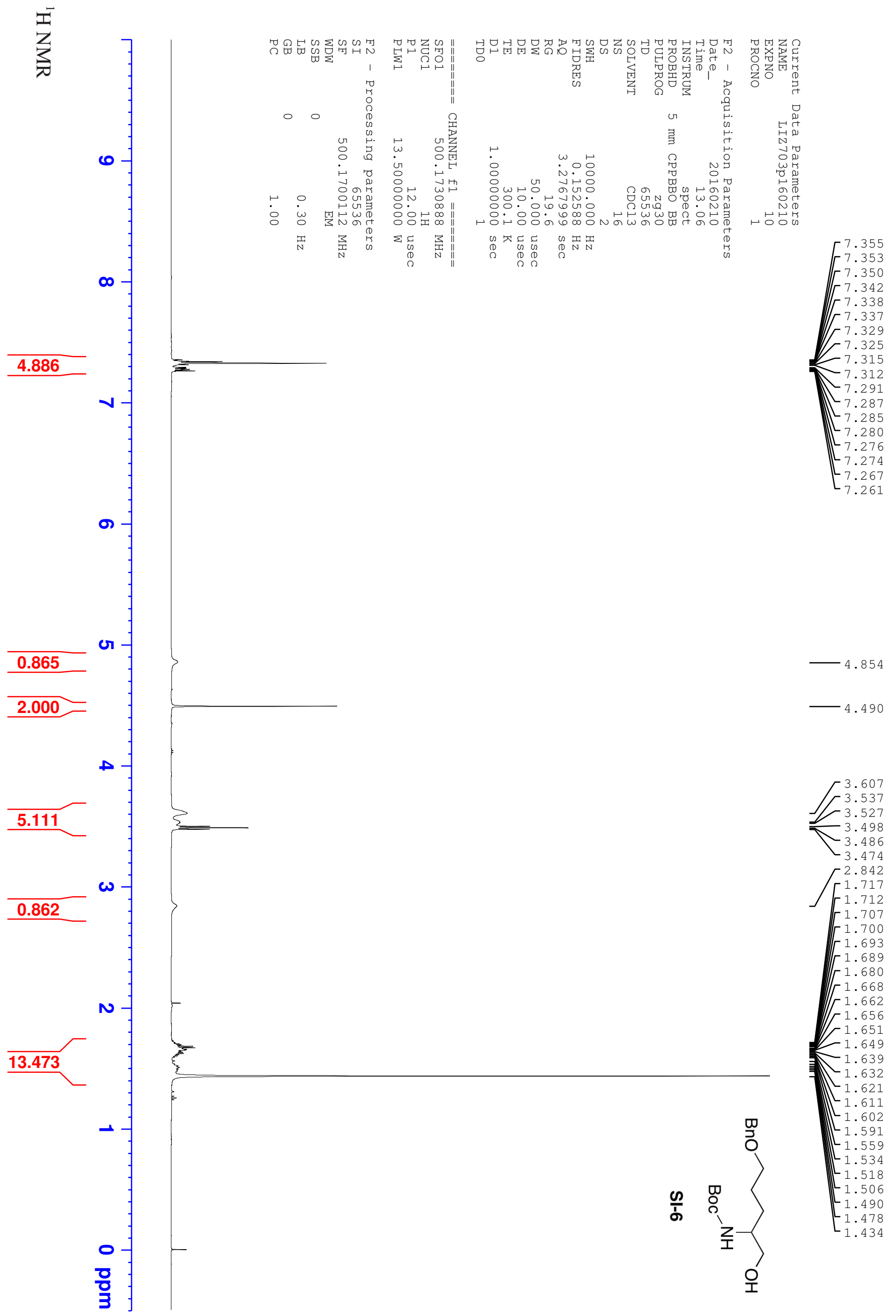




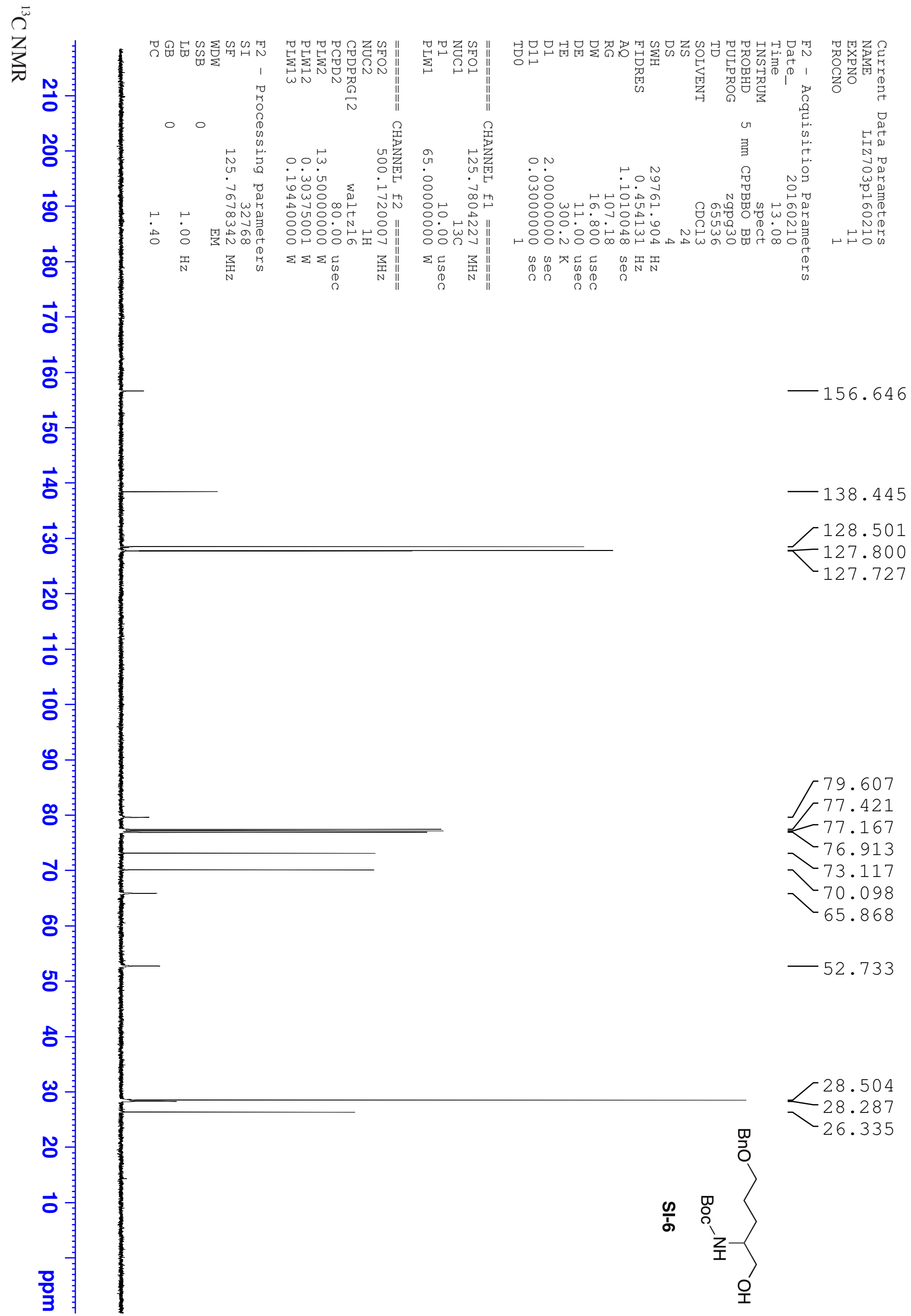

